\title{
Water-Quality and Lake-Stage Data for Wisconsin Lakes, Water Year 1999
}

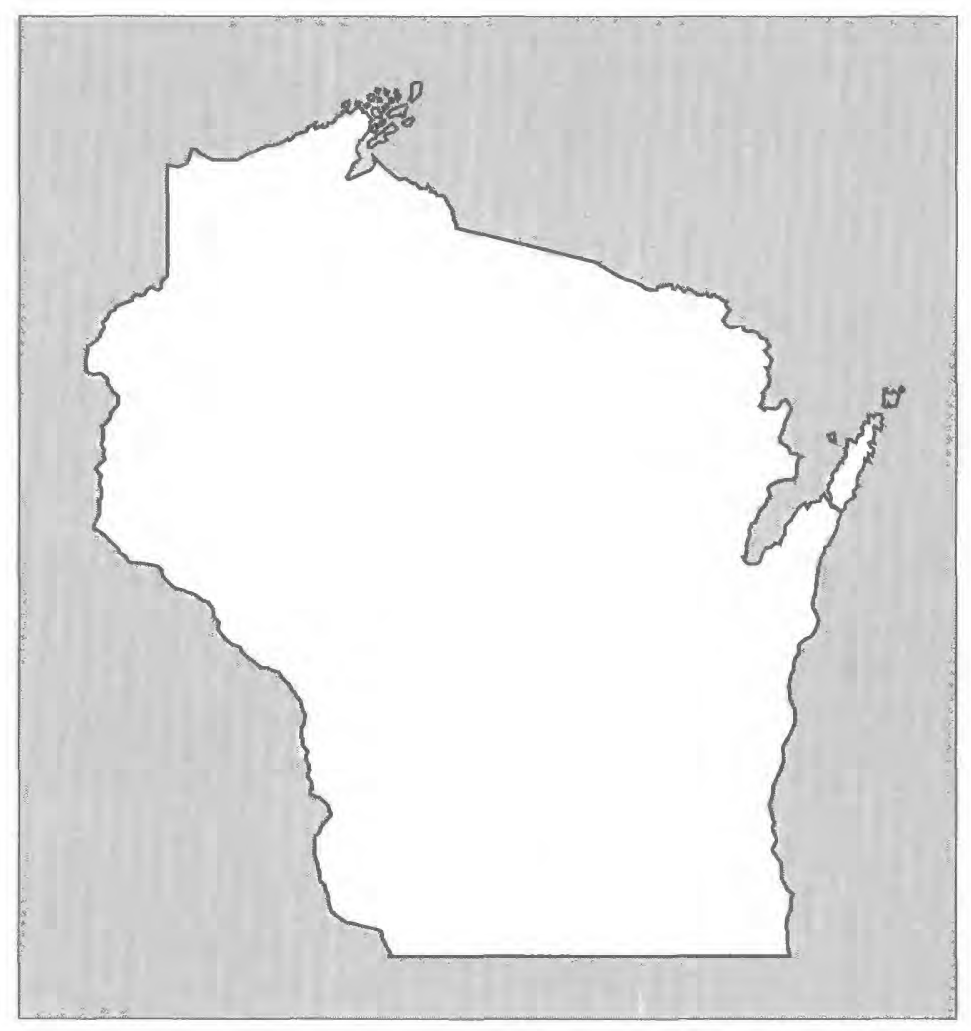

U.S. GEOLOGICAL SURVEY Open-File Report 00-89

Prepared in cooperation with the State of Wisconsin and local agencies 



\section{WATER-QUALITY AND LAKE-STAGE DATA FOR WISCONSIN LAKES, WATER YEAR 1999}

\section{By Wisconsin District Lake-Studies Team}

\section{U.S. GEOLOGICAL SURVEY}

Open-File Report 00-89
A report by the Wisconsin District Lake-Studies Team-
D.L. Olson (team leader), J.F. Elder, H.S. Garn, G.L. Goddard,
E.A. Mergener, D.M. Robertson, and W.J. Rose

Prepared in cooperation with

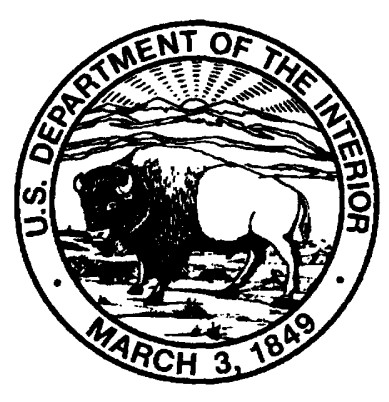

THE STATE OF WISCONSIN AND OTHER AGENCIES 


\title{
U.S. DEPARTMENT OF THE INTERIOR BRUCE BABBITT, Secretary
}

\author{
U.S. GEOLOGICAL SURVEY \\ Charles G. Groat, Director
}

District Chief

U.S. Geological Survey

8505 Research Way

Middleton, WI 53562
U.S. Geological Survey

Earth Science Information Center

Open-File Reports Section

Box 25286, MS 517

Denver Federal Center

Denver, CO 80225 


\section{CONTENTS}

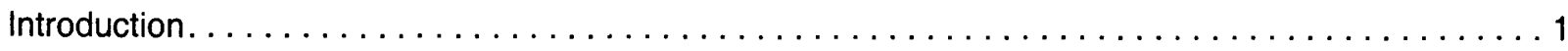

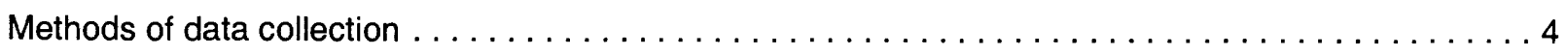

Explanation of physical and chemical characteristics of lakes $\ldots \ldots \ldots \ldots \ldots \ldots \ldots \ldots \ldots$

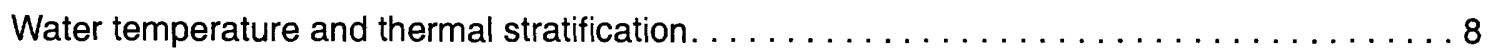

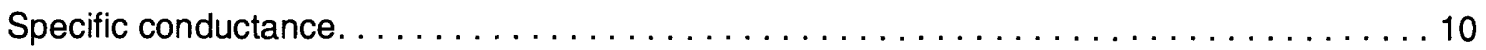

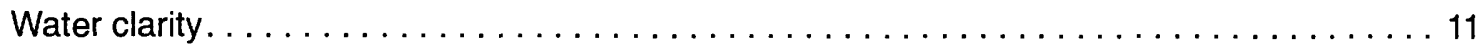

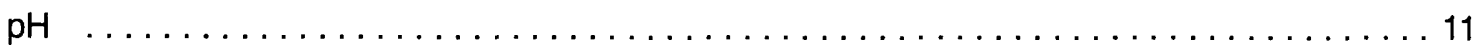

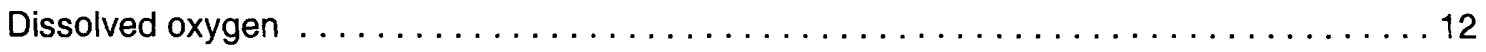

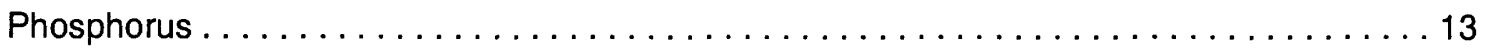

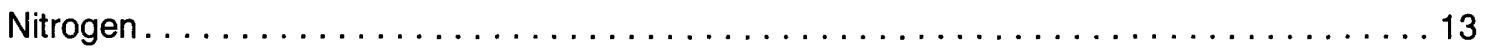

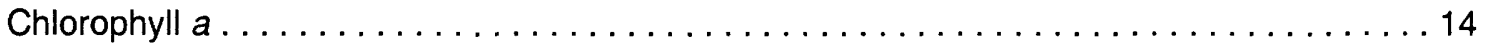

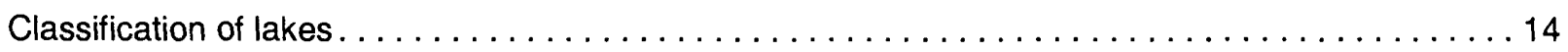

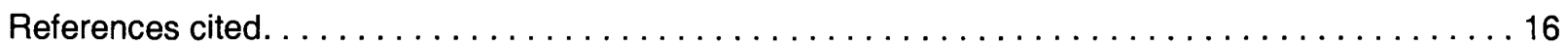

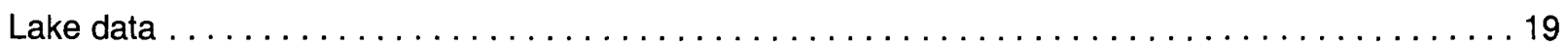

Benedict near Powers Lake, $423201088180800 \ldots \ldots \ldots \ldots \ldots \ldots \ldots \ldots \ldots \ldots \ldots . \ldots 21$

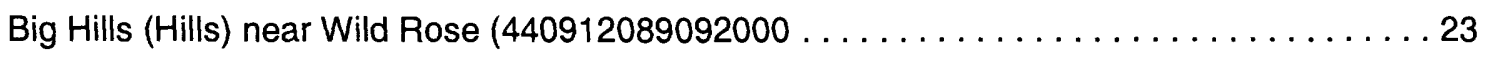

Big St. Germain near Lake Tomahawk, $05390750 \ldots \ldots \ldots \ldots \ldots \ldots \ldots \ldots \ldots \ldots \ldots 25$

Big Sissabagama

near Stone Lake, $454724091303600 \ldots \ldots \ldots \ldots \ldots \ldots \ldots \ldots \ldots \ldots \ldots \ldots . \ldots 26$

North Site, near Stone Lake, $454800091312900 \ldots \ldots \ldots \ldots \ldots \ldots \ldots \ldots \ldots . \ldots 29$

Buffalo

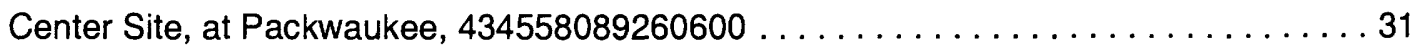

East End, at Montello, $434720089201600 \ldots \ldots \ldots \ldots \ldots \ldots \ldots \ldots \ldots \ldots \ldots \ldots$

West End, near Endeavor, $434414089282400 \ldots \ldots \ldots \ldots \ldots \ldots \ldots \ldots \ldots \ldots$

Delavan

near Delavan, $423706088363400 \ldots \ldots \ldots \ldots \ldots \ldots \ldots \ldots \ldots \ldots \ldots \ldots \ldots \ldots$

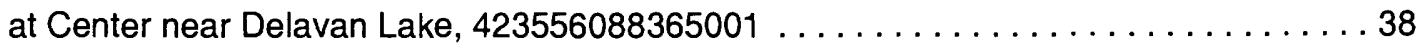

at North End near Lake Lawn, $423659088354401 \ldots \ldots \ldots \ldots \ldots \ldots \ldots \ldots \ldots . \ldots 43$

at SW End near Delavan Lake, $423526088380101 \ldots \ldots \ldots \ldots \ldots \ldots \ldots \ldots . \ldots 43$

Devils near Baraboo, $05404500 \ldots \ldots \ldots \ldots \ldots \ldots \ldots \ldots \ldots \ldots \ldots \ldots \ldots . \ldots \ldots$

Eagle Spring at Eagleville, $425103088261500 \ldots \ldots \ldots \ldots \ldots \ldots \ldots \ldots \ldots \ldots \ldots 45$

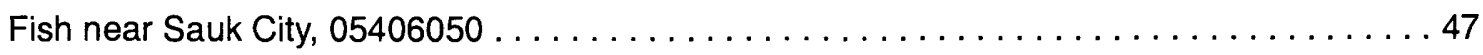

Geneva

at Lake Geneva, $423525088260400 \ldots \ldots \ldots \ldots \ldots \ldots \ldots \ldots \ldots \ldots \ldots \ldots$

Geneva Bay, at Lake Geneva, $423455088263800 \ldots \ldots \ldots \ldots \ldots \ldots \ldots \ldots \ldots . \ldots 50$

Williams Bay, at Williams Bay, $423420088320500 \ldots \ldots \ldots \ldots \ldots \ldots \ldots \ldots \ldots . \ldots \ldots 1$

Center, near Lake Geneva, $423402088301400 \ldots \ldots \ldots \ldots \ldots \ldots \ldots \ldots \ldots \ldots 2$

East End, near Lake Geneva, $423421088272300 \ldots \ldots \ldots \ldots \ldots \ldots \ldots \ldots \ldots \ldots$

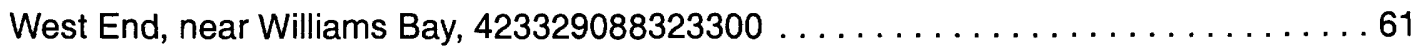

Green at County Trunk Highway A near Green Lake, 434928088553601 . . . . . . . . . . 67

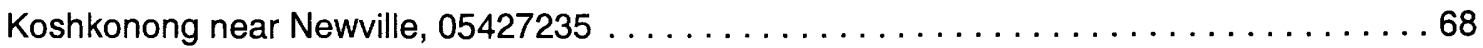

Little Arbor Vitae near Woodruff, 455446089370300 . . . . . . . . . . . . . . . . . . 69 


\section{CONTENTS--Continued}

Little Cedar

North Site, near West Bend, $432255088134700 \ldots \ldots \ldots \ldots \ldots \ldots \ldots \ldots$

South Site, near West Bend, $432249088134500 \ldots \ldots \ldots \ldots \ldots \ldots \ldots \ldots \ldots$. . . . . . . . 72

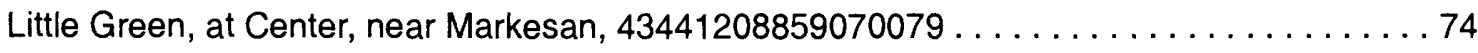

Little Muskego at Muskego, $425425088083500 \ldots \ldots \ldots \ldots \ldots \ldots \ldots \ldots$

Little St. Germain

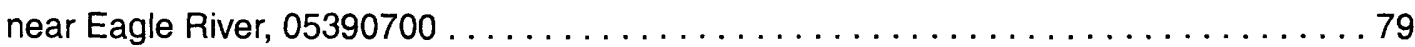

Northeast Bay, near St. Germain, $455545089262500 \ldots \ldots \ldots \ldots \ldots \ldots \ldots \ldots$

South Bay, near St. Germain, 455437089270800 . . . . . . . . . . . . . . . . . . . . 82

West Bay, at St. Germain, $455428089282400 \ldots \ldots \ldots \ldots \ldots \ldots \ldots \ldots$. . . . . . . . . 84

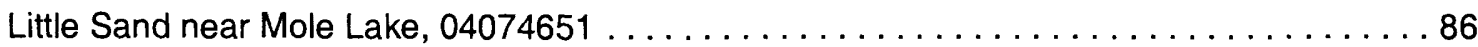

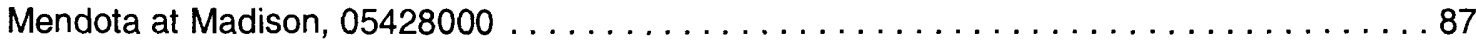

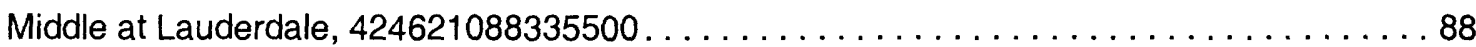

Middle Genesee near Oconomowoc, $430309088284800 \ldots \ldots \ldots \ldots \ldots \ldots$. . . . . . . . . 90

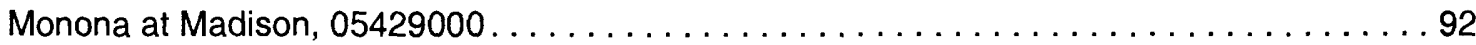

Muskego (Big Muskego)

Bass Bay, near Muskego, $425344088070100 \ldots \ldots \ldots \ldots \ldots \ldots \ldots . \ldots . \ldots . \ldots 9$

Muskego, near Wind Lake, $425109088075000 \ldots \ldots \ldots \ldots \ldots \ldots \ldots$

Muskego, South Site, near Muskego, $425112088072800 \ldots \ldots \ldots \ldots$. . . . . . . . . 97

Namekagon Lakes

Garden, near Cable, $461224091030200 \ldots \ldots \ldots \ldots \ldots \ldots$. . . . . . . . . . . . . 99

Jackson, near Cable, $461457091065900 \ldots \ldots \ldots \ldots \ldots \ldots \ldots \ldots \ldots \ldots$

Namekagon

Deep Hole, near Cable, $461308091065100 \ldots \ldots \ldots \ldots \ldots \ldots$. . . . . . . . . 103

East Basin, near Cable, 461228091044300 . . . . . . . . . . . . . . . . . . . . . 105

Northeast Basin, near Cable, $461410091050700 \ldots \ldots \ldots \ldots \ldots \ldots$. . . . . . . . 107

Oconomowoc

No. 1 (Center) at Oconomowoc, $430551088273500 \ldots \ldots \ldots \ldots \ldots \ldots$. . . . . . . . 109

No. 2 (off Hewitt Point) at Oconomowoc, $430609088262200 \ldots \ldots \ldots \ldots \ldots \ldots . \ldots . \ldots 111$ Okauchee

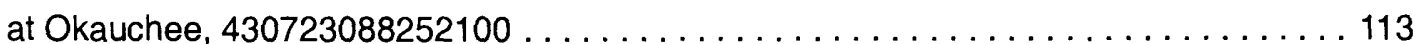

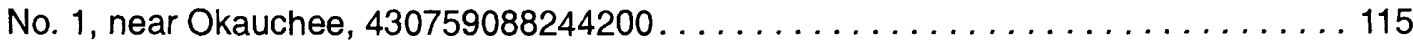

No. 2, at Okauchee, 430645088264500 . . . . . . . . . . . . . . . . . . . . . . . . . . . . 115

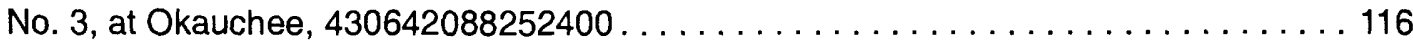

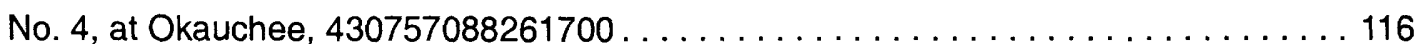

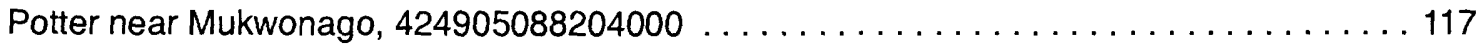

Powers at Powers Lake, $423246088175800 \ldots \ldots$. . . . . . . . . . . . . . . . . . . . . . . . 119

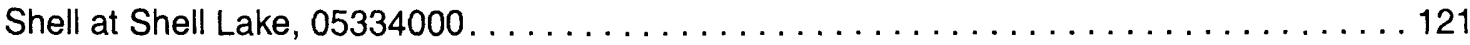

Tombeau near Powers Lake, $423153088184800 \ldots \ldots$. . . . . . . . . . . . . . . . . . . . . . . 123

Whitefish

North Site, near Gordon, 461321091520900 . . . . . . . . . . . . . . . . . . . . . . . . . . 125

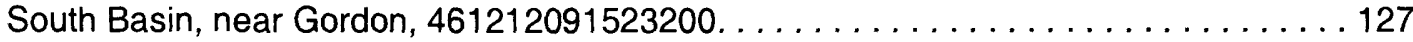




\section{CONTENTS--Continued}

Whitewater near Whitewater, $424608088414800 \ldots \ldots \ldots \ldots \ldots \ldots \ldots \ldots \ldots \ldots$

Wind

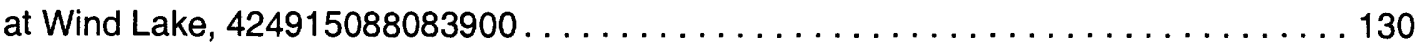

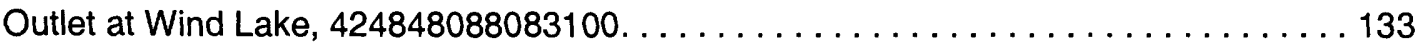

Winnebago

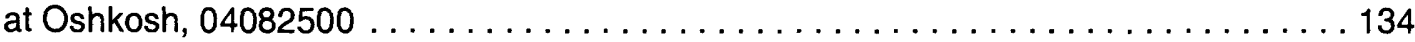

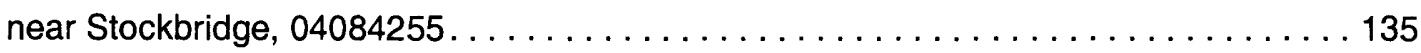

Wisconsin District publications pertaining to lakes $\ldots \ldots \ldots \ldots \ldots \ldots \ldots \ldots \ldots \ldots \ldots \ldots$

\section{FIGURE}

Figure 1. Map showing location of lake water-quality and lake-stage stations in Wisconsin

.2

\section{TABLES}

Table 1. Discontinued lake stations.

.5

2. WATSTORE parameter codes, minimum reporting limits, and laborator identifying codes for chemical parameters commonly measured in lakes, and analyzed at the National Water-Quality Laboratory or the Wisconsin State Laboratory of Hygiene .9 


\begin{tabular}{rcl}
\hline Multiply & By & To Obtain \\
\hline mile (mi) & 1.609 & kilometer \\
pound (lb) & 453.6 & gram \\
acre & 0.4048 & hectare \\
foot (ft) & 0.3048 & meter \\
gallon (gal) & 3.785 & liter \\
square mile (mi $\left.{ }^{2}\right)$ & 2.590 & square kilometer
\end{tabular}

Temperature, in degrees Celsius $\left({ }^{\circ} \mathrm{C}\right)$ can be converted to degrees Fahrenheit $\left({ }^{\circ} \mathrm{F}\right)$ by use of the following equation:

$$
{ }^{\circ} \mathrm{F}=1.8\left({ }^{\circ} \mathrm{C}\right)+32 \text {. }
$$

Sea level: In this report "sea level" refers to the National Geodetic Vertical Datum of 1929 (NGVD of 1929)-a geodetic datum derived from a general adjustment of the first-order level nets of both the United States and Canada, formerly called Sea Level Datum of 1929.

\begin{abstract}
Abbreviated water-quality units: Chemical concentrations and water temperature are given in metric units. Chemical concentration is given in milligrams per liter $(\mathrm{mg} / \mathrm{L})$ or micrograms per liter $(\mu \mathrm{g} / \mathrm{L})$. Milligrams per liter is a unit expressing the concentration of chemical constituents in solution as weight (milligrams) of solute per unit volume (liter) of water. One thousand micrograms per liter is equivalent to one milligram per liter. For water with dissolved-solids concentrations less than 7,000 $\mathrm{mg} / \mathrm{L}$, the numerical values for concentrations expressed as $\mathrm{mg} / \mathrm{L}$ and $\mu \mathrm{g} / \mathrm{L}$ are the same as for concentrations in parts per million and parts per billion, respectively.
\end{abstract}

Specific conductance of water is expressed in microsiemens per centimeter at 25 degrees Celsius $(\mu \mathrm{S} / \mathrm{cm})$. This unit is equivalent to micromhos per centimeter at 25 degrees Celsius $(\mu \mathrm{mho} / \mathrm{cm})$, formerly used by the U.S. Geological Survey. 


\section{WATER-QUALITY AND LAKE-STAGE DATA FOR WISCONSIN LAKES, WATER YEAR 1999}

By Wisconsin District Lake-Studies Team

\section{INTRODUCTION}

The U.S. Geological Survey (USGS), in cooperation with local and other agencies, collects data at selected lakes throughout Wisconsin. These data, accumulated over many years, provide a data base for developing an improved understanding of the water quality of lakes. To make these data available to interested parties outside the USGS, the data are published annually in this report series. The location of water-quality and lake-stage stations in Wisconsin for water year 1999 are shown in figure 1. A water year is the 12-month period from October 1 through September 30. It is designated by the calendar year in which it ends. Thus, the period October 1 , 1998 through September 30, 1999 is called "water year 1999."

The purpose of this report is to provide information about the chemical and physical characteristics of Wisconsin lakes. Data that have been collected at specific lakes, and information to aid in the interpretation of those data, are included in this report. Data collected include measurements of in-lake water quality and lake stage. Time series of Secchi depths, surface total phosphorus and chlorophyll a concentrations collected during non-frozen periods are usually included for lakes with two or more years of data. Graphs of vertical profiles of temperature, dissolved oxygen, $\mathrm{pH}$, and specific conductance are included for sites where these parameters were measured. Descriptive information for each lake includes: location of the lake, area of the lake's watershed, period for which data are available, revisions to previously published records, and pertinent remarks. Additional data, such as streamflow and water quality in tributary and outlet streams of some of the lakes, are published in another volume: "Water Resources DataWisconsin, 1999."

Water-resources data, including stage and discharge data at most streamflow-gaging stations, are available throught the World Wide Web on the Internet. The Wisconsin District's home page is at http://wi.water.usgs.gov/. Information on the Wisconsin District's Lakes Program is found at wi.water.usgs.gov/lake/index.html. 


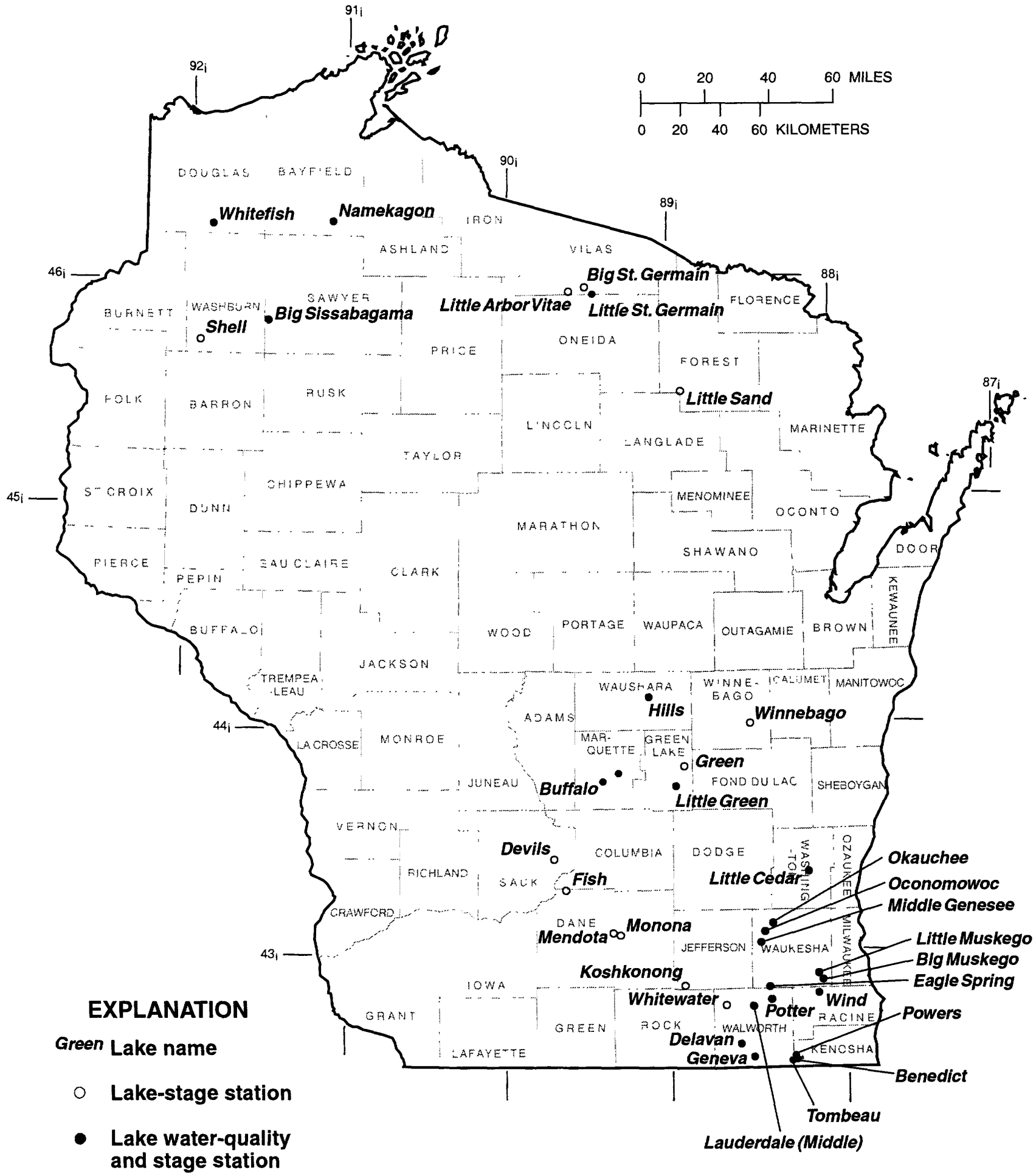

Note: at some lakes more than one site may be monitored.

Figure 1. Location of lake water-quality and lake-stage stations in Wisconsin. 
The USGS has done cooperative lake monitoring with local and other agencies since 1983. Cooperators in 1999 included:

Benedict/Tombeau Lake District

Big Muskego Lake District

Buffalo Lake District

City of Muskego

Dane County Department of Public Works

Eagle Spring Lake Management District

Geneva Lake Environmental Agency

Green Lake Sanitary District

Little Cedar Lake Protection and Rehabilitation District

Little Green Lake Protection and Rehabilitation District

Little Muskego Lake Protection and Rehabilitation District

Little St. Germain Lake Protection and Rehabilitation District

Middle Genesee Lake District

Okauchee Lake Management District

Potters Lake Protection and Rehabilitation District

Powers Lake District

Rock County Public Works Department

Town of Auburn

Town of Delavan (Delavan Lake)

Town of Namekagon (Namekagon Lakes Association)

Town of Sand Lake (Big Sissabagama Lake Association)

Town of Wascott (Whitefish Lake Association)

U.S. Army Corps of Engineers

Village of Oconomowoc Lake

Whitewater Lake Management District

Wind Lake Management District

Wisconsin Department of Natural Resources 
Lake data-collection sites are identified by a unique identification number. Lake waterquality sites are identified by a 15-digit number that is a concatenation of the site's latitude, longitude, and a two-digit sequence number. The sequence number is used to distinguish between sites located at the same latitude-longitude designation. The site identification number is permanently assigned to the site; actual latitude and longitude of the site are subject to update and are stored separately. For some lakes, which have historical records of lake stage, an eightto-ten digit number is assigned according to downstream order. Gaps are left in the numerical series to allow for new stations; hence, the numbers are not consecutive. The first two digits of the complete eight-to-ten digit number, such as 04087000 or 054310157 , designate the major river basin. For example, "04" designates the St. Lawrence River Basin and "05" designates the Upper Mississippi River Basin.

The water-quality lake stations that were discontinued prior to water year 1999 are listed in table 1. Discontinued lake-stage stations are not included in this table.

This report is the culmination of a concerted effort by a number of people who collected, compiled, analyzed, verified, and organized the data, and who typed, edited, and assembled the report. The authors had primary responsibility for assuring that the information contained herein is accurate, complete, and adheres to USGS policy and established guidelines. Technicians in charge of the field offices are: T.J. Popowski (Rice Lake), J.J. Hanig (Merrill), and J. Habale (Middleton). The data were collected and processed by G.L. Goddard, B.M. Gray, J.J. Hanig, D.E. Housner, J.A. Jasinski, S.B. Marsh, E.A. Mergener, D.L. Olson, and J.G. Schuler. P.A. Stark assembled, edited, and formatted the report. Additional assistance in preparation of the report was provided by M.M. Greenwood.

\section{METHODS OF DATA COLLECTION}

Depth profiles of water temperature, dissolved oxygen, $\mathrm{pH}$, and specific conductance were collected using multi-parameter meters. Prior to measurements, the meters were calibrated using standards for $\mathrm{pH}$ and conductance, and dissolved oxygen was calibrated using the air calibration method. Generally, field measurements in profiles were made at $0.5-\mathrm{m}$ intervals if the maximum depth of the lake was $5 \mathrm{~m}$ or less and at 1.0-m intervals if the maximum depth was greater than $5 \mathrm{~m}$. 
Table 1. Discontinued lake stations

\begin{tabular}{|c|c|c|}
\hline Station name & Site identification number & Period of record \\
\hline Alma Lake near St. Germain & 455426089254700 & $\begin{array}{l}\text { Oct. } 1984-S e p t .1990 \\
\text { May } 1992-S e p t .1996\end{array}$ \\
\hline Balsam Lake, off Cedar Island, at Balsam Lake & 452755092264600 & Feb. 1991-Aug. 1994 \\
\hline off Little Narrows, near Balsam Lake & 452858092265300 & May 1991-Aug. 1994 \\
\hline off Rock Island, near Balsam Lake & 452754092234300 & May 1991-Aug. 1994 \\
\hline near Birchwood & 453907091345800 & $\begin{array}{l}\text { Mar. 1993-Aug. 1994, } \\
\text { Mar. 1996-Aug. } 1997\end{array}$ \\
\hline Bass Lake near Shawano & 445215088300300 & Feb. 1990-Aug. 1992 \\
\hline Bear Lake at Deep Hole near Haugen & 453754091490900 & Mar. 1992-Aug. 1993 \\
\hline Beaver Dam Lake, South end, at Beaver Dam & 432814088515000 & June-Oct. 1991 \\
\hline North end, near Beaver Dam & 433122088545700 & June-Oct. 1991 \\
\hline Big Blacksmith Lake near Keshena & 445401088334500 & Feb. 1990-Aug. 1992 \\
\hline Big Muskego Lake, at North Site, near Muskego & 425301088061300 & Feb.-Aug. 1988 \\
\hline Research Base, near Muskego & 425235088075300 & May June 1994 \\
\hline Big St. Germain Lake near St. Germain & 455557089311000 & Feb. 1992-Aug. 1996 \\
\hline Booth Lake near East Troy & 424800088254800 & Feb. 1992-Aug. 1994 \\
\hline Denoon Lake at Wind Lake & 425044088100300 & Feb. 1991-Aug. 1996 \\
\hline Druid Lake near Hartford & 431643088243300 & Feb. 1991-Sept. 1996 \\
\hline Eagle Lake near Kansasville & 05544500 & $\begin{array}{l}\text { 1936-64, 1975-77, } 1979 \\
\text { Feb. 1993-Sept. } 1996\end{array}$ \\
\hline Eagle Lake, at Deep Hole, near Kansasville & 424207088072400 & Feb. 1993-Aug. 1996 \\
\hline Elizabeth Lake near Twin Lakes & 423051088155300 & Feb. 1995-Sept. 1997 \\
\hline Forest Lake near Dundee & 433632088100200 & Mar. 1994-Aug. 1996 \\
\hline Fowler Lake, Center, at Oconomowoc & 430653088294601 & $\begin{array}{l}\text { Jan.-Dec. 1984, } \\
\text { Oct. 1986-Sept. } 1996\end{array}$ \\
\hline Fox Lake Deep Hole at Fox Lake & 433458088560600 & June 1991-Mar. 1993 \\
\hline Hemlock Lake near Mikana & 453421091333700 & $\begin{array}{l}\text { Mar. 1993-Aug. 1994, } \\
\text { Mar. 1996-Aug. } 1997\end{array}$ \\
\hline Hooker Lake at Salem & 423335088060300 & Feb. 1992-Aug. 1993 \\
\hline Kirby Lake near Cumberland & 453554092042101 & Nov. 1995-Oct. 1996 \\
\hline Balsam Lake, (Site 1) near Cumberland & 453608092035801 & Nov. 1995-Nov. 1996 \\
\hline (Site 2) near Cumberland & 453601092035301 & Nov. 1995-Nov. 1996 \\
\hline (Site 3) near Cumberland & 453612092034901 & Nov. 1995-Nov. 1996 \\
\hline (Site 4) near Cumberland & 453603092035701 & Nov. 1995-Nov. 1996 \\
\hline (Site 5) near Cumberland & 453608092041201 & Nov. 1995-Nov. 1996 \\
\hline (Site 6) near Cumberland & 453555092040901 & Nov. 1995-Nov. 1996 \\
\hline Lac La Belle, Center, at Oconomowoc & 430733088305900 & $\begin{array}{l}\text { Feb. 1984-Aug. } 1985 \text {, } \\
\text { Feb.-Sept. } 1991\end{array}$ \\
\hline NW, at Oconomowoc & 430809088313900 & Feb. 1984-Aug. 1985 \\
\hline SE at Oconomowoc & 430707088301400 & Feb. 1984-Aug. 1985 \\
\hline Lake Blass at Lake Delton & 433545089482400 & Mar. 1989-Aug. 1990 \\
\hline Lake Keesus, East Bay, near Merton & 4309570088183400 & Apr. 1991-Aug. 1995 \\
\hline North Bay, near Merton & 431006088191000 & Apr. 1991-Aug. 1995 \\
\hline Lake Morris at Mount Morris & 440654089120500 & Jun. 1983-Sept. 1989 \\
\hline Lake Nebagamon, Northeast Bay, at Lake Nebagamon & 463050091412300 & May 1992-Aug. 1995 \\
\hline
\end{tabular}


Table 1. Discontinued lake stations

\begin{tabular}{|c|c|c|}
\hline Station name & Site identification number & Period of record \\
\hline Southeast Bay, at Lake Nebagamon & 462928091413500 & Mar. 1992-Sept. 1995 \\
\hline West Bay, at Lake Nebagamon & 463034091425300 & May 1992-Aug. 1995 \\
\hline Lake Noquebay near Crivitz & 451511087550900 & $\begin{array}{l}\text { Feb. 1987-Aug. 1988, } \\
\text { Apr. 1991-Aug. } 1994\end{array}$ \\
\hline East End, near Crivitz & 451540087525700 & Apr. 1991-Aug. 1994 \\
\hline Lamotte Lake near Shawano & 445305088361200 & Feb. 1990-Aug. 1992 \\
\hline \multicolumn{3}{|l|}{ Lauderdale Lakes } \\
\hline at Lauderdale & 424554088332700 & Oct. 1993-Oct. 1994 \\
\hline Green near Lauderdale & 424652088341500 & Nov. 1993-Nov. 1994 \\
\hline Mill at Lauderdale & 424555088335700 & Nov. 1993-Nov. 1994 \\
\hline Legend Lake (site 1) near Shawano & 445342088312700 & Feb. 1990-Feb. 1992 \\
\hline Little Rock Lake near Woodruff & 455946089415702 & Oct. 1983-Sept. 1996 \\
\hline Long (Kee Nong Go-Mong) Lake at Wind Lake & 424937088103400 & $\begin{array}{l}\text { Feb. 1988-Aug. } 1989, \\
\text { Feb. 1991-Aug. } 1996\end{array}$ \\
\hline Loon Lake near Shawano & 445009088303700 & Feb. 1991-Aug. 1993 \\
\hline Lost Lake near Beaver Dam & 432640088580500 & June-Oct. 1991 \\
\hline \multicolumn{3}{|l|}{ McKenzie Lakes } \\
\hline \multicolumn{3}{|l|}{ McKenzie (Big McKenzie) } \\
\hline Deep Hole, near Spooner & 455507092013500 & Feb. 1987-Aug. 1998 \\
\hline Northern Site, near Spooner & 455540092022000 & June 1997-Aug. 1998 \\
\hline South Site, near Spooner & 455437092022300 & June 1997-Aug. 1998 \\
\hline Lower McKenzie, near Webb Lake & 455902092011900 & June 1997-Aug. 1998 \\
\hline Middle McKenzie, near Spooner & 455635092021800 & June 1997-Aug. 1998 \\
\hline Mary (Marie) Lake at Twin Lakes & 423128088151200 & Feb. 1995-Aug. 1997 \\
\hline Max Lake near Woodruff & 460128089423501 & Mar. 1988-Dec. 1996 \\
\hline Mead Lake, East Bay near Willard & 444720090445000 & Apr. 1991-Aug. 1995 \\
\hline West Bay near Willard & 444733090460100 & Feb. 1991-Sept. 1995 \\
\hline Montello Lake at Montello & 434748089195800 & Feb. 1995-Aug. 1998 \\
\hline Moon Lake near St. Germain & 455504089260500 & Feb. 1992-Aug. 1996 \\
\hline Morgan Lake near Fence & 454622088324801 & Oct. 1987-Sept. 1998. \\
\hline Moshawquit Lake near Shawano & 445352088295800 & Feb. 1990-Aug. 1992 \\
\hline Park Lake (site 1) at Pardeeville & 433239089175800 & $\begin{array}{l}\text { Feb. 1986-Aug. 1987, } \\
\text { May-Nov. } 1993\end{array}$ \\
\hline (site 2) at Pardeeville & 433226089175500 & May-Nov. 1993 \\
\hline (site 3) at Pardeeville & 433245089173000 & May-Nov. 1993 \\
\hline (site 4) at Pardeeville & 433257089165100 & May-Nov. 1993 \\
\hline Pretty Lake, at Deep Hole, near Dousman & 425722088295000 & Feb. 1993-Aug. 1997 \\
\hline Red Cedar Lake, Deep Hole, near Mikana & 453725091345100 & $\begin{array}{l}\text { Mar. 1993-Aug. 1994, } \\
\text { Mar. 1996-Aug. } 1997\end{array}$ \\
\hline South End, at Mikana & 453519091352500 & $\begin{array}{l}\text { Mar. 1993-Aug. 1994, } \\
\text { Mar. 1996-Aug. } 1997\end{array}$ \\
\hline Rice Lake at Deep Hole near Whitewater & 424629088415700 & Apr.-Nov. 1991 \\
\hline Round Lake near Shawano & 445328088335000 & Feb. 1990-Aug. 1992 \\
\hline
\end{tabular}


Table 1. Discontinued lake stations

\begin{tabular}{lcl}
\hline \multicolumn{1}{c}{ Station name } & Site identification number & Period of record \\
\hline Sand Lake (Deep Hole) near Keshena & 445321088323101 & June-Aug. 1992 \\
Silver Lake near Oconomowoc & 430436088293300 & Apr. 1992-Aug. 1996 \\
Silver Lake near West Bend & 432322088125000 & Feb. 1996-Aug. 1997 \\
Sinissippi Lake, off Anthony Is., at Hustisford & 432113088361100 & Feb. 1991-Aug. 1993 \\
$\quad$ off Butternut Is., near Hustisford & 432240088363900 & Apr. 1991-Aug. 1993 \\
$\quad$ off Sam Point, near Hustisford & 432300088374200 & Apr. 1991-Aug. 1993 \\
Spirit Lake near Keshena & 445400088320100 & Apr.-Aug. 1992 \\
Stewart Lake at Mt. Horeb & 430117089442701 & May 1992-Sept. 1993 \\
Tichigan Lake near Waterford & 424854088123300 & Mar. 1994-Aug. 1996 \\
Upper Nemahbin Lake, Center, near Delafield & 430400088254900 & June 1993-Aug. 1995 \\
$\quad$ South Site, near Delafield & 430339088254800 & June 1993-Aug. 1995 \\
Outlet near Delafield & 430334088255400 & June 1993-Aug. 1995 \\
Vandercook Lake near Woodruff & 455909089405602 & Nov. 1980-Aug. 1998 \\
Watosah-skice Lake near Keshena & 445330088361400 & Feb. 1990-Aug. 1992 \\
Waubeesee Lake at Wind Lake & 424857088101500 & Feb. 1988-Aug. 1989, \\
& & Feb. 1991-Aug. 1996 \\
Whitewater Lake, off Heart Prairie, near Whitewater & 424533088420100 & Apr.-Nov. 1991 \\
$\quad$ near Whitewater & 424608088414800 & Apr.-Oct. 1991 \\
North Bay, near Whitewater & 424625088405500 & Apr.-Nov. 1991 \\
South Bay, near Whitewater & 424501088422300 & Apr.-Nov. 1991 \\
Wind Lake, Northeast Basin, at Wind Lake & 424938088080800 & Feb. 1997-Aug. 1998 \\
Wolf Lake near Mt. Calvary & 435152088123100 & Nov. 1983-Sept. 1986, \\
& & Nov. 1992-Sept. 1997 \\
\hline
\end{tabular}


In most lakes, water samples were collected at two depths - near the surface and near the bottom. Chemical analyses of water samples were performed using standard analytical methods by either the USGS National Water Quality Laboratory (Wershaw and others, 1987; Fishman and Friedman, 1989; Fishman, 1993) or the Wisconsin State Laboratory of Hygiene (Wisconsin State Laboratory of Hygiene, 1993). Analyses for dissolved constituents were performed on samples that were filtered in the field through a $0.45-\mu \mathrm{m}$ (micrometer) pore-size filter. Total or total recoverable constituents were determined by analyzing unfiltered water samples. Preservation and shipment of samples followed standard protocols established by the laboratories. Water-quality data were archived in the Water Quality Data Base (QWDATA) of the National Water Information System (NWIS). Additional descriptive information about water-quality data is available in the data report: "Water Resources Data - Wisconsin, 1999". NWIS parameter codes and minimum laboratory reporting limits for chemical constituents are given in table 2.

Records of lake stage are considered complete when one or more manual or automatic measurements were obtained per day. Partial records of lake stage result when measurements were less frequent than daily. A complete description of manual or automatic measurements of lake stage is described by Rantz and others (1982).

\section{EXPLANATION OF PHYSICAL AND CHEMICAL CHARACTERISTICS OF LAKES}

Following are brief, generalized explanations of some of the common measurements of water quality and some of the physical processes occurring in lakes that influence these measures of water quality. More detailed explanations of water-quality data and lake processes are given by Wetzel (1983), Hem (1985), and Shaw and others (1993).

\section{Water Temperature and Thermal Stratification}

Water temperature in lakes is important because of its role in stratification and because of the temperature dependence of many chemical reactions and life processes of aquatic organisms. The extent of thermal stratification in lakes depends on the interaction between the lake's shape, water clarity, solar heating, and wind-driven mixing. Complete mixing of the lake is usually inhibited by thermal stratification in summer and by ice cover in winter. Thermal stratification affects water quality and the distribution of organisms in the lake. Summer thermal stratification can occur in any lake, but in Wisconsin it commonly occurs in lakes deeper than about $6 \mathrm{~m}$ (Shaw and others, 1993). 
Table 2. Parameter identification numbers and laboratory reporting levels (LRL) for chemical parameters commonly measured in lakes, and analyzed at the National Water Quality Laboratory (NWQL) or the Wisconsin State Laboratory of Hygiene (WSLH)

\begin{tabular}{|c|c|c|c|c|c|c|c|c|c|}
\hline \multirow[b]{3}{*}{ Parameter Name } & \multirow[b]{3}{*}{ Units } & \multirow[b]{3}{*}{$\begin{array}{c}\text { CAS } \\
\text { Number } \\
\text { (1) }\end{array}$} & \multirow[b]{3}{*}{$\begin{array}{c}\text { Parameter } \\
\text { Code } \\
(2)\end{array}$} & \multicolumn{4}{|c|}{ (NWQL) } & \multicolumn{2}{|c|}{ (WSLH) } \\
\hline & & & & \multicolumn{2}{|c|}{$\underline{\text { Standard Analysis }}$} & \multicolumn{2}{|c|}{ Low-Level Analysis } & \multirow[b]{2}{*}{ MRL } & \multirow[b]{2}{*}{$\begin{array}{l}\text { Test } \\
\text { Code }\end{array}$} \\
\hline & & & & LRL & $\begin{array}{l}\text { Lab } \\
\text { Code }\end{array}$ & LRL & $\begin{array}{l}\text { Lab } \\
\text { Code }\end{array}$ & & \\
\hline Calcium, diss. (Ca) & $\mathrm{mg} / \mathrm{L}$ & $7440-70-2$ & 00915 & 0.020 & 659 & 0.002 & 1895 & 0.02 & I230IUD \\
\hline Magnesium, diss. (Mg) & $\mathrm{mg} / \mathrm{L}$ & $7439-95-4$ & 00925 & 0.004 & 663 & 0.001 & 1897 & 0.02 & I390IUD \\
\hline Sodium, diss. (Na) & $\mathrm{mg} / \mathrm{L}$ & $7440-23-5$ & 00930 & 0.09 & 675 & 0.025 & 1898 & 0.09 & I80IUD \\
\hline Potassium, diss. (K) & $\mathrm{mg} / \mathrm{L}$ & $7440-09-7$ & 00935 & 0.24 & 54 & 0.01 & 833 & 0.3 & I540IUD \\
\hline Sulfate, diss. $\left(\mathrm{SO}_{4}\right)$ & $\mathrm{mg} / \mathrm{L}$ & $14808-79-8$ & 00945 & 0.31 & 1572 & 0.01 & 1263 & 1.0 & I600DLD \\
\hline Chloride, diss. (Cl) & $\mathrm{mg} / \mathrm{L}$ & $16887-00-6$ & 00940 & 0.29 & 1571 & 0.01 & 1259 & 0.1 & I240ELD \\
\hline Fluoride, diss. (F) & $\mathrm{mg} / \mathrm{L}$ & $16984-48-8$ & 00950 & 0.100 & 31 & 0.01 & 1260 & 0.03 & I330FLD \\
\hline Iron, diss. $(\mathrm{Fe})$ & $\mu \mathrm{g} / \mathrm{L}$ & $7439-89-6$ & 01046 & 10 & 645 & 3 & 1896 & 10 & I370IUD \\
\hline Manganese, diss. (Mn) & $\mu g / L$ & $7439-96-5$ & 01056 & 2.2 & 648 & 1 & 1793 & 0.4 & I400IUD \\
\hline Silica, diss. $\left(\mathrm{SiO}_{2}\right)$ & $\mathrm{mg} / \mathrm{L}$ & $7631-86-9$ & 00955 & 0.1 & 56 & 0.02 & 1899 & 0.008 & I560LLD \\
\hline Nitrogen, $\mathrm{NO}_{2}+\mathrm{NO}_{3}$, diss. & $\mathrm{mg} / \mathrm{L}$ & & 00631 & 0.05 & 1975 & 0.005 & 1979 & 0.01 & I460MLD \\
\hline Nitrogen, ammonia, diss. & $\mathrm{mg} / \mathrm{L}$ & $7664-41-7$ & 00608 & 0.02 & 1976 & 0.002 & 1980 & 0.013 & I440NLD \\
\hline Nitrogen, organic, total (3) & $\mathrm{mg} / \mathrm{L}$ & & & & & & & & \\
\hline Nitrogen, amm.+org., total (4) & $\mathrm{mg} / \mathrm{L}$ & $17778-88-0$ & 00623 & 0.100 & 1985 & & & 0.2 & I470BLT \\
\hline Nitrogen, total (5) & $\mathrm{mg} / \mathrm{L}$ & & & & & & & & \\
\hline Phosphorus, total & $\mathrm{mg} / \mathrm{L}$ & $7723-14-0$ & 00665 & 0.05 & 1984 & 0.001 & 837 & 0.005 & I520PLT \\
\hline Phosphorus, ortho, diss. & $\mathrm{mg} / \mathrm{L}$ & $14265-44-2$ & 00671 & 0.01 & 1262 & 0.001 & 1978 & 0.002 & $1530 \mathrm{CLD}$ \\
\hline Chlorophyll a, phytoplankton & $\mu \mathrm{g} / \mathrm{L}$ & $479-61-8$ & 70953 & 0.1 & 586 & & & & \\
\hline Chlorophyll a, phytoplankton & $\mu \mathrm{g} / \mathrm{L}$ & $479-61-8$ & 32210 & & & & & 1 & I250UNF \\
\hline
\end{tabular}

\section{Footnotes:}

1: CAS (Chemical Abstracting Services) number= unique identification for each constituent

2: Parameter Code - unique number for storage of data in database

3: Calculated as difference between total ammonia + organic nitrogen and ammonia nitrogen

4: Also known as Total Kjeldahl Nitrogen (TKN)

5: Calculated as sum of TKN + Nitrogen as $\left(\mathrm{NO}_{2}+\mathrm{NO}_{3}\right)$ 
The density of water increases with decreasing temperature down to a temperature of $4^{\circ} \mathrm{C}$, then decreases with decreasing temperature between $4^{\circ} \mathrm{C}$ and the freezing point of water $\left(0^{\circ} \mathrm{C}\right)$. For a brief period in the spring after the ice is out, water temperature is usually uniform through the entire water column and wind action causes the lake to mix completely. This process is known as "spring turnover." As the lake absorbs the sun's energy, the surface water becomes warmer and its density decreases, making it more resistant to complete mixing. The difference in density caused by different water temperatures can prevent warm and cold water from mixing. In most lakes, therefore, a density "barrier" forms between the warmer surface water (epilimnion) and the underlying colder water (hypolimnion). This barrier is often marked by a sharp temperature gradient known as the "thermocline (metalimnion)." During the stratified summer period, these three distinct layers of lake water are often present. As the temperature difference between surface and deep water increases, this "stratified" condition stabilizes and can persist until surface temperatures decrease in the fall, which decreases the stability of the stratification. The mixing of the lake water in the fall is known as "fall turnover."

Thermal stratification may also occur under ice cover in the winter. In the winter, the coldest water (near $0^{\circ} \mathrm{C}$ ) under the ice at the surface of the lake is less dense than water deeper in the lake with warmer temperatures.

\section{Specific Conductance}

Specific conductance is a measure of the ability of water to conduct an electrical current and is an indicator of the concentration of dissolved solids in the water. Because conductance is temperature related, reported values are normalized at $25^{\circ} \mathrm{C}$ and are termed specific conductance. As the concentration of dissolved minerals increases, specific conductance increases. During winter and summer thermal stratification, concentrations of dissolved constituents near the lake bottom increase due to the decomposition of materials settling from the epilimnion, or release of dissolved materials (such as iron, manganese, and phosphorus) from the bottom sediments during anoxic periods. Therefore, differences in specific conductance with depth indicate differences in concentrations of dissolved solids. 


\section{Water Clarity}

Water clarity, or transparency, is commonly measured using a Secchi disc. The range of depths within which photosynthetic activity occurs depends largely on depth of light penetration, which is influenced by water clarity. A Secchi disc, most commonly an $20-\mathrm{cm}$.-diameter disc with alternating black-and-white quadrants, is lowered to a depth at which it is no longer visible. This depth is referred to as the Secchi depth. Clarity can be reduced by algae, zooplankton, water color, and suspended sediment. Algae are often the most dominant influence on clarity in lakes and, therefore, Secchi depth is usually correlated with the algal abundance. Secchi depths are generally the least during summer when algal populations are largest.

\section{pH}

The $\mathrm{pH}$ is a measure of the acidity of the water. It is defined as the negative logarithm of hydrogen-ion concentration and varies over a 14-unit log scale, with a pH of 7 being neutral. Values less than 7 indicate acidic conditions; the lower the value, the stronger the acidity. Values greater than 7 indicate alkaline conditions. The $\mathrm{pH}$ of water is influenced in part by photosynthesis and respiration of planktonic algae and aquatic plants. It is important because it affects the solubility of many chemical constituents, and because aquatic organisms have limited $\mathrm{pH}$ tolerances. Planktonic algae and aquatic plants produce oxygen and consume carbon dioxide as they photosynthesize during daytime; they consume oxygen and produce carbon dioxide when they respire at night. Carbon dioxide combines with the water molecule to form carbonic acid; therefore respiration causes a decrease in $\mathrm{pH}$ at night and photosynthesis during the day causes an increase in $\mathrm{pH}$. The result is a daily cycle in $\mathrm{pH}$. Because phytoplankton are usually concentrated in the near-surface water, changes in $\mathrm{pH}$ in the epilimnion are more extreme than in the hypolimnion, where less photosynthesis usually occurs.

Lakes having good fish populations and productivity generally have a pH between 6.7 and 8.2. Values of $\mathrm{pH}$ greater than 8.5 have been shown to cause the release of phosphorus from lake sediments (James and Barko, 1991). 


\section{Dissolved Oxygen}

Dissolved oxygen is one of the most critical factors affecting a lake ecosystem because it is essential to most aquatic organisms, and it is involved in many chemical reactions. Very low dissolved oxygen concentrations can control some types of chemical reactions. The solubility of oxygen in water is inversely related to temperature-that is, oxygen solubility decreases as water temperature increases. This relation is important because at warmer temperatures the metabolic rate of organisms increases but less oxygen is available for respiration. The primary sources of dissolved oxygen are from the air and from photosynthesis. The minimum dissolved oxygen concentration specified in national water-quality criteria for early life stages of warmwater aquatic life is 5.0 $\mathrm{mg} / \mathrm{L}$ (U.S. Environmental Protection Agency, 1986).

In early summer, if thermal stratification develops, the metalimnion restricts the surface supply of dissolved oxygen to the hypolimnion. The hypolimnion can become isolated from the atmosphere. Thus, as summer progresses, the dissolved oxygen concentration can decrease in response to decomposition of dead algae that settle from the epilimnion and in response to the biological and chemical oxygen demand of the sediments. The oxygen demand from these processes may completely deplete the oxygen (anoxia) in the water near the lake bottom. The oxygen depletion then progresses upward but usually is confined to the hypolimnion.

Anoxia in the hypolimnion is common in stratified eutrophic (nutrient-rich) lakes in Wisconsin. Complete anoxia, however, is often not detected because of meter constraints. During anoxic conditions, many aquatic organisms cannot survive, but many other species (primarily bacteria) actually function only in such conditions. Therefore, a shift from oxic to anoxic conditions produces a rapid and dramatic change in the biological community and chemical environment. Anoxia also can cause release of phosphorus from the bottom sediments. This phosphorus then mixes throughout the water column during spring and fall turnover. 


\section{Phosphorus}

Phosphorus is one of the essential nutrients for plant growth. High phosphorus concentrations can cause dense algal populations (blooms) and can therefore be a major cause of eutrophication in lakes. When phosphorus concentrations exceed $0.025 \mathrm{mg} / \mathrm{L}$ at the time of spring overturn in lakes and reservoirs, these water bodies may occasionally experience excess or nuisance growth of algae or other aquatic plants (U.S. Environmental Protection Agency, 1986). In many regions of the country, including the upper Midwest, other nutrients, particularly nitrogen, tend to be in abundant supply. Phosphorus is often the nutrient in shortest supply, therefore limiting or controlling plant growth. About 90 percent of the lakes in Wisconsin are limited by phosphorus (Shaw and others, 1993). In water, dissolved orthophosphate is that part of total phosphorus that is most readily available for use by algae.

Internal phosphorus recycling occurs in many lakes. Phosphorus used by algae, aquatic plants, fish, and zooplankton is stored within these organisms. As these organisms die and decompose, this phosphorus is returned to the lake water and sediments. Anoxia in the hypolimnion makes phosphorus more soluble, adding further to the release of phosphorus from the falling particles and the lake sediments. During spring and fall turnover the phosphorus, which was released from the bottom sediments into the hypolimnion during anoxia, is mixed throughout the lake. The phosphorus is then available for algal growth. These phenomena are part of the internal-recycling processes of lakes.

\section{Nitrogen}

Nitrogen, like phosphorus, is an essential nutrient for plant and algal growth. Usually in Wisconsin lakes, nitrogen is in abundant supply from the atmosphere and other sources. If phosphorus is abundant relative to algal needs, nitrogen can become the limiting nutrient. In that case, algal blooms are more likely to be triggered by increases in nitrogen than by increases in phosphorus. Some bluegreen algal species can fix nitrogen from the atmosphere (Wetzel, 1983). Therefore, in situations where other types of algae are excluded because of a shortage of nitrogen, the nitrogen-fixing bluegreen algae have a competitive advantage and may be present in abundance.

Lakes with a nitrogen to phosphorus ratio larger than 15 to 1 near the surface may generally be considered phosphorus limited; a ratio from 10 to 1 to 15 to 1 indicates a transition situation; and a ratio smaller than 10 to 1 generally indicates nitrogen limitation. Total nitrogen is the sum of ammonia, organic nitrogen, and nitrate-plus-nitrite nitrogen. The near-surface concentration is commonly used to compute the total nitrogen to phosphorus ratio because most algal species grow near the lake surface. 


\section{Chlorophyll a}

Chlorophyll $a$ is a photosynthetic pigment found in algae (Wetzel, 1983) and other green plants. Its concentration, therefore, is commonly used as a measure of the density of the algal population in a lake. Chlorophyll a concentrations are generally highest during summer when algal populations are highest. Moderate populations of desirable algae are important in the food chain; however, excessive populations or algal blooms are undesirable. Algal blooms can cause taste and odor problems, and limit light penetration needed to support growth of submerged aquatic plants. Certain species of bluegreen algae can produce toxins (Rapavich and others, 1987).

\section{CLASSIFICATION OF LAKES}

Two methods are commonly used to classify and evaluate Wisconsin lakes according to their water quality or trophic state: Lillie and Mason's (1983) water-quality index and a modification of Carlson's (1977) Trophic State Index (TSI) by Lillie and others (1993). Three waterquality measures are used in these classification systems: near-surface concentrations of total phosphorus and chlorophyll $a$, and water clarity as indicated by the Secchi depth.

Lillie and Mason's (1983) water-quality indices for Wisconsin lakes were developed based on random summer measurements of total phosphorus and chlorophyll a concentrations, and Secchi depth to classify the lakes' water quality as shown below:

\begin{tabular}{lccc}
\hline $\begin{array}{c}\text { Water-quality } \\
\text { index }\end{array}$ & $\begin{array}{c}\text { Total phosphorus range } \\
(\mathrm{mg} / \mathrm{L})\end{array}$ & $\begin{array}{c}\text { Chlorophyll a range } \\
(\mu \mathrm{g} / \mathrm{L})\end{array}$ & $\begin{array}{c}\text { Water clarity range } \\
(\text { Secchi depth, in meters })\end{array}$ \\
\hline "Excellent" & $<0.001$ & $<1.0$ & $>6.0$ \\
"Very good" & $.001-.009$ & $1.0-4.9$ & $3.0-6.0$ \\
"Good" & $.010-.029$ & $5.0-9.9$ & $2.0-2.9$ \\
"Fair" & $.030-.049$ & $10.0-14.9$ & $1.5-1.9$ \\
"Poor" & $.050-.149$ & $15.0-30.0$ & $1.0-1.4$ \\
"Very poor" & $>.150$ & $>30.0$ & $<1.0$ \\
\hline
\end{tabular}


The TSI approach to lake classification assigns numerical ranges to the three trophic conditions generally used to describe the wide range of lake water-quality conditions. Oligotrophic lakes are typically clear, algal populations and phosphorus concentrations are low, and the deepest water is likely to contain oxygen throughout the year. Mesotrophic lakes typically have a moderate supply of nutrients, experience moderate algal blooms, and have occasional oxygen depletions at depth. Eutrophic lakes are nutrient rich with relatively severe water-quality problems, such as frequent seasonal algal blooms, oxygen depletion in lower parts of the lakes, and poor clarity. When eutrophic conditions are very severe, the lake is considered hypereutrophic.

The WDNR modified the lakes classification scheme developed by Carlson (1977) to apply specifically to Wisconsin lakes. The WDNR system (Lillie and others, 1993) uses surface total phosphorus and chlorophyll a concentrations, and Secchi depth for ice-free periods to calculate values for TSI's. The WDNR has adopted the following TSI ranges to classify Wisconsin lakes: indices of less than 40 define oligotrophic conditions, 40 to 50 define mesotrophic conditions, greater than 50 to define eutrophic conditions, and greater than 70 define hypereutrophic conditions (Wisconsin Department of Natural Resources, 1992). These ranges are used to make relative comparisons in Wisconsin lake trophic-state evaluations by the WDNR and others.

The TSI for a lake can be calculated using the following equations (Lillie and others, 1993):

$\mathrm{TSI}_{\text {Secchi }}=60.0-33.2 \times\left(\log _{10}\right.$ Secchi depth $)$

$\mathrm{TSI}_{\text {chlorophyll } a}=34.82+\left(17.41 \times\left(\log _{10}\right.\right.$ chlorophyll a concentration $\left.)\right)$

$\mathrm{TSI}_{\text {total phosphorus }}=28.24+\left(17.81 \times\left(\log _{10}\right.\right.$ total phosphorus concentration $\left.\left.\times 1,000\right)\right)$

where: Secchi depth is in meters,

chlorophyll $a$ is in micrograms per liter, and

total phosphorus is in milligrams per liter.

The three trophic conditions are defined with the following boundaries for total phosphorus, Secchi disc, and chlorophyll a:

\begin{tabular}{|c|c|c|c|c|}
\hline $\begin{array}{l}\text { Trophic } \\
\text { Level }\end{array}$ & $\begin{array}{c}\text { Trophic State } \\
\text { Index }\end{array}$ & $\begin{array}{c}\text { Total phosphorus } \\
(\mathrm{mg} / \mathrm{L})\end{array}$ & $\begin{array}{l}\text { Secchi depth } \\
(\mathrm{m})\end{array}$ & $\begin{array}{c}\text { Chlorophyll } a \\
(\mu \mathrm{g} / \mathrm{L})\end{array}$ \\
\hline \multicolumn{5}{|c|}{ Eutrophic } \\
\hline Mesotrop & & & & \\
\hline
\end{tabular}




\section{REFERENCES CITED}

Carlson, R.E., 1977, A trophic state index for lakes: Limnology and Oceanography, March, v. 22, no. 2, p. 361-369.

Fishman, M.J., ed., 1993, Methods of analysis by the U.S. Geological Survey National Water Quality Laboratory-Determination of inorganic and organic constituents in water and fluvial sediments: U.S. Geological Survey Open-File Report 93-125, 217 p.

Fishman, M.J., and Friedman, L.C., eds., 1989, Methods for determination of inorganic substances in water and fluvial sediments ( $3 d$ ed.): U.S. Geological Survey Techniques of Water-Resources Investigations, book 5, chap. A1, 545 p.

Hem, J.D., 1985, Study and interpretation of the chemical characteristics of natural water (3rd ed.): U.S. Geological Survey Water-Supply Paper 2254, 263 p.

James, W.F., and Barko, J.W., 1991, Littoral-pelagic phosphorus dynamics during nighttime convective circulation: Limnology and Oceanography, v. 36, no. 5, p. 946-960.

Lillie, R.A., Graham, S., and Rasmussen, P., 1993, Trophic-State Index equations and regional predictive equations for Wisconsin lakes: Wisconsin Department of Natural Resources Research Management Findings No. 35, 4 p.

Lillie, R.A., and Mason, J.W., 1983, Limnological characteristics of Wisconsin lakes: Wisconsin Department of Natural Resources Technical Bulletin No. 138, 116 p.

Rantz, S.E., and others, 1982, Measurement and computation of streamflow: U.S. Geological Survey Water-Supply Paper 2175, $631 \mathrm{p}$.

Rapavich, W.M., Sonzogni, W.C., Standridge, J.H., Vennie J.G., and Wedepohl, R.E., 1987, Incidence of algal toxins in Wisconsin water experiencing blue-green algae blooms: Wisconsin State Laboratory of Hygiene and Wisconsin Department of Natural Resources, Informational Paper, 8 p.

Shaw, B., Mechenich, C., and Klessig, L., 1993, Understanding Lake Data: G3582: University of Wisconsin Extension, Madison, Wis., $19 \mathrm{p}$.

U.S. Environmental Protection Agency, 1986, Quality Criteria for Water 1986: U.S. Environmental Protection Agency publication, EPA 440/5-86-001 [variously paged].

Wershaw, R.L., Fishman, M.J., Grabbe, R.R., and Lowe, L.E., eds., 1987, Methods for the determination of organic substances in water and fluvial sediments: U.S. Geological Survey Techniques of Water-Resources Investigations, book 5, chap. A3, 80 p.

Wetzel, R.G., 1983, Limnology (2d ed.): New York, 767 p.

Wisconsin Department of Natural Resources, 1992, Wisconsin water quality assessment-Report to Congress, 1992: Wisconsin Department of Natural Resources Publ-WR254-92-REV, $220 \mathrm{p}$. 
Wisconsin State Laboratory of Hygiene, Environmental Sciences Section, 1993, Manual of analytical methods, inorganic chemistry unit: Wisconsin State Laboratory of Hygiene, revised November 1993 [variously paged]. 

LAKE DATA 



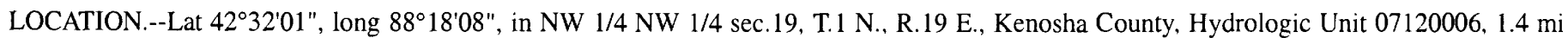
southwest of Powers Lake.

PERIOD OF RECORD.--May 1998 to current year

REMARKS.--Lake sampled near center at deep hole. Lake ice-covered during February sampling. Water-quality analyses done by Wisconsin State Laboratory of Hygiene.

WATER-QUALITY DATA, FEBRUARY 19 TO AUGUST 04, 1999

(Milligrams per Iiter unless otherwise indicated)
Lake stage (ft)

Secchi-depth $(\mathrm{m})$

Chlorophyll a, phytoplankton (ug/L)

Depth of sample (m)

Water temperature $\left({ }^{\circ} \mathrm{C}\right)$

Specific conductance ( $\mu \mathrm{S} / \mathrm{cm}$ )

$\mathrm{pH}$ (units)

Dissolved oxygen (mg/L)

Phosphorus, total (as

Phosphorus, ortho, dissolved (as P)

Nitron, No2 + NO3, diss. (as N)

Nitrogen, ammonia, dissolved (as N)

Nitrogen, amm. + org., total (as N)

Nitrogen, total (as $\mathrm{N}$ )

Color (Pt-Co. scale)

Turbidity (NTU)

Hardiness, (as $\mathrm{CaCO}_{3}$

Calcium, dissolved $\mathrm{CCa}$

Magnesium, dissolved (Mg)

Sodium, dissolved (Na)

Potassium, dissolved (K)

Potium, dissolved (

Sulfate, dissolved ( $\mathrm{SO}_{4}$ )

Chloride, dissolved (Cl)

Silica, dissolved (SiO

Solids, dissolved, at $180^{\circ}$

Iron, dissolved ( $\mathrm{Fe}$ ) $\mu \mathrm{g} / \mathrm{L}$

'anganese, dissolved (Mn) $\mu \mathrm{g} / \mathrm{L}$

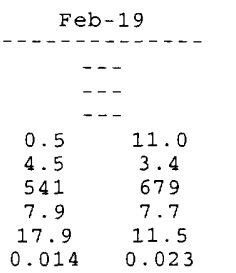

$\begin{array}{lll}0.014 & 0.023\end{array}$

-.

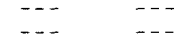

n-

n-

$\ldots$

-

$\begin{array}{ll}-- & -- \\ -- & --\end{array}$

n.

- - $\quad-$.

-

-

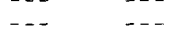

- -

$\cdots+-$

\begin{tabular}{|c|c|}
\hline & \\
\hline & \\
\hline 0.5 & 11.0 \\
\hline 11.6 & 9.2 \\
\hline 654 & 660 \\
\hline 8.2 & 8.1 \\
\hline 12.0 & 9.3 \\
\hline 0.018 & 0.021 \\
\hline$<0.002$ & $\ldots$ \\
\hline 0.557 & $\ldots$ \\
\hline 0.353 & $\ldots$ \\
\hline 1.2 & $\ldots$ \\
\hline 1.7 & $\ldots$ \\
\hline 5 & $\ldots$ \\
\hline 1.5 & $\ldots$ \\
\hline 260 & $\ldots$ \\
\hline 46 & $\ldots$ \\
\hline 36 & $\ldots$ \\
\hline 26 & - . - \\
\hline 2.2 & $\ldots$ \\
\hline 222 & -- \\
\hline 34 & $\ldots$ \\
\hline 59 & $\ldots$ \\
\hline 5.8 & $\ldots$ \\
\hline 356 & $\cdots$ \\
\hline$<10$ & $\ldots$ \\
\hline 0.7 & $\ldots$ \\
\hline
\end{tabular}

\begin{tabular}{|c|c|}
\hline \multicolumn{2}{|c|}{ Jun -10} \\
\hline \multicolumn{2}{|c|}{$\begin{array}{c}826.26 \\
2.3 \\
1.70\end{array}$} \\
\hline 0.5 & 11.0 \\
\hline 26.8 & 9.5 \\
\hline 612 & 691 \\
\hline 8.2 & 7.6 \\
\hline 9.0 & 0.7 \\
\hline 0.007 & 0.075 \\
\hline--- & -- \\
\hline$\ldots$ & 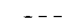 \\
\hline-- & $\ldots$ \\
\hline$\ldots$ & $\ldots$ \\
\hline--- & -- \\
\hline$\ldots$ & -- \\
\hline-- & $-\cdots$ \\
\hline-- & $\ldots$ \\
\hline$\cdots$ & $\cdots$ \\
\hline$\cdots$ & $\cdots$ \\
\hline$\cdots$ & $\cdots$ \\
\hline - - - & $\cdots$ \\
\hline-- & $\cdots$ \\
\hline--- & $\cdots$ \\
\hline-- & - \\
\hline-1 & $-\cdots$ \\
\hline$\ldots$ & $\ldots$ \\
\hline & \\
\hline
\end{tabular}

\begin{tabular}{|c|c|}
\hline \multicolumn{2}{|c|}{ Jul-7 } \\
\hline \multicolumn{2}{|c|}{$\begin{array}{c}825.65 \\
1.9 \\
1.64\end{array}$} \\
\hline 0.5 & 11.0 \\
\hline 27.5 & 9.5 \\
\hline 569 & 695 \\
\hline 8.2 & 7.5 \\
\hline 8.6 & 0.4 \\
\hline 0.010 & 0.102 \\
\hline 0.004 & $\ldots$ \\
\hline 0.512 & $\ldots$ \\
\hline 0.128 & $\ldots$ \\
\hline 0.92 & --- \\
\hline 1.4 & --- \\
\hline--- & -- \\
\hline$\ldots$ & --- \\
\hline--- & -.- \\
\hline$\ldots$ & $\ldots$ \\
\hline$\ldots$ & $\ldots$ \\
\hline$\ldots$ & --- \\
\hline$\cdots$ & -- \\
\hline-- & $\cdots$ \\
\hline$\cdots$ & -- \\
\hline$\cdots$ & --- \\
\hline$\cdots$ & -- \\
\hline-- & -1 \\
\hline- & -- \\
\hline
\end{tabular}

\begin{tabular}{|c|c|c|}
\hline & Aug-4 & \\
\hline & $\begin{array}{c}825.71 \\
1.9 \\
3.00\end{array}$ & \\
\hline 0.5 & 8.0 & 11.0 \\
\hline 27.4 & 14.0 & 9.1 \\
\hline 561 & 610 & 733 \\
\hline 8.1 & 8.0 & 7.2 \\
\hline 9.5 & 9.0 & 0.0 \\
\hline 0.020 & 0.030 & 0.077 \\
\hline$-\ldots$ & $\ldots$ & $\ldots$ \\
\hline - - & $\ldots$ & - - - \\
\hline$\ldots$ & $\ldots$ & $\ldots$ \\
\hline - - - & $\ldots$ & $\ldots$ \\
\hline - - - & $\ldots$ & $\ldots$ \\
\hline -. & $\ldots$ & $\ldots$ \\
\hline - - - & $\ldots$ & $\ldots$ \\
\hline$\ldots$ & $\ldots$ & $\ldots$ \\
\hline$\ldots$ & $\ldots$ & ... \\
\hline$\ldots$ & $\ldots$ & $\ldots$ \\
\hline$\ldots$ & ... & - - - \\
\hline$\ldots$ & $\ldots$ & $\ldots$ \\
\hline$-\ldots$ & $\ldots$ & - - \\
\hline$\ldots$ & $\ldots$ & - - - \\
\hline--- & $\ldots$ & $\ldots$ \\
\hline - - & $\ldots$ & $\ldots$ \\
\hline$\ldots$ & $\ldots$ & $\ldots$ \\
\hline - - & $\ldots$ & -.- \\
\hline--- & $\ldots$ & $\ldots$ \\
\hline
\end{tabular}

DISSOLVED OXYGEN (D.O.), IN MILLIGRAMS PER LITER
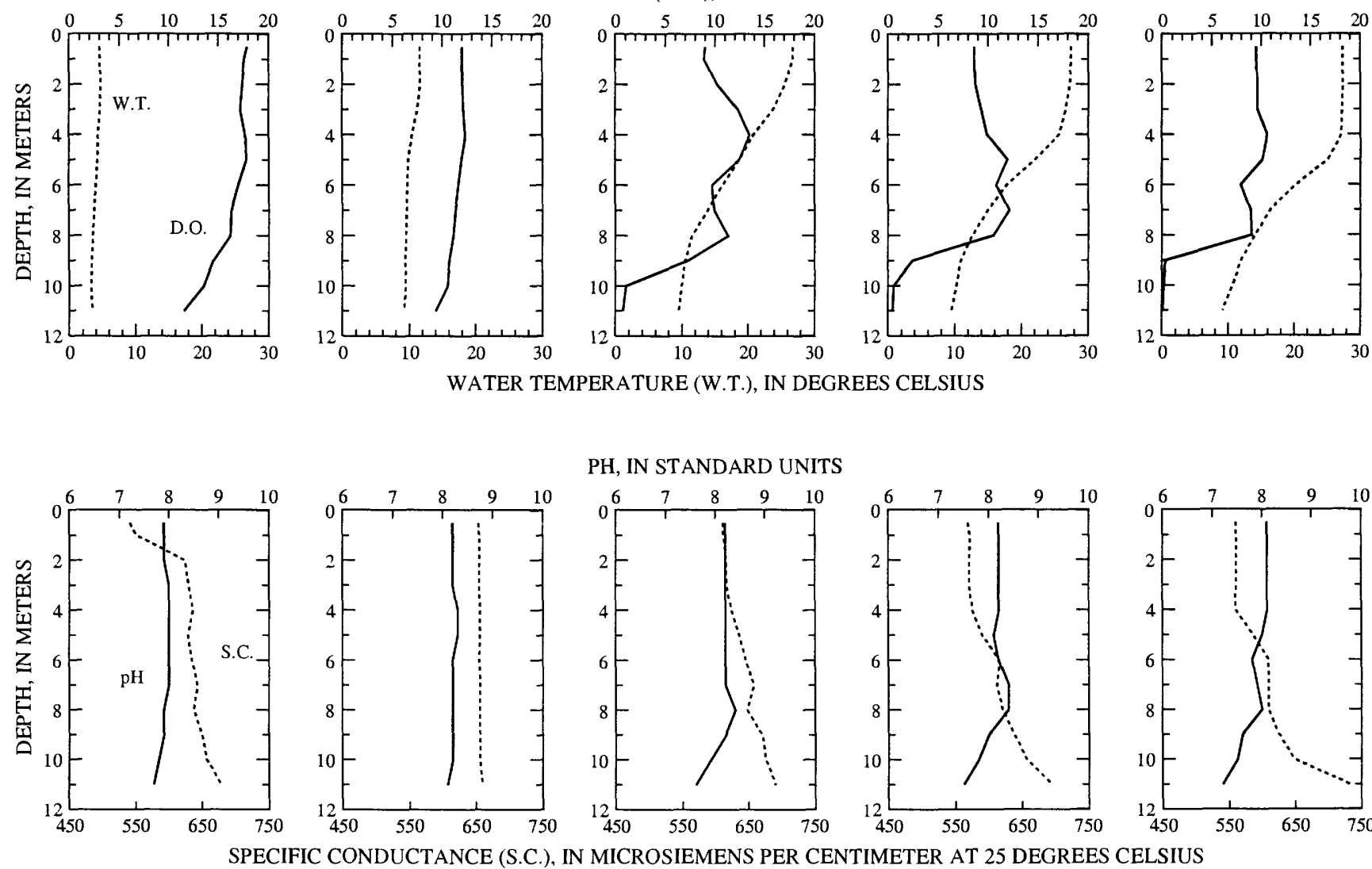

PH, IN STANDARD UNITS
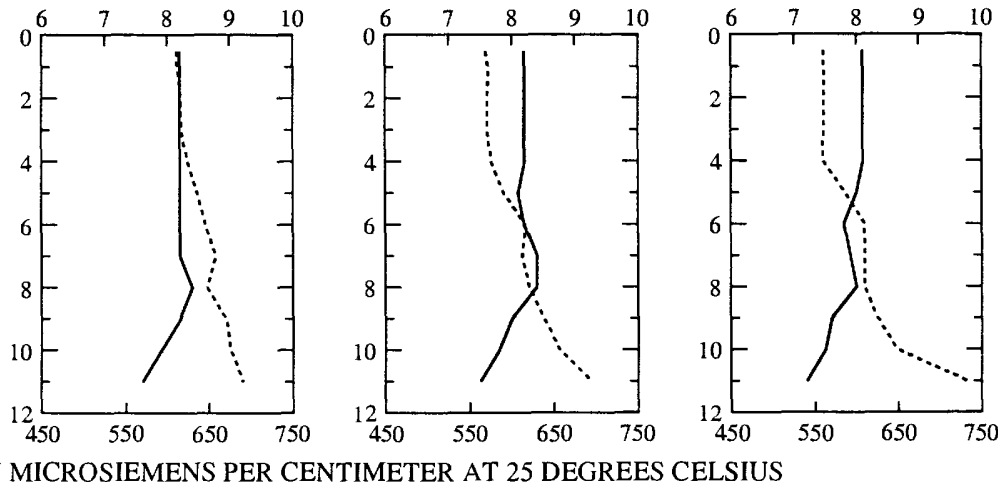

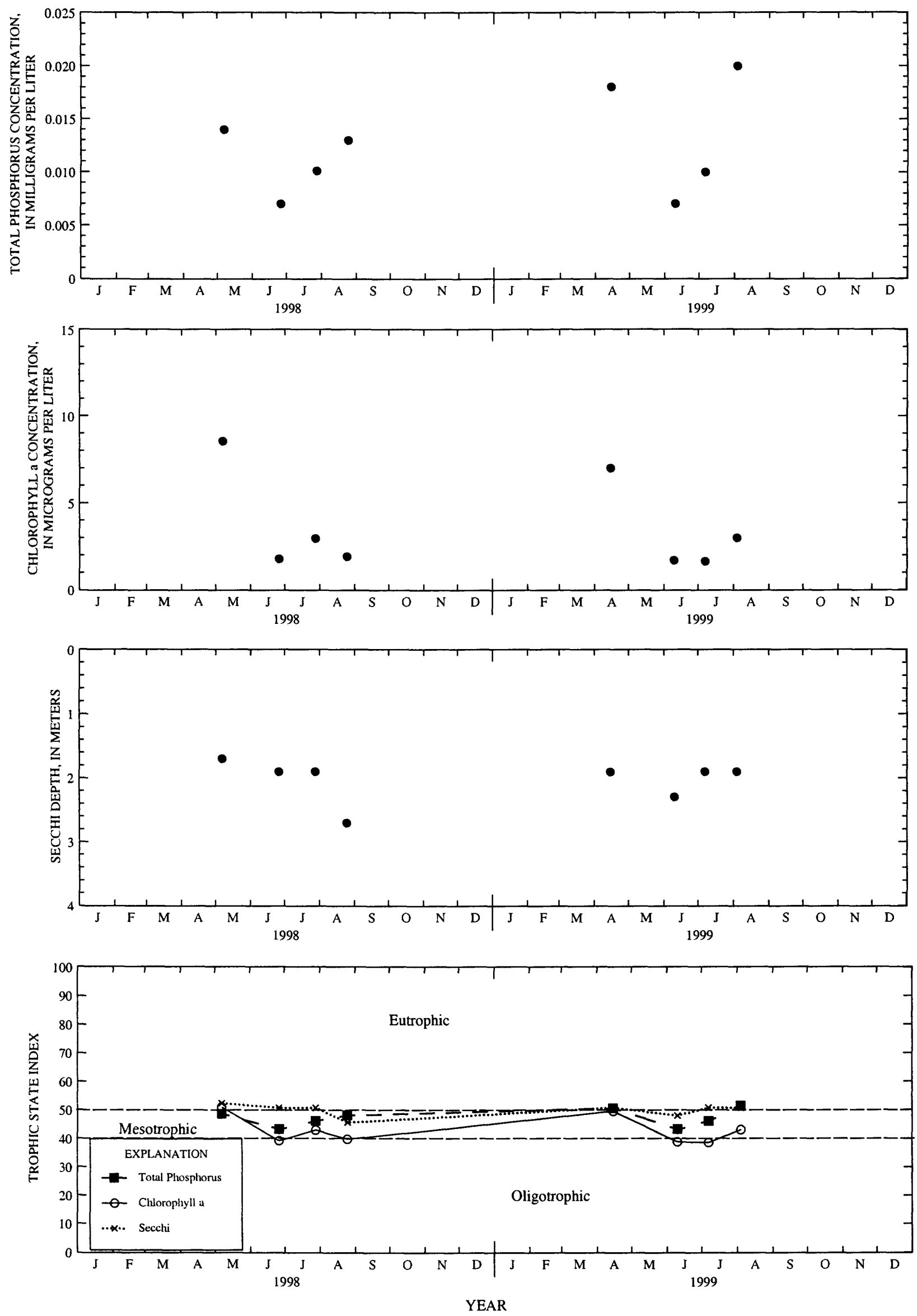

Surface total phosphorus, chlorophyll a concentrations, Secchi depths, and TSI data for Benedict Lake near Powers Lake, Wisconsin. 
LOCATION.--Lat $44^{\circ} 09^{\prime} 12^{\prime \prime}$, long $89^{\circ} 09^{\prime 20}$ ", in SW 1/4 NE 1/4 sec.2, T.19 N., R.11 E., Waushara County, Hydrologic Unit 04030202 , 4.6 mi southeast of Wild Rose.

DRAINAGE AREA.--0.78 $\mathrm{mi}^{2}$

PERIOD OF RECORD.--June 1983 to August 1984, February to August 1987, February to August 1990, February to August 1993, February to August 1996, and February to August 1999.

REMARKS.--Lake sampled at east end at deep hole. Lake ice-covered during February sampling. Water-quality analyses done by Wisconsin State Laboratory of Hygiene.

WATER-QUALITY DATA, FEBRUARY 23 TO AUGUST 18, 1999

(Milligrams per liter unless otherwise indicated)

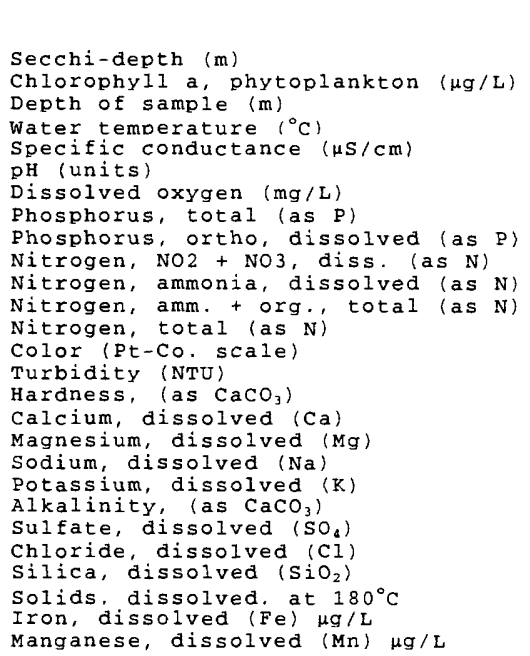

\begin{tabular}{|c|c|c|c|}
\hline $\mathrm{Fe}$ & & Ap & 28 \\
\hline & & & \\
\hline 0.5 & 5.5 & 0.5 & 5.5 \\
\hline 3.9 & 4.3 & 11.7 & 11.6 \\
\hline 250 & 269 & 233 & 234 \\
\hline 7.1 & 7.2 & 8.3 & 8.3 \\
\hline 7.1 & 6.6 & 12.3 & 12.1 \\
\hline 0.006 & 0.010 & 0.008 & 0.016 \\
\hline$\cdots$ & -- & $<0.002$ & $\cdots$ \\
\hline$-\cdots$ & $-\ldots$ & 0.113 & $-\ldots$ \\
\hline$-\cdots$ & $\ldots$ & 0.436 & $-\cdots$ \\
\hline--- & $\ldots$ & 1.0 & $\ldots$ \\
\hline-- & -- & 1.1 & -- \\
\hline$\ldots$ & $\ldots$ & 10 & $\ldots$ \\
\hline--- & $\cdots$ & 1.1 & $\cdots$ \\
\hline-- & -- & 110 & $-\ldots$ \\
\hline-- & -- & 23 & -- \\
\hline-- & --- & 13 & $-\cdots$ \\
\hline$\ldots$ & $\ldots$ & 1.8 & $\ldots$ \\
\hline-- & $\ldots$ & $<0.30$ & $\cdots$ \\
\hline-- & -- & 102 & $\ldots$ \\
\hline-- & -- & 5.9 & $\ldots$ \\
\hline-- & -- & 5.7 & -- \\
\hline$\ldots$ & -- & 0.7 & -- \\
\hline-- & - - & 126 & $-\ldots$ \\
\hline 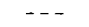 & $\cdots$ & $<10$ & $\cdots$ \\
\hline- & -- & 0.8 & -- \\
\hline
\end{tabular}

\begin{tabular}{|c|c|}
\hline & \\
\hline 0.5 & 5.5 \\
\hline 21.5 & 21.0 \\
\hline 224 & 232 \\
\hline 8.2 & 7.9 \\
\hline 8.7 & 6.8 \\
\hline 0.010 & 0.015 \\
\hline$\ldots$ & $\cdots$ \\
\hline - - - & $\ldots$ \\
\hline$\cdots$ & $\cdots$ \\
\hline$\ldots$ & $\ldots$ \\
\hline$\ldots$ & $\ldots$ \\
\hline$\ldots$ & $\ldots$ \\
\hline$\ldots$ & $\ldots$ \\
\hline-- & $\ldots$ \\
\hline--- & $-\ldots$ \\
\hline-- & $-\cdots$ \\
\hline--- & --- \\
\hline-- & $\cdots$ \\
\hline$\ldots$ & $\ldots$ \\
\hline$\ldots$ & $\ldots$ \\
\hline$\ldots$ & $-\ldots$ \\
\hline$\cdots$ & $\cdots$ \\
\hline$-\bar{s}$ & -- \\
\hline
\end{tabular}

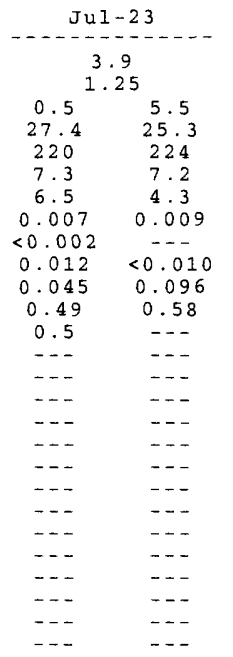

\begin{tabular}{|c|c|}
\hline \multicolumn{2}{|c|}{ Aug -18} \\
\hline \multicolumn{2}{|c|}{$\begin{array}{l}3.86 \\
0.500\end{array}$} \\
\hline $\begin{array}{c}0.5 \\
24.0 \\
229 \\
7.4 \\
6.2\end{array}$ & $\begin{array}{c}5.5 \\
24.0 \\
228 \\
7.3 \\
5.2\end{array}$ \\
\hline 0.013 & 0.012 \\
\hline$\cdots$ & $\cdots$ \\
\hline--- & --- \\
\hline--- & -- \\
\hline-- & -- \\
\hline$\ldots$ & $\ldots$ \\
\hline-- & -- \\
\hline--- & $\cdots$ \\
\hline$\cdots$ & - \\
\hline-- & $--n$ \\
\hline-- & --- \\
\hline--- & $\ldots$ \\
\hline--- & $\cdots$ \\
\hline--- & $\cdots$ \\
\hline-1 & -1 \\
\hline & \\
\hline- & \\
\hline
\end{tabular}

DISSOLVED OXYGEN (D.O.), IN MILLIGRAMS PER LITER
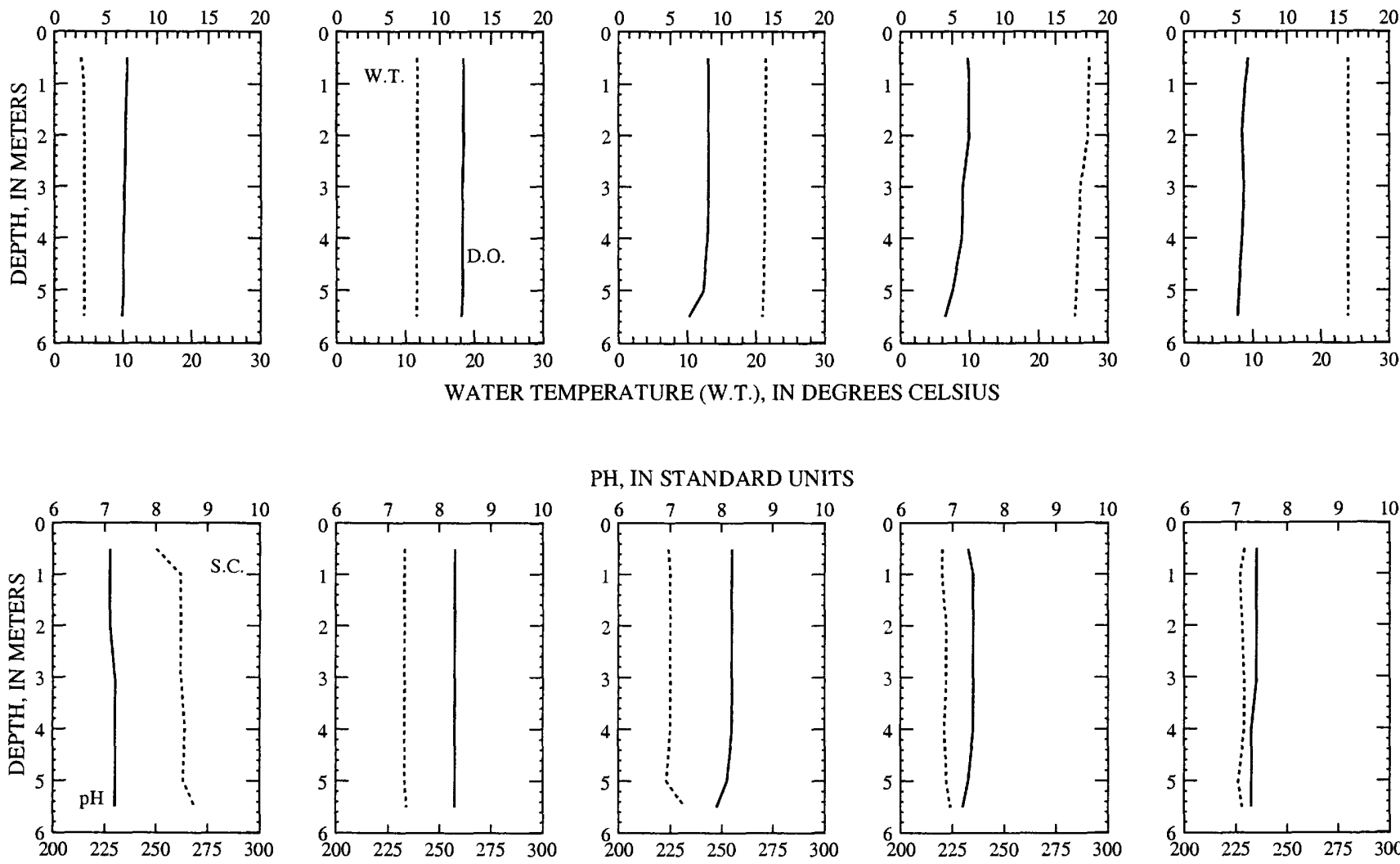

PH, IN STANDARD UNITS
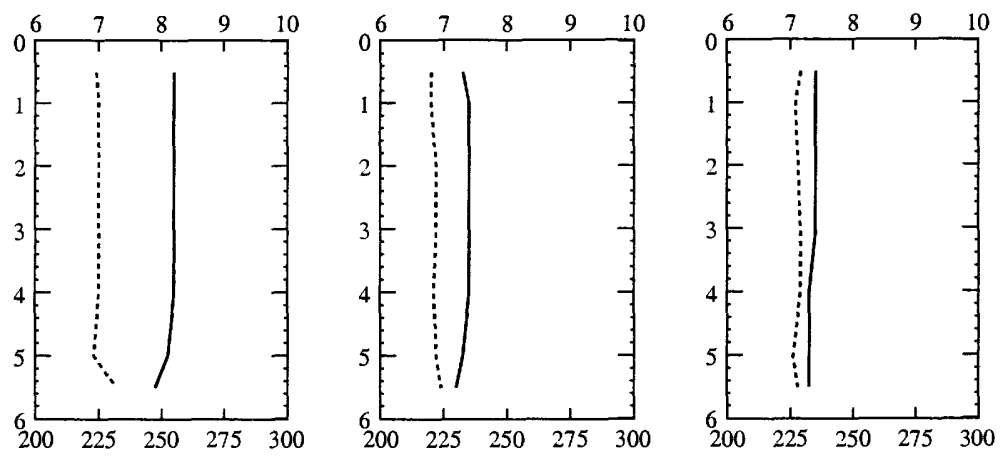

SPECIFIC CONDUCTANCE (S.C.), IN MICROSIEMENS PER CENTIMETER AT 25 DEGREES CELSIUS 

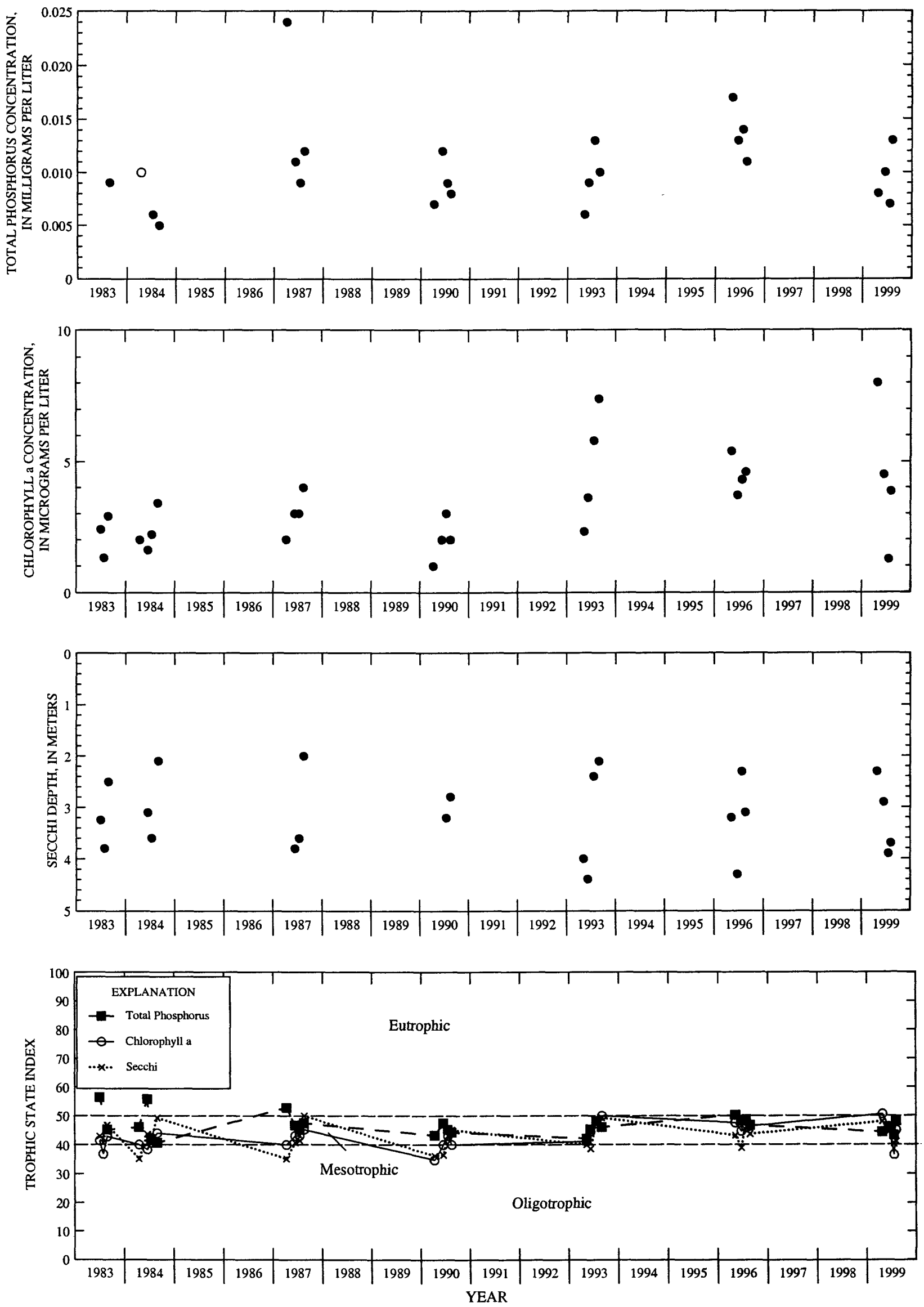

Surface total phosphorus, chlorophyll a concentrations, Secchi depths, and TSI data for Big Hills Lake near Wild Rose, Wisconsin.

(Circles on the first three plots indicate laboratory detection limit for selected analyses.

Actual concentrations for these particular analyses are less than the plotted circles.) 


\section{BIG ST. GERMAIN LAKE NEAR LAKE TOMAHAWK, WI}

LOCATION.--Lat $45^{\circ} 55^{\prime} 00^{\prime \prime}$, long $89^{\circ} 31^{\prime} 55^{\prime \prime}$ in NE 1/4 SE 1/4 sec.30. T.40 N., R.8 E., Vilas County, Hydrologic Unit 07070001, at dam outlet. 7.7 mi northeast of Lake Tomahawk.

DRAINAGE AREA.--73.1 $\mathrm{mi}^{2}$.

PERIOD OF RECORD.--October 1991 to current year. Lake stages for previous years were recorded by Wisconsin Valley Improvement Company.

GAGE.--Nonrecording gage. Datum of gage is $1,580 \mathrm{ft}$, above sea level.

COOPERATION.--Lake stages provided by Wisconsin Valley Improvement Company.

EXTREMES FOR PERIOD OF RECORD.--Maximum gage height observed, $10.98 \mathrm{ft}$, May 9, 1999; minimum observed, $8.16 \mathrm{ft}$, Jan. 26. 1996.

EXTREMES FOR CURRENT YEAR.--Maximum gage height observed, $10.98 \mathrm{ft}$, May 9; minimum observed, $8.84 \mathrm{ft}$, Jan. 25.

GAGE HEIGHT, FEET, WATER YEAR OCTOBER 1998 TO SEPTEMBER 1999

DAILY MEAN VALUES

\begin{tabular}{|c|c|c|c|c|c|c|c|c|c|c|c|c|}
\hline DAY & OCT & NOV & DEC & JAN & FEB & MAR & APR & MAY & JUN & JUL & AUG & SEP \\
\hline $\begin{array}{l}1 \\
2 \\
3 \\
4 \\
5\end{array}$ & $\begin{array}{l}10.56 \\
10.56 \\
10.58 \\
10.56 \\
10.54\end{array}$ & $\begin{array}{l}10.46 \\
10.38 \\
10.34 \\
10.30 \\
10.26\end{array}$ & $\begin{array}{c}9.46 \\
-\ldots \\
--38 \\
-\end{array}$ & $\begin{array}{r}9.02 \\
--- \\
--- \\
9.00\end{array}$ & $\begin{array}{r}9.02 \\
-.- \\
--- \\
9.04\end{array}$ & $\begin{array}{r}9.10 \\
9.05 \\
--- \\
-- \\
9.04\end{array}$ & $\begin{array}{l}8.96 \\
9.00 \\
9.12 \\
9.26 \\
9.32\end{array}$ & $\begin{array}{l}10.52 \\
10.54 \\
10.54 \\
10.54 \\
10.54\end{array}$ & $\begin{array}{l}10.50 \\
10.50 \\
10.48 \\
10.50 \\
10.50\end{array}$ & $\begin{array}{l}10.62 \\
10.66 \\
10.72 \\
10.72 \\
10.78\end{array}$ & $\begin{array}{l}10.48 \\
10.48 \\
10.48 \\
10.50 \\
10.52\end{array}$ & $\begin{array}{l}10.48 \\
10.48 \\
10.48 \\
10.48 \\
10.50\end{array}$ \\
\hline $\begin{array}{r}6 \\
7 \\
8 \\
9 \\
10\end{array}$ & $\begin{array}{l}10.62 \\
10.62 \\
10.62 \\
10.64 \\
10.64\end{array}$ & $\begin{array}{r}10.20 \\
10.12 \\
--- \\
10.00\end{array}$ & $\begin{array}{r}--- \\
9.34 \\
--- \\
---\end{array}$ & 8.98 & $\begin{array}{c}--- \\
--- \\
-\overline{-0} \\
.--\end{array}$ & 9.03 & $\begin{array}{l}9.40 \\
9.50 \\
9.58 \\
9.62 \\
9.66\end{array}$ & $\begin{array}{l}10.70 \\
10.88 \\
10.92 \\
10.98 \\
10.94\end{array}$ & $\begin{array}{l}10.62 \\
10.62 \\
10.60 \\
10.60 \\
10.60\end{array}$ & $\begin{array}{l}10.80 \\
10.76 \\
10.72 \\
10.94 \\
10.82\end{array}$ & $\begin{array}{l}10.52 \\
10.50 \\
10.50 \\
10.50 \\
10.48\end{array}$ & $\begin{array}{l}10.52 \\
10.52 \\
10.52 \\
10.52 \\
10.52\end{array}$ \\
\hline $\begin{array}{l}11 \\
12 \\
13 \\
14 \\
15\end{array}$ & $\begin{array}{l}10.64 \\
10.64 \\
10.64 \\
10.62 \\
10.62\end{array}$ & $\begin{array}{r}--- \\
9.98 \\
--\overline{8} \\
9.86 \\
---\end{array}$ & $\begin{array}{c}9.28 \\
--- \\
--- \\
-- \\
9.24\end{array}$ & $\begin{array}{r}--\overline{-} \\
8.96 \\
--- \\
--\overline{8} \\
8.96\end{array}$ & $\begin{array}{c}-.- \\
9.06 \\
--- \\
---\end{array}$ & $\begin{array}{c}--- \\
9.01 \\
--- \\
---\end{array}$ & $\begin{array}{l}9.68 \\
9.72 \\
9.78 \\
9.82 \\
9.86\end{array}$ & $\begin{array}{l}10.90 \\
10.86 \\
10.80 \\
10.76 \\
10.72\end{array}$ & $\begin{array}{l}10.62 \\
10.62 \\
10.68 \\
10.56 \\
10.52\end{array}$ & $\begin{array}{l}10.78 \\
10.70 \\
10.66 \\
10.58 \\
10.52\end{array}$ & $\begin{array}{l}10.48 \\
10.50 \\
10.70 \\
10.67 \\
10.67\end{array}$ & $\begin{array}{l}10.54 \\
10.56 \\
10.56 \\
10.56 \\
10.58\end{array}$ \\
\hline $\begin{array}{l}16 \\
17 \\
18 \\
19 \\
20\end{array}$ & $\begin{array}{l}10.62 \\
10.64 \\
10.64 \\
10.66 \\
10.66\end{array}$ & $\begin{array}{r}--- \\
9.82 \\
--- \\
9.74 \\
-.-\end{array}$ & $\begin{array}{r}--- \\
--- \\
--- \\
---\end{array}$ & $\begin{array}{r}--- \\
--- \\
--- \\
8.92 \\
---\end{array}$ & $\begin{array}{r}9.10 \\
- \\
-- \\
9.14 \\
---\end{array}$ & $\begin{array}{r}8.98 \\
--0 \\
8.97 \\
---\end{array}$ & $\begin{array}{r}9.90 \\
9.94 \\
9.98 \\
9.98 \\
10.10\end{array}$ & $\begin{array}{l}10.68 \\
10.66 \\
10.76 \\
10.74 \\
10.68\end{array}$ & $\begin{array}{l}10.50 \\
10.48 \\
10.44 \\
10.40 \\
10.42\end{array}$ & $\begin{array}{l}10.54 \\
10.54 \\
10.52 \\
10.54 \\
10.56\end{array}$ & $\begin{array}{l}10.70 \\
10.68 \\
10.66 \\
10.66 \\
10.64\end{array}$ & $\begin{array}{l}10.58 \\
10.56 \\
10.56 \\
10.55 \\
10.56\end{array}$ \\
\hline $\begin{array}{l}21 \\
22 \\
23 \\
24 \\
25\end{array}$ & $\begin{array}{l}10.68 \\
10.70 \\
10.68 \\
10.66 \\
10.68\end{array}$ & $\begin{array}{r}--- \\
9.66 \\
--- \\
9.62 \\
---\end{array}$ & $\begin{array}{r}9.12 \\
--- \\
--- \\
9.08 \\
---\end{array}$ & $\begin{array}{r}--- \\
8.92 \\
--- \\
8.84\end{array}$ & $\begin{array}{r}--- \\
--- \\
9.02 \\
--- \\
---\end{array}$ & $\begin{array}{l}--1 \\
8.98 \\
8.96 \\
8.96 \\
8.98\end{array}$ & $\begin{array}{l}10.22 \\
10.28 \\
10.32 \\
10.34 \\
10.34\end{array}$ & $\begin{array}{l}10.76 \\
10.76 \\
10.80 \\
10.72 \\
10.64\end{array}$ & $\begin{array}{l}10.42 \\
10.44 \\
10.46 \\
10.50 \\
10.52\end{array}$ & $\begin{array}{l}10.56 \\
10.56 \\
10.56 \\
10.56 \\
10.56\end{array}$ & $\begin{array}{l}10.62 \\
10.62 \\
10.60 \\
10.58 \\
10.56\end{array}$ & $\begin{array}{l}10.56 \\
10.56 \\
10.56 \\
10.56 \\
10.56\end{array}$ \\
\hline $\begin{array}{l}26 \\
27 \\
28 \\
29 \\
30 \\
31\end{array}$ & $\begin{array}{l}10.66 \\
10.62 \\
10.60 \\
10.58 \\
10.54 \\
10.48\end{array}$ & $\begin{array}{c}9.56 \\
--- \\
--- \\
9.50 \\
\ldots- \\
\ldots-\end{array}$ & $\begin{array}{c}--- \\
--- \\
--\overline{-} \\
9.04 \\
---\end{array}$ & $\begin{array}{r}--- \\
--- \\
8.96 \\
-0 . \\
9.00\end{array}$ & $\begin{array}{c}9.10 \\
--- \\
--- \\
--- \\
--- \\
---\end{array}$ & $\begin{array}{l}8.96 \\
8.96 \\
8.94 \\
8.94 \\
8.94 \\
8.94\end{array}$ & $\begin{array}{c}10.40 \\
10.52 \\
10.50 \\
10.50 \\
10.50 \\
\ldots . .\end{array}$ & $\begin{array}{l}10.56 \\
10.52 \\
10.52 \\
10.50 \\
10.50 \\
10.50\end{array}$ & $\begin{array}{c}10.52 \\
10.54 \\
10.54 \\
10.56 \\
10.56 \\
\end{array}$ & $\begin{array}{l}10.66 \\
10.62 \\
10.58 \\
10.56 \\
10.54 \\
10.54\end{array}$ & $\begin{array}{l}10.54 \\
10.54 \\
10.50 \\
10.50 \\
10.48 \\
10.50\end{array}$ & $\begin{array}{c}10.54 \\
10.56 \\
10.58 \\
10.58 \\
10.58 \\
.--\end{array}$ \\
\hline $\begin{array}{l}\text { MEAN } \\
\text { MAX } \\
\text { MIN }\end{array}$ & $\begin{array}{l}10.62 \\
10.70 \\
10.48\end{array}$ & $\begin{array}{l}\ldots- \\
\ldots-\end{array}$ & $\begin{array}{l}--- \\
-- \\
--\end{array}$ & $\begin{array}{l}--- \\
--- \\
---\end{array}$ & $\begin{array}{l}--- \\
--- \\
---\end{array}$ & $\begin{array}{l}--- \\
-- \\
--\end{array}$ & $\begin{array}{r}9.87 \\
10.52 \\
8.96\end{array}$ & $\begin{array}{l}10.69 \\
10.98 \\
10.50\end{array}$ & $\begin{array}{l}10.53 \\
10.68 \\
10.40\end{array}$ & $\begin{array}{l}10.64 \\
10.94 \\
10.52\end{array}$ & $\begin{array}{l}10.56 \\
10.70 \\
10.48\end{array}$ & $\begin{array}{l}10.54 \\
10.58 \\
10.48\end{array}$ \\
\hline
\end{tabular}




\section{BIG SISSABAGAMA LAKE NEAR STONE LAKE, WI}

LOCATION.--Lat 4547'24", long 9I $30^{\prime} 36^{\prime \prime}$, in NW 1/4 SE 1/4 sec.6, T.38 N., R.9 W., Sawyer County, Hydrologic Unit 07050001, near Stone Lake.

DRAINAGE AREA.--9.47 $\mathrm{mi}^{2}$

\section{LAKE-STAGE RECORDS}

PERIOD OF RECORD.--1986 to 1996, and October 1997 to current year, during open-water periods.

GAGE.--Water surface measured from reference point near lake outlet. Measurements were made by Richard Roehrich and James Eary.

EXTREMES FOR PERIOD OF RECORD: Maximum gage height observed, $6.09 \mathrm{ft}$, May 7 and Sept. 15, 1991; minimum observed, $4.78 \mathrm{ft}$, Sept. 15, 16, 1988

EXTREMES FOR CURRENT YEAR.--Maximum gage height observed, $5.95 \mathrm{ft}$, June 11; minimum observed, less than $5.07 \mathrm{ft}$, Oct. 1-16 (stages below $5.07 \mathrm{ft}$ cannot be measured from reference point).

GAGE HEIGHT, FEET, WATER YEAR OCTOBER 1998 TO SEPTEMBER 1999 DAILY MEAN VALUES

\begin{tabular}{|c|c|c|c|c|c|c|c|c|c|c|c|c|}
\hline DAY & OCT & Nov & $\mathrm{DEC}$ & JAN & FEB & MAR & APR & MAY & JUN & JUL & AUG & SEP \\
\hline $\begin{array}{l}1 \\
2 \\
3 \\
4 \\
5\end{array}$ & $\begin{array}{l}<5.07 \\
<5.07 \\
<5.07 \\
<5.07 \\
<5.07\end{array}$ & $\begin{array}{l}5.10 \\
5.08 \\
5.08 \\
5.08 \\
5.08\end{array}$ & $\begin{array}{l}--- \\
-- \\
-\cdots \\
\cdots-\end{array}$ & $\begin{array}{l}--- \\
-\cdots \\
-\cdots \\
--- \\
--\end{array}$ & $\begin{array}{l}\cdots- \\
-\cdots \\
--- \\
--\end{array}$ & $\begin{array}{l}--- \\
--- \\
--- \\
--- \\
---\end{array}$ & $\begin{array}{l}\ldots- \\
\ldots- \\
-\cdots\end{array}$ & $\begin{array}{l}-- \\
-- \\
-\cdots \\
--\end{array}$ & $\begin{array}{l}5.78 \\
5.75 \\
5.74 \\
5.74 \\
5.75\end{array}$ & $\begin{array}{l}5.75 \\
5.75 \\
5.79 \\
5.81 \\
5.84\end{array}$ & $\begin{array}{l}5.88 \\
5.86 \\
5.91 \\
5.90 \\
5.87\end{array}$ & $\begin{array}{l}5.76 \\
5.75 \\
5.74 \\
5.82\end{array}$ \\
\hline $\begin{array}{r}6 \\
7 \\
8 \\
9 \\
10\end{array}$ & $\begin{array}{l}<5.07 \\
<5.07 \\
<5.07 \\
<5.07 \\
<5.07\end{array}$ & $\begin{array}{r}5.08 \\
5.73 \\
\ldots- \\
\ldots- \\
\ldots\end{array}$ & $\begin{array}{l}--- \\
-\cdots \\
\cdots- \\
\cdots-\end{array}$ & $\begin{array}{l}--- \\
--- \\
--- \\
---\end{array}$ & $\begin{array}{l}--- \\
--- \\
--- \\
--- \\
---\end{array}$ & $\begin{array}{l}--- \\
--- \\
--- \\
--- \\
---\end{array}$ & $\begin{array}{l}--- \\
--- \\
--- \\
---\end{array}$ & \begin{tabular}{l}
--- \\
--- \\
--- \\
\hdashline .91
\end{tabular} & $\begin{array}{l}5.84 \\
5.91 \\
5.89 \\
5.88 \\
5.88\end{array}$ & $\begin{array}{l}5.83 \\
5.80 \\
5.84 \\
5.88 \\
5.84\end{array}$ & $\begin{array}{l}5.87 \\
5.91 \\
5.88 \\
5.86 \\
5.85\end{array}$ & $\begin{array}{l}5.92 \\
5.90 \\
5.96 \\
5.94 \\
5.91\end{array}$ \\
\hline $\begin{array}{l}11 \\
12 \\
13 \\
14 \\
15\end{array}$ & $\begin{array}{l}<5.07 \\
<5.07 \\
<5.07 \\
<5.07 \\
<5.07\end{array}$ & $\begin{array}{l}5.12 \\
5.15 \\
5.12 \\
5.11 \\
5.12\end{array}$ & $\begin{array}{l}--- \\
--- \\
--- \\
--- \\
---\end{array}$ & $\begin{array}{l}-\cdots \\
-\cdots \\
-\cdots \\
---\end{array}$ & $\begin{array}{l}--- \\
--- \\
--- \\
---\end{array}$ & $\begin{array}{l}--- \\
--- \\
--- \\
--- \\
---\end{array}$ & $\begin{array}{l}-\cdots \\
--- \\
--- \\
--- \\
---\end{array}$ & $\begin{array}{l}5.93 \\
5.91 \\
5.89 \\
5.88 \\
5.87\end{array}$ & $\begin{array}{l}5.95 \\
5.93 \\
5.91 \\
5.88 \\
5.86\end{array}$ & $\begin{array}{l}5.83 \\
5.82 \\
5.87 \\
5.84 \\
5.83\end{array}$ & $\begin{array}{l}5.85 \\
5.84 \\
5.91 \\
5.90 \\
5.88\end{array}$ & $\begin{array}{l}5.89 \\
5.95 \\
5.92 \\
5.91 \\
5.89\end{array}$ \\
\hline $\begin{array}{l}16 \\
17 \\
18 \\
19 \\
20\end{array}$ & $\begin{array}{r}<5.07 \\
5.10 \\
5.13 \\
5.12 \\
5.11\end{array}$ & $\begin{array}{l}5.13 \\
5.13 \\
5.12 \\
5.12 \\
5.13\end{array}$ & $\begin{array}{l}--- \\
--- \\
--- \\
--- \\
---\end{array}$ & $\begin{array}{l}\cdots \\
-\cdots \\
--- \\
--- \\
--\end{array}$ & $\begin{array}{l}\cdots \\
\cdots \\
\cdots- \\
-\cdots\end{array}$ & $\begin{array}{l}--- \\
--- \\
--- \\
--- \\
---\end{array}$ & $\begin{array}{l}--- \\
--- \\
--- \\
---\end{array}$ & $\begin{array}{l}5.85 \\
5.88 \\
5.90 \\
5.89 \\
5.91\end{array}$ & $\begin{array}{l}5.84 \\
5.82 \\
5.80 \\
5.77 \\
5.75\end{array}$ & $\begin{array}{r}--- \\
--- \\
--- \\
5.78 \\
5.75\end{array}$ & $\begin{array}{l}5.86 \\
5.85 \\
5.85 \\
5.88 \\
5.87\end{array}$ & $\begin{array}{l}5.88 \\
5.88 \\
5.87 \\
5.87 \\
5.86\end{array}$ \\
\hline $\begin{array}{l}21 \\
22 \\
23 \\
24 \\
25\end{array}$ & $\begin{array}{l}5.11 \\
5.10 \\
5.10 \\
5.09 \\
5.11\end{array}$ & $\begin{array}{l}5.13 \\
5.14 \\
5.14 \\
5.15 \\
5.15\end{array}$ & $\begin{array}{l}--- \\
--- \\
--- \\
--- \\
---\end{array}$ & $\begin{array}{l}--- \\
--- \\
--- \\
--\end{array}$ & $\begin{array}{l}-\cdots \\
-\cdots \\
-\cdots \\
---\end{array}$ & $\begin{array}{l}-\cdots- \\
--- \\
--- \\
--- \\
---\end{array}$ & $\begin{array}{l}--- \\
--- \\
--- \\
--- \\
---\end{array}$ & $\begin{array}{l}5.91 \\
5.90 \\
5.91 \\
--- \\
---\end{array}$ & $\begin{array}{l}5.74 \\
5.74 \\
5.80 \\
5.78 \\
5.77\end{array}$ & $\begin{array}{l}5.75 \\
5.74 \\
5.72 \\
5.70 \\
5.70\end{array}$ & $\begin{array}{l}5.87 \\
5.86 \\
5.86 \\
5.90 \\
5.93\end{array}$ & $\begin{array}{l}5.84 \\
5.83 \\
5.82 \\
5.80 \\
5.78\end{array}$ \\
\hline $\begin{array}{l}26 \\
27 \\
28 \\
29 \\
30 \\
31\end{array}$ & $\begin{array}{l}5.11 \\
5.11 \\
5.11 \\
5.11 \\
5.10 \\
5.10\end{array}$ & $\begin{array}{l}5.16 \\
5.16 \\
5.16 \\
5.17 \\
5.18 \\
\ldots-\end{array}$ & $\begin{array}{l}--- \\
--- \\
--- \\
--- \\
--- \\
---\end{array}$ & $\begin{array}{l}--- \\
--- \\
--- \\
--- \\
--- \\
---\end{array}$ & $\begin{array}{l}\cdots- \\
--- \\
-- \\
-- \\
-- \\
--\end{array}$ & $\begin{array}{l}--- \\
--- \\
--- \\
--- \\
-\cdots \\
---\end{array}$ & $\begin{array}{l}--- \\
--- \\
-\cdots \\
-\cdots \\
--\end{array}$ & $\begin{array}{l}5.88 \\
5.84 \\
5.83 \\
5.83 \\
5.82 \\
5.80\end{array}$ & $\begin{array}{l}5.72 \\
5.70 \\
5.75 \\
5.74 \\
5.73 \\
-.-\end{array}$ & $\begin{array}{l}5.95 \\
5.94 \\
5.92 \\
5.90 \\
5.88 \\
5.93\end{array}$ & $\begin{array}{l}5.90 \\
5.88 \\
5.86 \\
5.83 \\
5.81 \\
5.79\end{array}$ & $\begin{array}{l}5.76 \\
5.75 \\
5.72 \\
5.69 \\
5.66 \\
-.-\end{array}$ \\
\hline $\begin{array}{l}\text { MEAN } \\
\text { MAX } \\
\text { MIN }\end{array}$ & $\begin{array}{l}5.09 \\
5.13 \\
5.07\end{array}$ & $\begin{array}{l}-- \\
-- \\
--\end{array}$ & $\begin{array}{l}--- \\
--- \\
---\end{array}$ & $\begin{array}{l}--- \\
--- \\
---\end{array}$ & $\begin{array}{l}--- \\
--- \\
---\end{array}$ & $\begin{array}{l}-\cdots \\
--- \\
-\cdots\end{array}$ & $\cdots$ & $\begin{array}{l}--- \\
--- \\
---\end{array}$ & $\begin{array}{l}5.80 \\
5.95 \\
5.70\end{array}$ & -- & $\begin{array}{l}5.87 \\
5.93 \\
5.79\end{array}$ & $\cdots$ \\
\hline
\end{tabular}




\section{WATER-QUALITY RECORDS}

PERIOD OF RECORD.--1986 to 1996, and March 1998 to current year.

REMARKS.--Lake sampled near center at the deep hole. Lake ice-covered during March sampling. Water-quality analyses done by Wisconsin State Laboratory of Hygiene.

WATER-QUALITY DATA, MARCH 04 TO AUGUST 16, 1999

(Milligrams per liter unless otherwise indicated)

Lake stage $(\mathrm{ft})$

Secchi-depth (m)

Chlorophyll a, phytoplankton ( $\mu \mathrm{g} / \mathrm{L})$

Depth of sample (m)

Water temperature $\left({ }^{\circ} \mathrm{C}\right)$

Specific conductance $(\mu \mathrm{s} / \mathrm{cm})$

$\mathrm{pH}$ (units)

Dissolved oxygen (mg/L)

Phosphorus, total (as P)

Phosphorus, ortho, dissolved (as P)

Nitrogen, $\mathrm{NO} 2+\mathrm{NO} 3$, diss. (as N)

Nitrogen, ammonia, dissolved (as N)

Nitrogen, amm. + org., total (as $\mathrm{N}$ )

Nitrogen, total (as N)

Color (Pt-Co. scale)

Turbidity (NTU)

Hardness, (as $\mathrm{CaCO}_{3}$ )

Calcium, dissolved ( $\mathrm{Ca}$ )

Magnesium, dissolved (Mg)

Sodium, dissolved (Na)

Potassium, dissolved (K)

Alkalinity, (as $\mathrm{CaCO}_{3}$ )

Sulfate, dissolved $\left(\mathrm{SO}_{4}\right)$

Chloride, dissolved (Cl)

Silica, dissolved $\left(\mathrm{SiO}_{2}\right)$

Solids, dissolved, at $180^{\circ} \mathrm{C}$

Iron, dissolved $(\mathrm{Fe}) \mu \mathrm{g} / \mathrm{L}$

Manganese, dissolved (Mn) $\mu \mathrm{g} / \mathrm{L}$

Apr -13

-.-

$\begin{array}{cc}0.5 & 13.0 \\ 0.1 & 4.1\end{array}$

$\begin{array}{ll}0.1 & 4.1 \\ 72 & 104\end{array}$

$\begin{array}{ll}7.4 & 6.8\end{array}$

$\begin{array}{ll}11.8 & 0.3\end{array}$

$0.008 \quad 0.071$

$\begin{array}{ll}0.008 & 0.071 \\ --- & -\cdots\end{array}$

$\cdots$

$\begin{array}{ll}-2 & -2 \\ -\cdots & --\end{array}$

$---\quad-\cdots$

-

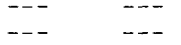

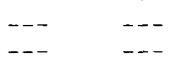

$\begin{array}{ll}-2 & -1 \\ --- & -2-\end{array}$

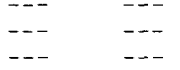

$--$

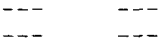

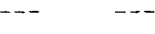

$---$

$--$

$--$
5.92

\begin{tabular}{cc} 
Apr -13 \\
\hline \multicolumn{2}{c}{5.92} \\
2.4 \\
0.5 & 14.0 \\
0.6 & 5.0 \\
69 & 69 \\
7.0 & 7.0 \\
11.4 & 10.2 \\
0.019 & 0.020
\end{tabular}

0.003

0.056

0.063

0.47

0.53

10

1.4
33

33
8.5

2.8

1.6

0.8

34

3
0.9
7.1

$\begin{array}{ll}-.- & 7.1 \\ \ldots & 56\end{array}$

$-$

220
Jun-2

5.75

1.9

9.43

$\begin{array}{ll}0.5 & 14.0\end{array}$

$\begin{array}{cc}0.5 & 14.0 \\ 16.5 & 9.3\end{array}$

$68 \quad 113$

9.10 .1

$0.023 \quad 0.159$

---

---

$---$

$--$

---
---

$---$

$---$

---

$---$

$---$

---
---
---
---
---

-.-

$--$

$\begin{array}{lll}-2 & -1 \\ -\cdots & --\end{array}$

$---\quad--$

$\cdots$

$\begin{array}{lll}- & - & - \\ -\cdots & --\end{array}$

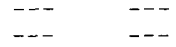

-

$\begin{array}{lll}--- & -1 \\ --- & ---\end{array}$

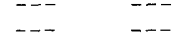

- n

$\begin{array}{cc}--- \\ --- & -1-\end{array}$

DISSOLVED OXYGEN (D.O.), IN MILLIGRAMS PER LITER
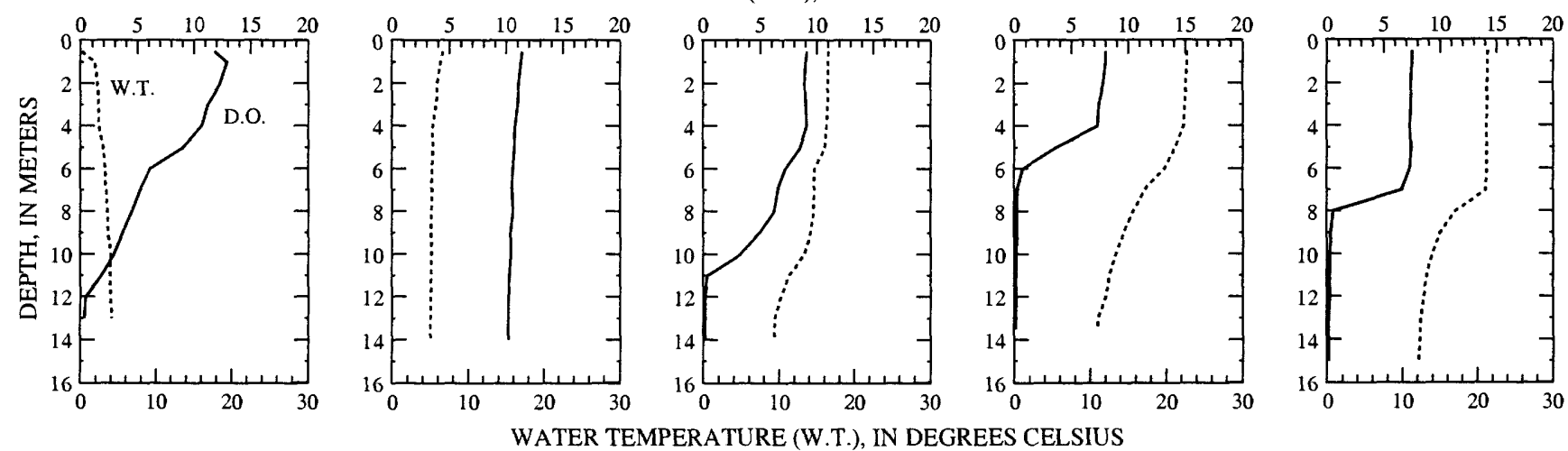

PH, IN STANDARD UNITS
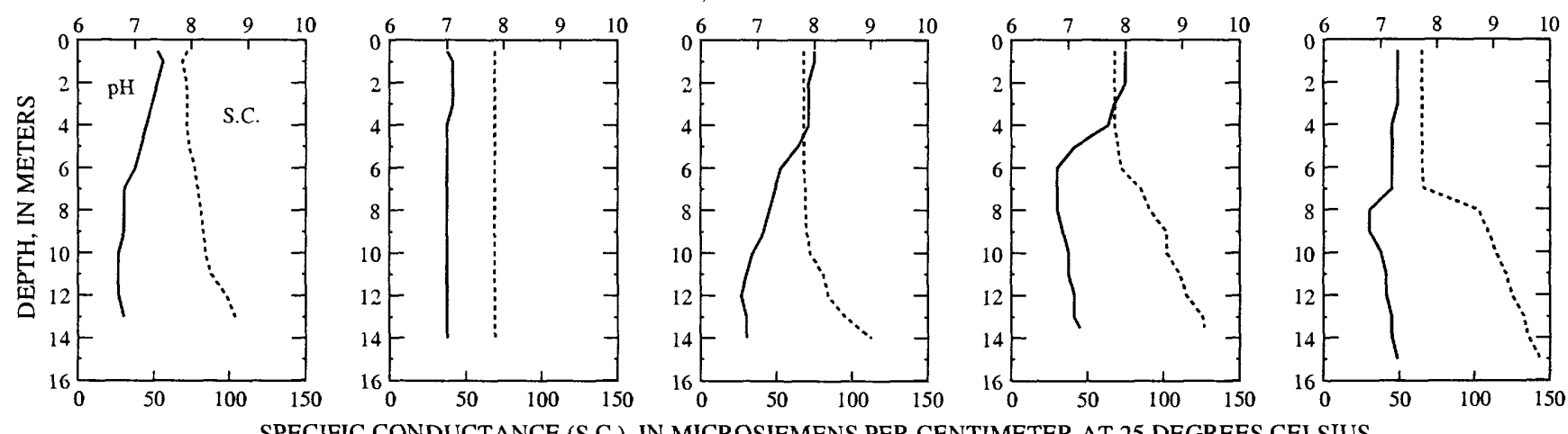

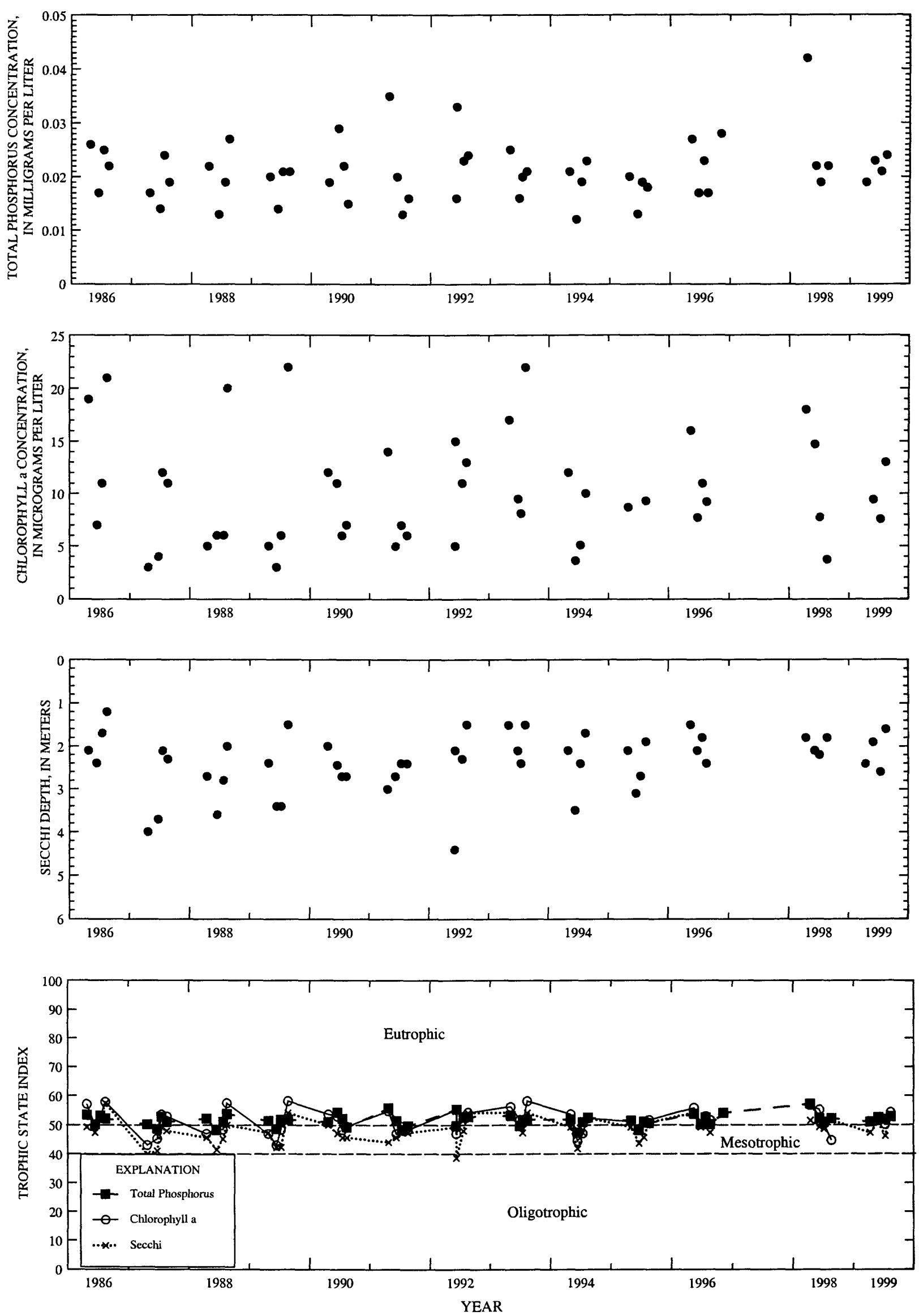

Surface total phosphorus, chlorophyll a concentrations, Secchi depths, and TSI data for Big Sissabagama Lake near Stone Lake, Wisconsin. 
LOCATION.--Lat 42 48'00", long 91'31'29", in NE 1/4 SE 1/4 sec.6, T.38 N., R.9 W., Sawyer County, Hydrologic Unit 07050001, near Stone Lake.

DRAINAGE AREA.--9.47 $\mathrm{mi}^{2}$.

PERIOD OF RECORD.--March 1998 to current year.

REMARKS.--Lake sampled near the deepest part of the North Bay. Lake ice-covered during March sampling. Water-quality analyses done by Wisconsin State Laboratory of Hygiene.

WATER-QUALITY DATA, MARCH 04 TO AUGUST 16, 1999

(Milligrams per liter unless otherwise indicated)

Lake stage (ft)

Secchi-depth (m)

Chlorophyll a, phytoplankton $(\mu \mathrm{g} / \mathrm{L})$

Depth of sample (m)

Water temperature $\left({ }^{\circ} \mathrm{C}\right)$

Specific conductance $(\mu \mathrm{S} / \mathrm{cm})$

$\mathrm{pH}$ (units)

Dissolved oxygen (mg/L)

Phosphorus, total (as P)

\begin{tabular}{ccc} 
Mar-4 & Apr-13 & Jun-2 \\
\hdashline-- & 5.92 & 5.75 \\
-- & 2.6 & 1.9 \\
-- & 3.28 & 5.88 \\
0.5 & 0.5 & 0.5 \\
0.7 & 8.0 & 16.1 \\
76 & 68 & 68 \\
7.5 & 7.4 & 7.8 \\
14.0 & 12.0 & 8.6 \\
0.014 & 0.014 & 0.017
\end{tabular}

0.017

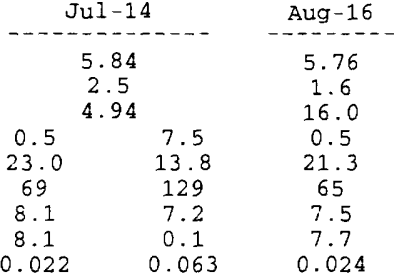

7-14-99

8-16-99

DISSOLVED OXYGEN (D.O.), IN MILLIGRAMS PER LITER
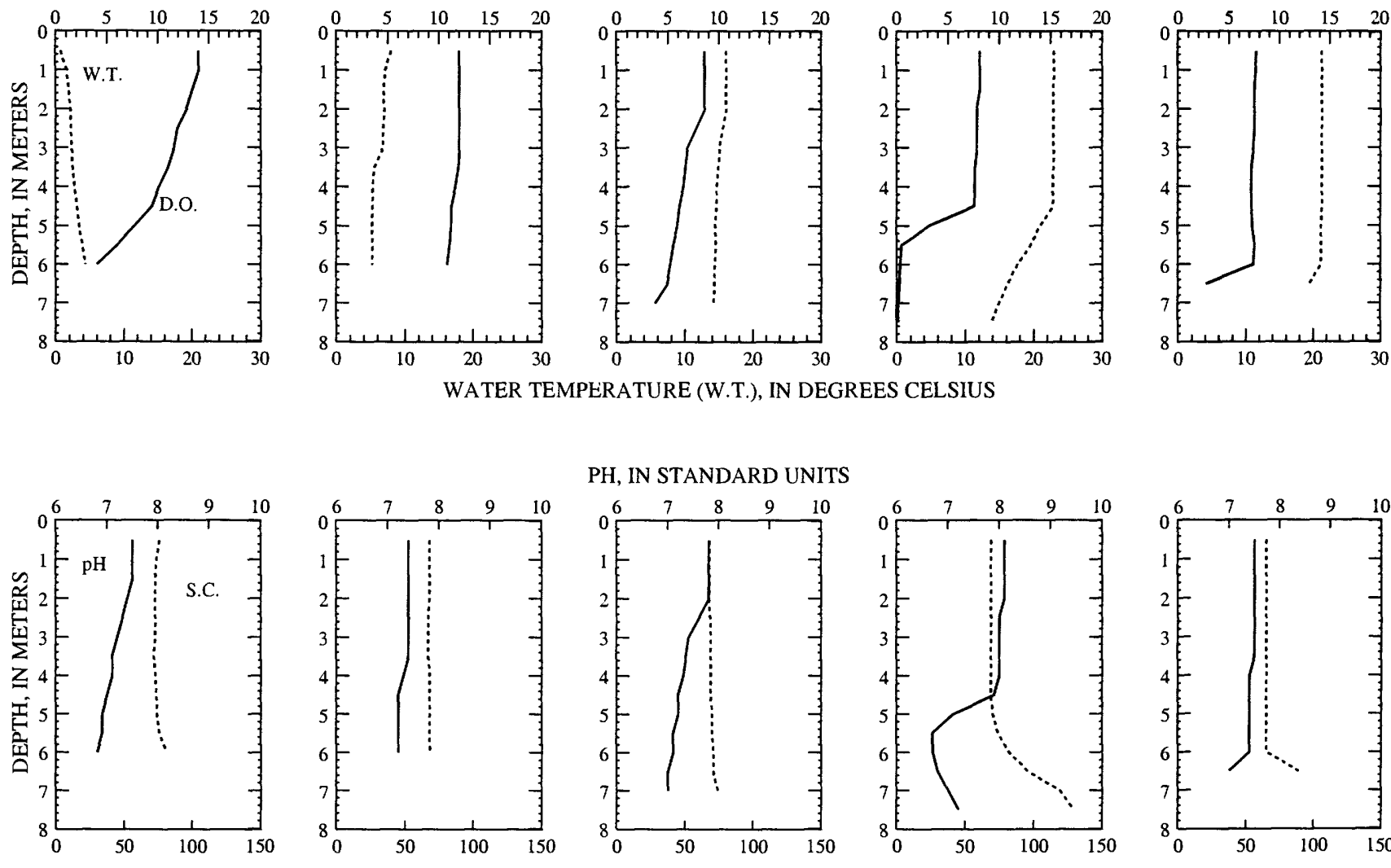

PH, IN STANDARD UNITS
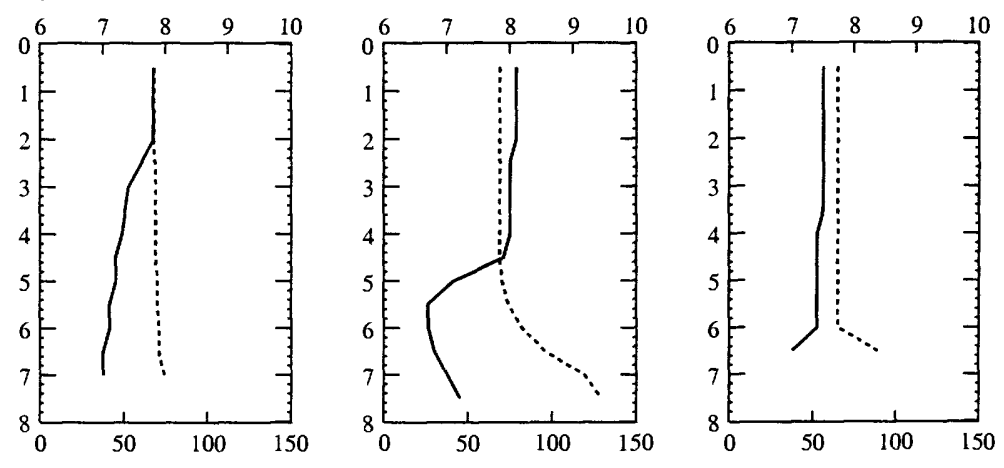

SPECIFIC CONDUCTANCE (S.C.), IN MICROSIEMENS PER CENTIMETER AT 25 DEGREES CELSIUS 

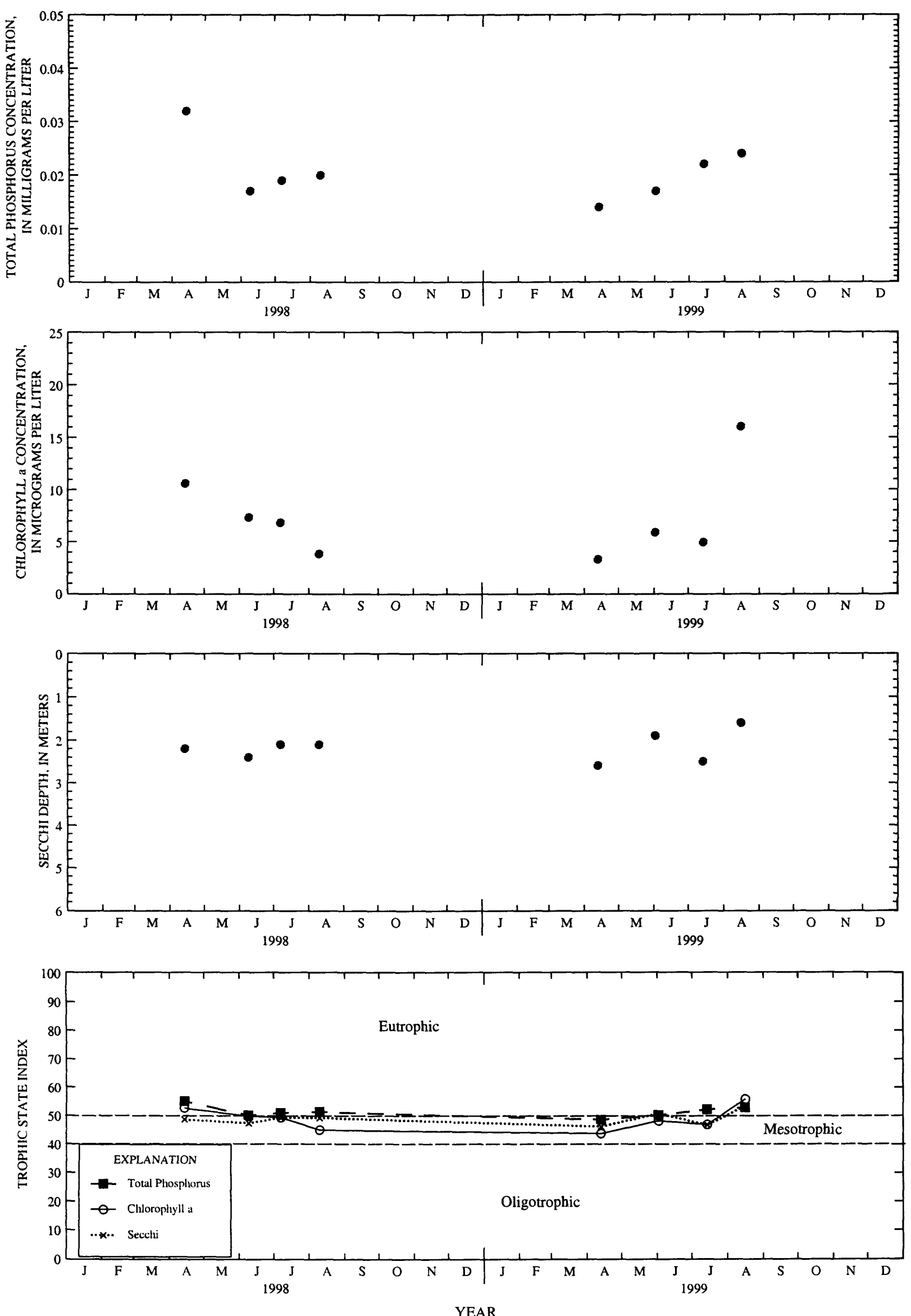

Surface total phosphorus, chlorophyll a concentrations, Secchi depths, and TSI data for Big Sissabagama Lake, North Site, near Stone Lake, Wisconsin. 
LOCATION.--Lat $43^{\circ} 45^{\prime} 58^{\prime \prime}$, long $89^{\circ} 26^{\prime} 06^{\prime \prime}$, in NW 1/4 SE 1/4 sec.21, T.15 N., R.9 E., Marquette County, Hydrologic Unit 04030201, $1.1 \mathrm{mi}$ northeast of Packwaukee.

PERIOD OF RECORD.--May 1998 to current year. Data collected 1991-94 by Wisconsin Department of Natural Resources are available.

REMARKS.--Site sampled near center of lake. Lake ice-covered during February sampling. Water-quality analyses done by Wisconsin State Laboratory of Hygiene.

WATER-QUALITY DATA, FEBRUARY 26 TO AUGUST 19, 1999

(Milligrams per liter unless otherwise indicated)

Secchi-depth (m)

Chlorophyll a, phytoplankton ( $\mu \mathrm{g} / \mathrm{L}$ )

Depth of sample $(m)$

Water temperature $\left({ }^{\circ} \mathrm{C}\right)$

Specific conductance $(\mu \mathrm{S} / \mathrm{cm})$

$\mathrm{pH}$ (units)

Dissolved oxygen (mg/L)

Phosphorus, total (as P)

Phosphorus, ortho, dissolved (as P)

Nitrogen, NO2 + NO3, diss. (as N)

Nitrogen, ammonia, dissolved (as N)
Nitrogen, amm. + org. total (as N)

Nitrogen, amm. + org.

Color (Pt-Co. scale)

Turbidity (NTU)

Hardness, (as $\mathrm{CaCO}_{3}$ )

Calcium, dissolved (Ca)

Magnesium, dissolved (Mg)

Sodium, dissolved (Na)

Potassium, dissolved (K)

Alkalinity, (as $\mathrm{CaCO}_{3}$ )

sulfate, dissolved $\left(\mathrm{SO}_{4}\right)$

Chloride, dissolved (CI)

Chloride, dissolved ( $\mathrm{Cl})$
Silica, dissolved ( $\left.\mathrm{SiO}_{2}\right)$

Solids, dissolved, at $180^{\circ} \mathrm{C}$

Iron, dissolved (Fe) $\mu \mathrm{g} / \mathrm{L}$

Manganese, dissolved (Mn) $\mu g / L$

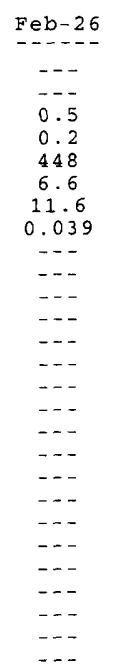

$+-$

\begin{tabular}{l} 
May-3 \\
\hdashline 0.7 \\
18.8 \\
0.5 \\
18.7 \\
371 \\
8.5 \\
13.6 \\
0.069 \\
0.003 \\
0.399 \\
0.021 \\
1.2 \\
1.6 \\
30 \\
6.9 \\
190 \\
38 \\
22 \\
4.9 \\
1.2 \\
166 \\
18 \\
10 \\
2.2 \\
230 \\
60 \\
4.6
\end{tabular}

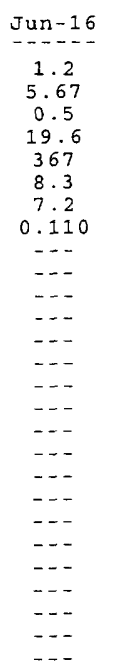

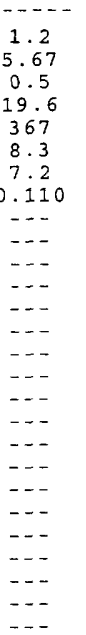

7-15-99

8-19-99

DISSOLVED OXYGEN (D.O.), IN MILLIGRAMS PER LITER
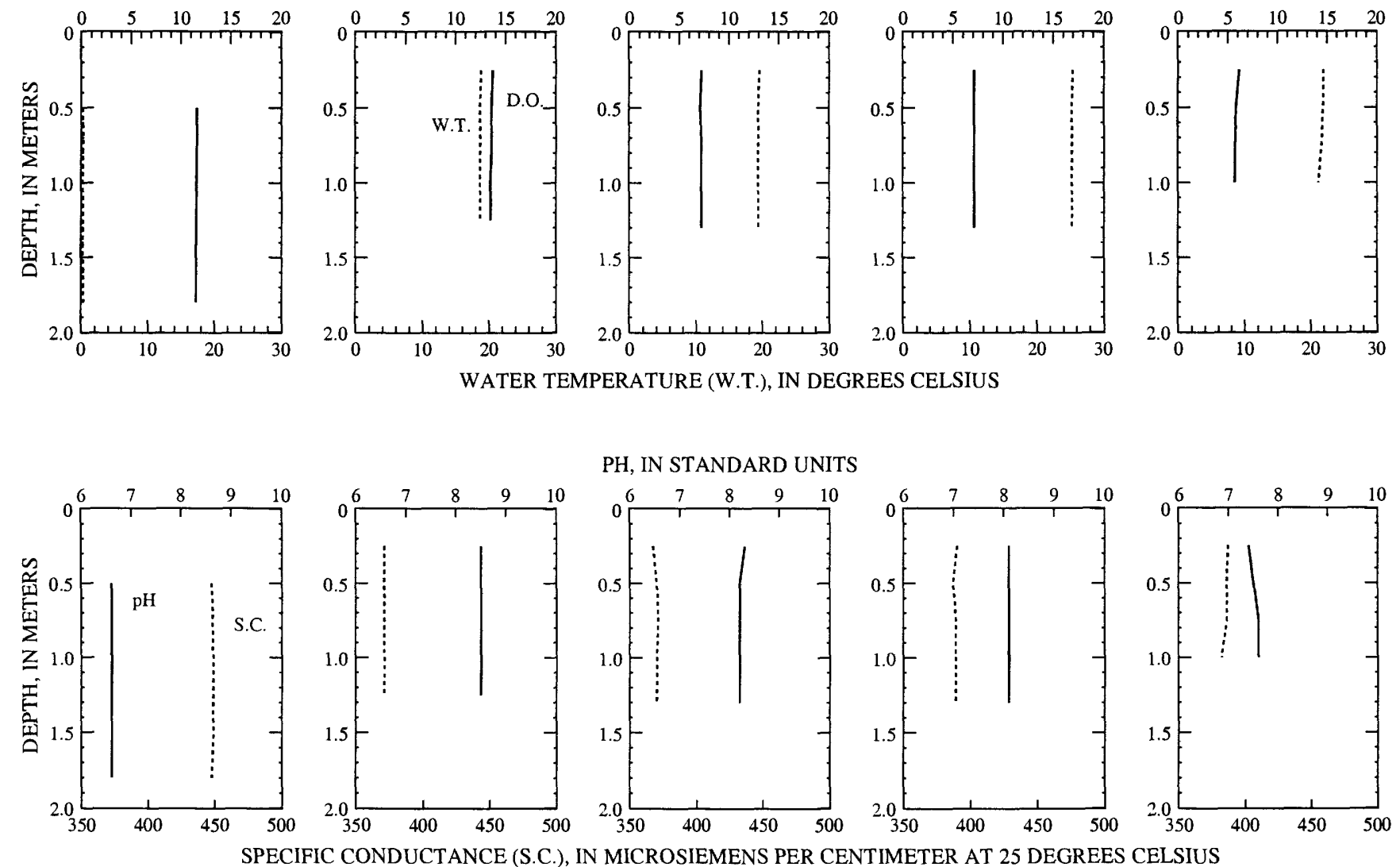

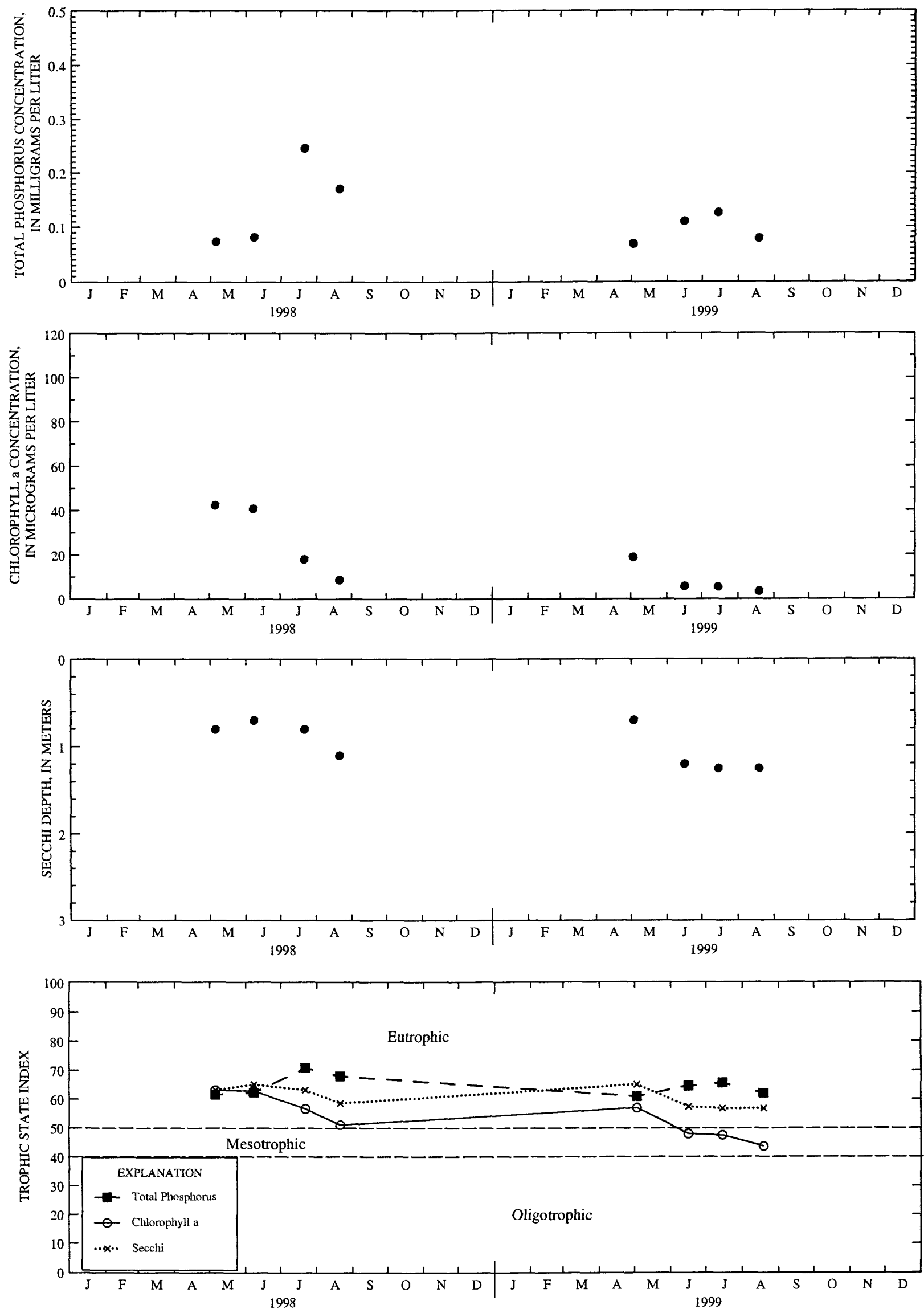

YEAR

Surface total phosphorus, chlorophyll a concentrations, Secchi depths, and TSI data for Buffalo Lake, Center Site, near Packwaukee, Wisconsin. 

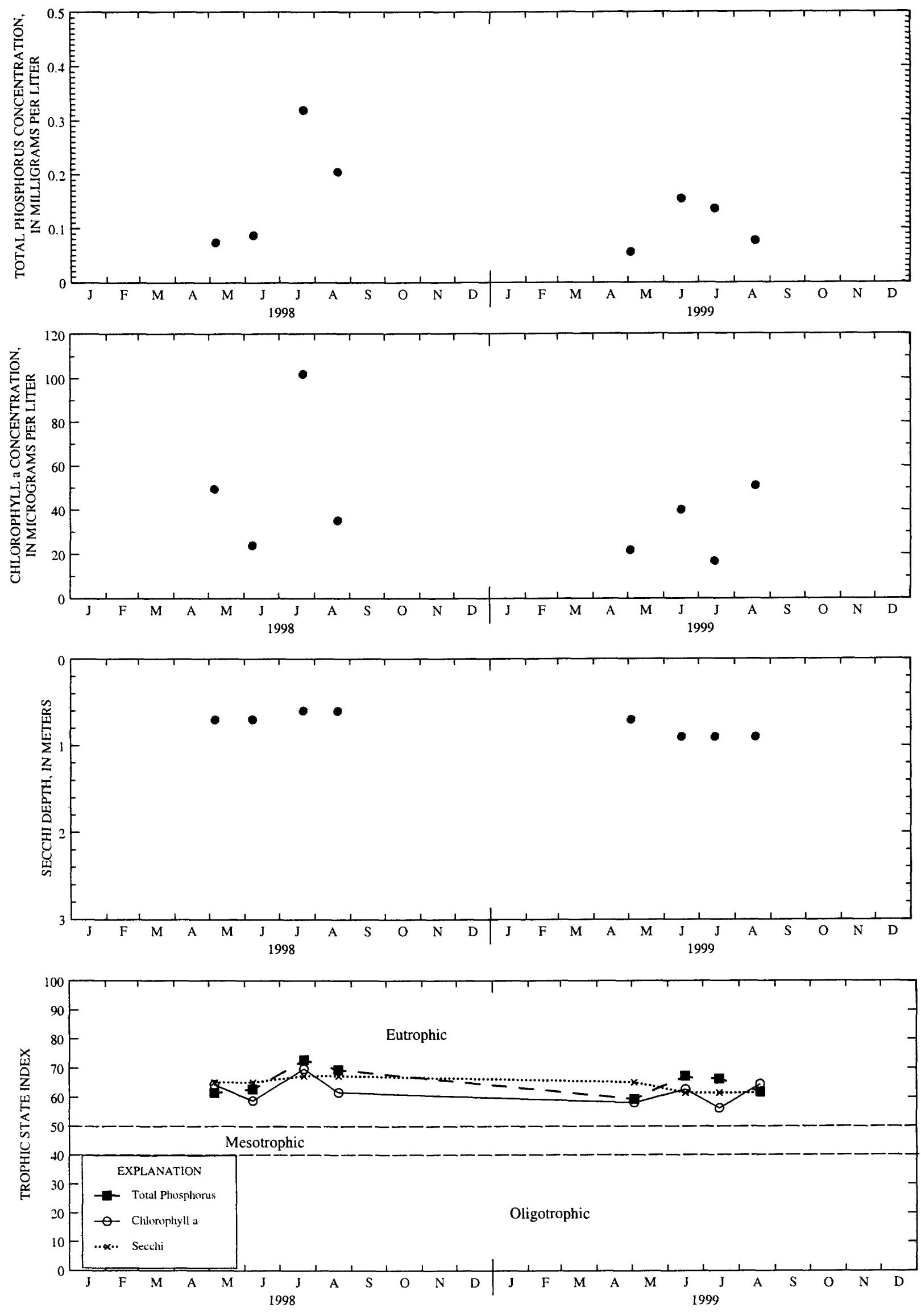

YEAR

Surface total phosphorus, chlorophyll a concentrations, Secchi depths, and TSI data for Buffalo Lake, East Site, at Montello, Wisconsin. 
LOCATION.--Lat $43^{\circ} 44^{\prime} 14^{\prime \prime}$, long $89^{\circ} 28^{\prime} 24^{\prime \prime}$, in NW 1/4 SE $1 / 4$ sec.31, T.15 N., R.9 E., Marquette County, Hydrologic Unit 04030201 , 1.5 mi north of Endeavor.

PERIOD OF RECORD.--May 1998 to current year. Data collected 1991-94 by Wisconsin Department of Natural Resources are available. REMARKS.--Site sampled near west end of lake. Lake ice-covered during February sampling. Water-quality analyses done by Wisconsin State Laboratory of Hygiene.

WATER-QUALITY DATA, FEBRUARY 26 TO AUGUST 19, 1999

(Milligrams per liter unless otherwise indicated)

\begin{tabular}{|c|c|c|c|c|c|}
\hline & Feb-26 & May -3 & Jun-16 & Jul-15 & Aug-19 \\
\hline Secchi-depth (m) & --- & 0.6 & 0.8 & 0.5 & 0.9 \\
\hline Chlorophyll a, phytoplankton $(\mu \mathrm{g} / \mathrm{L})$ & --- & 43.9 & 8.86 & 30.7 & 11.0 \\
\hline Depth of sample $(\mathrm{m})$ & 0.5 & 0.5 & 0.5 & 0.5 & 0.5 \\
\hline Water temperature $\left({ }^{\circ} \mathrm{C}\right)$ & 0.9 & 18.2 & 19.4 & 26.4 & 22.1 \\
\hline Specific conductance $(\mu \mathrm{S} / \mathrm{cm})$ & 448 & 398 & 391 & 398 & 393 \\
\hline pH (units) & 7.6 & 8.0 & 7.9 & 8.3 & 7.9 \\
\hline Dissolved oxygen (mg/L) & 12.8 & 9.5 & 8.3 & 10.0 & 10.0 \\
\hline Phosphorus, total (as P) & 0.054 & 0.093 & 0.090 & 0.142 & 0.090 \\
\hline
\end{tabular}
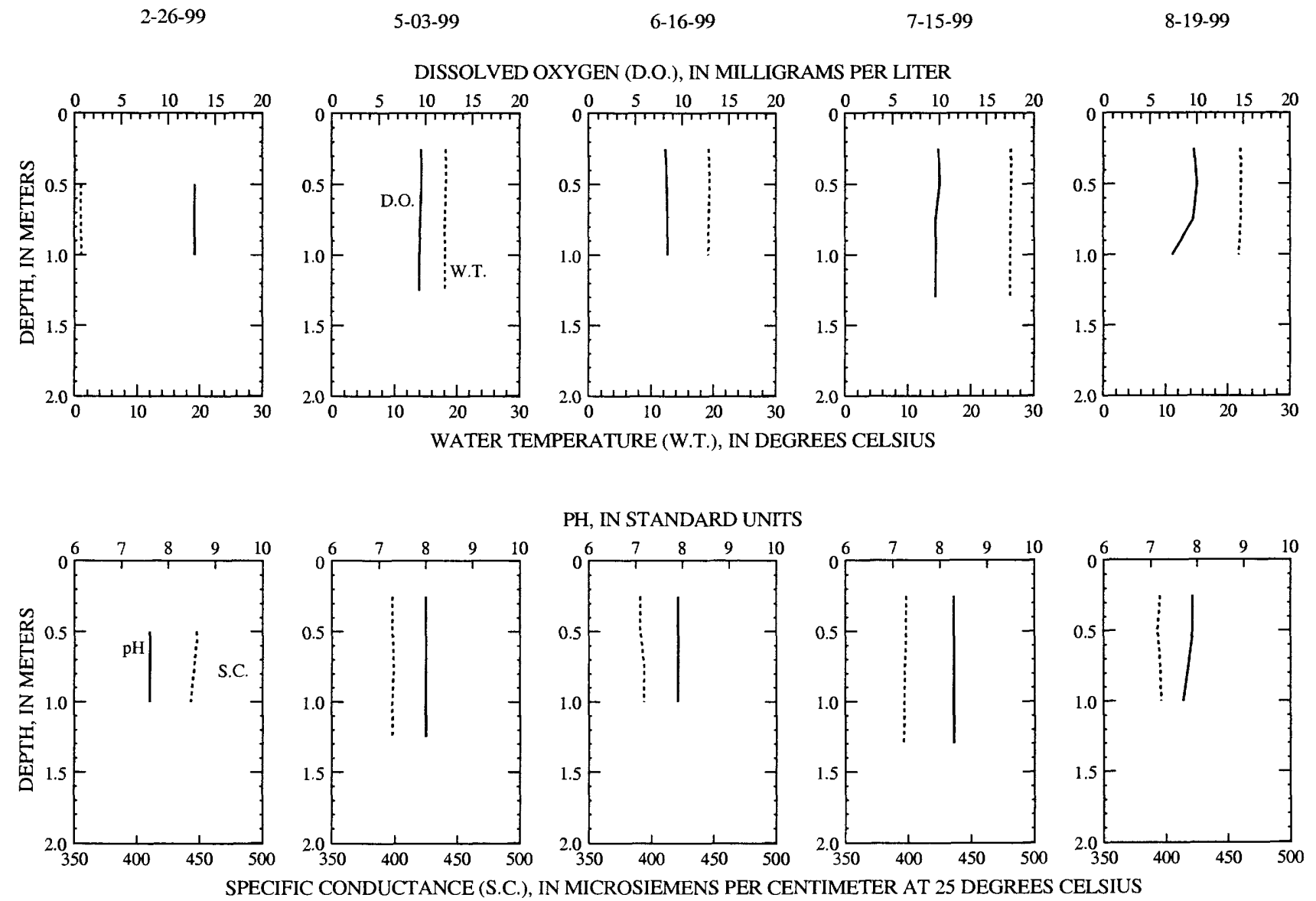

PH, IN STANDARD UNITS
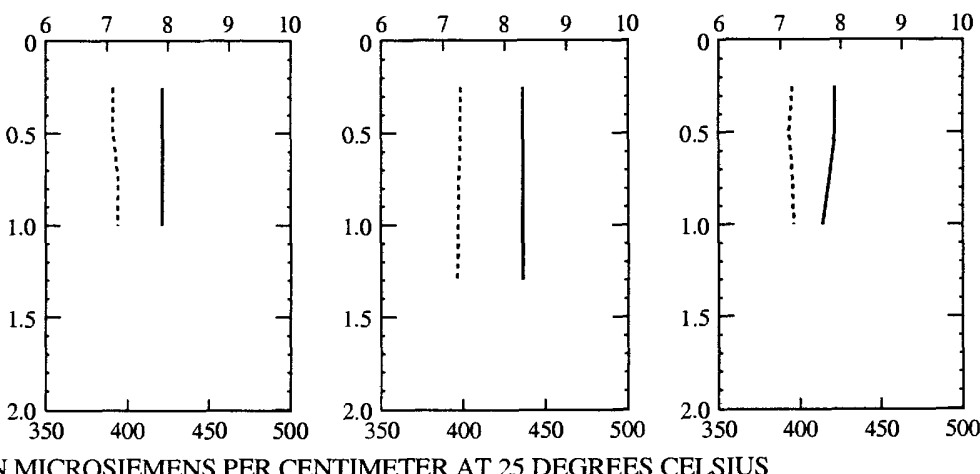

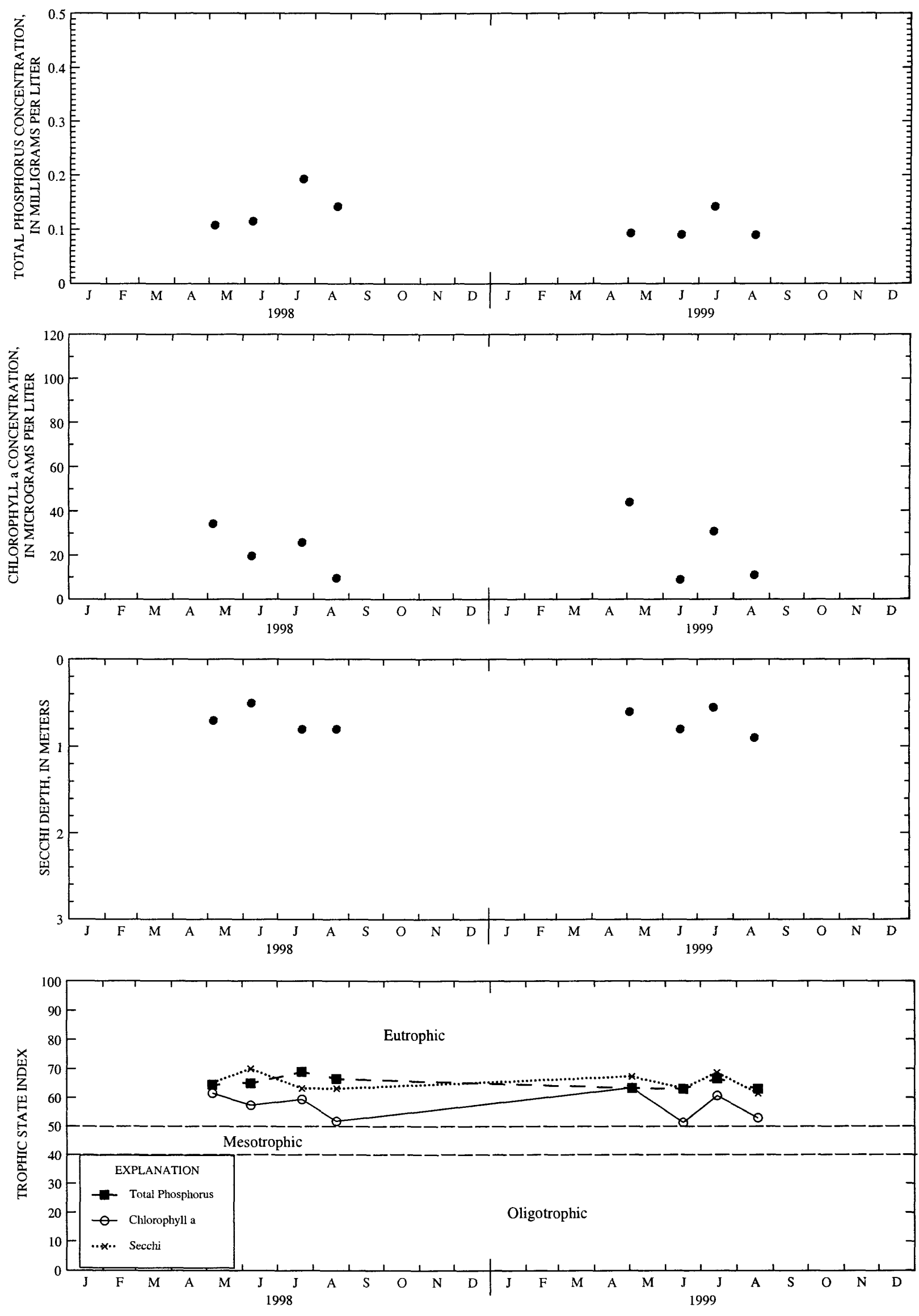

YEAR

Surface total phosphorus, chlorophyll a concentrations, Secchi depths, and TSI data for Buffalo Lake, West Site, near Endeavor, Wisconsin. 
LOCATION.--Lat $42^{\circ} 36^{\prime 2} 27^{\prime \prime}$, long $88^{\circ} 36^{\prime} 19^{\prime \prime}$, in SW 1/4 NE 1/4 sec.28, T.2 N., R.16 E., Walworth County, Hydrologic Unit 07090001, at Delavan Lake Sanitary District Lift Station No. 2 at Delavan Lake Yacht Club, $1.0 \mathrm{mi}$ southeast of outlet. and $2.7 \mathrm{mi}$ southeast of Delavan.

DRAINAGE AREA.--41.4 $\mathrm{mi}^{2}$, of which $2.3 \mathrm{mi}^{2}$ is non-contributing. Area of Delavan Lake, 2,072 acres.

PERIOD OF RECORD.--October 1983 to current year. October 1983 to September 1985 data published in Water Resources Investigation series report "Water Quality and Hydrology of Delavan Lake in Southeastern Wisconsin" by S. J. Field and M. D. Duerk (1988).

GAGE.--Water-stage recorder. Datum of gage is $922.92 \mathrm{ft}$ above sea level. Prior to Sept. 5, 1989, staff gage at bridge on North Shore Drive at same datum.

REMARKS.--No estimated daily gage heights. Records good. Lake was ice covered from Dec. 30 to Mar. 21. Lake levels controlled by Delavan Lake Sanitary District. Gage-height telemeter at station.

EXTREMES FOR PERIOD OF RECORD.--Maximum gage height observed, 6.19 ft, Feb. 21, 1994; minimum daily, -4.44 ft, Nov. 6, 1989 (lake drawn down for lake rehabilitation program).

EXTREMES FOR CURRENT YEAR.--Maximum gage height, 5.77 ft, June 14; minimum, 4.58 ft, Sept. 26.

GAGE HEIGHT, FEET, WATER YEAR OCTOBER 1998 TO SEPTEMBER 1999

\section{DAILY MEAN VALUES}

\begin{tabular}{|c|c|c|c|c|c|c|c|c|c|c|c|c|}
\hline DAY & OCT & NOV & $\mathrm{DEC}$ & JAN & $\mathrm{FEB}$ & MAR & $\mathrm{APR}$ & MAY & JUN & JUL & AUG & SEP \\
\hline $\begin{array}{l}1 \\
2 \\
3 \\
4 \\
5\end{array}$ & $\begin{array}{l}4.96 \\
4.95 \\
5.00 \\
5.01 \\
5.06\end{array}$ & $\begin{array}{l}4.97 \\
4.96 \\
4.94 \\
4.92 \\
4.91\end{array}$ & $\begin{array}{l}5.03 \\
5.02 \\
5.02 \\
5.01 \\
5.00\end{array}$ & $\begin{array}{l}4.92 \\
4.96 \\
5.01 \\
5.01 \\
5.02\end{array}$ & $\begin{array}{l}4.97 \\
4.99 \\
5.04 \\
5.07 \\
5.06\end{array}$ & $\begin{array}{l}4.92 \\
4.93 \\
4.94 \\
4.93 \\
4.94\end{array}$ & $\begin{array}{l}4.94 \\
4.95 \\
4.96 \\
4.98 \\
4.98\end{array}$ & $\begin{array}{l}4.98 \\
4.98 \\
4.98 \\
4.97 \\
4.97\end{array}$ & $\begin{array}{l}4.99 \\
5.06 \\
5.06 \\
5.05 \\
5.03\end{array}$ & $\begin{array}{l}4.97 \\
4.94 \\
4.95 \\
4.96 \\
4.96\end{array}$ & $\begin{array}{l}4.90 \\
4.88 \\
4.86 \\
4.84 \\
4.82\end{array}$ & $\begin{array}{l}4.83 \\
4.82 \\
4.82 \\
4.81 \\
4.80\end{array}$ \\
\hline $\begin{array}{r}6 \\
7 \\
8 \\
9 \\
10\end{array}$ & $\begin{array}{l}5.12 \\
5.12 \\
5.09 \\
5.04 \\
5.00\end{array}$ & $\begin{array}{l}4.91 \\
4.90 \\
4.91 \\
4.91 \\
5.09\end{array}$ & $\begin{array}{l}5.01 \\
5.06 \\
5.05 \\
5.04 \\
5.03\end{array}$ & $\begin{array}{l}5.02 \\
5.02 \\
5.02 \\
5.02 \\
5.02\end{array}$ & $\begin{array}{l}5.03 \\
4.99 \\
4.95 \\
4.92 \\
4.93\end{array}$ & $\begin{array}{l}4.96 \\
4.96 \\
4.95 \\
4.97 \\
4.98\end{array}$ & $\begin{array}{l}4.95 \\
4.93 \\
4.94 \\
5.04 \\
5.03\end{array}$ & $\begin{array}{l}5.00 \\
5.01 \\
5.00 \\
5.00 \\
5.00\end{array}$ & $\begin{array}{l}5.03 \\
5.04 \\
5.02 \\
4.99 \\
5.01\end{array}$ & $\begin{array}{l}4.96 \\
4.94 \\
4.92 \\
4.91 \\
4.89\end{array}$ & $\begin{array}{l}4.80 \\
4.79 \\
4.79 \\
4.78 \\
4.77\end{array}$ & $\begin{array}{l}4.79 \\
4.78 \\
4.75 \\
4.74 \\
4.71\end{array}$ \\
\hline $\begin{array}{l}11 \\
12 \\
13 \\
14 \\
15\end{array}$ & $\begin{array}{l}4.96 \\
4.95 \\
4.94 \\
4.93 \\
4.92\end{array}$ & $\begin{array}{l}5.17 \\
5.12 \\
5.04 \\
4.97 \\
4.95\end{array}$ & $\begin{array}{l}5.02 \\
5.01 \\
5.00 \\
4.99 \\
4.98\end{array}$ & $\begin{array}{l}5.03 \\
5.03 \\
5.03 \\
5.02 \\
5.00\end{array}$ & $\begin{array}{l}4.97 \\
5.06 \\
5.03 \\
4.99 \\
4.95\end{array}$ & $\begin{array}{l}4.97 \\
4.97 \\
4.96 \\
4.96 \\
4.96\end{array}$ & $\begin{array}{l}5.02 \\
5.00 \\
4.98 \\
4.97 \\
4.95\end{array}$ & $\begin{array}{l}5.00 \\
5.09 \\
5.11 \\
5.09 \\
5.06\end{array}$ & $\begin{array}{l}5.11 \\
5.10 \\
5.33 \\
5.74 \\
5.61\end{array}$ & $\begin{array}{l}4.87 \\
4.86 \\
4.85 \\
4.83 \\
4.81\end{array}$ & $\begin{array}{l}4.76 \\
4.78 \\
4.79 \\
4.78 \\
4.77\end{array}$ & $\begin{array}{l}4.70 \\
4.70 \\
4.72 \\
4.71 \\
4.70\end{array}$ \\
\hline $\begin{array}{l}16 \\
17 \\
18 \\
19 \\
20\end{array}$ & $\begin{array}{l}4.92 \\
4.98 \\
5.16 \\
5.17 \\
5.12\end{array}$ & $\begin{array}{l}4.95 \\
4.95 \\
4.95 \\
4.94 \\
4.95\end{array}$ & $\begin{array}{l}4.98 \\
4.98 \\
4.97 \\
4.97 \\
4.97\end{array}$ & $\begin{array}{l}4.97 \\
4.96 \\
4.97 \\
4.95 \\
4.93\end{array}$ & $\begin{array}{l}4.91 \\
4.90 \\
4.88 \\
4.86 \\
4.86\end{array}$ & $\begin{array}{l}4.96 \\
4.97 \\
4.98 \\
4.97 \\
4.95\end{array}$ & $\begin{array}{l}4.96 \\
4.94 \\
4.92 \\
4.91 \\
4.91\end{array}$ & $\begin{array}{l}5.05 \\
5.13 \\
5.19 \\
5.21 \\
5.18\end{array}$ & $\begin{array}{l}5.41 \\
5.23 \\
5.08 \\
4.99 \\
4.96\end{array}$ & $\begin{array}{l}4.80 \\
4.84 \\
4.87 \\
4.88 \\
4.89\end{array}$ & $\begin{array}{l}4.76 \\
4.76 \\
4.75 \\
4.79 \\
4.80\end{array}$ & $\begin{array}{l}4.70 \\
4.69 \\
4.69 \\
4.68 \\
4.68\end{array}$ \\
\hline $\begin{array}{l}21 \\
22 \\
23 \\
24 \\
25\end{array}$ & $\begin{array}{l}5.06 \\
4.99 \\
4.93 \\
4.93 \\
4.93\end{array}$ & $\begin{array}{l}4.95 \\
4.94 \\
4.95 \\
4.96 \\
4.96\end{array}$ & $\begin{array}{l}4.98 \\
4.97 \\
4.96 \\
4.95 \\
4.94\end{array}$ & $\begin{array}{l}4.91 \\
4.97 \\
5.07 \\
5.21 \\
5.27\end{array}$ & $\begin{array}{l}4.87 \\
4.88 \\
4.89 \\
4.89 \\
4.89\end{array}$ & $\begin{array}{l}4.94 \\
4.94 \\
4.94 \\
4.94 \\
4.94\end{array}$ & $\begin{array}{l}4.93 \\
4.98 \\
5.34 \\
5.38 \\
5.25\end{array}$ & $\begin{array}{l}5.13 \\
5.16 \\
5.13 \\
5.09 \\
5.02\end{array}$ & $\begin{array}{l}4.94 \\
4.94 \\
4.96 \\
4.97 \\
4.97\end{array}$ & $\begin{array}{l}4.90 \\
4.90 \\
4.92 \\
4.96 \\
4.96\end{array}$ & $\begin{array}{l}4.79 \\
4.79 \\
4.81 \\
4.87 \\
4.87\end{array}$ & $\begin{array}{l}4.67 \\
4.66 \\
4.64 \\
4.63 \\
4.62\end{array}$ \\
\hline $\begin{array}{l}26 \\
27 \\
28 \\
29 \\
30 \\
31\end{array}$ & $\begin{array}{l}4.94 \\
4.96 \\
4.98 \\
4.97 \\
4.98 \\
4.98\end{array}$ & $\begin{array}{l}4.97 \\
4.97 \\
4.98 \\
4.99 \\
5.02 \\
-\ldots\end{array}$ & $\begin{array}{l}4.94 \\
4.93 \\
4.93 \\
4.94 \\
4.94 \\
4.94\end{array}$ & $\begin{array}{l}5.24 \\
5.19 \\
5.15 \\
5.12 \\
5.07 \\
5.02\end{array}$ & $\begin{array}{r}4.89 \\
4.90 \\
4.92 \\
--- \\
--- \\
---\end{array}$ & $\begin{array}{l}4.94 \\
4.94 \\
4.94 \\
4.94 \\
4.94 \\
4.93\end{array}$ & $\begin{array}{l}5.15 \\
5.10 \\
5.14 \\
5.06 \\
4.99 \\
---\end{array}$ & $\begin{array}{l}4.95 \\
4.95 \\
4.96 \\
4.96 \\
4.97 \\
4.97\end{array}$ & $\begin{array}{l}4.97 \\
4.97 \\
5.05 \\
5.03 \\
5.00 \\
-.-\end{array}$ & $\begin{array}{l}4.96 \\
4.96 \\
4.94 \\
4.93 \\
4.92 \\
4.92\end{array}$ & $\begin{array}{l}4.87 \\
4.87 \\
4.87 \\
4.87 \\
4.85 \\
4.84\end{array}$ & $\begin{array}{l}4.60 \\
4.66 \\
4.93 \\
5.04 \\
5.00 \\
-.-\end{array}$ \\
\hline $\begin{array}{l}\text { MEAN } \\
\text { MAX } \\
\text { MIN }\end{array}$ & $\begin{array}{l}5.00 \\
5.17 \\
4.92\end{array}$ & $\begin{array}{l}4.97 \\
5.17 \\
4.90\end{array}$ & $\begin{array}{l}4.99 \\
5.06 \\
4.93\end{array}$ & $\begin{array}{l}5.04 \\
5.27 \\
4.91\end{array}$ & $\begin{array}{l}4.95 \\
5.07 \\
4.86\end{array}$ & $\begin{array}{l}4.95 \\
4.98 \\
4.92\end{array}$ & $\begin{array}{l}5.02 \\
5.38 \\
4.91\end{array}$ & $\begin{array}{l}5.04 \\
5.21 \\
4.95\end{array}$ & $\begin{array}{l}5.09 \\
5.74 \\
4.94\end{array}$ & $\begin{array}{l}4.91 \\
4.97 \\
4.80\end{array}$ & $\begin{array}{l}4.82 \\
4.90 \\
4.75\end{array}$ & $\begin{array}{l}4.74 \\
5.04 \\
4.60\end{array}$ \\
\hline
\end{tabular}

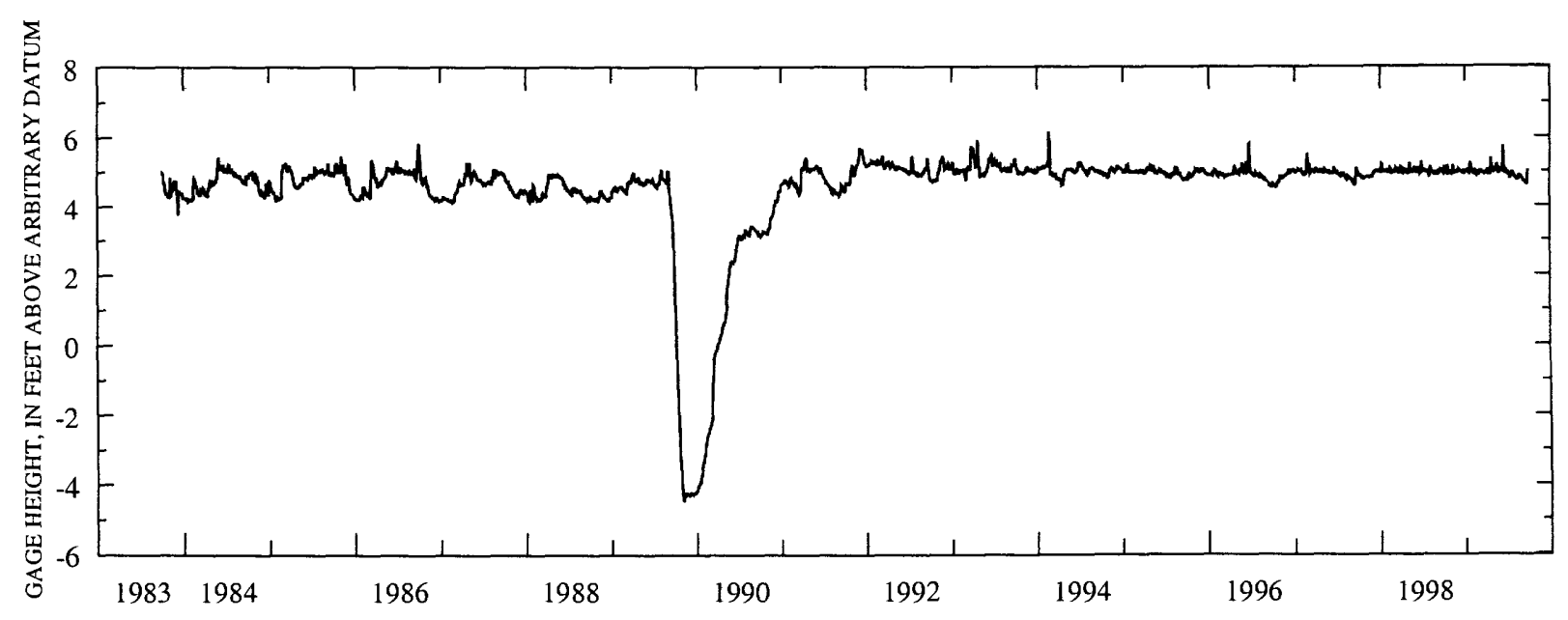




\section{DELAVAN LAKE AT CENTER NEAR DELAVAN LAKE, WI}

LOCATION.--Lat $42^{\circ} 35^{\prime} 56^{\prime \prime}$, long $88^{\circ} 36^{\prime} 50^{\prime \prime}$, in SE $1 / 4$ SW 1/4 sec.28, T.2 N., R.16 E., Walworth County, Hydrologic Unit 07090001, 2.6 mi southeast of Delavan.

DRAINAGE AREA.--41.4 $\mathrm{mi}^{2}$, of which $2.3 \mathrm{mi}^{2}$ is non-contributing.

PERIOD OF RECORD.--October 1983 to current year.

REMARKS.--Lake ice-covered during February measurements. Water-quality analyses done by the U.S. Geological Survey National Water Quality Laboratory. Samples for determination of chlorophyll-a concentration are collected from the top $1.5 \mathrm{ft}$ of the lake.

WATER-QUALITY DATA, NOVEMBER 17, 1998 TO APRIL 13, 1999

(Milligrams per liter unless otherwise indicated)

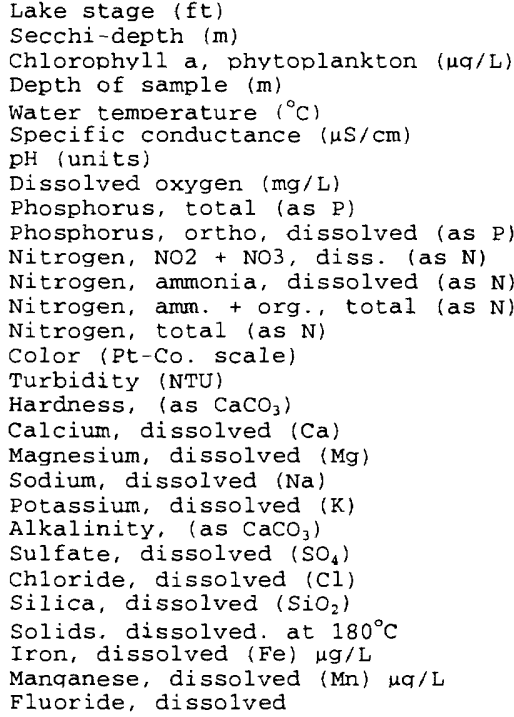

11-17-98

\begin{tabular}{|c|c|c|}
\hline \multicolumn{2}{|c|}{ Nov -17} & \\
\hline & $\begin{array}{c}4.95 \\
4.6\end{array}$ & \\
\hline 0.5 & 16.0 & 0.5 \\
\hline 8.1 & 8.1 & 2.0 \\
\hline 556 & 558 & 552 \\
\hline 8.1 & 8.2 & 7.7 \\
\hline 10.4 & 10.0 & 12.1 \\
\hline 0.110 & 0.110 & 0.140 \\
\hline 0.076 & 0.083 & 0.12 \\
\hline 0.13 & $\ldots$ & 0.58 \\
\hline 0.118 & -- & 0.186 \\
\hline 0.8 & $\cdots$ & 0.7 \\
\hline 0.93 & --- & 1.3 \\
\hline$\cdots$ & -- & $\cdots$ \\
\hline-- & -- & $\ldots$ \\
\hline$-\ldots$ & $\ldots$ & $\ldots$ \\
\hline--- & $\ldots$ & $\ldots$ \\
\hline-- & -- & $-\cdots$ \\
\hline$-\ldots$ & $\ldots$ & $\ldots$ \\
\hline--- & $\ldots$ & $-\cdots$ \\
\hline$\cdots$ & -- & $-\cdots$ \\
\hline$\ldots$ & $\ldots$ & --- \\
\hline$\ldots$ & -- & $-\cdots$ \\
\hline--- & -- & $\cdots$ \\
\hline--- & --- & $\ldots$ \\
\hline-- & --- & $-\cdots$ \\
\hline$\ldots$ & --- & --- \\
\hline--- & -- & $\ldots$ \\
\hline
\end{tabular}

2-09-99

\begin{tabular}{cc}
\multicolumn{2}{c}{ Apr -13} \\
\multicolumn{2}{c}{4.98} \\
\multicolumn{3}{c}{0.6} \\
0.5 & 0.2 \\
9.1 & 15.5 \\
580 & 8.0 \\
8.2 & 582 \\
10.9 & 8.3 \\
0.065 & 10.2 \\
0.037 & 0.073 \\
0.33 & 0.035 \\
0.061 & 0.33 \\
0.7 & 0.05 \\
1.0 & 1.2 \\
5 & 5 \\
2.0 & 2.4 \\
230 & 240 \\
42 & 42 \\
32 & 32 \\
24 & 25 \\
3 & 3 \\
185 & 186 \\
27 & 27 \\
56 & 57 \\
0.8 & 0.8 \\
336 & 344 \\
$<10$ & $<10$ \\
$E 2.6$ & $E 2.9$ \\
0.140 & 0.149
\end{tabular}

4-13-99

DISSOLVED OXYGEN (D.O.), IN MILLIGRAMS PER LITER
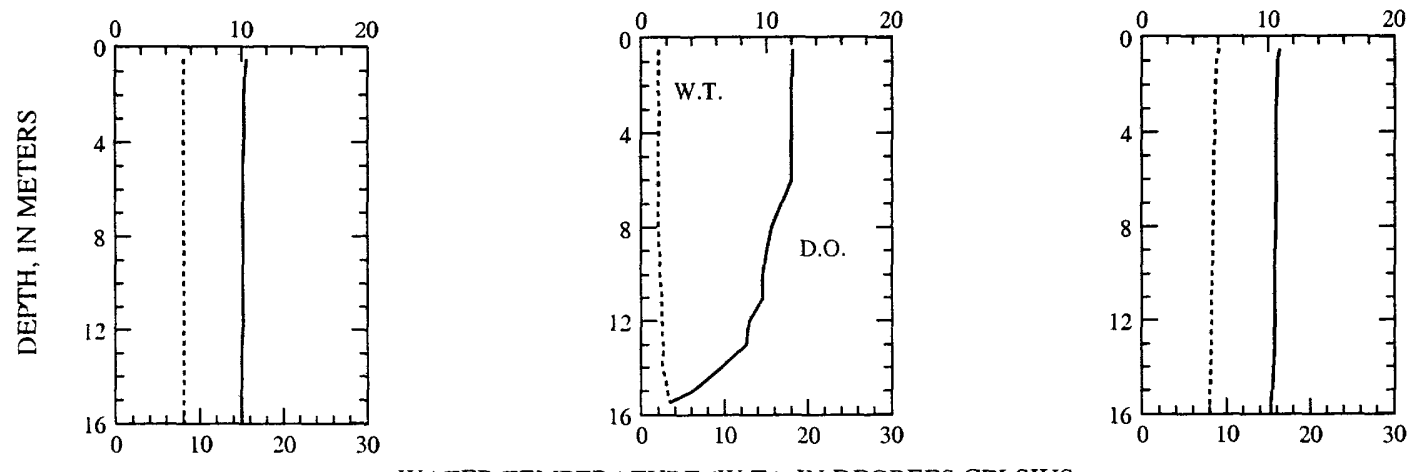

WATER TEMPERATURE (W.T.), IN DEGREES CELSIUS

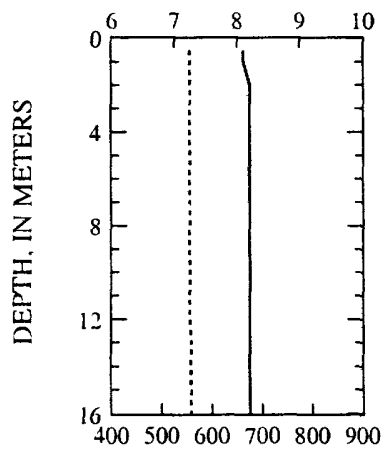

PH, IN STANDARD UNITS
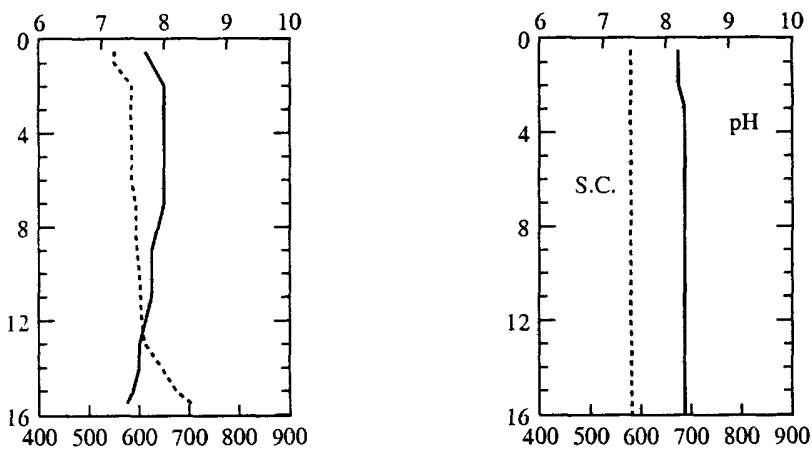

SPECIFIC CONDUCTANCE (S.C.), IN MICROSIEMENS PER CENTIMETER AT 25 DEGREES CELSIUS 
WATER-QUALITY DATA, MAY 19 TO JULY 13, 1999

(Milligrams per liter unless otherwise indicated)

Lake stage (ft)

Secchi-depth (m)

Chlorophyll a, phytoplankton $(\mu \mathrm{g} / \mathrm{L})$

Depth of sample (m)

water temperature $\left({ }^{\circ} \mathrm{C}\right)$

Specific conductance $(\mu \mathrm{S} / \mathrm{cm})$

$\mathrm{pH}$ (units)

Dissolved oxygen ( $\mathrm{mg} / \mathrm{L}$ )

Phosphorus, total (as P)

Phosphorus, ortho, dissolved (as P)

Nitrogen, NO2 + NO3, diss. (as N)

Nitrogen, ammonia, dissolved (as $\mathrm{N}$ )

Nitrogen, amm. + org., total (as $N$ )

Nitrogen, total (as N)

May -1

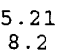

8.2
0.8

$0.5 \quad 7.0 .82 .00$

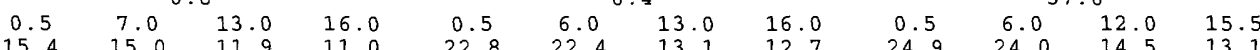

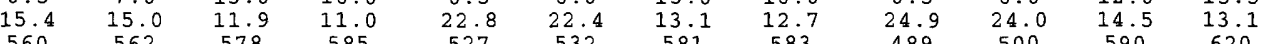

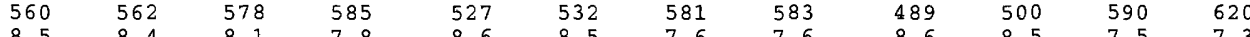

$\begin{array}{cccccccccccc}8.5 & 8.4 & 8.1 & 7.8 & 8.6 & 8.5 & 7.6 & 7.6 & 8.6 & 8.5 & 7.5 & 7.3\end{array}$

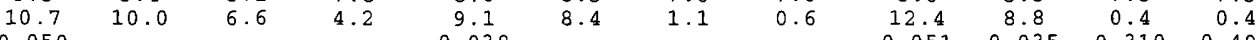

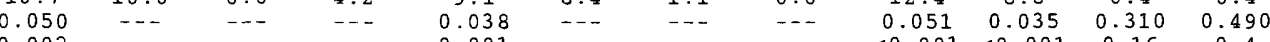

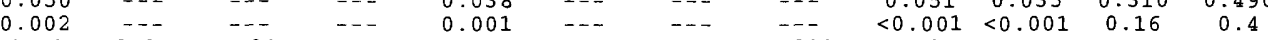

$\begin{array}{llllllll}0.19 & 0.26 & 0.31 & 0.26 & 0.055 & 0.068 & 0.22 & 0.093\end{array}$

$\begin{array}{llllllll}0.011 & 0.009 & 0.174 & 0.48 & 0.009 & 0.042 & 0.236 & 0.318\end{array}$

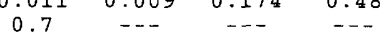

0.86

0.011

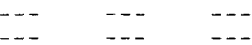

0.017

$--$

-

6-14-99

7-13-99

DISSOLVED OXYGEN (D.O.), IN MLLIGRAMS PER LITER
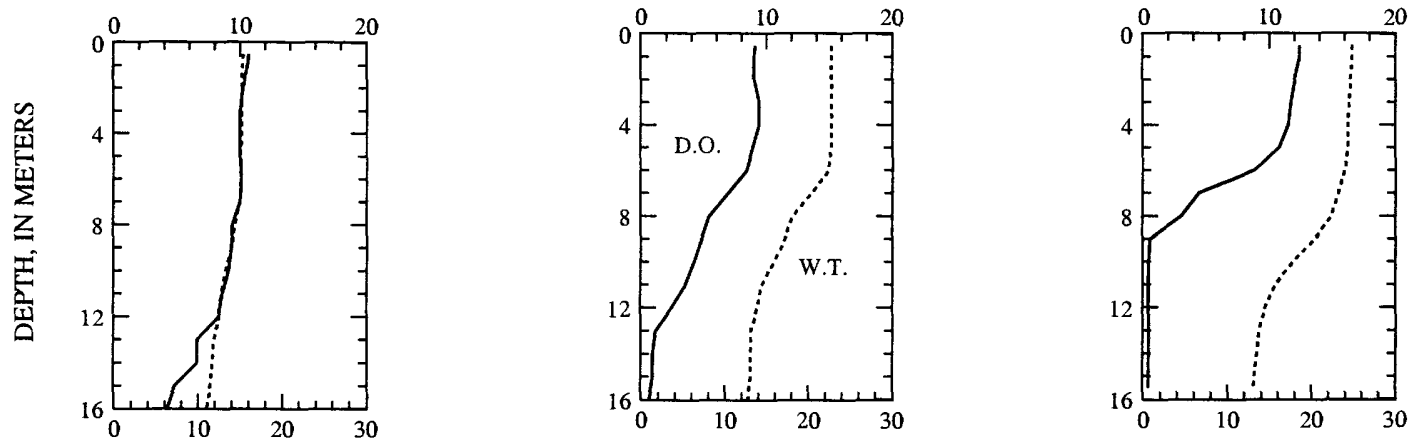

WATER TEMPERATURE (W.T.), IN DEGREES CELSIUS

PH, IN STANDARD UNITS
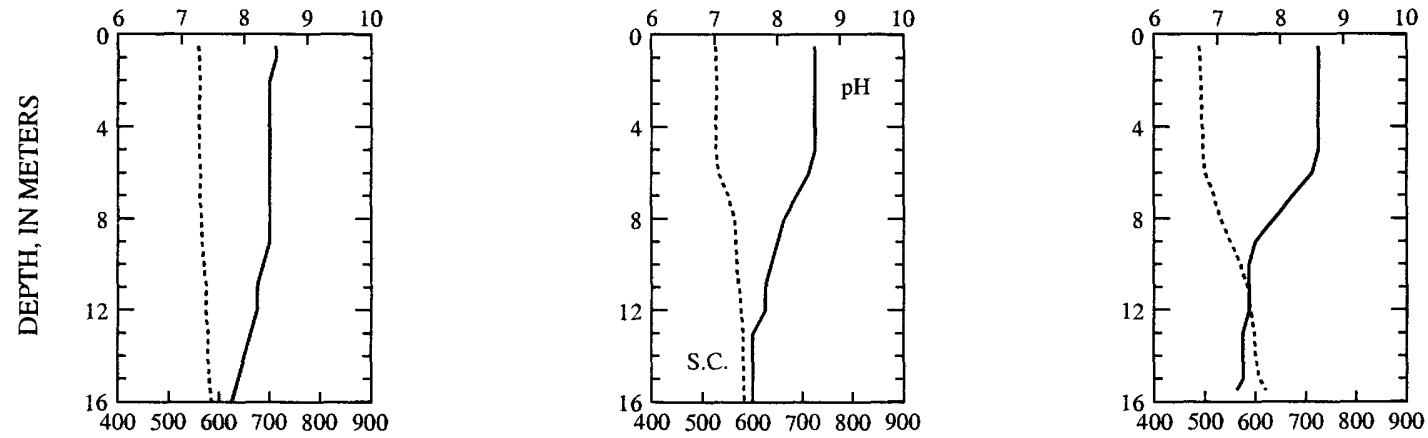

SPECIFIC CONDUCTANCE (S.C.), IN MICROSIEMENS PER CENTIMETER AT 25 DEGREES CELSIUS 
Lake stage ( $\mathrm{ft}$ )

Secchi-depth (m)

Chlorophylla, phytoplankton ( $\mu \mathrm{g} / \mathrm{L}$ )

Depth of sample (m)

Water temperature $\left({ }^{\circ} \mathrm{C}\right)$

Specific conductance $(\mu \mathrm{s} / \mathrm{cm})$

$\mathrm{pH}$ (units)

Dissolved oxygen ( $\mathrm{mg} / \mathrm{L}$ )

Phosphorus, total (as P)

phosphorus, ortho, dissolved (as P)

Nitrogen, NO2 + NO3, diss. (as N)

Nitrogen, ammonia, dissolved (as $N$ )

Nitrogen, amm. + org., total (as N)

Nitrogen, total (as N)
WATER-QUALITY DATA, AUGUST 17 TO SEPTEMBER 14, 1999

(Milligrams per liter unless otherwise indicated)

Aug -17

Sep-14

4.76

4.71

14

$\begin{array}{cccccccc}0.5 & 2.0 & 5.0 & 9.0 & 11.0 & 13.0 & 14.0 & 15.5 \\ 23.2 & 23.2 & 23.1 & 22.9 & 18.5 & 14.7 & 13.9 & 13.3\end{array}$

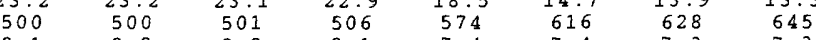

$\begin{array}{llllllll}8.1 & 8.2 & 8.2 & 8.1 & 7.4 & 7.4 & 7.3 & 7.3 \\ 7.8 & 7.7 & 7.0 & 5.8 & 0.7 & 0.8 & 0.7 & 0.7\end{array}$

0.0450 .0410 .0370 .8

$\begin{array}{cccccccc}0.045 & 0.041 & 0.037 & 0.043 & 0.150 & 0.440 & 0.540 & 0.670 \\ 0.001 & -- & --- & <0.001 & -\ldots & -. & 0.37 & 0.58\end{array}$

$<0.005 \ldots$

$0.02 \quad \cdots+\cdots$

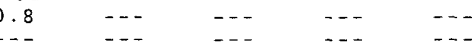

$8-17-99$

0.370 .58

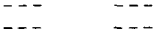

- -

9-14-99

DISSOLVED OXYGEN (D.O.), IN MILLIGRAMS PER LITER
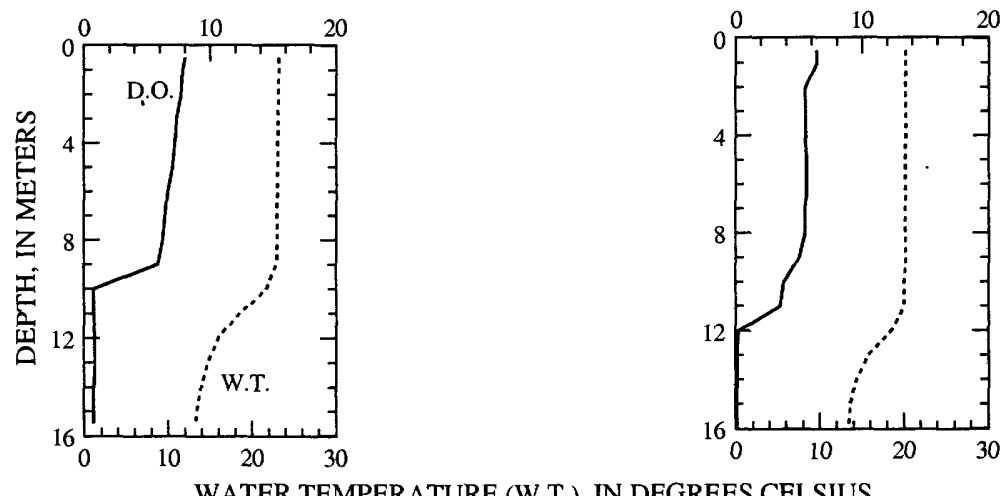

WATER TEMPERATURE (W.T.), IN DEGREES CELSIUS

PH, IN STANDARD UNITS
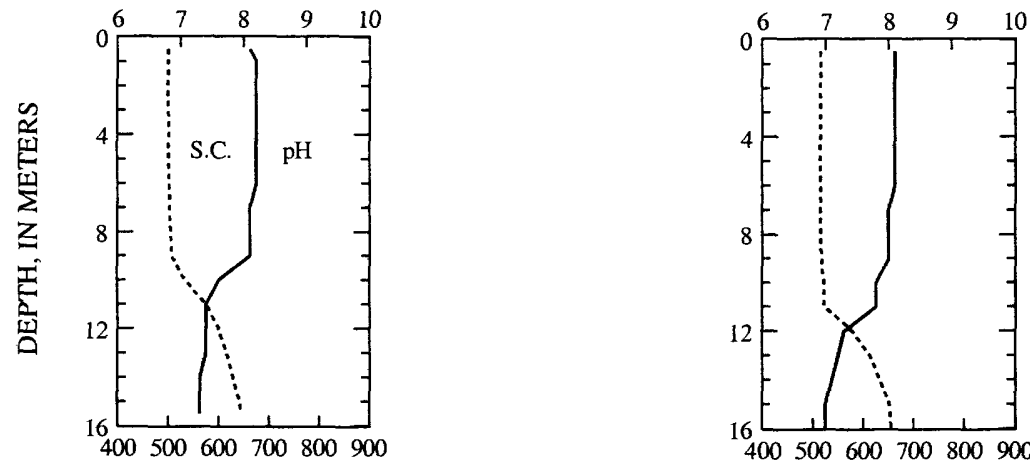

SPECIFIC CONDUCTANCE (S.C.), IN MICROSIEMENS PER CENTIMETER AT 25 DEGREES CELSIUS 

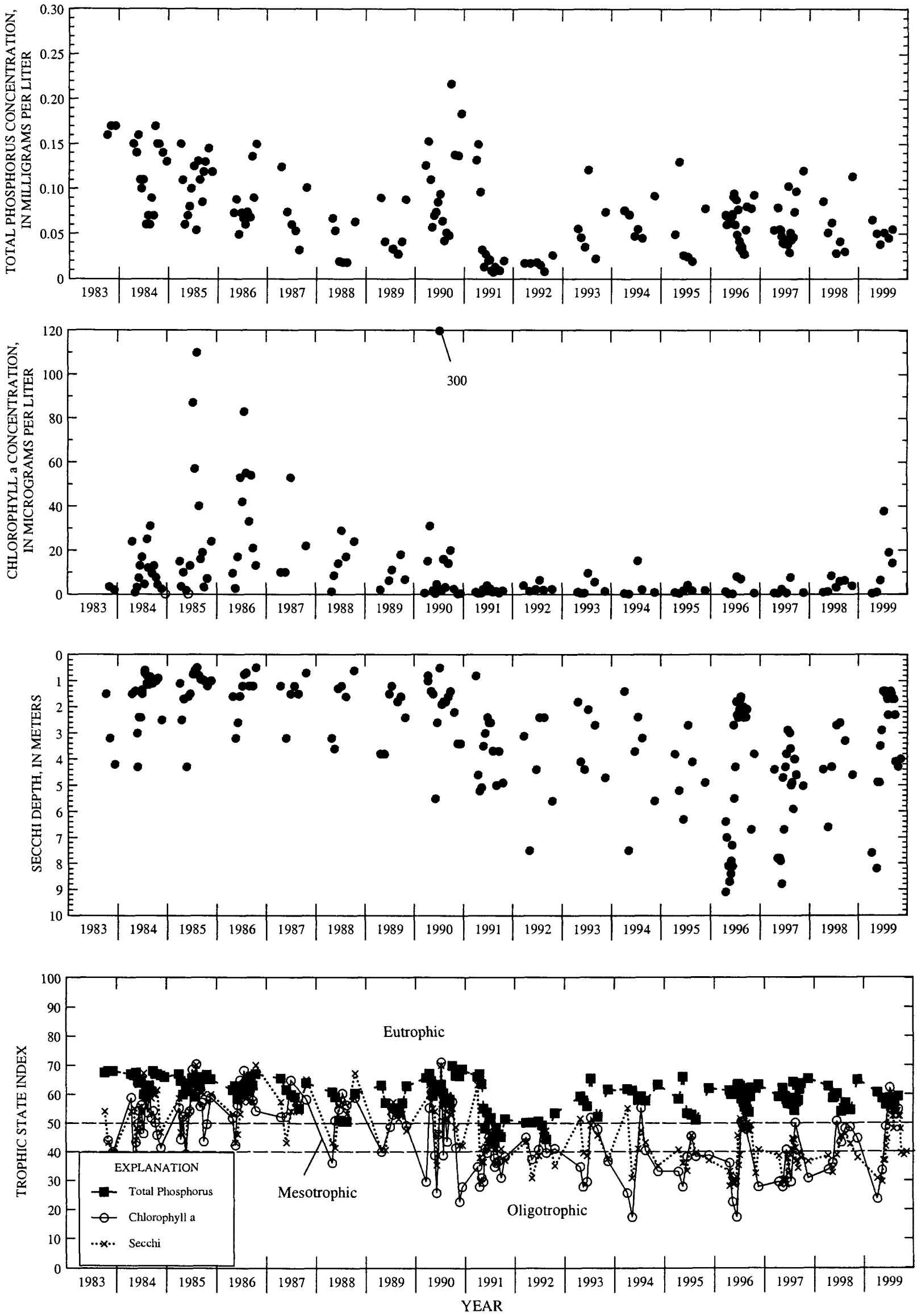

Surface total phosphorus, chlorophyll a concentrations, Secchi depths, and TSI data for Delavan Lake at Center near Delavan, Wisconsin. 
ADDITIONAL WATER-QUALITY DATA, OCTOBER 9, 1998 TO SEPTEMBER 24, 1999

(Milligrams per liter unless otherwise indicated)

Lake stage (ft)

Secchi-depth (meters)

Depth of sample (meters)

Water temperature $\left({ }^{\circ} \mathrm{C}\right)$

Phosphorus, total (as P)

Lake stage (ft)

Secchi-depth (meters)

Depth of sample (meters)

water temperature $\left({ }^{\circ} \mathrm{C}\right)$

Phosphorus, total (as P)

Lake stage (ft)

Secchi-depth (meters)

Depth of sample (meters)

Water temperature $\left({ }^{\circ} \mathrm{C}\right)$

Phosphorus, total (as P)

oct. 9
5.04
3.8
0.5
17.8

Qct. 18

5.17

2.8

0.5

15.5

0.084

0.115

ect. 26
4.94
3.1
0.5
14.0
0.059

May 27
4.95
4.9

4.9

16.1

0.047

June 3
5.06
4.9
0.5
19.4
0.043

June 8

5.02

4.9

0.5

22.5

0.043

$\begin{array}{cc}\text { July } 7 & \text { July } 22 \\ 4.94 & 4.90 \\ 1.4 & 1.4 \\ 0.5 & 0.5 \\ 25.0 & 26.5 \\ 0.054 & 0.046\end{array}$

Aug. 26
4.87
1.5
0.5
22.0
0.022

Sept. 1
4.83
1.4
0.5
25.0
0.033

July 28
4.94
1.5
0.5
29.0
0.035

Aug. 5
4.82
1.7
0.5
26.5
0.031

Aug. 11
4.76
2.3
0.5
25.0
0.046

\begin{tabular}{c} 
Sept. 14 \\
\hline 4.71 \\
1.7 \\
0.5 \\
20.2 \\
0.055
\end{tabular}

0.035
Seat. 24

4.63

1.7

0.5

21.1

0.082 


\section{DELAVAN LAKE, AT NORTH END, NEAR LAKE LAWN, WI}

LOCATION.--Lat $42^{\circ} 36^{\prime} 59^{\prime \prime}$, long $88^{\circ} 35^{\prime} 44^{\prime \prime}$, in NW 1/4 SW 1/4, sec.22, T.2 N., R.16 E., Walworth County, Hydrologic Unit 07090001, $2.6 \mathrm{mi}$ southeast of Delavan.

DRAINAGE AREA.--41.4 $\mathrm{mi}^{2}$, of which $2.3 \mathrm{mi}^{2}$ is non-contributing.

PERIOD OF RECORD.--October 1983 to current year.

WATER-QUALITY DATA, APRIL 13 TO AUGUST 17, 1999

$\begin{array}{ccccc}\text { Apr. } 13 & \text { May } 19 & \text { June } 14 & \text { July } 13 & \text { Aug. } 17 \\ 7.0 & 7.6 & 3.4 & 1.2 & 1.8\end{array}$

\section{DELAVAN LAKE, AT SW END, NEAR DELAVAN LAKE, WI}

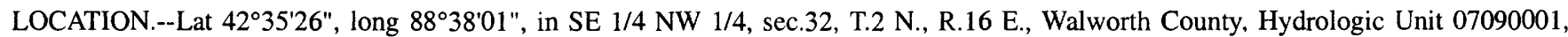
2.6 mi southeast of Delavan.

DRAINAGE AREA.--41.4 $\mathrm{mi}^{2}$, of which $2.3 \mathrm{mi}^{2}$ is non-contributing.

PERIOD OF RECORD.--October 1983 to current year.

WATER-QUALITY DATA, APRIL 13 TO AUGUST 17, 1999

$\begin{array}{ccccc}\text { Apr. } 13 & \text { May } 19 & \text { June 14 } & \text { July } 13 & \text { Aug. } 17 \\ 8.2 & 7.3 & 3.0 & 1.2 & 1.4\end{array}$


LOCATION.--Lat 43²5'18", long 8943'38", in SW 1/4 SE 1/4 sec.13, T.11 N., R.6 E., Sauk County, Hydrologic Unit 07070004, in Devils Lake State Park, $3.5 \mathrm{mi}$ south of Baraboo.

DRAINAGE AREA.--4.79 $\mathrm{mi}^{2}$. Area of Devils Lake, 361 acres.

PERIOD OF RECORD.--June 1922 to August 1930, June to August 1932, June 1934 to September 1981 (fragmentary). October 1981 to September 1984, data unpublished in district files. October 1984 to current year.

REVISED RECORDS.--WDR WI-78-1: Drainage area.

GAGE.--Water-stage recorder installed July 17, 1991. Datum of gage is $955.00 \mathrm{ft}$, above sea level.

REMARKS.--No estimated daily gage heights. Records good. Lake has no surface outlet.

EXTREMES FOR PERIOD OF RECORD.--Maximum gage height observed, $14.13 \mathrm{ft}$, July 18, 1993; minimum observed, $1.49 \mathrm{ft}$ Feb. 8, 1965.

EXTREMES FOR CURRENT YEAR.--Maximum recorded gage height, $11.06 \mathrm{ft}$, June 11 and 13; minimum recorded, $8.76 \mathrm{ft}$, Jan. 1.

GAGE HEIGHT, FEET, WATER YEAR OCTOBER 1998 TO SEPTEMBER 1999

DAILY MEAN VALUES

\begin{tabular}{|c|c|c|c|c|c|c|c|c|c|c|c|c|}
\hline DAY & OCT & NOV & DEC & JAN & FEB & MAR & APR & MAY & JUN & JUL & AUG & SEP \\
\hline $\begin{array}{l}1 \\
2 \\
3 \\
4 \\
5\end{array}$ & $\begin{array}{l}9.53 \\
9.50 \\
9.49 \\
9.48 \\
9.51\end{array}$ & $\begin{array}{l}9.24 \\
9.21 \\
9.19 \\
9.17 \\
9.15\end{array}$ & $\begin{array}{l}9.09 \\
9.08 \\
9.07 \\
9.07 \\
9.06\end{array}$ & $\begin{array}{l}8.77 \\
8.81 \\
8.85 \\
8.84 \\
8.84\end{array}$ & $\begin{array}{l}8.88 \\
8.89 \\
8.89 \\
8.88 \\
8.88\end{array}$ & $\begin{array}{l}9.16 \\
9.16 \\
9.15 \\
9.15 \\
9.15\end{array}$ & $\begin{array}{l}9.12 \\
9.12 \\
9.28 \\
9.48 \\
9.55\end{array}$ & $\begin{array}{l}10.75 \\
10.74 \\
10.73 \\
10.72 \\
10.72\end{array}$ & $\begin{array}{l}10.85 \\
10.89 \\
10.87 \\
10.87 \\
10.87\end{array}$ & $\begin{array}{l}10.68 \\
10.67 \\
10.66 \\
10.66 \\
10.64\end{array}$ & $\begin{array}{l}10.38 \\
10.34 \\
10.31 \\
10.29 \\
10.26\end{array}$ & $\begin{array}{l}9.97 \\
9.95 \\
9.93 \\
9.91 \\
9.88\end{array}$ \\
\hline $\begin{array}{r}6 \\
7 \\
8 \\
9 \\
10\end{array}$ & $\begin{array}{l}9.55 \\
9.53 \\
9.51 \\
9.50 \\
9.48\end{array}$ & $\begin{array}{l}9.13 \\
9.11 \\
9.10 \\
9.11 \\
9.26\end{array}$ & $\begin{array}{l}9.06 \\
9.05 \\
9.04 \\
9.02 \\
9.01\end{array}$ & $\begin{array}{l}8.85 \\
8.84 \\
8.84 \\
8.84 \\
8.84\end{array}$ & $\begin{array}{l}8.87 \\
8.87 \\
8.87 \\
8.87 \\
8.87\end{array}$ & $\begin{array}{l}9.15 \\
9.15 \\
9.15 \\
9.18 \\
9.18\end{array}$ & $\begin{array}{r}9.62 \\
9.65 \\
9.68 \\
9.94 \\
10.07\end{array}$ & $\begin{array}{l}10.75 \\
10.80 \\
10.81 \\
10.81 \\
10.80\end{array}$ & $\begin{array}{l}10.87 \\
10.85 \\
10.87 \\
11.02 \\
11.02\end{array}$ & $\begin{array}{l}10.62 \\
10.59 \\
10.56 \\
10.56 \\
10.52\end{array}$ & $\begin{array}{l}10.23 \\
10.23 \\
10.21 \\
10.19 \\
10.17\end{array}$ & $\begin{array}{l}9.86 \\
9.83 \\
9.80 \\
9.77 \\
9.74\end{array}$ \\
\hline $\begin{array}{l}11 \\
12 \\
13 \\
14 \\
15\end{array}$ & $\begin{array}{l}9.46 \\
9.44 \\
9.41 \\
9.39 \\
9.37\end{array}$ & $\begin{array}{l}9.26 \\
9.25 \\
9.24 \\
9.23 \\
9.21\end{array}$ & $\begin{array}{l}9.00 \\
8.99 \\
8.98 \\
8.97 \\
8.95\end{array}$ & $\begin{array}{l}8.84 \\
8.85 \\
8.86 \\
8.86 \\
8.85\end{array}$ & $\begin{array}{l}8.94 \\
9.09 \\
9.11 \\
9.13 \\
9.14\end{array}$ & $\begin{array}{l}9.17 \\
9.17 \\
9.16 \\
9.15 \\
9.15\end{array}$ & $\begin{array}{l}10.12 \\
10.15 \\
10.17 \\
10.19 \\
10.19\end{array}$ & $\begin{array}{l}10.79 \\
10.78 \\
10.76 \\
10.75 \\
10.74\end{array}$ & $\begin{array}{l}11.03 \\
11.04 \\
11.04 \\
11.02 \\
10.99\end{array}$ & $\begin{array}{l}10.49 \\
10.47 \\
10.44 \\
10.41 \\
10.37\end{array}$ & $\begin{array}{l}10.15 \\
10.14 \\
10.11 \\
10.09 \\
10.06\end{array}$ & $\begin{array}{l}9.71 \\
9.69 \\
9.67 \\
9.64 \\
9.61\end{array}$ \\
\hline $\begin{array}{l}16 \\
17 \\
18 \\
19 \\
20\end{array}$ & $\begin{array}{l}9.36 \\
9.39 \\
9.42 \\
9.40 \\
9.37\end{array}$ & $\begin{array}{l}9.20 \\
9.20 \\
9.19 \\
9.19 \\
9.16\end{array}$ & $\begin{array}{l}8.94 \\
8.93 \\
8.92 \\
8.91 \\
8.89\end{array}$ & $\begin{array}{l}8.85 \\
8.86 \\
8.87 \\
8.87 \\
8.86\end{array}$ & $\begin{array}{l}9.15 \\
9.16 \\
9.15 \\
9.15 \\
9.15\end{array}$ & $\begin{array}{l}9.14 \\
9.15 \\
9.16 \\
9.16 \\
9.16\end{array}$ & $\begin{array}{l}10.20 \\
10.20 \\
10.20 \\
10.20 \\
10.21\end{array}$ & $\begin{array}{l}10.74 \\
10.89 \\
10.94 \\
10.95 \\
10.95\end{array}$ & $\begin{array}{l}10.96 \\
10.94 \\
10.91 \\
10.89 \\
10.86\end{array}$ & $\begin{array}{l}10.36 \\
10.39 \\
10.38 \\
10.43 \\
10.43\end{array}$ & $\begin{array}{r}10.03 \\
10.00 \\
9.99 \\
10.13 \\
10.13\end{array}$ & $\begin{array}{l}9.59 \\
9.57 \\
9.54 \\
9.52 \\
9.50\end{array}$ \\
\hline $\begin{array}{l}21 \\
22 \\
23 \\
24 \\
25\end{array}$ & $\begin{array}{l}9.35 \\
9.33 \\
9.31 \\
9.29 \\
9.28\end{array}$ & $\begin{array}{r}9.15 \\
9.14 \\
9.12 \\
9.12 \\
9.10\end{array}$ & $\begin{array}{l}8.89 \\
8.87 \\
8.86 \\
8.85 \\
8.84\end{array}$ & $\begin{array}{l}8.85 \\
8.89 \\
8.90 \\
8.91 \\
8.92\end{array}$ & $\begin{array}{l}9.14 \\
9.14 \\
9.13 \\
9.13 \\
9.14\end{array}$ & $\begin{array}{l}9.16 \\
9.16 \\
9.16 \\
9.15 \\
9.15\end{array}$ & $\begin{array}{l}10.21 \\
10.30 \\
10.59 \\
10.69 \\
10.73\end{array}$ & $\begin{array}{l}10.94 \\
10.94 \\
10.95 \\
10.94 \\
10.92\end{array}$ & $\begin{array}{l}10.84 \\
10.82 \\
10.82 \\
10.82 \\
10.80\end{array}$ & $\begin{array}{l}10.52 \\
10.51 \\
10.51 \\
10.53 \\
10.51\end{array}$ & $\begin{array}{l}10.12 \\
10.10 \\
10.11 \\
10.14 \\
10.13\end{array}$ & $\begin{array}{l}9.48 \\
9.45 \\
9.43 \\
9.41 \\
9.39\end{array}$ \\
\hline $\begin{array}{l}26 \\
27 \\
28 \\
29 \\
30 \\
31\end{array}$ & $\begin{array}{l}9.27 \\
9.28 \\
9.29 \\
9.28 \\
9.27 \\
9.25\end{array}$ & $\begin{array}{l}9.09 \\
9.09 \\
9.09 \\
9.09 \\
9.09 \\
---\end{array}$ & $\begin{array}{l}8.83 \\
8.82 \\
8.80 \\
8.81 \\
8.80 \\
8.79\end{array}$ & $\begin{array}{l}8.91 \\
8.91 \\
8.90 \\
8.90 \\
8.89 \\
8.89\end{array}$ & $\begin{array}{r}9.14 \\
9.15 \\
9.16 \\
\ldots- \\
\ldots- \\
\ldots-\end{array}$ & $\begin{array}{l}9.14 \\
9.14 \\
9.14 \\
9.13 \\
9.13 \\
9.13\end{array}$ & $\begin{array}{c}10.75 \\
10.76 \\
10.76 \\
10.76 \\
10.76 \\
\\
\end{array}$ & $\begin{array}{l}10.90 \\
10.88 \\
10.86 \\
10.85 \\
10.83 \\
10.84\end{array}$ & $\begin{array}{r}10.77 \\
10.75 \\
10.72 \\
10.69 \\
10.67 \\
\ldots\end{array}$ & $\begin{array}{l}10.50 \\
10.48 \\
10.45 \\
10.43 \\
10.41 \\
10.41\end{array}$ & $\begin{array}{l}10.11 \\
10.10 \\
10.08 \\
10.06 \\
10.03 \\
10.00\end{array}$ & $\begin{array}{r}9.37 \\
9.45 \\
9.46 \\
9.45 \\
9.42 \\
---\end{array}$ \\
\hline $\begin{array}{l}\text { MEAN } \\
\text { MAX } \\
\text { MIN }\end{array}$ & $\begin{array}{l}9.40 \\
9.55 \\
9.25\end{array}$ & $\begin{array}{l}9.16 \\
9.26 \\
9.09\end{array}$ & $\begin{array}{l}8.94 \\
9.09 \\
8.79\end{array}$ & $\begin{array}{l}8.86 \\
8.92 \\
8.77\end{array}$ & $\begin{array}{l}9.04 \\
9.16 \\
8.87\end{array}$ & $\begin{array}{l}9.15 \\
9.18 \\
9.13\end{array}$ & $\begin{array}{r}10.12 \\
10.76 \\
9.12\end{array}$ & $\begin{array}{l}10.83 \\
10.95 \\
10.72\end{array}$ & $\begin{array}{l}10.88 \\
11.04 \\
10.67\end{array}$ & $\begin{array}{l}10.50 \\
10.68 \\
10.36\end{array}$ & $\begin{array}{r}10.14 \\
10.38 \\
9.99\end{array}$ & $\begin{array}{l}9.63 \\
9.97 \\
9.37\end{array}$ \\
\hline
\end{tabular}




\section{EAGLE SPRING LAKE AT EAGLEVILLE, WI}

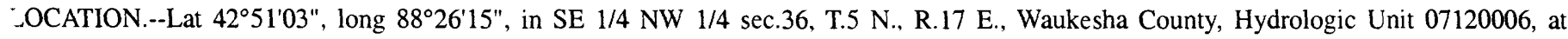
Eagleville.

TRAINAGE AREA.--33.2 $\mathrm{mi}^{2}$.

PERIOD OF RECORD.--April 1991 to current year.

REMARKS.--Lake sampled near southeast end at a lake depth of about $3 \mathrm{~m}$. Lake ice-covered during February sampling. Water-quality analyses done by Wisconsin State Laboratory of Hygiene. Lake-stage readings from 1991 to 1993 (except 2/4/93 and 4/19/93) were previously reported $1 \mathrm{ft}$ too high.

WATER-QUALITY DATA, FEBRUARY 19 TO AUGUST 04, 1999

(Milligrams per liter unless otherwise indicated

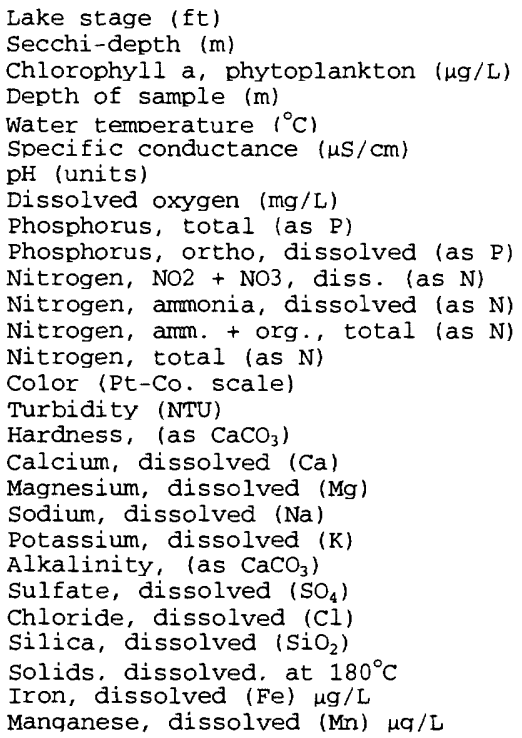

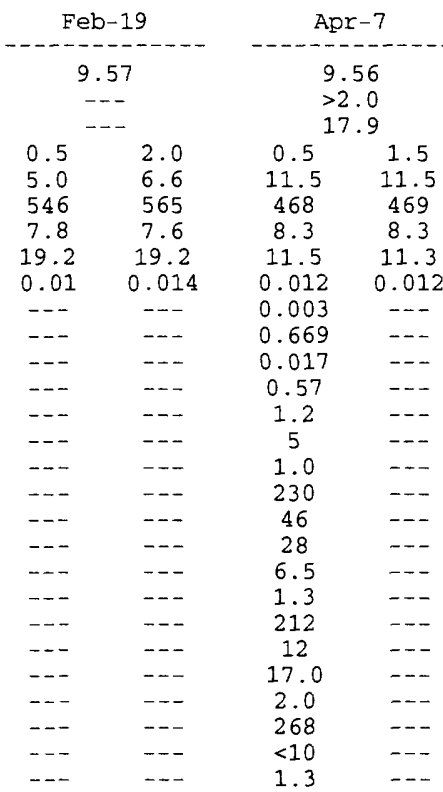

6-11-99

\begin{tabular}{|c|c|c|}
\hline Jun-11 & Jul-7 & Aug- 4 \\
\hline $\begin{array}{c}9.56 \\
2.3 \\
2.09 \\
0.5 \\
26.8 \\
457 \\
7.9 \\
8.2 \\
0.021\end{array}$ & $\begin{array}{c}9.49 \\
1.5 \\
2.95 \\
0.5 \\
28.6 \\
451 \\
8.2 \\
9.3 \\
0.017\end{array}$ & $\begin{array}{c}--- \\
1.7 \\
3.21 \\
0.5 \\
28.0 \\
445 \\
8.1 \\
9.8 \\
0.038\end{array}$ \\
\hline -. & -- & --- \\
\hline--- & --- & -- \\
\hline$\cdots$ & -- & $\cdots$ \\
\hline-- & --- & $\cdots$ \\
\hline$\ldots$ & - - & -.. \\
\hline$\ldots$ & $\ldots$ & -- \\
\hline-- & $-\cdots$ & -- \\
\hline-- & -- & -- \\
\hline$\ldots$ & -- & - \\
\hline -. & $\cdots$ & -- \\
\hline-- & -- & -- \\
\hline-- & $\cdots$ & - \\
\hline$\ldots$ & $\ldots$ & $\ldots$ \\
\hline$\ldots$ & -- & -- \\
\hline-- & $--\cdots$ & --- \\
\hline -.. & -- & -- \\
\hline
\end{tabular}

7-07-99

DISSOLVED OXYGEN (D.O.), IN MILLIGRAMS PER LITER
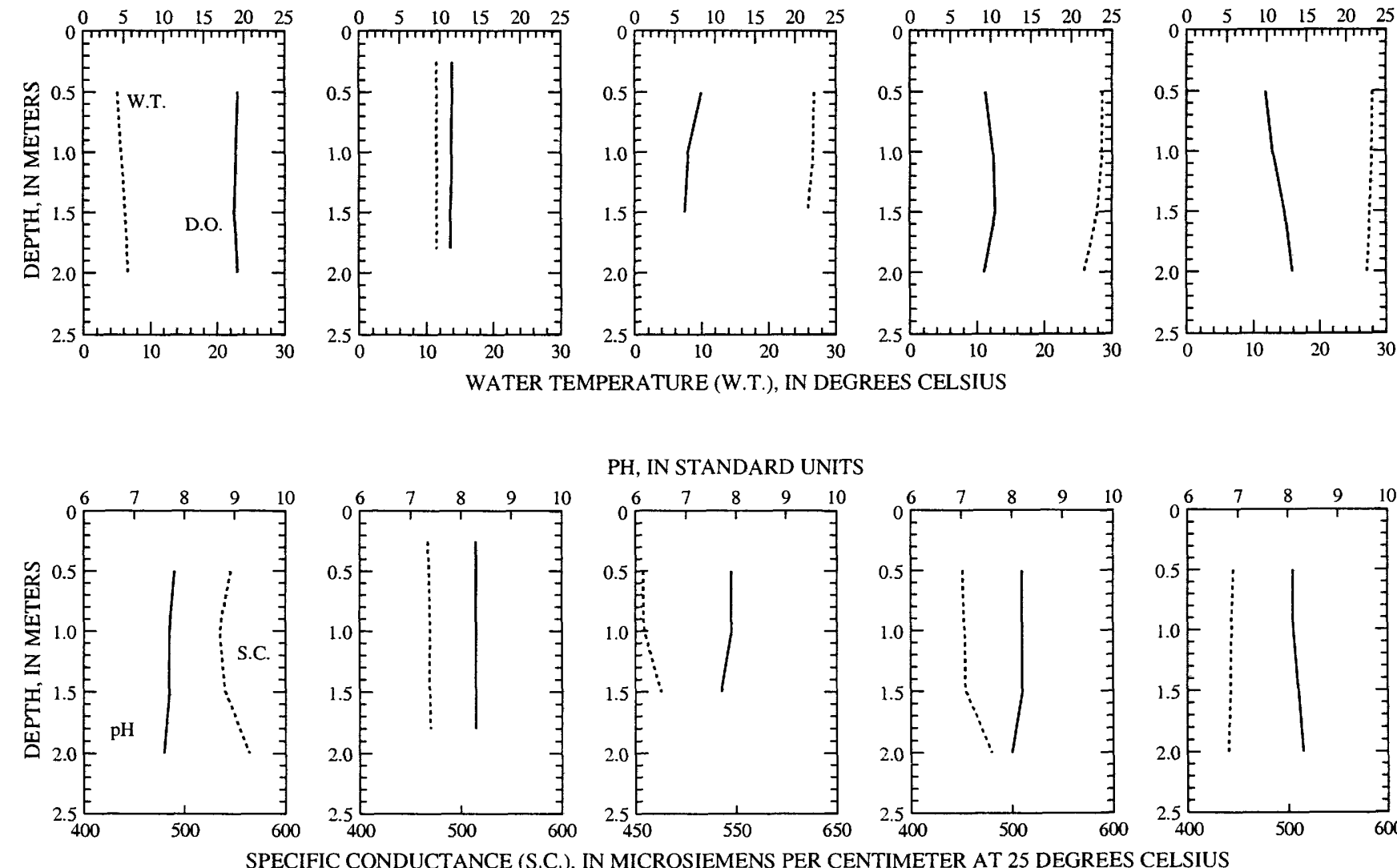

PH, IN STANDARD UNITS
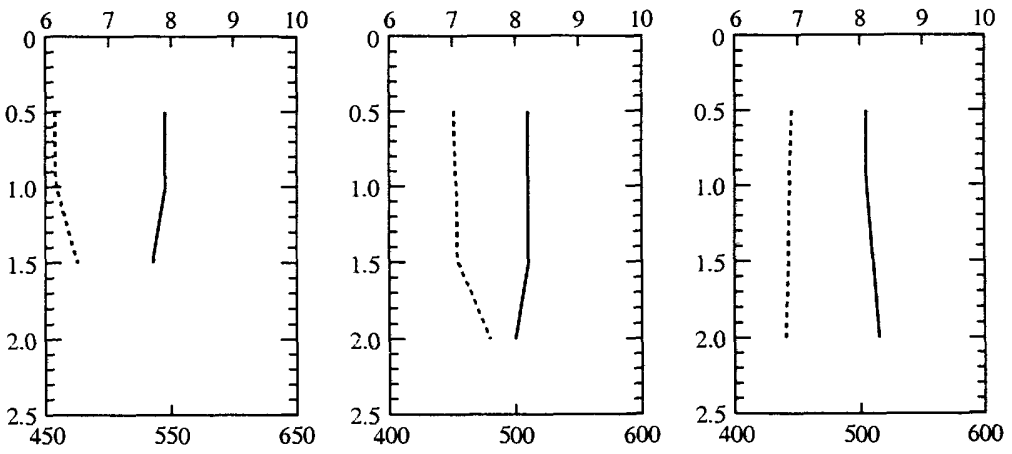

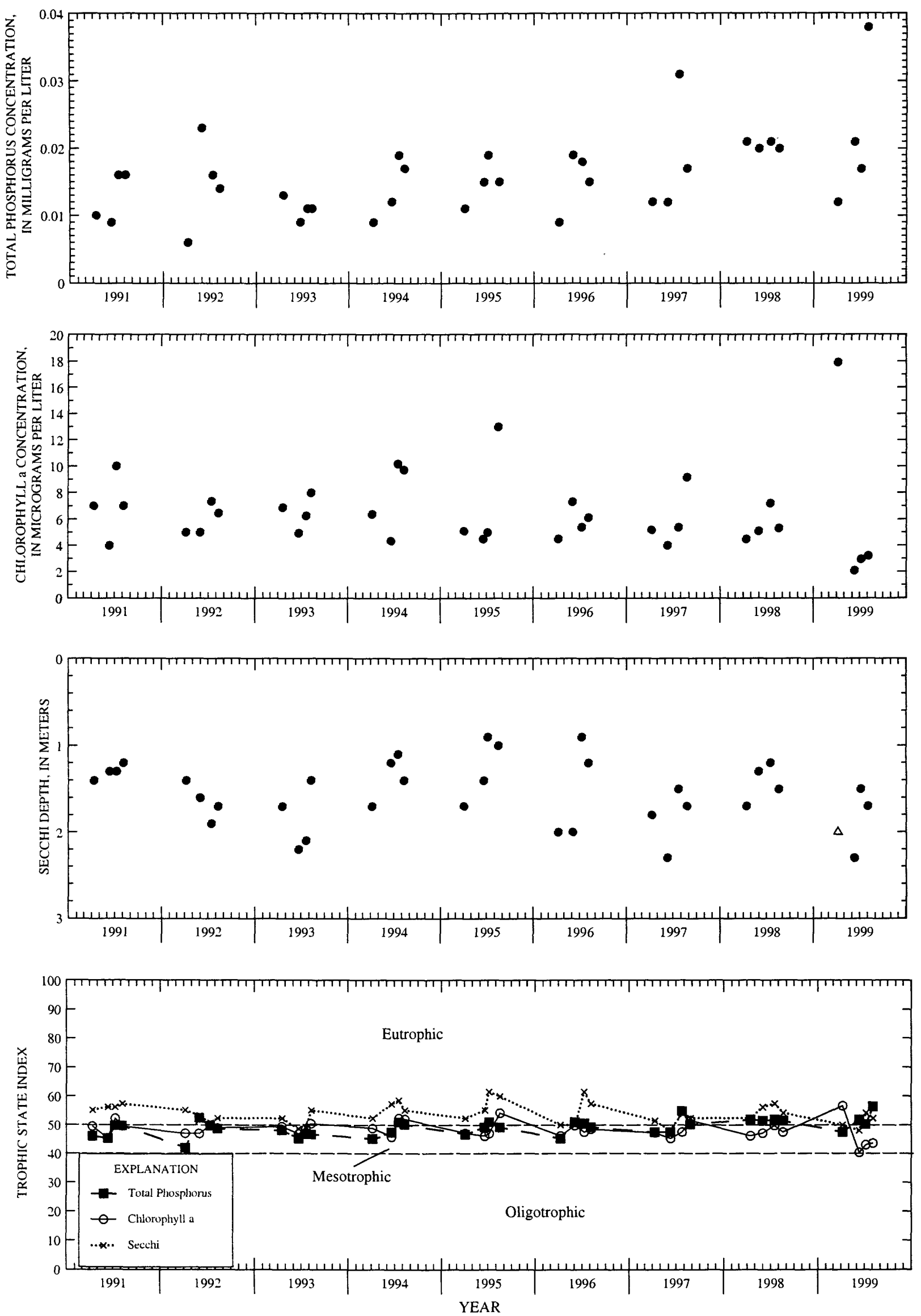

Surface total phosphorus, chlorophyll a concentrations, Secchi depths, and TSI data for Eagle Spring Lake at Eagleville, Wisconsin. (Triangles indicate maximum depth at sampling site. Actual secchi depth on these days was greater than plotted triangles.) 
LOCATION.--Lat $43^{\circ} 17^{\prime} 27^{\prime \prime}$, long $89^{\circ} 39^{\prime} 09^{\prime \prime}$ in NE 1/4 SW 1/4 sec.3, T.9 N., R.7 E., Dane County, Hydrologic Unit 07070005 , on north side of lake, 0.4 mi southwest of Crystal Lake, and 3.1 mi east of Sauk City.

DRAINAGE AREA.--2.23 $\mathrm{mi}^{2}$. Area of Fish Lake, 252 acres.

PERIOD OF RECORD.--November 1966 to September 1981, April 1985 to May 1987, May 1988, April 1989 to October 11, 1990 (fragmentary); continuous record from Oct. 23, 1990 to Nov. 22, 1996; nonrecording gage Nov. 23, 1996 to current year.

REVISED RECORDS.--WDR WI-92-1: Drainage area. WDR WI-87-1: All published values for the 1987 water year are invalid. Two valid values for water years 1987 and 1988 are available: May 7, 1987, water surface $10.52 \mathrm{ft}$, and May 16, 1988, water surface 10.83 ft.

GAGE.--Nonrecording gage. Datum of gage is $848.07 \mathrm{ft}$ above sea level. Prior to Oct. 23, 1990, nonrecording gage. Local observer, Richard Lillie, reads staff gage 2-3 times per week when lake is ice-free.

REMARKS.--Lake has no surface outlet.

EXTREMES FOR PERIOD OF RECORD.--Maximum gage height observed, $11.96 \mathrm{ft}$, July 4, 1999; minimum observed, $3.02 \mathrm{ft}$, Aug. 29 , 1970.

EXTREMES FOR CURRENT YEAR.--Maximum gage height observed, $11.96 \mathrm{ft}$, July 4; minimum observed, $11.07 \mathrm{ft}$, Jan. 7.

GAGE HEIGHT, FEET, WATER YEAR OCTOBER 1998 TO SEPTEMBER 1999

DAILY MEAN VALUES

\begin{tabular}{|c|c|c|c|c|c|c|c|c|c|c|c|c|}
\hline DAY & OCT & NOV & DEC & JAN & FEB & MAR & APR & MAY & JUN & JUL & AUG & SEP \\
\hline 1 & -- & --- & -- & -- & -- & -- & --- & 11.82 & 11.83 & 11.95 & 11.87 & -- \\
\hline 2 & 11.23 & -- & $\ldots$ & -- & -- & -- & -- & 11.81 & 11.86 & 11.94 & --- & -. \\
\hline 3 & -- & 11.19 & $\ldots$ & --. & -.. & 11.32 & 11.46 & -- & -. & 11.95 & -- & 11.64 \\
\hline 4 & 11.20 & $-\cdots$ & $\ldots$ & $\cdots$ & $\cdots$ & $\cdots$ & 11.51 & 11.79 & 11.84 & 11.96 & 11.83 & --- \\
\hline 5 & $\ldots$ & $\ldots$ & -- & 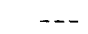 & -- & $\ldots$ & $\ldots$ & 11.79 & 11.83 & 11.94 & 11.81 & 11.62 \\
\hline 6 & 11.35 & --- & --- & -.- & -- & -. & -- & --- & 11.82 & -. & 11.79 & 11.61 \\
\hline 7 & $-\cdots$ & -- & -- & 11.07 & -- & -- & -- & -.- & 11.84 & 11.90 & --- & 11.58 \\
\hline 8 & $-\ldots$ & $\ldots$ & -- & -- & -- & -- & 11.48 & $\ldots$ & 11.82 & 11.86 & 11.79 & 11.56 \\
\hline 9 & 11.32 & $\ldots$ & -.- & -.- & -- & -- & 11.62 & 11.84 & 11.84 & 11.86 & 11.82 & $\cdots$ \\
\hline 10 & --- & -- & $-\cdots$ & -- & --- & -- & 11.62 & -- & $-\cdot$ & -.- & 11.80 & -- \\
\hline 11 & 11.30 & $\ldots$ & $-\ldots$ & -- & ... & -.. & 11.65 & 11.83 & -- & $\cdots$ & 11.79 & 11.51 \\
\hline 12 & $\ldots$ & $\ldots$ & -.- & $\cdots$ & -. & -- & $\ldots$ & -- & -- &.- & 11.79 & -- \\
\hline 13 & -- & 11.22 & $\ldots$ & -- & -- & -- & 11.65 & 11.81 & 11.85 & $-\cdots$ & $\cdots$ & -- \\
\hline 14 & 11.28 & -. & -.- & -- & -- & -.. & -- & $\cdots$ & 11.84 & -.- & 11.77 & 11.45 \\
\hline 15 & -- & -- & --- & $\cdots$ & -- & $-\cdots$ & 11.65 & 11.71 & -. & --- & 11.75 & 11.44 \\
\hline 16 & 11.26 &.- & -- & -. & -- & -.. & 11.64 & 11.71 & 11.82 & --- & 11.74 & 11.43 \\
\hline 17 & $\ldots$ & -- & $\ldots$ & -.- & -- & -- & --- & 11.88 & 11.80 & --- & 11.73 & --- \\
\hline 18 & 11.30 & $\cdots$ & -- & $\cdots$ & -- & $\cdots$ & 11.63 & -. & 11.78 & --- & -- & 11.40 \\
\hline 19 & $\ldots$ & $\ldots$ & --- & $\cdots$ &.- & $\ldots$ & $\ldots$ & 11.90 & 11.75 & -- & 11.74 & 11.39 \\
\hline 20 & -- & -- & $\cdots$ & -- & -- & -- & --- & --- & 11.74 & 11.76 & 11.73 & 11.37 \\
\hline 21 & 11.28 & $\ldots$ & -. & $\ldots$ & -- & $-\cdots$ & -- & --- & 11.73 & 11.89 & -- & --- \\
\hline 22 & -- &.- & $\ldots$ & -- & -- & -- & -- & 11.90 & -.. & 11.89 & 11.70 & -- \\
\hline 23 & -- & --- & $\ldots$ & -- & -- & -- & 11.84 & 11.94 & 11.75 & -- & 11.77 & 11.35 \\
\hline 24 & 11.22 & $\ldots$ & -- & $-\cdots$ & -- & -- & 11.86 & 11.92 & 11.74 & 11.88 & 11.76 & 11.33 \\
\hline 25 & 11.22 & --- & -- & --- & -- & -- & 11.85 & 11.90 & 11.73 & 11.87 & -- & --- \\
\hline 26 & $\ldots$ & - - - & -.. & $\ldots$ & -.. & 11.28 & 11.84 & --- & $\ldots$ & 11.89 &.- & $\ldots$ \\
\hline 27 & -.. & -.- & -.- & -- & -- &.- & -- & --- & -- & 11.87 & -- & -- \\
\hline 28 & -.- & -- & -.. & -- & -- & $\ldots$ & 11.83 & -.. & -.. & 11.85 & 11.74 & -- \\
\hline 29 & -- & -- & $\ldots$ & -- & -- & 11.25 & 11.82 & 11.84 & $\ldots$ & 11.83 & -- & 11.37 \\
\hline 30 & -.. & -- & $\ldots$ & -- & -- &.- & -- & 11.83 & 11.70 & $\cdots$ & $\cdots$ & 11.45 \\
\hline 31 & 11.26 & -- & -.- & -- & --- & -. & $\ldots$ & $\ldots$ & -- & 11.91 & 11.66 & -- \\
\hline
\end{tabular}

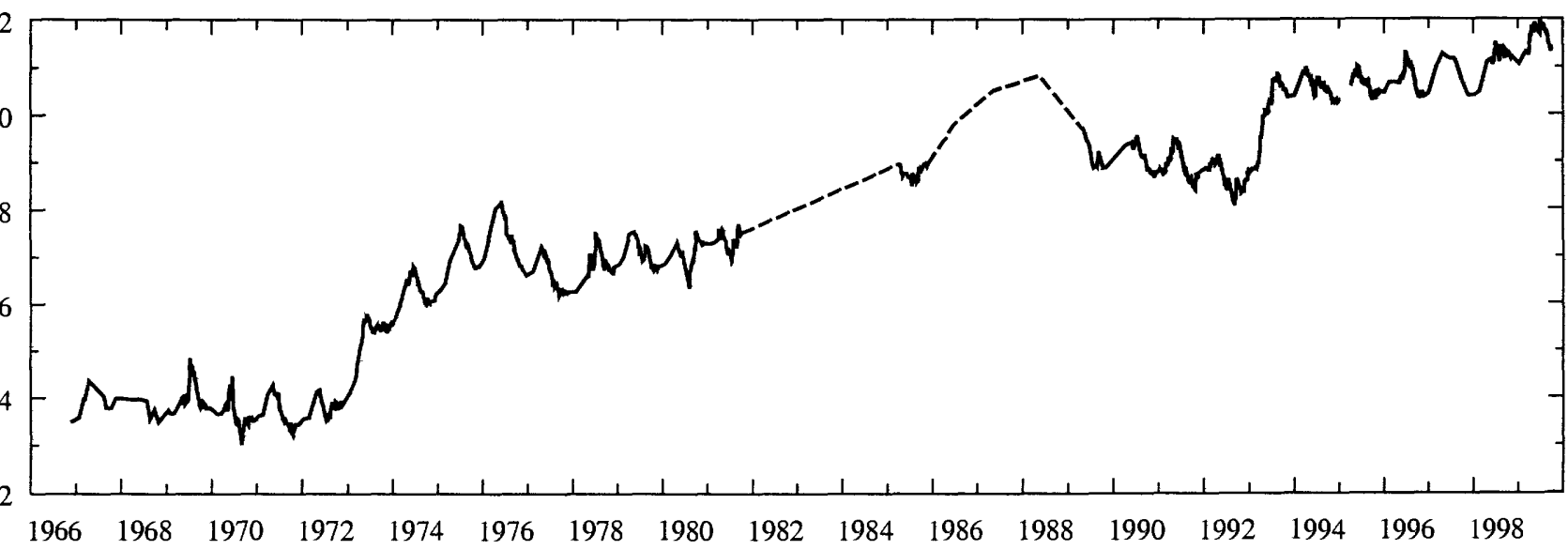




\section{GENEVA LAKE AT LAKE GENEVA, WI}

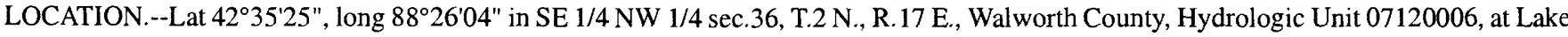
Geneva dam at Center Street at Lake Geneva.

DRAINAGE AREA. - $-28.7 \mathrm{mi}^{2}$. Area of Lake Geneva, 5,262 acres.

PERIOD OF RECORD.--October 1997 to September 1999.

GAGE.--Water-stage recorder. Datum of gage is $862.08 \mathrm{ft}$ above sea level.

REMARKS.--Estimated daily gage heights: Jan. 10-11, Apr. 9, and May 27, 1998. Recording rain gage and gage-height telemeter at station.

EXTREMES FOR CURRENT PERIOD.--Water year 1998: Maximum gage height observed, $3.02 \mathrm{ft}$, June 28; minimum gage height observed, $1.86 \mathrm{ft}$, Jan. 2.

Water year 1999: Maximum gage height observed, $3.23 \mathrm{ft}$, May 17; minimum gage height observed, $1.84 \mathrm{ft}$, Sept. 27.

GAGE HEIGHT, FEET, WATER YEAR OCTOBER 1997 TO SEPTEMBER 1998

DAILY MEAN VALUES

\begin{tabular}{|c|c|c|c|c|c|c|c|c|c|c|c|c|}
\hline DAY & OCT & NOV & $\mathrm{DEC}$ & JAN & FEB & MAR & APR & MAY & JUN & JUL & AUG & SEP \\
\hline $\begin{array}{l}1 \\
2 \\
3 \\
4 \\
5\end{array}$ & $\begin{array}{l}2.40 \\
2.40 \\
2.39 \\
2.40 \\
2.40\end{array}$ & $\begin{array}{l}2.31 \\
2.30 \\
2.28 \\
2.28 \\
2.27\end{array}$ & $\begin{array}{l}2.26 \\
2.26 \\
2.29 \\
2.31 \\
2.30\end{array}$ & $\begin{array}{l}2.02 \\
2.02 \\
2.03 \\
2.05 \\
2.10\end{array}$ & $\begin{array}{l}2.06 \\
2.08 \\
2.07 \\
2.06 \\
2.06\end{array}$ & $\begin{array}{l}2.03 \\
2.04 \\
2.05 \\
2.05 \\
2.06\end{array}$ & $\begin{array}{l}2.49 \\
2.50 \\
2.49 \\
2.49 \\
2.49\end{array}$ & $\begin{array}{l}2.65 \\
2.65 \\
2.64 \\
2.63 \\
2.63\end{array}$ & $\begin{array}{l}2.67 \\
2.65 \\
2.62 \\
2.60 \\
2.57\end{array}$ & $\begin{array}{l}2.77 \\
2.75 \\
2.73 \\
2.74 \\
2.71\end{array}$ & $\begin{array}{l}2.35 \\
2.34 \\
2.33 \\
2.34 \\
2.36\end{array}$ & $\begin{array}{l}2.35 \\
2.32 \\
2.30 \\
2.29 \\
2.29\end{array}$ \\
\hline $\begin{array}{r}6 \\
7 \\
8 \\
9 \\
10\end{array}$ & $\begin{array}{l}2.38 \\
2.38 \\
2.39 \\
2.41 \\
2.38\end{array}$ & $\begin{array}{l}2.29 \\
2.30 \\
2.30 \\
2.30 \\
2.29\end{array}$ & $\begin{array}{l}2.29 \\
2.28 \\
2.28 \\
2.28 \\
2.31\end{array}$ & $\begin{array}{l}2.12 \\
2.14 \\
2.16 \\
2.24 \\
2.21\end{array}$ & $\begin{array}{l}2.05 \\
2.05 \\
2.04 \\
2.03 \\
2.03\end{array}$ & $\begin{array}{l}2.07 \\
2.06 \\
2.08 \\
2.13 \\
2.13\end{array}$ & $\begin{array}{l}2.49 \\
2.49 \\
2.55 \\
2.59 \\
2.60\end{array}$ & $\begin{array}{l}2.64 \\
2.73 \\
2.79 \\
2.77 \\
2.75\end{array}$ & $\begin{array}{l}2.56 \\
2.54 \\
2.52 \\
2.52 \\
2.54\end{array}$ & $\begin{array}{l}2.71 \\
2.69 \\
2.68 \\
2.66 \\
2.64\end{array}$ & $\begin{array}{l}2.40 \\
2.41 \\
2.43 \\
2.43 \\
2.42\end{array}$ & $\begin{array}{l}2.29 \\
2.34 \\
2.31 \\
2.29 \\
2.29\end{array}$ \\
\hline $\begin{array}{l}11 \\
12 \\
13 \\
14 \\
15\end{array}$ & $\begin{array}{l}2.37 \\
2.37 \\
2.42 \\
2.39 \\
2.36\end{array}$ & $\begin{array}{l}2.29 \\
2.27 \\
2.25 \\
2.25 \\
2.27\end{array}$ & $\begin{array}{l}2.31 \\
2.31 \\
2.28 \\
2.26 \\
2.26\end{array}$ & $\begin{array}{l}2.17 \\
2.14 \\
2.15 \\
2.14 \\
2.14\end{array}$ & $\begin{array}{l}2.06 \\
2.10 \\
2.09 \\
2.07 \\
2.06\end{array}$ & $\begin{array}{l}2.13 \\
2.14 \\
2.14 \\
2.12 \\
2.11\end{array}$ & $\begin{array}{l}2.60 \\
2.60 \\
2.60 \\
2.62 \\
2.60\end{array}$ & $\begin{array}{l}2.74 \\
2.72 \\
2.76 \\
2.74 \\
2.73\end{array}$ & $\begin{array}{l}2.57 \\
2.62 \\
2.64 \\
2.62 \\
2.61\end{array}$ & $\begin{array}{l}2.63 \\
2.61 \\
2.59 \\
2.58 \\
2.57\end{array}$ & $\begin{array}{l}2.40 \\
2.38 \\
2.37 \\
2.38 \\
2.37\end{array}$ & $\begin{array}{l}2.28 \\
2.25 \\
2.25 \\
2.30 \\
2.38\end{array}$ \\
\hline $\begin{array}{l}16 \\
17 \\
18 \\
19 \\
20\end{array}$ & $\begin{array}{l}2.35 \\
2.34 \\
2.34 \\
2.33 \\
2.31\end{array}$ & $\begin{array}{l}2.28 \\
2.27 \\
2.25 \\
2.24 \\
2.23\end{array}$ & $\begin{array}{l}2.23 \\
2.21 \\
2.20 \\
2.18 \\
2.17\end{array}$ & $\begin{array}{l}2.13 \\
2.14 \\
2.13 \\
2.12 \\
2.11\end{array}$ & $\begin{array}{l}2.05 \\
2.07 \\
2.07 \\
2.06 \\
2.05\end{array}$ & $\begin{array}{l}2.11 \\
2.12 \\
2.18 \\
2.21 \\
2.21\end{array}$ & $\begin{array}{l}2.67 \\
2.67 \\
2.66 \\
2.65 \\
2.65\end{array}$ & $\begin{array}{l}2.77 \\
2.73 \\
2.71 \\
2.69 \\
2.68\end{array}$ & $\begin{array}{l}2.61 \\
2.61 \\
2.61 \\
2.66 \\
2.63\end{array}$ & $\begin{array}{l}2.53 \\
2.52 \\
2.51 \\
2.51 \\
2.51\end{array}$ & $\begin{array}{l}2.36 \\
2.36 \\
2.34 \\
2.33 \\
2.33\end{array}$ & $\begin{array}{l}2.37 \\
2.37 \\
2.37 \\
2.37 \\
2.37\end{array}$ \\
\hline $\begin{array}{l}21 \\
22 \\
23 \\
24 \\
25\end{array}$ & $\begin{array}{l}2.30 \\
2.28 \\
2.28 \\
2.29 \\
2.29\end{array}$ & $\begin{array}{l}2.22 \\
2.24 \\
2.22 \\
2.21 \\
2.22\end{array}$ & $\begin{array}{l}2.15 \\
2.14 \\
2.13 \\
2.12 \\
2.14\end{array}$ & $\begin{array}{l}2.11 \\
2.11 \\
2.12 \\
2.11 \\
2.10\end{array}$ & $\begin{array}{l}2.04 \\
2.02 \\
2.02 \\
2.03 \\
2.01\end{array}$ & $\begin{array}{l}2.22 \\
2.23 \\
2.24 \\
2.24 \\
2.25\end{array}$ & $\begin{array}{l}2.68 \\
2.69 \\
2.68 \\
2.67 \\
2.65\end{array}$ & $\begin{array}{l}2.65 \\
2.61 \\
2.60 \\
2.66 \\
2.67\end{array}$ & $\begin{array}{l}2.63 \\
2.61 \\
2.60 \\
2.59 \\
2.62\end{array}$ & $\begin{array}{l}2.55 \\
2.53 \\
2.51 \\
2.48 \\
2.46\end{array}$ & $\begin{array}{l}2.32 \\
2.32 \\
2.34 \\
2.33 \\
2.36\end{array}$ & $\begin{array}{l}2.35 \\
2.33 \\
2.32 \\
2.31 \\
2.31\end{array}$ \\
\hline $\begin{array}{l}26 \\
27 \\
28 \\
29 \\
30 \\
31\end{array}$ & $\begin{array}{l}2.28 \\
2.31 \\
2.31 \\
2.30 \\
2.30 \\
2.30\end{array}$ & $\begin{array}{l}2.22 \\
2.22 \\
2.23 \\
2.24 \\
2.26 \\
-\ldots\end{array}$ & $\begin{array}{l}2.13 \\
2.10 \\
2.09 \\
2.08 \\
2.07 \\
2.05\end{array}$ & $\begin{array}{l}2.09 \\
2.08 \\
2.08 \\
2.08 \\
2.07 \\
2.06\end{array}$ & $\begin{array}{r}2.01 \\
2.05 \\
2.04 \\
--- \\
--- \\
-\end{array}$ & $\begin{array}{l}2.29 \\
2.30 \\
2.32 \\
2.34 \\
2.35 \\
2.44\end{array}$ & $\begin{array}{r}2.67 \\
2.67 \\
2.65 \\
2.65 \\
2.64 \\
-\ldots\end{array}$ & $\begin{array}{l}2.66 \\
2.64 \\
2.68 \\
2.71 \\
2.68 \\
2.70\end{array}$ & $\begin{array}{l}2.75 \\
2.73 \\
2.84 \\
2.84 \\
2.82 \\
-\end{array}$ & $\begin{array}{l}2.44 \\
2.44 \\
2.43 \\
2.40 \\
2.38 \\
2.36\end{array}$ & $\begin{array}{l}2.35 \\
2.35 \\
2.37 \\
2.38 \\
2.36 \\
2.35\end{array}$ & $\begin{array}{r}2.33 \\
2.31 \\
2.30 \\
2.30 \\
2.29 \\
-\end{array}$ \\
\hline $\begin{array}{l}\text { MEAN } \\
\text { MAX } \\
\text { MIN }\end{array}$ & $\begin{array}{l}2.35 \\
2.42 \\
2.28\end{array}$ & $\begin{array}{l}2.26 \\
2.31 \\
2.21\end{array}$ & $\begin{array}{l}2.21 \\
2.31 \\
2.05\end{array}$ & $\begin{array}{l}2.11 \\
2.24 \\
2.02\end{array}$ & $\begin{array}{l}2.05 \\
2.10 \\
2.01\end{array}$ & $\begin{array}{l}2.17 \\
2.44 \\
2.03\end{array}$ & $\begin{array}{l}2.61 \\
2.69 \\
2.49\end{array}$ & $\begin{array}{l}2.69 \\
2.79 \\
2.60\end{array}$ & $\begin{array}{l}2.63 \\
2.84 \\
2.52\end{array}$ & $\begin{array}{l}2.57 \\
2.77 \\
2.36\end{array}$ & $\begin{array}{l}2.36 \\
2.43 \\
2.32\end{array}$ & $\begin{array}{l}2.32 \\
2.38 \\
2.25\end{array}$ \\
\hline
\end{tabular}


GAGE HEIGHT, FEET, WATER YEAR OCTOBER 1998 TO SEPTEMBER 1999

\section{DAILY MEAN VALUES}

\begin{tabular}{|c|c|c|c|c|c|c|c|c|c|c|c|c|}
\hline DAY & OCT & NOV & DEC & JAN & FEB & MAR & APR & MAY & JUN & JUL & AUG & SEP \\
\hline $\begin{array}{l}1 \\
2 \\
3 \\
4 \\
5\end{array}$ & $\begin{array}{l}2.27 \\
2.26 \\
2.28 \\
2.27 \\
2.32\end{array}$ & $\begin{array}{l}2.40 \\
2.36 \\
2.35 \\
2.34 \\
2.34\end{array}$ & $\begin{array}{l}2.46 \\
2.45 \\
2.45 \\
2.44 \\
2.45\end{array}$ & $\begin{array}{l}2.37 \\
2.38 \\
2.45 \\
2.43 \\
2.43\end{array}$ & $\begin{array}{l}2.57 \\
2.57 \\
2.57 \\
2.56 \\
2.55\end{array}$ & $\begin{array}{l}2.40 \\
2.40 \\
2.40 \\
2.38 \\
2.37\end{array}$ & $\begin{array}{l}2.35 \\
2.36 \\
2.36 \\
2.38 \\
2.37\end{array}$ & $\begin{array}{l}2.74 \\
2.73 \\
2.71 \\
2.69 \\
2.70\end{array}$ & $\begin{array}{l}2.85 \\
2.89 \\
2.86 \\
2.83 \\
2.83\end{array}$ & $\begin{array}{l}2.69 \\
2.67 \\
2.69 \\
2.68 \\
2.67\end{array}$ & $\begin{array}{l}2.44 \\
2.41 \\
2.40 \\
2.37 \\
2.35\end{array}$ & $\begin{array}{l}2.32 \\
2.31 \\
2.32 \\
2.31 \\
2.31\end{array}$ \\
\hline $\begin{array}{r}6 \\
7 \\
8 \\
9 \\
10\end{array}$ & $\begin{array}{l}2.38 \\
2.39 \\
2.37 \\
2.37 \\
2.36\end{array}$ & $\begin{array}{l}2.33 \\
2.32 \\
2.32 \\
2.32 \\
2.58\end{array}$ & $\begin{array}{l}2.47 \\
2.50 \\
2.50 \\
2.49 \\
2.48\end{array}$ & $\begin{array}{l}2.43 \\
2.44 \\
2.44 \\
2.45 \\
2.45\end{array}$ & $\begin{array}{l}2.55 \\
2.54 \\
2.54 \\
2.53 \\
2.52\end{array}$ & $\begin{array}{l}2.39 \\
2.38 \\
2.36 \\
2.39 \\
2.39\end{array}$ & $\begin{array}{l}2.43 \\
2.41 \\
2.40 \\
2.48 \\
2.50\end{array}$ & $\begin{array}{l}2.76 \\
2.77 \\
2.75 \\
2.72 \\
2.70\end{array}$ & $\begin{array}{l}2.82 \\
2.83 \\
2.79 \\
2.77 \\
2.77\end{array}$ & $\begin{array}{l}2.63 \\
2.61 \\
2.59 \\
2.58 \\
2.53\end{array}$ & $\begin{array}{l}2.33 \\
2.32 \\
2.31 \\
2.30 \\
2.29\end{array}$ & $\begin{array}{l}2.30 \\
2.28 \\
2.27 \\
2.25 \\
2.22\end{array}$ \\
\hline $\begin{array}{l}11 \\
12 \\
13 \\
14 \\
15\end{array}$ & $\begin{array}{l}2.36 \\
2.36 \\
2.34 \\
2.32 \\
2.31\end{array}$ & $\begin{array}{l}2.53 \\
2.47 \\
2.46 \\
2.47 \\
2.46\end{array}$ & $\begin{array}{l}2.48 \\
2.46 \\
2.45 \\
2.45 \\
2.45\end{array}$ & $\begin{array}{l}2.45 \\
2.45 \\
2.45 \\
2.45 \\
2.45\end{array}$ & $\begin{array}{l}2.56 \\
2.60 \\
2.58 \\
2.56 \\
2.55\end{array}$ & $\begin{array}{l}2.37 \\
2.35 \\
2.34 \\
2.33 \\
2.32\end{array}$ & $\begin{array}{l}2.52 \\
2.52 \\
2.52 \\
2.52 \\
2.52\end{array}$ & $\begin{array}{l}2.69 \\
2.76 \\
2.75 \\
2.76 \\
2.76\end{array}$ & $\begin{array}{l}2.85 \\
2.90 \\
3.04 \\
3.15 \\
3.08\end{array}$ & $\begin{array}{l}2.52 \\
2.51 \\
2.51 \\
2.50 \\
2.47\end{array}$ & $\begin{array}{l}2.28 \\
2.29 \\
2.31 \\
2.28 \\
2.27\end{array}$ & $\begin{array}{l}2.19 \\
2.19 \\
2.19 \\
2.17 \\
2.14\end{array}$ \\
\hline $\begin{array}{l}16 \\
17 \\
18 \\
19 \\
20\end{array}$ & $\begin{array}{l}2.32 \\
2.39 \\
2.50 \\
2.47 \\
2.45\end{array}$ & $\begin{array}{l}2.45 \\
2.44 \\
2.44 \\
2.47 \\
2.42\end{array}$ & $\begin{array}{l}2.43 \\
2.42 \\
2.42 \\
2.41 \\
2.40\end{array}$ & $\begin{array}{l}2.45 \\
2.48 \\
2.53 \\
2.52 \\
2.51\end{array}$ & $\begin{array}{l}2.55 \\
2.53 \\
2.52 \\
2.51 \\
2.50\end{array}$ & $\begin{array}{l}2.31 \\
2.33 \\
2.29 \\
2.29 \\
2.30\end{array}$ & $\begin{array}{l}2.55 \\
2.56 \\
2.57 \\
2.56 \\
2.57\end{array}$ & $\begin{array}{l}2.79 \\
2.92 \\
2.94 \\
2.93 \\
2.92\end{array}$ & $\begin{array}{l}3.03 \\
2.97 \\
2.92 \\
2.87 \\
2.84\end{array}$ & $\begin{array}{l}2.46 \\
2.47 \\
2.48 \\
2.48 \\
2.48\end{array}$ & $\begin{array}{l}2.27 \\
2.25 \\
2.24 \\
2.29 \\
2.29\end{array}$ & $\begin{array}{l}2.12 \\
2.12 \\
2.11 \\
2.12 \\
2.10\end{array}$ \\
\hline $\begin{array}{l}21 \\
22 \\
23 \\
24 \\
25\end{array}$ & $\begin{array}{l}2.43 \\
2.41 \\
2.41 \\
2.40 \\
2.39\end{array}$ & $\begin{array}{l}2.41 \\
2.43 \\
2.41 \\
2.40 \\
2.40\end{array}$ & $\begin{array}{l}2.43 \\
2.45 \\
2.41 \\
2.42 \\
2.42\end{array}$ & $\begin{array}{l}2.51 \\
2.58 \\
2.64 \\
2.66 \\
2.66\end{array}$ & $\begin{array}{l}2.49 \\
2.47 \\
2.45 \\
2.44 \\
2.43\end{array}$ & $\begin{array}{l}2.30 \\
2.30 \\
2.31 \\
2.31 \\
2.31\end{array}$ & $\begin{array}{l}2.58 \\
2.61 \\
2.77 \\
2.80 \\
2.78\end{array}$ & $\begin{array}{l}2.92 \\
2.99 \\
3.00 \\
2.98 \\
2.96\end{array}$ & $\begin{array}{l}2.81 \\
2.79 \\
2.77 \\
2.77 \\
2.76\end{array}$ & $\begin{array}{l}2.48 \\
2.46 \\
2.50 \\
2.53 \\
2.51\end{array}$ & $\begin{array}{l}2.29 \\
2.29 \\
2.30 \\
2.36 \\
2.36\end{array}$ & $\begin{array}{l}2.08 \\
2.07 \\
2.06 \\
2.03 \\
2.02\end{array}$ \\
\hline $\begin{array}{l}26 \\
27 \\
28 \\
29 \\
30 \\
31\end{array}$ & $\begin{array}{l}2.39 \\
2.40 \\
2.41 \\
2.40 \\
2.41 \\
2.41\end{array}$ & $\begin{array}{r}2.40 \\
2.39 \\
2.40 \\
2.42 \\
2.45 \\
\ldots\end{array}$ & $\begin{array}{l}2.41 \\
2.39 \\
2.39 \\
2.39 \\
2.39 \\
2.39\end{array}$ & $\begin{array}{l}2.64 \\
2.63 \\
2.62 \\
2.61 \\
2.59 \\
2.57\end{array}$ & $\begin{array}{r}2.42 \\
2.42 \\
2.42 \\
\ldots- \\
\ldots- \\
\ldots-\end{array}$ & $\begin{array}{l}2.31 \\
2.32 \\
2.34 \\
2.34 \\
2.35 \\
2.36\end{array}$ & $\begin{array}{l}2.77 \\
2.75 \\
2.78 \\
2.77 \\
2.76 \\
-\end{array}$ & $\begin{array}{l}2.92 \\
2.89 \\
2.86 \\
2.84 \\
2.82 \\
2.83\end{array}$ & $\begin{array}{r}2.74 \\
2.72 \\
2.77 \\
2.74 \\
2.71 \\
-\end{array}$ & $\begin{array}{l}2.51 \\
2.50 \\
2.50 \\
2.50 \\
2.48 \\
2.48\end{array}$ & $\begin{array}{l}2.36 \\
2.37 \\
2.37 \\
2.35 \\
2.32 \\
2.32\end{array}$ & $\begin{array}{r}2.03 \\
2.04 \\
2.27 \\
2.34 \\
2.34 \\
-\end{array}$ \\
\hline $\begin{array}{l}\text { MEAN } \\
\text { MAX } \\
\text { MIN }\end{array}$ & $\begin{array}{l}2.37 \\
2.50 \\
2.26\end{array}$ & $\begin{array}{l}2.41 \\
2.58 \\
2.32\end{array}$ & $\begin{array}{l}2.44 \\
2.50 \\
2.39\end{array}$ & $\begin{array}{l}2.51 \\
2.66 \\
2.37\end{array}$ & $\begin{array}{l}2.52 \\
2.60 \\
2.42\end{array}$ & $\begin{array}{l}2.34 \\
2.40 \\
2.29\end{array}$ & $\begin{array}{l}2.56 \\
2.80 \\
2.35\end{array}$ & $\begin{array}{l}2.82 \\
3.00 \\
2.69\end{array}$ & $\begin{array}{l}2.85 \\
3.15 \\
2.71\end{array}$ & $\begin{array}{l}2.54 \\
2.69 \\
2.46\end{array}$ & $\begin{array}{l}2.32 \\
2.44 \\
2.24\end{array}$ & $\begin{array}{l}2.19 \\
2.34 \\
2.02\end{array}$ \\
\hline
\end{tabular}




\section{GENEVA LAKE AT GENEVA BAY AT LAKE GENEVA, WI}

LOCATION.--Lat $42^{\circ} 34^{\prime} 55^{\prime \prime}$, long $88^{\circ} 26^{\prime} 38^{\prime \prime}$, in NE $1 / 4$ NE 1/4, sec.2, T.1 N., R.17 E., Walworth County, Hydrologic Unit 07120006, 0.7 mi southwest of outlet at Lake Geneva.

DRAINAGE AREA.--28.7 $\mathrm{mi}^{2}$.

PERIOD OF RECORD.--April 1997 to February 1999 (discontinued).

REMARKS.--Lake sampled at a depth of about $15 \mathrm{~m}$. Lake ice-covered during February sampling. Water-quality analyses done by Wisconsin State Laboratory of Hygiene. Samples for determination of chlorophyll-a concentration are collected from the top $1.5 \mathrm{ft}$ of the lake.

WATER-QUALITY DATA, FEBRUARY 08, 1999

(Milligrams per liter unless otherwise indicated)

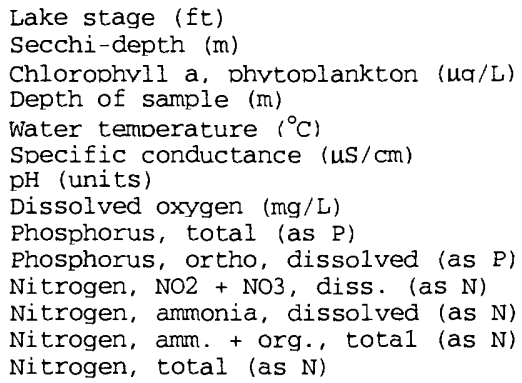

\begin{tabular}{cc}
\multicolumn{2}{c}{ Feb-8 } \\
\hline \multicolumn{2}{c}{5.54} \\
\multicolumn{2}{c}{2.6} \\
0.5 & 14.0 \\
1.9 & 2.1 \\
505 & 506 \\
8.3 & 8.4 \\
13.4 & 13.1 \\
$<0.005$ & 0.007 \\
0.002 & 0.002 \\
0.087 & 0.055 \\
0.013 & $<0.013$ \\
0.42 & 0.62 \\
0.51 & 0.68
\end{tabular}

02-08-99

DISSOLVED OXYGEN (D.O.), IN MILLIGRAMS PER LITER

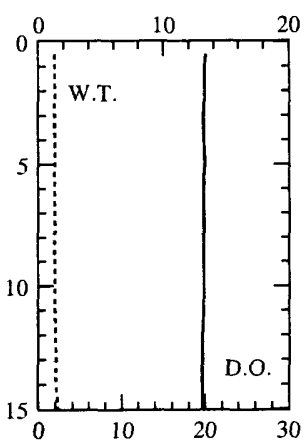

WATER TEMPERATURE (W.T.), IN DEGREES CELSIUS

PH, IN STANDARD UNITS

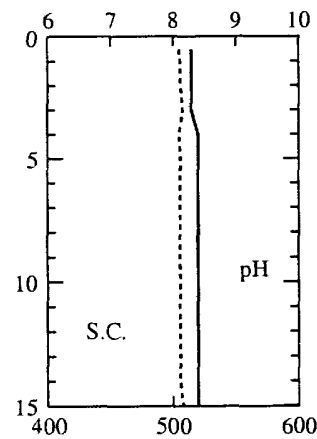

SPECIFIC CONDUCTANCE (S.C.), IN MICROSIEMENS PER CENTIMETER AT 25 DEGREES CELSIUS 


\section{GENEVA LAKE AT WILLIAMS BAY AT WILLIAMS BAY, WI}

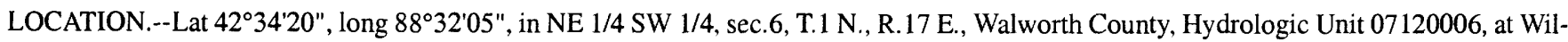
liams Bay.

DRAINAGE AREA. - $-28.7 \mathrm{mi}^{2}$.

PERIOD OF RECORD.--April 1997 to February 1999 (discontinued).

REMARKS.--Lake sampled at a depth of about $18 \mathrm{~m}$. Lake ice-covered during February sampling. Water-quality analyses done by Wisconsin State Laboratory of Hygiene. Samples for determination of chlorophyll-a concentration are collected from the top $1.5 \mathrm{ft}$ of the lake.

WATER-QUALITY DATA, FEBRUARY 08, 1999 (Milligrams per liter unless otherwise indicated)

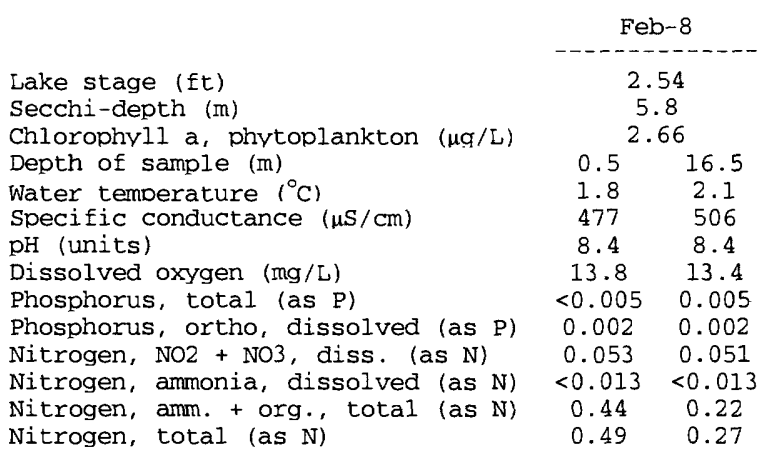

\begin{tabular}{cc}
\multicolumn{2}{c}{ Feb-8 } \\
2.54 \\
\multicolumn{2}{c}{5.8} \\
\multicolumn{2}{c}{2.66} \\
0.5 & 16.5 \\
1.8 & 2.1 \\
477 & 506 \\
8.4 & 8.4 \\
13.8 & 13.4 \\
$<0.005$ & 0.005 \\
0.002 & 0.002 \\
0.053 & 0.051 \\
$<0.013$ & $<0.013$ \\
0.44 & 0.22 \\
0.49 & 0.27
\end{tabular}

02-08-99

DISSOLVED OXYGEN (D.O.), IN MILLIGRAMS PER LITER

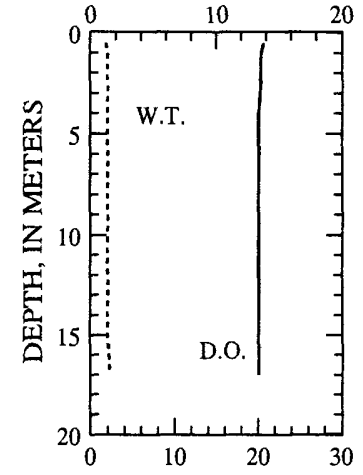

WATER TEMPERATURE (W.T.), IN DEGREES CELSIUS

PH, IN STANDARD UNITS

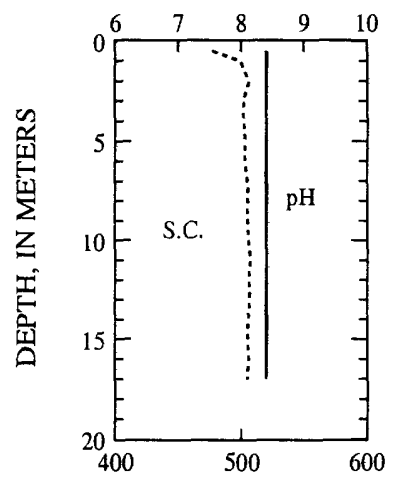

SPECIFIC CONDUCTANCE (S.C.), IN MICROSIEMENS PER CENTIMETER AT 25 DEGREES CELSIUS 


\section{GENEVA LAKE AT CENTER NEAR LAKE GENEVA, WI}

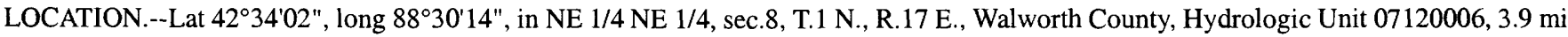
southwest of outlet at Lake Geneva.

DRAINAGE AREA.--28.7 $\mathrm{mi}^{2}$.

PERIOD OF RECORD.--April 1997 to March 1999 (discontinued).

REMARKS.--Lake sampled at a depth of about $29 \mathrm{~m}$. Lake ice-covered during February sampling. Water-quality analyses done by Wisconsin State Laboratory of Hygiene. Samples for determination of chlorophyll-a concentration are collected from the top $1.5 \mathrm{ft}$ of the lake.

WATER-QUALITY DATA, OCTOBER 13 AND 26, 1998

(Milligrams per liter unless otherwise indicated)

Lake stage (ft)

Secchi-depth (m)

Chlorophyll a, phytoplankton ( $\mu \mathrm{g} / \mathrm{L}$ )

Depth of sample (m)

Water temperature $\left({ }^{\circ} \mathrm{C}\right)$

Specific conductance $(\mu \mathrm{S} / \mathrm{cm})$

$\mathrm{pH}$ (units)

Dissolved oxygen (mg/L)

Phosphorus, total (as P)

Phosphorus, ortho, dissolved (as P)

Nitrogen, NO2 + NO3, diss. (as N)

Nitrogen, ammonia, dissolved (as $\mathrm{N}$ )

Nitrogen, amm. + org., total (as N)

Nitrogen, total (as N) oct -13

\subsection{4}

3.8

3.28

0.514.

16.7

$<0.002$

0.019

$<0.013$

0.71

0.73

$8.9 \quad 0.7$

$0.007 \quad 0.006$

$<0.002$

0.01

0.013

0.64

0.65

$<0.013$

$\begin{array}{ll}0.58 & 0.47 \\ 0.59 & 0.60\end{array}$

$10-13-98$

oct -26

2.39

5.2

2.18

19.0

9.7

516

7.6

1.9

0.007

$<0.002$

0.021

$<0.013$

0.43

0.43
0.45

$\begin{array}{cc}23.0 & 28.0 \\ 8.8 & 7.8 \\ 516 & 520 \\ 7.5 & 7.5 \\ 1.2 & 0.6 \\ 0.008 & 0.014 \\ 0.002 & 0.003 \\ 0.073 & 0.031 \\ <0.013 & 0.059 \\ 0.51 & 0.63 \\ 0.58 & 0.66\end{array}$

10-26-98

DISSOLVED OXYGEN (D.O.), IN MILLIGRAMS PER LITER
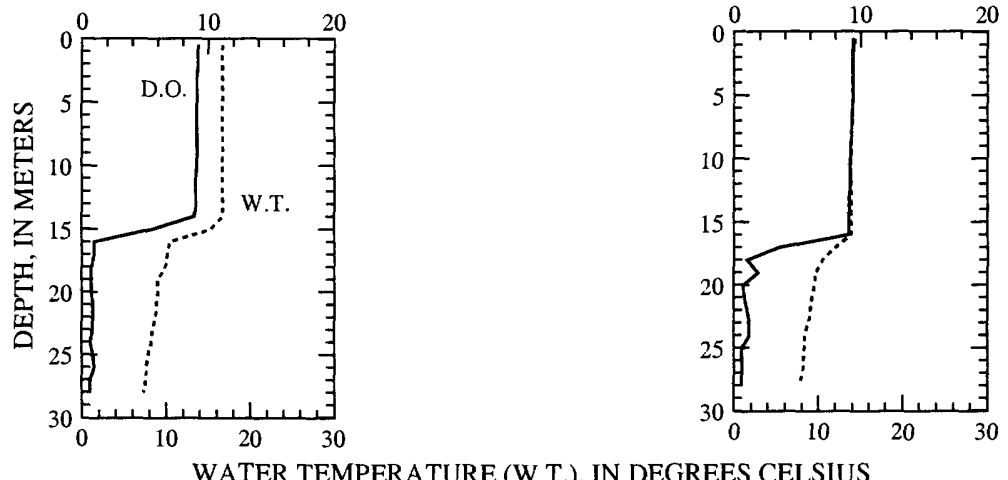

PH, IN STANDARD UNITS
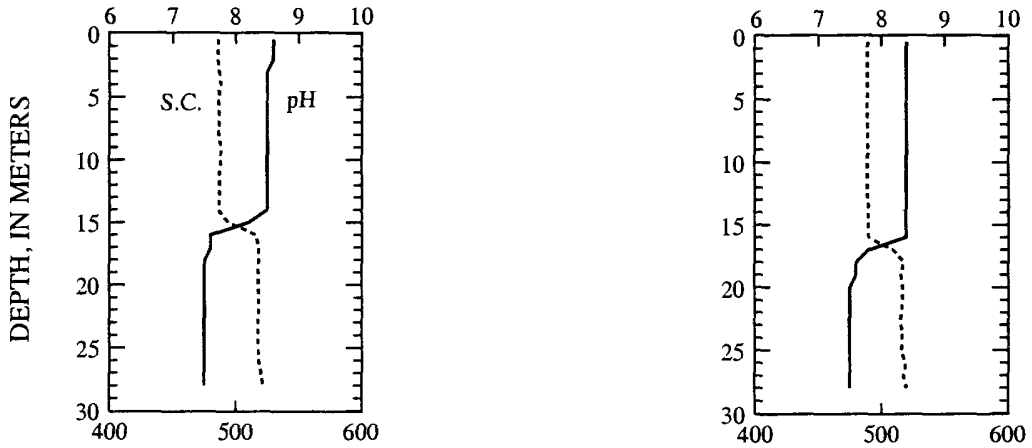

SPECIFIC CONDUCTANCE (S.C.), IN MICROSIEMENS PER CENTIMETER AT 25 DEGREES CELSIUS 
WATER-QUALITY DATA, NOVEMBER 17, 1998 TO MARCH 22, 1999

(Milligrams per liter unless otherwise indicated)

Lake stage (ft)

$\therefore$ ecchi-depth (m)

hlorophyll a, phytoplankton ( $\mu \mathrm{q} / \mathrm{L})$

lepth of sample (m)

Water temoerature $\left({ }^{\circ} \mathrm{C}\right)$

recific conductance ( $\mu \mathrm{S} / \mathrm{cm}$ )

$\mathrm{TH}$ (units)

Tissolved oxygen (mg/L)

'hosphorus, total (as P)

'hosphorus, ortho, dissolved (as P)

1-itrogen, $\mathrm{NO} 2+\mathrm{NO} 3$, diss. (as N)

litrogen, ammonia, dissolved (as N)

itrogen, amm. + org., total (as $\mathrm{N}$ )

litrogen, total (as N)

$11-17-98$

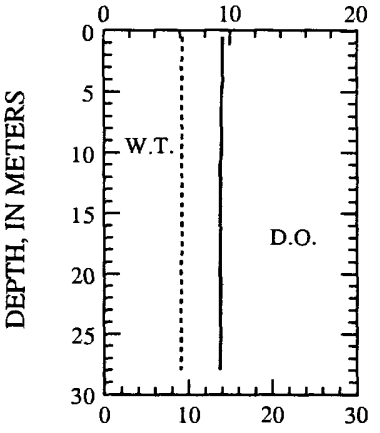

Dec-16
2.43
7.3
1.73

0.5

7.2
495

495
8.2

11.3

0.014

0.003

0.063

0.019

0.50

0.56

$12-16-98$

\begin{tabular}{cc}
\multicolumn{2}{c}{ Feb-8 } \\
\multicolumn{3}{c}{2.54} \\
\multicolumn{3}{c}{6.6} \\
0.5 & 3.40 \\
1.7 & 27.0 \\
500 & 2.5 \\
8.4 & 502 \\
13.4 & 8.3 \\
0.006 & 11.4 \\
$<0.002$ & 0.014 \\
0.057 & 0.003 \\
$<0.013$ & 0.066 \\
0.48 & $<0.013$ \\
0.54 & 0.18 \\
0.25 & 0.25
\end{tabular}

03-22-99

DISSOLVED OXYGEN (D.O.), IN MILLIGRAMS PER LITER
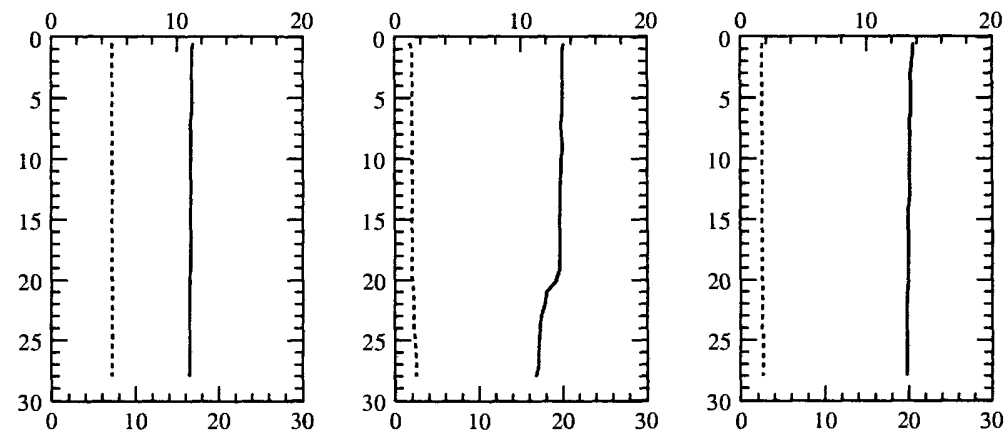

WATER TEMPERATURE (W.T.), IN DEGREES CELSIUS

PH, IN STANDARD UNITS

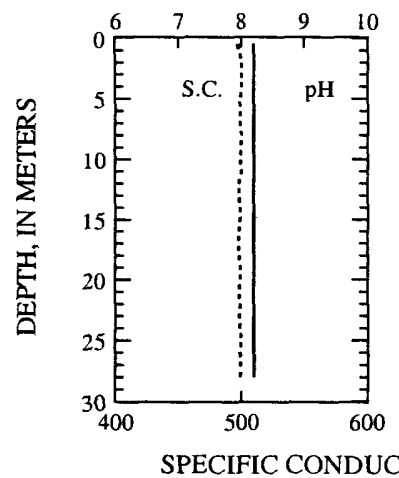

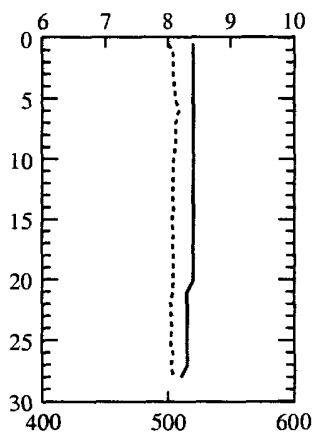

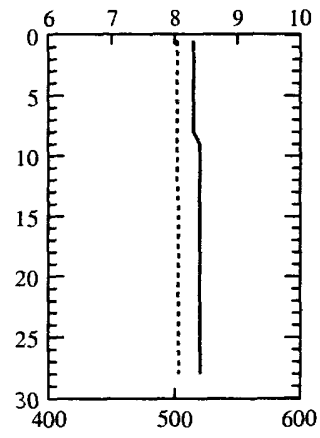

Mar -22

2.30

5.0

28.0

2.6

503

8.4

13.2

0.009

$<0.002$

0.077

$<0.013$

0.40

SPECIFIC CONDUCTANCE (S.C.), IN MICROSIEMENS PER CENTIMETER AT 25 DEGREES CELSIUS 

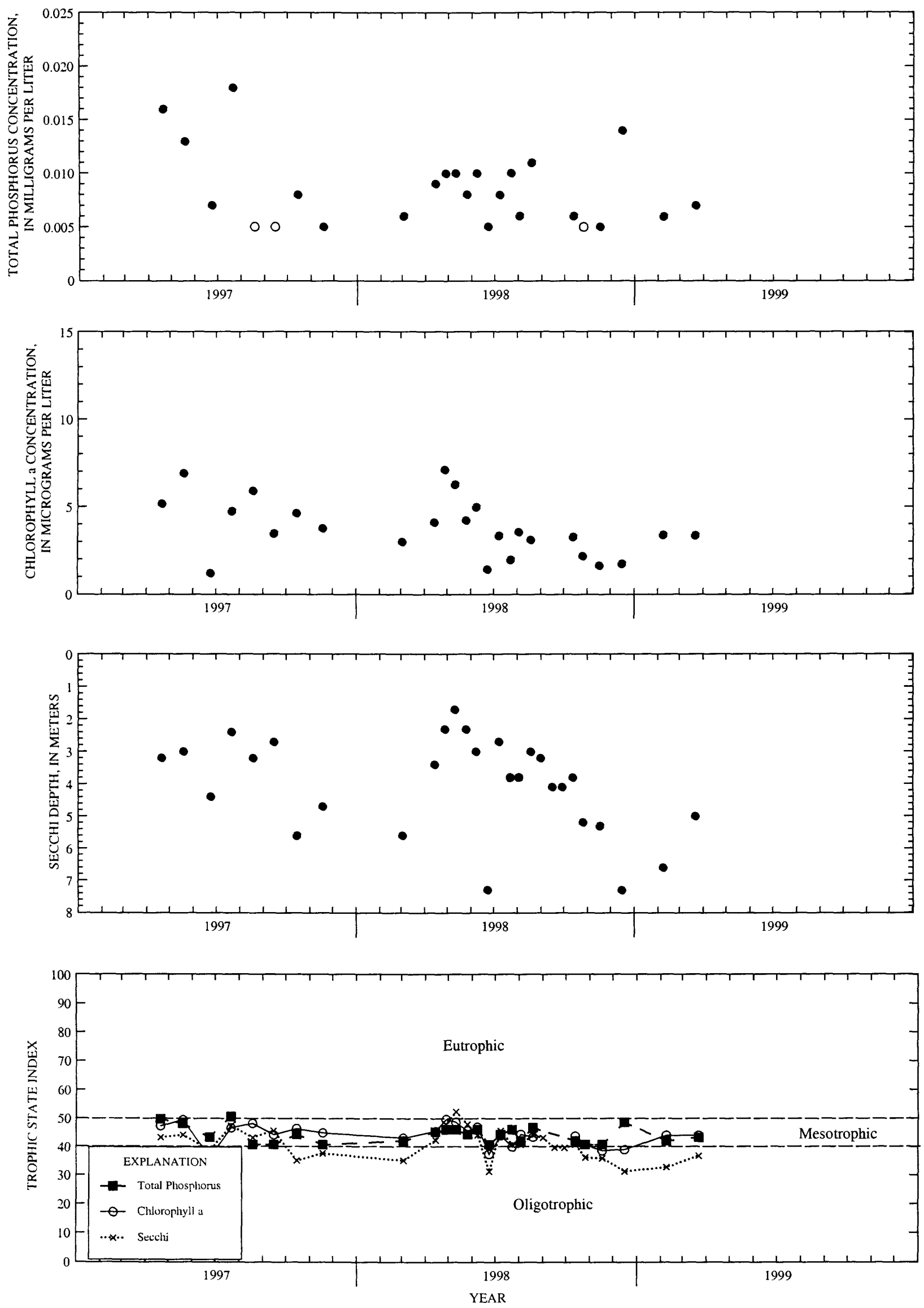

Surface total phosphorus, chlorophyll a concentrations, Secchi depths, and TSI data for Geneva Lake, Center Site, near Lake Geneva, Wisconsin.

(Circles on the first three plots indicate laboratory detection limit for selected analy ses. Actual concentrations for these particular analyses are less than the plotted circles.) 


\section{GENEVA LAKE AT EAST END NEAR LAKE GENEVA, WI}

LOCATION.--Lat $42^{\circ} 34^{\prime 2} 21^{\prime \prime}$, long 88 $27^{\circ} 23^{\prime \prime}$, in NE 1/4 SW 1/4, sec.2, T.1 N., R. 17 E., Walworth County, Hydrologic Unit 07120006, 1.6 mi southwest of outlet at Lake Geneva.

DRAINAGE AREA.--28.7 $\mathrm{mi}^{2}$.

PERIOD OF RECORD.--April 1997 to current year.

REMARKS.--Lake sampled at a depth of about $23 \mathrm{~m}$. Lake ice-covered during February sampling. Water-quality analyses done by Wisconsin State Laboratory of Hygiene. Samples for determination of chlorophyll-a concentration are collected from the top $1.5 \mathrm{ft}$ of the lake.

WATER-QUALITY DATA, OCTOBER 13 TO NOVEMBER 17, 1998

(Milligrams per liter unless otherwise indicated)

T.ake stage (ft)

"ecchi-depth (m)

(hloroohvl1 a, ohvtoolankton (Ma/L)

"epth of sample (m)

inater temperature $\left({ }^{\circ} \mathrm{C}\right)$

"roecific conductance (us/cm)

$\mathrm{r} H$ (units)

rissolved oxygen (mg/L)

hosphorus, total (as P)

":hosphorus, ortho, dissolved (as P)

litrogen, $\mathrm{NO} 2+\mathrm{NO} 3$, diss. (as N)

jitrogen, ammonia, dissolved (as N)

litrogen, amm. +org., total (as N)

litrogen, total (as N)

$10-13-98$
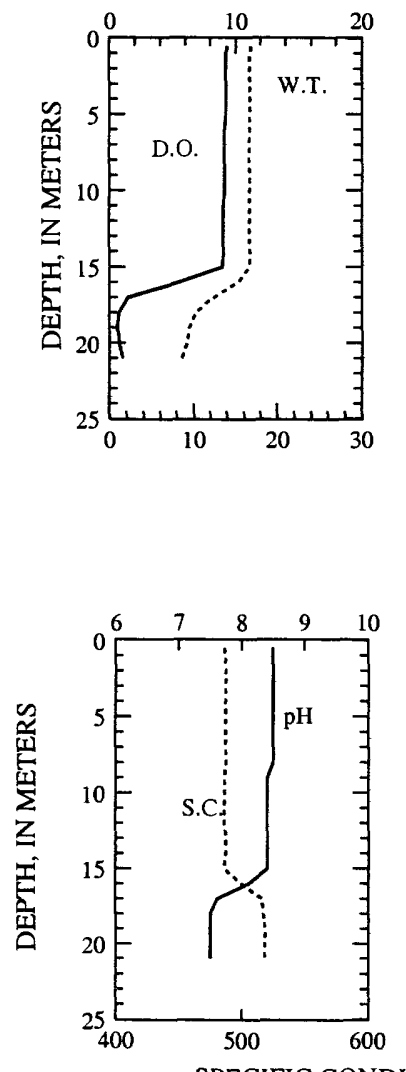

Oct -13

Oct -26

2.39

5.5
1.75

2.34
4.0

3.37

$\begin{array}{ccccc}0.5 & 15.0 & 17.0 & 19.0 & 21.0 \\ 16.8 & 16.6 & 12.3 & 9.5 & 8.6\end{array}$

$\begin{array}{ccccc}16.8 & 16.6 & 12.3 & 9.5 & 8.6 \\ 486 & 486 & 516 & 518 & 518\end{array}$

$\begin{array}{lllll}8.5 & 8.4 & 7.6 & 7.5 & 7.5 \\ 9.3 & 8.9 & 1.4 & 0.6 & 1.0\end{array}$

$\begin{array}{lllll}9.3 & 8.9 & 1.4 & 0.6 & 1.0\end{array}$

$\begin{array}{ccccc}<0.005 & 0.006 & 0.007 & 0.007 & 0.008\end{array}$

$\begin{array}{ccccc}0.02 & 0.02 & 0.02 & 0.017 & 0.09\end{array}$

$<0.013<0.013<0.013 \quad 0.031 \quad 0.017$

$\begin{array}{lllll}0.57 & 0.51 & 0.53 & 0.52 & 0.45\end{array}$

$\begin{array}{lllll}0.59 & 0.53 & 0.55 & 0.54 & 0.54\end{array}$

$10-26-98$

11-17-98

DISSOLVED OXYGEN (D.O.), IN MILLIGRAMS PER LITER
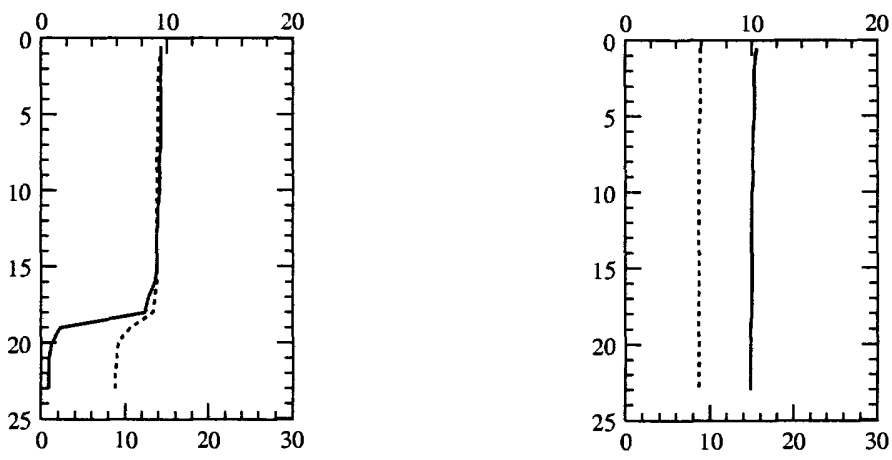

WATER TEMPERATURE (W.T.), IN DEGREES CELSIUS

PH. IN STANDARD UNITS

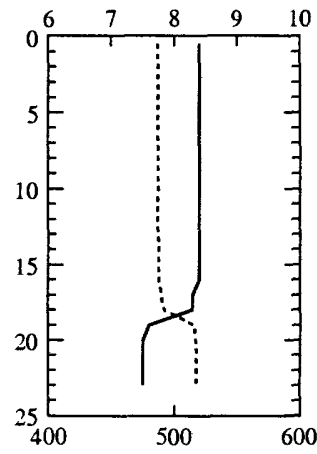

Nov -17

2.44

5.0
1.78

$0.5 \quad 23.0$

$8.9 \quad 8.7$

$495 \quad 497$

$8.3 \quad 8.3$

$10.4 \quad 9.9$

$0.008 \quad 0.008$

$0.002 \quad 0.002$

$0.03 \quad 0.026$

$\begin{array}{ll}0.03 & 0.026 \\ 0.021 & 0.025\end{array}$

$0.45 \quad 0.46$

$0.48 \quad 0.49$

SPECIFIC CONDUCTANCE (S.C.), IN MICROSIEMENS PER CENTIMETER AT 25 DEGREES CELSIUS 
WATER-QUALITY DATA, DECEMBER 16, 1998 TO APRIL 13, 1999

(Milligrams per liter unless otherwise indicated)

Lake stage (ft)

Secchi-depth (m)

Chlorophv11 a, phytoplankton (Ha/L)

Depth of sample (m)

water temperature $\left({ }^{\circ} \mathrm{C}\right)$

Specific conductance $\{$ us/cm

$\mathrm{pH}$ (units)

Dissolved oxygen (mg/L)

Phosphorus, total (as P)

Phosphorus, ortho, dissolved (as P)

Nitrogen, NO2 + NO3, diss. (as N)

Nitrogen, ammonia, dissolved (as N)

Nitrogen, amm. + orq.. total (as $\mathrm{N}$ )

Nitrogen, total (as N)

Color (Pt-Co. scale)

Turbidity (NTU)

Hardness, (as $\mathrm{CaCO}_{3}$ )

Calcium, dissolved (Ca)

Magnesium, dissolved (Mg)

Sodium, dissolved (Na)

Potassium, dissolved $(\mathrm{K})$

Alkalinity, (as $\mathrm{CaCO}_{3}$ )

Sulfate, dissolved $\left(\mathrm{SO}_{4}\right)$

Chloride, dissolved (Cl)

Silica, dissolved ( $\left.\mathrm{SiO}_{2}\right)$

Solids, dissolved, at $180^{\circ} \mathrm{C}$

Iron, dissolved ( $\mathrm{Fe}$ ) $\mathrm{ua} / \mathrm{L}$

Manqanese, dissolved (Mn) $\mu \mathrm{a} / \mathrm{L}$

\begin{tabular}{|c|c|c|c|c|c|c|c|}
\hline \multicolumn{2}{|c|}{ Dec-16 } & \multicolumn{2}{|c|}{ Feb- 8} & \multicolumn{2}{|c|}{$\operatorname{Mar}-22$} & \multicolumn{2}{|c|}{ Apr -13} \\
\hline \multicolumn{2}{|c|}{$\begin{array}{c}2.43 \\
6.5 \\
2.55\end{array}$} & \multicolumn{2}{|c|}{$\begin{array}{c}2.54 \\
5.8 \\
2.64\end{array}$} & \multicolumn{2}{|c|}{$\begin{array}{c}2.30 \\
4.9 \\
2.9\end{array}$} & \multicolumn{2}{|c|}{$\begin{array}{c}2.52 \\
6.7 \\
1.02\end{array}$} \\
\hline 0.5 & 22.0 & 0.5 & 20.5 & 0.5 & 21.0 & 0.5 & 21.5 \\
\hline 6.7 & 6.7 & 1.7 & 2.3 & 3.1 & 3.0 & 7.5 & 5.1 \\
\hline 496 & 497 & 499 & 512 & 500 & 500 & 508 & 509 \\
\hline 8.3 & 8.3 & 8.4 & 8.3 & 8.3 & 8.4 & 8.4 & 8.3 \\
\hline 11.7 & 11.4 & 13.5 & 11.7 & 13.6 & 13.0 & 12.8 & 12.2 \\
\hline 0.012 & 0.013 & $<0.005$ & 0.026 & 0.008 & 0.008 & 0.005 & 0.008 \\
\hline 0.003 & 0.003 & $<0.002$ & 0.003 & 0.001 & $<0.002$ & $<0.002$ & $<0.002$ \\
\hline 0.052 & 0.049 & 0.016 & 0.085 & 0.079 & 0.072 & 0.077 & 0.077 \\
\hline 0.023 & 0.022 & $<0.013$ & 0.014 & $<0.013$ & $<0.013$ & $<0.013$ & 0.014 \\
\hline $\begin{array}{l}0.61 \\
0.66\end{array}$ & $\begin{array}{l}0.54 \\
0.59\end{array}$ & $\begin{array}{l}0.41 \\
0.43\end{array}$ & $\begin{array}{l}0.25 \\
0.34\end{array}$ & $\begin{array}{l}0.39 \\
0.47\end{array}$ & $\begin{array}{l}0.5 \\
0.57\end{array}$ & $\begin{array}{l}0.47 \\
0.55\end{array}$ & $\begin{array}{l}0.49 \\
0.57\end{array}$ \\
\hline$\ldots$ & $\ldots$ & $\ldots$ & $\ldots$ & $\ldots$ & $\ldots$ & $<5$ & $\ldots$ \\
\hline$\ldots$ & $\ldots$ & $\ldots$ & $\ldots$ & $\ldots$ & $\ldots$ & 0.3 & $\ldots$ \\
\hline$\ldots \ldots$ & $\ldots$ & $\ldots$ & $\ldots$ & $\ldots$ & $\ldots$ & 220 & $\ldots$ \\
\hline--- & $\ldots$ & -- & --- & $\ldots$ & $\ldots$ & 33 & --- \\
\hline$\ldots$ & $\ldots$ & $\ldots$ & $-\ldots$ & - - - & $\ldots$ & 33 & $\ldots$ \\
\hline$-\ldots$ & $-\ldots$ & --- & --- & -- & -- & 17 & --- \\
\hline$\ldots$ & -- & $\ldots$ & $\ldots$ & $\ldots$ & -- & 1.9 & -- \\
\hline$\ldots$ & -- & $-\cdots$ & -- & -- & -- & 186 & --- \\
\hline$\ldots$ & $\ldots$ & -- & $\rightarrow-$ & $\ldots$ & $\ldots$ & 31 & --- \\
\hline--- & $\ldots$ & --- & --- & --- & $\ldots$ & 35 & -- \\
\hline--- & $\ldots$ & $\ldots$ & $\ldots$ & -- & $\ldots$ & 0.3 & -- \\
\hline-- & $\ldots$ & $-\cdots$ & -- & $-\cdots$ & -- & 272 & $\cdots$ \\
\hline$\ldots$ & $-\ldots$ & $\ldots$ & $\ldots$ & -- & -- & $<10$ & -- \\
\hline$\ldots$ & $\ldots$ & $\ldots$ & $\ldots$ & $\ldots$ & $\ldots$ & 0.5 & -- \\
\hline
\end{tabular}

DISSOLVED OXYGEN (D.O.), IN MILLIGRAMS PER LITER
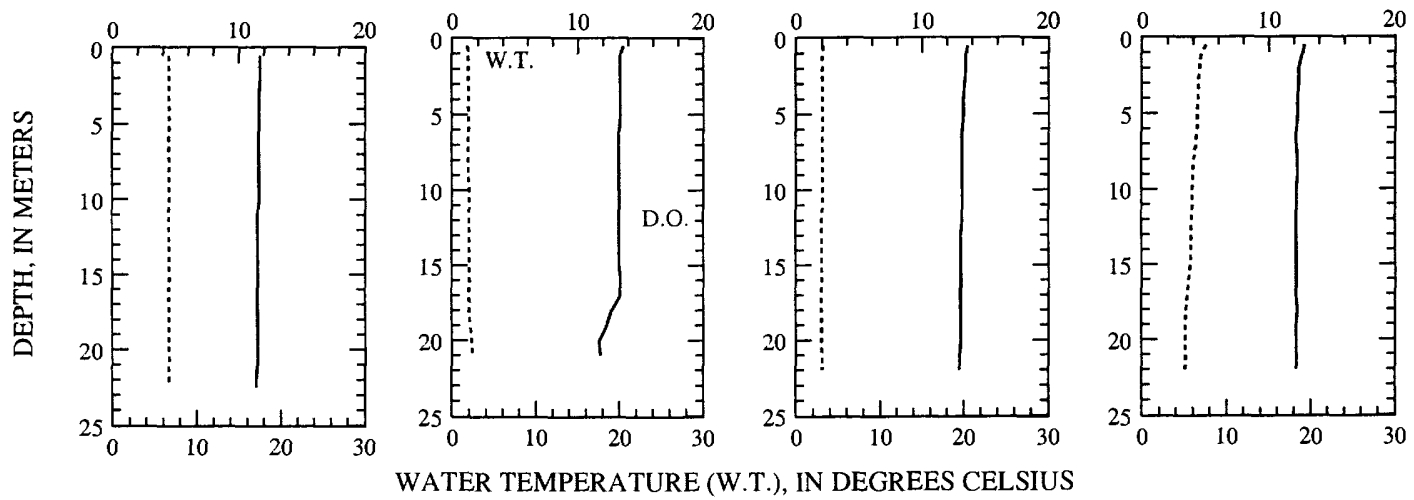

WATER TEMPERATURE (W.T.), IN DEGREES CELSIUS

PH, IN STANDARD UNITS
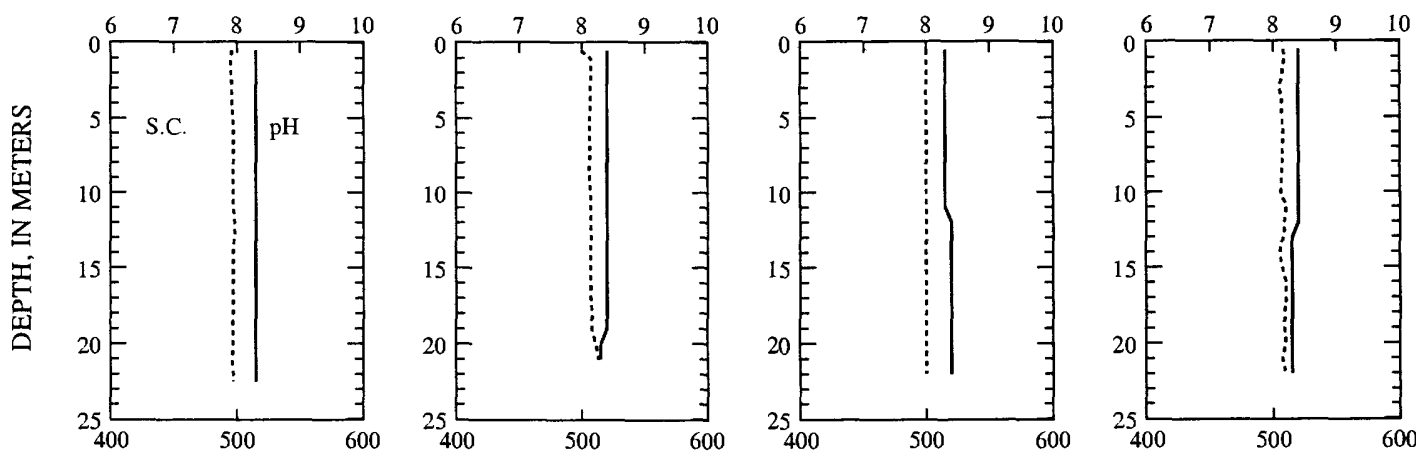

SPECIFIC CONDUCTANCE (S.C.), IN MICROSIEMENS PER CENTIMETER AT 25 DEGREES CELSIUS 
WATER-QUALITY DATA, MAY 19 TO JUNE 14, 1999

(Milligrams per liter, unless otherwise indicated)

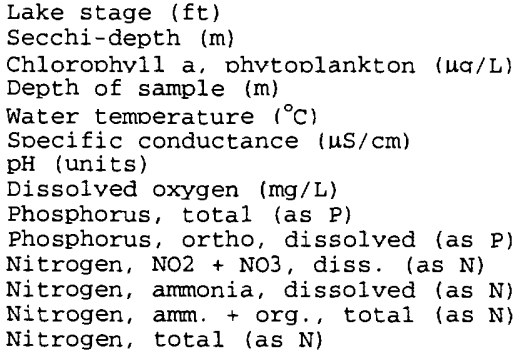

May -19

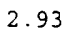

5.0

0.43

$\begin{array}{lll}0.5 & 11.0 & 16.0\end{array}$

$14.2 \quad 12.3$

$497 \quad 499$

8.48 .4

$10.8 \quad 10.7$

$<0.005$

$<0.002$

$<0.002$

$<0.013$

$<0.013$
0.56

0.56
0.64
9.0

8.3
10.8

0.005

$<0.002$

0.079

0.006

0.5

0.58

18.0
8.6
505
8.3
10.9
$<0.005$
$<0.002$
0.083
0.009
0.56
0.64

21.0
8.6
505
8.3
10.9
$<0.005$
$<0.002$
0.081
0.014
0.57
0.65

8.3

10.9

$<0.005$

.081

0.014

0.65

DISSOLVED OXYGEN (D.O.), IN MILLIGRAMS PER LITER
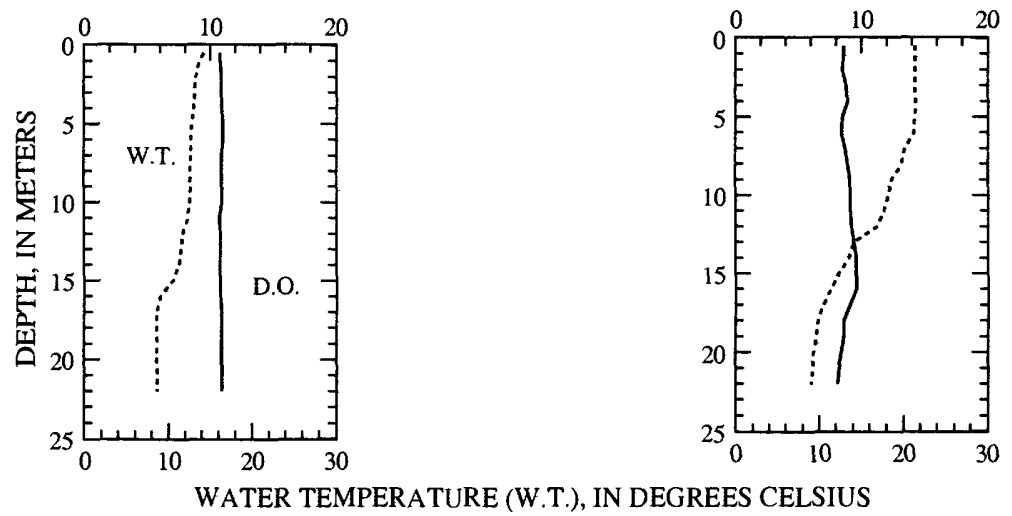

PH, IN STANDARD UNITS
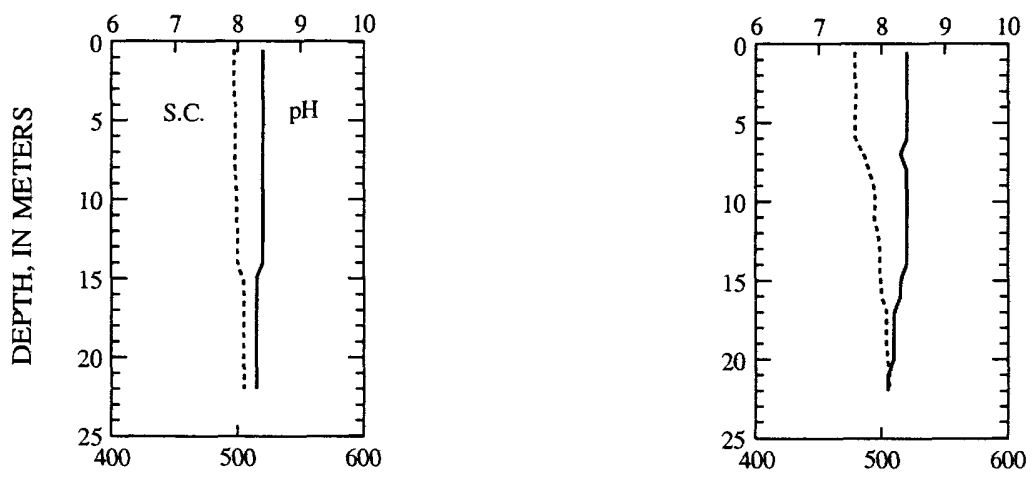

SPECIFIC CONDUCTANCE (S.C.), IN MICROSIEMENS PER CENTIMETER AT 25 DEGREES CELSIUS 
WATER-QUALITY DATA, JULY 13 TO AUGUST 17, 1999

(Milligrams per liter unless otherwise indicated)

Lake stage ( $f t)$

Secchi-depth $(m)$

Chlorophyll a, phytoplankton ( $\mathrm{dq} / \mathrm{L}$ )

Depth of sample (m)

Water temperature $\left({ }^{\circ} \mathrm{C}\right)$

Specific conductance (uS/ cm)

$\mathrm{pH}$ (units)

Dissolved oxygen (mg/L)

Phosphorus, total (as P)

Phosphorus, ortho, dissolved (as $\mathrm{P}$ )

Nitrogen, $\mathrm{NO} 2+\mathrm{NO} 3$, diss. (as $\mathrm{N}$ )

Nitrogen, ammonia, dissolved (as N)

Nitrogen, amm. + org., total (as N)

Nitrogen, total (as N)

JuI -13

\begin{tabular}{ccccc}
\multicolumn{5}{c}{2.51} \\
5.5 \\
& 1.78 & & \\
0.5 & 9.0 & 13.0 & 18.0 & 23.0 \\
24.8 & 23.0 & 13.8 & 10.5 & 9.3 \\
487 & 492 & 505 & 508 & 513 \\
8.4 & 8.3 & 8.1 & 8.0 & 7.9 \\
9.1 & 7.9 & 8.1 & 7.6 & 6.0 \\
$<0.005$ & $<0.005$ & 0.009 & $<0.005$ & 0.011 \\
0.002 & 0.003 & 0.002 & 0.002 & 0.003 \\
$<0.010$ & 0.018 & 0.073 & 0.083 & 0.066 \\
$<0.013$ & 0.017 & 0.022 & 0.053 & 0.098 \\
0.39 & 0.37 & 0.57 & 0.53 & 0.65 \\
--- & 0.39 & 0.64 & 0.61 & 0.72
\end{tabular}

$7-13-99$
Aug- 17

\begin{tabular}{ccccc}
\multicolumn{5}{c}{2.25} \\
4.6 \\
& 1.65 & & \\
0.5 & 11.0 & 15.0 & 18.0 & 22.5 \\
23.7 & 22.9 & 11.8 & 10.1 & 9.5 \\
492 & 494 & 512 & 514 & 516 \\
8.3 & 8.3 & 7.8 & 7.7 & 7.6 \\
8.6 & 7.5 & 4.9 & 3.6 & 2.8 \\
0.009 & 0.010 & 0.008 & 0.011 & 0.010 \\
$<0.002$ & $<0.002$ & $<0.002$ & $<0.002$ & $<0.002$ \\
$<0.005$ & $<0.005$ & 0.138 & 0.184 & 0.225 \\
0.012 & $<0.002$ & $<0.002$ & $<0.002$ & 0.006 \\
0.45 & 0.41 & 0.46 & 0.39 & 0.43 \\
--- & --- & 0.6 & 0.57 & 0.65
\end{tabular}

8-17-99

DISSOLVED OXYGEN (D.O.), IN MILLIGRAMS PER LITER
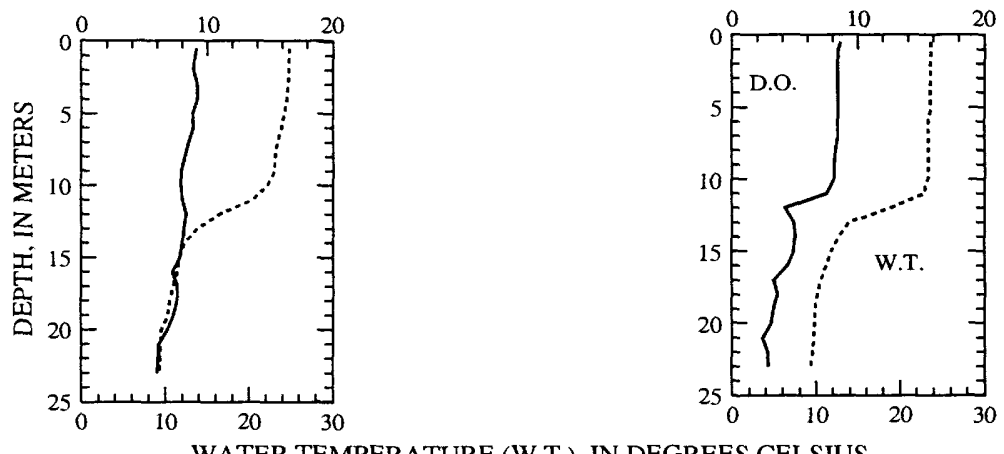

PH, IN STANDARD UNITS
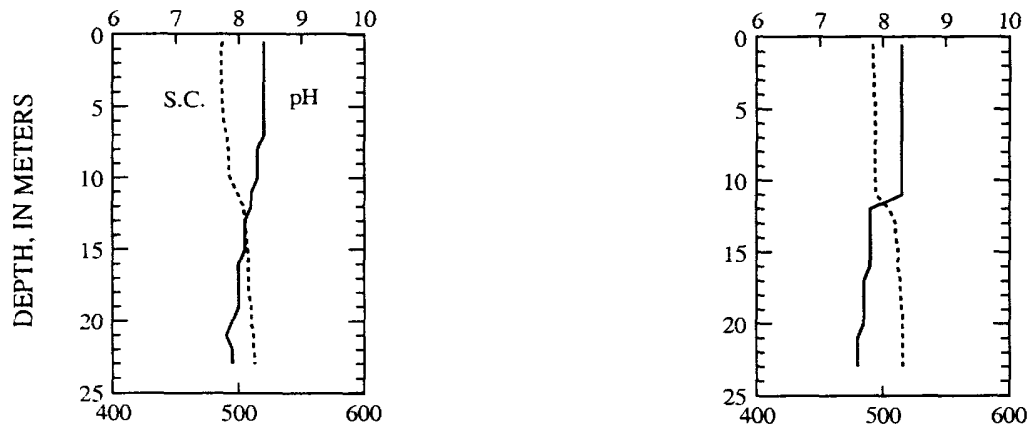

SPECIFIC CONDUCTANCE (S.C.), IN MICROSIEMENS PER CENTIMETER AT 25 DEGREES CELSIUS 
WATER-QUALITY DATA, SEPTEMBER 15, 1999

(Milligrams per liter unless otherwise indicated)

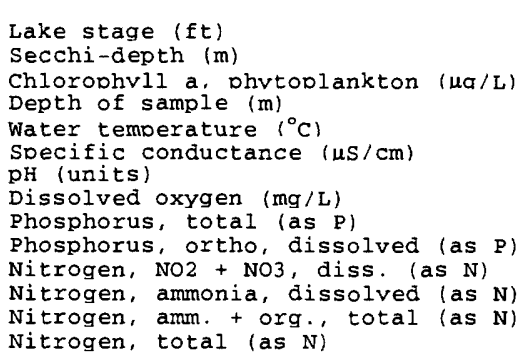

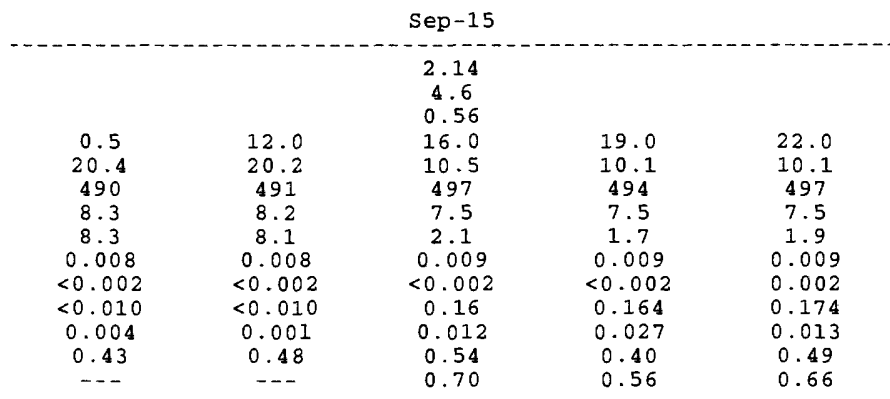

9-15-99

\begin{abstract}
DISSOLVED OXYGEN (D.O.), IN MILLIGRAMS PER LITER
\end{abstract}

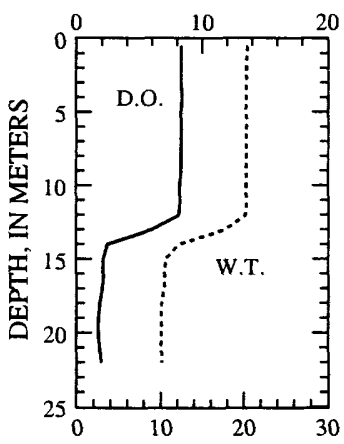

WATER TEMPERATURE (W.T.), IN DEGREES CELSIUS

PH, IN STANDARD UNITS

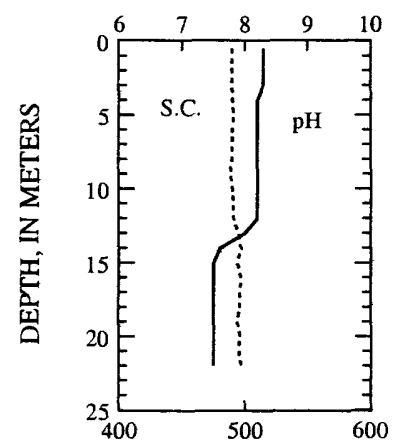

SPECIFIC CONDUCTANCE (S.C.), IN MICROSIEMENS PER CENTIMETER AT 25 DEGREES CELSIUS 

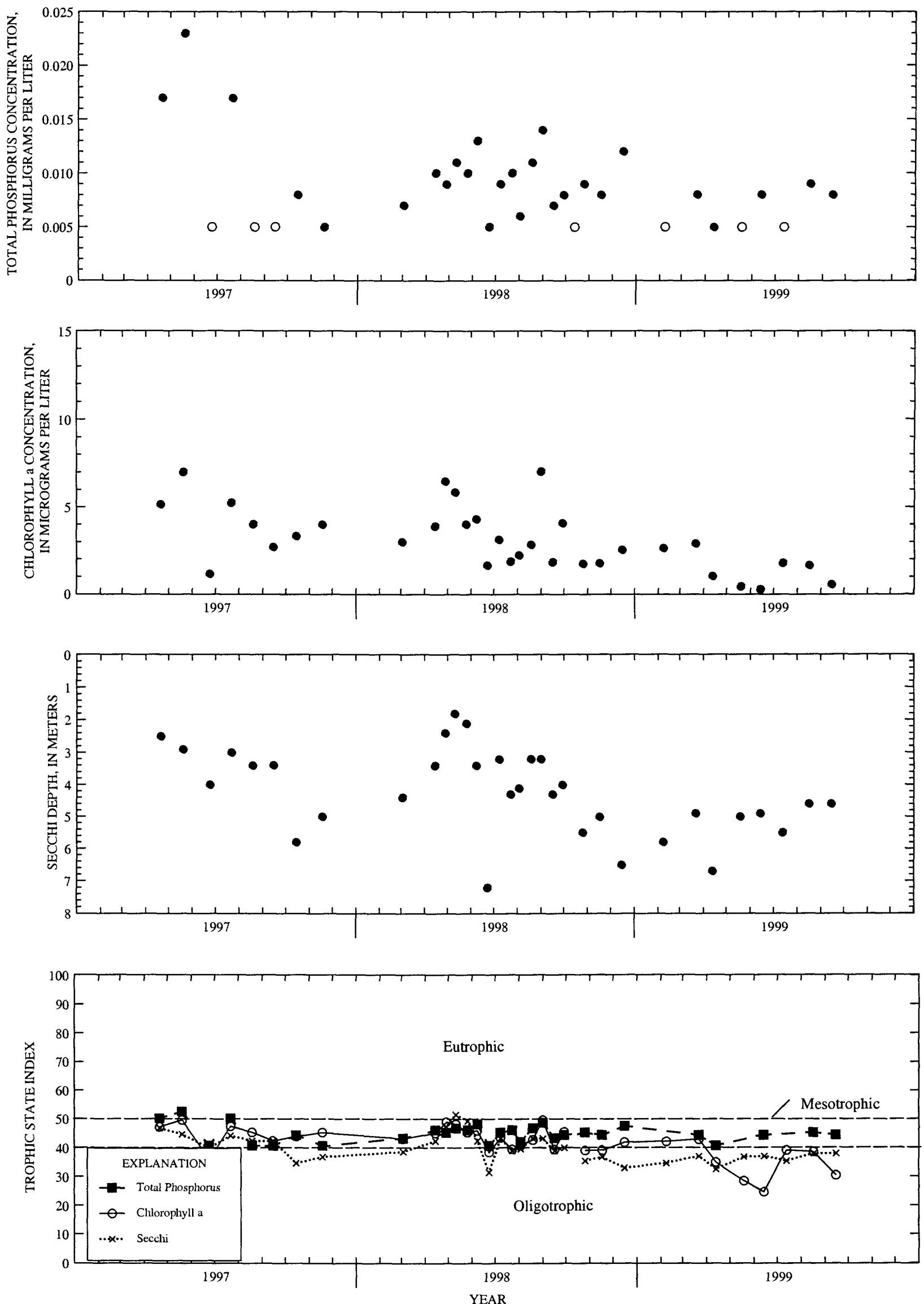

Surface total phosphorus, chlorophyll a concentrations, Secchi depths, and TSI data for Geneva Lake, East End, near Lake Geneva, Wisconsin.

(Circles on the first three plots indicate laboratory detection limit for selected analyses. Actual concentrations for these particular analyses are less than the plotted circles.) 


\section{GENEVA LAKE AT WEST END NEAR WILLIAMS BAY, WI}

LOCATION.--Lat 42³3'29", long 88³2'33", in NE 1/4 SE 1/4, sec.12, T.1 N., R.16 E., Walworth County, Hydrologic Unit 07120006, 1.3 mi south of Williams Bay.

DRAINAGE AREA.--28.7 $\mathrm{mi}^{2}$.

PERIOD OF RECORD.--April 1997 to current year.

REMARKS.--Lake sampled at deep hole at a depth of about $43 \mathrm{~m}$. Lake ice-covered during February sampling. Water-quality analyses done by Wisconsin State Laboratory of Hygiene. Samples for determination of chlorophyll-a concentration are collected from the top $1.5 \mathrm{ft}$ of the lake.

WATER-QUALITY DATA, OCTOBER I3 AND 26, 1998

(Milligrams per liter unless otherwise indicated)

ake stage (ft)

Secchi-depth (m)

Chlorophyll a, phytoplankton ( $\mu \mathrm{g} / \mathrm{L})$

respth of sample (m)

Whater temoerature $\left({ }^{\circ} \mathrm{C}\right)$

mecific conductance $(\mu \mathrm{S} / \mathrm{cm})$

"H (units)

I'ssolved oxygen (mg/L)

T'hosphorus, total (as P)

'hosphorus, ortho, dissolved (as $\mathrm{P}$ )

-itrogen, $\mathrm{NO} 2+\mathrm{NO} 3$, diss. (as N)

-itrogen, armonia, dissolved (as N)

itrogen, anm. + org., total (as $\mathrm{N}$ )

-itrogen, total (as N)
Oct -13

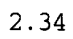

4.1

\begin{tabular}{|c|c|c|c|c|c|}
\hline \multicolumn{6}{|c|}{ Oct -13} \\
\hline \multirow{2}{*}{\multicolumn{6}{|c|}{$\begin{array}{c}2.34 \\
4.1 \\
-.-\end{array}$}} \\
\hline & & & & & \\
\hline 0.5 & 14.0 & 22.0 & 33.0 & 38.0 & 43.0 \\
\hline 16.7 & 16.2 & 8.9 & 7.2 & 6.9 & 6.8 \\
\hline 487 & 491 & 515 & 520 & 525 & 529 \\
\hline 8.5 & 8.4 & 7.6 & 7.5 & 7.5 & 7.5 \\
\hline 9.4 & 7.9 & 3.7 & 1.3 & 0.6 & 0.6 \\
\hline 0.005 & 0.007 & $<0.005$ & $<0.005$ & 0.043 & 0.061 \\
\hline$<0.002$ & $<0.002$ & $<0.002$ & $<0.002$ & 0.03 & 0.047 \\
\hline 0.025 & 0.025 & 0.045 & 0.247 & 0.03 & $<0.010$ \\
\hline$<0.013$ & $<0.013$ & $<0.013$ & $<0.013$ & 0.228 & 0.27 \\
\hline 0.57 & 0.69 & 0.53 & 0.48 & 1.6 & 1.1 \\
\hline 0.6 & 0.71 & 0.57 & 0.73 & 1.7 & -- \\
\hline
\end{tabular}

$10-13-98$
Oct-26

\begin{tabular}{cccccc}
\multicolumn{7}{c}{2.39} \\
\multicolumn{7}{c}{1.97} \\
0.5 & 17.0 & 30.0 & 34.0 & 38.0 & 43.0 \\
14.0 & 13.7 & 7.6 & 7.2 & 7.0 & 6.9 \\
491 & 494 & 518 & 522 & 525 & 527 \\
8.4 & 8.3 & 7.5 & 7.5 & 7.5 & 7.5 \\
9.2 & 8.3 & 1.2 & 0.6 & 0.6 & 0.6 \\
0.009 & 0.013 & $<0.005$ & 0.014 & 0.041 & 0.067 \\
$<0.002$ & $<0.002$ & 0.002 & 0.011 & 0.037 & 0.066 \\
$<0.010$ & $<0.010$ & 0.179 & 0.012 & $<0.010$ & $<0.010$ \\
$<0.013$ & $<0.013$ & $<0.013$ & 0.102 & 0.238 & 0.355 \\
0.44 & 0.54 & 0.66 & 0.56 & 0.81 & 1.2 \\
-- & -- & 0.84 & 0.57 & -- & --
\end{tabular}

$10-26-98$

\section{DISSOLVED OXYGEN (D.O.), IN MILLIGRAMS PER LITER}
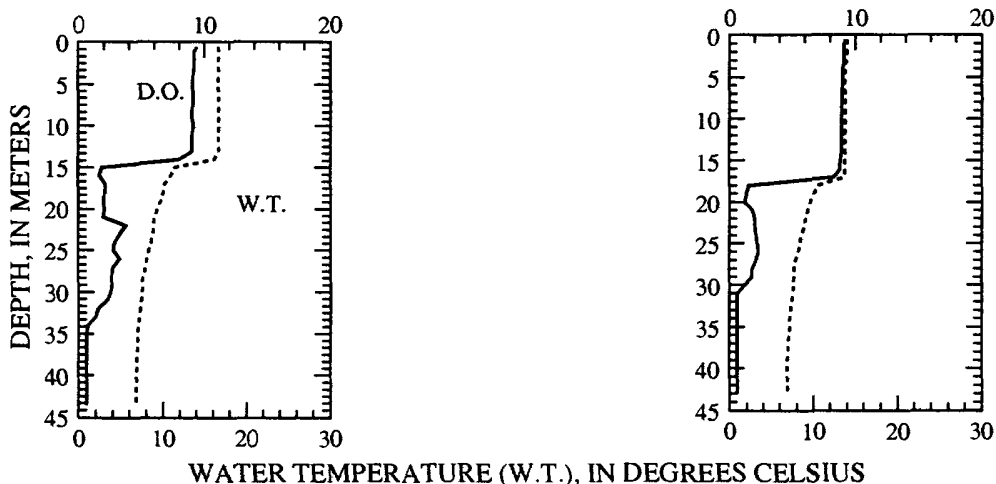

PH, IN STANDARD UNITS
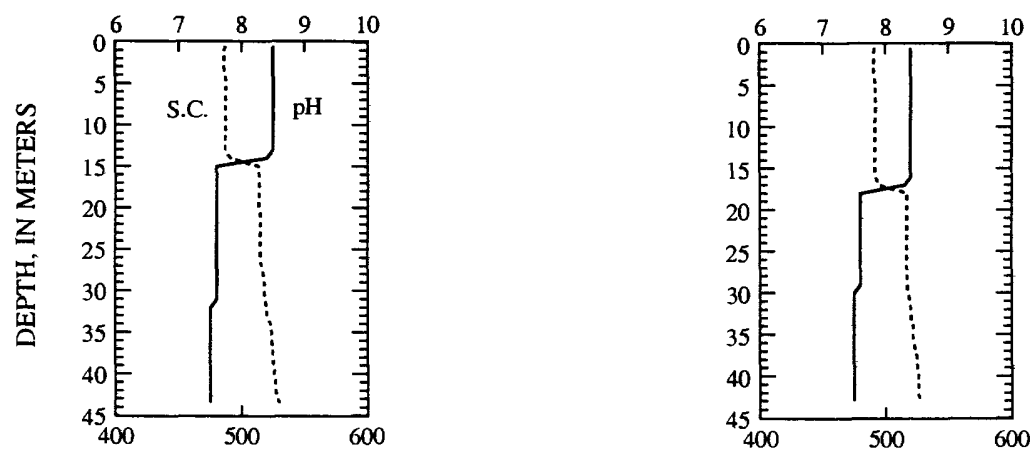

SPECIFIC CONDUCTANCE (S.C.), IN MICROSIEMENS PER CENTIMETER AT 25 DEGREES CELSIUS 
WATER-QUALITY DATA, NOVEMBER 17, 1998 TO MARCH 22, 1999

(Milligrams per liter unless otherwise indicated)

Lake stage (ft)

Secchi-depth (m)

Chlorophyll a, phytoplankton $(\mu g / L)$ Depth of sample (m)

Water temperature $\left({ }^{\circ} \mathrm{C}\right)$

Specific conductance $(\mu \mathrm{S} / \mathrm{cm})$ $\mathrm{pH}$ (units)

Dissolved oxygen (mg/L)

Phosphorus, total (as P)

Phosphorus, ortho, dissolved (as P)

Nitrogen, NO2 + NO3, diss. (as N)

Nitrogen, ammonia, dissolved (as N)

Nitrogen, amm. + org., total (as N)

Nitrogen, total (as N)
Nov -17

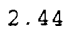

5.4

1.04

$0.5 \quad 40.0$

9.3

501

8.1

0.006

0.003

0.037

0.037

0.42

0.46
8.5

510

7.7

2.6

0.023

0.013

0.042

0.104

0.50

0.54

43.0
8.0
51
7.5
0.0
0.054
0.02
0.006
0.203
0.69
0.70

43.0
8.0
517
7.5
0.6
.054
0.02
.006
.203
0.69
0.70

Dec-16

Feb- 8

$\operatorname{Mar}-22$

\subsection{3}

6.2

$0.5^{2.92} 44.0$

0.5
7.4

4.467 .4

496

8.1
11.2

0.014

0.003

0.061

0.012

0.012
0.51

0.57

498
8.1
10.
0.0
0.0
0.0
0.0
0.5
0.5

498
8.1

8.1
10.6

0.015

0.003

0.066

0.011

0.59

\begin{tabular}{cccc}
\multicolumn{4}{c}{ Feb-8 } \\
\multicolumn{4}{c}{2.54} \\
\multicolumn{4}{c}{2.7} \\
0.5 & 10.0 & 30.0 & 42.0 \\
1.0 & 2.0 & 2.7 & 3.3 \\
480 & 505 & 504 & 549 \\
8.4 & 8.4 & 8.2 & 7.8 \\
13.9 & 13.4 & 10.7 & 6.0 \\
0.005 & 0.005 & 0.007 & 0.025 \\
$<0.002$ & 0.002 & 0.002 & 0.019 \\
0.052 & 0.052 & 0.071 & 0.154 \\
$<0.013$ & $<0.013$ & $<0.013$ & 0.113 \\
0.36 & 0.28 & 0.31 & 0.44 \\
0.41 & 0.33 & 0.38 & 0.59
\end{tabular}

\begin{tabular}{cc} 
Mar -22 \\
2.30 \\
\multicolumn{2}{c}{4.9} \\
\multicolumn{2}{c}{3.87} \\
0.5 & 43.0 \\
2.3 & 2.4 \\
504 & 503 \\
7.6 & 8.3 \\
13.8 & 13.1 \\
0.007 & 0.008 \\
0.002 & $<0.00$ \\
0.076 & 0.07 \\
$<0.013$ & $<0.01$ \\
0.42 & 0.50 \\
0.50 & 0.58
\end{tabular}

11-17-98

12-16-98

$2-08-99$

3-22-99

DISSOLVED OXYGEN (D.O.), IN MILLIGRAMS PER LITER
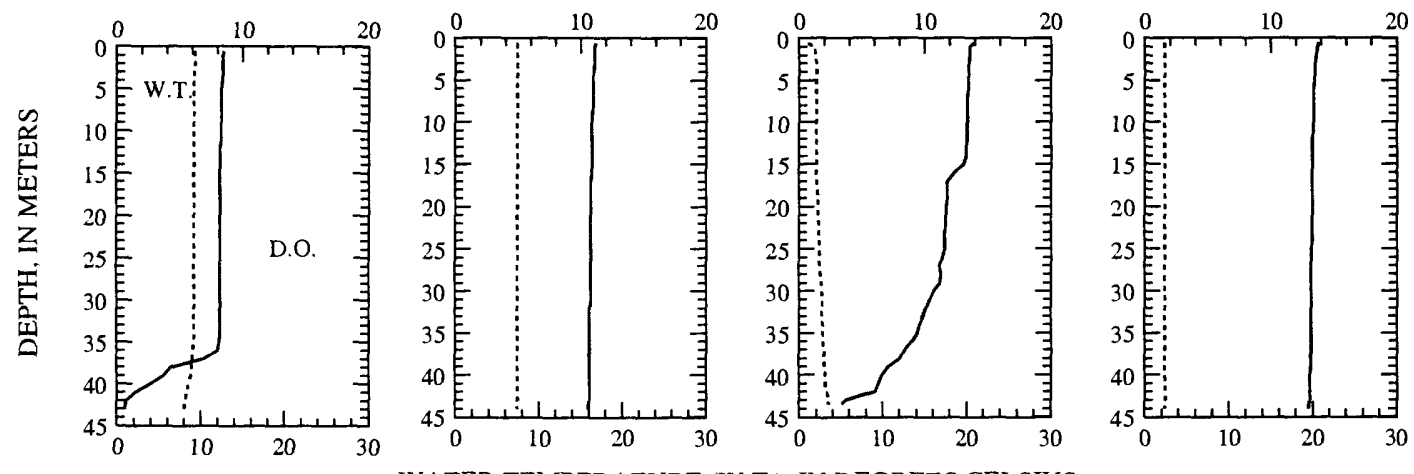

WATER TEMPERATURE (W.T.), IN DEGREES CELSIUS

PH, IN STANDARD UNITS
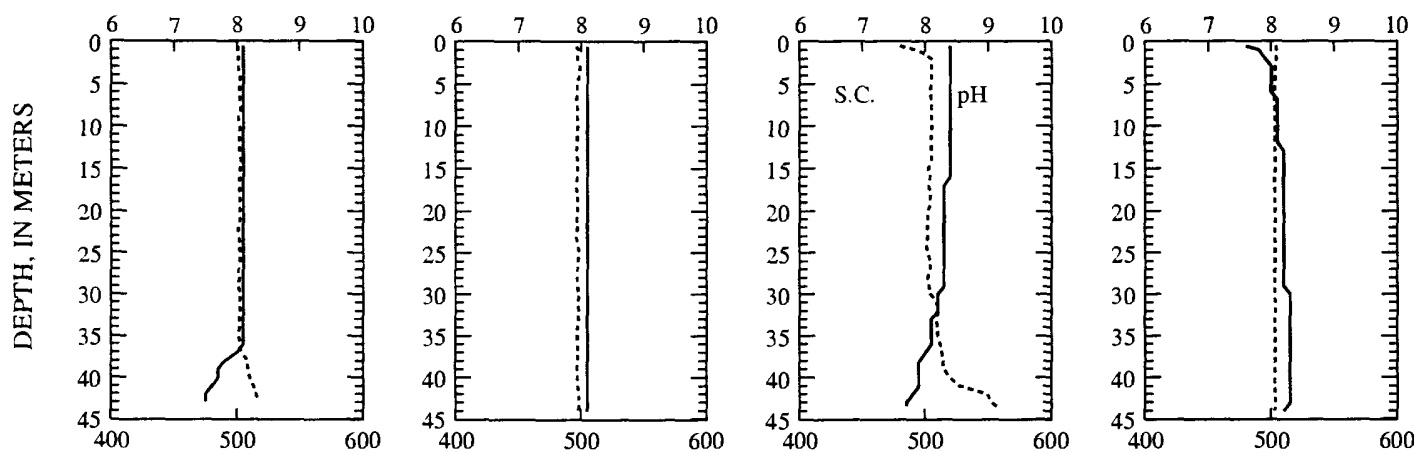

SPECIFIC CONDUCTANCE (S.C.), IN MICROSIEMENS PER CENTIMETER AT 25 DEGREES CELSIUS 
WATER-QUALITY DATA, APRIL 13 TO MAY 19, 1999

(Milligrams per liter unless otherwise indicated)

Lake stage ( $f t$ )

Secchi-depth (m)

Chlorophyll a, phytoplankton $(\mu \mathrm{g} / \mathrm{L})$

Depth of sample (m)

water temperature $\left({ }^{\circ} \mathrm{C}\right)$

Specific conductance $(\mu \mathrm{S} / \mathrm{cm})$

$\mathrm{pH}$ (units)

Dissolved oxygen (mg/L)

Phosphorus, total (as P)

Phosphorus, ortho, dissolved (as P)

Nitrogen, $\mathrm{NO} 2+\mathrm{NO} 3$, diss. (as N)

Nitrogen, ammonia, dissolved (as N)

Nitrogen, amm. + org., total (as N)

Nitrogen, total (as N)

Color (Pt-Co. scale)

Turbidity (NTU)

Hardness, (as $\mathrm{CaCO}_{3}$ )

Calcium, dissolved (Ca)

Magnesium, dissolved (Mg)

Sodium, dissolved (Na)

Potassium, dissolved $(\mathrm{K})$

Alkalinity, (as $\mathrm{CaCO}_{3}$ )

Sulfate, dissolved $\left(\mathrm{SO}_{4}\right)$

Chloride, dissolved (Cl)

Silica, dissolved $\left(\mathrm{SiO}_{2}\right)$

Solids, dissolved, at $180^{\circ} \mathrm{C}$

Iron, dissolved (Fe) $\mu \mathrm{g} / \mathrm{L}$

Manganese, dissolved (Mn) $\mu \mathrm{g} / \mathrm{L}$

\begin{tabular}{c} 
Apr \\
\multicolumn{1}{c}{2.52} \\
6.7 \\
0.5 \\
6.6 \\
510 \\
8.2 \\
13.4 \\
0.006 \\
$<0.002$ \\
0.083 \\
0.017 \\
0.45 \\
0.53 \\
5 \\
0.4 \\
220 \\
34 \\
34 \\
17 \\
2.0 \\
185 \\
31 \\
35 \\
0.38 \\
272 \\
$<10$ \\
$<0.40$ \\
\end{tabular}

pr -1

.52

28

43.0

5.1

12.1

0.010

$<0.002$

0.079

$<0.013$

0.49

0.57

5

0.5
220

220

34
33
17

33
17
1.7

1.7
185

31
35

35
0.50

274

$<10$

$<0.40$

04-13-99
May-19

\begin{tabular}{|c|c|c|c|c|}
\hline & & $\begin{array}{c}2.93 \\
7.3 \\
0.51\end{array}$ & & \\
\hline 0.5 & 7.0 & 34.0 & 39.0 & 43.0 \\
\hline 13.0 & 12.3 & 6.8 & 6.6 & 6.6 \\
\hline 500 & 500 & 507 & 508 & 510 \\
\hline 8.3 & 8.3 & 8.2 & 8.1 & 8.0 \\
\hline 11.5 & 11.0 & 10.7 & 9.8 & 8.8 \\
\hline$<0.005$ & 0.008 & 0.006 & 0.008 & 0.011 \\
\hline$<0.002$ & $<0.002$ & $<0.002$ & $<0.002$ & 0.002 \\
\hline 0.077 & 0.081 & 0.072 & 0.074 & 0.062 \\
\hline 0.001 & $<0.013$ & 0.025 & 0.049 & 0.085 \\
\hline 0.44 & 0.49 & 0.49 & 0.57 & 0.69 \\
\hline 0.52 & 0.57 & 0.56 & 0.64 & 0.75 \\
\hline--- & --- & $-\cdots$ & --- & --- \\
\hline--- & --- & --- & --- & -- \\
\hline--- & -- & -- & --- & --- \\
\hline$\ldots$ & --- & --- & --- & --- \\
\hline-- & -- & $\cdots$ & $\ldots$ & --- \\
\hline--- & -- & -- & $\cdots$ & $-\cdots$ \\
\hline$\ldots$ & --- & --- & --- & --- \\
\hline--- & -- & --- & -- & --- \\
\hline--- & -- & --- & --- & --- \\
\hline-- & --- & --- & --- & $\cdots$ \\
\hline--- & $\cdots$ & $-\cdots$ & -- & $\cdots$ \\
\hline--- & --- & $-\cdots-$ & --- & --- \\
\hline--- & -- & --- & --- & --- \\
\hline--- & --- & -- & -- & $\cdots$ \\
\hline
\end{tabular}

05-19-99

DISSOLVED OXYGEN (D.O.), IN MILLIGRAMS PER LITER
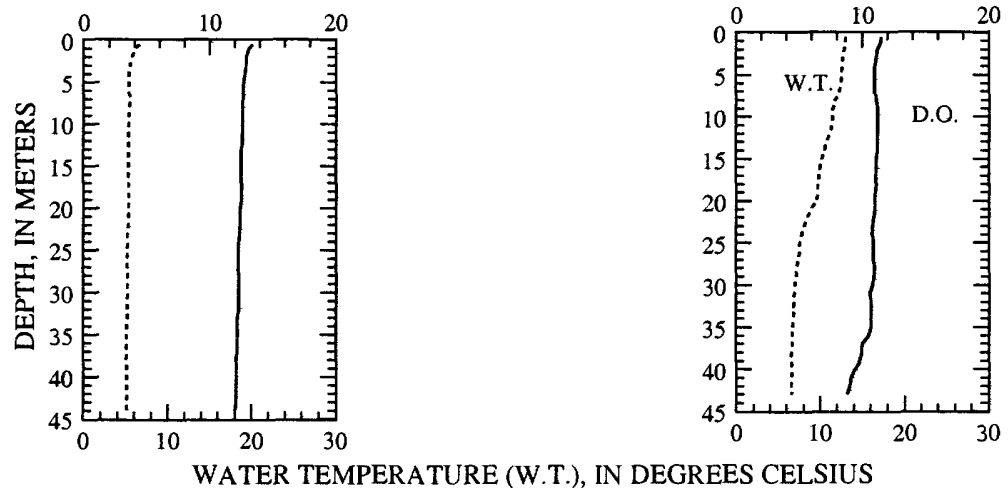

PH, IN STANDARD UNITS
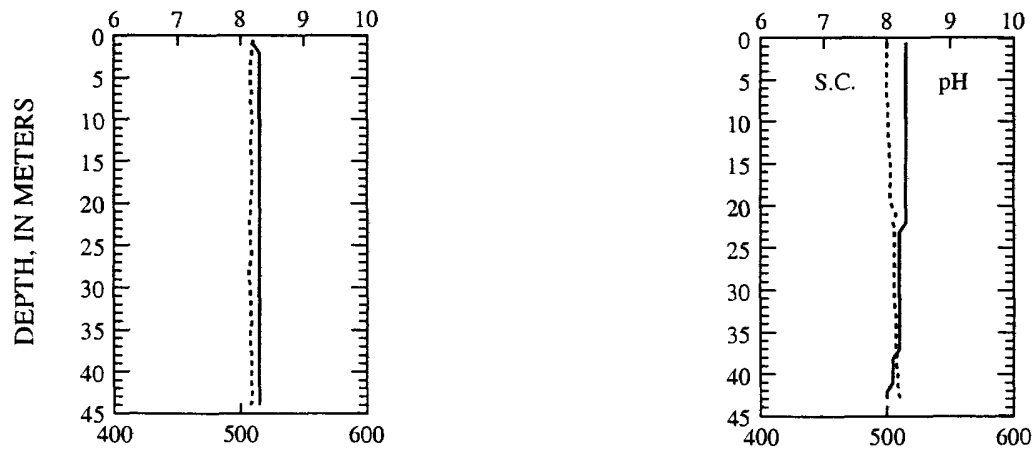

SPECIFIC CONDUCTANCE (S.C.), IN MICROSIEMENS PER CENTIMETER AT 25 DEGREES CELSIUS 
WATER-QUALITY DATA, JUNE 14 TO JULY 13, 1999

(Milligrams per liter unless otherwise indicated)

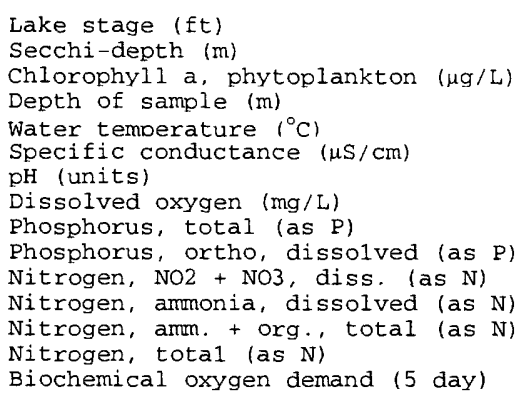

Jun-14

\begin{tabular}{|c|c|c|c|c|c|}
\hline \multicolumn{6}{|c|}{$\begin{array}{c}3.15 \\
4.6 \\
0.39\end{array}$} \\
\hline 0.5 & 7.0 & 22.0 & 34.0 & 39.0 & 43.0 \\
\hline 21.5 & 21.3 & 9.2 & 7.2 & 7.1 & 6.9 \\
\hline 483 & 484 & 503 & 505 & 507 & 509 \\
\hline 8.3 & 8.3 & 8.2 & 8.1 & 8.1 & 8.0 \\
\hline 8.9 & 8.8 & 9.5 & 8.6 & 8.0 & 7.1 \\
\hline 0.006 & 0.007 & 0.007 & 0.007 & $<0.005$ & 0.009 \\
\hline 0.003 & 0.003 & 0.003 & 0.003 & 0.004 & 0.008 \\
\hline 0.072 & 0.071 & 0.075 & 0.069 & 0.077 & 0.073 \\
\hline$<0.013$ & 0.009 & 0.042 & 0.071 & 0.11 & 0.169 \\
\hline 0.75 & 0.56 & 0.56 & 0.70 & 0.74 & 0.81 \\
\hline 0.82 & 0.63 & 0.63 & 0.77 & 0.82 & 0.88 \\
\hline 1.5 & -- & 1.6 & -- & -- & 1.7 \\
\hline
\end{tabular}

6-14-99
Jul -13

\begin{tabular}{cccccc}
\multicolumn{7}{c}{2.51} \\
\multicolumn{7}{c}{2.5} \\
0.5 & 8.0 & 28.0 & 33.0 & 38.0 & 42.5 \\
24.6 & 23.8 & 8.1 & 7.4 & 7.1 & 7.0 \\
488 & 488 & 510 & 513 & 514 & 516 \\
8.3 & 8.3 & 8.0 & 7.8 & 7.8 & 7.7 \\
9.4 & 9.4 & 7.8 & 6.3 & 5.0 & 4.1 \\
$<0.005$ & $<0.005$ & 0.005 & $<0.005$ & 0.008 & 0.017 \\
0.002 & 0.002 & 0.002 & 0.002 & 0.003 & 0.006 \\
$<0.010$ & $<0.010$ & 0.089 & 0.084 & 0.075 & 0.066 \\
$<0.013$ & $<0.013$ & 0.061 & 0.147 & 0.2 & 0.275 \\
0.38 & 0.32 & 0.43 & 0.5 & 0.82 & 0.76 \\
--- & --- & 0.52 & 0.58 & 0.89 & 0.83 \\
--- &.-- &.-- & --- &.-- & ---
\end{tabular}

7-13-99

DISSOLVED OXYGEN (D.O.), IN MILLIGRAMS PER LITER

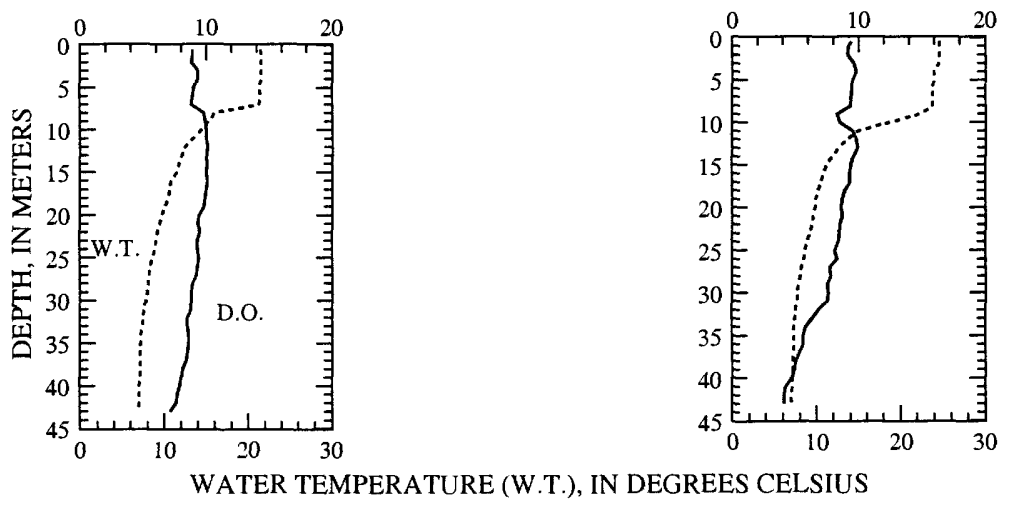

PH, IN STANDARD UNITS
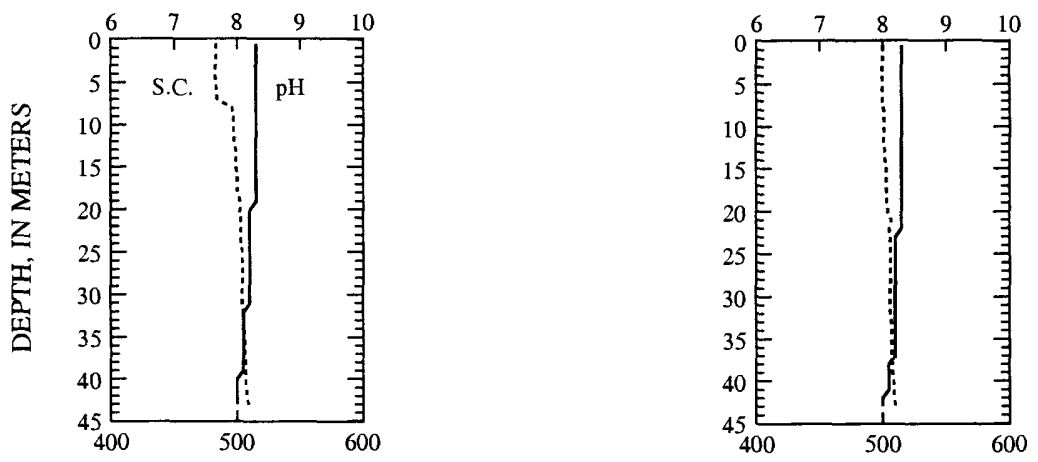

SPECIFIC CONDUCTANCE (S.C.), IN MICROSIEMENS PER CENTIMETER AT 25 DEGREES CELSIUS 
WATER-QUALITY DATA, AUGUST 17 TO SEPTEMBER 15, 1999

(Milligrams per liter unless otherwise indicated)

\begin{tabular}{|c|c|c|c|c|c|c|c|c|c|c|c|}
\hline \multicolumn{6}{|c|}{ Aug-17 } & \multicolumn{6}{|c|}{ Sep-15 } \\
\hline \multicolumn{6}{|c|}{$\begin{array}{c}2.25 \\
4.6 \\
2.24\end{array}$} & \multicolumn{6}{|c|}{$\begin{array}{c}2.14 \\
4.6 \\
1.45\end{array}$} \\
\hline 0.5 & 10.0 & 17.0 & 33.0 & 38.0 & 42.5 & 0.5 & 12.0 & 25.0 & 34.0 & 39.0 & 42.5 \\
\hline 23.4 & 23.2 & 10.8 & 7.6 & 7.3 & 7.2 & 20.0 & 19.9 & 9.1 & 7.6 & 7.2 & 7.2 \\
\hline 493 & 494 & 512 & 514 & 517 & 520 & 490 & 491 & 490 & 492 & 498 & 498 \\
\hline 8.3 & 8.3 & 8.1 & 7.7 & 7.5 & 7.5 & 8.1 & 8.1 & 7.7 & 7.5 & 7.4 & 7.4 \\
\hline 8.7 & 8.3 & 7.4 & 4.4 & 1.6 & 0.8 & 8.2 & 8.0 & 5.3 & 1.8 & 0.1 & 0.1 \\
\hline 0.009 & 0.011 & 0.007 & 0.009 & 0.032 & 0.057 & 0.008 & 0.008 & 0.008 & 0.006 & 0.059 & 0.085 \\
\hline 0.002 & $<0.002$ & $<0.002$ & 0.004 & 0.023 & 0.044 & 0.002 & 0.002 & $<0.002$ & $<0.002$ & 0.046 & 0.072 \\
\hline$<0.010$ & $<0.010$ & 0.078 & 0.319 & 0.357 & 0.143 & $<0.010$ & 0.01 & 0.204 & 0.305 & 0.12 & $<0.010$ \\
\hline 0.003 & 0.002 & 0.021 & 0.002 & 0.074 & 0.277 & 0.009 & 0.006 & 0.004 & 0.003 & 0.256 & 0.38 \\
\hline 0.49 & 0.47 & 0.45 & 0.35 & 0.51 & 0.89 & 0.62 & 0.54 & 0.52 & 0.49 & 0.73 & 0.94 \\
\hline--- & --- & 0.53 & 0.67 & 0.87 & 1.00 & --- & 0.55 & 0.72 & 0.80 & 0.85 & $\cdots$ \\
\hline
\end{tabular}

DISSOLVED OXYGEN (D.O.), IN MILLIGRAMS PER LITER
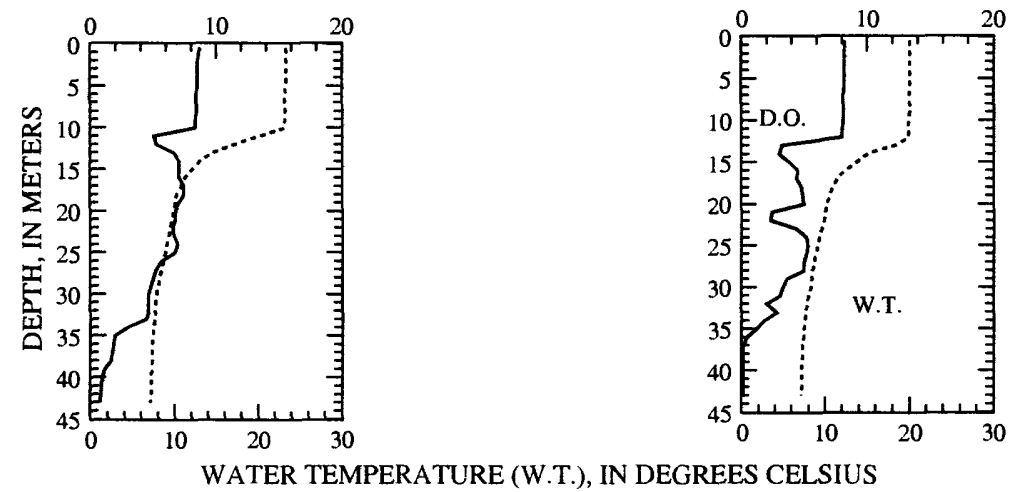

PH, IN STANDARD UNITS
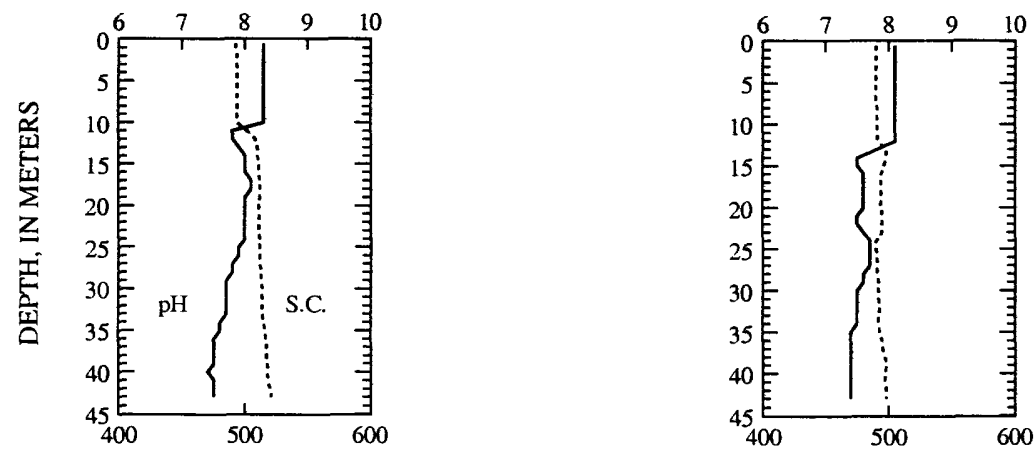

SPECIFIC CONDUCTANCE (S.C.), IN MICROSIEMENS PER CENTIMETER AT 25 DEGREES CELSIUS 

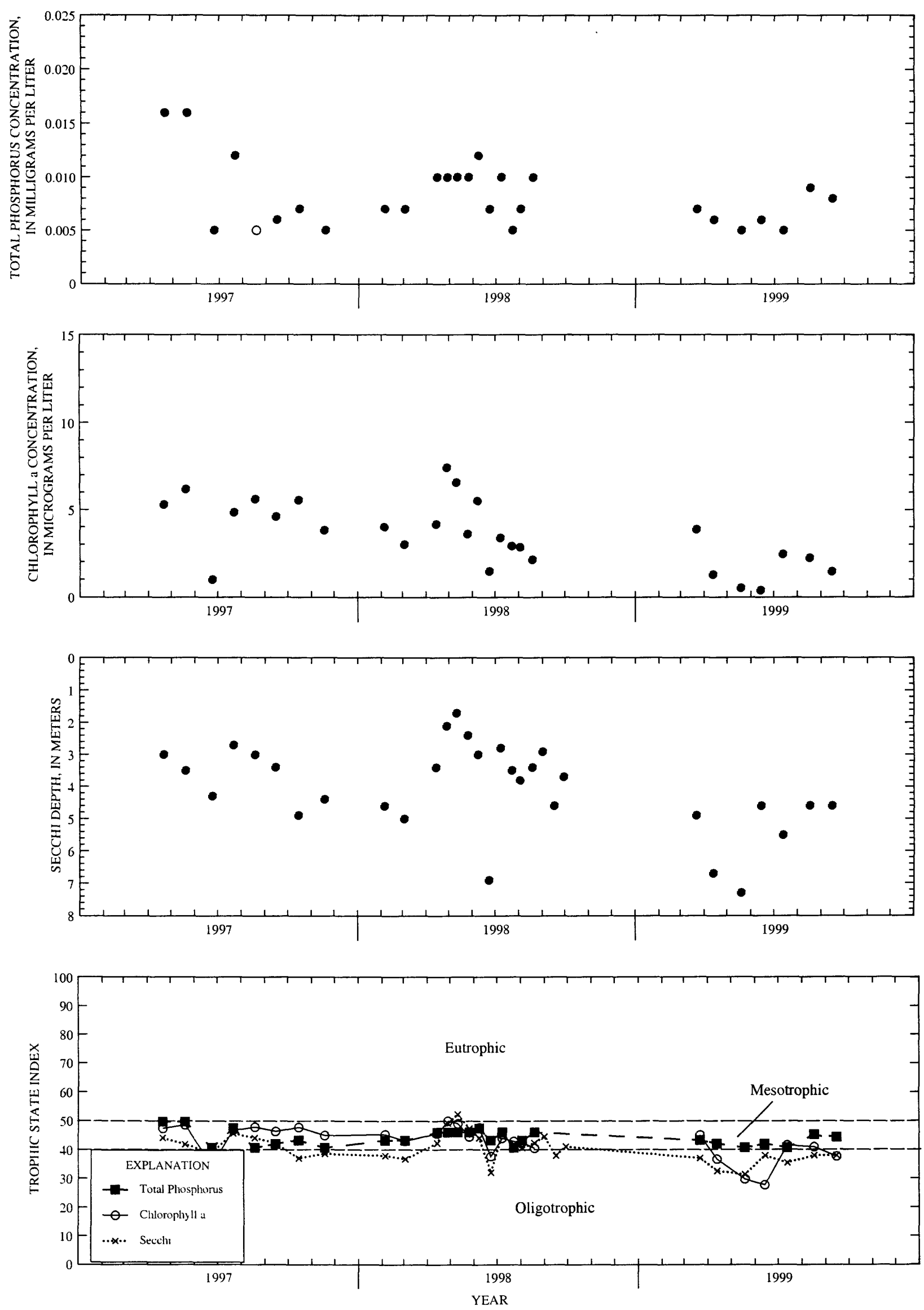

Surface total phosphorus, chlorophyll a concentrations, Secchi depths, and TSI data for Geneva Lake, West End, near Williams Bay, Wisconsin. 


\section{GREEN LAKE AT COUNTY TRUNK HIGHWAY A NEAR GREEN LAKE, WI}

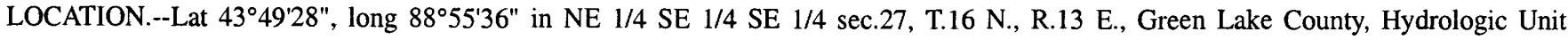
04030201, on left bank at downstream side of County Trunk Highway A, 2.3 mi southeast of Green Lake.

DRAINAGE AREA.--103 $\mathrm{mi}^{2}$.

PERIOD OF RECORD.--October 1993 to current year.

GAGE.--Water-stage recorder. Datum of gage is $790.00 \mathrm{ft}$ above sea level.

REMARKS.--Estimated daily gage heights: Apr. 3-6. Records good except those for estimated daily gage heights, which are fair. Lake level regulated by dam at outlet at Green Lake. Gage-height telemeter at station.

EXTREMES FOR PERIOD OF RECORD.--Maximum recorded gage height, $7.03 \mathrm{ft}$, June 20, 1996; minimum recorded, $5.41 \mathrm{ft}$, Jan. 17 , 1995.

EXTREMES FOR CURRENT YEAR.--Maximum recorded gage height, $7.00 \mathrm{ft}$, July 21, 22, and 23; minimum recorded, $5.79 \mathrm{ft}$, Oct. 5 and Nov. 10.

GAGE HEIGHT, FEET, WATER YEAR OCTOBER I998 TO SEPTEMBER 1999

DAILY MEAN VALUES

\begin{tabular}{|c|c|c|c|c|c|c|c|c|c|c|c|c|}
\hline DAY & OCT & NOV & DEC & JAN & FEB & MAR & APR & MAY & JUN & JUL & AUG & SEP \\
\hline $\begin{array}{l}1 \\
2 \\
3 \\
4 \\
5\end{array}$ & $\begin{array}{l}5.90 \\
5.88 \\
5.83 \\
5.81 \\
5.82\end{array}$ & $\begin{array}{l}5.88 \\
5.85 \\
5.83 \\
5.83 \\
5.82\end{array}$ & $\begin{array}{l}6.05 \\
6.04 \\
6.05 \\
6.05 \\
6.06\end{array}$ & $\begin{array}{l}5.94 \\
5.94 \\
5.99 \\
5.98 \\
5.97\end{array}$ & $\begin{array}{l}6.19 \\
6.20 \\
6.20 \\
6.20 \\
6.19\end{array}$ & $\begin{array}{l}6.37 \\
6.38 \\
6.38 \\
6.37 \\
6.38\end{array}$ & $\begin{array}{l}6.37 \\
6.38 \\
6.45 \\
6.55 \\
6.65\end{array}$ & $\begin{array}{l}6.58 \\
6.56 \\
6.54 \\
6.51 \\
6.52\end{array}$ & $\begin{array}{l}6.54 \\
6.64 \\
6.66 \\
6.64 \\
6.65\end{array}$ & $\begin{array}{l}6.51 \\
6.53 \\
6.56 \\
6.60 \\
6.61\end{array}$ & $\begin{array}{l}6.69 \\
6.64 \\
6.60 \\
6.60 \\
6.58\end{array}$ & $\begin{array}{l}6.36 \\
6.35 \\
6.35 \\
6.35 \\
6.33\end{array}$ \\
\hline $\begin{array}{r}6 \\
7 \\
8 \\
9 \\
10\end{array}$ & $\begin{array}{l}5.90 \\
5.91 \\
5.90 \\
5.89 \\
5.89\end{array}$ & $\begin{array}{l}5.82 \\
5.81 \\
5.81 \\
5.82 \\
6.00\end{array}$ & $\begin{array}{l}6.07 \\
6.06 \\
6.06 \\
6.06 \\
6.06\end{array}$ & $\begin{array}{l}5.97 \\
5.96 \\
5.96 \\
5.96 \\
5.96\end{array}$ & $\begin{array}{l}6.19 \\
6.19 \\
6.19 \\
6.20 \\
6.20\end{array}$ & $\begin{array}{l}6.37 \\
6.36 \\
6.36 \\
6.38 \\
6.37\end{array}$ & $\begin{array}{l}6.68 \\
6.63 \\
6.62 \\
6.68 \\
6.68\end{array}$ & $\begin{array}{l}6.57 \\
6.61 \\
6.61 \\
6.59 \\
6.56\end{array}$ & $\begin{array}{l}6.65 \\
6.66 \\
6.62 \\
6.60 \\
6.59\end{array}$ & $\begin{array}{l}6.63 \\
6.61 \\
6.58 \\
6.68 \\
6.64\end{array}$ & $\begin{array}{l}6.55 \\
6.54 \\
6.51 \\
6.48 \\
6.50\end{array}$ & $\begin{array}{l}6.32 \\
6.30 \\
6.30 \\
6.28 \\
6.25\end{array}$ \\
\hline $\begin{array}{l}11 \\
12 \\
13 \\
14 \\
15\end{array}$ & $\begin{array}{l}5.89 \\
5.89 \\
5.87 \\
5.83 \\
5.82\end{array}$ & $\begin{array}{l}6.04 \\
5.96 \\
5.96 \\
5.97 \\
5.96\end{array}$ & $\begin{array}{l}6.05 \\
6.04 \\
6.04 \\
6.05 \\
6.05\end{array}$ & $\begin{array}{l}5.97 \\
5.98 \\
6.00 \\
6.00 \\
6.00\end{array}$ & $\begin{array}{l}6.24 \\
6.31 \\
6.32 \\
6.33 \\
6.34\end{array}$ & $\begin{array}{l}6.37 \\
6.36 \\
6.36 \\
6.36 \\
6.35\end{array}$ & $\begin{array}{l}6.73 \\
6.77 \\
6.75 \\
6.73 \\
6.71\end{array}$ & $\begin{array}{l}6.55 \\
6.54 \\
6.52 \\
6.52 \\
6.51\end{array}$ & $\begin{array}{l}6.58 \\
6.58 \\
6.57 \\
6.56 \\
6.52\end{array}$ & $\begin{array}{l}6.62 \\
6.61 \\
6.58 \\
6.58 \\
6.56\end{array}$ & $\begin{array}{l}6.49 \\
6.47 \\
6.47 \\
6.44 \\
6.43\end{array}$ & $\begin{array}{l}6.22 \\
6.22 \\
6.22 \\
6.20 \\
6.18\end{array}$ \\
\hline $\begin{array}{l}16 \\
17 \\
18 \\
19 \\
20\end{array}$ & $\begin{array}{l}5.83 \\
5.85 \\
5.92 \\
5.88 \\
5.85\end{array}$ & $\begin{array}{l}5.99 \\
6.00 \\
6.01 \\
6.07 \\
6.02\end{array}$ & $\begin{array}{l}6.04 \\
6.03 \\
6.03 \\
6.03 \\
6.02\end{array}$ & $\begin{array}{l}6.01 \\
6.02 \\
6.06 \\
6.06 \\
6.06\end{array}$ & $\begin{array}{l}6.36 \\
6.36 \\
6.36 \\
6.36 \\
6.35\end{array}$ & $\begin{array}{l}6.36 \\
6.38 \\
6.36 \\
6.36 \\
6.37\end{array}$ & $\begin{array}{l}6.69 \\
6.67 \\
6.65 \\
6.64 \\
6.62\end{array}$ & $\begin{array}{l}6.52 \\
6.63 \\
6.68 \\
6.67 \\
6.66\end{array}$ & $\begin{array}{l}6.49 \\
6.48 \\
6.46 \\
6.43 \\
6.42\end{array}$ & $\begin{array}{l}6.56 \\
6.61 \\
6.62 \\
6.68 \\
6.67\end{array}$ & $\begin{array}{l}6.43 \\
6.42 \\
6.40 \\
6.42 \\
6.43\end{array}$ & $\begin{array}{l}6.16 \\
6.15 \\
6.14 \\
6.13 \\
6.13\end{array}$ \\
\hline $\begin{array}{l}21 \\
22 \\
23 \\
24 \\
25\end{array}$ & $\begin{array}{l}5.82 \\
5.82 \\
5.82 \\
5.81 \\
5.81\end{array}$ & $\begin{array}{l}6.01 \\
6.01 \\
6.03 \\
6.00 \\
6.01\end{array}$ & $\begin{array}{l}6.05 \\
6.04 \\
6.01 \\
6.00 \\
5.98\end{array}$ & $\begin{array}{l}6.06 \\
6.10 \\
6.14 \\
6.18 \\
6.19\end{array}$ & $\begin{array}{l}6.34 \\
6.33 \\
6.33 \\
6.33 \\
6.34\end{array}$ & $\begin{array}{l}6.37 \\
6.36 \\
6.37 \\
6.36 \\
6.36\end{array}$ & $\begin{array}{l}6.60 \\
6.62 \\
6.68 \\
6.68 \\
6.67\end{array}$ & $\begin{array}{l}6.65 \\
6.65 \\
6.67 \\
6.68 \\
6.65\end{array}$ & $\begin{array}{l}6.41 \\
6.40 \\
6.42 \\
6.43 \\
6.43\end{array}$ & $\begin{array}{l}6.95 \\
6.99 \\
6.99 \\
6.98 \\
6.93\end{array}$ & $\begin{array}{l}6.43 \\
6.43 \\
6.43 \\
6.43 \\
6.43\end{array}$ & $\begin{array}{l}6.11 \\
6.10 \\
6.09 \\
6.08 \\
6.06\end{array}$ \\
\hline $\begin{array}{l}26 \\
27 \\
28 \\
29 \\
30 \\
31\end{array}$ & $\begin{array}{l}5.81 \\
5.84 \\
5.87 \\
5.86 \\
5.89 \\
5.89\end{array}$ & $\begin{array}{l}6.01 \\
6.00 \\
6.01 \\
6.03 \\
6.06 \\
. .-\end{array}$ & $\begin{array}{l}5.97 \\
5.97 \\
5.96 \\
5.97 \\
5.96 \\
5.96\end{array}$ & $\begin{array}{l}6.19 \\
6.19 \\
6.19 \\
6.19 \\
6.19 \\
6.19\end{array}$ & $\begin{array}{r}6.34 \\
6.37 \\
6.38 \\
--- \\
- \\
-\end{array}$ & $\begin{array}{l}6.36 \\
6.36 \\
6.37 \\
6.37 \\
6.36 \\
6.37\end{array}$ & $\begin{array}{l}6.66 \\
6.64 \\
6.62 \\
6.61 \\
6.60 \\
\ldots\end{array}$ & $\begin{array}{l}6.62 \\
6.62 \\
6.61 \\
6.59 \\
6.55 \\
6.55\end{array}$ & $\begin{array}{l}6.42 \\
6.42 \\
6.43 \\
6.45 \\
6.44 \\
-\end{array}$ & $\begin{array}{l}6.95 \\
6.91 \\
6.86 \\
6.81 \\
6.76 \\
6.75\end{array}$ & $\begin{array}{l}6.43 \\
6.44 \\
6.44 \\
6.42 \\
6.38 \\
6.37\end{array}$ & $\begin{array}{l}6.06 \\
6.07 \\
6.08 \\
6.10 \\
6.11 \\
-\end{array}$ \\
\hline $\begin{array}{l}\text { MEAN } \\
\text { MAX } \\
\text { MIN }\end{array}$ & $\begin{array}{l}5.86 \\
5.92 \\
5.81\end{array}$ & $\begin{array}{l}5.95 \\
6.07 \\
5.81\end{array}$ & $\begin{array}{l}6.03 \\
6.07 \\
5.96\end{array}$ & $\begin{array}{l}6.05 \\
6.19 \\
5.94\end{array}$ & $\begin{array}{l}6.29 \\
6.38 \\
6.19\end{array}$ & $\begin{array}{l}6.37 \\
6.38 \\
6.35\end{array}$ & $\begin{array}{l}6.63 \\
6.77 \\
6.37\end{array}$ & $\begin{array}{l}6.59 \\
6.68 \\
6.51\end{array}$ & $\begin{array}{l}6.52 \\
6.66 \\
6.40\end{array}$ & $\begin{array}{l}6.71 \\
6.99 \\
6.51\end{array}$ & $\begin{array}{l}6.47 \\
6.69 \\
6.37\end{array}$ & $\begin{array}{l}6.19 \\
6.36 \\
6.06\end{array}$ \\
\hline
\end{tabular}




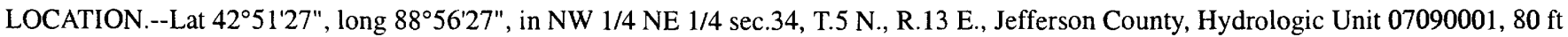
east of Pottawatomi Trail Bridge at Bingham Point Estates, and 4.5 mi northeast of Newville.

DRAINAGE AREA.--2,560 $\mathrm{mi}^{2}$, at lake outlet. Area of Lake Koshkonong, $16.3 \mathrm{mi}^{2}$.

PERIOD OF RECORD.--July 1987 to current year.

GAGE.--Water-stage recorder. Datum of gage is $770.00 \mathrm{ft}$ above sea level.

REMARKS.--No estimated daily gage heights. Records good except those for July 14 to Aug. 3, which are fair. Lake level regulated by dam at Indianford. Gage-height telemeter at station.

EXTREMES FOR PERIOD OF RECORD.--Maximum recorded gage height, $12.23 \mathrm{ft}$, Apr. 25, 1993; minimum recorded, $5.40 \mathrm{ft}$, Dec. 26 , 27, 1989.

EXTREMES FOR CURRENT YEAR.--Maximum recorded gage height, $9.73 \mathrm{ft}$, Apr. 28; minimum recorded, $5.48 \mathrm{ft}$, Jan. 5.

GAGE HEIGHT, FEET, WATER YEAR OCTOBER 1998 TO SEPTEMBER 1999

DAILY MEAN VALUES

\begin{tabular}{|c|c|c|c|c|c|c|c|c|c|c|c|c|}
\hline DAY & OCT & NOV & DEC & JAN & FEB & MAR & APR & MAY & JUN & JUL & AUG & SEP \\
\hline $\begin{array}{l}1 \\
2 \\
3 \\
4 \\
5\end{array}$ & $\begin{array}{l}6.28 \\
6.25 \\
6.27 \\
6.27 \\
6.37\end{array}$ & $\begin{array}{l}5.99 \\
5.93 \\
5.86 \\
5.84 \\
5.80\end{array}$ & $\begin{array}{l}6.28 \\
6.28 \\
6.28 \\
6.26 \\
6.24\end{array}$ & $\begin{array}{l}5.52 \\
5.52 \\
5.53 \\
5.51 \\
5.49\end{array}$ & $\begin{array}{l}6.93 \\
7.02 \\
7.11 \\
7.20 \\
7.29\end{array}$ & $\begin{array}{l}7.24 \\
7.19 \\
7.15 \\
7.10 \\
7.06\end{array}$ & $\begin{array}{l}6.30 \\
6.28 \\
6.29 \\
6.39 \\
6.39\end{array}$ & $\begin{array}{l}9.61 \\
9.54 \\
9.44 \\
9.33 \\
9.22\end{array}$ & $\begin{array}{l}8.13 \\
8.10 \\
7.99 \\
7.89 \\
7.80\end{array}$ & $\begin{array}{l}7.16 \\
7.07 \\
6.99 \\
6.95 \\
6.88\end{array}$ & $\begin{array}{l}7.80 \\
7.84 \\
7.86 \\
7.89 \\
7.90\end{array}$ & $\begin{array}{l}6.30 \\
6.24 \\
6.18 \\
6.11 \\
6.05\end{array}$ \\
\hline $\begin{array}{r}6 \\
7 \\
8 \\
9 \\
10\end{array}$ & $\begin{array}{l}6.45 \\
6.46 \\
6.46 \\
6.45 \\
6.45\end{array}$ & $\begin{array}{l}5.77 \\
5.74 \\
5.72 \\
5.69 \\
5.80\end{array}$ & $\begin{array}{l}6.25 \\
6.24 \\
6.22 \\
6.20 \\
6.20\end{array}$ & $\begin{array}{l}5.52 \\
5.55 \\
5.61 \\
5.65 \\
5.69\end{array}$ & $\begin{array}{l}7.35 \\
7.39 \\
7.42 \\
7.43 \\
7.45\end{array}$ & $\begin{array}{l}7.05 \\
6.97 \\
6.90 \\
6.85 \\
6.77\end{array}$ & $\begin{array}{l}6.58 \\
6.68 \\
6.80 \\
6.96 \\
7.14\end{array}$ & $\begin{array}{l}9.16 \\
9.07 \\
9.01 \\
8.91 \\
8.79\end{array}$ & $\begin{array}{l}7.73 \\
7.71 \\
7.68 \\
7.64 \\
7.59\end{array}$ & $\begin{array}{l}6.83 \\
6.72 \\
6.60 \\
6.55 \\
6.45\end{array}$ & $\begin{array}{l}7.90 \\
7.90 \\
7.91 \\
7.87 \\
7.89\end{array}$ & $\begin{array}{l}5.97 \\
5.89 \\
5.94 \\
6.00 \\
6.03\end{array}$ \\
\hline $\begin{array}{l}11 \\
12 \\
13 \\
14 \\
15\end{array}$ & $\begin{array}{l}6.43 \\
6.43 \\
6.41 \\
6.35 \\
6.31\end{array}$ & $\begin{array}{l}5.97 \\
6.01 \\
6.12 \\
6.27 \\
6.36\end{array}$ & $\begin{array}{l}6.16 \\
6.14 \\
6.11 \\
6.08 \\
6.07\end{array}$ & $\begin{array}{l}5.75 \\
5.78 \\
5.82 \\
5.85 \\
5.86\end{array}$ & $\begin{array}{l}7.50 \\
7.59 \\
7.66 \\
7.71 \\
7.77\end{array}$ & $\begin{array}{l}6.71 \\
6.66 \\
6.62 \\
6.57 \\
6.53\end{array}$ & $\begin{array}{l}7.35 \\
7.54 \\
7.70 \\
7.82 \\
7.90\end{array}$ & $\begin{array}{l}8.67 \\
8.56 \\
8.43 \\
8.31 \\
8.19\end{array}$ & $\begin{array}{l}7.55 \\
7.54 \\
7.64 \\
7.86 \\
8.05\end{array}$ & $\begin{array}{l}6.39 \\
6.34 \\
6.29 \\
6.27 \\
6.24\end{array}$ & $\begin{array}{l}7.85 \\
7.81 \\
7.79 \\
7.72 \\
7.64\end{array}$ & $\begin{array}{l}6.07 \\
6.13 \\
6.18 \\
6.22 \\
6.23\end{array}$ \\
\hline $\begin{array}{l}16 \\
17 \\
18 \\
19 \\
20\end{array}$ & $\begin{array}{l}6.28 \\
6.32 \\
6.46 \\
6.47 \\
6.48\end{array}$ & $\begin{array}{l}6.44 \\
6.49 \\
6.50 \\
6.59 \\
6.60\end{array}$ & $\begin{array}{l}6.05 \\
6.03 \\
5.97 \\
5.98 \\
5.95\end{array}$ & $\begin{array}{l}5.86 \\
5.87 \\
5.89 \\
5.85 \\
5.81\end{array}$ & $\begin{array}{l}7.81 \\
7.85 \\
7.84 \\
7.83 \\
7.78\end{array}$ & $\begin{array}{l}6.51 \\
6.56 \\
6.55 \\
6.50 \\
6.48\end{array}$ & $\begin{array}{l}7.98 \\
7.98 \\
7.97 \\
7.96 \\
7.93\end{array}$ & $\begin{array}{l}8.09 \\
8.31 \\
8.53 \\
8.71 \\
8.84\end{array}$ & $\begin{array}{l}8.24 \\
8.36 \\
8.39 \\
8.38 \\
8.33\end{array}$ & $\begin{array}{l}6.22 \\
6.32 \\
6.39 \\
6.40 \\
6.45\end{array}$ & $\begin{array}{l}7.56 \\
7.51 \\
7.43 \\
7.37 \\
7.28\end{array}$ & $\begin{array}{l}6.21 \\
6.17 \\
6.13 \\
6.12 \\
6.12\end{array}$ \\
\hline $\begin{array}{l}21 \\
22 \\
23 \\
24 \\
25\end{array}$ & $\begin{array}{l}6.47 \\
6.42 \\
6.39 \\
6.34 \\
6.30\end{array}$ & $\begin{array}{l}6.55 \\
6.53 \\
6.55 \\
6.49 \\
6.46\end{array}$ & $\begin{array}{l}5.94 \\
5.87 \\
5.83 \\
5.79 \\
5.76\end{array}$ & $\begin{array}{l}5.78 \\
5.80 \\
5.86 \\
5.97 \\
6.10\end{array}$ & $\begin{array}{l}7.71 \\
7.64 \\
7.58 \\
7.51 \\
7.46\end{array}$ & $\begin{array}{l}6.52 \\
6.48 \\
6.48 \\
6.48 \\
6.45\end{array}$ & $\begin{array}{l}7.93 \\
7.97 \\
8.34 \\
8.68 \\
9.06\end{array}$ & $\begin{array}{l}8.92 \\
8.98 \\
8.97 \\
8.93 \\
8.86\end{array}$ & $\begin{array}{l}8.26 \\
8.17 \\
8.08 \\
8.03 \\
7.90\end{array}$ & $\begin{array}{l}6.50 \\
6.52 \\
6.71 \\
6.85 \\
7.02\end{array}$ & $\begin{array}{l}7.16 \\
7.04 \\
6.94 \\
6.88 \\
6.79\end{array}$ & $\begin{array}{l}6.07 \\
6.05 \\
6.09 \\
6.10 \\
6.11\end{array}$ \\
\hline $\begin{array}{l}26 \\
27 \\
28 \\
29 \\
30 \\
31\end{array}$ & $\begin{array}{l}6.25 \\
6.21 \\
6.18 \\
6.12 \\
6.09 \\
6.04\end{array}$ & $\begin{array}{l}6.43 \\
6.39 \\
6.36 \\
6.31 \\
6.33 \\
-\ldots\end{array}$ & $\begin{array}{l}5.72 \\
5.68 \\
5.63 \\
5.60 \\
5.56 \\
5.54\end{array}$ & $\begin{array}{l}6.22 \\
6.36 \\
6.49 \\
6.62 \\
6.75 \\
6.85\end{array}$ & $\begin{array}{r}7.39 \\
7.33 \\
7.29 \\
-\ldots \\
- \\
\ldots-\end{array}$ & $\begin{array}{l}6.43 \\
6.41 \\
6.39 \\
6.40 \\
6.37 \\
6.32\end{array}$ & $\begin{array}{c}9.36 \\
9.54 \\
9.67 \\
9.69 \\
9.67 \\
---\end{array}$ & $\begin{array}{l}8.77 \\
8.69 \\
8.60 \\
8.49 \\
8.36 \\
8.24\end{array}$ & $\begin{array}{c}7.76 \\
7.62 \\
7.50 \\
7.36 \\
7.23 \\
-\end{array}$ & $\begin{array}{l}7.11 \\
7.27 \\
7.37 \\
7.49 \\
7.59 \\
7.72\end{array}$ & $\begin{array}{l}6.71 \\
6.65 \\
6.59 \\
6.51 \\
6.41 \\
6.35\end{array}$ & $\begin{array}{c}6.12 \\
6.26 \\
6.38 \\
6.41 \\
6.39 \\
-\end{array}$ \\
\hline $\begin{array}{l}\text { MEAN } \\
\text { MAX } \\
\text { MIN }\end{array}$ & $\begin{array}{l}6.34 \\
6.48 \\
6.04\end{array}$ & $\begin{array}{l}6.20 \\
6.60 \\
5.69\end{array}$ & $\begin{array}{l}6.00 \\
6.28 \\
5.54\end{array}$ & $\begin{array}{l}5.89 \\
6.85 \\
5.49\end{array}$ & $\begin{array}{l}7.49 \\
7.85 \\
6.93\end{array}$ & $\begin{array}{l}6.67 \\
7.24 \\
6.32\end{array}$ & $\begin{array}{l}7.80 \\
9.69 \\
6.28\end{array}$ & $\begin{array}{l}8.79 \\
9.61 \\
8.09\end{array}$ & $\begin{array}{l}7.88 \\
8.39 \\
7.23\end{array}$ & $\begin{array}{l}6.76 \\
7.72 \\
6.22\end{array}$ & $\begin{array}{l}7.38 \\
7.91 \\
6.35\end{array}$ & $\begin{array}{l}6.14 \\
6.41 \\
5.89\end{array}$ \\
\hline
\end{tabular}


LOCATION.--Lat $45^{\circ} 54^{\prime} 46^{\prime \prime}$ long $89^{\circ} 37^{\prime} 03^{\prime \prime}$, in SW 1/4 SE 1/4 sec.28, T.40 N., R.7 E., Vilas County, Hydrologic Unit 07070001, 4 mi northeast of Woodruff.

PERIOD OF RECORD.--February 1991 to current year.

GAGE.--Staff gage read by Glyn A. Roberts.

EXTREMES FOR PERIOD OF RECORD.--Maximum gage height observed, $8.02 \mathrm{ft}$, May 7, 1999; minimum observed, $7.72 \mathrm{ft}$, Feb. 28 , June 12, 1991, Oct. 13, 1994, Sept.. 1-30, and Oct. 4, 1998.

EXTREMES FOR CURRENT YEAR.--Maximum gage height observed, $8.02 \mathrm{ft}$, May 7; minimum observed, $7.72 \mathrm{ft}$, Oct. 4.

GAGE HEIGHT, FEET, WATER YEAR OCTOBER 1998 TO SEPTEMBER 1999 DAILY MEAN VALUES

\begin{tabular}{|c|c|c|c|c|c|c|c|c|c|c|c|c|}
\hline DAY & OCT & NOV & $\mathrm{DEC}$ & JAN & FEB & MAR & APR & MAY & JUN & JUL & AUG & SEP \\
\hline 1 & --- & --- & 7.78 & $\ldots$ & -- & 7.82 & -.- & $\ldots$ & - - & $\ldots$ & -.- & $\ldots$ \\
\hline 2 & $-\cdots$ & -- & $\cdots$ & -- & $\cdots$ & -- & $\cdots$ & - - & -- & 7.86 & -- & -- \\
\hline 3 & --- & -- & $\cdots$ & -- & -- & -- & --- & -- & -- & $\cdots$ & 7.84 & 7.78 \\
\hline 4 & 7.72 & $\ldots$ & -- & -- & --- & $-\cdots$ & -- & -- & -- & $\ldots$ & 7.88 & $\ldots$ \\
\hline 5 & $\cdots$ & 7.82 & -- & -- & -- & -- & 7.84 & $\cdots$ & $\cdots$ & $\cdots$ & --- & $\cdots$ \\
\hline 6 & -- & -- & 7.80 & 7.82 & 7.88 & 7.80 & -- & 7.84 & 7.82 & $-\cdots$ & -- & -- \\
\hline 7 & -- & -- & $\ldots$ & $\ldots$ & $\ldots$ & - & 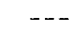 & 8.02 & -- & 7.86 & -- & -- \\
\hline 8 & --- & - & -- & --- & -- & --- & $-\cdots$ & -- & -- & $\cdots$ & $\cdots$ & 7.78 \\
\hline 9 & -- & 7.76 & -- & --- & -- & --- & -- & $-\cdots$ & $\cdots$ & $\ldots$ & $\ldots$ & \\
\hline 10 & 7.74 & $\ldots$ & 7.78 & 7.82 & -- & 7.80 & 7.84 & 7.90 & --- & $-\cdots$ & 7.80 & $\ldots$ \\
\hline 11 & -. & -- & -- & --- & 7.90 & --. & --- & $\ldots$ & 7.80 & $\ldots$ & -.. & $\ldots$ \\
\hline 12 & --- & -- & -- & $\cdots$ & --- & $\cdots$ & --- & $\ldots$ & --- & 7.84 & -- & $\cdots$ \\
\hline 13 & -- & -- & $\ldots$ & --- & -- & $-\cdots$ & -. & 7.84 & $-\cdots$ & $\ldots$ & $\ldots$ & $\cdots$ \\
\hline 14 & -- & 7.80 & -- & --- & -- & 7.80 & $-\cdots$ & $\cdots$ & $\cdots$ & $\cdots$ & --- & -. \\
\hline 15 & 7.76 & $\ldots$ & -- & -- & 7.94 & - & 7.82 & $\ldots$ & --- & $\ldots$ & -- & 7.76 \\
\hline 16 & -.- & - - & - - & 7.84 & --- & $\ldots$ & -.. & -. & --- & $\ldots$ & -.. & -. \\
\hline 17 & $\ldots$ & --. & 7.80 & -.. & $\ldots$ & $\ldots$ & $\ldots$ & $\ldots$ & 7.80 & 7.86 & 7.80 & $\ldots$ \\
\hline 18 & -.- & - - & $\ldots$ & - & -- & -.- & - - & 7.88 & -- & $\cdots$ & - . & $\cdots$ \\
\hline 19 & 7.78 & -- & - - & -.. & -- & 7.82 &.-- & $\ldots$ & -- & $\ldots$ & -.. & $\ldots$ \\
\hline 20 & $\ldots$ & 7.82 & --- & -.- & --- & $\ldots$ & 7.80 & - - & $-\cdots$ & - & $\ldots$ & -- \\
\hline 21 & -- & -.. & $\cdots$ & -- & $\ldots$ & -- & $-\cdots$ & $\ldots$ & --- & -- & -.. & $\cdots$ \\
\hline 22 & --- & -- & - - & 7.84 & 7.82 & --- & $\cdots$ & -- & 7.86 & 7.82 & $\cdots$ & \\
\hline 23 & -- & --- & --- & -- & -- & --- & -- & -.- & --- & --- & $\ldots$ & 7.76 \\
\hline 24 & 7.76 & $\cdots$ & 7.80 & -- & -- & 7.80 & -- & 7.86 & $\cdots$ & $-\cdots$ & 7.80 & $\cdots$ \\
\hline 25 & -.. & 7.82 & -. & --- & -.. & - & 7.80 & $\ldots$ & --- & --- & $\ldots$ & -- \\
\hline 26 & $\ldots$ & -- & $\ldots$ & -- & $\ldots$ & --- & -.- & $\ldots$ & $-\ldots$ & -- & -. & $\ldots$ \\
\hline 27 & 7.82 & $\cdots$ & -- & 7.86 & -- & 7.80 & $\cdots$ & $\cdots$ & 7.88 & 7.78 & --- & \\
\hline 28 & --- & --- & $-\cdots$ & --- & 7.82 & --- & -- & -.- & --- & --- & -.. & $\cdots$ \\
\hline 29 & -- & $\ldots$ & -- & -- & $\ldots$ & 7.82 & --- & $\ldots$ & -- & -- & $-\cdots$ & 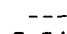 \\
\hline 30 & --- & 7.80 & $\ldots$ & $\ldots$ & --- & - & 7.80 & 7.82 & 7.90 & $-\cdots$ & -- & 7.74 \\
\hline 31 & 7.88 & -- & 7.82 & 7.88 & -- & 7.82 & $\ldots$ & -- & -- & -- & 7.80 & $\cdots$ \\
\hline
\end{tabular}




\section{LITTLE CEDAR LAKE, NORTH SITE, NEAR WEST BEND, WI}

LOCATION.--Lat $43^{\circ} 22^{\prime} 55^{\prime \prime}$, long $88^{\circ} 13^{\prime} 47^{\prime \prime}$, in NW 1/4 NE 1/4 sec.33, T.11 N., R.19 E., Washington County, Hydrologic Unit $04040003,2.6$ mi southwest of West Bend.

PERIOD OF RECORD.--February 1997 to current year.

REMARKS.--Lake sampled at center of northern basin at deep hole. Lake ice-covered during February sampling. Water-quality analyses done by Wisconsin State Laboratory of Hygiene.

WATER-QUALITY DATA, FEBRUARY 10 TO AUGUST 18, 1998

(Milligrams per liter unless otherwise indicated)

Lake stage (ft)

Secchi-depth (m)

Chlorophv1l a, ohvtoolankton ( $\mathrm{Ha} / \mathrm{L}$ )

Depth of sample (m)

Water temperature $\left({ }^{\circ} \mathrm{C}\right)$

Specific conductance (us $/ \mathrm{cm}$ )

$\mathrm{pH}$ (units)

Dissolved oxygen (mg/L)

Phosphorus, total (as P)

Phosphorus, ortho, dissolved (as P)

Nitrogen, NO2 + NO3 diss. (as N)

Nitrogen, ammonia, dissolved (as N)

Nitrogen, amm. + org., total (as N)
Feb-10

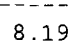

8.19

$0.5^{---} 7.0$

3.0

7.8

15.3

0.0110 .046

0.046

‥

- n

-

\begin{tabular}{cc}
\multicolumn{2}{c}{ Jun-17 } \\
\multicolumn{2}{c}{8.70} \\
\multicolumn{2}{c}{4.3} \\
\multicolumn{2}{c}{1.49} \\
0.5 & 7.5 \\
21.1 & 13.6 \\
482 & 519 \\
8.0 & 7.4 \\
7.6 & 0.5 \\
0.014 & 0.101 \\
.-- & $-\ldots-$ \\
.-- &.-- \\
.-- &.-- \\
.- &.--
\end{tabular}

\begin{tabular}{cc}
\multicolumn{2}{c}{ Jul -22} \\
\multicolumn{2}{c}{9.23} \\
\multicolumn{2}{c}{2.7} \\
\multicolumn{2}{c}{3.87} \\
0.5 & 8.0 \\
25.6 & 14.1 \\
450 & 521 \\
8.4 & 7.3 \\
8.6 & 0.0 \\
0.016 & 0.105 \\
0.004 &.-- \\
$<0.010$ & $\ldots$ \\
0.008 & $\ldots-$. \\
0.6 & $\ldots$
\end{tabular}

\begin{tabular}{ccc}
\multicolumn{3}{c}{ Aug-18 } \\
\multicolumn{3}{c}{8.25} \\
& 1.1 \\
& 43.0 \\
0.5 & 6.0 & 7.5 \\
22.5 & 17.2 & 15.3 \\
471 & 534 & 551 \\
8.6 & 7.2 & 7.2 \\
9.3 & 0.0 & 0.0 \\
0.040 & 0.194 & 0.361 \\
--- & --- & --- \\
--- &.-- &.-- \\
--- & --- & --- \\
--- & --- & ---
\end{tabular}

DISSOLVED OXYGEN (D.O.), IN MILLIGRAMS PER LITER
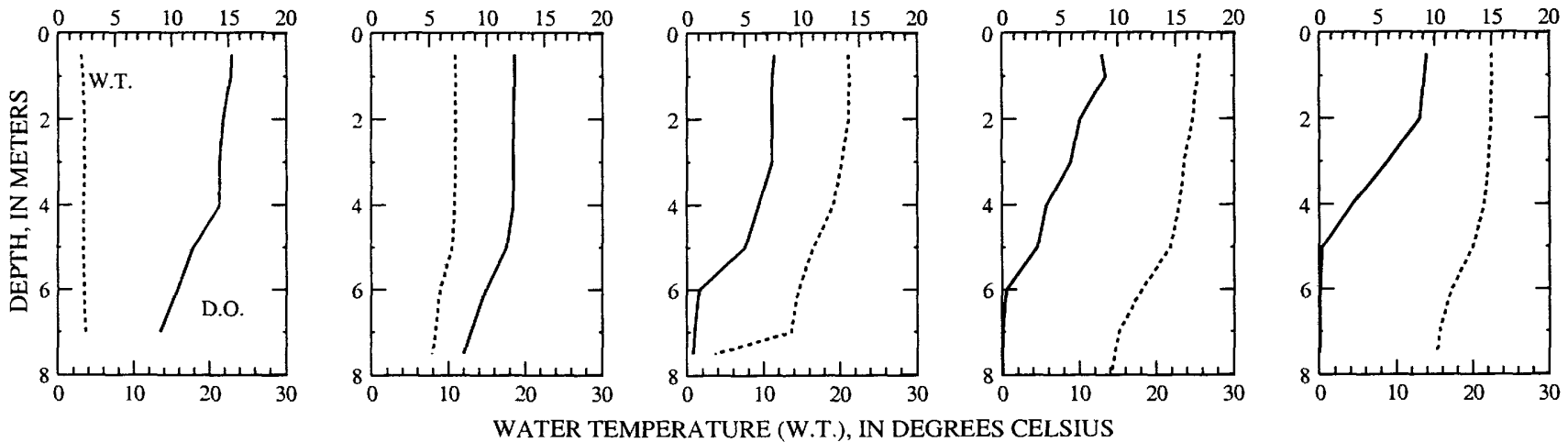

WATER TEMPERATURE (W.T.), IN DEGREES CELSIUS
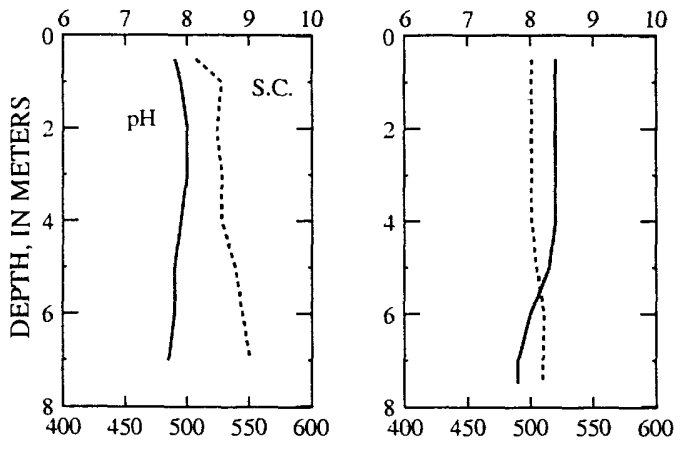

PH, IN STANDARD UNITS
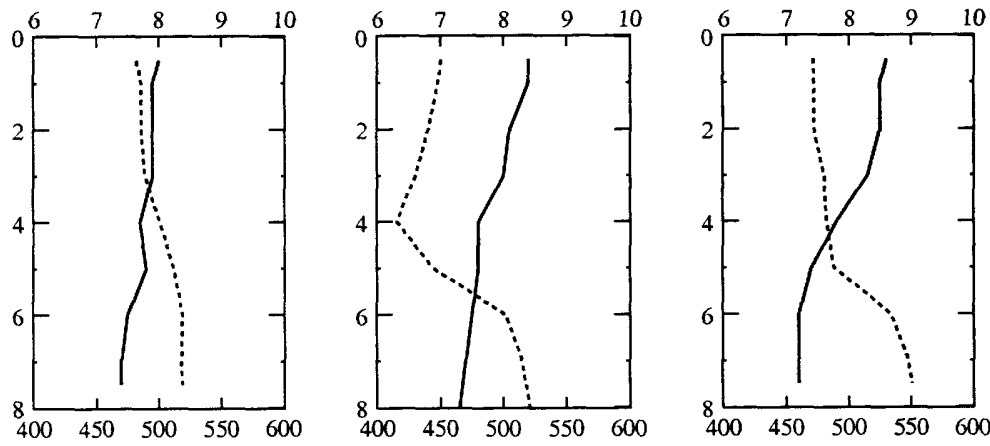

SPECIFIC CONDUCTANCE (S.C.), IN MICROSIEMENS PER CENTIMETER AT 25 DEGREES CELSIUS 

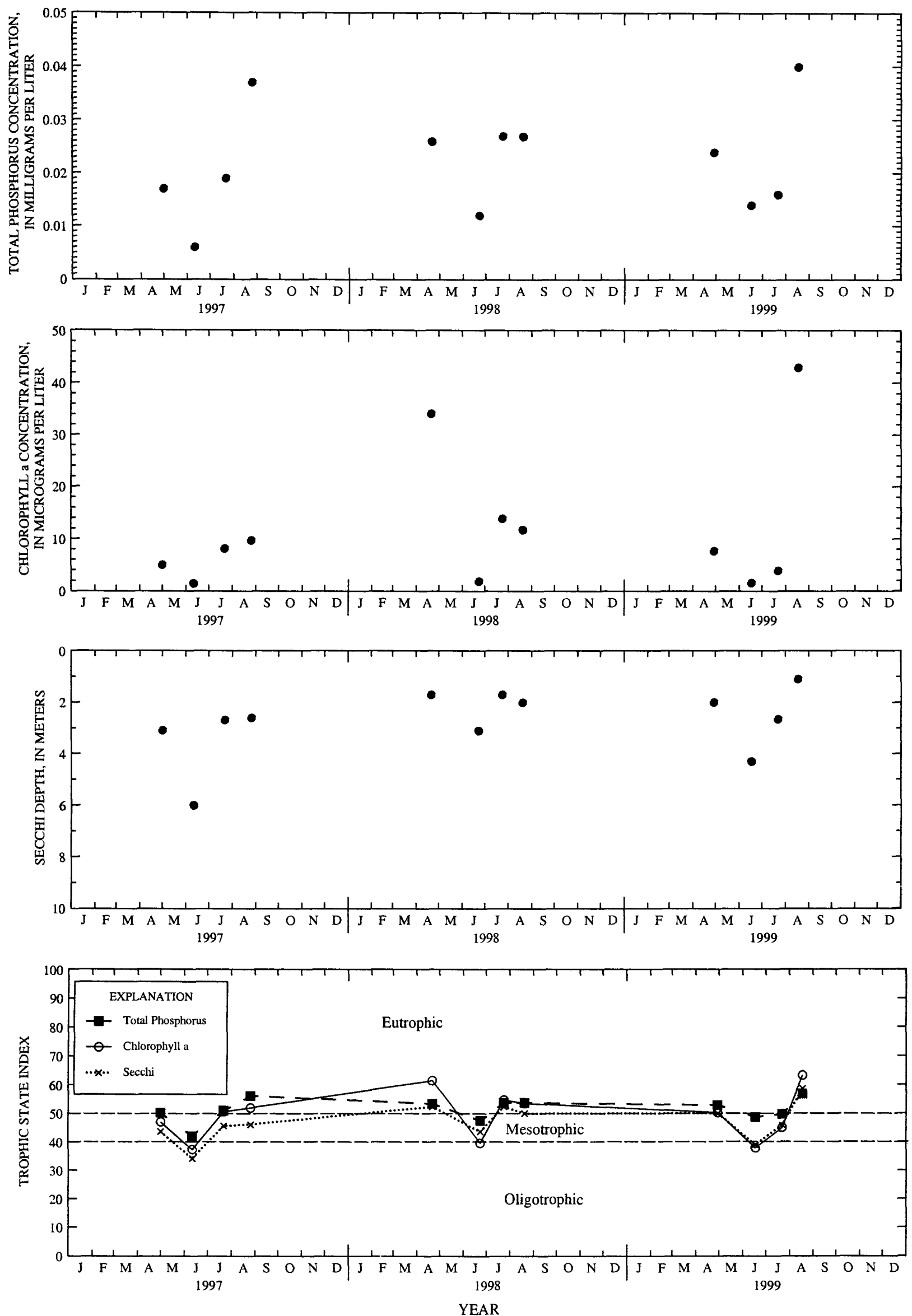

Surface total phosphorus, chlorophyll a concentrations, Secchi depths, and TSI data for Little Cedar Lake, North Site, near West Bend, Wisconsin. 
LOCATION.--Lat $43^{\circ} 22^{\prime} 49^{\prime \prime}$, long $88^{\circ} 13^{\prime} 45^{\prime \prime}$, in NW 1/4 SE 1/4 sec.33, T.11 N., R.19 E., Washington County, Hydrologic Unit $04040003,2.8$ mi southwest of West Bend.

PERIOD OF RECORD.--February 1997 to current year.

REMARKS.--Lake sampled in southern basin at deep hole. Lake ice-covered during February sampling. Water-quality analyses done by Wisconsin State Laboratory of Hygiene.

WATER-QUALITY DATA, FEBRUARY 10 TO AUGUST 18, I999

(Milligrams per liter unless otherwise indicated)

Lake stage (ft)

Secchi-depth (m)

Chlorophyll a, phytoplankton ( $\mu \mathrm{g} / \mathrm{L})$

Depth of sample (m)

water temoerature $\left({ }^{\circ} \mathrm{C}\right)$

Specific conductance $(\mu \mathrm{S} / \mathrm{cm})$

$\mathrm{pH}$ (units)

Disso1ved oxygen (mg/L)

Phosphorus, total (as P)

Phosphorus, ortho, dissolved (as P)

Nitrogen, NO2 + NO3, diss. (as N)

Nitrogen, ammonia, dissolved (as N)

Nitrogen, anm. + org., total (as N)

Nitrogen, total (as $\mathrm{N}$ )

Color (Pt-Co. scale)

Turbidity (NTU)

Hardness, (as $\mathrm{CaCO}_{3}$ )

Calcium, dissolved ( $\mathrm{Ca}$ )

Magnesium, dissolved $(\mathrm{Mg})$

Sodium, dissolved ( $\mathrm{Na}$ )

Potassium, dissolved (K)

Alkalinity, (as $\mathrm{CaCO}_{3}$ )

Sulfate, dissolved $\left(\mathrm{SO}_{4}\right)$

Chloride, dissolved (Cl)

Silica, dissolved $\left(\mathrm{SiO}_{2}\right)$

Solids, dissolved, at $180^{\circ} \mathrm{C}$

Iron, dissolved ( $\mathrm{Fe}) \mu \mathrm{g} / \mathrm{L}$

Manqanese, dissolved (Mn) $\mu \mathrm{q} / \mathrm{L}$
Feb-10

\begin{tabular}{|c|c|c|c|}
\hline \multicolumn{2}{|c|}{ Feb-10 } & \multicolumn{2}{|c|}{ Apr -29} \\
\hline \multicolumn{2}{|c|}{8.19} & \multicolumn{2}{|c|}{$\begin{array}{c}8.48 \\
4.5 \\
4.71\end{array}$} \\
\hline $\begin{array}{c}0.5 \\
2.4 \\
435 \\
7.9 \\
15.2 \\
0.009\end{array}$ & $\begin{array}{c}15.5 \\
3.6 \\
527 \\
7.6 \\
7.8 \\
0.125\end{array}$ & $\begin{array}{c}0.5 \\
10.8 \\
498 \\
8.2 \\
11.8 \\
0.017\end{array}$ & $\begin{array}{c}16.0 \\
8.3 \\
504 \\
8.0 \\
9.0 \\
0.038\end{array}$ \\
\hline$\ldots$ & $\ldots$ & 0.003 & - - \\
\hline$\ldots$ & --- & 0.071 & -- \\
\hline--- & --- & 0.073 & $\rightarrow--$ \\
\hline$\ldots$ & --- & 0.67 & -- \\
\hline-- & $-\cdots$ & 0.74 & $\ldots$ \\
\hline--- & -- & 10 & $\ldots$ \\
\hline--- & -- & 6.6 & -- \\
\hline$\cdots$ & --- & 210 & -- \\
\hline--- & --- & 34 & --- \\
\hline-- & --- & 31 & --- \\
\hline-- & --- & 18 & --- \\
\hline-- & $\cdots$ & 1.7 & -- \\
\hline--- & --- & 185 & -- \\
\hline-- & --- & 20 & -- \\
\hline-- & -- & 41 & -- \\
\hline--- & -- & 0.3 & --- \\
\hline--- & -- & 286 & $-\cdots$ \\
\hline-- & --- & $<10$ & $\cdots$ \\
\hline-- & --- & 9.4 & -- \\
\hline
\end{tabular}

Jun -17

8.70
4.1
1.82
$0.5^{17.0}$

$0.5 \quad 17.0$

21.9

464
8.2

7.4

$9.2 \quad 0.6$

0.0110 .168

$\begin{array}{ll}0 .-- \\ --- & --\end{array}$

-

-n

‥

$\begin{array}{lll}--- & -1 \\ -\cdots & -\cdots\end{array}$

$\begin{array}{ll}--- & -- \\ --- & ---\end{array}$

-

$--$

$\cdots$

$--$

$--$

$--$

$--$

$-\cdots$

$\cdots$

\section{---}

---

$--$

$--$

$---$

$\cdots$

6-17-99

Jul -22

9.23
4.1
$0.5^{1.92} 17.0$

$\begin{array}{cc}0.5 & 17.0 \\ 25.5 & 8.6\end{array}$

$452 \quad 510$

8.27 .5

$8.5 \quad 0.0$

$0.010 \quad 0.251$

0.005

0.012

$<0.013$

0.52

0.53

-..-

-.

--

---

$--$

$---$

$-\cdots$

--

$---$

$---$
-.-

$--$

$--$

-..

-..

--

$--$

$---$

-..

$--$

$-\cdots$

$---$

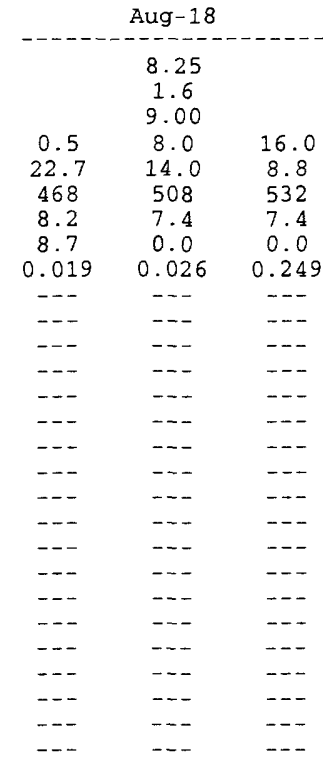

8-18-99

DISSOLVED OXYGEN (D.O.), IN MILLIGRAMS PER LITER
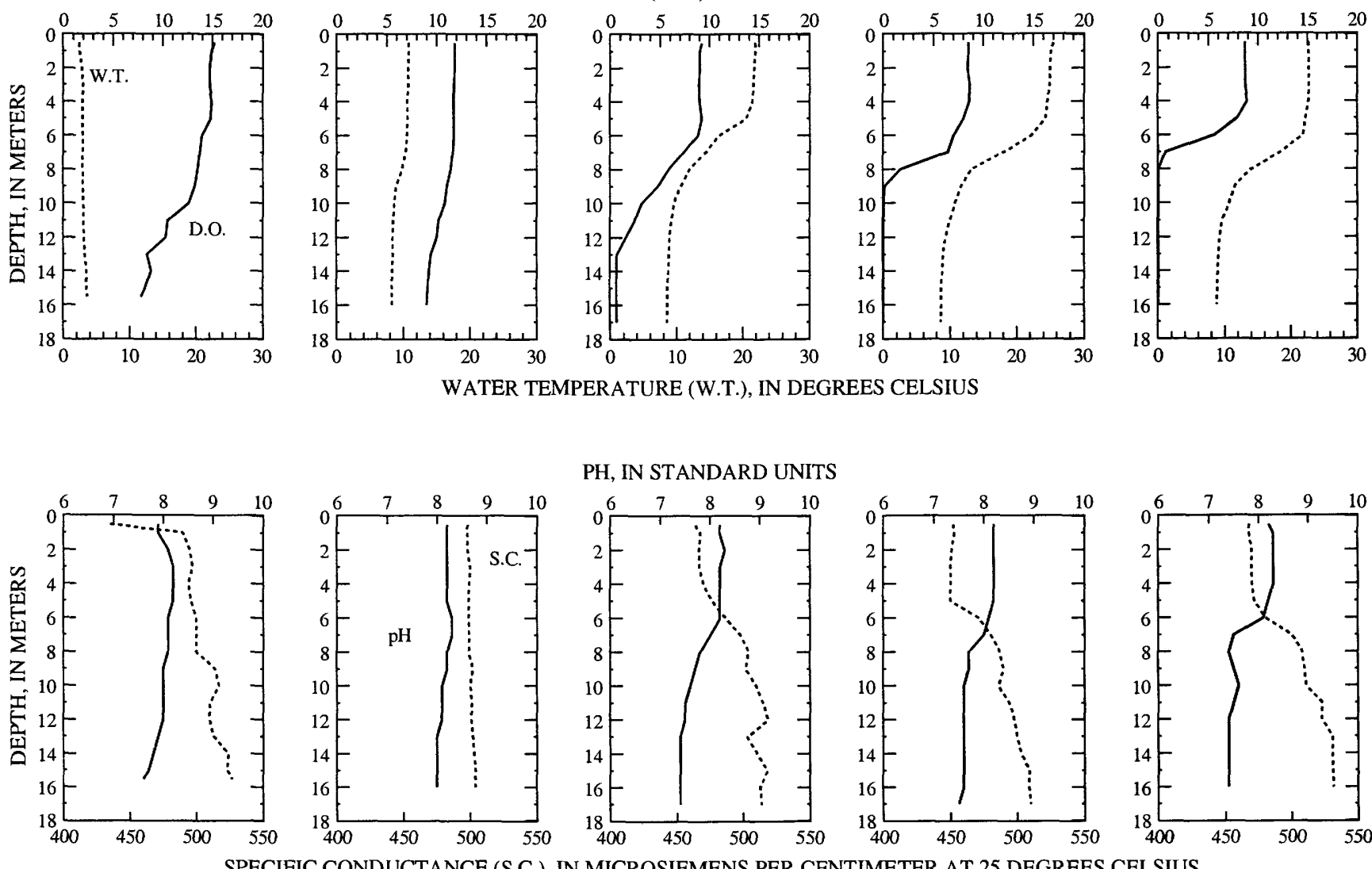

PH, IN STANDARD UNITS
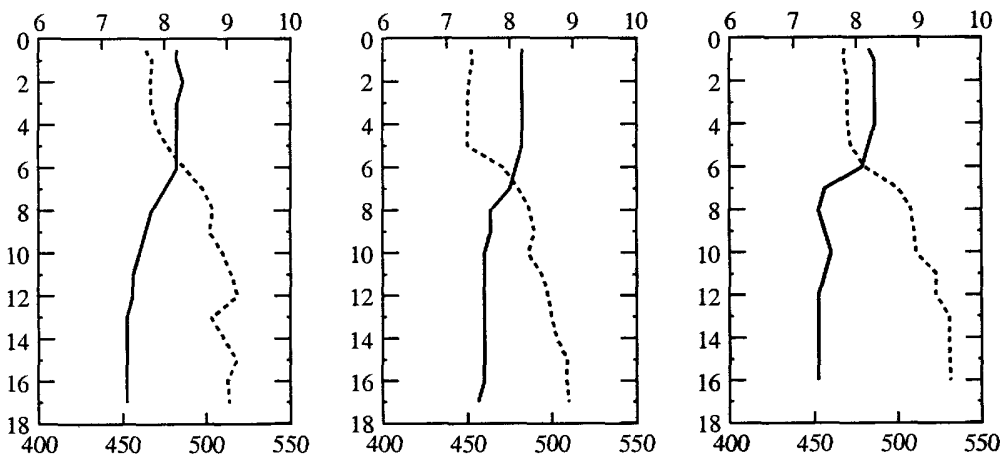

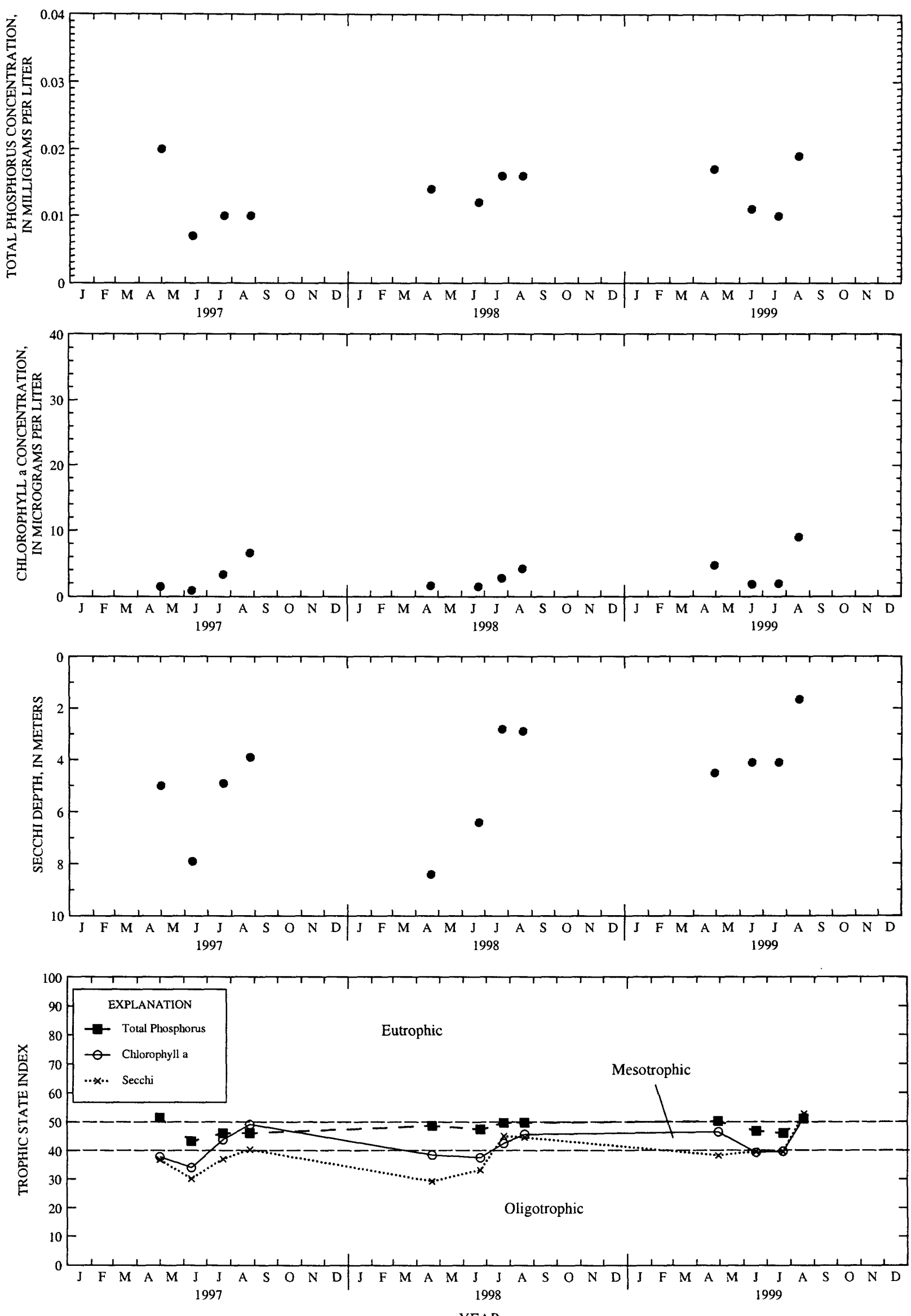

Surface total phosphorus, chlorophyll a concentrations, Secchi depths, and TSI data for Little Cedar Lake, South Site, near West Bend, Wisconsin. 


\section{LITTLE GREEN LAKE, AT CENTER, NEAR MARKESAN, WI}

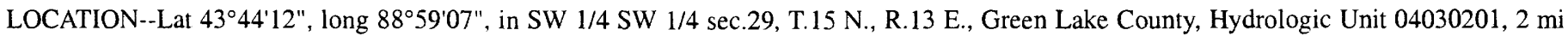
north of Markesan

PERIOD OF RECORD.--February 1991 to current year.

REMARKS.--Lake sampled near center at the deep hole. Lake ice-covered during February sampling. Water-quality analyses done by Wisconsin State Laboratory of Hygiene.

WATER-QUALITY DATA, FEBRUARY 23 TO JUNE 16, 1999

(Milligrams per liter unless otherwise indicated)

Lake stage $(f t)$

chioroohyli a, ohvtoolankton ( $\mu$ g/L)

Depth of sample $(m)$

water temoerature $1^{\circ} \mathrm{Cl}$

Specific conductance $(\mu \mathrm{S} / \mathrm{cm})$

$\mathrm{pH}$ (units)

Dissolved oxyaen (ma/L)

Phosphorus, total (as P)

Phosphorus, ortho, dissolved (as P)

Nitroqen, NO2 + NO3, diss. (as N)

Nitrogen, ammonia, dissolved (as $N$ )

Nitrogen, amm. + ora., total (as N)

Nitrogen, total (as $N$ )

Color (Pt-Co. scale)

Turbidity (NTU)

Hardness, (as $\mathrm{CaCO}_{3}$ )

Calcium, dissolved (Ca)

Magnesium, dissolved (Ma)

Sodium, dissolved (Na)

Potassium, dissolved (K)

Alkalinity, (as $\mathrm{CaCO}_{3}$ )

sulfate, dissolved (SO,

Chloride, dissolved (C1)

Silica, dissolved ( $\mathrm{SiO}_{2}$ )

Sollds, dissolved. at $180^{\circ} \mathrm{C}$

Iron, dissolved (Fe) $\mu \mathrm{q} / \mathrm{L}$

Manqanese, dissolved (Mn) $\mu \mathrm{q} / \mathrm{L}$

\begin{tabular}{|c|c|c|c|}
\hline & 23 & Ap & 28 \\
\hline & & & \\
\hline 0.5 & 7.5 & 0.5 & 7.5 \\
\hline 1.5 & 4.4 & 10.9 & 10.6 \\
\hline 320 & 423 & 324 & 327 \\
\hline 7.3 & 7.3 & 8.3 & 8.4 \\
\hline 19.4 & 0.9 & 12.0 & 11.3 \\
\hline 0.026 & 0.283 & 0.045 & 0.051 \\
\hline$\cdots$ & $\cdots$ & 0.003 & $-\cdots$ \\
\hline$-\cdots$ & $\cdots$ & $<0.010$ & \\
\hline-- & $\ldots$ & $<0.013$ & -- \\
\hline-- & -- & 1 & $-\ldots$ \\
\hline$-\cdots$ & $\cdots$ & 1.0 & -- \\
\hline$\cdots$ & -- & 10 & $\cdots$ \\
\hline-- & -- & 5.3 & $\ldots$ \\
\hline-- & $\ldots$ & 140 & $\ldots$ \\
\hline$\cdots$ & --- & 24 & $-\cdots$ \\
\hline-- & -- & 20 & $-\cdots$ \\
\hline$\cdots$ & -- & 7.3 & $\ldots$ \\
\hline$\cdots$ & $\ldots$ & 3.1 & -- \\
\hline$\cdots$ & -- & 132 & $\ldots$ \\
\hline$\cdots$ & $\cdots$ & 7.6 & $\ldots$ \\
\hline$\cdots$ & -- & 17 & -- \\
\hline$\cdots$ & $\ldots$ & 0.4 & $\cdots$ \\
\hline$-\cdots$ & -- & 186 & $\cdots$ \\
\hline- & $-\cdots$ & $<10$ & -- \\
\hline$\ldots$ & - & & \\
\hline
\end{tabular}

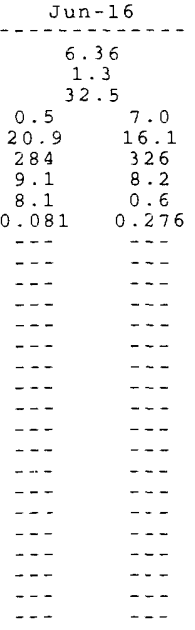

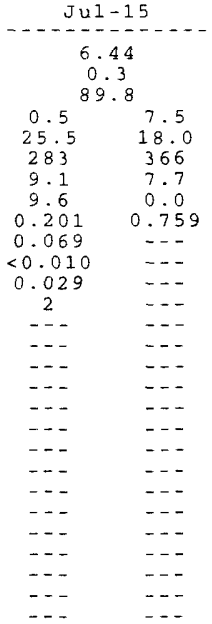

\begin{tabular}{|c|c|c|}
\hline & $\begin{array}{c}6.66 \\
0.3 \\
90.0\end{array}$ & \\
\hline $\begin{array}{c}0.5 \\
22.2 \\
271 \\
8.9 \\
6.8 \\
0.411\end{array}$ & $\begin{array}{c}4.0 \\
22.2 \\
274 \\
9.0 \\
6.7 \\
0.396\end{array}$ & $\begin{array}{c}7.5 \\
22.0 \\
273 \\
9.0 \\
5.6 \\
0.402\end{array}$ \\
\hline$-\ldots$ & $\ldots$ & ... \\
\hline-- & -- & -- \\
\hline--- & $\cdots$ & $\cdots$ \\
\hline$\cdots$ & $\cdots$ & -- \\
\hline$--\overline{-}$ & $\cdots$ & $\cdots$ \\
\hline$\cdots$ & $\ldots$ & - - - \\
\hline$-\cdots$ & -- & -- \\
\hline$\cdots$ & $\cdots$ & $\cdots$ \\
\hline--- & $-\bar{x}$ & $-\overline{-}$ \\
\hline - . - & $\ldots$ & $\ldots$ \\
\hline$-\ldots$ & $\cdots$ & -- \\
\hline$\cdots$ & -- & -- \\
\hline$\ldots$ & - & -1 \\
\hline$\cdots$ & -- & $\ldots$ \\
\hline--- & --- & -- \\
\hline
\end{tabular}

2-23-99

4-28-99

6-16-99

7-15-99

8-18-99

DISSOLVED OXYGEN (D.O.), IN MILLIGRAMS PER LITER
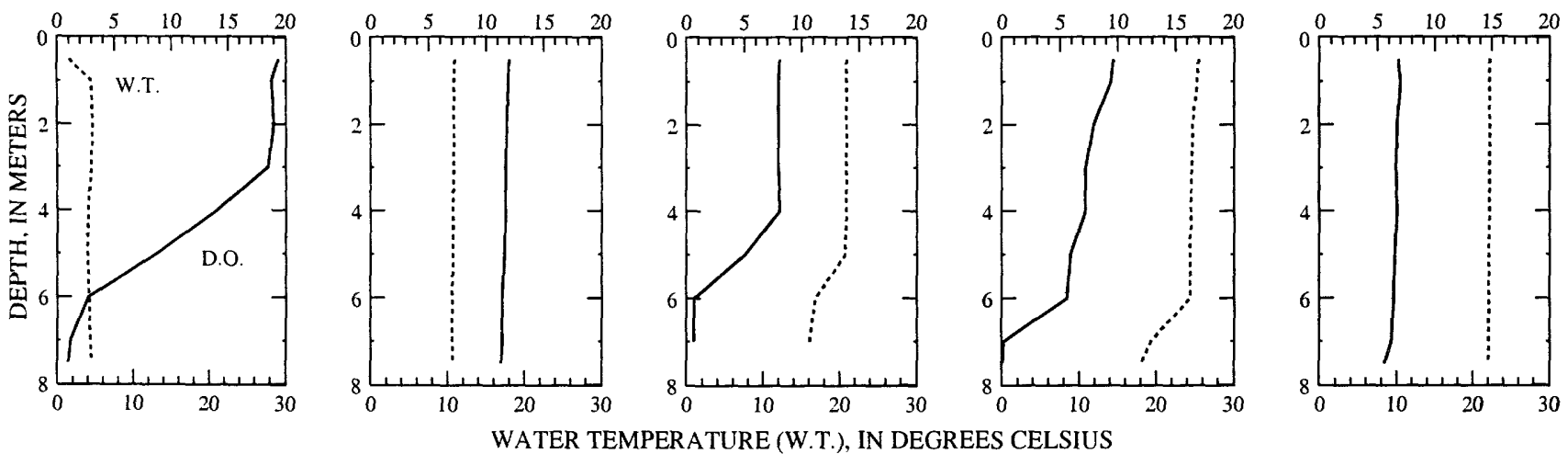

WATER TEMPERATURE (W.T.), IN DEGREES CELSIUS
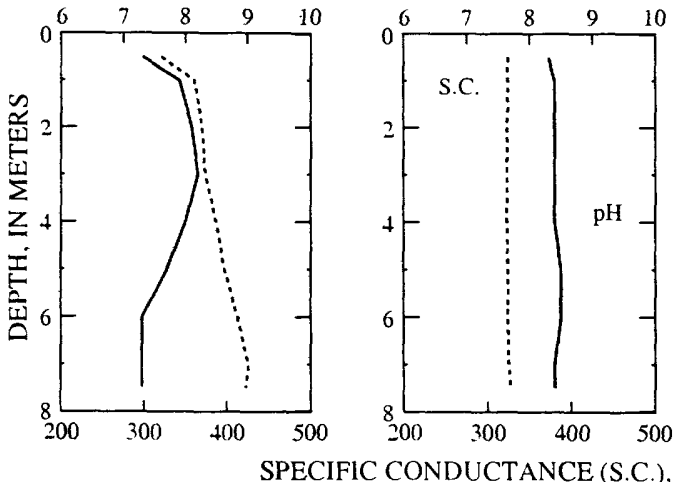

PH, IN STANDARD UNITS
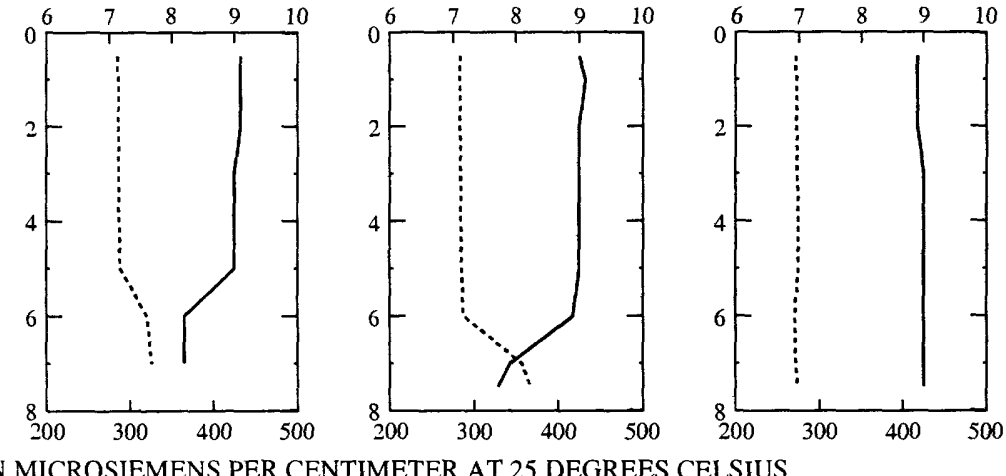

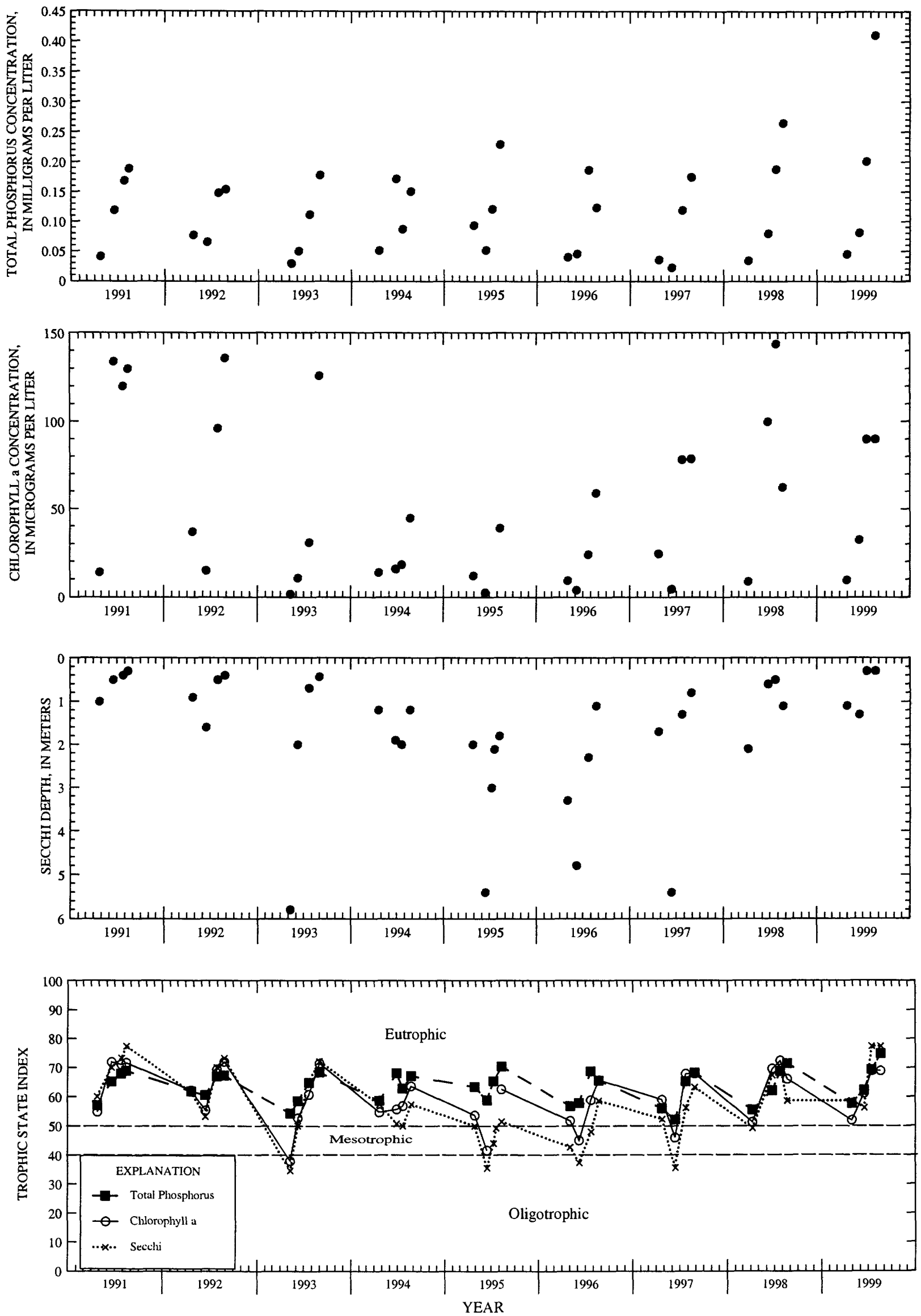

Surface total phosphorus, chlorophyll a concentrations, Secchi depths, and TSI data for Little Green Lake near Markesan, Wisconsin. 
LOCATION.--Lat $42^{\circ} 54^{\prime} 25^{\prime \prime}$, long $88^{\circ} 08^{\prime} 35^{\prime \prime}$, in SE 1/4 NW 1/4 sec.9, T.5 N., R.20 E., Waukesha County, Hydrologic Unit 07120006, at Muskego.

DRAINAGE AREA.-- $11.6 \mathrm{mi}^{2}$.

PERIOD OF RECORD.--October 1986 to current year.

LAKE-STAGE GAGE.--Datum of gage is $693.40 \mathrm{ft}$ above sea level.

REMARKS.--Lake sampled at the deep hole about 1,000 ft north-northwest of dam at outlet. Lake ice-covered during February sampling. An aeration system was operated from April to November for the years 1987-91. Water-quality analyses done by Wisconsin State Laboratory of Hygiene. Prior to October 1987, published under station number 425450088083500.

WATER-QUALITY DATA, FEBRUARY 11 TO JUNE 08, 1999

(Milligrams per liter unless otherwise indicated)

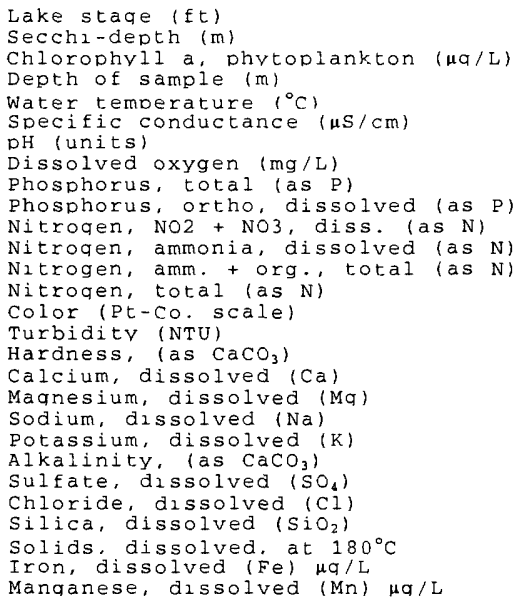

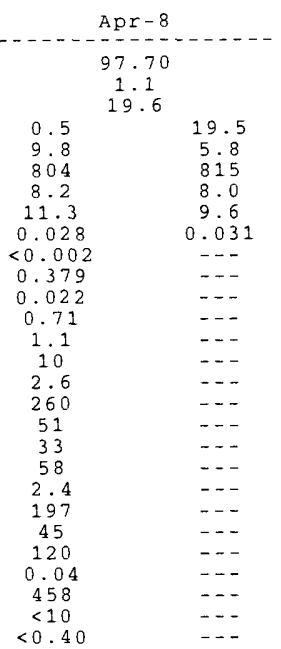

4-08-99

6-08-99

DISSOLVED OXYGEN (D.O.), IN MILLIGRAMS PER LITER
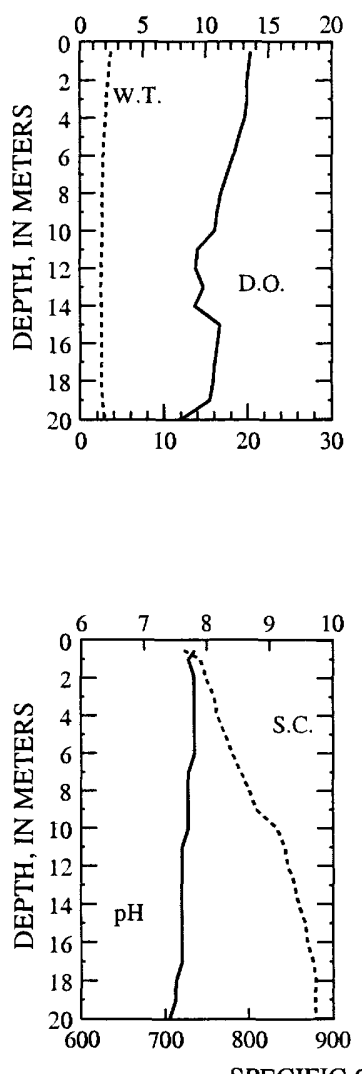
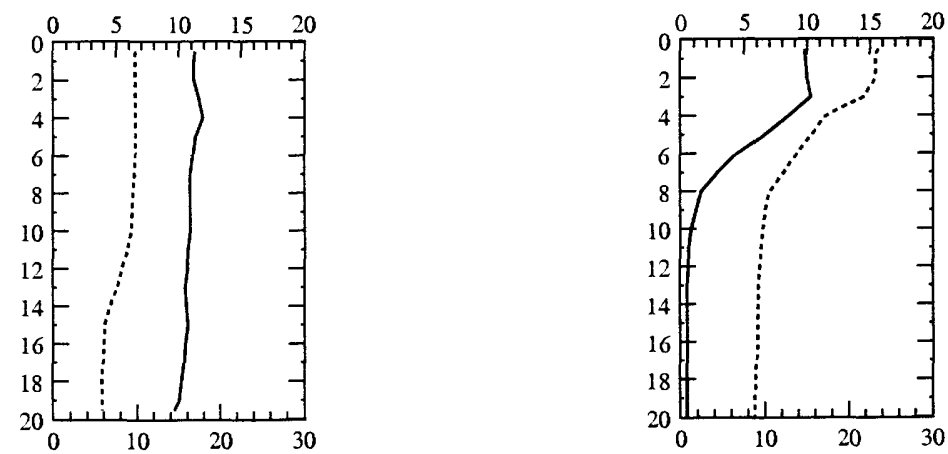

WATER TEMPERATURE (W.T.), IN DEGREES CELSIUS

PH, IN STANDARD UNITS
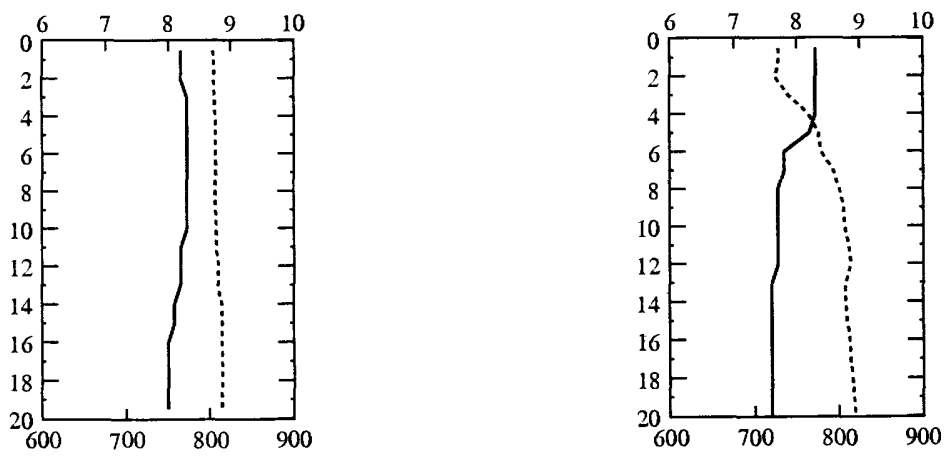

SPECIFIC CONDUCTANCE (S.C.), IN MICROSIEMENS PER CENTIMETER AT 25 DEGREES CELSIUS 
WATER-QUALITY DATA, JULY 06 TO AUGUST 05, 1999

(Milligrams per liter unless otherwise indicated)

Lake stage (ft)

Secchi-depth (m)

Chlorophyll a, phytoplankton $(\mu \mathrm{g} / \mathrm{L})$

Depth of sample (m)

Water temperature $\left({ }^{\circ} \mathrm{C}\right)$

specific conductance $(\mu \mathrm{s} / \mathrm{cm})$

$\mathrm{pH}$ (units)

Dissolved oxygen (mg/L)

Phosphorus, total (as P)
Jul-6

\begin{tabular}{cc}
\multicolumn{3}{c}{ Jul-6 } \\
\multicolumn{3}{c}{98.80} \\
0.9 \\
0.5 & 13.8 \\
26.9 & 17.5 \\
617 & 9.1 \\
8.4 & 823 \\
9.2 & 7.5 \\
0.022 & 0.4 \\
& 0.129
\end{tabular}

Aug - 5

\begin{tabular}{|c|c|c|c|c|}
\hline & & $\begin{array}{c}98.60 \\
1.1 \\
8.00\end{array}$ & & \\
\hline $\begin{array}{c}0.5 \\
26.1 \\
650 \\
8.2 \\
7.4 \\
0.031\end{array}$ & $\begin{array}{c}4.0 \\
24.4 \\
657 \\
8.1 \\
3.6 \\
0.027\end{array}$ & $\begin{array}{c}7.0 \\
15.3 \\
764 \\
7.4 \\
0.1 \\
0.019\end{array}$ & $\begin{array}{c}18.0 \\
9.1 \\
801 \\
7.4 \\
0.0 \\
0.233\end{array}$ & $\begin{array}{c}20.0 \\
9.0 \\
805 \\
7.3 \\
0.0 \\
0.277\end{array}$ \\
\hline
\end{tabular}

7-06-99

8-05-99

DISSOLVED OXYGEN (D.O.), IN MILLIGRAMS PER LITER
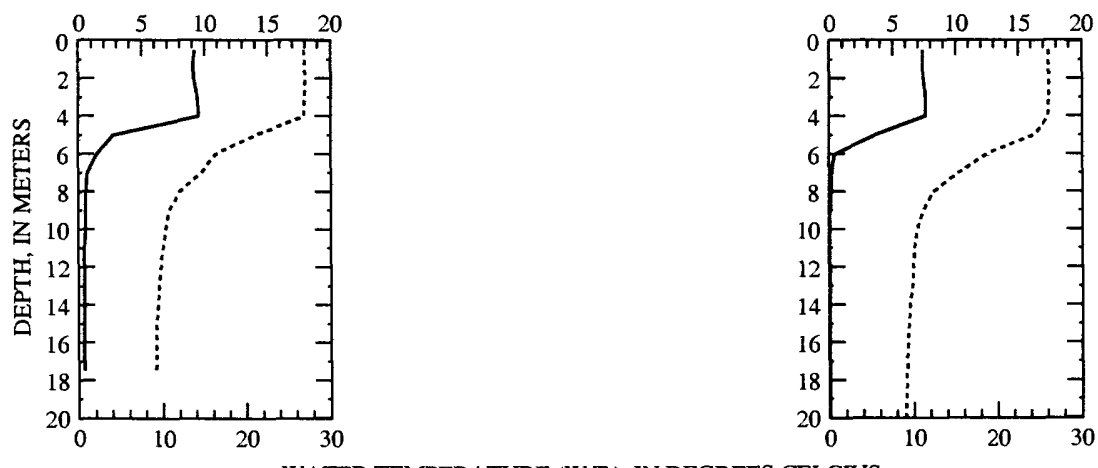

WATER TEMPERATURE (W.T.), IN DEGREES CELCIUS

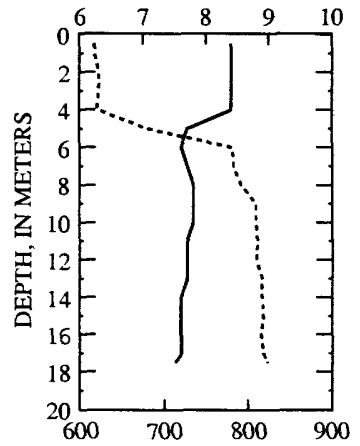

PH, IN STANDARD UNITS

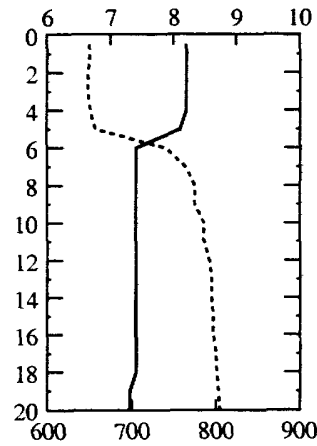

SPECIFIC CONDUCTANCE (S.C.), IN MICROSIEMENS PER CENTIMETER AT 25 DEGREES CELCIUS 

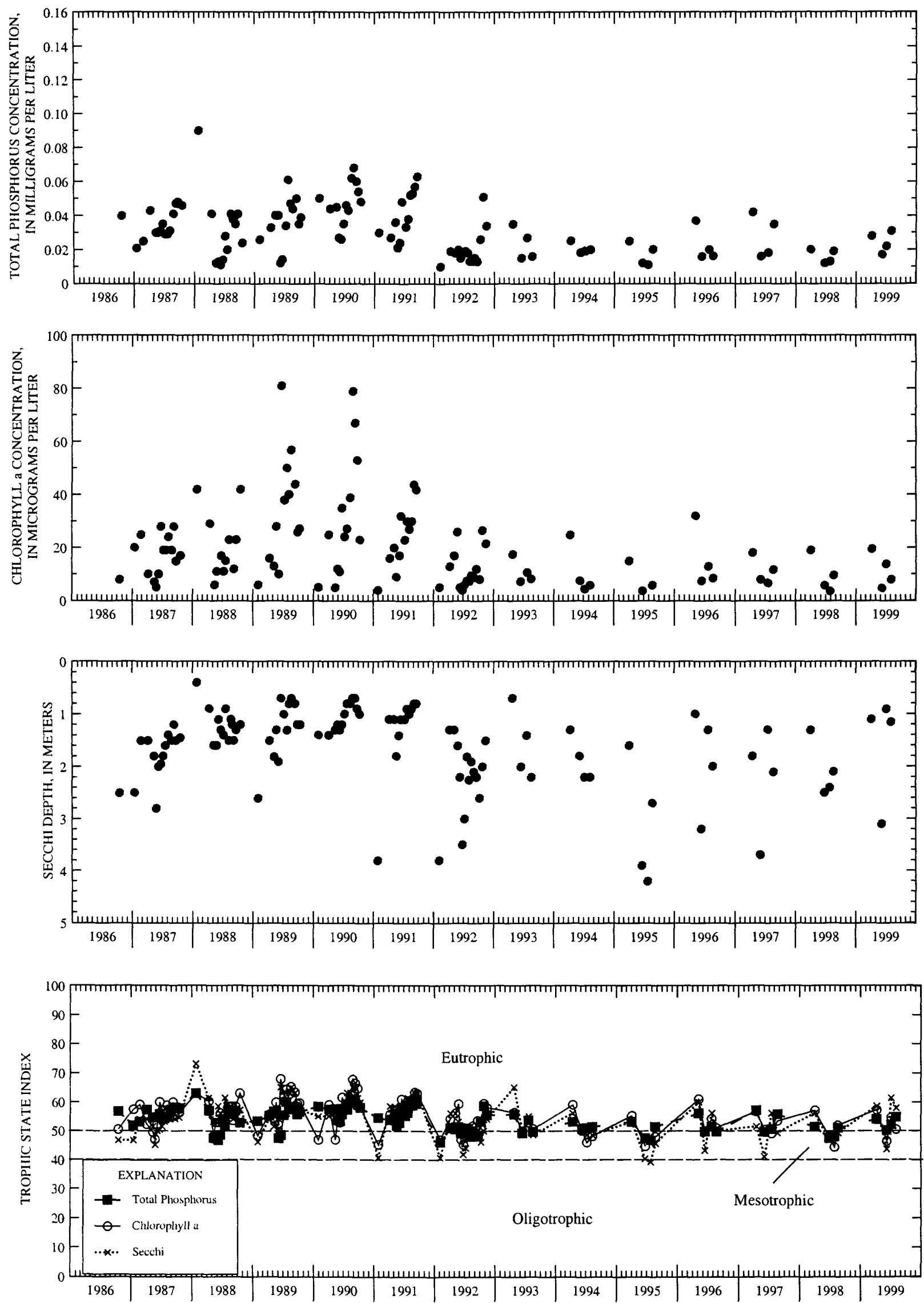

YEAR

Surface total phosphorus, chlorophyll a concentrations, Secchi depths, and TSI data for Little Muskego Lake at Muskego, Wisconsin. 
LOCATION--Lat $45^{\circ} 53^{\prime} 55^{\prime \prime}$, long 89 27'10", in SW 1/4 SE 1/4 sec.35, T.40 N., R.8 E., Vilas County, Hydrologic Unit 07070001, 9.6 mi west of Eagle River.

DRAINAGE AREA.--19.0 $\mathrm{mi}^{2}$.

PERIOD OF RECORD.--October 1990 to current year.

GAGE.--Staff gage mounted on the dam wall at lake outlet. Datum of gage is $1,600 \mathrm{ft}$, above sea level.

REMARKS.--Lake level regulated by dam at outlet.

COOPERATION.--Gage readings furnished by Wisconsin Valley Improvement Company.

EXTREMES FOR PERIOD OF RECORD.--Maximum gage height observed, $14.00 \mathrm{ft}$, June 6, 1997; minimum observed, $12.00 \mathrm{ft}$, Jan. 3 and

Feb. 3, 1992.

EXTREMES FOR CURRENT YEAR.--Maximum gage height observed, 13.88, July 9; minimum observed, 12.02 ft, Feb. 9.

GAGE HEIGHT, FEET, WATER YEAR OCTOBER 1998 TO SEPTEMBER 1999.

DAILY MEAN VALUE

\begin{tabular}{|c|c|c|c|c|c|c|c|c|c|c|c|c|}
\hline DAY & OCT & NOV & DEC & JAN & FEB & MAR & APR & MAY & JUN & JUL & AUG & SEP \\
\hline $\begin{array}{l}1 \\
2 \\
3 \\
4 \\
5\end{array}$ & $\begin{array}{l}13.06 \\
13.04 \\
13.06 \\
13.06 \\
13.04\end{array}$ & $\begin{array}{l}13.00 \\
12.94 \\
12.94 \\
12.94 \\
12.92\end{array}$ & $\begin{array}{r}12.62 \\
- \\
12.58 \\
-\end{array}$ & $\begin{array}{r}12.26 \\
--- \\
12.24 \\
12.22\end{array}$ & $\begin{array}{r}--- \\
--- \\
--- \\
12.10\end{array}$ & $\begin{array}{r}12.40 \\
12.36 \\
--- \\
12.32\end{array}$ & $\begin{array}{l}12.52 \\
12.56 \\
12.58 \\
12.62 \\
12.66\end{array}$ & $\begin{array}{l}12.84 \\
12.84 \\
12.82 \\
12.82 \\
12.80\end{array}$ & $\begin{array}{l}13.76 \\
13.76 \\
13.74 \\
13.72 \\
13.72\end{array}$ & $\begin{array}{l}13.56 \\
13.56 \\
13.56 \\
13.64 \\
13.64\end{array}$ & $\begin{array}{l}13.78 \\
13.74 \\
13.72 \\
13.72 \\
13.72\end{array}$ & $\begin{array}{l}13.64 \\
13.62 \\
13.60 \\
13.60 \\
13.62\end{array}$ \\
\hline $\begin{array}{r}6 \\
7 \\
8 \\
9 \\
10\end{array}$ & $\begin{array}{l}13.08 \\
13.10 \\
13.12 \\
13.12 \\
13.14\end{array}$ & $\begin{array}{r}12.90 \\
12.88 \\
\ldots \\
12.98\end{array}$ & $\begin{array}{r}--- \\
12.48 \\
--- \\
---\end{array}$ & $\begin{array}{r}--- \\
12.22 \\
--- \\
---\end{array}$ & $\begin{array}{r}--- \\
--- \\
--0 \\
-\end{array}$ & $\begin{array}{r}--- \\
12.38 \\
---\end{array}$ & $\begin{array}{l}12.74 \\
12.76 \\
12.76 \\
12.80 \\
12.80\end{array}$ & $\begin{array}{l}12.98 \\
12.98 \\
13.16 \\
13.34 \\
13.38\end{array}$ & $\begin{array}{l}13.86 \\
13.82 \\
13.80 \\
13.78 \\
13.78\end{array}$ & $\begin{array}{l}13.70 \\
13.68 \\
13.66 \\
13.88 \\
13.84\end{array}$ & $\begin{array}{l}13.72 \\
13.70 \\
13.70 \\
13.68 \\
13.70\end{array}$ & $\begin{array}{l}13.60 \\
13.60 \\
13.62 \\
13.60 \\
13.58\end{array}$ \\
\hline $\begin{array}{l}11 \\
12 \\
13 \\
14 \\
15\end{array}$ & $\begin{array}{l}13.14 \\
13.16 \\
13.16 \\
13.16 \\
13.18\end{array}$ & $\begin{array}{r}12.88 \\
12.84 \\
---\end{array}$ & $\begin{array}{r}12.40 \\
- \\
12.30\end{array}$ & $\begin{array}{r}12 .-\overline{18} \\
--- \\
---\end{array}$ & $\begin{array}{r}12 .-\overline{18} \\
--- \\
---\end{array}$ & $\begin{array}{r}--- \\
12.40 \\
--- \\
---\end{array}$ & $\begin{array}{l}12.80 \\
12.80 \\
12.80 \\
12.82 \\
12.84\end{array}$ & $\begin{array}{l}13.40 \\
13.40 \\
13.38 \\
13.38 \\
13.36\end{array}$ & $\begin{array}{l}13.76 \\
13.78 \\
13.72 \\
13.72 \\
13.70\end{array}$ & $\begin{array}{l}13.80 \\
13.78 \\
13.74 \\
13.72 \\
13.70\end{array}$ & $\begin{array}{l}13.68 \\
13.68 \\
13.80 \\
13.80 \\
13.78\end{array}$ & $\begin{array}{l}13.60 \\
13.62 \\
13.62 \\
13.60 \\
13.60\end{array}$ \\
\hline $\begin{array}{l}16 \\
17 \\
18 \\
19 \\
20\end{array}$ & $\begin{array}{l}13.18 \\
13.20 \\
13.22 \\
13.26 \\
13.24\end{array}$ & $\begin{array}{r}12.86 \\
12.94 \\
-\end{array}$ & 12.20 & 12.14 & $\begin{array}{r}12.28 \\
- \\
12.32 \\
---\end{array}$ & $\begin{array}{r}12.40 \\
--- \\
12.45 \\
\end{array}$ & $\begin{array}{l}12.86 \\
12.88 \\
12.86 \\
12.86 \\
12.86\end{array}$ & $\begin{array}{l}13.36 \\
13.44 \\
13.56 \\
13.56 \\
13.56\end{array}$ & $\begin{array}{l}13.68 \\
13.66 \\
13.64 \\
13.62 \\
13.62\end{array}$ & $\begin{array}{l}13.68 \\
13.70 \\
13.70 \\
13.70 \\
13.68\end{array}$ & $\begin{array}{l}13.76 \\
13.74 \\
13.70 \\
13.72 \\
13.72\end{array}$ & $\begin{array}{l}13.60 \\
13.58 \\
13.58 \\
13.58 \\
13.62\end{array}$ \\
\hline $\begin{array}{l}21 \\
22 \\
23 \\
24 \\
25\end{array}$ & $\begin{array}{l}13.24 \\
13.22 \\
13.22 \\
13.20 \\
13.18\end{array}$ & $\begin{array}{r}12.84 \\
12.82 \\
-\end{array}$ & $\begin{array}{r}12.14 \\
- \\
12.10 \\
-\end{array}$ & $\begin{array}{r}--- \\
12.10 \\
--- \\
--- \\
---\end{array}$ & $\begin{array}{r}--- \\
--\overline{-} \\
12.34 \\
----\end{array}$ & $\begin{array}{l}12.48 \\
12.46 \\
12.48 \\
12.46 \\
12.46\end{array}$ & $\begin{array}{l}12.88 \\
12.90 \\
12.90 \\
12.86 \\
12.86\end{array}$ & $\begin{array}{l}13.68 \\
13.72 \\
13.82 \\
13.80 \\
13.78\end{array}$ & $\begin{array}{l}13.62 \\
13.60 \\
13.62 \\
13.62 \\
13.60\end{array}$ & $\begin{array}{l}13.70 \\
13.68 \\
13.70 \\
13.70 \\
13.68\end{array}$ & $\begin{array}{l}13.72 \\
13.72 \\
13.72 \\
13.72 \\
13.72\end{array}$ & $\begin{array}{l}13.60 \\
13.60 \\
13.60 \\
13.58 \\
13.58\end{array}$ \\
\hline $\begin{array}{l}26 \\
27 \\
28 \\
29 \\
30 \\
31\end{array}$ & $\begin{array}{l}13.18 \\
13.14 \\
13.10 \\
13.08 \\
13.06 \\
13.02\end{array}$ & $\begin{array}{r}12.80 \\
12.70 \\
- \\
-\end{array}$ & 12.30 & $\begin{array}{r}12.12 \\
-. .4 \\
12.14 \\
12.10\end{array}$ & $\begin{array}{r}12.28 \\
--- \\
--- \\
--- \\
---\end{array}$ & $\begin{array}{l}12.48 \\
12.48 \\
12.48 \\
12.50 \\
12.50 \\
12.52\end{array}$ & $\begin{array}{r}12.86 \\
12.86 \\
12.86 \\
12.84 \\
12.84 \\
\end{array}$ & $\begin{array}{l}13.70 \\
13.74 \\
13.74 \\
13.76 \\
13.74 \\
13.72\end{array}$ & $\begin{array}{r}13.60 \\
13.60 \\
13.60 \\
13.58 \\
13.56 \\
---\end{array}$ & $\begin{array}{l}13.76 \\
13.76 \\
13.74 \\
13.76 \\
13.76 \\
13.84\end{array}$ & $\begin{array}{l}13.72 \\
13.72 \\
13.70 \\
13.68 \\
13.66 \\
13.64\end{array}$ & $\begin{array}{l}13.54 \\
13.56 \\
13.58 \\
13.58 \\
13.56 \\
---\end{array}$ \\
\hline $\begin{array}{l}\text { MEAN } \\
\text { MAX } \\
\text { MIN }\end{array}$ & $\begin{array}{l}13.14 \\
13.26 \\
13.02\end{array}$ & 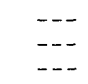 & $\begin{array}{l}--- \\
\cdots- \\
---\end{array}$ & $\begin{array}{l}--- \\
--- \\
---\end{array}$ & $m$ & --- & $\begin{array}{l}12.79 \\
12.90 \\
12.52\end{array}$ & $\begin{array}{l}13.41 \\
13.82 \\
12.80\end{array}$ & $\begin{array}{l}13.69 \\
13.86 \\
13.56\end{array}$ & $\begin{array}{l}13.71 \\
13.88 \\
13.56\end{array}$ & $\begin{array}{l}13.72 \\
13.80 \\
13.64\end{array}$ & $\begin{array}{l}13.60 \\
13.64 \\
13.54\end{array}$ \\
\hline
\end{tabular}

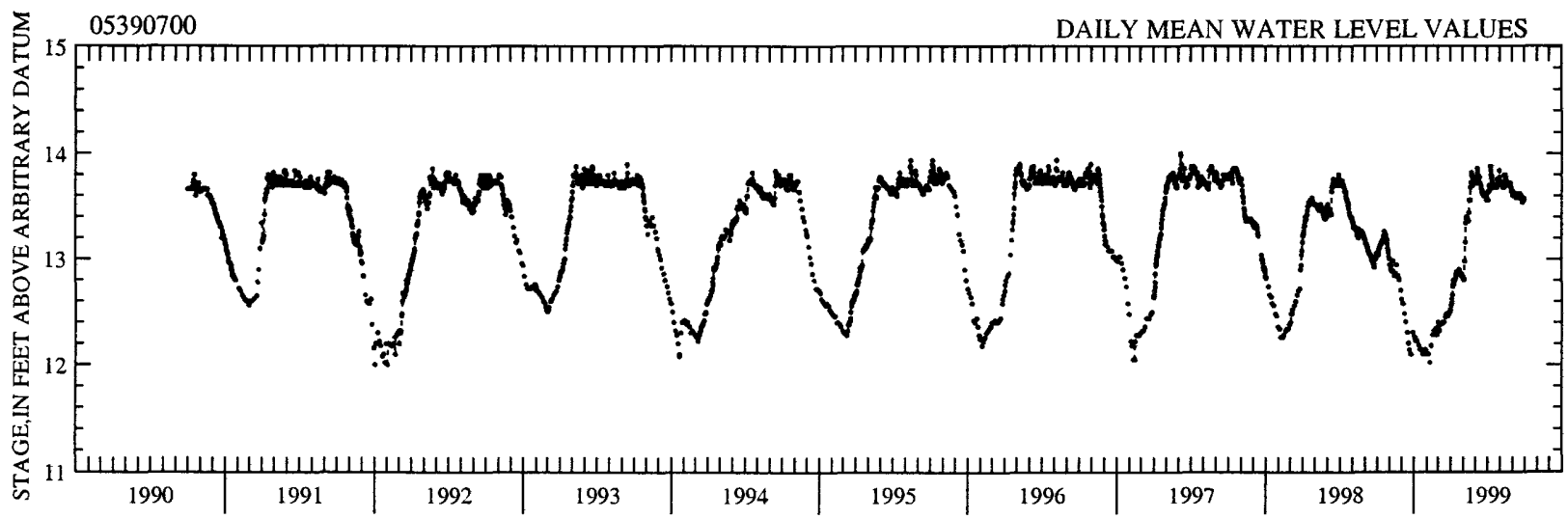




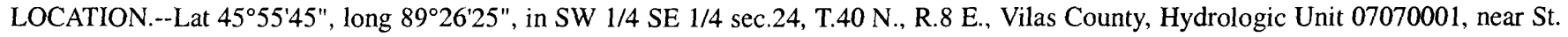
Germain.

PERIOD OF RECORD.--April 1991 to August 1994, August 1996 to August 1997, March to October 1999.

REMARKS.--Lake sampled in northeast bay at a lake depth of about $4 \mathrm{~m}$. Lake ice-covered during March sampling. Water-quality analyses by Wisconsin State Laboratory of Hygiene.

WATER-QUALITY DATA, MARCH 18 TO OCTOBER 06, 1999

(Milligrams per liter unless otherwise indicated)

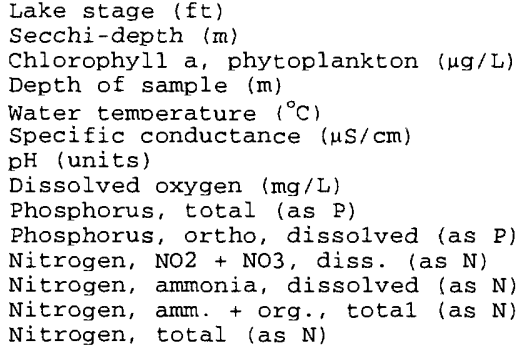

\begin{tabular}{|c|c|c|c|c|c|c|c|}
\hline \multicolumn{2}{|c|}{ Mar-18 } & May-13 & Jun-15 & \multicolumn{2}{|c|}{ Jul-22 } & \multicolumn{2}{|c|}{ Oct-06 } \\
\hline \multicolumn{2}{|c|}{$\begin{array}{l}-- \\
-- \\
--\end{array}$} & $\begin{array}{c}13.38 \\
1.7 \\
4.1\end{array}$ & $\begin{array}{c}13.70 \\
1.4 \\
8.89\end{array}$ & \multicolumn{2}{|c|}{$\begin{array}{c}13.68 \\
0.8 \\
31.1\end{array}$} & \multicolumn{2}{|c|}{$\begin{array}{c}13.50 \\
1.6 \\
15.0\end{array}$} \\
\hline 0.5 & 2.5 & 0.5 & 0.5 & 0.5 & 3.0 & 0.5 & 3.3 \\
\hline 0.3 & 3.6 & 14.7 & 20.3 & 25.4 & 23.6 & 10.6 & 9.8 \\
\hline 98 & 93 & 74 & 75 & 79 & 72 & 7.9 & 7.7 \\
\hline 7.3 & 6.7 & 7.4 & 7.2 & 9.3 & 8.3 & 79.0 & 79.0 \\
\hline 7.2 & 0.8 & 9.5 & 7.6 & 12.5 & 8.4 & 10.2 & 8.9 \\
\hline 0.054 & 0.034 & 0.030 & 0.029 & 0.033 & 0.039 & 0.060 & 0.052 \\
\hline$\ldots$ & $\ldots$ & -- & - - & 0.003 & --- & --- & -- \\
\hline$\ldots$ & - - & --- & -- & 0.154 & -- & --- & --- \\
\hline--- & --- & -- & -- & 0.062 & --- & --- & $-\cdots$ \\
\hline--- & - - & $\ldots$ & -- & 1.1 & $\cdots$ & --- & $\ldots$ \\
\hline--- & --- & --- & --- & 1.3 & --- & --- & $\cdots$ \\
\hline
\end{tabular}

DISSOLVED OXYGEN (D.O.), IN MILLIGRAMS PER LITER
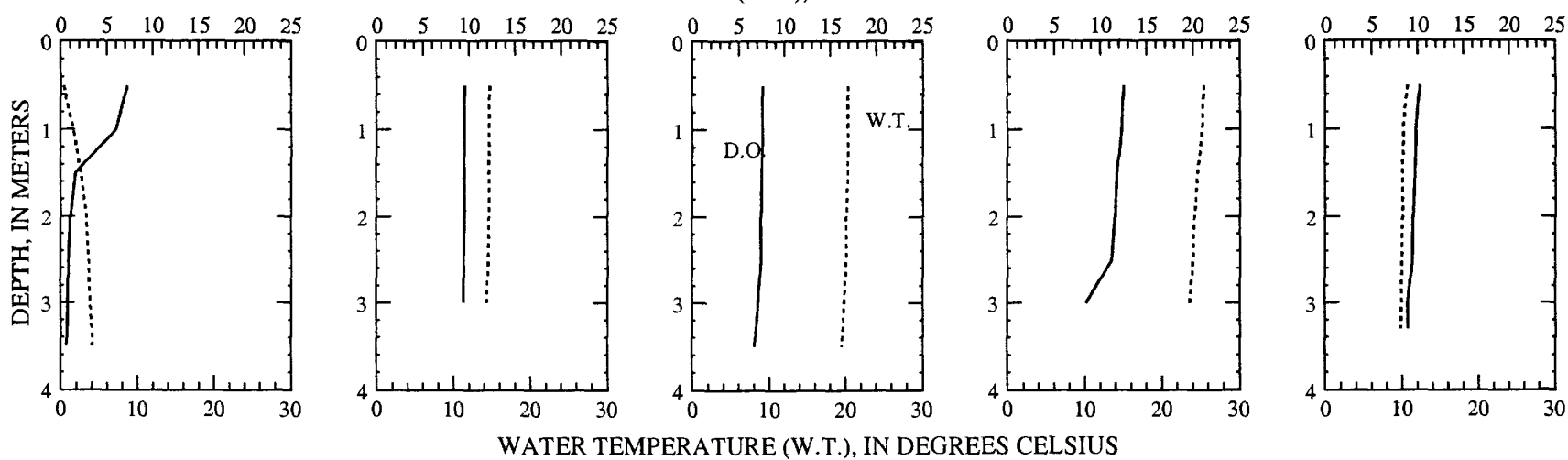

WATER TEMPERATURE (W.T.), IN DEGREES CELSIUS
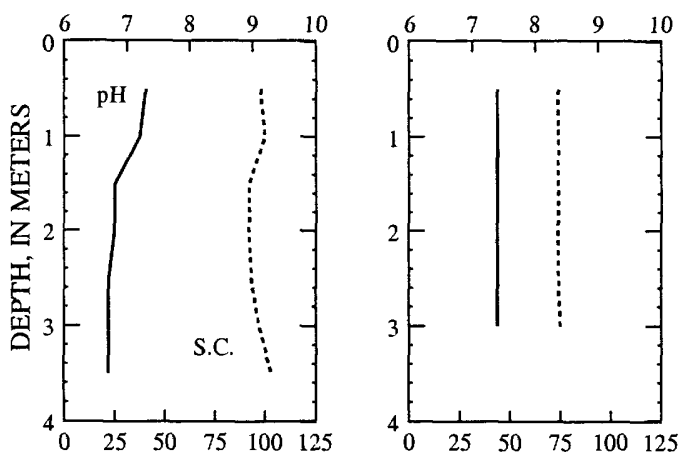

PH, IN STANDARD UNITS
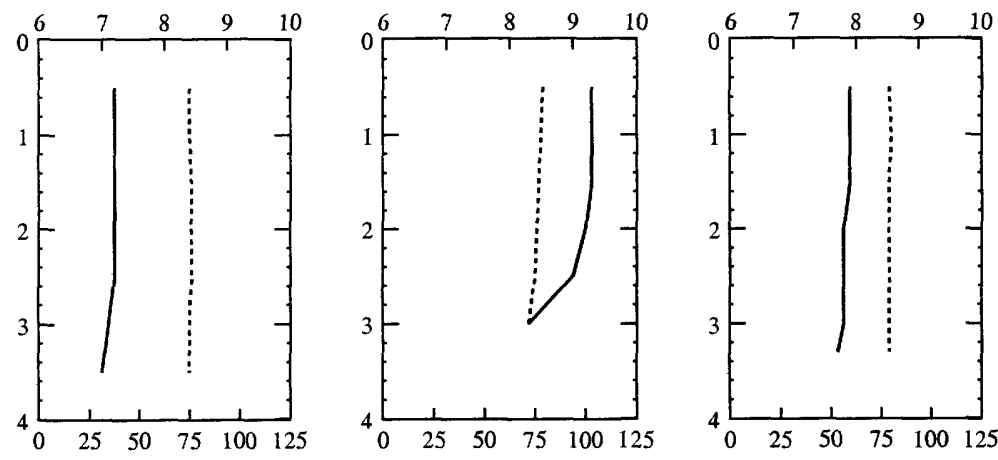

SPECIFIC CONDUCTANCE (S.C.), IN MICROSIEMENS PER CENTIMETER AT 25 DEGREES CELSIUS 

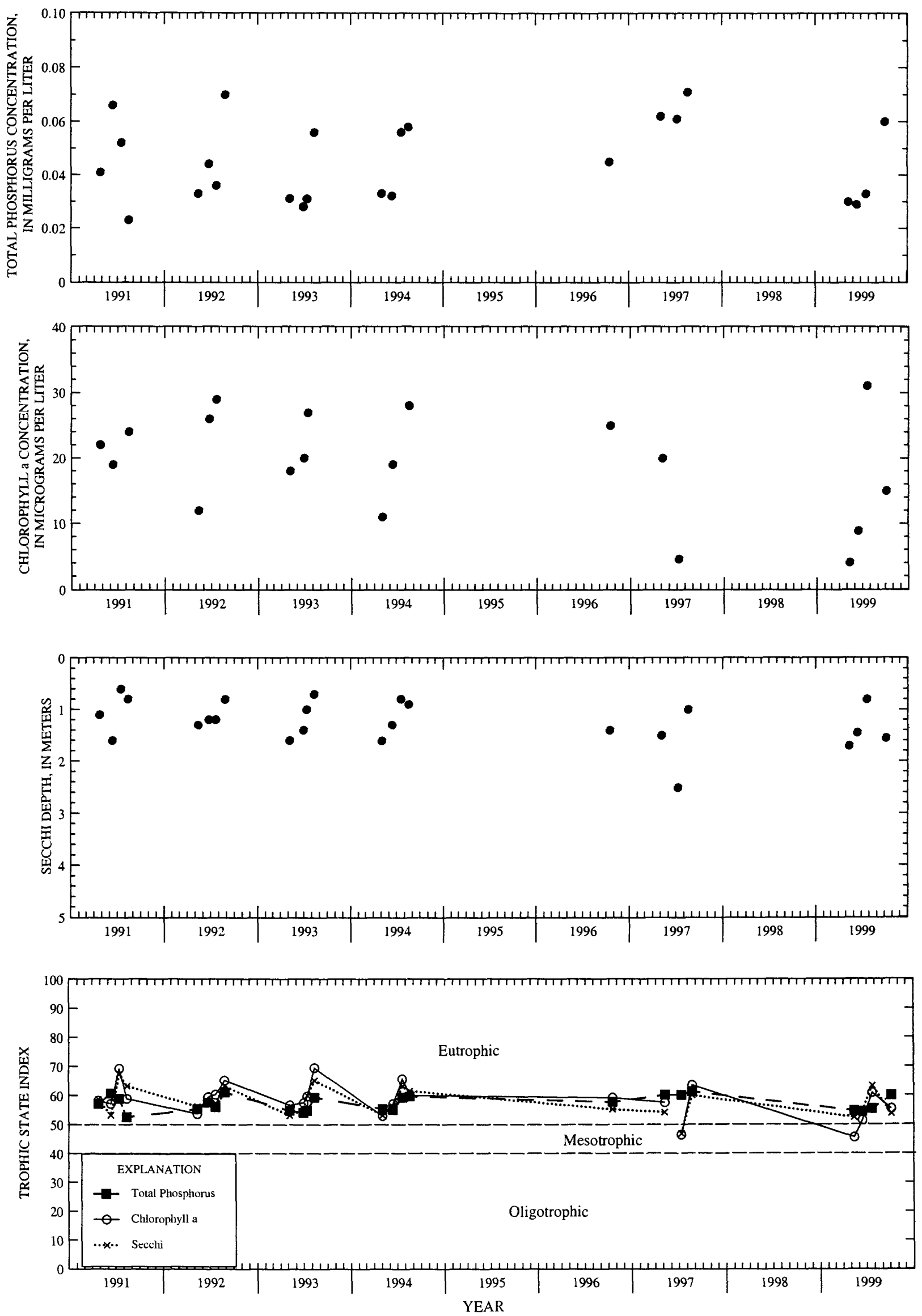

Surface total phosphorus, chlorophyll a concentrations, Secchi depths, and TSI data for Little St. Germain Lake, NE Bay, at St. Germain, Wisconsin. 
LOCATION.--Lat $45^{\circ} 54^{\prime} 37^{\prime \prime}$, long $89^{\circ} 27^{\prime} 08^{\prime \prime}$, in NW 1/4 NE 1/4 sec.35, T.40 N., R.8 E., Vilas County, Hydrologic Unit 07070001, $1.7 \mathrm{mi}$ east of St. Germain.

PERIOD OF RECORD.--April 1991 to August 1994, August 1996 to August 1997, March to October 1999.

REMARKS.--Lake sampled in south bay at a lake depth of about $7 \mathrm{~m}$. Lake ice-covered during March sampling. Water-quality analyses by Wisconsin State Laboratory of Hygiene.

WATER-QUALITY DATA, MARCH 18 TO OCTOBER 06, 1999

(Milligrams per liter unless otherwise indicated)

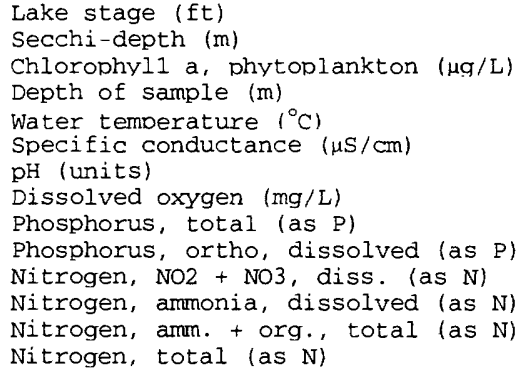

3-18-99

5-13-99

$\begin{array}{cccc}\text { Mar-18 } & \text { May-13 } & \text { Jun-15 } \\ & & 13.38 & 13.70 \\ & & 2.1 & 2.5 \\ & & 4.99 & 9.30 \\ 0.5 & 5.5 & 0.5 & 0.5 \\ 0.4 & 5.0 & 14.5 & 20.0 \\ 99 & 149 & 77 & 73 \\ 6.6 & 6.9 & 7.9 & 8.0 \\ 1.8 & 0.5 & 10.4 & 8.8 \\ 0.029 & 0.045 & 0.027 & 0.028 \\ --- & --- & --- & --- \\ --- & --- & --- & --- \\ \cdots- & --- & --- & --- \\ \cdots- & --- & --- & --- \\ --- & --- & --- & --\end{array}$

6-15-99

\begin{tabular}{cccc} 
Jul-22 & \multicolumn{2}{c}{ Oct-06 } \\
\hline \multicolumn{2}{c}{13.68} & \multicolumn{2}{c}{13.50} \\
2.4 & \multicolumn{2}{c}{2.8} \\
\multicolumn{2}{c}{7.26} & \multicolumn{2}{c}{5.00} \\
0.5 & 5.5 & 0.5 & 5.8 \\
25.7 & 19.2 & 11.1 & 10.5 \\
71 & 72 & 79 & 78 \\
8.7 & 6.7 & 8.1 & 8.0 \\
9.7 & 5.5 & 10.5 & 9.8 \\
0.021 & 0.092 & 0.022 & 0.026 \\
0.002 & --- & --- & -- \\
0.029 & --- & --- & --- \\
0.012 & --- & --- & -- \\
0.57 & -- & -- & -- \\
0.60 &.-- & -- & --
\end{tabular}

7-22-99
10-06-99
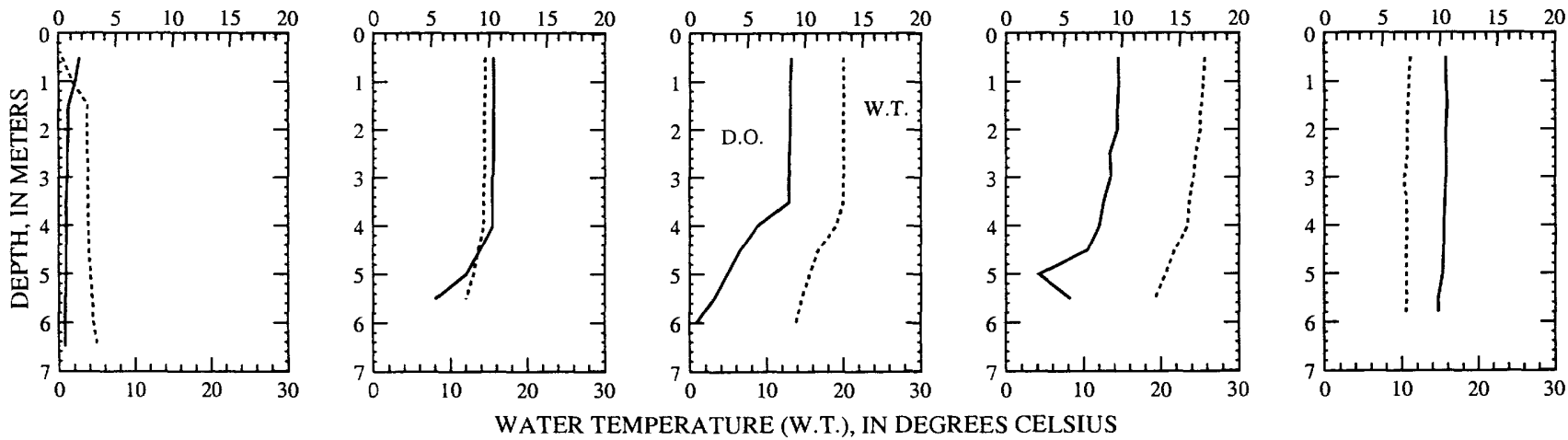

WATER TEMPERATURE (W.T.), IN DEGREES CELSIUS
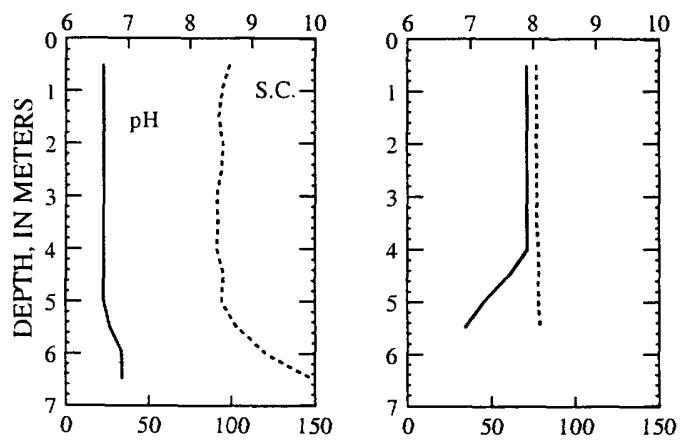

PH, IN STANDARD UNITS
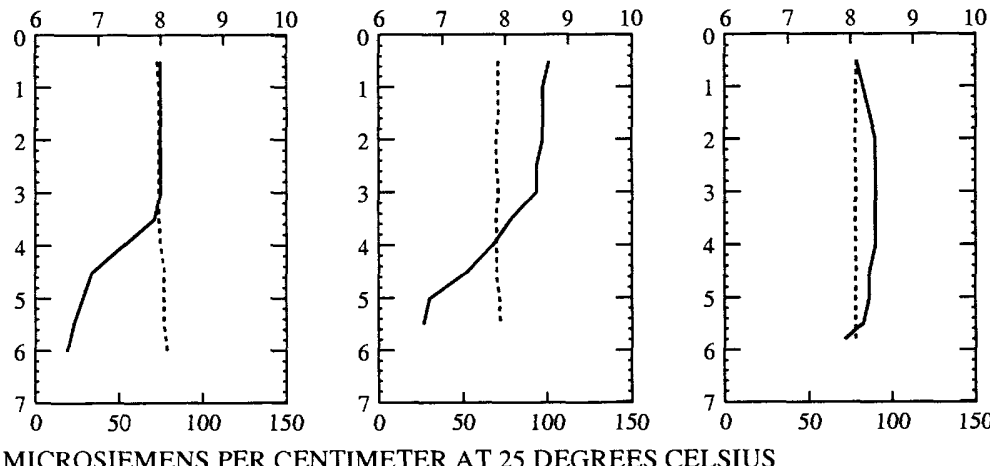

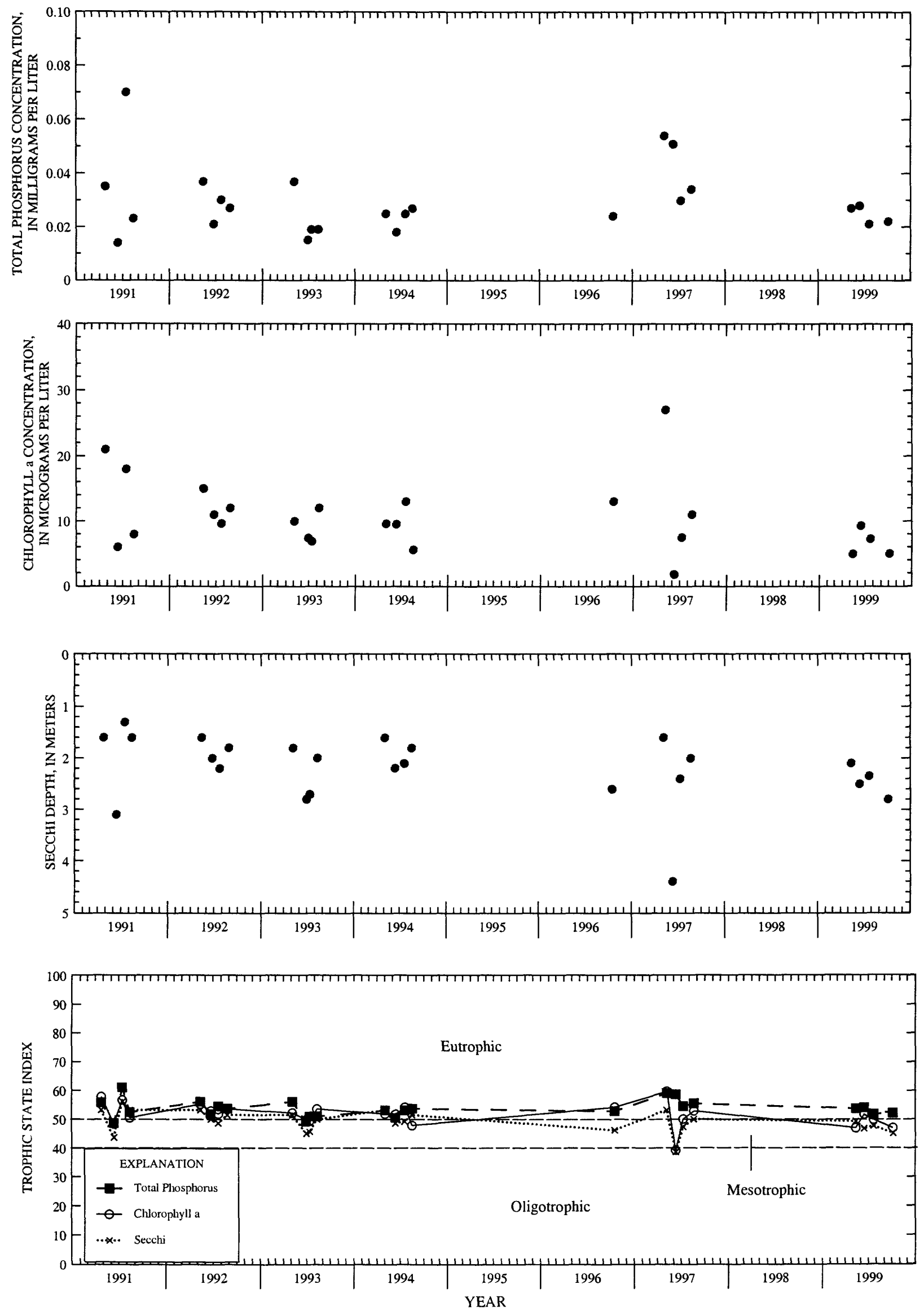

Surface total phosphorus, chlorophyll a concentrations, Secchi depths, and TSI data for Little St. Germain Lake, South Bay, at St. Germain, Wisconsin. 
LOCATION.--Lat $45^{\circ} 54^{\prime} 28^{\prime \prime}$, long $89^{\circ} 28^{\prime} 24^{\prime \prime}$, in SW 1/4 NE 1/4 sec.34, T.40 N., R.8 E., Vilas County, Hydrologic Unit 0707000I, at St. Germain.

PERIOD OF RECORD.--April 1991 to August 1994, August 1996 to August 1997, March to October 1999.

REMARKS.--Lake sampled in west bay at a lake depth of about $18 \mathrm{~m}$. Lake ice-covered during March sampling. Water-quality analyses by Wisconsin State Laboratory of Hygiene.

WATER-QUALITY DATA, MARCH 18 TO OCTOBER 06, 1999

(Milligrams per liter unless otherwise indicated)

Lake stage (ft)

Secchi-depth (m)

Chlorophyl1 a, phytoplankton ( $\mu \mathrm{q} / \mathrm{L})$

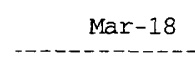

May-13

Jun-15

Jul -22

Oct -06

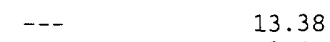

13.70

$---$

3.4

2.8

$\begin{array}{llllll}0.5 & 13.0 & 0.5 & 15.5 & 0.5 & 15.0\end{array}$

Depth of sample (m)

water temoerature $\left({ }^{\circ} \mathrm{C}\right)$

Specific conductance $(\mu \mathrm{S} / \mathrm{cm})$

$\mathrm{pH}$ (units)

Dissolved oxygen (mg/L)

Phosphorus, total (as P)

$87 \quad 80$

$7.1 \quad 6.5$

$12.8 \quad 0.9$

0.013

0.9
0.019

$13.9 \quad 5.6$

$20.0 \quad 6.0$

73

$7.7 \quad 6.5$

$10.5 \quad 4.5$

0.015

0.044

$8.1-6.4$

9.2

0.015

0.1

0.030

\begin{tabular}{cccc}
\multicolumn{2}{c}{ Jul-22 } & \multicolumn{2}{c}{ Oct -06} \\
\hline 13.68 & \multicolumn{2}{c}{13.50} \\
2.9 & \multicolumn{2}{c}{3.4} \\
2.84 & 5.00 \\
0.5 & 15.5 & 0.5 & 15.5 \\
25.3 & 6.3 & 12.3 & 6.9 \\
71 & 99 & 76 & 125 \\
7.8 & 6.7 & 7.4 & 7.0 \\
8.7 & 0.1 & 8.5 & 0.1 \\
0.009 & 0.145 & 0.014 & 0.322
\end{tabular}

3-18-99

5-13-99

6-15-99

7-22-99

10-06-99

DISSOLVED OXYGEN (D.O.), IN MILLIGRAMS PER LITER
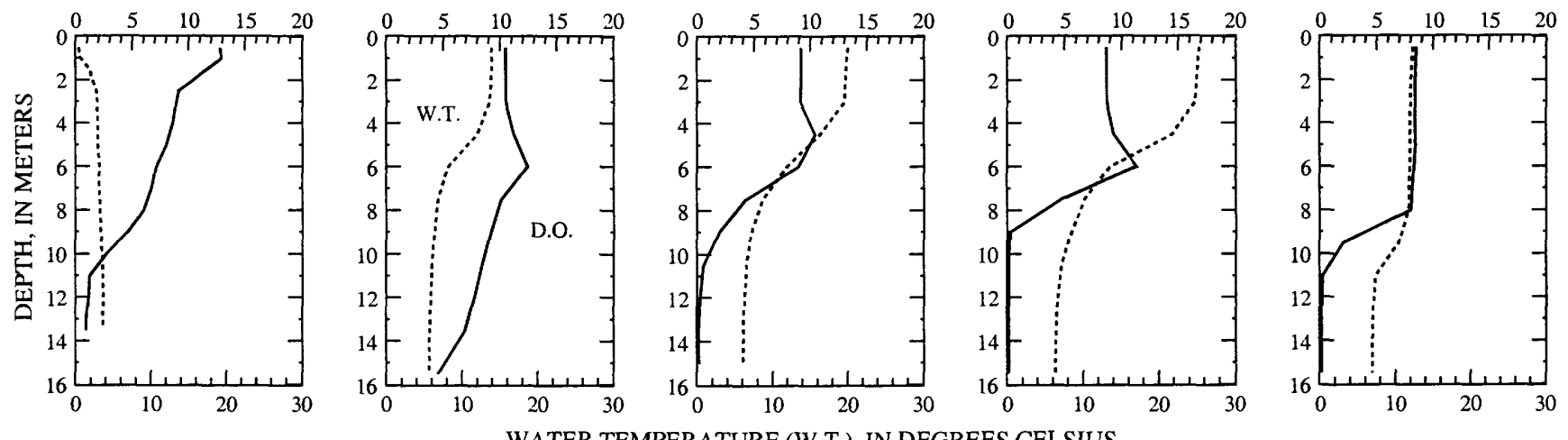

WATER TEMPERATURE (W.T.), IN DEGREES CELSIUS
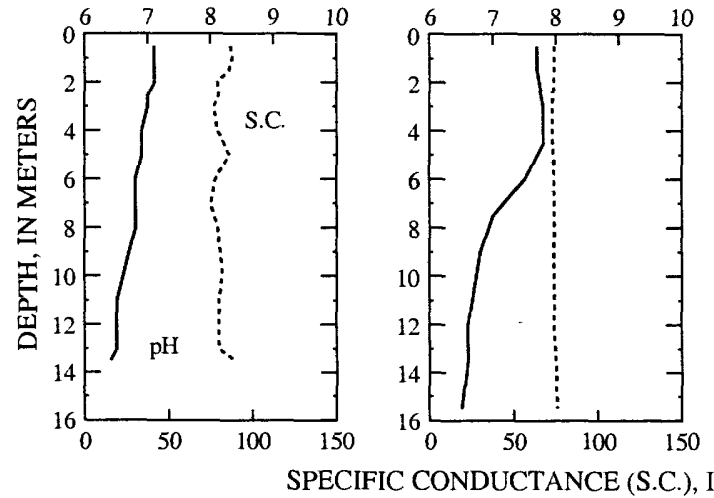

PH, IN STANDARD UNITS
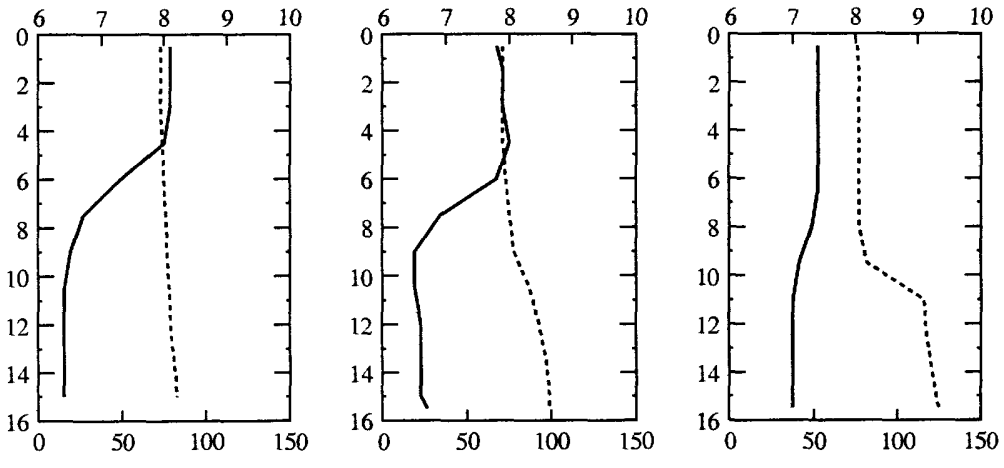

SPECIFIC CONDUCTANCE (S.C.), IN MICROSIEMENS PER CENTIMETER AT 25 DEGREES CELSIUS 

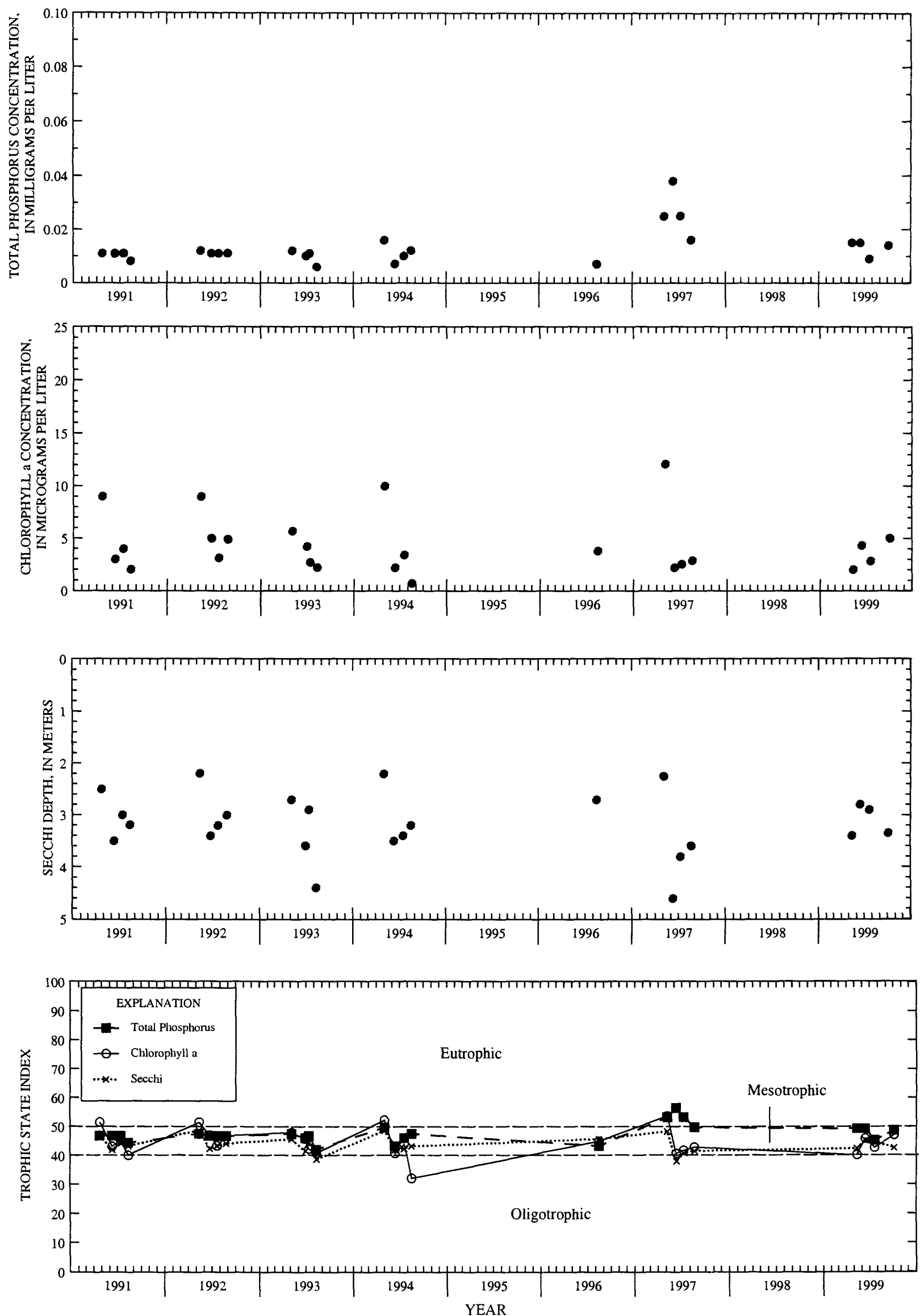

Surface total phosphorus, chlorophyll a concentrations, Secchi depths, and TSI data for Little St. Germain Lake, West Bay, at St. Germain, Wisconsin. 


\section{LITTLE SAND LAKE NEAR MOLE LAKE, WI}

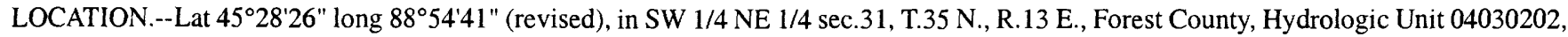
on left bank $1 \mathrm{mi}$ upstream of outlet, $3 \mathrm{mi}$ southeast of Mole Lake.

PERIOD OF RECORD.--May 1996 to current year.

GAGE.--Water-stage recorder. Datum of gage is $1,587.32 \mathrm{ft}$ above sea level.

REMARKS.--Recorder removed during period of ice, Nov. 20, 1998 to Apr. 28, 1999. Gage heights are obtained from reference point during this period.

EXTREMES FOR PERIOD OF RECORD.--Maximum gage height observed, $5.97 \mathrm{ft}$, May 25, 1997; minimum observed, $3.78 \mathrm{ft}$, Nov. 7-9, 1999.

EXTREMES FOR CURRENT YEAR.-- Maximum gage height observed, $5.01 \mathrm{ft}$, July 23, 24, 26; minimum observed, $3.78 \mathrm{ft}$, Nov. $7-9$.

GAGE HEIGHT, FEET, WATER YEAR OCTOBER 1998 TO SEPTEMBER 1999 DAILY MEAN VALUES

\begin{tabular}{|c|c|c|c|c|c|c|c|c|c|c|c|c|}
\hline DAY & OCT & Nov & DEC & JAN & FEB & MAR & APR & MAY & JUN & JUL & AUG & SEP \\
\hline $\begin{array}{l}1 \\
2 \\
3 \\
4 \\
5\end{array}$ & $\begin{array}{l}3.88 \\
3.87 \\
3.86 \\
3.85 \\
3.84\end{array}$ & $\begin{array}{l}3.81 \\
3.80 \\
3.80 \\
3.80 \\
3.80\end{array}$ & $\begin{array}{l}--- \\
-\cdots- \\
--- \\
--- \\
---\end{array}$ & $\begin{array}{l}--- \\
--- \\
--- \\
--- \\
---\end{array}$ & $\begin{array}{l}--- \\
--- \\
--- \\
--- \\
---\end{array}$ & $\begin{array}{l}--- \\
--- \\
--- \\
--- \\
---\end{array}$ & $\begin{array}{l}--- \\
--- \\
--- \\
--- \\
---\end{array}$ & $\begin{array}{l}4.13 \\
4.11 \\
4.10 \\
4.10 \\
4.10\end{array}$ & $\begin{array}{l}4.25 \\
4.21 \\
4.20 \\
4.21 \\
4.21\end{array}$ & $\begin{array}{l}4.63 \\
4.61 \\
4.63 \\
4.63 \\
4.62\end{array}$ & $\begin{array}{l}4.87 \\
4.86 \\
4.85 \\
4.81 \\
4.81\end{array}$ & $\begin{array}{l}4.62 \\
4.61 \\
4.59 \\
4.56 \\
4.55\end{array}$ \\
\hline $\begin{array}{r}6 \\
7 \\
8 \\
9 \\
10\end{array}$ & $\begin{array}{l}3.86 \\
3.86 \\
3.86 \\
3.85 \\
3.85\end{array}$ & $\begin{array}{l}3.79 \\
3.78 \\
3.78 \\
3.78 \\
3.85\end{array}$ & $\begin{array}{l}-- \\
-- \\
--- \\
--- \\
---\end{array}$ & $\begin{array}{l}--- \\
--- \\
--- \\
--- \\
---\end{array}$ & $\begin{array}{l}--- \\
--- \\
--- \\
--- \\
---\end{array}$ & $\begin{array}{l}--- \\
--- \\
--- \\
--- \\
---\end{array}$ & $\begin{array}{l}--- \\
--- \\
--- \\
--- \\
---\end{array}$ & $\begin{array}{l}4.15 \\
4.29 \\
4.32 \\
4.30 \\
4.32\end{array}$ & $\begin{array}{l}4.39 \\
4.43 \\
4.46 \\
4.60 \\
4.68\end{array}$ & $\begin{array}{l}4.60 \\
4.58 \\
4.64 \\
4.81 \\
4.84\end{array}$ & $\begin{array}{l}4.79 \\
4.78 \\
4.76 \\
4.74 \\
4.74\end{array}$ & $\begin{array}{l}4.53 \\
4.50 \\
4.45 \\
4.46 \\
4.42\end{array}$ \\
\hline $\begin{array}{l}11 \\
12 \\
13 \\
14 \\
15\end{array}$ & $\begin{array}{l}3.85 \\
3.84 \\
3.83 \\
3.82 \\
3.81\end{array}$ & $\begin{array}{l}3.87 \\
3.85 \\
3.85 \\
3.84 \\
3.85\end{array}$ & $\begin{array}{l}--- \\
--- \\
--- \\
--- \\
---\end{array}$ & $\begin{array}{l}-- \\
-- \\
-- \\
-- \\
--\end{array}$ & $\begin{array}{l}--- \\
--- \\
--- \\
--- \\
---\end{array}$ & $\begin{array}{l}--- \\
--- \\
--- \\
--- \\
---\end{array}$ & $\begin{array}{r}--- \\
--- \\
--- \\
4.27 \\
---\end{array}$ & $\begin{array}{l}4.30 \\
4.29 \\
4.25 \\
4.23 \\
4.21\end{array}$ & $\begin{array}{l}4.69 \\
4.71 \\
4.68 \\
4.67 \\
4.68\end{array}$ & $\begin{array}{l}4.84 \\
4.83 \\
4.83 \\
4.84 \\
4.82\end{array}$ & $\begin{array}{l}4.73 \\
4.73 \\
4.84 \\
4.83 \\
4.83\end{array}$ & $\begin{array}{l}4.43 \\
4.45 \\
4.42 \\
4.40 \\
4.39\end{array}$ \\
\hline $\begin{array}{l}16 \\
17 \\
18 \\
19 \\
20\end{array}$ & $\begin{array}{l}3.81 \\
3.82 \\
3.87 \\
3.85 \\
3.84\end{array}$ & $\begin{array}{r}3.85 \\
3.85 \\
3.87 \\
3.92 \\
\ldots \ldots\end{array}$ & $\begin{array}{l}-\cdots \\
\cdots- \\
--- \\
--- \\
---\end{array}$ & $\begin{array}{c}--- \\
--- \\
--- \\
--- \\
3.92\end{array}$ & $\begin{array}{l}--- \\
--- \\
--- \\
--- \\
---\end{array}$ & $\begin{array}{l}--- \\
--- \\
-- \\
--- \\
---\end{array}$ & $\begin{array}{l}--- \\
-\cdots \\
\cdots- \\
\cdots- \\
\cdots-\end{array}$ & $\begin{array}{l}4.22 \\
4.27 \\
4.31 \\
4.25 \\
4.26\end{array}$ & $\begin{array}{l}4.68 \\
4.66 \\
4.64 \\
4.62 \\
4.62\end{array}$ & $\begin{array}{l}4.86 \\
4.93 \\
4.94 \\
4.95 \\
4.96\end{array}$ & $\begin{array}{l}4.85 \\
4.83 \\
4.83 \\
4.82 \\
4.80\end{array}$ & $\begin{array}{l}4.39 \\
4.39 \\
4.36 \\
4.39 \\
4.41\end{array}$ \\
\hline $\begin{array}{l}21 \\
22 \\
23 \\
24 \\
25\end{array}$ & $\begin{array}{l}3.83 \\
3.82 \\
3.81 \\
3.81 \\
3.80\end{array}$ & $\begin{array}{l}--- \\
--- \\
--- \\
--- \\
---\end{array}$ & $\begin{array}{l}-- \\
-- \\
--- \\
--- \\
---\end{array}$ & $\begin{array}{l}--- \\
--- \\
--- \\
--- \\
---\end{array}$ & $\begin{array}{l}--- \\
--- \\
--- \\
--- \\
---\end{array}$ & $\begin{array}{l}--- \\
--- \\
--- \\
--- \\
---\end{array}$ & $\begin{array}{l}-\cdots \\
-\cdots \\
-\cdots \\
-\cdots \\
-\cdots\end{array}$ & $\begin{array}{l}4.28 \\
4.27 \\
4.31 \\
4.32 \\
4.31\end{array}$ & $\begin{array}{l}4.60 \\
4.59 \\
4.61 \\
4.63 \\
4.59\end{array}$ & $\begin{array}{l}4.97 \\
4.99 \\
5.01 \\
5.01 \\
4.96\end{array}$ & $\begin{array}{l}4.79 \\
4.78 \\
4.77 \\
4.74 \\
4.73\end{array}$ & $\begin{array}{l}4.37 \\
4.35 \\
4.33 \\
4.32 \\
4.31\end{array}$ \\
\hline $\begin{array}{l}26 \\
27 \\
28 \\
29 \\
30 \\
31\end{array}$ & $\begin{array}{l}3.80 \\
3.80 \\
3.79 \\
3.79 \\
3.80 \\
3.80\end{array}$ & $\begin{array}{l}--- \\
--- \\
--- \\
--- \\
--- \\
---\end{array}$ & $\begin{array}{l}--- \\
--- \\
-\cdots \\
-- \\
-\cdots \\
-\cdots\end{array}$ & $\begin{array}{l}--- \\
--- \\
-- \\
-- \\
-\cdots \\
--\end{array}$ & $\begin{array}{l}-\cdots \\
\cdots- \\
\cdots- \\
-\cdots \\
\cdots- \\
\cdots-\end{array}$ & $\begin{array}{l}--- \\
--- \\
--- \\
--- \\
--- \\
---\end{array}$ & $\begin{array}{r}\ldots \\
\ldots .12 \\
4.12 \\
\ldots\end{array}$ & $\begin{array}{l}4.27 \\
4.26 \\
4.26 \\
4.25 \\
4.24 \\
4.23\end{array}$ & $\begin{array}{r}4.58 \\
4.58 \\
4.59 \\
4.63 \\
4.61 \\
---\end{array}$ & $\begin{array}{l}5.01 \\
4.98 \\
4.94 \\
4.92 \\
4.89 \\
4.91\end{array}$ & $\begin{array}{l}4.75 \\
4.73 \\
4.70 \\
4.67 \\
4.65 \\
4.62\end{array}$ & $\begin{array}{c}4.31 \\
4.33 \\
4.34 \\
4.32 \\
4.31 \\
-\end{array}$ \\
\hline $\begin{array}{l}\text { MEAN } \\
\text { MAX } \\
\text { MIN }\end{array}$ & $\begin{array}{l}3.83 \\
3.88 \\
3.79\end{array}$ & --- & $-\cdots$ & $\begin{array}{l}--- \\
--- \\
--\end{array}$ & $\begin{array}{l}--- \\
--- \\
---\end{array}$ & $\begin{array}{l}--- \\
--- \\
---\end{array}$ & $-\cdots$ & $\begin{array}{l}4.24 \\
4.32 \\
4.10\end{array}$ & $\begin{array}{l}4.54 \\
4.71 \\
4.20\end{array}$ & $\begin{array}{l}4.84 \\
5.01 \\
4.58\end{array}$ & $\begin{array}{l}4.78 \\
4.87 \\
4.62\end{array}$ & $\begin{array}{l}4.42 \\
4.62 \\
4.31\end{array}$ \\
\hline
\end{tabular}


LOCATION.--Lat $43^{\circ} 05^{\prime} 42^{\prime \prime}$, long $89^{\circ} 22^{\prime} 12^{\prime \prime}$, in SE $1 / 4 \mathrm{sec} .12$, T.7 N., R.9 E., Dane County, Hydrologic Unit 07090001 , in city boat house at dam at outlet, in Madison.

DRAINAGE AREA.--233 $\mathrm{mi}^{2}$. Area of Lake Mendota, $15.2 \mathrm{mi}^{2}$.

PERIOD OF RECORD.--December 1902 to May 1903, January 1916 to current year (incomplete).

REVISED RECORDS.--WDR WI-73-1: Drainage area.

GAGE.--Water-stage recorder. Datum of gage is $840.00 \mathrm{ft}$ above sea level, or $5.60 \mathrm{ft}$ below City of Madison datum. Prior to Oct. 1, 1979 , at datum $7.82 \mathrm{ft}$ higher; prior to Nov. 15, 1971, nonrecording gage at same site.

REMARKS.--No estimated daily gage heights. Records are good. Lake level regulated by concrete dam with two 12-foot gates and 20-foot lock at outlet. Gage-height telemeter at station.

EXTREMES FOR PERIOD OF RECORD.--Maximum gage height observed, $12.20 \mathrm{ft}$, July 14-15, 1993; minimum observed, $8.02 \mathrm{ft}$, Feb. 24 to Mar. 10, 1920, current datum.

EXTREMES FOR CURRENT YEAR.--Maximum recorded gage height, $10.83 \mathrm{ft}$, May 18; minimum recorded, $9.10 \mathrm{ft}$, Mar. 2 .

GAGE HEIGHT, FEET, WATER YEAR OCTOBER 1998 TO SEPTEMBER 1999

DAILY MEAN VALUES

\begin{tabular}{|c|c|c|c|c|c|c|c|c|c|c|c|c|}
\hline DAY & OCT & NOV & $\mathrm{DEC}$ & JAN & $F E B$ & MAR & APR & MAY & JUN & JUL & AUG & SEP \\
\hline $\begin{array}{l}1 \\
2 \\
3 \\
4 \\
5\end{array}$ & $\begin{array}{l}10.14 \\
10.12 \\
10.14 \\
10.14 \\
10.20\end{array}$ & $\begin{array}{l}9.73 \\
9.68 \\
9.62 \\
9.57 \\
9.52\end{array}$ & $\begin{array}{l}9.43 \\
9.44 \\
9.44 \\
9.44 \\
9.44\end{array}$ & $\begin{array}{l}9.29 \\
9.33 \\
9.36 \\
9.37 \\
9.37\end{array}$ & $\begin{array}{l}9.45 \\
9.44 \\
9.44 \\
9.43 \\
9.43\end{array}$ & $\begin{array}{l}9.16 \\
9.15 \\
9.16 \\
9.17 \\
9.19\end{array}$ & $\begin{array}{l}9.43 \\
9.43 \\
9.46 \\
9.55 \\
9.56\end{array}$ & $\begin{array}{l}10.52 \\
10.51 \\
10.49 \\
10.48 \\
10.48\end{array}$ & $\begin{array}{l}10.26 \\
10.31 \\
10.28 \\
10.27 \\
10.28\end{array}$ & $\begin{array}{l}10.41 \\
10.40 \\
10.39 \\
10.40 \\
10.40\end{array}$ & $\begin{array}{l}10.37 \\
10.33 \\
10.30 \\
10.29 \\
10.26\end{array}$ & $\begin{array}{l}9.99 \\
9.97 \\
9.96 \\
9.94 \\
9.94\end{array}$ \\
\hline $\begin{array}{r}6 \\
7 \\
8 \\
9 \\
10\end{array}$ & $\begin{array}{l}10.31 \\
10.33 \\
10.30 \\
10.27 \\
10.25\end{array}$ & $\begin{array}{l}9.47 \\
9.42 \\
9.38 \\
9.34 \\
9.47\end{array}$ & $\begin{array}{l}9.46 \\
9.45 \\
9.44 \\
9.43 \\
9.43\end{array}$ & $\begin{array}{l}9.37 \\
9.37 \\
9.38 \\
9.39 \\
9.39\end{array}$ & $\begin{array}{l}9.42 \\
9.40 \\
9.38 \\
9.37 \\
9.36\end{array}$ & $\begin{array}{l}9.21 \\
9.22 \\
9.23 \\
9.28 \\
9.29\end{array}$ & $\begin{array}{l}9.61 \\
9.60 \\
9.61 \\
9.77 \\
9.82\end{array}$ & $\begin{array}{l}10.56 \\
10.59 \\
10.61 \\
10.60 \\
10.59\end{array}$ & $\begin{array}{l}10.30 \\
10.38 \\
10.40 \\
10.42 \\
10.43\end{array}$ & $\begin{array}{l}10.41 \\
10.38 \\
10.34 \\
10.35 \\
10.31\end{array}$ & $\begin{array}{l}10.23 \\
10.23 \\
10.23 \\
10.20 \\
10.22\end{array}$ & $\begin{array}{l}9.92 \\
9.89 \\
9.88 \\
9.85 \\
9.81\end{array}$ \\
\hline $\begin{array}{l}11 \\
12 \\
13 \\
14 \\
15\end{array}$ & $\begin{array}{l}10.22 \\
10.20 \\
10.16 \\
10.11 \\
10.07\end{array}$ & $\begin{array}{l}9.51 \\
9.51 \\
9.51 \\
9.53 \\
9.52\end{array}$ & $\begin{array}{l}9.41 \\
9.40 \\
9.40 \\
9.39 \\
9.38\end{array}$ & $\begin{array}{l}9.41 \\
9.41 \\
9.43 \\
9.43 \\
9.44\end{array}$ & $\begin{array}{l}9.39 \\
9.47 \\
9.48 \\
9.47 \\
9.46\end{array}$ & $\begin{array}{l}9.30 \\
9.31 \\
9.33 \\
9.34 \\
9.35\end{array}$ & $\begin{array}{l}9.85 \\
9.87 \\
9.87 \\
9.87 \\
9.86\end{array}$ & $\begin{array}{l}10.57 \\
10.56 \\
10.53 \\
10.48 \\
10.43\end{array}$ & $\begin{array}{l}10.45 \\
10.46 \\
10.51 \\
10.53 \\
10.49\end{array}$ & $\begin{array}{l}10.28 \\
10.26 \\
10.23 \\
10.19 \\
10.17\end{array}$ & $\begin{array}{l}10.20 \\
10.19 \\
10.20 \\
10.16 \\
10.13\end{array}$ & $\begin{array}{l}9.78 \\
9.77 \\
9.76 \\
9.74 \\
9.72\end{array}$ \\
\hline $\begin{array}{l}16 \\
17 \\
18 \\
19 \\
20\end{array}$ & $\begin{array}{l}10.04 \\
10.06 \\
10.14 \\
10.11 \\
10.08\end{array}$ & $\begin{array}{l}9.52 \\
9.51 \\
9.50 \\
9.52 \\
9.50\end{array}$ & $\begin{array}{l}9.39 \\
9.38 \\
9.37 \\
9.38 \\
9.37\end{array}$ & $\begin{array}{l}9.44 \\
9.46 \\
9.49 \\
9.49 \\
9.48\end{array}$ & $\begin{array}{l}9.44 \\
9.43 \\
9.41 \\
9.39 \\
9.36\end{array}$ & $\begin{array}{l}9.36 \\
9.38 \\
9.40 \\
9.42 \\
9.43\end{array}$ & $\begin{array}{l}9.86 \\
9.85 \\
9.83 \\
9.83 \\
9.82\end{array}$ & $\begin{array}{l}10.40 \\
10.62 \\
10.67 \\
10.64 \\
10.60\end{array}$ & $\begin{array}{l}10.48 \\
10.45 \\
10.44 \\
10.41 \\
10.40\end{array}$ & $\begin{array}{l}10.16 \\
10.26 \\
10.28 \\
10.29 \\
10.31\end{array}$ & $\begin{array}{l}10.11 \\
10.11 \\
10.09 \\
10.09 \\
10.08\end{array}$ & $\begin{array}{l}9.70 \\
9.69 \\
9.67 \\
9.67 \\
9.66\end{array}$ \\
\hline $\begin{array}{l}21 \\
22 \\
23 \\
24 \\
25\end{array}$ & $\begin{array}{r}10.04 \\
10.00 \\
9.97 \\
9.94 \\
9.91\end{array}$ & $\begin{array}{l}9.46 \\
9.45 \\
9.47 \\
9.45 \\
9.44\end{array}$ & $\begin{array}{l}9.39 \\
9.36 \\
9.34 \\
9.34 \\
9.32\end{array}$ & $\begin{array}{l}9.47 \\
9.52 \\
9.56 \\
9.59 \\
9.60\end{array}$ & $\begin{array}{l}9.33 \\
9.30 \\
9.28 \\
9.26 \\
9.24\end{array}$ & $\begin{array}{l}9.46 \\
9.46 \\
9.47 \\
9.48 \\
9.49\end{array}$ & $\begin{array}{r}9.83 \\
9.96 \\
10.27 \\
10.41 \\
10.47\end{array}$ & $\begin{array}{l}10.56 \\
10.55 \\
10.55 \\
10.54 \\
10.50\end{array}$ & $\begin{array}{l}10.38 \\
10.37 \\
10.39 \\
10.42 \\
10.41\end{array}$ & $\begin{array}{l}10.38 \\
10.39 \\
10.38 \\
10.38 \\
10.36\end{array}$ & $\begin{array}{l}10.07 \\
10.05 \\
10.06 \\
10.12 \\
10.11\end{array}$ & $\begin{array}{l}9.64 \\
9.63 \\
9.62 \\
9.62 \\
9.61\end{array}$ \\
\hline $\begin{array}{l}26 \\
27 \\
28 \\
29 \\
30 \\
31\end{array}$ & $\begin{array}{l}9.89 \\
9.88 \\
9.88 \\
9.84 \\
9.82 \\
9.78\end{array}$ & $\begin{array}{l}9.44 \\
9.43 \\
9.43 \\
9.43 \\
9.46 \\
\ldots-\end{array}$ & $\begin{array}{l}9.32 \\
9.32 \\
9.31 \\
9.32 \\
9.30 \\
9.29\end{array}$ & $\begin{array}{l}9.58 \\
9.56 \\
9.54 \\
9.52 \\
9.50 \\
9.48\end{array}$ & $\begin{array}{r}9.21 \\
9.20 \\
9.18 \\
--- \\
--- \\
---\end{array}$ & $\begin{array}{l}9.49 \\
9.48 \\
9.47 \\
9.47 \\
9.45 \\
9.42\end{array}$ & $\begin{array}{c}10.50 \\
10.51 \\
10.54 \\
10.53 \\
10.53 \\
.-\end{array}$ & $\begin{array}{l}10.44 \\
10.41 \\
10.38 \\
10.35 \\
10.31 \\
10.28\end{array}$ & $\begin{array}{l}10.38 \\
10.37 \\
10.38 \\
10.39 \\
10.35 \\
-\end{array}$ & $\begin{array}{l}10.39 \\
10.38 \\
10.36 \\
10.35 \\
10.33 \\
10.38\end{array}$ & $\begin{array}{l}10.10 \\
10.10 \\
10.09 \\
10.06 \\
10.02 \\
10.00\end{array}$ & $\begin{array}{c}9.60 \\
9.66 \\
9.72 \\
9.75 \\
9.74 \\
\ldots--\end{array}$ \\
\hline $\begin{array}{l}\text { MEAN } \\
\text { MAX } \\
\text { MIN }\end{array}$ & $\begin{array}{r}10.08 \\
10.33 \\
9.78\end{array}$ & $\begin{array}{l}9.49 \\
9.73 \\
9.34\end{array}$ & $\begin{array}{l}9.38 \\
9.46 \\
9.29\end{array}$ & $\begin{array}{l}9.45 \\
9.60 \\
9.29\end{array}$ & $\begin{array}{l}9.37 \\
9.48 \\
9.18\end{array}$ & $\begin{array}{l}9.35 \\
9.49 \\
9.15\end{array}$ & $\begin{array}{r}9.93 \\
10.54 \\
9.43\end{array}$ & $\begin{array}{l}10.51 \\
10.67 \\
10.28\end{array}$ & $\begin{array}{l}10.39 \\
10.53 \\
10.26\end{array}$ & $\begin{array}{l}10.33 \\
10.41 \\
10.16\end{array}$ & $\begin{array}{l}10.15 \\
10.37 \\
10.00\end{array}$ & $\begin{array}{l}9.76 \\
9.99 \\
9.60\end{array}$ \\
\hline
\end{tabular}




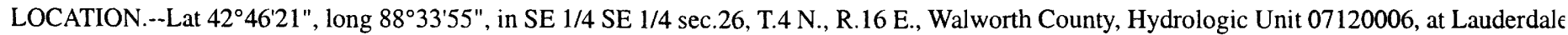
PERIOD OF RECORD.--November 1993 to November 1994, and February to August 1999.

REMARKS.--Lake sampled near east end of lake at lake depth of about $52 \mathrm{ft}$. Lake ice-covered during February sampling. Water-quality analyse done by Wisconsin State Laboratory of Hygiene.

WATER-QUALITY DATA, FEBRUARY 19 TO AUGUST 11, 1999

(Milligrams per liter unless otherwise indicated)

Secchi-depth (m)

Chlorophy11 a, phytoplankton $(\mu \mathrm{g} / \mathrm{L})$

Depth of sample (m)

Water temperature $\left({ }^{\circ} \mathrm{C}\right)$

Specific conductance $(\mu \mathrm{S} / \mathrm{cm})$

$\mathrm{pH}$ (units)

Dissolved oxygen (mg/L)

Phosphorus, total (as P)

Phosphorus, ortho, dissolved (as P)

Nitrogen, NO2 + NO3, diss. (as N)

Nitrogen, ammonia, dissolved (as N)

Nitrogen, amm. + org., total (as N)

Nitrogen, total (as N)

Color (Pt-Co. scale)

Turbidity (NTU)

Hardness, (as $\left.\mathrm{CaCO}_{3}\right)$

Calcium, dissolved (Ca)

Magnesium, dissolved (Mg)

Sodium, dissolved (Na)

Potassium, dissolved $(\mathrm{K})$

Alkalinity, (as $\mathrm{CaCO}_{3}$ )

Sulfate, dissolved $\left(\mathrm{SO}_{4}\right)$

Chloride, dissolved (C1)

Silica, dissolved $\left(\mathrm{SiO}_{2}\right)$

Solids, dissolved, at $180^{\circ} \mathrm{C}$

Iron, dissolved (Fe) $\mu \mathrm{g} / \mathrm{L}$

Manganese, dissolved (Mn) $\mu \mathrm{g} / \mathrm{L}$

Feb-19

$\begin{array}{cc} & \\ 0.5 & 13.5 \\ 5.0 & 3.7 \\ 431 & 618 \\ 8.1 & 7.6 \\ 16.1 & 9.1 \\ 0.006 & 0.008\end{array}$

2-19-99

-n

-

$\begin{array}{ll}-2 & -1 \\ -\cdots & -1\end{array}$

--- ---

--

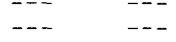

-

‥

$-\cdots$

\begin{tabular}{cc}
\multicolumn{2}{c}{ Apr-14 } \\
\multicolumn{2}{c}{9.8} \\
\multicolumn{2}{c}{0.330} \\
0.5 & 14.0 \\
11.0 & 9.0 \\
535 & 539 \\
8.1 & 8.1 \\
10.8 & 9.9 \\
0.006 & 0.009 \\
$<0.002$ & --- \\
1.11 & -- \\
0.244 & -- \\
0.7 & -- \\
1.8 & -- \\
5 & --- \\
0.5 & --- \\
250 & --- \\
47 & --- \\
33 & --- \\
7.6 & --- \\
1.9 & --- \\
217 & --- \\
35 & --- \\
22 & --- \\
3.8 & --- \\
298 & --- \\
$<10$ & --- \\
0.8 & ---
\end{tabular}

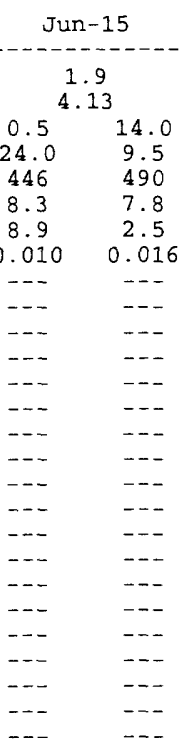

Ju1-14

\begin{tabular}{cc}
\multicolumn{2}{c}{2.0} \\
4.47 \\
0.5 & 14.0 \\
25.4 & 9.6 \\
465 & 483 \\
8.0 & 7.6 \\
8.4 & 0.0 \\
0.010 & 0.022
\end{tabular}

-.- - -

-

-.-

--

$--$

$--$
Aug-11

2.9

3.00

$11.0 \quad 14.0$

$25.2 \quad 11.4 \quad 9.7$

$450 \quad 478 \quad 493$

$\begin{array}{lll}8.1 & 7.5 & 7.5\end{array}$

$\begin{array}{lll}8.8 & 0.0 & 0.0\end{array}$

$\begin{array}{ccc}8.8 & 0.0 & 0.0 \\ 0.010 & 0.018 & 0.020\end{array}$

$0.010 \quad 0.018$

-

n-

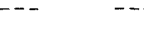

$\cdots$

--

$--$

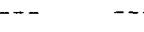

n-n

$\cdots$

no

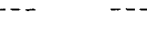

$\cdots$

$-\cdots$

DISSOLVED OXYGEN (D.O.), IN MILLIGRAMS PER LITER
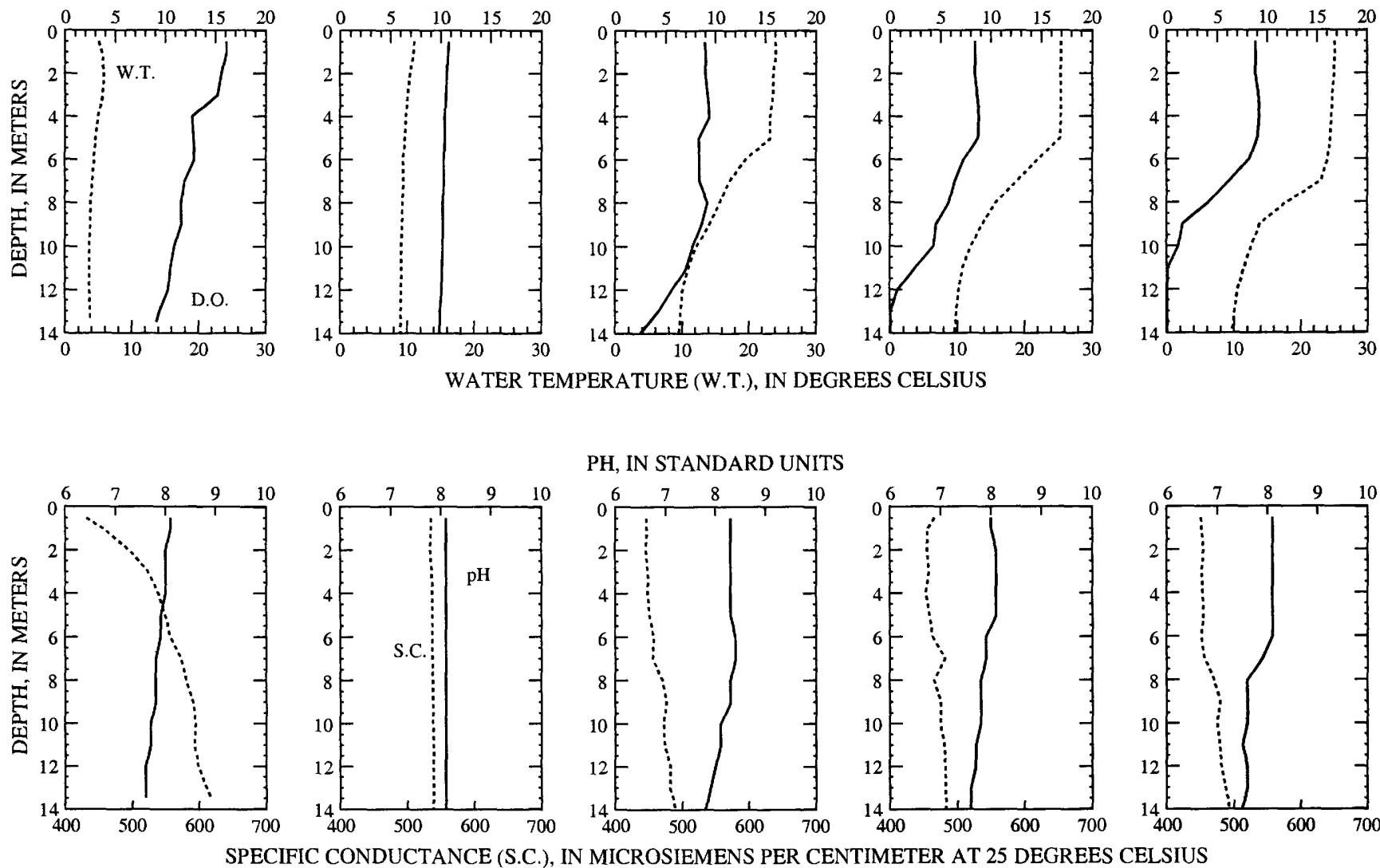

PH, IN STANDARD UNITS
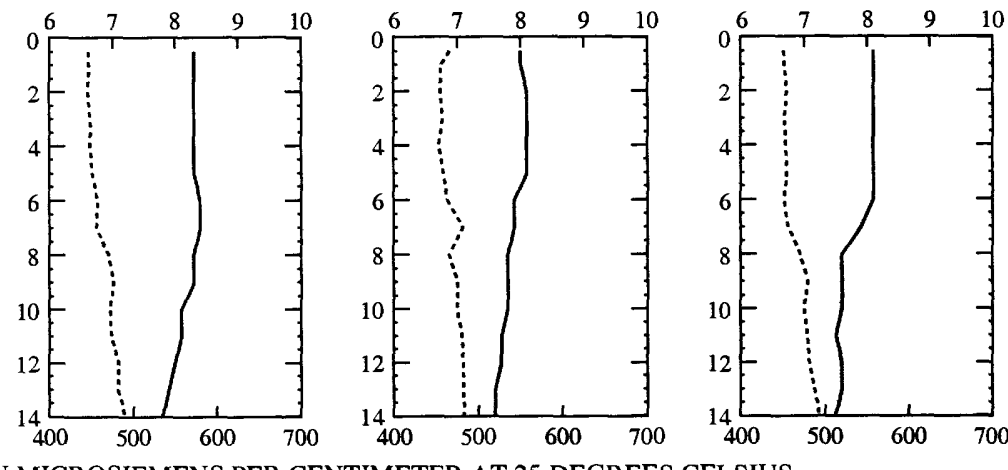

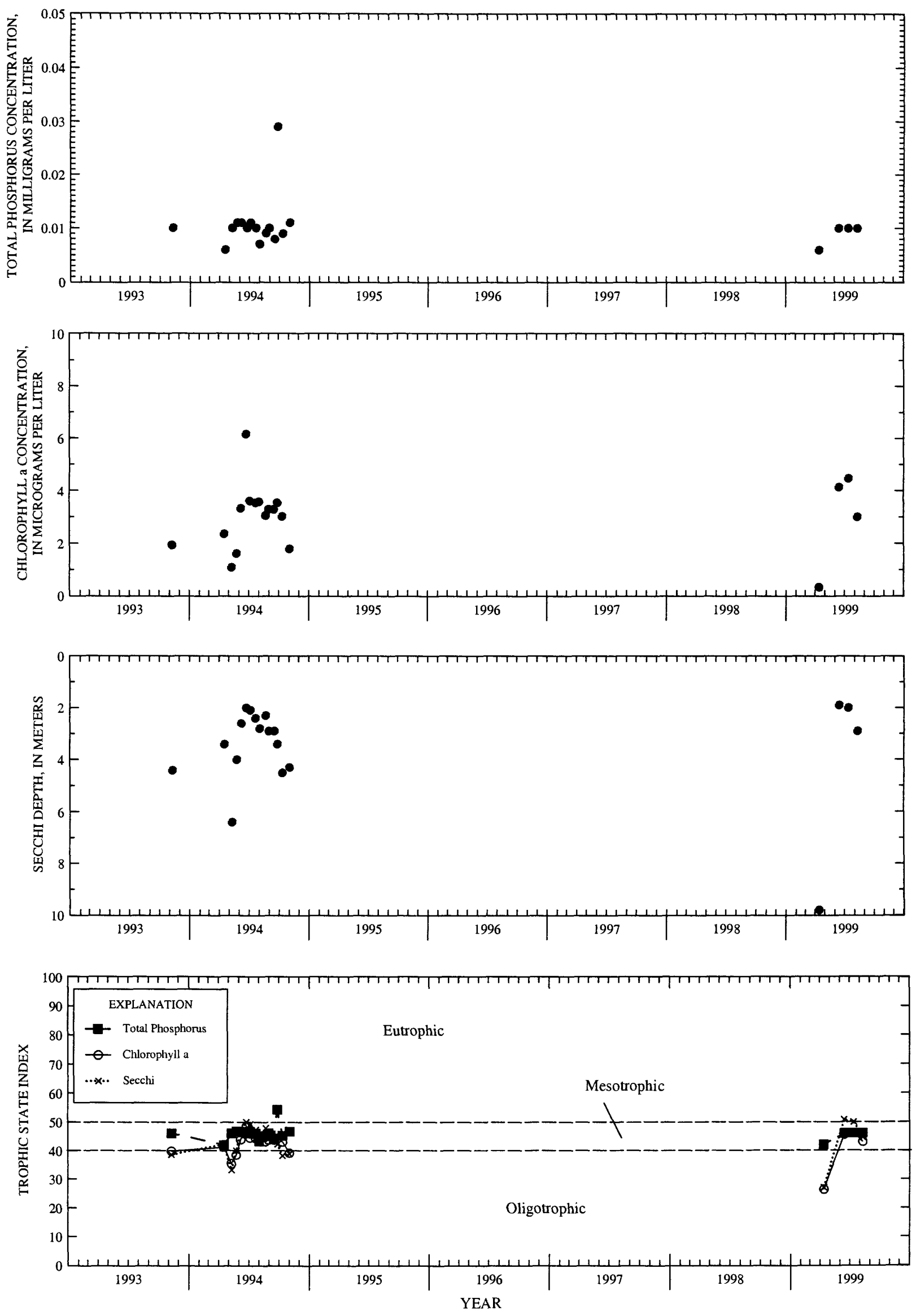

Surface total phosphorus, chlorophyll a concentrations, Secchi depths, and TSI data for Lauderdale Lake (Middle) near Lauderdale, Wisconsin. 


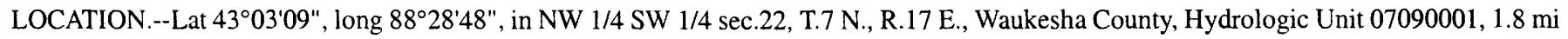
south of Oconomowoc.

PERIOD OF RECORD.--February 1996 to current year.

REMARKS.--Lake sampled near center at the deep hole. Lake ice-covered during February sampling. Water-quality analyses done by Wisconsin State Laboratory of Hygiene.

WATER-QUALITY DATA, FEBRUARY 10 TO AUGUST 03, 1999

(Milligrams per liter unless otherwise indicated)

Lake stage ( $f t)$

Secchi-depth (m)

Chlorophyll a, phytoplankton ( $\mu \mathrm{g} / \mathrm{L}$ )

Depth of sample (m)

water temperature $\left({ }^{\circ} \mathrm{C}\right)$

Specific conductance $(\mu \mathrm{S} / \mathrm{cm})$

$\mathrm{pH}$ (units)

Dissolved oxygen (mg/L)

Phosphorus, total (as $P$ )

Phosphorus, ortho, dissolved (as P)

Nitrogen, NO2 + NO3, diss. (as N)

Nitrogen, ammonia, dissolved (as N)

Nitrogen, amm. + org.. total (as N)

Nitrogen, total (as N)

Color (Pt-Co. scale)

Turbidity (NTU)

Hardness, (as $\mathrm{CaCO}_{3}$ )

Calcium, dissolved ( $\mathrm{Ca}$ )

Magnesium, dissolved (Mg)

Sodium, dissolved (Na)

potassium, dissolved (K)

Alkalinity, (as $\mathrm{CaCO}_{3}$ )

sulfate, dissolved $\left(\mathrm{SO}_{4}\right)$

Chloride, dissolved (CI)

Silica, dissolved $\left(\mathrm{SiO}_{2}\right)$

Solids, dissolved, at $180^{\circ} \mathrm{C}$

Iron, dissolved ( $\mathrm{Fe}$ ) $\mu \mathrm{g} / \mathrm{L}$

Manganese, dissolved (Mn) $\mu \mathrm{g} / \mathrm{L}$

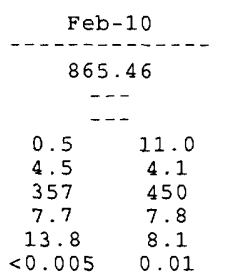

$0.005 \quad 0.01$

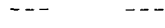

n-

$\cdots$

$\begin{array}{ll}--- & -1 \\ -\cdots & --\end{array}$

-

- -

--

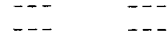

-- $\quad-\cdots$

$\begin{array}{ll}-- & -- \\ --- & --\end{array}$

$\cdots$

$\cdots$

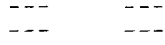

$\cdots$

Apr -7
865.48
4.0

4.0

$\begin{array}{ll}0.5 & 11.5 \\ 9.2 & 8.5\end{array}$

$416 \quad 415$

$8.2 \quad 8.3$

$11.7 \quad 10.9$

0.013

0.002

0.023

0.141

0.84
0.86

5
0.6
180

180
29

29
26
10

1.7
164
17

164

25
1.6
228

1.6
228
$<10$
1.0

1.0
0.016

-.-

---

$--$

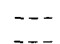

-

$\ldots$

-.-

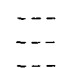

$\ldots$

-

-

$--$

\begin{tabular}{cc} 
Jun-2 \\
\hline 866.86 \\
5.7 \\
\multicolumn{2}{c}{1.49} \\
0.5 & 11.0 \\
20.2 & 11.3 \\
408 & 432 \\
8.1 & 7.7 \\
9.0 & 0.5 \\
0.008 & 0.011
\end{tabular}

$\begin{array}{ll}0.008 & 0.011\end{array}$

‥

$\cdots$

-.

$---$

-

-

$--$

$---$

-

--

$--$

$--$

$\cdots$

-

-

-.

-.

-

-..

-

-.-

$--$

\begin{tabular}{|c|c|}
\hline Jul-6 & Aug -3 \\
\hline $\begin{array}{c}867.02 \\
3.7 \\
2.07\end{array}$ & $\begin{array}{c}866.91 \\
3.3 \\
1.15\end{array}$ \\
\hline $0.5 \quad 11.5$ & 11.0 \\
\hline
\end{tabular}

$26.7 \quad 12.7 \quad 27.7 \quad 13$.

$\begin{array}{llll}415 & 463 & 399 & 461 \\ 8.2 & 7.7 & 8.3 & 7.5\end{array}$

$\begin{array}{llll}8.0 & 0.4 & 8.5 & 0.0\end{array}$

$\begin{array}{llll}0.007 & 0.023 & 0.015 & 0.041\end{array}$

--

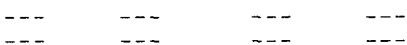

$\cdots$

n-

n-

-

$\cdots$

-- $\quad--$

$\begin{array}{ll}--- & -- \\ --- & --\end{array}$

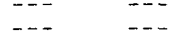

-.

$\cdots$

-

$\cdots$

-..

-

- -

-.-

- -

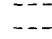

$--$

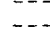

-.

-..

-

DISSOLVED OXYGEN (D.O.), IN MILLIGRAMS PER LITER
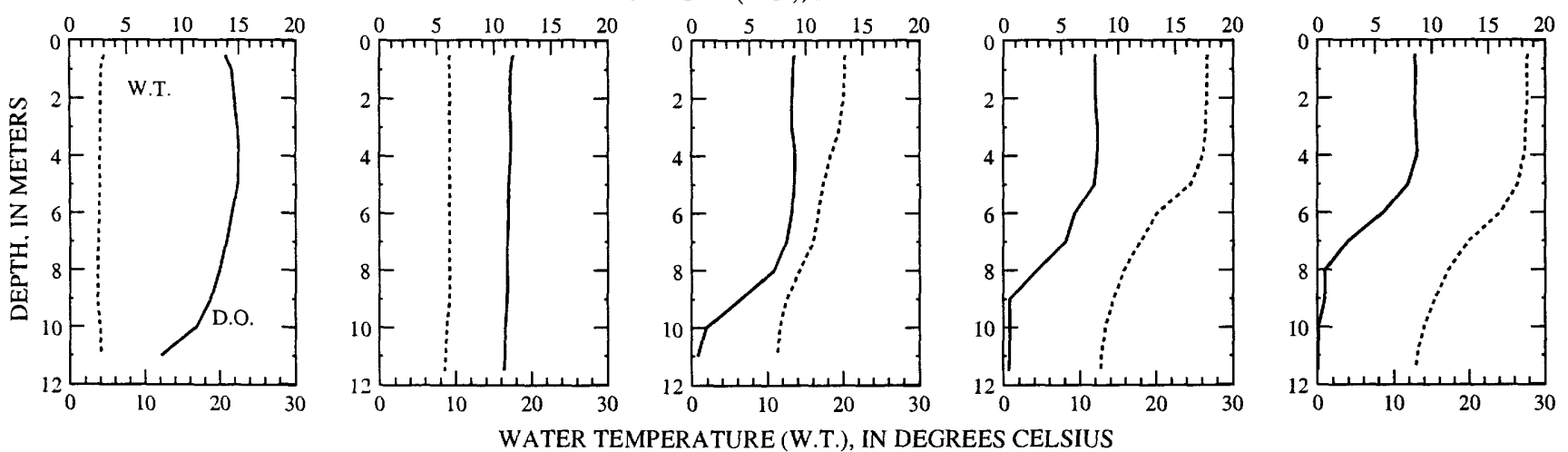

PH, IN STANDARD UNITS
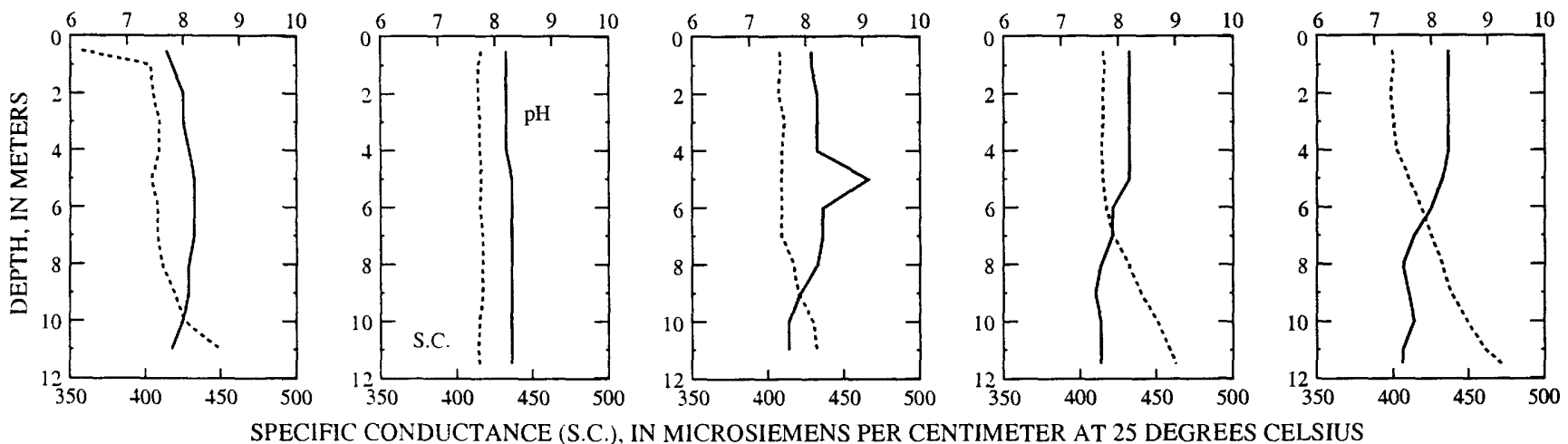

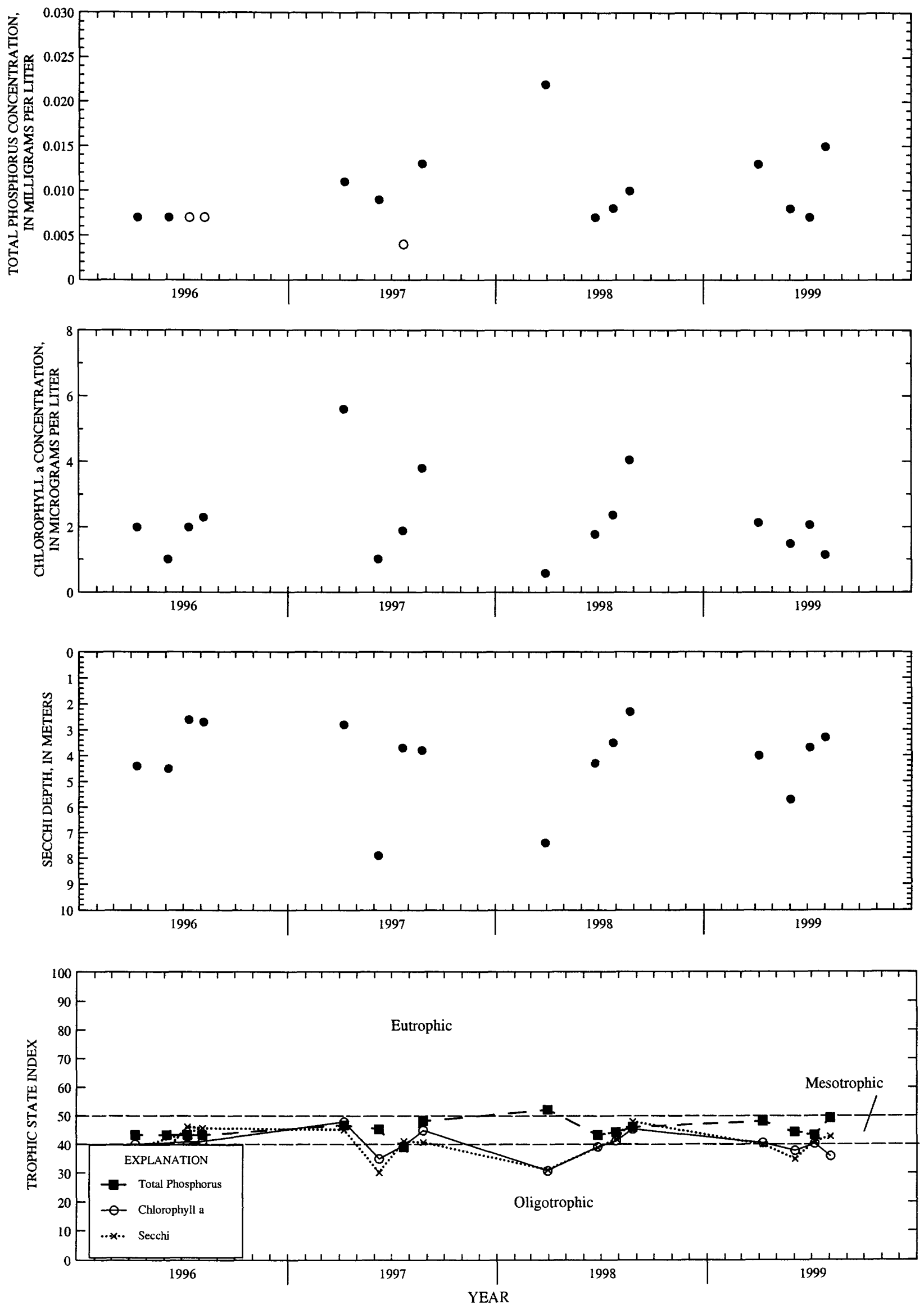

Surface total phosphorus, chlorophyll a concentrations, Secchi depths, and TSI data for Middle Genesee Lake near Oconomowoc, Wisconsin. 
LOCATION.--Lat 4303'48", long 89²3'49', in SW 1/4 sec.23, T.7 N., R.9 E., Dane County, Hydrologic Unit 07090001, in Brittingham Park, in Madison.

DRAINAGE AREA.--279 $\mathrm{mi}^{2}$. Area of Lake Monona, $5.3 \mathrm{mi}^{2}$.

PERIOD OF RECORD.--September 1915 to current year (fragmentary) in reports of the Geological Survey. For 1856 to March 1917 in reports of Wisconsin Railroad Commission, volume 19.

REVISED RECORDS.--WSP 1338: Lake area. WDR WI-73-1: Drainage area.

GAGE.--Water-stage recorder. Datum of gage is $840.00 \mathrm{ft}$ above sea level, or $5.60 \mathrm{ft}$ below City of Madison datum. Prior to Oct. 1, 1979, datum $3.61 \mathrm{ft}$ higher; prior to Nov. 15, 1971, nonrecording gage at same site.

REMARKS.--No estimated daily gage heights. Records good. Lake level regulated by concrete dam with four 12 -foot stop-log sections and 12-foot lock at outlet of Lake Waubesa. Gage-height telemeter at station.

EXTREMES FOR PERIOD OF RECORD.--Maximum gage height observed, $7.28 \mathrm{ft}$, June 19, 1996; minimum observed, $3.22 \mathrm{ft}$, Jan. 20 , 1965, current datum.

EXTREMES FOR CURRENT YEAR.--Maximum recorded gage height, $5.99 \mathrm{ft}$, June 13, 14, July 25-27, and 31; minimum recorded, $3.79 \mathrm{ft}$, Mar. 26.

GAGE HEIGHT, FEET, WATER YEAR OCTOBER 1998 TO SEPTEMBER 1999

DAILY MEAN VALUES

\begin{tabular}{|c|c|c|c|c|c|c|c|c|c|c|c|c|}
\hline DAY & OCT & NOV & $\mathrm{DEC}$ & JAN & $\mathrm{F} E \mathrm{~B}$ & MAR & APR & MAY & $\pi N$ & JUL & AUG & SEP \\
\hline $\begin{array}{l}1 \\
2 \\
3 \\
4 \\
5\end{array}$ & $\begin{array}{l}4.93 \\
4.90 \\
4.90 \\
4.89 \\
4.97\end{array}$ & $\begin{array}{l}5.20 \\
5.20 \\
5.18 \\
5.15 \\
5.14\end{array}$ & $\begin{array}{l}4.46 \\
4.44 \\
4.42 \\
4.41 \\
4.39\end{array}$ & $\begin{array}{l}4.00 \\
4.03 \\
4.07 \\
4.07 \\
4.07\end{array}$ & $\begin{array}{l}4.65 \\
4.68 \\
4.71 \\
4.73 \\
4.75\end{array}$ & $\begin{array}{l}4.88 \\
4.85 \\
4.74 \\
4.66 \\
4.61\end{array}$ & $\begin{array}{l}4.07 \\
4.10 \\
4.20 \\
4.31 \\
4.38\end{array}$ & $\begin{array}{l}5.47 \\
5.41 \\
5.35 \\
5.31 \\
5.26\end{array}$ & $\begin{array}{l}5.56 \\
5.62 \\
5.61 \\
5.60 \\
5.58\end{array}$ & $\begin{array}{l}5.83 \\
5.82 \\
5.81 \\
5.79 \\
5.77\end{array}$ & $\begin{array}{l}5.94 \\
5.89 \\
5.86 \\
5.86 \\
5.84\end{array}$ & $\begin{array}{l}5.76 \\
5.75 \\
5.74 \\
5.73 \\
5.71\end{array}$ \\
\hline $\begin{array}{r}6 \\
7 \\
8 \\
9 \\
10\end{array}$ & $\begin{array}{l}5.06 \\
5.06 \\
5.11 \\
5.14 \\
5.17\end{array}$ & $\begin{array}{l}5.12 \\
5.12 \\
5.12 \\
5.14 \\
5.33\end{array}$ & $\begin{array}{l}4.38 \\
4.38 \\
4.36 \\
4.34 \\
4.31\end{array}$ & $\begin{array}{l}4.07 \\
4.07 \\
4.07 \\
4.07 \\
4.07\end{array}$ & $\begin{array}{l}4.76 \\
4.77 \\
4.78 \\
4.79 \\
4.80\end{array}$ & $\begin{array}{l}4.54 \\
4.47 \\
4.43 \\
4.40 \\
4.34\end{array}$ & $\begin{array}{l}4.43 \\
4.48 \\
4.57 \\
4.86 \\
5.00\end{array}$ & $\begin{array}{l}5.27 \\
5.27 \\
5.21 \\
5.15 \\
5.11\end{array}$ & $\begin{array}{l}5.60 \\
5.64 \\
5.65 \\
5.68 \\
5.72\end{array}$ & $\begin{array}{l}5.75 \\
5.71 \\
5.69 \\
5.68 \\
5.64\end{array}$ & $\begin{array}{l}5.81 \\
5.83 \\
5.82 \\
5.80 \\
5.82\end{array}$ & $\begin{array}{l}5.68 \\
5.67 \\
5.64 \\
5.60 \\
5.55\end{array}$ \\
\hline $\begin{array}{l}11 \\
12 \\
13 \\
14 \\
15\end{array}$ & $\begin{array}{l}5.19 \\
5.20 \\
5.19 \\
5.19 \\
5.20\end{array}$ & $\begin{array}{l}5.35 \\
5.28 \\
5.20 \\
5.13 \\
5.07\end{array}$ & $\begin{array}{l}4.29 \\
4.28 \\
4.27 \\
4.26 \\
4.24\end{array}$ & $\begin{array}{l}4.07 \\
4.07 \\
4.07 \\
4.06 \\
4.06\end{array}$ & $\begin{array}{l}4.87 \\
4.99 \\
5.00 \\
5.02 \\
5.02\end{array}$ & $\begin{array}{l}4.30 \\
4.25 \\
4.21 \\
4.18 \\
4.14\end{array}$ & $\begin{array}{l}5.03 \\
5.05 \\
5.08 \\
5.12 \\
5.14\end{array}$ & $\begin{array}{l}5.09 \\
5.11 \\
5.10 \\
5.10 \\
5.14\end{array}$ & $\begin{array}{l}5.83 \\
5.86 \\
5.95 \\
5.97 \\
5.95\end{array}$ & $\begin{array}{l}5.61 \\
5.59 \\
5.56 \\
5.54 \\
5.52\end{array}$ & $\begin{array}{l}5.81 \\
5.83 \\
5.83 \\
5.81 \\
5.80\end{array}$ & $\begin{array}{l}5.52 \\
5.49 \\
5.44 \\
5.39 \\
5.36\end{array}$ \\
\hline $\begin{array}{l}16 \\
17 \\
18 \\
19 \\
20\end{array}$ & $\begin{array}{l}5.20 \\
5.26 \\
5.33 \\
5.31 \\
5.28\end{array}$ & $\begin{array}{l}5.02 \\
4.96 \\
4.92 \\
4.84 \\
4.79\end{array}$ & $\begin{array}{l}4.20 \\
4.17 \\
4.16 \\
4.12 \\
4.10\end{array}$ & $\begin{array}{l}4.06 \\
4.07 \\
4.09 \\
4.09 \\
4.12\end{array}$ & $\begin{array}{l}5.01 \\
4.99 \\
4.98 \\
4.97 \\
4.95\end{array}$ & $\begin{array}{l}4.11 \\
4.05 \\
4.01 \\
3.99 \\
3.96\end{array}$ & $\begin{array}{l}5.13 \\
5.12 \\
5.12 \\
5.13 \\
5.14\end{array}$ & $\begin{array}{l}5.21 \\
5.62 \\
5.73 \\
5.78 \\
5.80\end{array}$ & $\begin{array}{l}5.93 \\
5.90 \\
5.90 \\
5.89 \\
5.86\end{array}$ & $\begin{array}{l}5.50 \\
5.76 \\
5.84 \\
5.85 \\
5.87\end{array}$ & $\begin{array}{l}5.79 \\
5.78 \\
5.78 \\
5.80 \\
5.80\end{array}$ & $\begin{array}{l}5.33 \\
5.31 \\
5.28 \\
5.25 \\
5.22\end{array}$ \\
\hline $\begin{array}{l}21 \\
22 \\
23 \\
24 \\
25\end{array}$ & $\begin{array}{l}5.25 \\
5.23 \\
5.21 \\
5.19 \\
5.18\end{array}$ & $\begin{array}{l}4.75 \\
4.72 \\
4.67 \\
4.64 \\
4.60\end{array}$ & $\begin{array}{l}4.06 \\
4.04 \\
4.05 \\
4.05 \\
4.05\end{array}$ & $\begin{array}{l}4.14 \\
4.23 \\
4.31 \\
4.37 \\
4.40\end{array}$ & $\begin{array}{l}4.94 \\
4.93 \\
4.92 \\
4.91 \\
4.91\end{array}$ & $\begin{array}{l}3.91 \\
3.88 \\
3.86 \\
3.83 \\
3.81\end{array}$ & $\begin{array}{l}5.18 \\
5.37 \\
5.82 \\
5.92 \\
5.89\end{array}$ & $\begin{array}{l}5.81 \\
5.79 \\
5.76 \\
5.70 \\
5.65\end{array}$ & $\begin{array}{l}5.84 \\
5.83 \\
5.89 \\
5.90 \\
5.87\end{array}$ & $\begin{array}{l}5.91 \\
5.89 \\
5.88 \\
5.88 \\
5.89\end{array}$ & $\begin{array}{l}5.79 \\
5.78 \\
5.81 \\
5.88 \\
5.88\end{array}$ & $\begin{array}{l}5.17 \\
5.12 \\
5.08 \\
5.04 \\
5.01\end{array}$ \\
\hline $\begin{array}{l}26 \\
27 \\
28 \\
29 \\
30 \\
31\end{array}$ & $\begin{array}{l}5.16 \\
5.18 \\
5.20 \\
5.20 \\
5.18 \\
5.19\end{array}$ & $\begin{array}{r}4.56 \\
4.53 \\
4.51 \\
4.49 \\
4.48 \\
---\end{array}$ & $\begin{array}{l}4.04 \\
4.04 \\
4.04 \\
4.03 \\
4.01 \\
4.01\end{array}$ & $\begin{array}{l}4.45 \\
4.50 \\
4.54 \\
4.58 \\
4.60 \\
4.63\end{array}$ & $\begin{array}{r}4.91 \\
4.90 \\
4.89 \\
--- \\
--- \\
---\end{array}$ & $\begin{array}{l}3.80 \\
3.85 \\
3.89 \\
3.92 \\
3.96 \\
4.02\end{array}$ & $\begin{array}{l}5.83 \\
5.78 \\
5.73 \\
5.64 \\
5.55 \\
\ldots \ldots\end{array}$ & $\begin{array}{l}5.62 \\
5.59 \\
5.57 \\
5.56 \\
5.56 \\
5.55\end{array}$ & $\begin{array}{l}5.85 \\
5.83 \\
5.82 \\
5.82 \\
5.79 \\
\ldots\end{array}$ & $\begin{array}{l}5.98 \\
5.99 \\
5.97 \\
5.94 \\
5.93 \\
5.97\end{array}$ & $\begin{array}{l}5.87 \\
5.86 \\
5.86 \\
5.85 \\
5.81 \\
5.78\end{array}$ & $\begin{array}{l}4.98 \\
5.04 \\
5.10 \\
5.09 \\
5.03 \\
\end{array}$ \\
\hline $\begin{array}{l}\text { MEAN } \\
\text { MAX } \\
\text { MIN }\end{array}$ & $\begin{array}{l}5.15 \\
5.33 \\
4.89\end{array}$ & $\begin{array}{l}4.94 \\
5.35 \\
4.48\end{array}$ & $\begin{array}{l}4.21 \\
4.46 \\
4.01\end{array}$ & $\begin{array}{l}4.20 \\
4.63 \\
4.00\end{array}$ & $\begin{array}{l}4.88 \\
5.02 \\
4.65\end{array}$ & $\begin{array}{l}4.19 \\
4.88 \\
3.80\end{array}$ & $\begin{array}{l}5.07 \\
5.92 \\
4.07\end{array}$ & $\begin{array}{l}5.44 \\
5.81 \\
5.09\end{array}$ & $\begin{array}{l}5.79 \\
5.97 \\
5.56\end{array}$ & $\begin{array}{l}5.79 \\
5.99 \\
5.50\end{array}$ & $\begin{array}{l}5.83 \\
5.94 \\
5.78\end{array}$ & $\begin{array}{l}5.37 \\
5.76 \\
4.98\end{array}$ \\
\hline
\end{tabular}


LOCATION.--Lat $42^{\circ} 53^{\prime} 44^{\prime \prime}$, long $88^{\circ} 07^{\prime} 01^{\prime \prime}$, in SW 1/4 NE 1/4 sec.15, T.5 N., R.20 E., Waukesha County, Hydrologic Unit $07120006,1.3$ mi southeast of Muskego.

PERIOD OF RECORD.--February 1988 to current year.

REMARKS.--Lake sampled near center at the deep hole. Lake ice-covered during February sampling. Water-quality analyses done by Wisconsin State Laboratory of Hygiene.

WATER-QUALITY DATA, FEBRUARY 11 TO JUNE 08, 1999

(Milligrams per liter unless otherwise indicated)

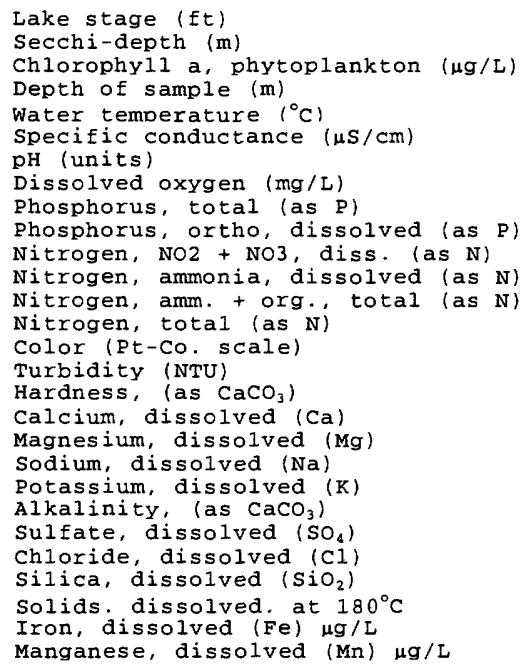

Apr -21

Feb-11

11.68

4.6

11.87

\begin{tabular}{cc}
0.5 & 7.0 \\
4.7 & 3.6 \\
600 & 715 \\
7.7 & 7.5 \\
12.8 & 9.6 \\
0.034 & 0.060 \\
\hline- & -
\end{tabular}

2.27

0.5
10.0

10.0

8.0

10.7

0.019

$<0.002$

0.558

0.078

0.85

1.4

20

0.9

260

260
54

54
30
26

26
2.8

2.8

183

65
61

0.31

0.31
432

$<10$

6.8

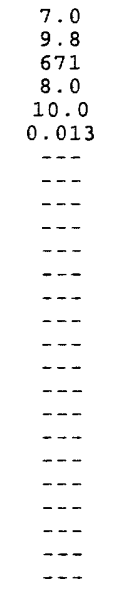

Jun -8

11.82

3.4

\begin{tabular}{|c|c|}
\hline \multicolumn{2}{|c|}{1.83} \\
\hline $\begin{array}{c}0.5 \\
25.2\end{array}$ & 7.0 \\
\hline 583 & 633 \\
\hline 8.6 & 7.5 \\
\hline 8.7 & 0.6 \\
\hline 0.013 & 0.030 \\
\hline-- & $\ldots$ \\
\hline$\cdots$ & -- \\
\hline-- & $-\ldots$ \\
\hline$\ldots$ & -- \\
\hline-- & -- \\
\hline-- & - - \\
\hline--- & $\cdots$ \\
\hline-- & -- \\
\hline-- & $\cdots$ \\
\hline--- & -- \\
\hline$\cdots$ & $\cdots$ \\
\hline-- & -- \\
\hline$\ldots$ & $\ldots$ \\
\hline-- & $\ldots$ \\
\hline$\cdots$ & $\ldots$ \\
\hline- & -- \\
\hline -. & -- \\
\hline-- & -- \\
\hline
\end{tabular}
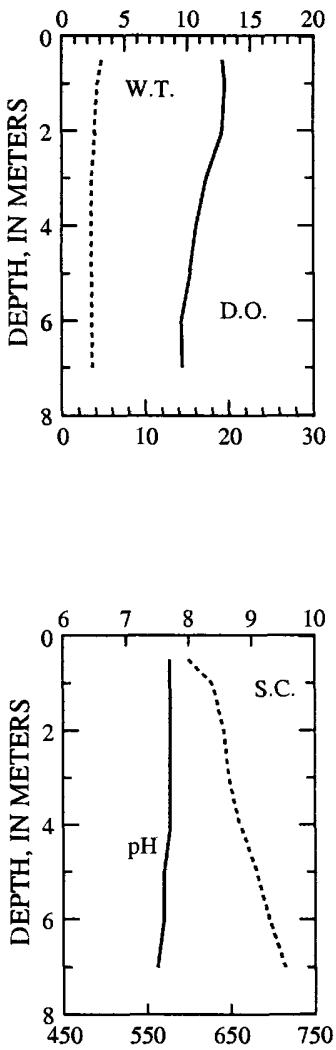

DISSOLVED OXYGEN (D.O.), IN MILLIGRAMS PER LITER
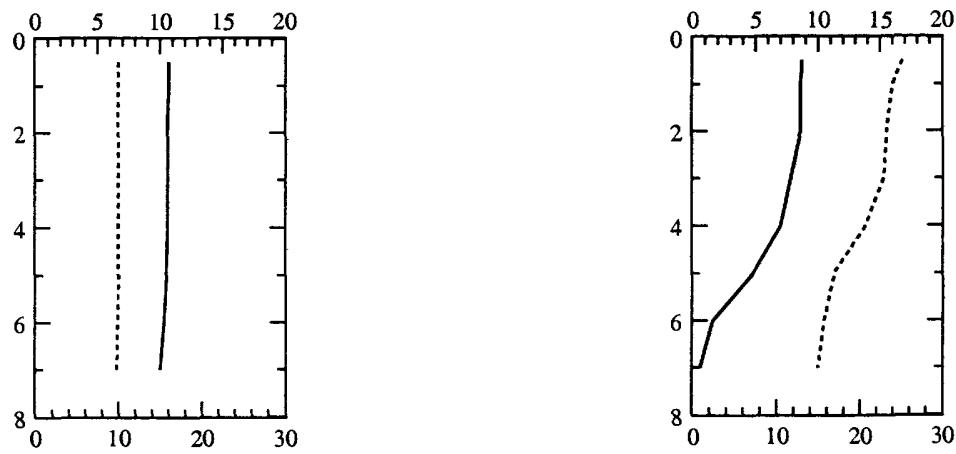

WATER TEMPERATURE (W.T.), IN DEGREES CELSIUS

PH, IN STANDARD UNITS

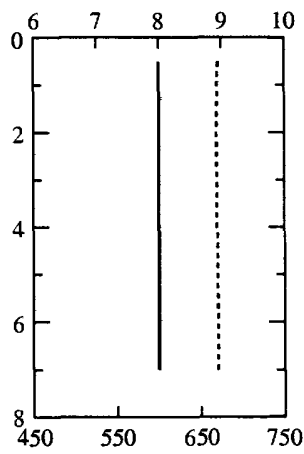

6-08-99 
WATER-QUALITY DATA, JULY 06 TO AUGUST 04, 1999

(Milligrams per liter unless otherwise indicated)

Lake stage (ft)

Secchi-depth (m)

Chlorophyll a, phytoplankton $(\mu \mathrm{g} / \mathrm{L})$

Depth of sample (m)

water temoerature $\left({ }^{\circ} \mathrm{C}\right.$

Specific conductance $(\mu \mathrm{S} / \mathrm{cm})$

$\mathrm{pH}$ (units)

Dissolved oxygen (mg/L)

Phosphorus, tota1 (as P)

\begin{tabular}{|c|c|}
\hline \multicolumn{2}{|c|}{ Jul-6 } \\
\hline & \\
\hline $\begin{array}{c}0.5 \\
27.5 \\
523 \\
8.4 \\
9.9 \\
0.030\end{array}$ & $\begin{array}{c}7.5 \\
15.4 \\
645 \\
7.3 \\
0.4 \\
0.150\end{array}$ \\
\hline
\end{tabular}

7-06-99

8-04-99

DISSOLVED OXYGEN (D.O.), IN MILLIGRAMS PER LITER
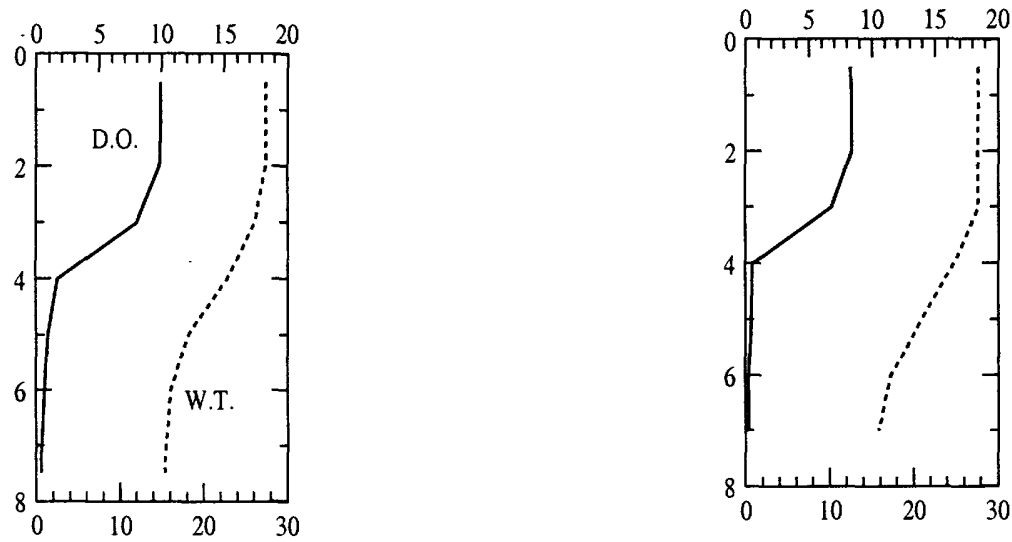

WATER TEMPERATURE (W.T.), IN DEGREES CELCIUS

PH, IN STANDARD UNITS
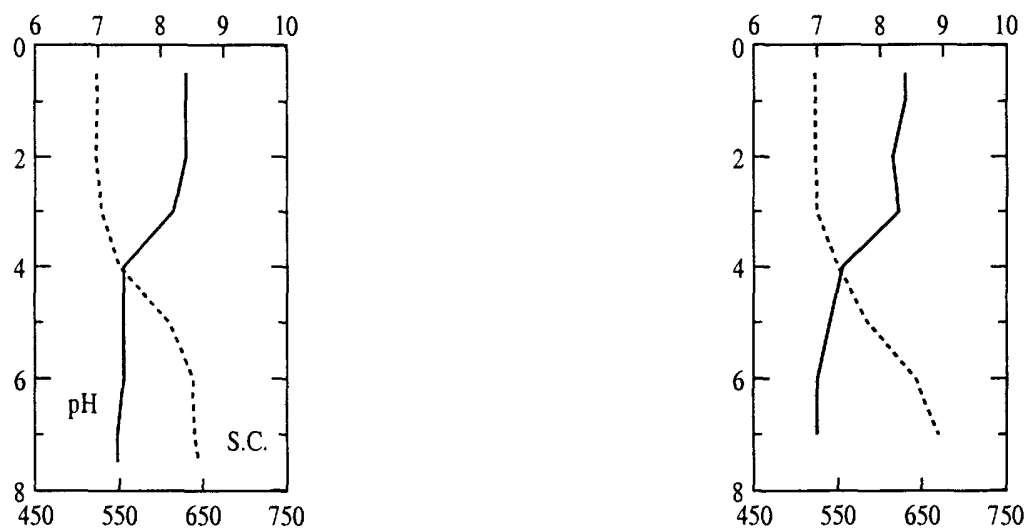

SPECIFIC CONDUCTANCE (S.C.), IN MICROSIEMENS PER CENTIMETER AT 25 DEGREES CELCIUS 

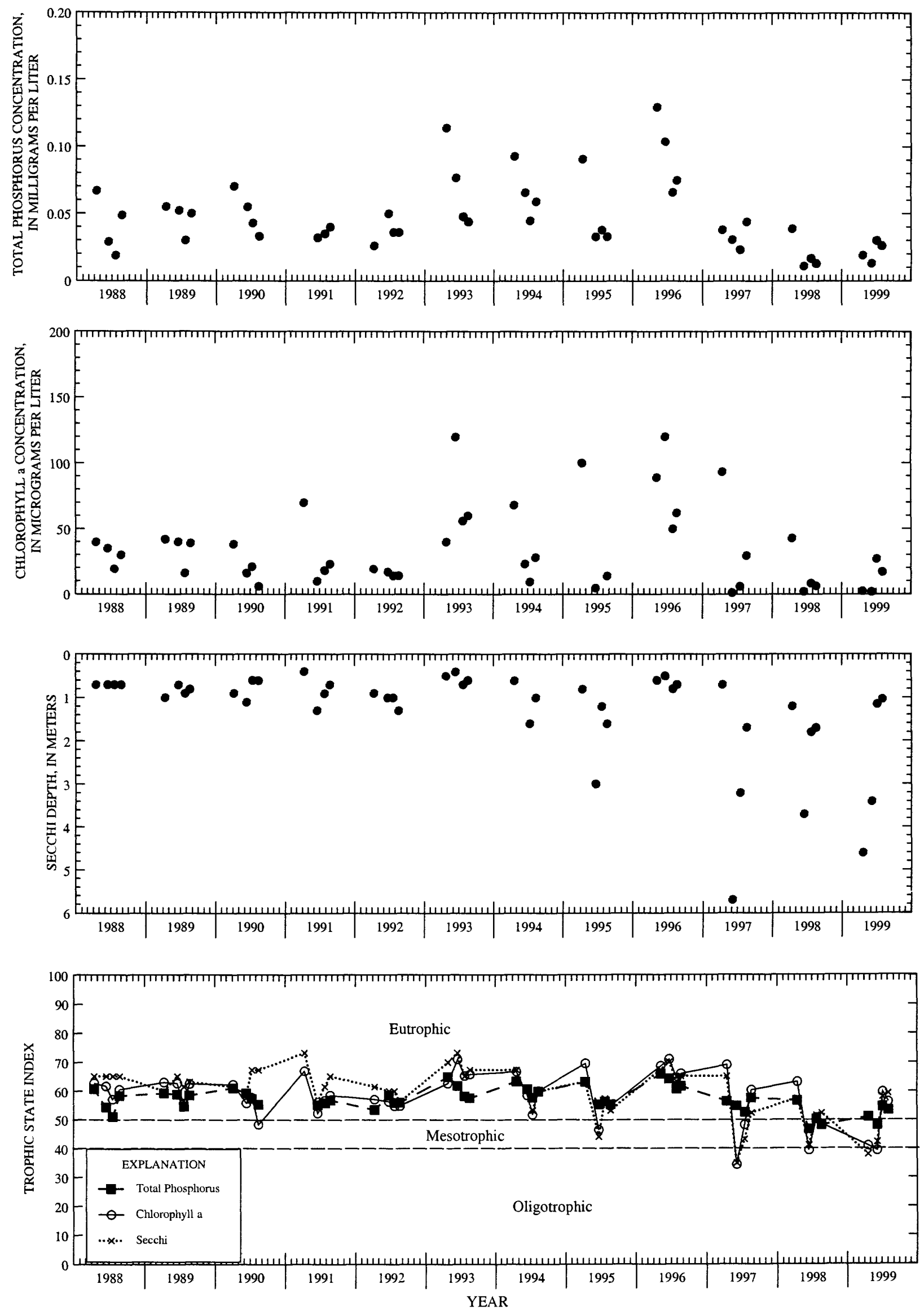

Surface total phosphorus, chlorophyll a concentrations, Secchi depths, and TSI data for Big Muskego Lake, Bass Bay, near Muskego, Wisconsin. 
LOCATION.--Lat 42 $51^{\prime} 09^{\prime \prime}$, long $88^{\circ} 07^{\prime} 50^{\prime \prime}$, in SE 1/4 NE 1/4 sec.33, T.5 N., R.20 E., Waukesha County, Hydrologic Unit 07120006, on left bank $8 \mathrm{ft}$ upstream of dam outlet of Muskego Lake, $700 \mathrm{ft}$ north of Muskego Dam Drive, 2 mi northeast of Wind Lake.

DRAINAGE AREA.--28.3 $\mathrm{mi}^{2}$.

PERIOD OF RECORD.--October 1987 to September 1989, January 1991 to current year.

GAGE.--Water-stage recorder. Datum of gage is $760.00 \mathrm{ft}$ above sea level. October to December 1987 and January 1991 to September 1995 , nonrecording gage at the same datum. December 1987 through September 1989, data collected using water-stage recorder at the same datum.

REMARKS.--Estimated daily gage heights: Dec. 17-20, May 20-26, and June 4, 5. Lake levels regulated by concrete dam with one 5-ft lift gate. Prior to October 1993, published as Muskego Lake Outlet near Wind Lake, WI.

EXTREMES FOR PERIOD OF RECORD.--Maximum observed gage height, $12.60 \mathrm{ft}$, Oct. 7, 1991 and Aug. 8, 1994; minimum instantaneous, less than $8.72 \mathrm{ft}$, July 12, 1996 to Feb. 18, I997, due to drawdown of lake.

EXTREMES FOR CURRENT YEAR.--Maximum observed gage-height, $12.49 \mathrm{ft}$, June 15; minimum observed, $11.01 \mathrm{ft}$, Sept. 26.

GAGE HEIGHT, FEET, WATER YEAR OCTOBER 1998 TO SEPTEMBER 1999

DAILY MEAN VALUES

\begin{tabular}{|c|c|c|c|c|c|c|c|c|c|c|c|c|}
\hline DAY & OCT & NOV & DEC & JAN & FEB & MAR & APR & MAY & JUN & JUL & AUG & SEP \\
\hline $\begin{array}{l}1 \\
2 \\
3 \\
4 \\
5\end{array}$ & $\begin{array}{l}11.61 \\
11.60 \\
11.64 \\
11.65 \\
11.68\end{array}$ & $\begin{array}{l}11.68 \\
11.71 \\
11.68 \\
11.64 \\
11.63\end{array}$ & $\begin{array}{l}11.66 \\
11.67 \\
11.68 \\
11.71 \\
11.70\end{array}$ & $\begin{array}{l}11.67 \\
11.71 \\
11.74 \\
11.73 \\
11.72\end{array}$ & $\begin{array}{l}11.95 \\
11.95 \\
11.95 \\
11.97 \\
11.96\end{array}$ & $\begin{array}{l}11.77 \\
11.78 \\
11.79 \\
11.78 \\
11.80\end{array}$ & $\begin{array}{l}11.74 \\
11.73 \\
11.76 \\
11.76 \\
11.76\end{array}$ & $\begin{array}{l}11.96 \\
11.90 \\
11.86 \\
11.84 \\
11.82\end{array}$ & $\begin{array}{l}11.87 \\
11.81 \\
11.82 \\
11.81 \\
11.79\end{array}$ & $\begin{array}{l}11.83 \\
11.82 \\
11.70 \\
11.68 \\
11.68\end{array}$ & $\begin{array}{l}11.54 \\
11.52 \\
11.48 \\
11.48 \\
11.47\end{array}$ & $\begin{array}{l}11 \cdot 34 \\
11.33 \\
11.32 \\
11.32 \\
11.30\end{array}$ \\
\hline $\begin{array}{r}6 \\
7 \\
8 \\
9 \\
10\end{array}$ & $\begin{array}{l}11.71 \\
11.72 \\
11.73 \\
11.72 \\
11.71\end{array}$ & $\begin{array}{l}11.62 \\
11.61 \\
11.62 \\
11.63 \\
11.57\end{array}$ & $\begin{array}{l}11.72 \\
11.76 \\
11.74 \\
11.73 \\
11.74\end{array}$ & $\begin{array}{l}11.72 \\
11.71 \\
11.71 \\
11.71 \\
11.71\end{array}$ & $\begin{array}{l}11.94 \\
11.92 \\
11.88 \\
11.87 \\
11.86\end{array}$ & $\begin{array}{l}11.82 \\
11.78 \\
11.78 \\
11.80 \\
11.81\end{array}$ & $\begin{array}{l}11.66 \\
11.68 \\
11.77 \\
11.97 \\
11.86\end{array}$ & $\begin{array}{l}11.86 \\
11.85 \\
11.92 \\
11.93 \\
11.92\end{array}$ & $\begin{array}{l}11.77 \\
11.81 \\
11.82 \\
11.79 \\
11.79\end{array}$ & $\begin{array}{l}11.69 \\
11.68 \\
11.65 \\
11.65 \\
11.65\end{array}$ & $\begin{array}{l}11.45 \\
11.44 \\
11.47 \\
11.43 \\
11.47\end{array}$ & $\begin{array}{l}11.30 \\
11.27 \\
11.25 \\
11.22 \\
11.20\end{array}$ \\
\hline $\begin{array}{l}11 \\
12 \\
13 \\
14 \\
15\end{array}$ & $\begin{array}{l}11.70 \\
11.69 \\
11.68 \\
11.68 \\
11.66\end{array}$ & $\begin{array}{l}11.81 \\
11.88 \\
11.86 \\
11.84 \\
11.82\end{array}$ & $\begin{array}{l}11.71 \\
11.72 \\
11.73 \\
11.72 \\
11.70\end{array}$ & $\begin{array}{l}11.71 \\
11.71 \\
11.72 \\
11.71 \\
11.71\end{array}$ & $\begin{array}{l}11.87 \\
11.95 \\
11.96 \\
11.94 \\
11.93\end{array}$ & $\begin{array}{l}11.81 \\
11.81 \\
11.81 \\
11.81 \\
11.80\end{array}$ & $\begin{array}{l}11.85 \\
11.81 \\
11.79 \\
11.76 \\
11.74\end{array}$ & $\begin{array}{l}11.90 \\
12.00 \\
12.00 \\
11.94 \\
11.88\end{array}$ & $\begin{array}{l}11.85 \\
11.92 \\
12.15 \\
12.40 \\
12.47\end{array}$ & $\begin{array}{l}11.61 \\
11.59 \\
11.56 \\
11.52 \\
11.51\end{array}$ & $\begin{array}{l}11.47 \\
11.46 \\
11.47 \\
11.47 \\
11.43\end{array}$ & $\begin{array}{l}11.21 \\
11.19 \\
11.19 \\
11.18 \\
11.19\end{array}$ \\
\hline $\begin{array}{l}16 \\
17 \\
18 \\
19 \\
20\end{array}$ & $\begin{array}{l}11.67 \\
11.69 \\
11.77 \\
11.81 \\
11.81\end{array}$ & $\begin{array}{l}11.81 \\
11.78 \\
11.74 \\
11.67 \\
11.69\end{array}$ & $\begin{array}{l}11.72 \\
11.72 \\
11.72 \\
11.71 \\
11.71\end{array}$ & $\begin{array}{l}11.71 \\
11.72 \\
11.75 \\
11.74 \\
11.73\end{array}$ & $\begin{array}{l}11.91 \\
11.88 \\
11.84 \\
11.81 \\
11.77\end{array}$ & $\begin{array}{l}11.81 \\
11.78 \\
11.87 \\
11.88 \\
11.85\end{array}$ & $\begin{array}{l}11.79 \\
11.68 \\
11.64 \\
11.63 \\
11.63\end{array}$ & $\begin{array}{l}11.86 \\
11.91 \\
11.99 \\
12.03 \\
12.02\end{array}$ & $\begin{array}{l}12.43 \\
12.35 \\
12.26 \\
12.18 \\
12.11\end{array}$ & $\begin{array}{l}11.49 \\
11.58 \\
11.60 \\
11.61 \\
11.61\end{array}$ & $\begin{array}{l}11.40 \\
11.41 \\
11.41 \\
11.45 \\
11.43\end{array}$ & $\begin{array}{l}11.19 \\
11.17 \\
11.16 \\
11.15 \\
11.21\end{array}$ \\
\hline $\begin{array}{l}21 \\
22 \\
23 \\
24 \\
25\end{array}$ & $\begin{array}{l}11.81 \\
11.77 \\
11.72 \\
11.68 \\
11.65\end{array}$ & $\begin{array}{l}11.68 \\
11.63 \\
11.66 \\
11.69 \\
11.68\end{array}$ & $\begin{array}{l}11.70 \\
11.70 \\
11.70 \\
11.69 \\
11.69\end{array}$ & $\begin{array}{l}11.73 \\
11.85 \\
11.95 \\
12.00 \\
12.02\end{array}$ & $\begin{array}{l}11.73 \\
11.69 \\
11.67 \\
11.69 \\
11.71\end{array}$ & $\begin{array}{l}11.88 \\
11.84 \\
11.80 \\
11.83 \\
11.81\end{array}$ & $\begin{array}{l}11.68 \\
11.82 \\
12.14 \\
12.18 \\
12.22\end{array}$ & $\begin{array}{l}11.99 \\
11.96 \\
11.93 \\
11.90 \\
11.87\end{array}$ & $\begin{array}{l}12.04 \\
11.98 \\
11.93 \\
11.90 \\
11.90\end{array}$ & $\begin{array}{l}11.61 \\
11.61 \\
11.62 \\
11.61 \\
11.61\end{array}$ & $\begin{array}{l}11.41 \\
11.40 \\
11.41 \\
11.45 \\
11.45\end{array}$ & $\begin{array}{l}11.19 \\
11.16 \\
11.16 \\
11.17 \\
11.15\end{array}$ \\
\hline $\begin{array}{l}26 \\
27 \\
28 \\
29 \\
30 \\
31\end{array}$ & $\begin{array}{l}11.64 \\
11.64 \\
11.66 \\
11.65 \\
11.66 \\
11.67\end{array}$ & $\begin{array}{r}11.68 \\
11.68 \\
11.68 \\
11.65 \\
11.68 \\
---\end{array}$ & $\begin{array}{l}11.69 \\
11.68 \\
11.68 \\
11.68 \\
11.68 \\
11.67\end{array}$ & $\begin{array}{l}12.02 \\
12.02 \\
12.02 \\
12.01 \\
11.99 \\
11.97\end{array}$ & $\begin{array}{r}11.73 \\
11.75 \\
11.76 \\
-.- \\
-.- \\
---\end{array}$ & $\begin{array}{l}11.78 \\
11.76 \\
11.72 \\
11.74 \\
11.71 \\
11.66\end{array}$ & $\begin{array}{r}12.22 \\
12.21 \\
12.19 \\
12.09 \\
12.01 \\
\quad---\end{array}$ & $\begin{array}{l}11.84 \\
11.80 \\
11.79 \\
11.77 \\
11.75 \\
11.73\end{array}$ & $\begin{array}{r}11.87 \\
11.86 \\
11.84 \\
11.84 \\
11.84 \\
\ldots\end{array}$ & $\begin{array}{l}11.60 \\
11.60 \\
11.57 \\
11.54 \\
11.54 \\
11.54\end{array}$ & $\begin{array}{l}11.43 \\
11.41 \\
11.41 \\
11.42 \\
11.37 \\
11.35\end{array}$ & $\begin{array}{r}11.10 \\
11.17 \\
11.45 \\
11.51 \\
11.49 \\
\end{array}$ \\
\hline $\begin{array}{l}\text { MEAN } \\
\text { MAX } \\
\text { MIN }\end{array}$ & $\begin{array}{l}11.69 \\
11.81 \\
11.60\end{array}$ & $\begin{array}{l}11.70 \\
11.88 \\
11.57\end{array}$ & $\begin{array}{l}11.70 \\
11.76 \\
11.66\end{array}$ & $\begin{array}{l}11.80 \\
12.02 \\
11.67\end{array}$ & $\begin{array}{l}11.85 \\
11.97 \\
11.67\end{array}$ & $\begin{array}{l}11.80 \\
11.88 \\
11.66\end{array}$ & $\begin{array}{l}11.86 \\
12.22 \\
11.63\end{array}$ & $\begin{array}{l}11.89 \\
12.03 \\
11.73\end{array}$ & $\begin{array}{l}11.97 \\
12.47 \\
11.77\end{array}$ & $\begin{array}{l}11.62 \\
11.83 \\
11.49\end{array}$ & $\begin{array}{l}11.44 \\
11.54 \\
11.35\end{array}$ & $\begin{array}{l}11.24 \\
11.51 \\
11.10\end{array}$ \\
\hline
\end{tabular}



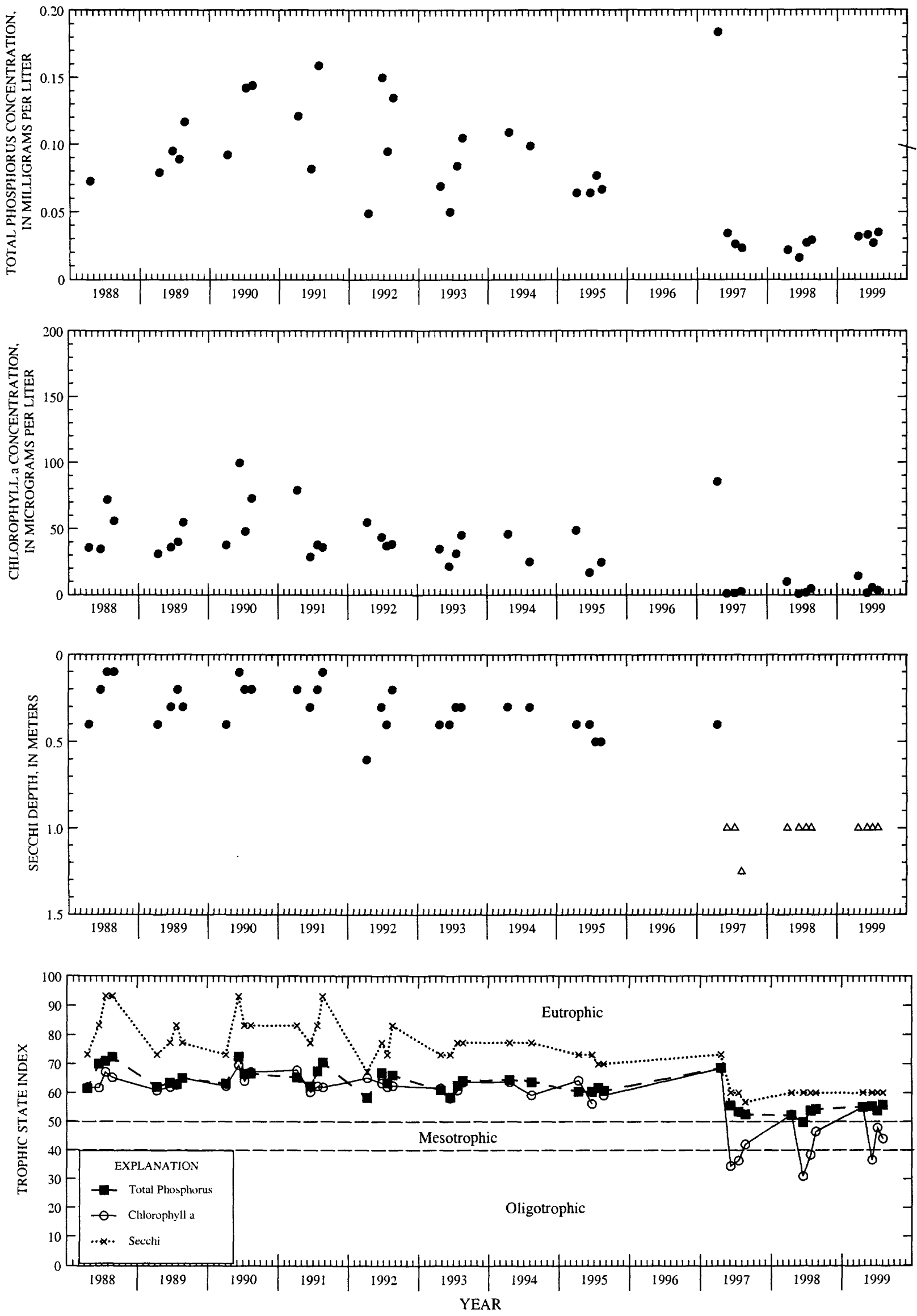

Surface total phosphorus, chlorophyll a concentrations, Secchi depths, and TSI data for Big Muskego Lake, South Site, near Muskego, Wisconsin. (Triangles indicate maximum depth at sampling site. Actual Secchi depth on these days was greater than plotted triangles.) 
LOCATION.--Lat $46^{\circ} 12^{\prime} 24^{\prime \prime}$, long $91^{\circ} 03 ' 32^{\prime \prime}$, in NW 1/4 SE 1/4 sec.13, T.43 N., R.6 W., Bayfield County, Hydrologic Unit 07030002, near Cable.

PERIOD OF RECORD.--March 1998 to current year.

REMARKS.--Lake sampled at deepest part of Garden Lake. Lake ice-covered during March measurements. Water-quality analyses done by Wisconsin State Laboratory of Hygiene.

WATER-QUALITY DATA, MARCH 04 TO AUGUST 17, 1999

(Milligrams per liter unless otherwise indicated)

Lake stage ( $\mathrm{ft}$ )

Secchi-depth (m)

Chlorophyll a, phytoplankton ( $\mu \mathrm{g} / \mathrm{L}$ )

Depth of sample (m)

Water temperature $\left({ }^{\circ} \mathrm{C}\right)$

Specific conductance ( $\mu \mathrm{S} / \mathrm{cm}$ )

$\mathrm{pH}$ (units)

Dissolved oxygen (mg/L)

Phosphorus, total (as P)

Phosphorus, ortho, dissolved (as P)

$\mathrm{Nitrogen,} \mathrm{NO} 2+\mathrm{NO} 3$, diss. (as N)

Nitrogen, ammonia, dissolved (as N)

Nitrogen, amm. + org., total (as N)

Nitrogen, total (as $N$ )

Color (Pt-Co. scale)

Turbidity (NTU)

Hardness, (as $\mathrm{CaCO}_{3}$ )

Calcium, dissolved ( $\mathrm{Ca}$

Magnesium, dissolved (Mg)

Sodium, dissolved (Na)

Potassium, dissolved $(K)$

Alkalinity, (as $\mathrm{CaCO}_{3}$ )

Sulfate, dissolved $\left(\mathrm{SO}_{4}\right)$

Chloride, dissolved (CI)

Silica, dissolved ( $\left.\mathrm{SiO}_{2}\right)$

Solids, dissolved, at $180^{\circ} \mathrm{C}$

Iron, dissolved ( $\mathrm{Fe}) \mu \mathrm{g} / \mathrm{L}$

Manganese, dissolved (Mn) $\mu \mathrm{g} / \mathrm{I}$

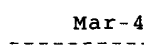

$\operatorname{Mar}-4$

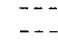

$-$

$0.5 \quad 6.0$

$\begin{array}{ll}0.2 & 4.7 \\ 100 & 126\end{array}$

$7.2 \quad 6.8$

$11.7 \quad 2.9$

$0.020 \quad 0.121$

-

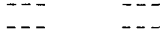

- -

$--\cdot \quad-\cdots$

- - -

--

‥

…

$\cdots$

$\cdots$

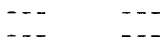

$\cdots$

$\cdots$

$\cdots$

\begin{tabular}{|c|c|c|c|}
\hline \multicolumn{2}{|c|}{ Apr -20} & \multicolumn{2}{|c|}{ Jun -2} \\
\hline \multicolumn{2}{|c|}{$\begin{array}{c}8.75 \\
2.2 \\
2.25\end{array}$} & \multicolumn{2}{|c|}{$\begin{array}{c}8.43 \\
1.8 \\
3.53\end{array}$} \\
\hline 0.5 & 6.0 & 0.5 & 6.0 \\
\hline 6.6 & 6.4 & 15.6 & 13.7 \\
\hline $\begin{array}{l}91 \\
7.1\end{array}$ & 7.1 & 7.7 & 7.0 \\
\hline 10.3 & 10.6 & 8.3 & 3.4 \\
\hline 0.022 & 0.021 & 0.026 & 0.028 \\
\hline 0.007 & $\cdots$ & --- & $\cdots$ \\
\hline 0.127 & -- & -- & $\ldots$ \\
\hline 0.013 & -- & $-\cdots$ & --- \\
\hline 0.39 & -- & --- & $\cdots$ \\
\hline 0.52 & -- & --- & --- \\
\hline 50 & $\cdots$ & --- & -- \\
\hline 2 & -- & $\cdots$ & $\cdots$ \\
\hline 45 & -- & -- & $\cdots$ \\
\hline 12 & -- & $\ldots$ & $\cdots$ \\
\hline 3.6 & -- & -- & --- \\
\hline 1.9 & -- & $\ldots$ & --- \\
\hline 0.9 & $\cdots$ & $\cdots$ & -- \\
\hline 41 & $\cdots$ & $\cdots$ & $\cdots$ \\
\hline$<45$ & $\ldots$ & $\cdots$ & -- \\
\hline 1.9 & -- & $\cdots$ & $\cdots$ \\
\hline 14 & -- & $\cdots$ & $\cdots$ \\
\hline 80 & $\ldots$ & $\ldots$ & $\cdots$ \\
\hline 330 & $\cdots$ & -- & $\ldots$ \\
\hline 180 & --- & $\cdots$ & $\cdots$ \\
\hline
\end{tabular}

\begin{tabular}{|c|c|c|c|}
\hline \multicolumn{2}{|c|}{ Ju 1-7 } & \multicolumn{2}{|c|}{ Aug - 17} \\
\hline \multicolumn{2}{|c|}{$\begin{array}{c}8.75 \\
1.8 \\
4.11\end{array}$} & \multicolumn{2}{|c|}{$\begin{array}{c}8.80 \\
1.1 \\
3.17\end{array}$} \\
\hline $\begin{array}{c}0.5 \\
22.4\end{array}$ & $\begin{array}{c}5.5 \\
19.9\end{array}$ & $\begin{array}{c}0.5 \\
20.5\end{array}$ & $\begin{array}{c}6.0 \\
19.9\end{array}$ \\
\hline $\begin{array}{l}87 \\
7.9\end{array}$ & $\begin{array}{c}91 \\
7.2\end{array}$ & $\begin{array}{c}83 \\
7.4\end{array}$ & \\
\hline $\begin{array}{l}8.1 \\
0.033\end{array}$ & $\begin{array}{c}4.2 \\
0.027\end{array}$ & $\begin{array}{l}7.4 \\
0.045\end{array}$ & $\begin{array}{c}5.5 \\
0.054\end{array}$ \\
\hline 0.004 & $\ldots$ & $\ldots$ & $\ldots$ \\
\hline 0.012 & -- & -- & $\cdots$ \\
\hline 0.019 & - - & $-\cdots$ & -- \\
\hline 0.59 & $\cdots$ & $\cdots$ & $\ldots$ \\
\hline 0.6 & -- & -- & --- \\
\hline$-\cdots$ & --- & --- & -- \\
\hline$\ldots$ & $\ldots$ & -- & --- \\
\hline$\ldots$ & --- & --- & --- \\
\hline$\cdots$ & --- & --- & -- \\
\hline--- & -- & $\ldots$ & $\cdots$ \\
\hline-- & - & $\ldots$ & $\cdots$ \\
\hline--- & -- & -- & -- \\
\hline-- & -- & $-\cdots$ & $\cdots$ \\
\hline$-\cdots$ & - & -- & -- \\
\hline-- & $\cdots$ & $-\cdots$ & -- \\
\hline$\cdots$ & -- & -- & -- \\
\hline-- & -- & -- & -- \\
\hline--- & --- & --- & $-\cdots$ \\
\hline$\cdots$ & --- & --- & $\cdots$ \\
\hline
\end{tabular}

7-07-99

DISSOLVED OXYGEN (D.O.), IN MILLIGRAMS PER LITER
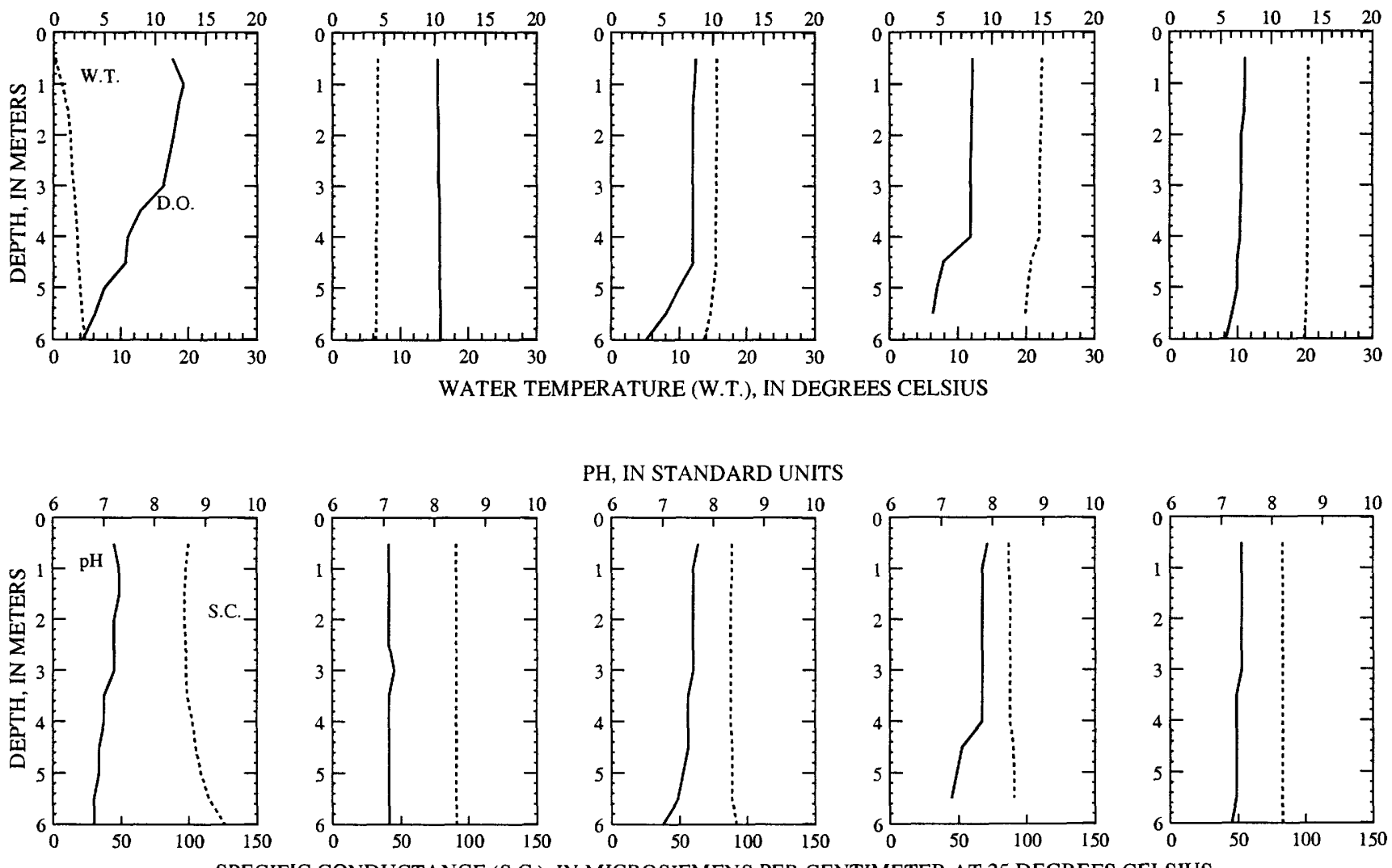

PH, IN STANDARD UNITS
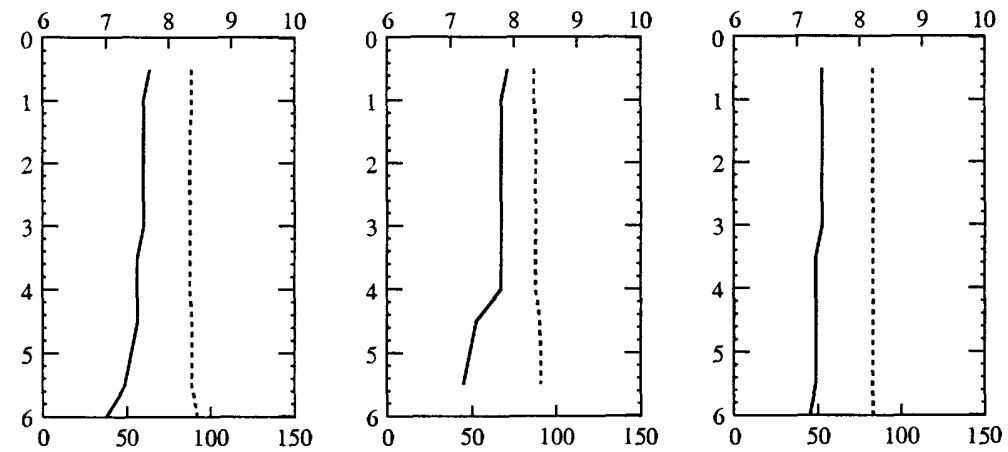

SPECIFIC CONDUCTANCE (S.C.), IN MICROSIEMENS PER CENTIMETER AT 25 DEGREES CELSIUS 

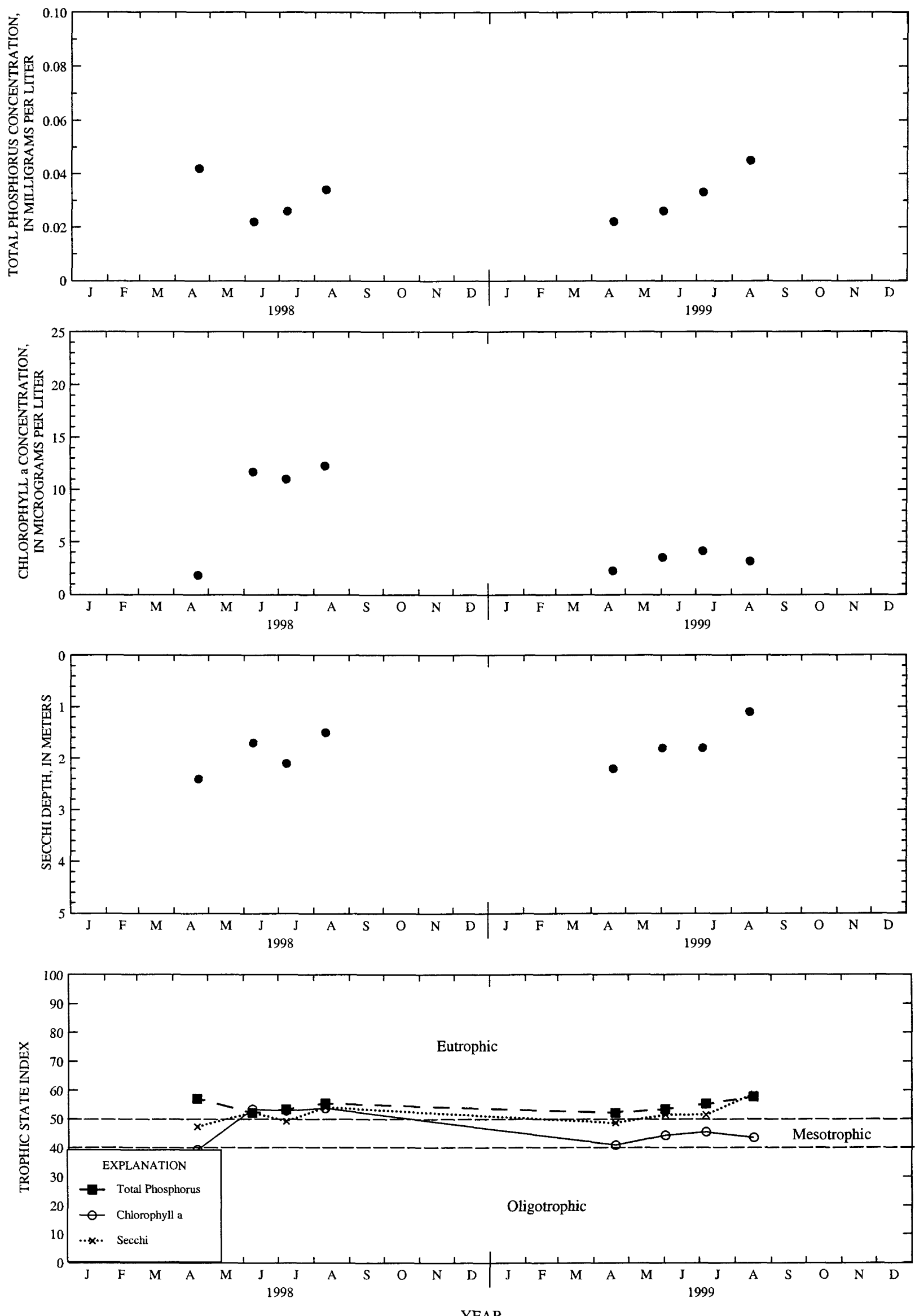

YEAR

Surface total phosphorus, chlorophyll a concentrations, Secchi depths, and TSI data for Namekagon Lake (Garden) near Cable, Wisconsin. 


\section{NAMEKAGON LAKES, JACKSON LAKE, NEAR CABLE, WI}

LOCATION.--Lat 46²'57", long 91 06'59", in NE 1/4 SE 1/4 sec.33, T.44 N., R.6 W., Bayfield County, Hydrologic Unit 07030002, near Cable.

PERIOD OF RECORD.--March 1998 to current year.

REMARKS.--Lake sampled at deepest part of Jackson Lake. Lake ice-covered during March measurements. Water-quality analyses done by Wisconsin State Laboratory of Hygiene.

WATER-QUALITY DATA, MARCH 03 TO AUGUST 17, 1999

(Milligrams per liter unless otherwise indicated)

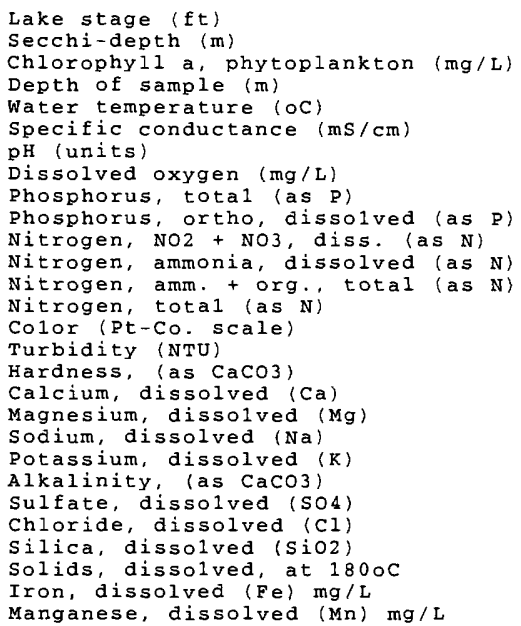

\begin{tabular}{|c|c|c|c|}
\hline \multicolumn{2}{|c|}{$\operatorname{Mar}-3$} & \multicolumn{2}{|c|}{ Apr -20} \\
\hline & & & \\
\hline 0.5 & 3.0 & 0.5 & 3.0 \\
\hline 0.0 & 4.6 & 8.4 & 7.1 \\
\hline 84 & 112 & 66 & 66 \\
\hline 6.4 & 6.6 & 7.0 & 6.8 \\
\hline 6.9 & 0.4 & 9.1 & 8.3 \\
\hline 0.039 & 0.061 & 0.041 & 0.039 \\
\hline$\ldots$ & $\ldots$ & 0.006 & $\ldots$ \\
\hline$\ldots$ & $-\ldots$ & 0.143 &.- \\
\hline$\ldots$ & $\ldots$ & 0.023 & $\ldots$ \\
\hline$\ldots$ & $\ldots$ & 0.72 & -- \\
\hline$\ldots$ & $\ldots$ & 0.86 & --- \\
\hline$-\ldots$ & $\ldots$ & 60 & -- \\
\hline$\cdots$ & -- & 3.9 & -- \\
\hline$\ldots$ & $\ldots$ & 30 & -- \\
\hline$\ldots$ & $\ldots$ & 8 & $\ldots$ \\
\hline$\ldots$ & -- & 2.5 & -- \\
\hline$\ldots$ & $\ldots$ & 2.3 & $\cdots$ \\
\hline-- & -- & 0.7 & -- \\
\hline$\cdots$ & -- & 27 & $\ldots$ \\
\hline$\ldots$ & $\ldots$ & 4.5 & -- \\
\hline$\cdots$ & $\cdots$ & 2.7 & -- \\
\hline$-\ldots$ & $\ldots$ & 9 & $\ldots$ \\
\hline-- & $-\cdots$ & 68 & $\cdots$ \\
\hline$\ldots$ & $\ldots$ & 880 & $\ldots$ \\
\hline-- & $\cdots$ & 78 & $\cdots$ \\
\hline
\end{tabular}

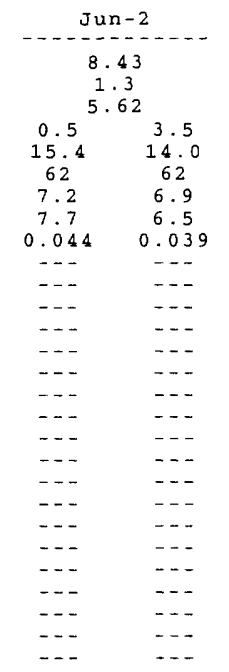

\begin{tabular}{|c|c|c|c|}
\hline & & & \\
\hline & & & \\
\hline 0.5 & 3.5 & 0.5 & \\
\hline 23.9 & 22.0 & 20.6 & 20.3 \\
\hline $\begin{array}{c}64 \\
7.4\end{array}$ & $\begin{array}{c}72 \\
6.7\end{array}$ & $\begin{array}{r}60 \\
7.2\end{array}$ & $\begin{array}{c}61 \\
7.0\end{array}$ \\
\hline 7.4 & 1.6 & 6.9 & 6.3 \\
\hline 0.034 & 0.037 & 0.062 & 0.054 \\
\hline 0.004 & $\cdots$ & -1 & -- \\
\hline $\begin{array}{c}<0.010 \\
0.016\end{array}$ & $\cdots$ & $\cdots$ & $\cdots$ \\
\hline 0.81 & -- & $\cdots$ & $\cdots$ \\
\hline 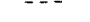 & - & & 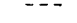 \\
\hline & 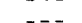 & - & \\
\hline$\ldots$ & & & \\
\hline$\ldots$ & $\ldots$ & - - & - \\
\hline-- & -- & $\cdots$ & -.. \\
\hline-- & $\cdots$ & $\cdots$ & -- \\
\hline$\cdots$ & -1 & $\cdots$ & $\ldots$ \\
\hline & $\cdots$ & $\ldots$ & \\
\hline & $\ldots$ & $\cdots$ & - \\
\hline - - - & $\cdots$ & & -. \\
\hline- & $\cdots$ & -- & $\cdots$ \\
\hline- & $\ldots$ & $\ldots$ & $\ldots$ \\
\hline
\end{tabular}

3-03-99

DISSOLVED OXYGEN (D.O.), IN MILLIGRAMS PER LITER
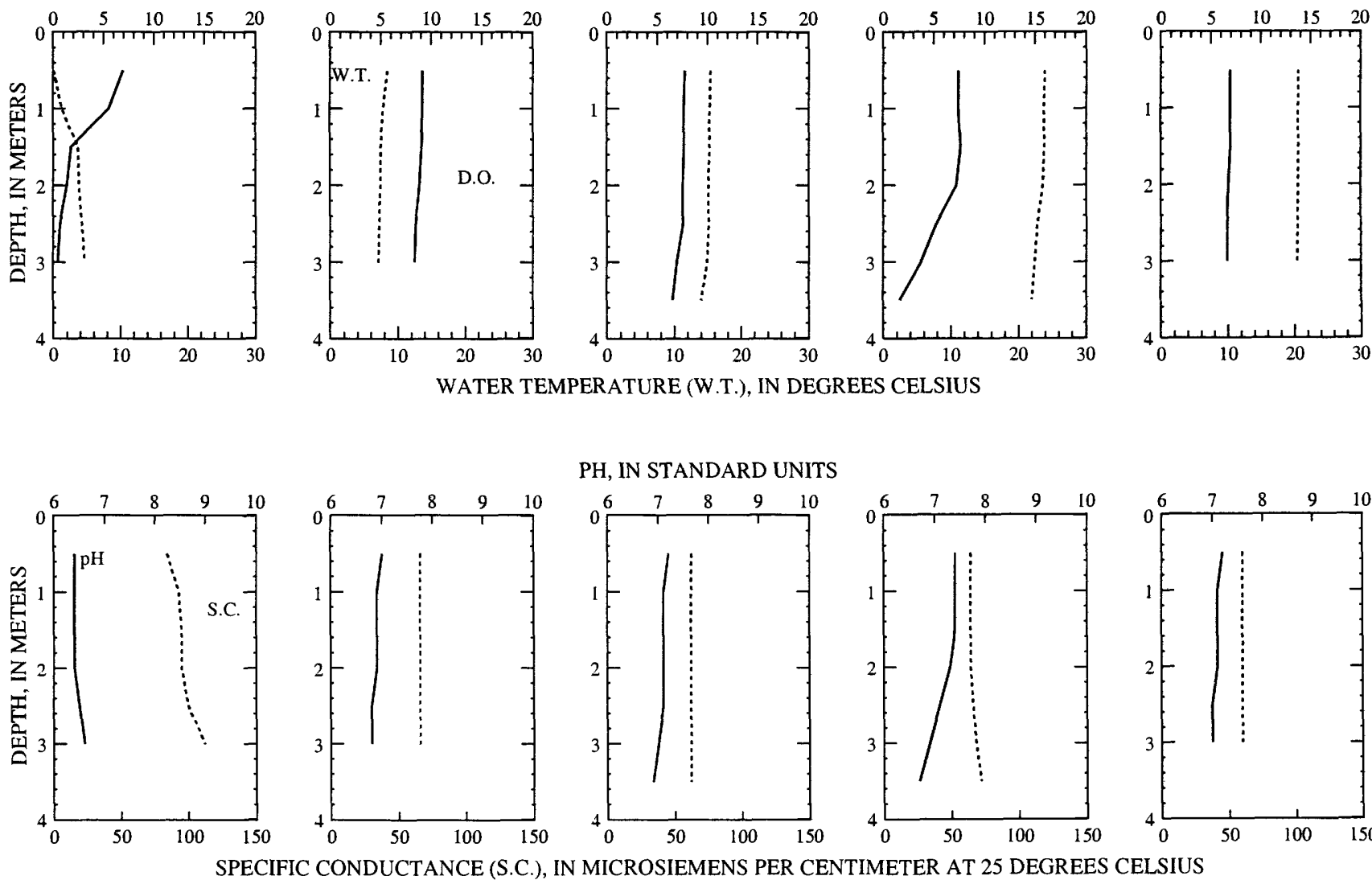

PH, IN STANDARD UNITS
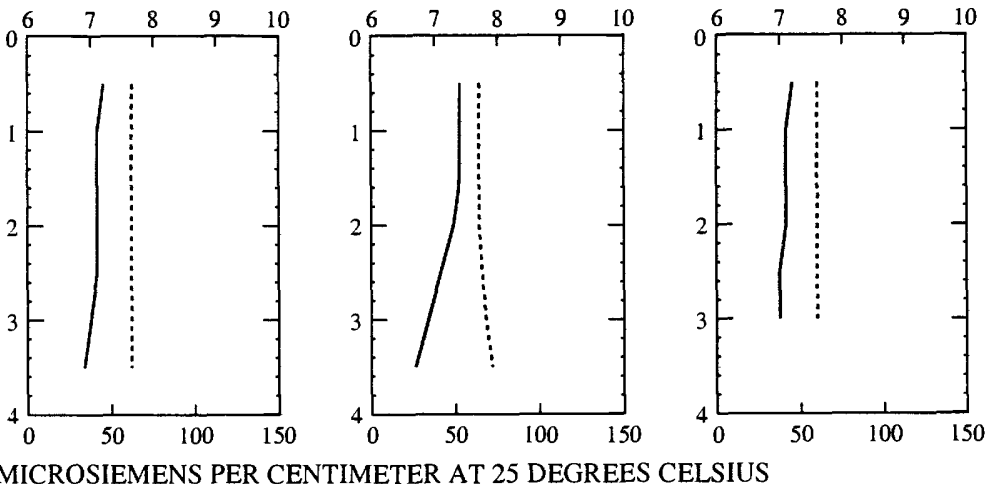

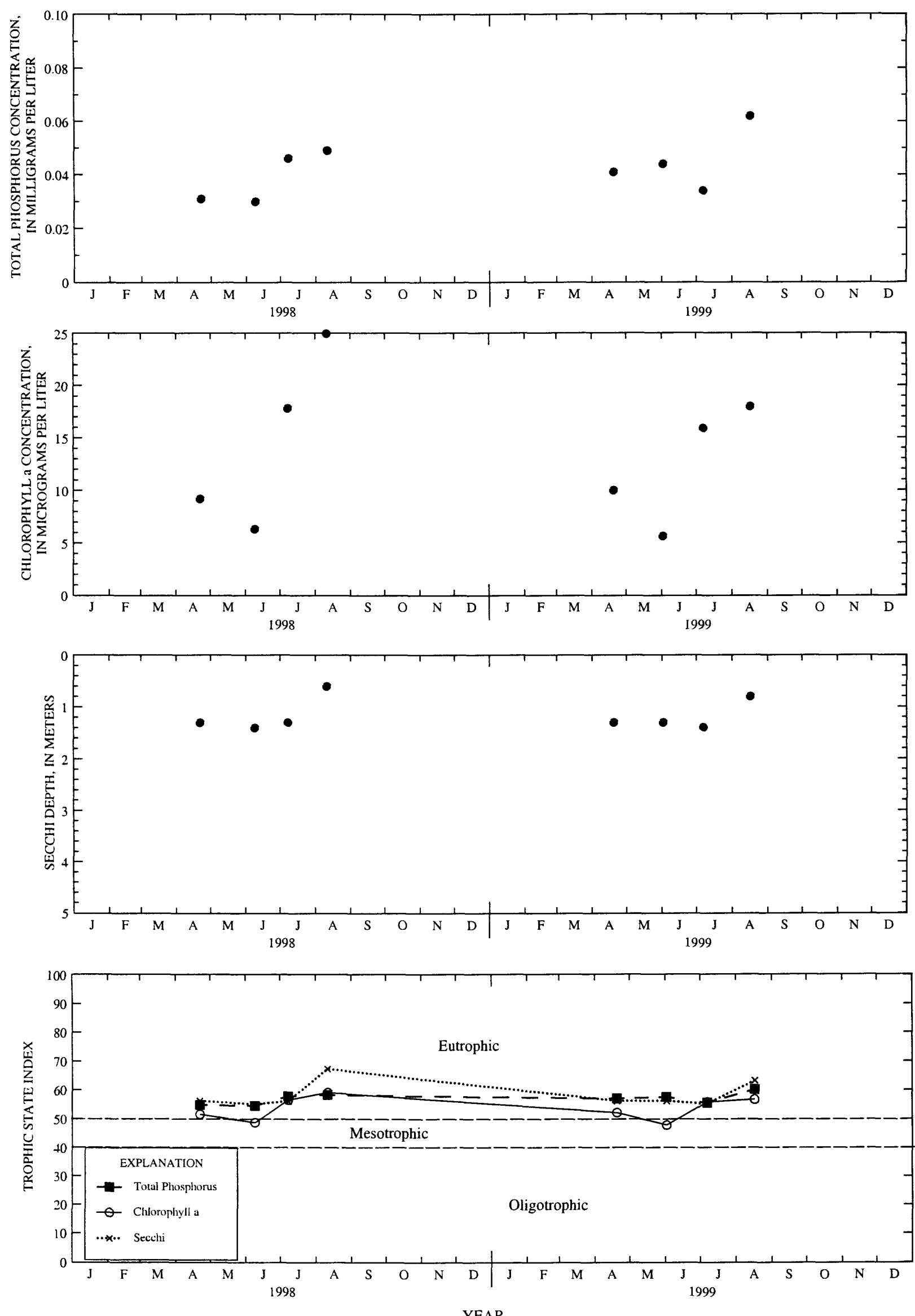

Surface total phosphorus, chlorophyll a concentrations, Secchi depths, and TSI data for Namekagon Lake (Jackson) near Cable, Wisconsin. 
LOCATION.--Lat 46 $13^{\prime} 08^{\prime \prime}$, long 91 06 '51", in NE 1/4 SE 1/4 sec.9, T.43 N., R.6 W., Bayfield County, Hydrologic Unit 07030002, near Cable. PERIOD OF RECORD.--March 1998 to current year.

REMARKS.--Lake sampled near center of the lake at the deep hole. Lake ice-covered during March sampling. Water-quality analyses done by Wisconsin State Laboratory of Hygiene.

Lake stage (ft)

Chlorophyll a, phytoplankton $(\mu \mathrm{g} / \mathrm{L})$

Depth of sample (m)

water temperature $\left({ }^{\circ} \mathrm{C}\right)$

pecific conductance $(\mu \mathrm{S} / \mathrm{cm})$

H (units)

Dissolved oxygen (mg/L)

hosphorus, total (as P)

phosphorus, ortho, dissolved (as P)

Nitrogen, NO2 + NO3, diss. (as N)

Nitrogen, ammonia, dissolved (as $N$ )

Nitrogen, amm. + org., total (as N)

Nitrogen, total (as N)

color (Pt-Co. scale)

Turbidity (NTU)

Hardness, (as $\mathrm{CaCO}_{3}$ )

Calcium, dissolved ( $\mathrm{Ca}$

Magnesium, dissolved (Mg)

Sodium, dissolved (Na)

Potassium, dissolved $(\mathrm{K})$

Alkalinity, (as $\mathrm{CaCO}_{3}$ )

Sulfate, dissolved $\left(\mathrm{SO}_{4}\right)$

Chloride, dissolved (CI)

Silica, dissolved $\left(\mathrm{SiO}_{2}\right)$

solids, dissolved, at $180^{\circ} \mathrm{C}$

Iron, dissolved (Fe) $\mu \mathrm{g} / \mathrm{I}$

Manganese, dissolved (Mn) $\mu \mathrm{g} / \mathrm{I}$
WATER-QUALITY DATA, MARCH 04 TO AUGUST 17, 1999

(Milligrams per liter unless otherwise indicated)
Mar -3

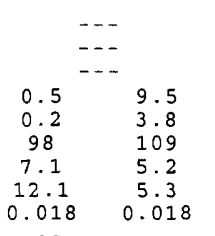

3-03-99

\begin{tabular}{|c|c|}
\hline \multicolumn{2}{|c|}{ Apr -20} \\
\hline \multicolumn{2}{|c|}{$\begin{array}{c}8.75 \\
3.2 \\
2.34\end{array}$} \\
\hline 0.5 & 14.0 \\
\hline 6.0 & 5.5 \\
\hline $\begin{array}{c}97 \\
7.3\end{array}$ & 98 \\
\hline $\begin{array}{c}7.3 \\
10.2\end{array}$ & 7.1 \\
\hline 0.011 & $\begin{array}{c}8.9 \\
0.015\end{array}$ \\
\hline$<0.002$ & $\cdots$ \\
\hline 0.09 & -- \\
\hline 0.015 & -- \\
\hline 0.33 & -- \\
\hline 0.42 & -- \\
\hline 15 & $\cdots$ \\
\hline 1.1 & -- \\
\hline 45 & -- \\
\hline 12 & -- \\
\hline 3.6 & $\cdots$ \\
\hline 2.4 & -- \\
\hline 0.6 & $\cdots$ \\
\hline 55 & $-\cdots$ \\
\hline$<4.5$ & $\cdots$ \\
\hline 2.8 & -- \\
\hline 12 & --- \\
\hline 72 & $\cdots$ \\
\hline 70 & -- \\
\hline 45 & $\cdots$ \\
\hline
\end{tabular}

\begin{tabular}{|c|c|}
\hline \multicolumn{2}{|c|}{ Jun-2 } \\
\hline \multicolumn{2}{|c|}{$\begin{array}{c}8.43 \\
3.8 \\
2.81\end{array}$} \\
\hline 0.5 & 14.0 \\
\hline 15.1 & 10.0 \\
\hline 95 & 108 \\
\hline 7.6 & 6.7 \\
\hline 8.4 & 0.3 \\
\hline 0.017 & 0.045 \\
\hline-- & -- \\
\hline$\ldots$ & $\ldots$ \\
\hline$\cdots$ & -- \\
\hline-- & -- \\
\hline$\ldots$ & -- \\
\hline$\cdots$ & -- \\
\hline-- & --- \\
\hline - - - & $\ldots$ \\
\hline-- & -- \\
\hline-- & -- \\
\hline$\cdots$ & -- \\
\hline-- & $\cdots$ \\
\hline$\ldots$ & -- \\
\hline-- & -- \\
\hline$\cdots$ & --- \\
\hline$\cdots$ & -- \\
\hline$\ldots$ & - \\
\hline$\ldots$ & $\ldots$ \\
\hline
\end{tabular}

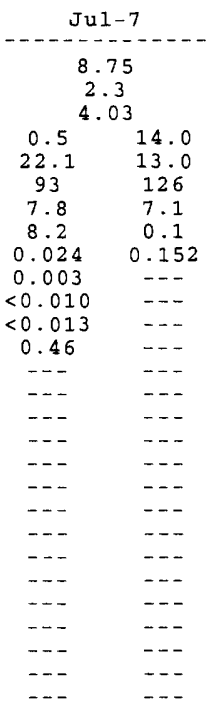

\begin{tabular}{|c|c|c|}
\hline & Aug -17 & \\
\hline & $\begin{array}{c}8.80 \\
1.6 \\
12.0\end{array}$ & \\
\hline $\begin{array}{c}0.5 \\
20.7\end{array}$ & $\begin{array}{l}10.0 \\
16.7\end{array}$ & $\begin{array}{l}14.0 \\
14.3\end{array}$ \\
\hline 91 & 119 & 146 \\
\hline 7.6 & 6.9 & 7.2 \\
\hline 7.8 & 0.3 & 0.1 \\
\hline 0.031 & 0.046 & 0.035 \\
\hline$-\cdots$ & -- & -- \\
\hline$\ldots$ & $\ldots$ &.-- \\
\hline$\ldots$ & $\ldots-$ & $\ldots$ \\
\hline-- & --- & $\ldots$ \\
\hline--- & $\ldots$ & $\ldots$ \\
\hline--- & $\ldots$ & --- \\
\hline$\ldots$ & $\ldots$ & -- \\
\hline--- & --- & --- \\
\hline$\ldots$ & $-\ldots$ & $\ldots$ \\
\hline$\cdots$ & --- & -- \\
\hline$\cdots$ & --- & $\cdots$ \\
\hline$\ldots$ & -- & --- \\
\hline$\cdots$ & $-\cdots$ & $\cdots$ \\
\hline$\cdots$ & -- & -. \\
\hline$\cdots$ & $-\ldots$ & $\cdots$ \\
\hline$\cdots$ & --- & --- \\
\hline$\cdots$ & --- & $\cdots$ \\
\hline$\cdots$ & $-\cdots$ & -- \\
\hline$\cdots$ & $\ldots$ & $\cdots$ \\
\hline
\end{tabular}
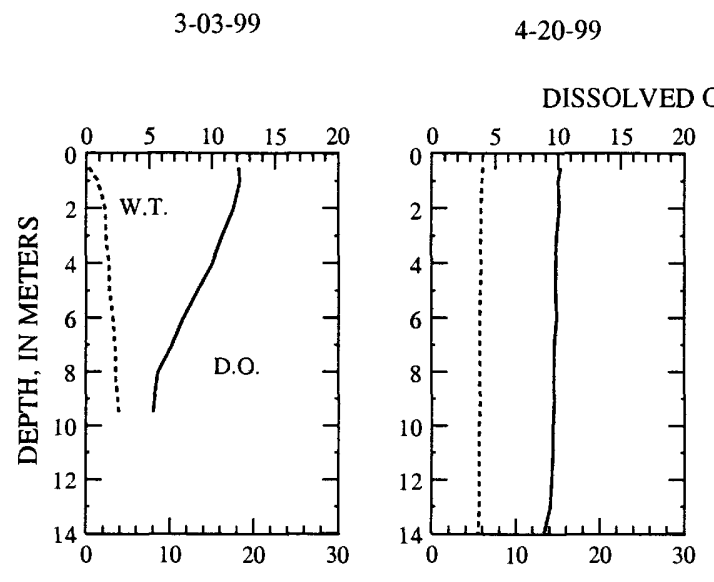

6-02-99

7-07-99

$8-17-99$
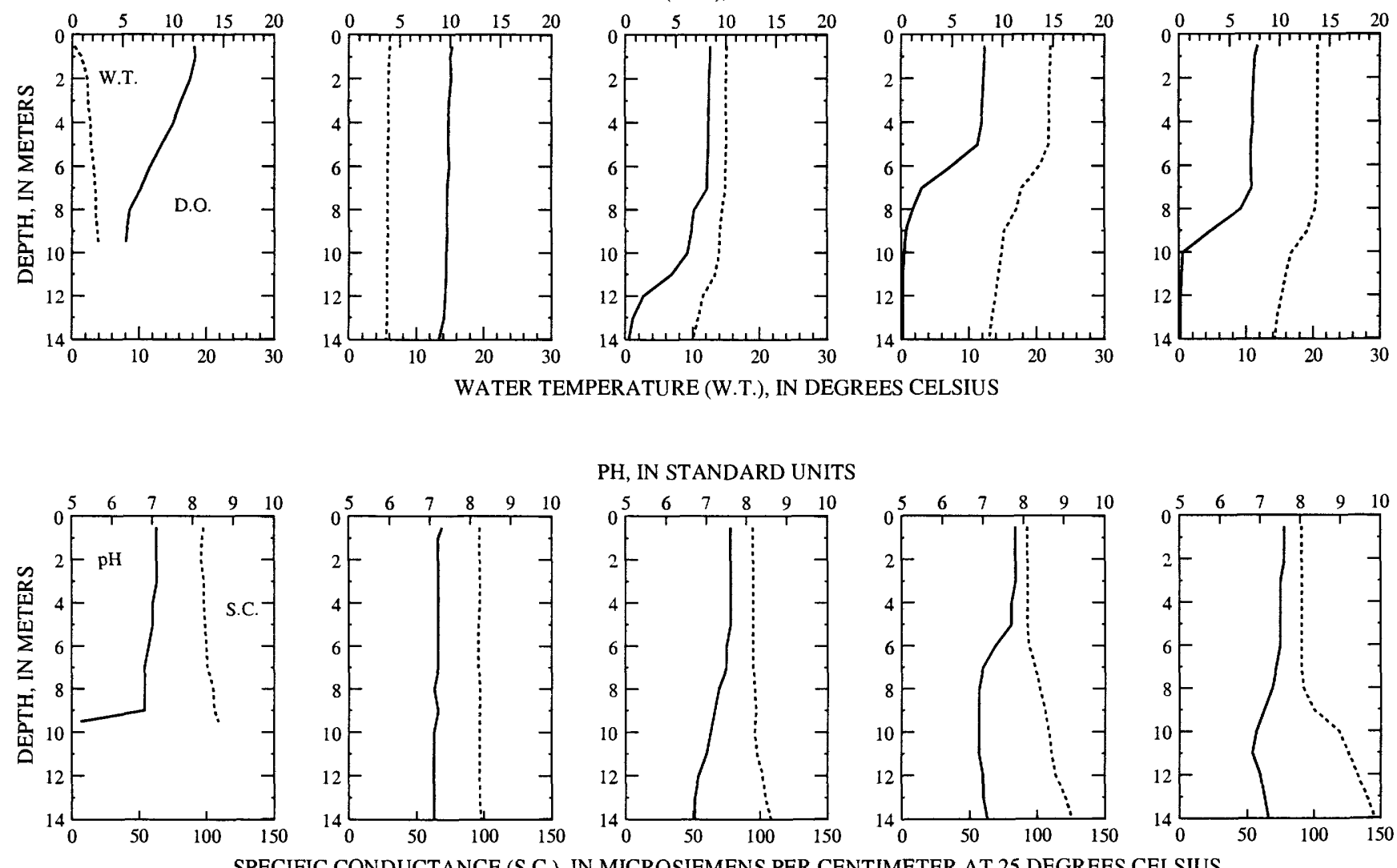

PH, IN STANDARD UNITS
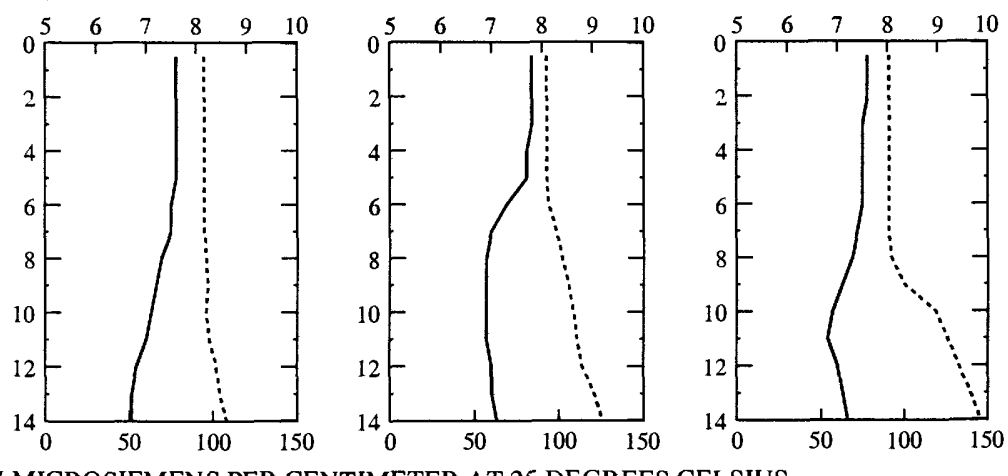

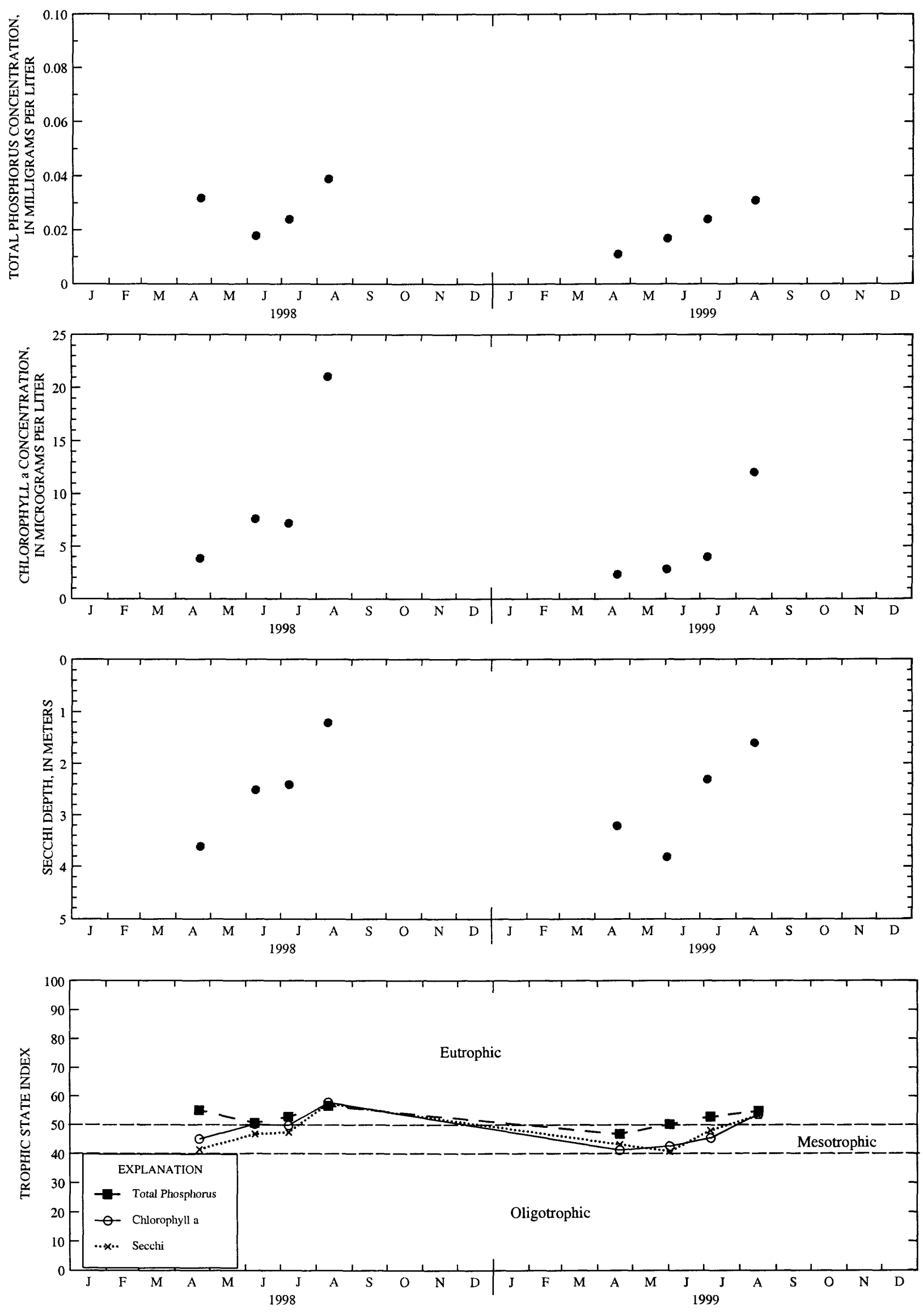

YEAR

Surface total phosphorus, chlorophyll a concentrations, Secchi depths, and TSI data for Namekagon Lake, Deep Hole, near Cable, Wisconsin. 


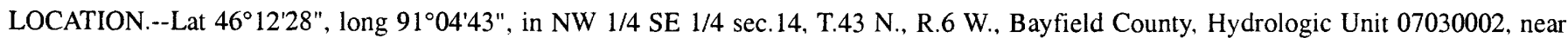
Cable.

PERIOD OF RECORD.--March 1998 to current year.

REMARKS.--Lake sampled at deepest part of the eastern basin. Lake ice-covered during March sampling. Water-quality analyses done by Wisconsin State Laboratory of Hygiene.

WATER-QUALITY DATA, MARCH 04 TO AUGUST 17, 1999

(Milligrams per liter unless otherwise indicated)

Lake stage (ft)

Secchi-depth (m)

Chlorophyll a, phytoplankton ( $\mathrm{g} / \mathrm{L}$ )

Depth of sample (m)

Water temoerature $\left({ }^{\circ} \mathrm{C}\right)$

Specific conductance $(\mu \mathrm{S} / \mathrm{cm})$

$\mathrm{pH}$ (units)

Dissolved oxygen (mg/L)

Phosphorus, total (as P)

\begin{tabular}{|c|c|c|c|c|c|}
\hline Mar-4 & Apr-20 & Jun-2 & \multicolumn{2}{|c|}{ Jul-7 } & Aug -17 \\
\hline-- & 8.75 & 8.43 & \multicolumn{2}{|c|}{8.75} & 8.80 \\
\hline--- & 2.0 & 2.5 & \multicolumn{2}{|c|}{2.1} & 1.3 \\
\hline--- & 7.11 & 4.7 & \multicolumn{2}{|c|}{6.95} & 18.0 \\
\hline 0.5 & 0.5 & 0.5 & 0.5 & 7.0 & 0.5 \\
\hline 0.1 & 6.5 & 15.3 & 22.4 & 14.5 & 21.0 \\
\hline 103 & 94 & 94 & 92 & 133 & 88 \\
\hline 7.0 & 7.3 & 7.5 & 7.8 & 7.2 & 7.5 \\
\hline 10.6 & 10.5 & 8.3 & 7.8 & 0.1 & 7.9 \\
\hline 0.026 & 0.021 & 0.021 & 0.020 & 0.120 & 0.040 \\
\hline
\end{tabular}

3-04-99

4-20-99

6-02-99

7-07-99

8-17-99
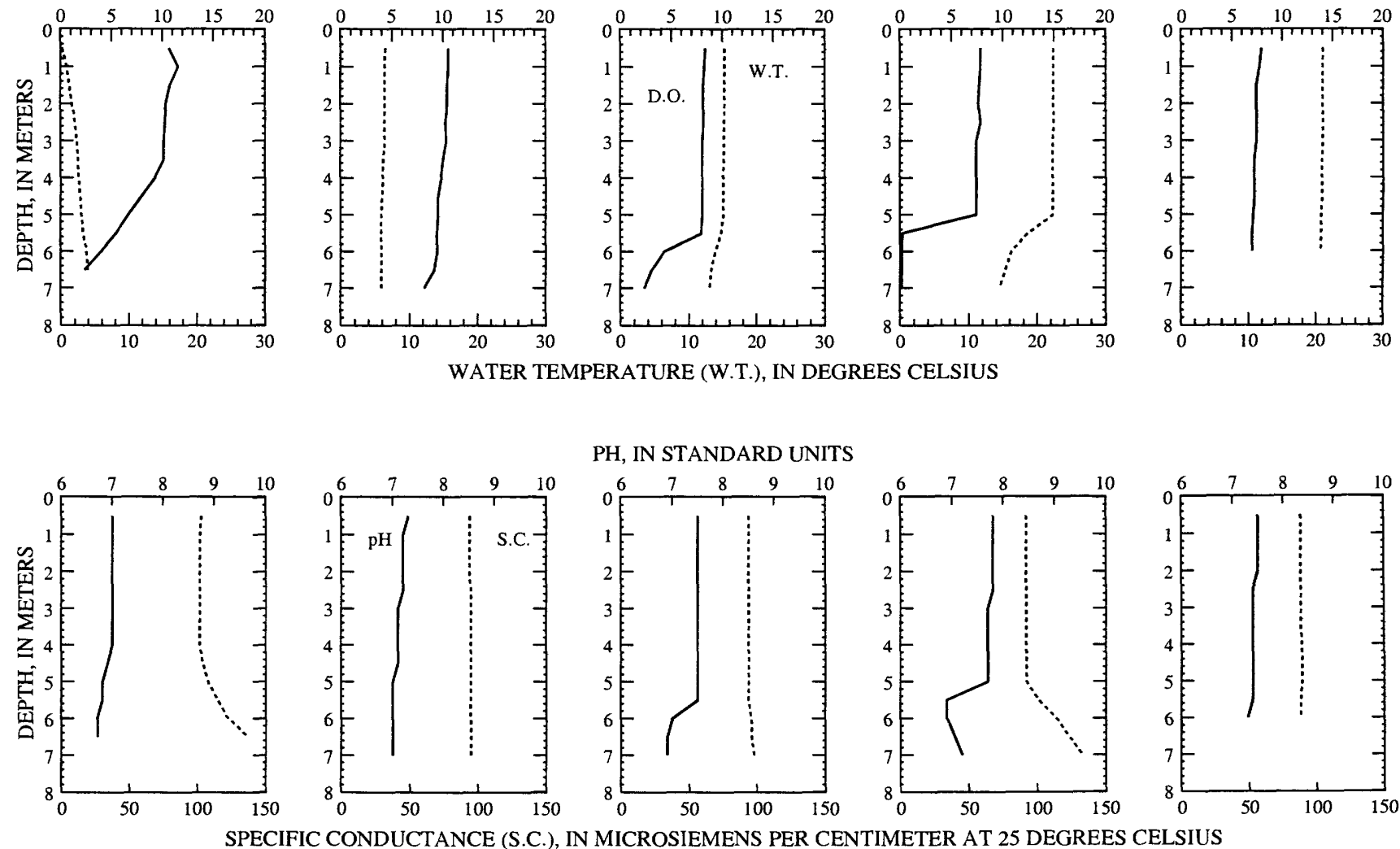

PH, IN STANDARD UNITS
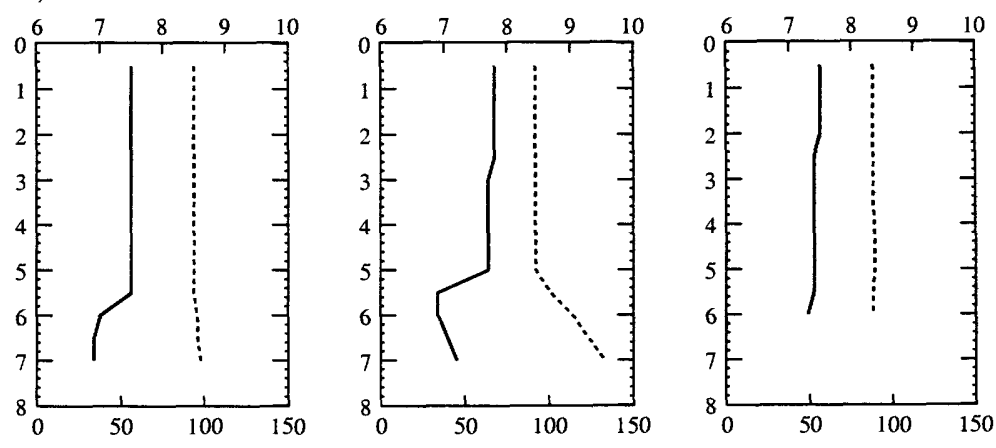

SPECIFIC CONDUCTANCE (S.C.), IN MICROSIEMENS PER CENTIMETER AT 25 DEGREES CELSIUS 

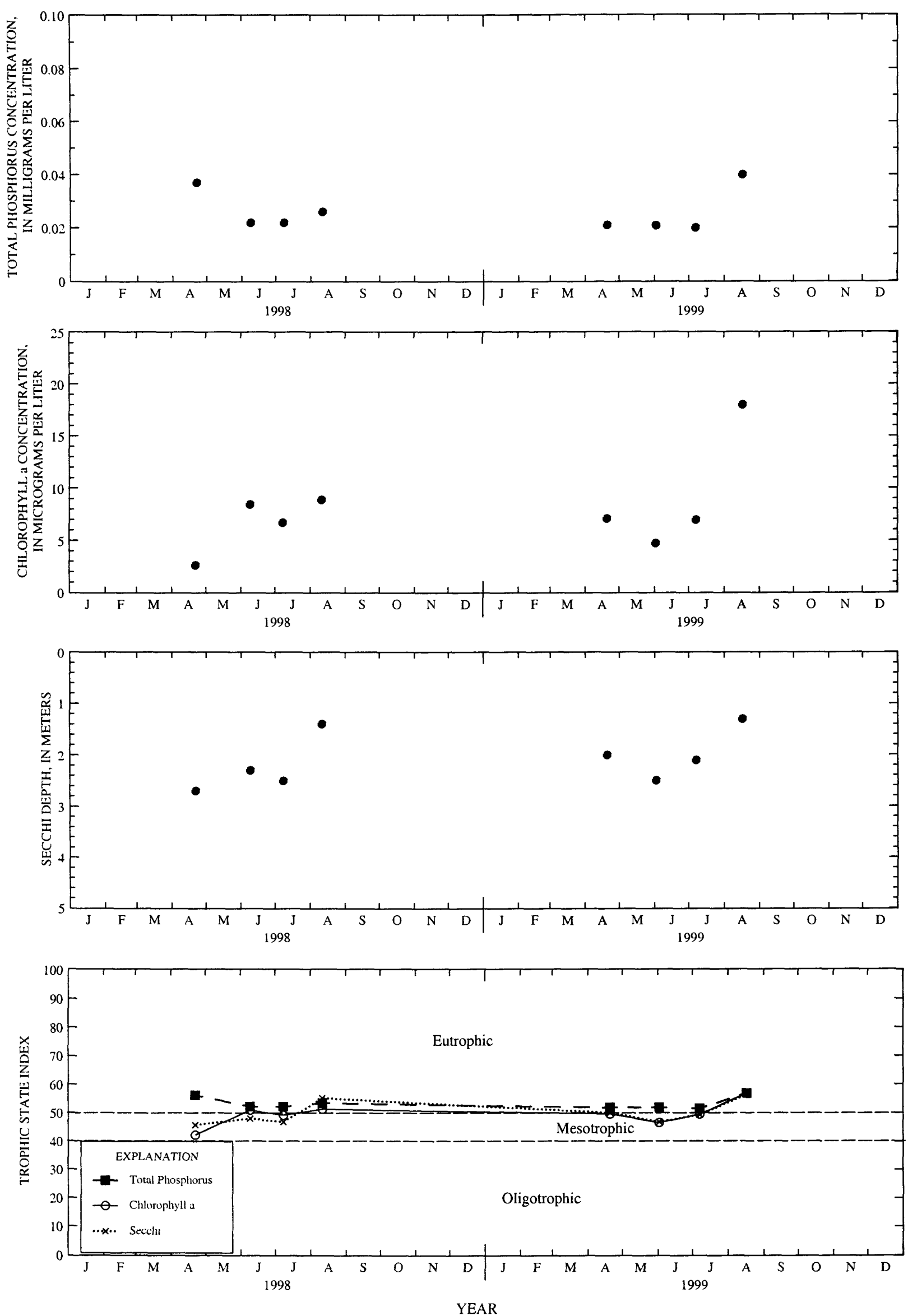

Surface total phosphorus, chlorophyll a concentrations, Secchi depths, and TSI data for Namekagon Lake, East Basin, near Cable, Wisconsin. 
LOCATION.--Lat 46 $14^{\prime} 10^{\prime \prime}$, long 91 05'07", in SE 1/4 NW 1/4 sec.2, T.43 N., R.6 W., Bayfield County, Hydrologic Unit 07030002, near Cable. PERIOD OF RECORD.--March 1998 to current year.

REMARKS.--Lake sampled at deepest part of the northeast bay. Lake ice-covered during March sampling. Water-quality analyses done by Wisconsin State Laboratory of Hygiene.

WATER-QUALITY DATA, MARCH 03 TO AUGUST 17, 1999

(Milligrams per liter unless otherwise indicated)

Lake stage ( $\mathrm{ft}$ )

Secchi-depth (m)

Chlorophyll a, phytoplankton ( $\mu \mathrm{g} / \mathrm{L}$ )

Depth of sample (m)

Water temperature $\left({ }^{\circ} \mathrm{C}\right)$

Specific conductance $(\mu \mathrm{S} / \mathrm{cm})$

pH (units)

Dissolved oxygen (mg/L)

Phosphorus, total (as P)

\begin{tabular}{|c|c|c|c|c|}
\hline \multicolumn{2}{|c|}{ Mar-3 } & Apr-20 & \multicolumn{2}{|c|}{ Jun-2 } \\
\hline \multicolumn{2}{|c|}{---} & 8.75 & \multicolumn{2}{|c|}{8.43} \\
\hline \multicolumn{2}{|c|}{---} & 2.3 & \multirow{2}{*}{\multicolumn{2}{|c|}{$\begin{array}{c}3.0 \\
3.16\end{array}$}} \\
\hline & & 5.04 & & \\
\hline 0.5 & 11.5 & 0.5 & 0.5 & 10.0 \\
\hline 0.4 & 4.6 & 6.9 & 15.7 & 9.0 \\
\hline 97 & 140 & 89 & 92 & 102 \\
\hline 7.0 & 7.2 & 7.2 & 7.5 & 6.6 \\
\hline 11.6 & 0.7 & 10.3 & 8.2 & 0.3 \\
\hline 0.015 & 0.032 & 0.018 & 0.019 & 0.034 \\
\hline
\end{tabular}

4-20-99

6-02-99

\begin{tabular}{cccc} 
Jul-7 & \multicolumn{2}{c}{ Aug-17 } \\
\hline \multicolumn{2}{c}{8.75} & \multicolumn{2}{c}{8.80} \\
2.4 & \multicolumn{2}{c}{1.3} \\
\multicolumn{2}{c}{5.21} & \multicolumn{2}{c}{18} \\
0.5 & 11.0 & 0.5 & 10.0 \\
22.9 & 11.3 & 21.1 & 12.8 \\
89 & 123 & 85 & 139 \\
7.6 & 7.1 & 7.7 & 7.1 \\
7.5 & 0.1 & 8.0 & 0.2 \\
0.130 & 0.164 & 0.038 & 0.265
\end{tabular}

7-07-99

$8-17-99$

DISSOLVED OXYGEN (D.O.), IN MILLIGRAMS PER LITER
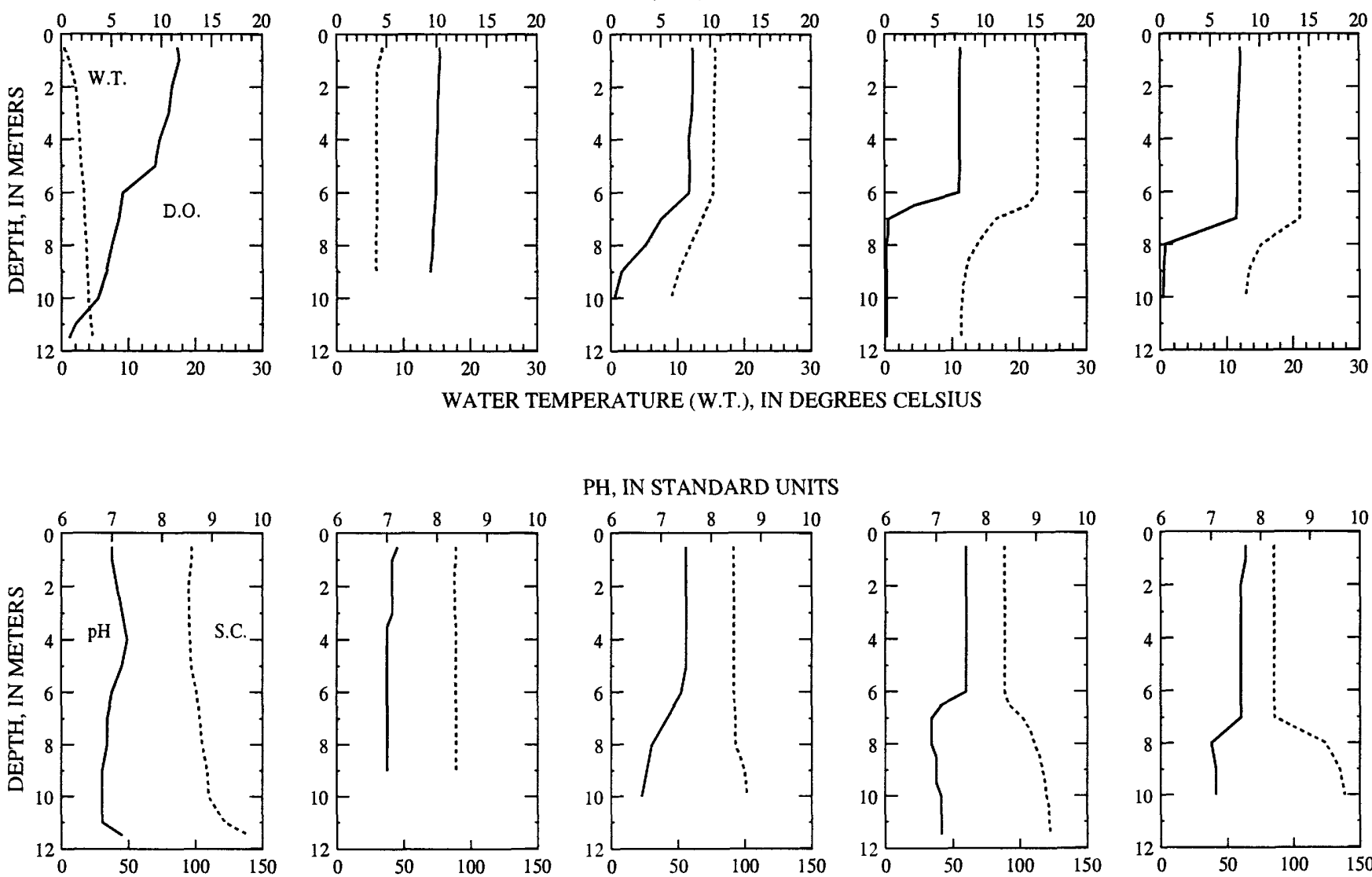

PH, IN STANDARD UNITS
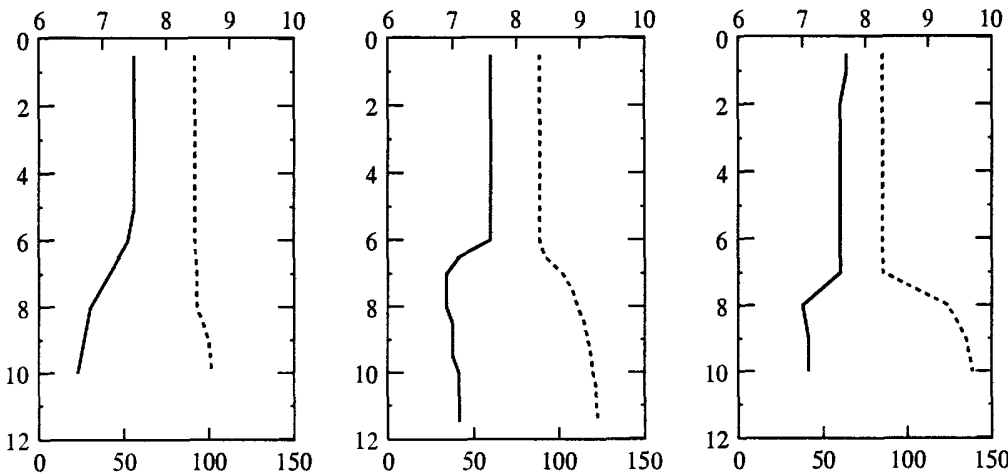

SPECIFIC CONDUCTANCE (S.C.), IN MICROSIEMENS PER CENTIMETER AT 25 DEGREES CELSIUS 

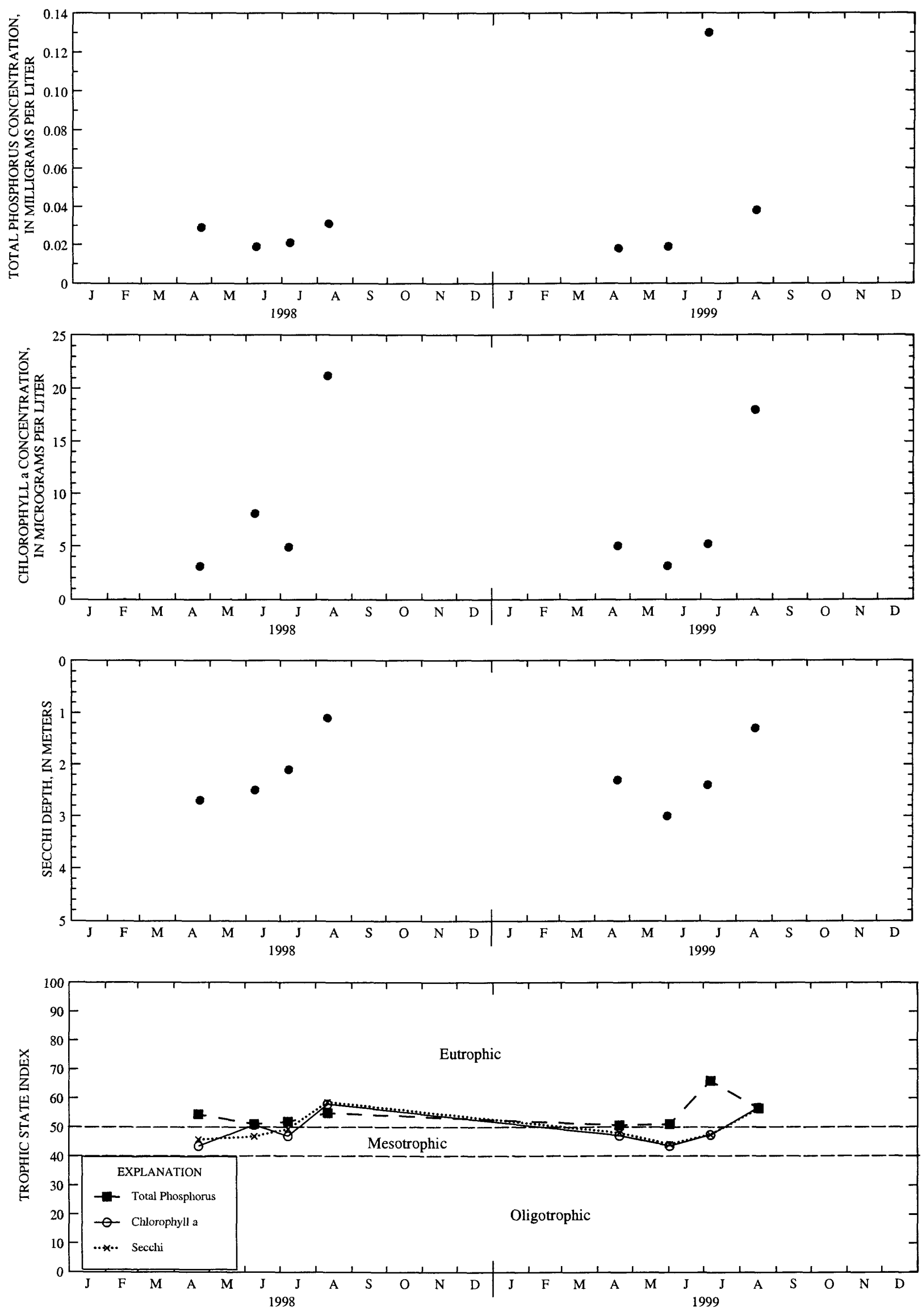

YEAR

Surface total phosphorus, chlorophyll a concentrations, Secchi depths, and TSI data for Namekagon Lake, Northeast Basin, near Cable, Wisconsin. 


\section{OCONOMOWOC LAKE NO. 1 (CENTER) AT OCONOMOWOC, WI}

LOCATION.--Lat $43^{\circ} 05^{\prime} 51^{\prime \prime}$, long $88^{\circ} 27^{\prime} 35^{\prime \prime}$, in NW 1/4 SE 1/4 sec.2, T.7 N., R.17 E., Waukesha County, Hydrologic Unit 07090001, at Oconomowoc.

PERIOD OF RECORD.--March 1986 to current year.

REMARKS.--Lake sampled near center at the deep hole. Lake ice-covered during February sampling. Water-quality analyses done by Wisconsin State Laboratory of Hygiene.

WATER-QUALITY DATA, FEBRUARY 10 TO AUGUST 09, 1999

(Milligrams per liter unless otherwise indicated)

Lake stage ( $f t)$

Secchi-depth (m)

Chiorophyli a, phytoplankton (mg/L)

Depth of sample (m)

specific conductance (ms/cm)

specific co

Dissolved oxygen ( $\mathrm{mg} / \mathrm{L}$ )

Phosphorus, total (as $P$ )

No2 + No3 diss (as N)

itrogen, ammonia, dissolved (as N)

Nitrogen, amm.+org., total (as N)

Nitrogen, total (as N)

Color (Pt-Co. scale)

Turbidity (NTU)

Hardness, (as $\mathrm{CaCO}^{3}$ )

Calcium, dissolved ( $\mathrm{Ca}$ )

Magnesium, dissolved (Mg)

Sodium, dissolved (Na)

Potassium, dissolved (K)

Alkalinity, (as $\mathrm{CaCO} 3$

Sulfate, dissolved (SO4)

Chloride, dissolved (Cl)

Silica, dissolved (Sion)

Solids, dissolved, at $1800 \mathrm{C}$

Iron, dissolved ( $\mathrm{Fe}$ ) $\mathrm{mg} / \mathrm{L}$

Manganese. dissolved (Mn) $\mathrm{mg} / \mathrm{L}$
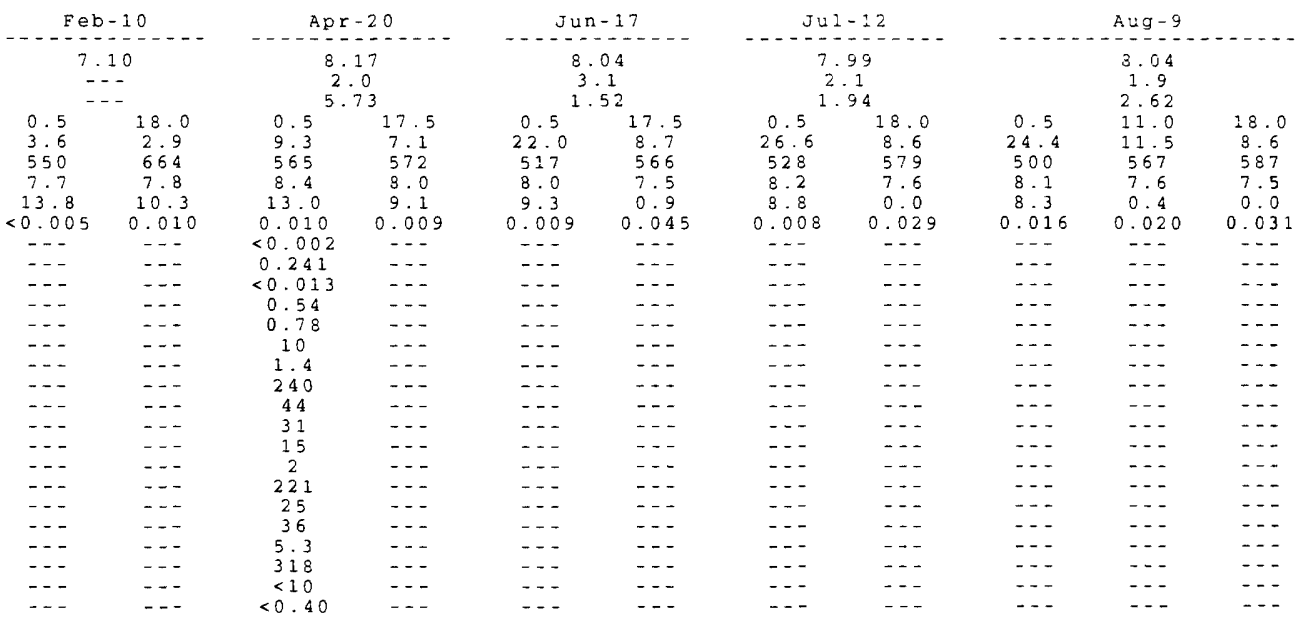

2-10-99

4-20-99

6-17-99

$7-12-99$

8-09-99

DISSOLVED OXYGEN (D.O.), IN MILLIGRAMS PER LITER
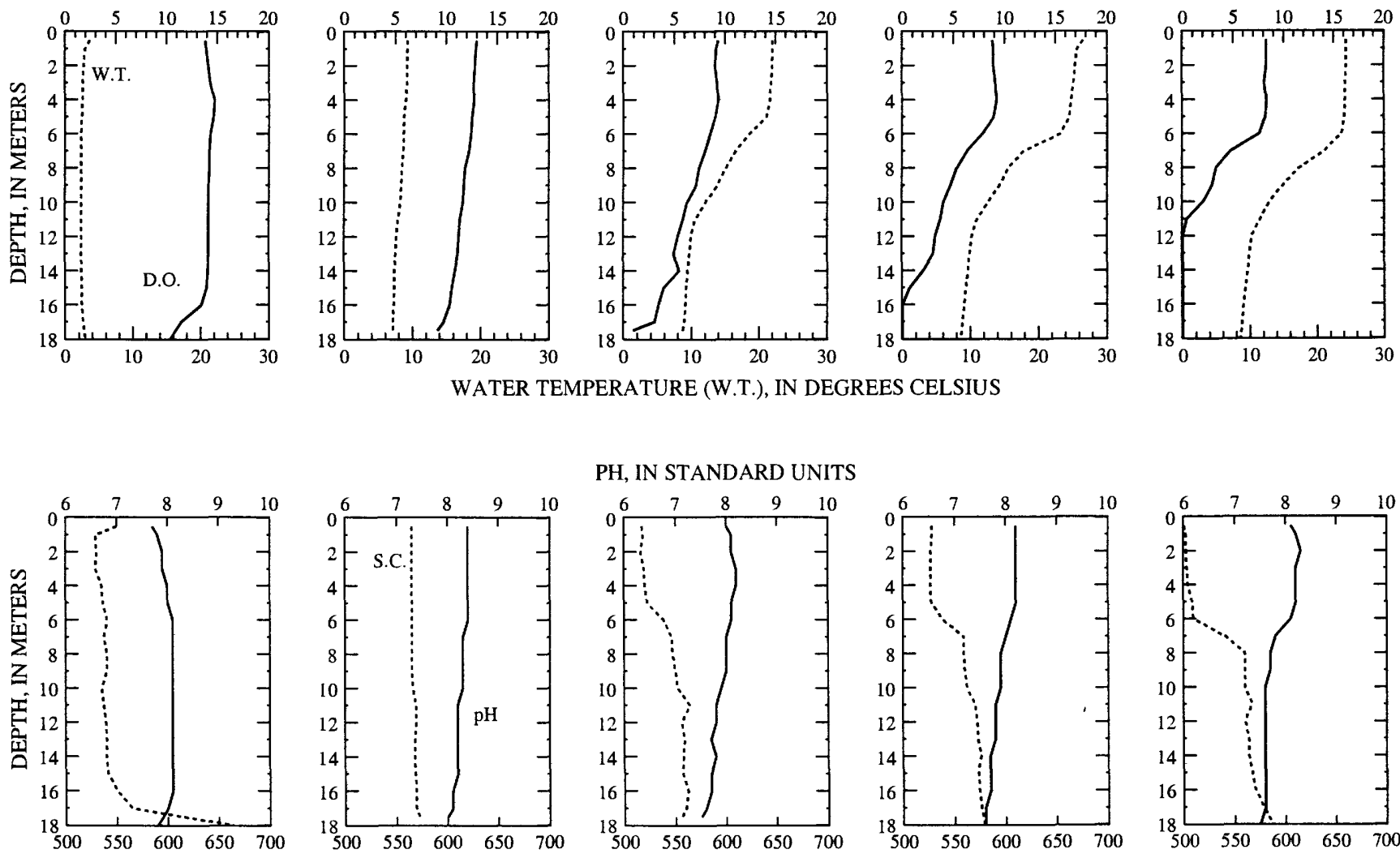

PH, IN STANDARD UNITS
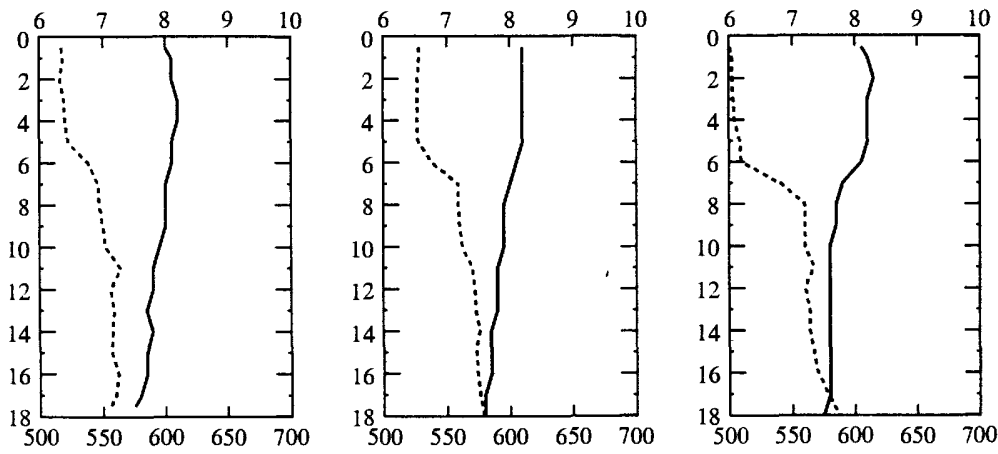

SPECIFIC CONDUCTANCE (S.C.), IN MICROSIEMENS PER CENTIMETER AT 25 DEGREES CELSIUS 

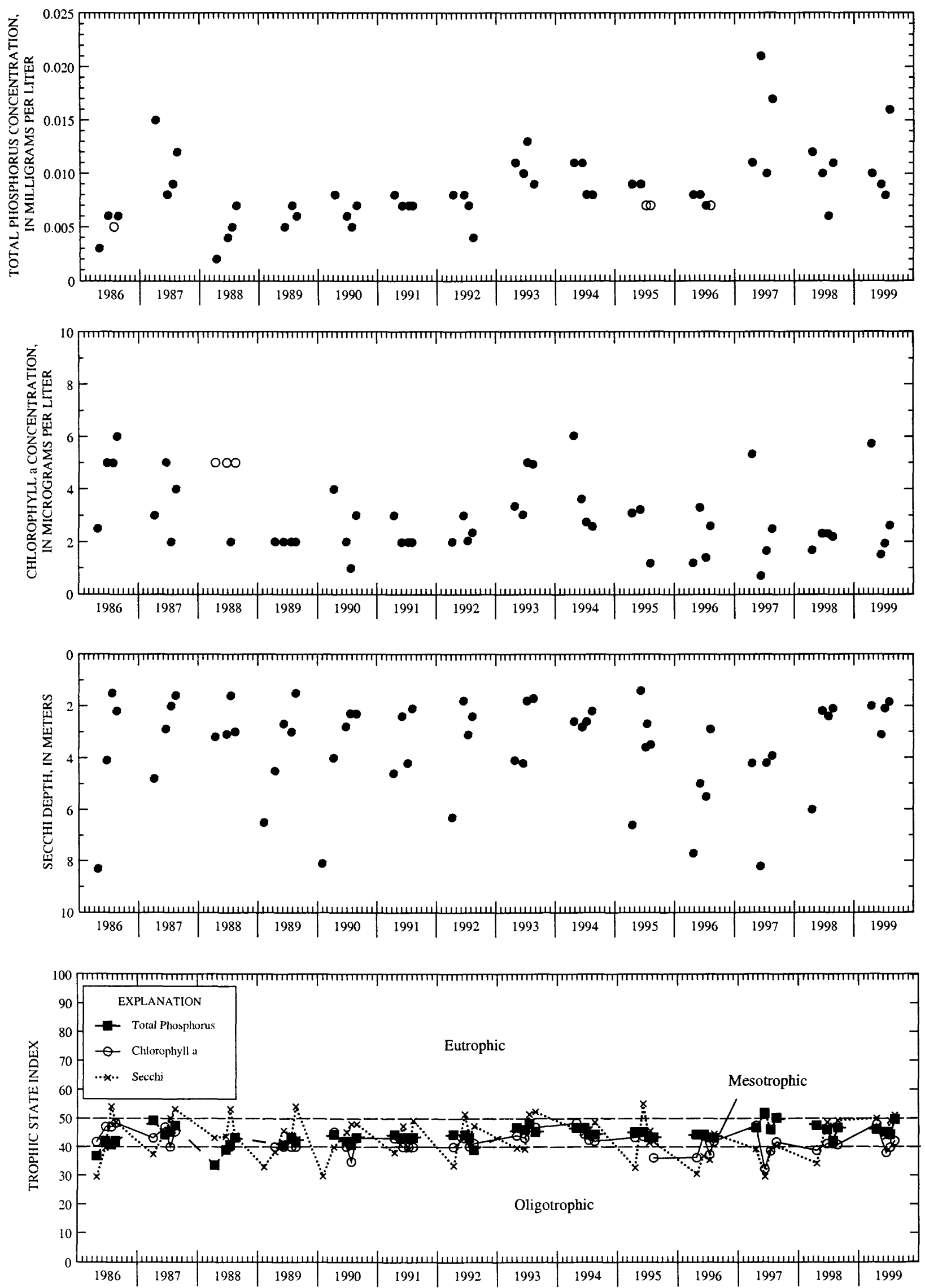

YEAR

Surface total phosphorus, chlorophyll a concentrations, Secchi depths, and TSI data for Oconomowoc Lake, No. 1 (Center) at Oconomowoc, Wisconsin.

(Circles on the first three plots indicate laboratory detection limit for selected analyses. Actual concentrations for these particular analyses are less than the plotted circles.) 
LOCATION.--Lat $43^{\circ} 06^{\prime} 09^{\prime \prime}$, long $88^{\circ} 26^{\prime} 22^{\prime \prime}$, in NW 1/4 NW 1/4 sec.1, T.7 N., R.17 E., Waukesha County, Hydrologic Unit 07090001, at Oconomowoc.

PERIOD OF RECORD.--March 1986 to current year.

REMARKS.--Lake sampled at the deepest point in northeast bay near Hewitt Point. Lake ice-covered during February sampling. Water-quality analyses done by Wisconsin State Laboratory of Hygiene.

WATER-QUALITY DATA, FEBRUARY 10 TO AUGUST 09, 1999

(Milligrams per liter unless otherwise indicated)

Lake stage (ft)

Secchi-depth (m)

Chlorophyll a, phvtoolankton ( $\mu / \mathrm{L} / \mathrm{L}$ ) Depth of sampie (m)

Water temperature $\left({ }^{\circ} \mathrm{C}\right)$

Specific conductance (us/cm) $\mathrm{pH}$ (units)

Dissolved oxygen (mg/L)

Phosphorus, total (as P)

Phosphorus, ortho, dissolved (as P) Nitrogen, $\mathrm{NO} 2+\mathrm{NO} 3$, diss. (as N) Nitrogen, ammonia, dissolved (as $N$ ) Nitrogen, amm. + org., total (as N) Nitrogen, total (as N)
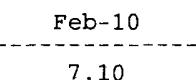

\begin{tabular}{|c|c|}
\hline \multicolumn{2}{|c|}{ Apr -20} \\
\hline \multicolumn{2}{|c|}{$\begin{array}{c}8.17 \\
4.5 \\
1.05\end{array}$} \\
\hline 0.5 & 14.5 \\
\hline 9.5 & 7.8 \\
\hline 606 & 612 \\
\hline 8.2 & 8.0 \\
\hline 11.6 & 9.1 \\
\hline$<0.005$ & 0.009 \\
\hline--- & --- \\
\hline 0.225 & -- \\
\hline 0.09 & $-\ldots$ \\
\hline 0.55 & --- \\
\hline 0.77 & -- \\
\hline
\end{tabular}

\begin{tabular}{|c|c|}
\hline \multicolumn{2}{|c|}{ Jun-17 } \\
\hline \multicolumn{2}{|c|}{$\begin{array}{c}8.04 \\
2.7 \\
0.94\end{array}$} \\
\hline 0.5 & 14.5 \\
\hline 22.1 & 8.7 \\
\hline 544 & 614 \\
\hline 8.2 & 7.4 \\
\hline 9.4 & 0.6 \\
\hline 0.008 & 0.041 \\
\hline--- & -- \\
\hline$\ldots$ & --- \\
\hline - - & -- \\
\hline--- & $\ldots$ \\
\hline-- & --- \\
\hline
\end{tabular}

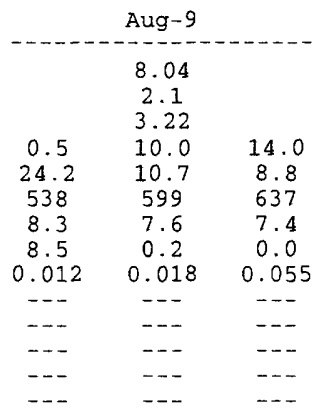

2-10-99

4-20-99

6-17-99

7-12-99

8-09-99

DISSOLVED OXYGEN (D.O.), IN MILLIGRAMS PER LITER
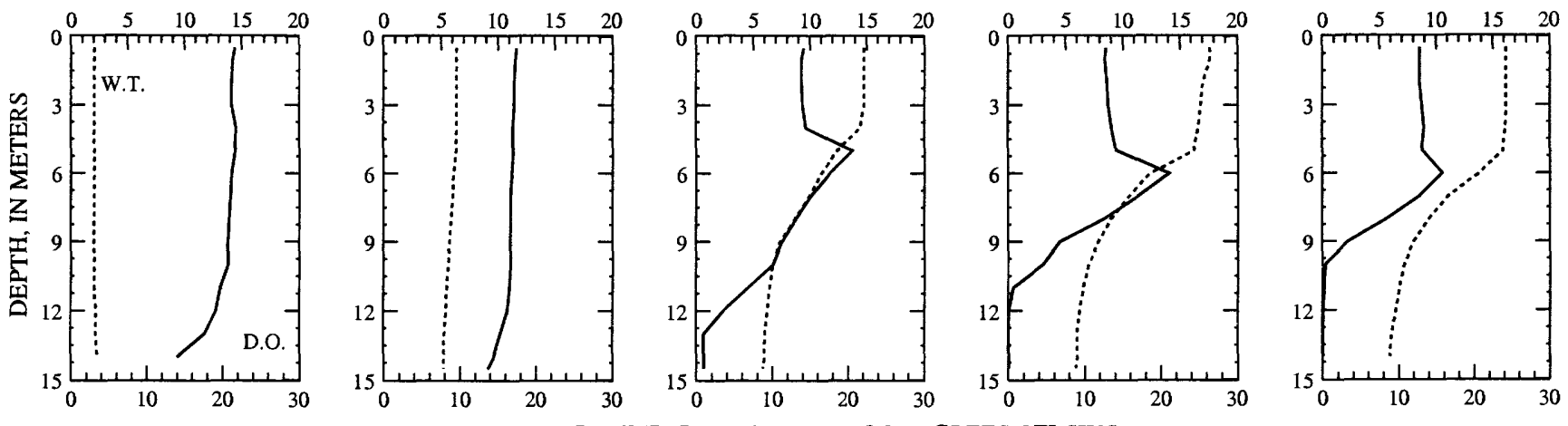

WATER TEMPERATURE (W.T.), IN DEGREES CELSIUS
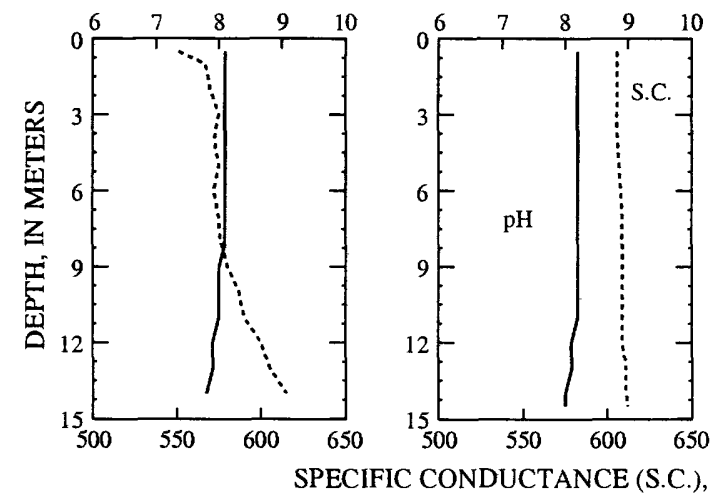

PH, IN STANDARD UNITS
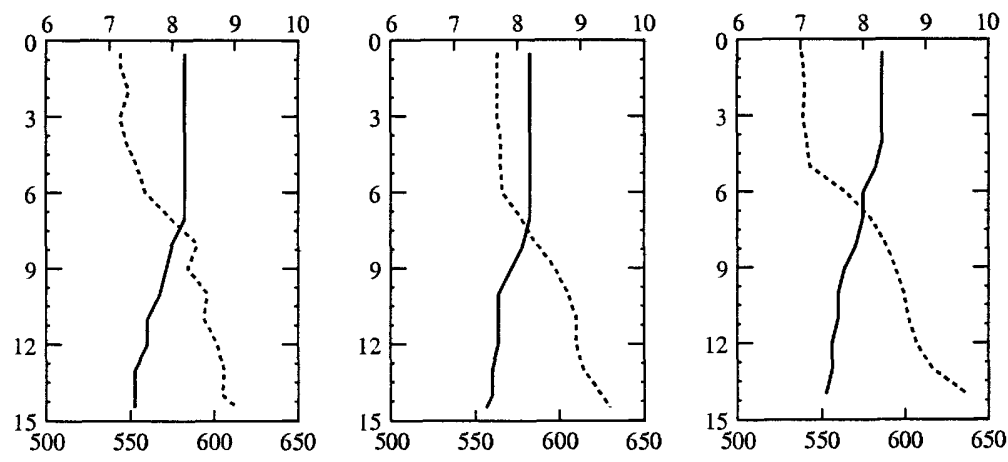

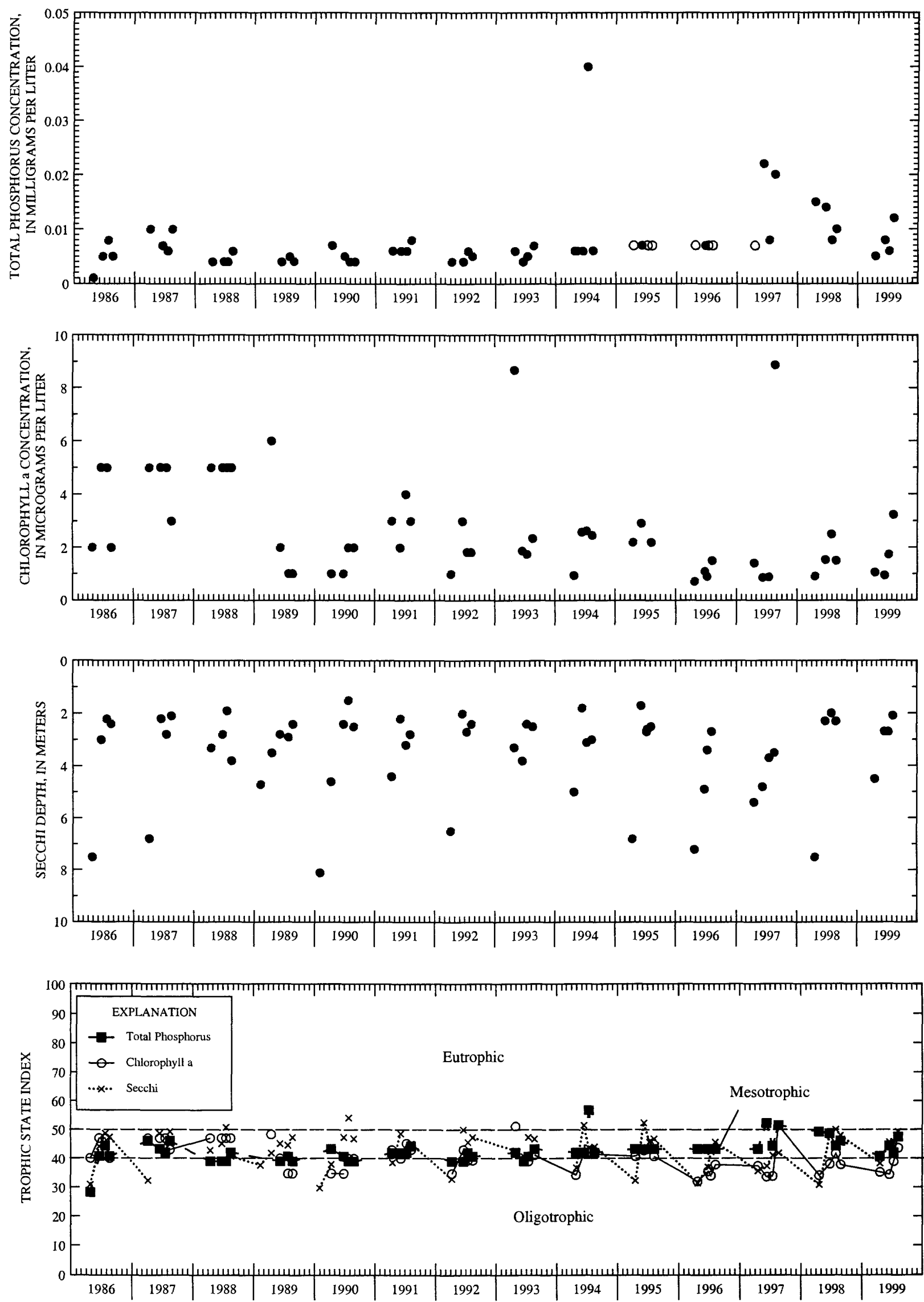

YEAR

Surface total phosphorus, chlorophyll a concentrations, Secchi depths, and TSI data for Oconomowoc Lake, No. 2 (Hewitt Pt.) at Oconomowoc, Wisconsin.

(Circles on the first three plots indicate laboratory detection limit for selected analyses. Actual concentrations for these particular analyses are less than the plotted circles.) 


\section{OKAUCHEE LAKE AT OKAUCHEE, WI}

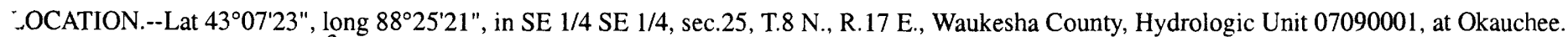
TRAINAGE AREA.--80.7 $\mathrm{mi}^{2}$.

गERIOD OF RECORD.--February 1984 to current year.

AKE-STAGE GAGE.--Datum of gage is $869.00 \mathrm{ft}$ above sea level.

'REMARKS.--A detailed water quality management plan has been developed for Okauchee Lake by Southeastern Wisconsin Regional Planning Commission; previous water-quality data are available in this report. Lake sampled near center at the deep hole. Lake ice-covered during February sampling. Water-quality analyses done by Wisconsin State Laboratory of Hygiene.

WATER-QUALITY DATA, FEBRUARY 10 TO AUGUST 09, 1999

(Milligrams per liter unless otherwise indicatedd)

Lake stage (ft)

Secchi-depth (m)

Chlorophyll a, phytoplankton $(\mu \mathrm{q} / \mathrm{L})$

Depth of sample (m)

water temperature $\left({ }^{\circ} \mathrm{C}\right)$

Specific conductance $(\mu \mathrm{S} / \mathrm{cm})$

$\mathrm{pH}$ (units)

Dissolved oxygen (mg/L)

Phosphorus, total (as P)

Phosphorus, ortho, dissolved (as P)

Nitrogen, NO2 + NO3, diss. (as N)

Nitrogen, ammonia, dissolved (as N)

Nitrogen, amm. + org., total (as N)

Nitrogen, total (as N)

Color (Pt-Co. scale)

Turbidity (NTU)

Hardness, (as $\mathrm{CaCO}_{3}$ )

Calcium, dissolved (Ca)

Magnesium, dissolved (Mg)

Sodium, dissolved (Na)

Potassium, dissolved (K)

Alkalinity, (as $\mathrm{CaCO}_{3}$ )

Sulfate, dissolved $\left(\mathrm{SO}_{4}\right)$

Chloride, dissolved (Cl)

Silica, dissolved $\left(\mathrm{SiO}_{2}\right.$ )

Solids, dissolved. at $180^{\circ} \mathrm{C}$

Iron, dissolved (Fe) $\mu \mathrm{q} / \mathrm{L}$

Manqanese, dissolved (Mn) $\mu \mathrm{q} / \mathrm{L}$

\begin{tabular}{|c|c|c|c|}
\hline \multicolumn{2}{|c|}{ Feb-10 } & \multicolumn{2}{|c|}{ Apr -20} \\
\hline \multicolumn{2}{|c|}{4.35} & & \\
\hline 0.5 & 27.5 & 0.5 & 28.5 \\
\hline 2.7 & 2.6 & 8.9 & 6.6 \\
\hline 740 & 569 & 568 & 577 \\
\hline 7.9 & 7.9 & 8.4 & 8.1 \\
\hline 14.5 & 8.7 & 13.9 & 9.8 \\
\hline 0.007 & 0.101 & 0.014 & 0.014 \\
\hline-- & $\ldots$ & $<0.002$ & $\ldots$ \\
\hline-- & -- & 0.378 & $\ldots$ \\
\hline$\ldots$ & $\ldots$ & $<0.013$ & $\ldots$ \\
\hline$\ldots$ &.-- & 0.66 & $\ldots$ \\
\hline-- & --- & 1.0 & $\cdots$ \\
\hline$-\cdots$ & -- & 10 & $\ldots$ \\
\hline-- & -- & 1.3 & $\ldots$ \\
\hline--- & -- & 260 & -- \\
\hline-- & -- & 50 & $\ldots$ \\
\hline$\ldots$ & $-\cdots$ & 32 & - - \\
\hline-- & -- & 13 & $\ldots$ \\
\hline$\ldots$ & $\ldots$ & 1.9 & $\ldots$ \\
\hline$\ldots$ & $\ldots$ & 234 & $\ldots$ \\
\hline$\ldots$ & -- & 25 & -- \\
\hline-- & -- & 32 & -- \\
\hline--- & --- & 3.1 & $\ldots$ \\
\hline$\ldots$ & -- & 328 & -- \\
\hline-- & -- & $<10$ & $\ldots$ \\
\hline-- & $\ldots$ & $<0.40$ & $\ldots$ \\
\hline
\end{tabular}

4-20-99

\begin{tabular}{|c|c|}
\hline \multicolumn{2}{|c|}{ Jun-18 } \\
\hline \multicolumn{2}{|c|}{$\begin{array}{c}4.79 \\
1.9 \\
5.08\end{array}$} \\
\hline 0.5 & 28 \\
\hline 21.6 & 7.5 \\
\hline 517 & 569 \\
\hline 8.2 & 7.5 \\
\hline 8.7 & 0.6 \\
\hline 0.012 & 0.024 \\
\hline$\ldots$ & $\ldots$ \\
\hline$=-$ & $\ldots$ \\
\hline$\ldots$ & $\ldots$ \\
\hline - - - & $\ldots$ \\
\hline - - & -- \\
\hline-- & -- \\
\hline -.. & - - - \\
\hline-- & $\ldots$ \\
\hline--- & -- \\
\hline$\cdots$ & $\cdots$ \\
\hline-- & -- \\
\hline$--\cdots$ & --- \\
\hline-- & $\cdots$ \\
\hline$\cdots$ & --- \\
\hline$\ldots$ & -- \\
\hline$\ldots$ & $\ldots$ \\
\hline$\ldots$ & $\ldots$ \\
\hline & $\ldots$ \\
\hline
\end{tabular}

\begin{tabular}{|c|c|}
\hline \multicolumn{2}{|c|}{ Jul-12 } \\
\hline \multicolumn{2}{|c|}{$\begin{array}{c}4.76 \\
1.3 \\
5.20\end{array}$} \\
\hline 0.5 & 27.5 \\
\hline 25.6 & 7.5 \\
\hline 541 & 576 \\
\hline 8.0 & 7.6 \\
\hline 8.4 & 0.0 \\
\hline 0.012 & 0.029 \\
\hline$\cdots$ & $\ldots$ \\
\hline$\ldots$ & --- \\
\hline$\ldots$ & $\cdots$ \\
\hline-- & -- \\
\hline--- & -- \\
\hline--- & --- \\
\hline--- & $\cdots$ \\
\hline$\cdots$ & $\cdots$ \\
\hline 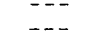 & -1 \\
\hline$\ldots$ & - \\
\hline$\ldots$ & $\ldots$ \\
\hline$\ldots$ & $\ldots$ \\
\hline$\ldots$ & $\ldots$ \\
\hline$\ldots$ & $\ldots$ \\
\hline$\cdots$ & -- \\
\hline.-- & $\ldots$ \\
\hline-- & 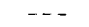 \\
\hline$\cdots$ & $\cdots$ \\
\hline
\end{tabular}

\begin{tabular}{|c|c|c|}
\hline & Aug -9 & \\
\hline & $\begin{array}{c}4.78 \\
1.3 \\
7.00\end{array}$ & \\
\hline $\begin{array}{c}0.5 \\
24.4 \\
517 \\
8.0 \\
7.4 \\
0.023\end{array}$ & $\begin{array}{c}11 \\
10.8 \\
562 \\
7.7 \\
1.0 \\
0.018\end{array}$ & $\begin{array}{c}26.5 \\
7.6 \\
570 \\
7.6 \\
0.0 \\
0.034\end{array}$ \\
\hline$\ldots$ & -- & $\ldots$ \\
\hline$\ldots$ & -.. & $\cdots$ \\
\hline -. & $-\ldots$ & $\ldots$ \\
\hline$\ldots$ & - - & $\ldots$ \\
\hline-- & -- & --- \\
\hline$\ldots$ & -- & $\ldots$ \\
\hline$\ldots$ & $\rightarrow$ & $\ldots$ \\
\hline$\ldots$ & $-\cdots$ & $\ldots$ \\
\hline$\ldots$ & $\cdots$ & $\cdots$ \\
\hline--- & -- & $\cdots$ \\
\hline$\ldots$ & -- & $\ldots$ \\
\hline$\cdots$ & -- & -- \\
\hline$\cdots$ & - - & $\cdots$ \\
\hline$\ldots$ & -- & $\ldots$ \\
\hline-- & $\cdots$ & -- \\
\hline-- & $\cdots$ & -- \\
\hline$\ldots$ & -- & $\cdots$ \\
\hline-- & --- & $\cdots$ \\
\hline-- & $\cdots$ & $\cdots$ \\
\hline
\end{tabular}

8-09-99

DISSOLVED OXYGEN (D.O.), IN MILLIGRAMS PER LITER
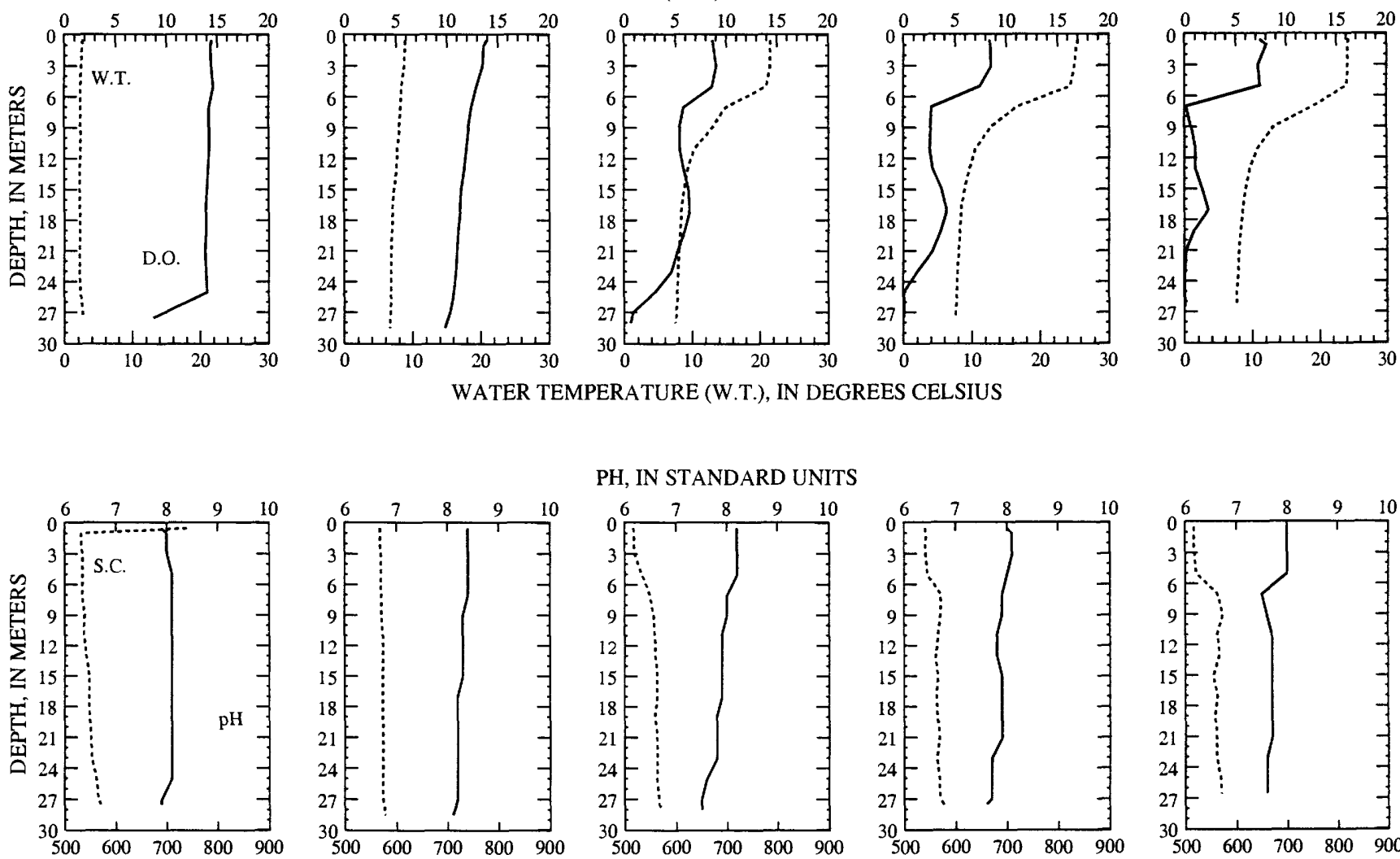

PH, IN STANDARD UNITS
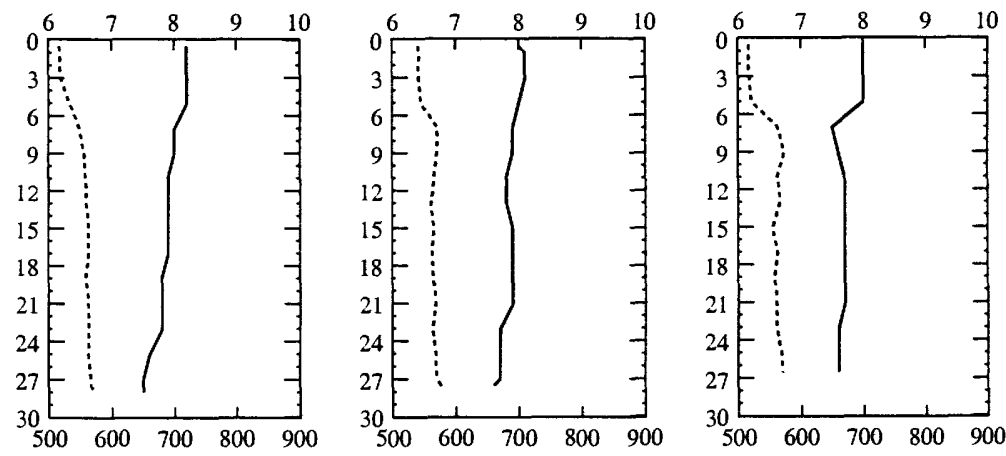

SPECIFIC CONDUCTANCE (S.C.), IN MICROSIEMENS PER CENTIMETER AT 25 DEGREES CELSIUS 

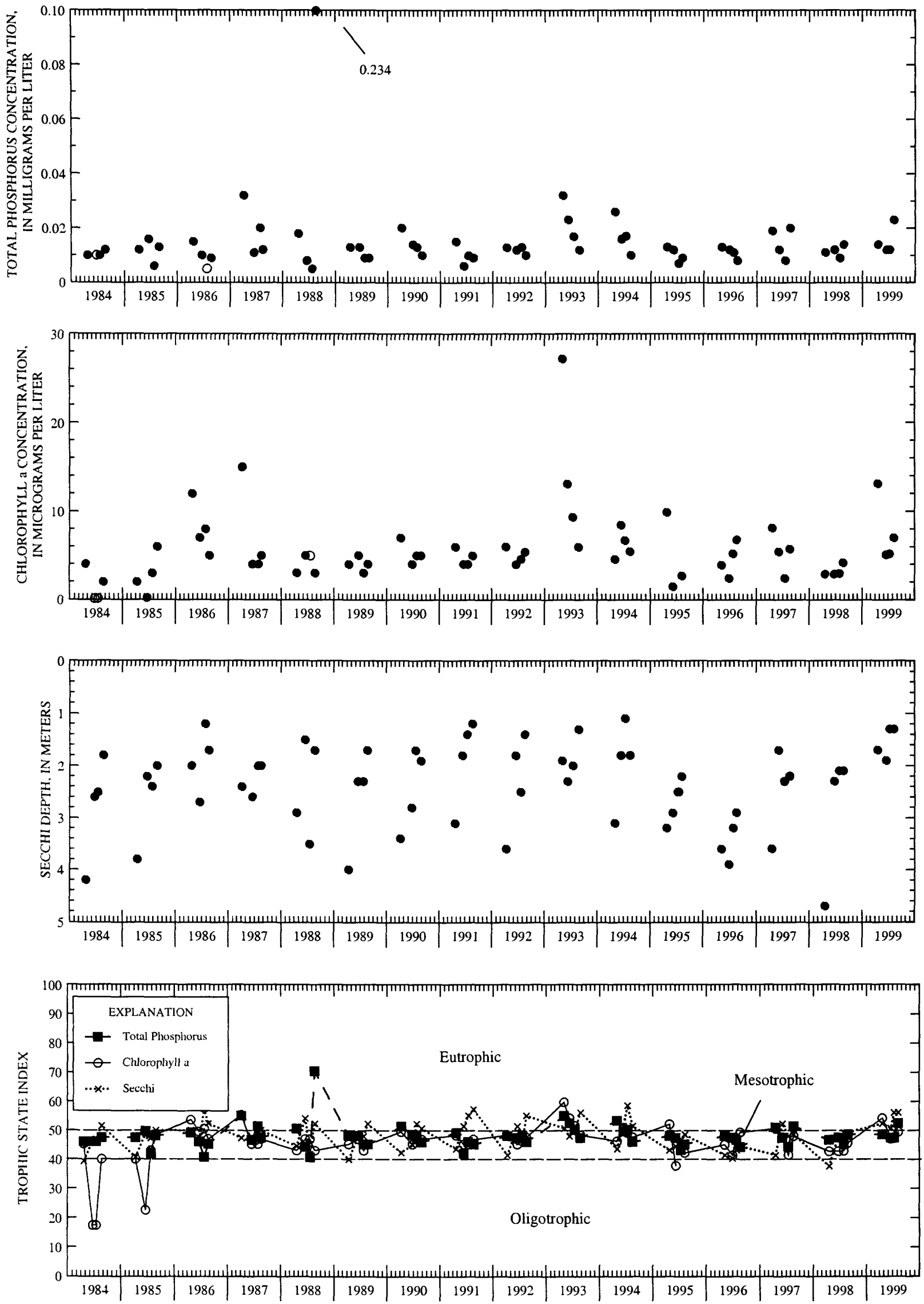

YEAR

Surface total phosphorus, chlorophyll a concentrations, Secchi depths, and TSI data for Okauchee Lake at Okauchee, Wisconsin. 
LOCATION.--Lat $43^{\circ} 07^{\prime} 59^{\prime \prime}$, long $88^{\circ} 24^{\prime} 42^{\prime \prime}$, in NE $1 / 4$ NW 1/4 sec.30, T.8 N., R. 18 E., Waukesha County, Hydrologic Unit 07090001 , near Okauchee.

PERIOD OF RECORD.--April 1986 to current year.

LAKE-STAGE GAGE.--Datum of gage is $869.00 \mathrm{ft}$ above sea level.

REMARKS.--Lake sampled in Crane's Nest Bay, in the northeast part of the lake, at an approximate depth of $2 \mathrm{~m}$. Water-quality analyses done by Wisconsin State Laboratory of Hygiene.

WATER-QUALITY DATA, APRIL 20 TO AUGUST 09, 1999

(Milligrams per liter unless otherwise indicated)

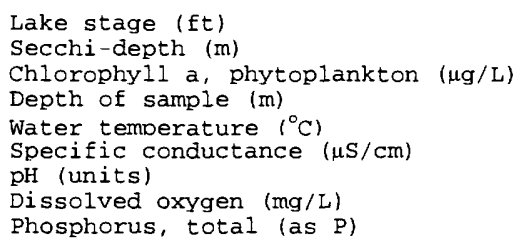

\begin{tabular}{cccc} 
Apr-20 & Jun-18 & Jul-12 & Aug-9 \\
\hline 4.65 & 4.79 & 4.76 & 4.78 \\
1.4 & 1.9 & 1.1 & 1.5 \\
15.3 & 2.69 & 3.58 & 10 \\
0.5 & 0.5 & 0.5 & 0.5 \\
9.0 & 21.9 & 25.9 & 24.4 \\
594 & 518 & 547 & 517 \\
8.6 & 8.3 & 8.2 & 8.3 \\
14.8 & 9.0 & 8.4 & 7.6 \\
0.021 & 0.012 & 0.011 & 0.022
\end{tabular}

\section{OKAUCHEE LAKE, NO. 2, AT OKAUCHEE, WI}

LOCATION.--Lat $43^{\circ} 06^{\prime} 45^{\prime \prime}$, long $88^{\circ} 26^{\prime} 45^{\prime \prime}$, in SE 1/4 NE 1/4 sec.35, T.8 N., R.17 E., Waukesha County, Hydrologic Unit 07090001, at Okauchee.

PERIOD OF RECORD.--April 1986 to current year.

LAKE-STAGE GAGE.--Datum of gage is $869.00 \mathrm{ft}$ above sea level.

REMARKS.--Lake sampled in Lower Okauchee Lake, at an approximate depth of $5 \mathrm{~m}$. Water-quality analyses done by Wisconsin State Laboratory of Hygiene.

WATER-QUALITY DATA, APRIL 20 TO AUGUST 09, 1999

(Milligrams per liter unless otherwise indicated)

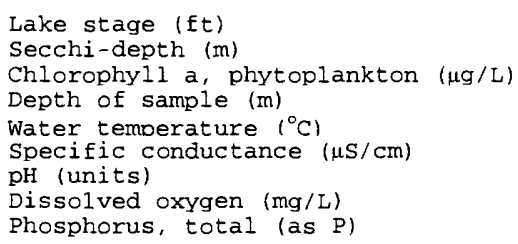

\begin{tabular}{cccc} 
Apr-20 & Jun-18 & Ju1-12 & Aug-9 \\
\hline 4.65 & 4.79 & 4.76 & 4.78 \\
1.1 & 1.4 & 1.6 & 2.1 \\
10.5 & 2.44 & 4.34 & 4.10 \\
0.5 & 0.5 & 0.5 & 0.5 \\
10.4 & 22.3 & 26.5 & 24.5 \\
563 & 504 & 520 & 498 \\
8.5 & 8.3 & 8.2 & 8.1 \\
13.4 & 8.7 & 9.0 & 7.5 \\
0.012 & 0.013 & 0.013 & 0.025
\end{tabular}




\section{OKAUCHEE LAKE, NO. 3, AT OKAUCHEE, WI}

LOCATION.--Lat $43^{\circ} 06^{\prime} 42^{\prime \prime}$, long $88^{\circ} 25^{\prime} 24^{\prime \prime}$, in NE 1/4 SE 1/4 sec.36, T.8 N., R.17 E., Waukesha County, Hydrologic Unit 07090001, at Okauchee.

PERIOD OF RECORD.--April 1986 to current year.

LAKE-STAGE GAGE.--Datum of gage is $869.00 \mathrm{ft}$ above sea level.

REMARKS.--Lake sampled in Ice House Bay, in the southern part of the lake, at an approximate depth of $4 \mathrm{~m}$. Water-quality analyses done by Wisconsin State Laboratory of Hygiene.

WATER-QUALITY DATA, APRIL 20 TO AUGUST 09, 1999

(Milligrams per liter unless otherwise indicated)

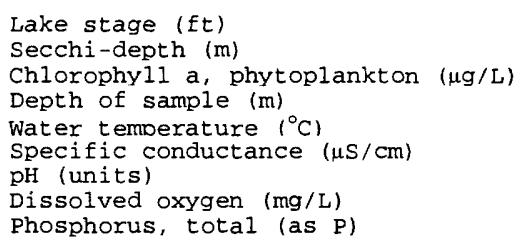

\begin{tabular}{cccc} 
Apr-20 & Jun-18 & Jul-12 & Aug-9 \\
\hline 4.65 & 4.79 & 4.76 & 4.78 \\
1.3 & 1.3 & 1.3 & 1.5 \\
9.61 & 5.59 & 6 & 7.00 \\
0.5 & 0.5 & 0.5 & 0.5 \\
9.6 & 21.7 & 26.1 & 24.1 \\
560 & 502 & 525 & 500 \\
8.6 & 8.3 & 8.2 & 8.2 \\
13.4 & 9.1 & 8.6 & 7.6 \\
0.011 & 0.019 & 0.015 & 0.026
\end{tabular}

\section{OKAUCHEE LAKE, NO. 4, AT OKAUCHEE, WI}

LOCATION.--Lat $43^{\circ} 07^{\prime} 57^{\prime \prime}$, long $88^{\circ} 26^{\prime} 17^{\prime \prime}$, in NW 1/4 NW 1/4 sec.25, T.8 N., R.17 E., Waukesha County, Hydrologic Unit 07090001, at Okauchee.

PERIOD OF RECORD.--June 1986 to current year.

LAKE-STAGE GAGE.--Datum of gage is $869.00 \mathrm{ft}$ above sea level.

REMARKS.--Lake sampled near McDowell (Crazyman's) Island, in the northwest bay of the lake, at an approximate depth of $2 \mathrm{~m}$. Waterquality analyses done by Wisconsin State Laboratory of Hygiene.

WATER-QUALITY DATA, APRIL 20 TO AUGUST 09, 1999

(Milligrams per liter unless otherwise indicated)

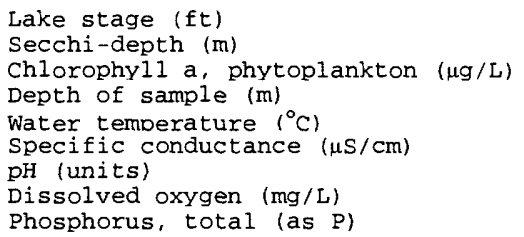

\begin{tabular}{cccc} 
Apr-20 & Jun-18 & Jul-12 & Aug-9 \\
\hline 4.65 & 4.79 & 4.76 & 4.78 \\
1.3 & 1.7 & 1.1 & 1.3 \\
8.90 & 2.62 & 4.42 & 6.00 \\
0.5 & 0.5 & 0.5 & 0.5 \\
9.1 & 21.9 & 26.4 & 23.9 \\
569 & 517 & 540 & 513 \\
8.5 & 8.3 & 8.1 & 8.4 \\
13.1 & 8.5 & 7.7 & 8.1 \\
0.011 & 0.015 & 0.011 & 0.021
\end{tabular}


LOCATION.--Lat $42^{\circ} 49^{\prime} 05^{\prime \prime}$, long 88 $20^{\prime} 40^{\prime \prime}$, in NW 1/4 SW 1/4 sec.11, T.4 N., R.18 E., Walworth County. Hydrologic Unit 07120006, $3.3 \mathrm{mi}$ south of Mukwonago.

PERIOD OF RECORD.--February 1993 to current year.

REMARKS.--Lake sampled at the deep hole. Lake ice-covered during February sampling. Water-quality analyses done by Wisconsin State Laboratory of Hygiene.

WATER-QUALITY DATA, FEBRUARY 11 TO AUGUST 05, 1999

(Milligrams per liter unless otherwise indicated)

Lake stage (ft)

Secchi-depth $(m)$

Chlorophyll a, phytoplankton $(\mu \mathrm{g} / \mathrm{L})$

Depth of sample (m)

Water temperature $\left({ }^{\circ} \mathrm{C}\right)$

Specific conductance $(\mu S / \mathrm{cm})$

$\mathrm{pH}$ (units)

Dissolved oxygen (mg/L)

Phosphorus, total (as P)

Phosphorus, ortho, dissolved (as P)

Nitrogen, $\mathrm{NO} 2+\mathrm{NO} 3$, diss. (as N)

Nitrogen, ammonia, dissolved (as N)

Nitrogen, amm. + org., total (as N)

Nitrogen, total (as N)

Color (Pt-Co. scale)

Turbidity (NTU)

Hardness, (as $\mathrm{CaCO}_{3}$ )

Calcium, dissolved (Ca)

Magnesium, dissolved (Mg)

Sodium, dissolved (Na)

Potassium, dissolved (K)

Alkalinity, (as $\mathrm{CaCO}_{3}$ )

Sulfate, dissolved $\left(\mathrm{SO}_{4}\right)$

Chloride, dissolved (Cl)

Silica, dissolved $\left(\mathrm{SiO}_{2}\right)$

Solids, dissolved, at $180^{\circ} \mathrm{C}$

Iron, dissolved (Fe) $\mu \mathrm{g} / \mathrm{L}$

Manganese, dissolved (Mn) $\mu \mathrm{g} / \mathrm{L}$

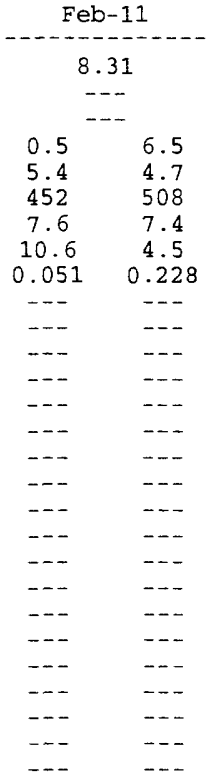

4-21-99

\begin{tabular}{cc}
\multicolumn{2}{c}{ Apr-21 } \\
\multicolumn{2}{c}{3.13} \\
\multicolumn{2}{c}{1.91} \\
0.5 & 7.0 \\
11.2 & 10.7 \\
486 & 487 \\
8.1 & 8.0 \\
11.2 & 10.7 \\
0.017 & 0.034 \\
0.011 & --- \\
0.048 & --- \\
0.196 & --- \\
1.3 & --- \\
1.3 &.-- \\
15 & -- \\
0.90 & --- \\
180 & --- \\
40 & --- \\
20 & --- \\
20 & --- \\
2.2 & --- \\
171 & --- \\
7.6 & --- \\
45 & -- \\
0.16 & --- \\
272 & --- \\
20 & $-\ldots$ \\
4.9 & --- \\
&
\end{tabular}

6-11-99

\begin{tabular}{|c|c|}
\hline \multicolumn{2}{|c|}{$\begin{array}{c}8.27 \\
1.1 \\
7.74\end{array}$} \\
\hline $\begin{array}{c}0.5 \\
26.9 \\
445 \\
8.3 \\
9.1 \\
0.022\end{array}$ & $\begin{array}{c}7.0 \\
15.8 \\
513 \\
7.4 \\
0.6 \\
0.047\end{array}$ \\
\hline--- & --- \\
\hline$\cdots$ & --- \\
\hline--- & --- \\
\hline--- & --- \\
\hline$-\cdots$ & --- \\
\hline$-\cdots$ & $\cdots$ \\
\hline-- & -- \\
\hline$\cdots$ & --- \\
\hline-- & --- \\
\hline-- & --- \\
\hline-- & -- \\
\hline$\cdots$ & $-\cdots$ \\
\hline--- & $-\cdots$ \\
\hline-- & --- \\
\hline--- & -- \\
\hline$\cdots$ & $\rightarrow--$ \\
\hline--- & --- \\
\hline-- & -- \\
\hline- & -- \\
\hline
\end{tabular}

7-08-99
Ju1-8

7.96

0.7

19.6

$\begin{array}{ll}0.5 & 7.0\end{array}$

$26.8 \quad 15.9$

$444 \quad 531$

$\begin{array}{ll}8.1 & 6.9 \\ 8.2 & 0.5\end{array}$

$0.046 \quad 0.062$

0.018

0.021

0.048

1.7

1.8

1.8

-

-..

$--$

$--$

-

$--$

$--$

$-\cdots$

- -

$---$

$--$

$--$

$--$

-.-

$--$

-

$--$

$--$

$--$

$--$

$---$

2-11-99
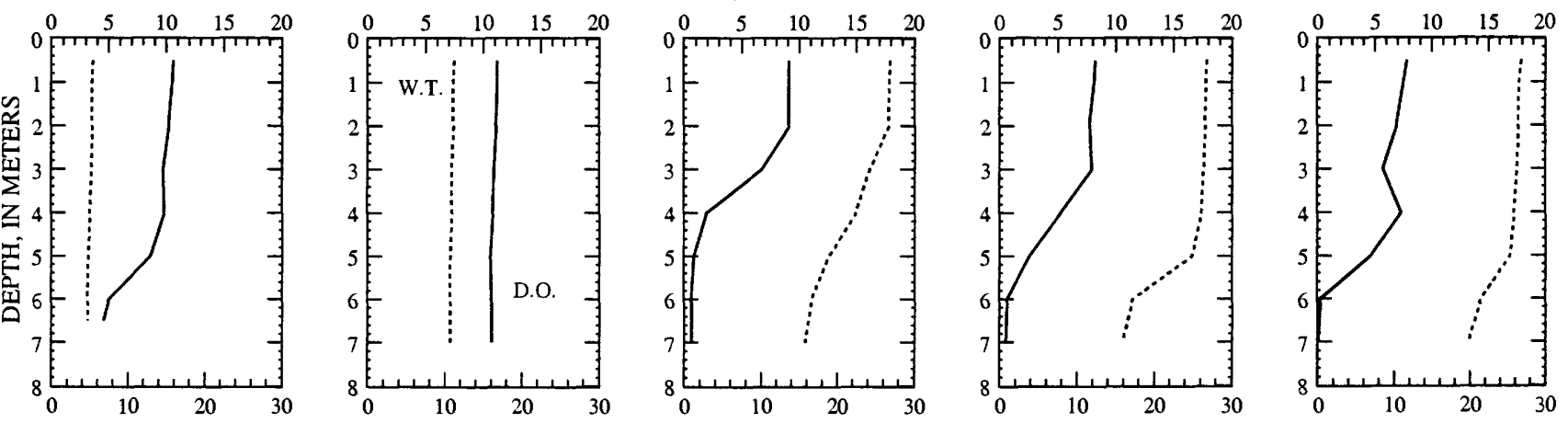

WATER TEMPERATURE (W.T.), IN DEGREES CELSIUS

\section{PH, IN STANDARD UNITS}
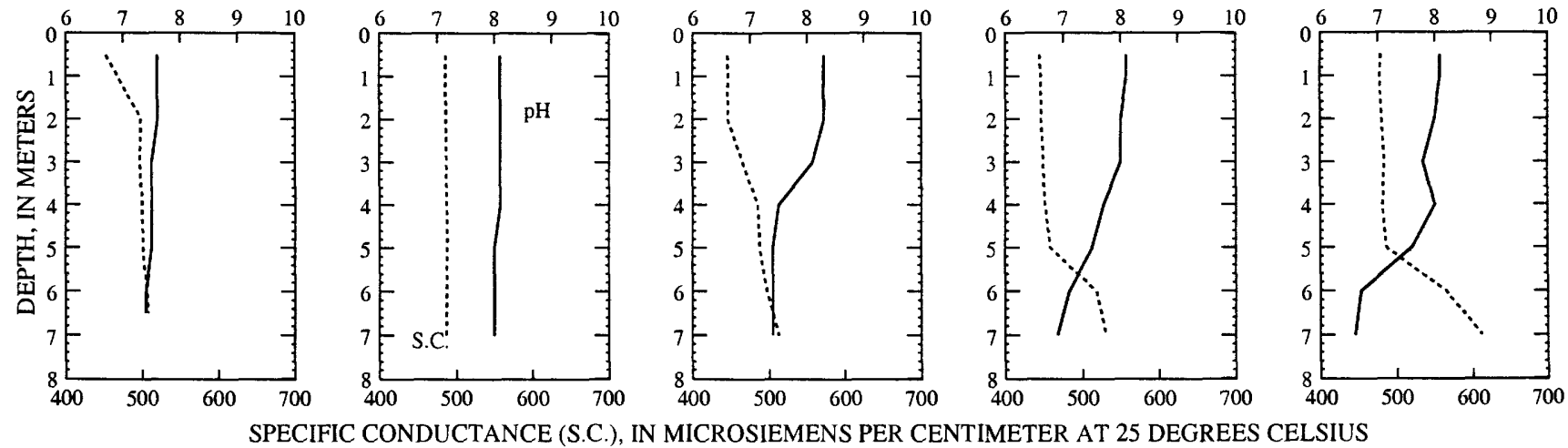

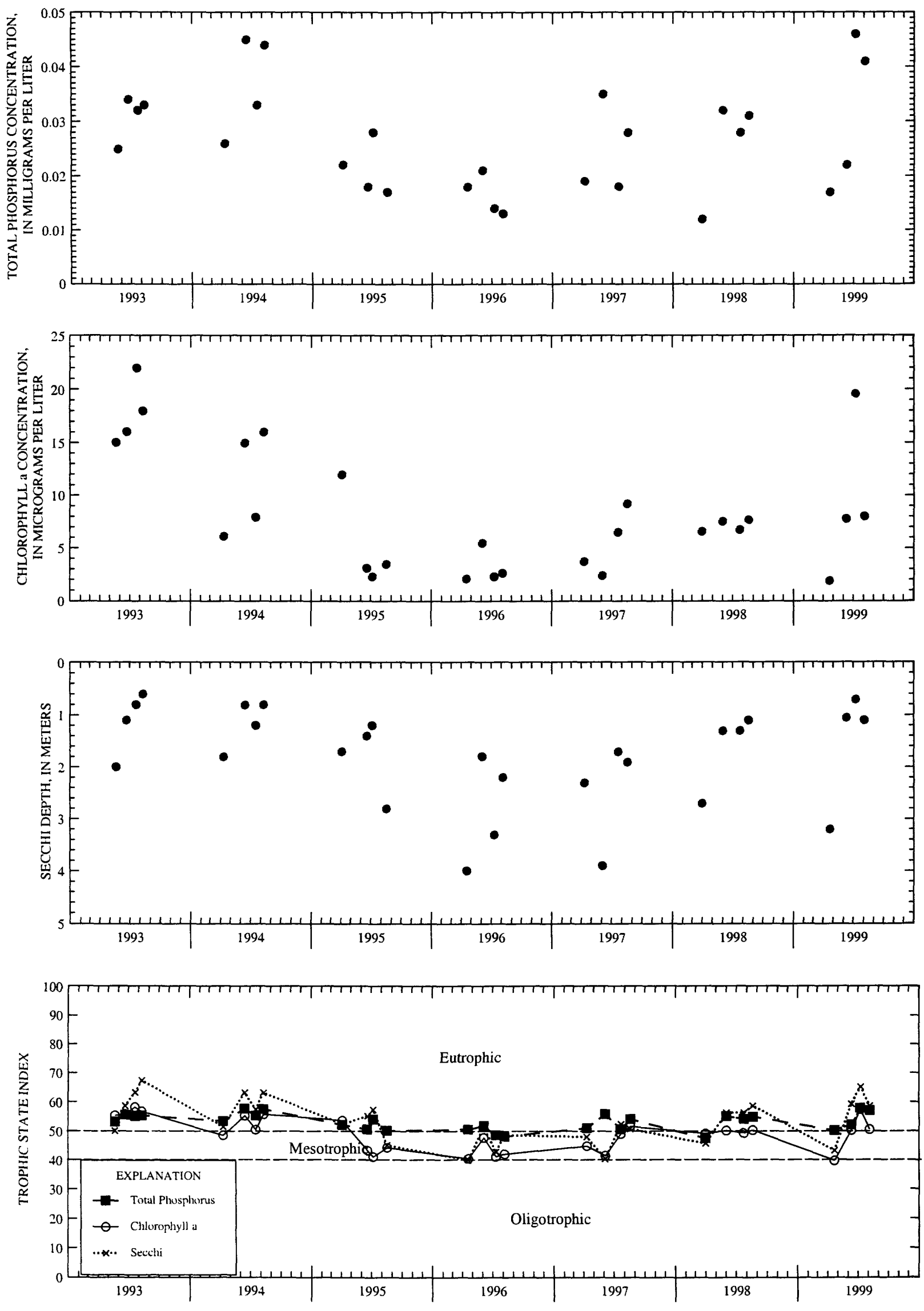

YEAR

Surface total phosphorus, chlorophyll a concentrations, Secchi depths, and TSI data for Potter Lake near Mukwonago, Wisconsin. 
LOCATION.--Lat $42^{\circ} 32^{\prime} 46^{\prime \prime}$, long $88^{\circ} 1^{\prime} 7^{\prime} 58^{\prime \prime}$, in NW 1/4 SE 1/4 sec.13, T.1 N., R.18 E., Walworth County, Hydrologic Unit 07120006, at Powers Lake.

DRAINAGE AREA.--3.42 $\mathrm{mi}^{2}$.

PERIOD OF RECORD.--March 1986 to August 1996, and April 1998 to current year.

REMARKS.--Lake sampled near center at the deep hole. Lake ice-covered during February sampling. Water-quality analyses done by Wisconsin State Laboratory of Hygiene.

$$
\begin{aligned}
& \text { WATER-QUALITY DATA, FEBRUARY } 19 \text { TO AUGUST 04, } 1999 \\
& \text { (Mililigrams per liter unless otherwise indicated) }
\end{aligned}
$$

Lake stage ( $f t$ )

Secchi-depth (m)

Chlorophyll a, phytoplankton ( $\mu \mathrm{q} / L)$

Depth of sample (m)

water temperature $\left({ }^{\circ} \mathrm{C}\right)$

Specific conductance $(\mu \mathrm{S} / \mathrm{cm})$

$\mathrm{pH}$ (units)

Dissolved oxygen (mg/L)

Phosphorus, total (as P)

Phosphorus, ortho, dissolved (as P)

$\mathrm{Nitrogen,} \mathrm{NO} 2+\mathrm{NO} 3$, diss. (as N)

Nitrogen, ammonia, dissolved (as N)

Nitrogen, amm. + org., total (as N)

Nitrogen, total (as $\mathrm{N}$ )

Color (Pt-Co. scale)

Turbidity (NTU)

Hardness, (as $\mathrm{CaCO}_{3}$ )

Calcium, dissolved ( $\mathrm{Ca}$

Magnesium, dissolved (Mg)

Sodium, dissolved (Na)

Potassium, dissolved (K)

Alkalinity, (as $\mathrm{CaCO}_{3}$ )

Sulfate, dissolved $\left(\mathrm{SO}_{4}\right)$

Chloride, dissolved (C1)

Silica, dissolved ( $\left.\mathrm{SiO}_{2}\right)$

Solids, dissolved. at $180^{\circ} \mathrm{C}$

Iron, dissolved ( $\mathrm{Fe}) \mu \mathrm{q} / \mathrm{L}$

\begin{tabular}{|c|c|}
\hline & 19 \\
\hline & \\
\hline & \\
\hline & \\
\hline 0.5 & 9.0 \\
\hline 4.9 & 3.8 \\
\hline 415 & 518 \\
\hline 7.8 & 7.9 \\
\hline 18.6 & 15.1 \\
\hline 0.011 & 0.009 \\
\hline$\ldots$ & $\cdots$ \\
\hline--- & -- \\
\hline--- & $\ldots$ \\
\hline.- & -.- \\
\hline--- & -- \\
\hline--- & --- \\
\hline$\ldots$ & --- \\
\hline$\ldots$ & --- \\
\hline$\ldots$ & --- \\
\hline--- & --- \\
\hline$\ldots$ & --- \\
\hline-- & $-\ldots$ \\
\hline--- & $-\ldots$ \\
\hline--- & - - \\
\hline-- & $\cdots$ \\
\hline-- & --- \\
\hline--- & --- \\
\hline$\cdots-$ & $\cdots$ \\
\hline- & $-\cdots$ \\
\hline
\end{tabular}

Manqanese, dissolved $(M n) \mu q / L$

\begin{tabular}{|c|c|}
\hline \multicolumn{2}{|c|}{ Apr-14 } \\
\hline \multicolumn{2}{|c|}{$\begin{array}{c}10.00 \\
6.1 \\
2.57\end{array}$} \\
\hline 0.5 & 10.0 \\
\hline 11.9 & 9.4 \\
\hline 507 & 511 \\
\hline 8.2 & 8.1 \\
\hline 10.7 & 9.4 \\
\hline 0.010 & 0.013 \\
\hline$<0.002$ & $\ldots$ \\
\hline 0.07 & $\ldots$ \\
\hline 0.037 & -.. \\
\hline 0.6 & $-\ldots$ \\
\hline 0.67 & -- \\
\hline 5 & $\ldots$ \\
\hline 0.5 & - - \\
\hline 220 & -- \\
\hline 36 & -- \\
\hline 31 & $\ldots$ \\
\hline 17 & -- \\
\hline 2.3 & $\cdots$ \\
\hline 183 & -.. \\
\hline 31 & $\ldots$ \\
\hline 38 & --- \\
\hline 5.8 & --- \\
\hline 282 & $\ldots$ \\
\hline$<10$ & --- \\
\hline 1.9 & $\cdots$ \\
\hline
\end{tabular}

4-14-99

\begin{tabular}{|c|c|}
\hline \multicolumn{2}{|c|}{ Jun-10 } \\
\hline \multicolumn{2}{|c|}{$\begin{array}{c}10.23 \\
3.1 \\
2.77\end{array}$} \\
\hline 0.5 & 10.0 \\
\hline 25.6 & 13.7 \\
\hline 482 & 528 \\
\hline 8.2 & 7.5 \\
\hline 9.2 & 0.6 \\
\hline 0.012 & 0.024 \\
\hline--- & $\ldots$ \\
\hline-- & $\ldots$ \\
\hline$\ldots$ & $\ldots$ \\
\hline$\ldots$ & --- \\
\hline--- & --- \\
\hline--- & $\ldots$ \\
\hline$\ldots$ & --- \\
\hline$\ldots$ & --- \\
\hline-- & --- \\
\hline$\ldots$ & --- \\
\hline--- & --- \\
\hline$\ldots$ & --- \\
\hline-- & $\cdots$ \\
\hline--- & --- \\
\hline--- & --- \\
\hline$-\cdots$ & -- \\
\hline$-\cdots$ & --- \\
\hline$\cdots$ & --- \\
\hline-- & --- \\
\hline
\end{tabular}

6-10-99

\begin{tabular}{|c|c|}
\hline & \\
\hline & \\
\hline 0.5 & 10.0 \\
\hline 26 & 14 \\
\hline 446 & 524 \\
\hline 8.2 & 7.5 \\
\hline 8.2 & 0.4 \\
\hline 0.013 & 0.084 \\
\hline 0.002 & $\ldots$ \\
\hline$<0.010$ & $\ldots$ \\
\hline 0.016 & $\ldots$ \\
\hline 0.58 & $\ldots$ \\
\hline-- & $\ldots$ \\
\hline--- & $\ldots$ \\
\hline$\ldots$ & $\ldots$ \\
\hline$\ldots$ & $\ldots$ \\
\hline$\ldots$ & $\ldots$ \\
\hline$\ldots$ & $\ldots$ \\
\hline-- & --- \\
\hline-- & $\ldots$ \\
\hline-- & $\ldots$ \\
\hline--- & $\ldots$ \\
\hline$\cdots$ & -- \\
\hline$-\cdots$ & --- \\
\hline-- & $\ldots$ \\
\hline$\ldots$ & $\ldots$ \\
\hline--- & -- \\
\hline
\end{tabular}

$7-07-99$

\begin{tabular}{|c|c|c|}
\hline \multicolumn{3}{|c|}{ Aug -4} \\
\hline & $\begin{array}{c}9.83 \\
1.9 \\
2.81\end{array}$ & \\
\hline $\begin{array}{c}0.5 \\
27.1 \\
455 \\
8.1 \\
8.1 \\
0.019\end{array}$ & $\begin{array}{c}8.0 \\
19.3 \\
490 \\
7.3 \\
0.6 \\
0.024\end{array}$ & $\begin{array}{c}9.5 \\
14.6 \\
530 \\
7.2 \\
0.0 \\
0.061\end{array}$ \\
\hline--- & $-\cdots$ & $\cdots$ \\
\hline--- & -- & $\cdots$ \\
\hline--- & -- & $\ldots$ \\
\hline-- & -- & -- \\
\hline-- & -- & --- \\
\hline-- & -- & $\cdots$ \\
\hline-- & -- & -- \\
\hline--- & -- & $\cdots$ \\
\hline-- & -- & $\cdots$ \\
\hline--- & --- & -- \\
\hline--- & --- & $\cdots$ \\
\hline$\cdots$ & -- & -- \\
\hline$-\infty$ & --- & -- \\
\hline$\cdots$ & $\cdots$ & -- \\
\hline$\cdots$ & -- & --- \\
\hline--- & --- & --- \\
\hline--- & --- & --- \\
\hline 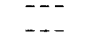 & $\begin{array}{l}--- \\
--\end{array}$ & --- \\
\hline$\cdots$ & -- & $\cdots$ \\
\hline
\end{tabular}

8-04-99

DISSOLVED OXYGEN (D.O.), IN MILLIGRAMS PER LITER
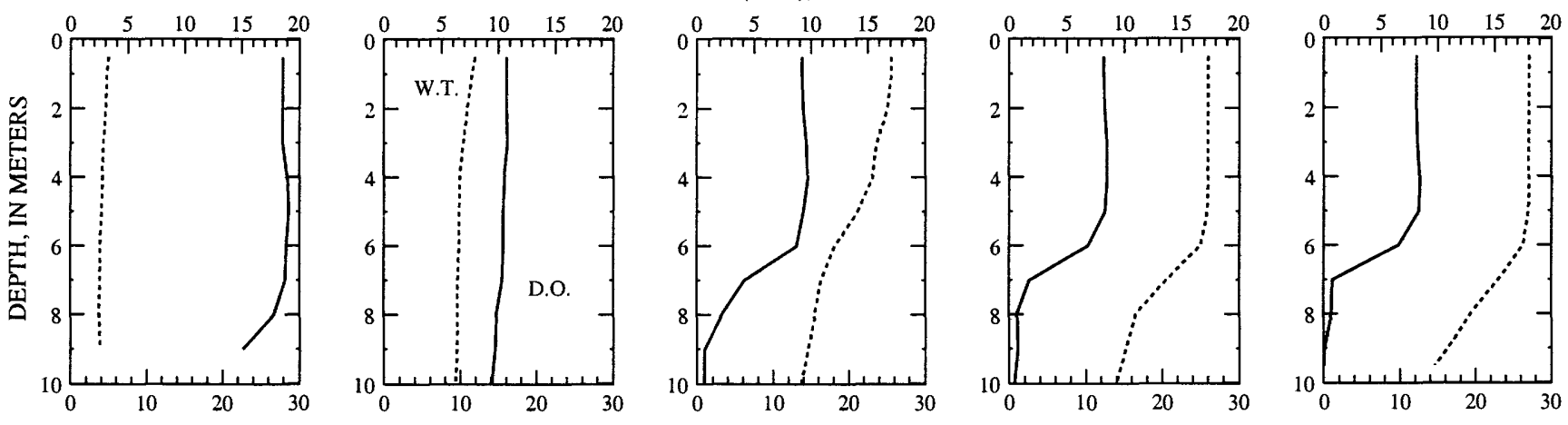

WATER TEMPERATURE (W.T.), IN DEGREES CELSIUS
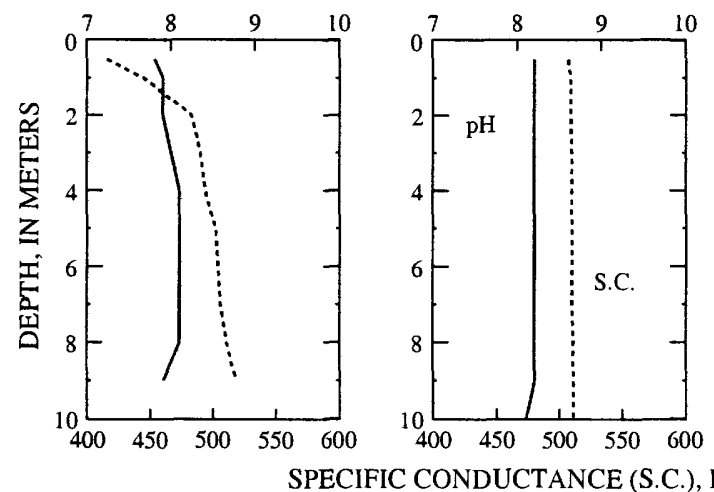

PH, IN STANDARD UNITS
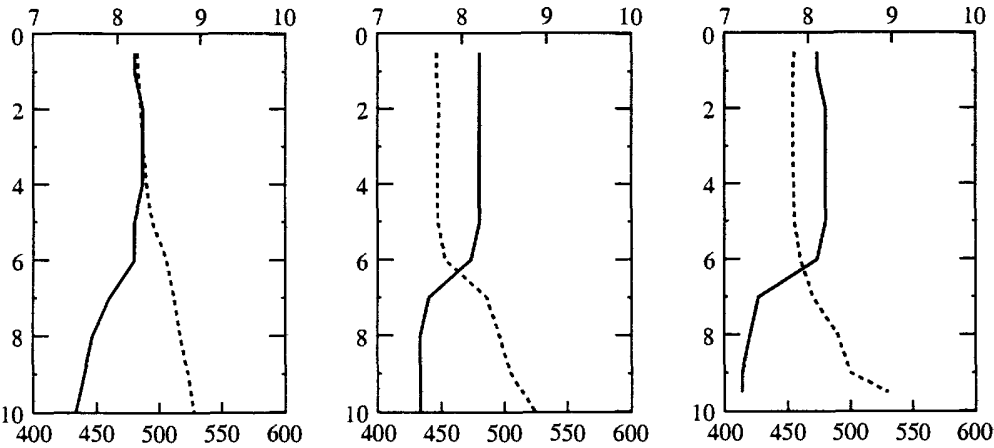

SPECIFIC CONDUCTANCE (S.C.), IN MICROSIEMENS PER CENTIMETER AT 25 DEGREES CELSIUS 

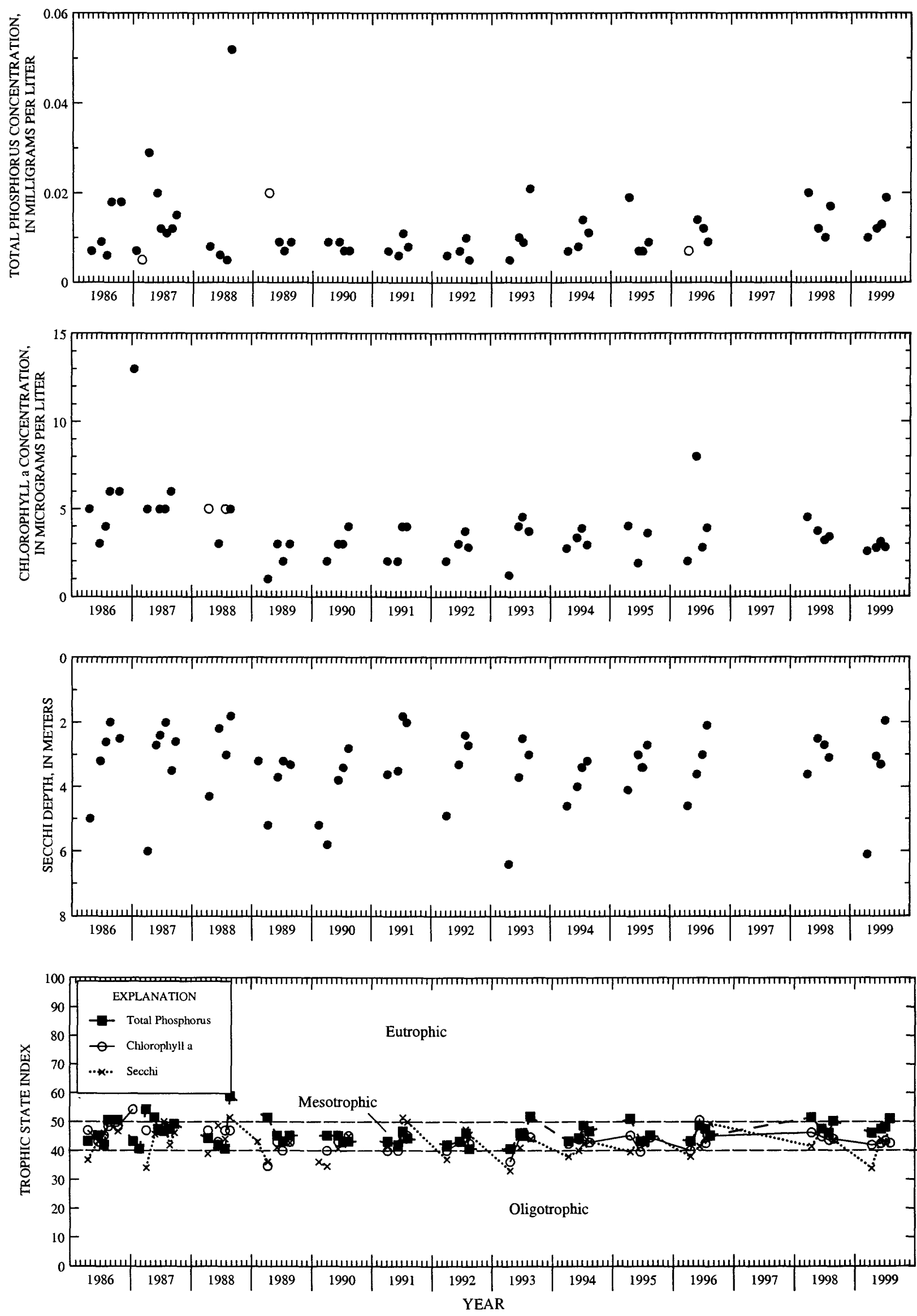

Surface total phosphorus, chlorophyll a concentrations, Secchi depths, and TSI data for Powers Lake at Powers Lake, Wisconsin. 
LOCATION--Lat $45^{\circ} 44^{\prime} 46^{\prime \prime}$, long $91^{\circ} 55^{\prime} 00^{\prime \prime}$, in NE 1/4 sec. 25 , T.38 N., R. 13 W., Washburn County, Hydrologic Unit 07030001.500 ft east of Peterson Boat Factory in the village of Shell Lake.

DRAINAGE AREA.--26.0 $\mathrm{mi}^{2}$. Area of Shell Lake, 3,200 acres.

PERIOD OF RECORD.--August 1936 to September 1999.

REVISED RECORD.--WDR WI-80-1: Drainage area.

GAGE.--Nonrecording gage. Datum of gage is $1,215.88 \mathrm{ft} \mathrm{ft}$, National Geodetic Vertical Datum of 1929. May 3, 1952 to Apr. 21, I961, $2.3 \mathrm{mi}$ southeast of village of Shell Lake at same datum.

REMARKS.--Stage measured on the west side of lake intermittently by an observer from 1936 to 1998 . In 1998-99 water year, stage was continuously measured during non-freezing periods.

EXTREMES FOR PERIOD OF RECORD.--Maximum gage height observed, $6.31 \mathrm{ft}$, June 27, 1998; minimum observed, -0.92 ft, Nov. 28 , 1949.

EXTREMES FOR CURRENT PERIOD.--1998 water year: Maximum gage height observed, 6.31, June 27; minimum observed, $5.32 \mathrm{ft}$, Sept. 24. 1999 water year: Maximum gage height observed, 5.34, Oct. 1; minimum observed, $3.40 \mathrm{ft}$, Sept. 29, 30 .

GAGE HEIGHT, FEET, WATER YEAR OCTOBER 1997 TO SEPTEMBER 1998

DAILY MEAN VALUE

\begin{tabular}{|c|c|c|c|c|c|c|c|c|c|c|c|c|}
\hline DAY & OCT & NOV & DEC & JAN & FEB & MAR & APR & MAY & $\pi \mathbb{N}$ & JUL & AUG & SEP \\
\hline $\begin{array}{l}1 \\
2 \\
3 \\
4 \\
5\end{array}$ & $\begin{array}{l}--- \\
--- \\
--- \\
--- \\
---\end{array}$ & $\begin{array}{l}--- \\
--- \\
--- \\
--- \\
---\end{array}$ & $\begin{array}{l}--- \\
--- \\
--- \\
--- \\
---\end{array}$ & $\begin{array}{l}--- \\
-\cdots \\
-\cdots \\
--- \\
-\cdots\end{array}$ & $\begin{array}{l}-- \\
-- \\
-- \\
-- \\
---\end{array}$ & $\begin{array}{l}--- \\
--- \\
--- \\
--- \\
---\end{array}$ & $\begin{array}{r}--- \\
6.28 \\
--- \\
--- \\
---\end{array}$ & $\begin{array}{r}--- \\
--- \\
--- \\
6.18\end{array}$ & $\begin{array}{l}6.22 \\
6.22 \\
6.20 \\
6.18 \\
6.16\end{array}$ & $\begin{array}{l}6.24 \\
6.24 \\
6.23 \\
6.21 \\
6.19\end{array}$ & $\begin{array}{l}5.79 \\
5.77 \\
5.78 \\
5.78 \\
5.77\end{array}$ & $\begin{array}{l}5.70 \\
5.68 \\
5.67 \\
5.64 \\
5.62\end{array}$ \\
\hline $\begin{array}{r}6 \\
7 \\
8 \\
9 \\
10\end{array}$ & $\begin{array}{c}5.90 \\
-- \\
\ldots \\
- \\
\ldots\end{array}$ & $\begin{array}{l}-- \\
--- \\
--- \\
--- \\
---\end{array}$ & $\begin{array}{l}--- \\
--- \\
--- \\
--- \\
---\end{array}$ & $\begin{array}{l}--- \\
-- \\
-\cdots \\
--- \\
---\end{array}$ & $\begin{array}{l}--- \\
-\cdots \\
--- \\
--- \\
---\end{array}$ & $\begin{array}{l}--- \\
--- \\
--- \\
--- \\
---\end{array}$ & $\begin{array}{r}--- \\
--- \\
--- \\
--- \\
6.34\end{array}$ & $\begin{array}{l}6.17 \\
6.17 \\
6.16 \\
6.15 \\
6.14\end{array}$ & $\begin{array}{l}6.14 \\
6.13 \\
6.12 \\
6.11 \\
6.10\end{array}$ & $\begin{array}{l}6.21 \\
6.22 \\
6.21 \\
6.20 \\
6.19\end{array}$ & $\begin{array}{l}5.77 \\
5.77 \\
5.79 \\
5.78 \\
5.76\end{array}$ & $\begin{array}{l}5.61 \\
5.59 \\
5.57 \\
5.55 \\
5.53\end{array}$ \\
\hline $\begin{array}{l}11 \\
12 \\
13 \\
14 \\
15\end{array}$ & $\begin{array}{c}\ldots . \\
\ldots .96 \\
\ldots-\end{array}$ & $\begin{array}{r}-- \\
5.67 \\
--- \\
--- \\
---\end{array}$ & $\begin{array}{l}\cdots \\
--- \\
-- \\
-\cdots \\
--\end{array}$ & $\begin{array}{l}--- \\
--- \\
--- \\
--- \\
-\cdots\end{array}$ & $\begin{array}{l}--- \\
--- \\
--- \\
--- \\
---\end{array}$ & $\begin{array}{l}--- \\
-- \\
--- \\
--- \\
---\end{array}$ & $\begin{array}{l}--- \\
--- \\
--- \\
--- \\
---\end{array}$ & $\begin{array}{l}6.13 \\
6.12 \\
6.12 \\
6.11 \\
6.12\end{array}$ & $\begin{array}{l}6.11 \\
6.14 \\
6.14 \\
6.13 \\
6.13\end{array}$ & $\begin{array}{l}6.18 \\
6.16 \\
6.15 \\
6.14 \\
6.17\end{array}$ & $\begin{array}{l}5.74 \\
5.72 \\
5.71 \\
5.69 \\
5.67\end{array}$ & $\begin{array}{l}5.50 \\
5.50 \\
5.49 \\
5.48 \\
5.47\end{array}$ \\
\hline $\begin{array}{l}16 \\
17 \\
18 \\
19 \\
20\end{array}$ & $\begin{array}{c}--- \\
-\cdots \\
--- \\
--\overline{-} \\
5.84\end{array}$ & $\begin{array}{r}-.- \\
5.65 \\
--- \\
--- \\
---\end{array}$ & $\begin{array}{l}--- \\
--- \\
--- \\
--- \\
-\cdots\end{array}$ & $\begin{array}{l}--- \\
--- \\
--- \\
--- \\
---\end{array}$ & $\begin{array}{r}--- \\
--- \\
--- \\
--- \\
5.79\end{array}$ & $\begin{array}{l}--- \\
--- \\
--- \\
--- \\
---\end{array}$ & $\begin{array}{r}--- \\
--- \\
--- \\
--1- \\
6.38\end{array}$ & $\begin{array}{l}6.16 \\
6.15 \\
6.16 \\
6.22 \\
6.21\end{array}$ & $\begin{array}{l}6.12 \\
6.11 \\
6.14 \\
6.21 \\
6.24\end{array}$ & $\begin{array}{l}6.15 \\
6.13 \\
6.11 \\
6.09 \\
6.07\end{array}$ & $\begin{array}{l}5.65 \\
5.83 \\
5.83 \\
5.80 \\
5.83\end{array}$ & $\begin{array}{l}5.45 \\
5.44 \\
5.42 \\
5.41 \\
5.41\end{array}$ \\
\hline $\begin{array}{l}21 \\
22 \\
23 \\
24 \\
25\end{array}$ & $\begin{array}{c}--- \\
5.78 \\
--- \\
-\cdots \\
\ldots-\end{array}$ & $\begin{array}{l}--- \\
--- \\
--- \\
-\cdots \\
---\end{array}$ & $\begin{array}{l}--- \\
--- \\
--- \\
--- \\
---\end{array}$ & $\begin{array}{l}--- \\
--- \\
--- \\
--- \\
---\end{array}$ & $\begin{array}{l}--- \\
--- \\
--- \\
--- \\
---\end{array}$ & $\begin{array}{l}--- \\
--- \\
--- \\
--- \\
---\end{array}$ & $\begin{array}{l}-\cdots \\
--- \\
--- \\
-- \\
---\end{array}$ & $\begin{array}{l}6.20 \\
6.18 \\
6.16 \\
6.14 \\
6.12\end{array}$ & $\begin{array}{l}6.25 \\
6.23 \\
6.22 \\
6.23 \\
6.26\end{array}$ & $\begin{array}{l}6.05 \\
6.03 \\
5.99 \\
5.95 \\
5.93\end{array}$ & $\begin{array}{l}5.82 \\
5.83 \\
5.85 \\
5.84 \\
5.82\end{array}$ & $\begin{array}{l}5.38 \\
5.37 \\
5.34 \\
5.32 \\
5.34\end{array}$ \\
\hline $\begin{array}{l}26 \\
27 \\
28 \\
29 \\
30 \\
31\end{array}$ & 5.72 & $\begin{array}{l}--- \\
--- \\
--- \\
-- \\
-- \\
--\end{array}$ & $\begin{array}{l}--- \\
--- \\
--- \\
--- \\
--- \\
---\end{array}$ & $\begin{array}{l}--- \\
--- \\
--- \\
--- \\
-- \\
---\end{array}$ & $\begin{array}{l}--- \\
--- \\
--- \\
--- \\
-- \\
---\end{array}$ & $\begin{array}{l}--- \\
--- \\
--- \\
--- \\
-- \\
---\end{array}$ & $\begin{array}{r}6.30 \\
-- \\
- \\
-\end{array}$ & $\begin{array}{l}6.12 \\
6.11 \\
6.10 \\
6.09 \\
6.14 \\
6.25\end{array}$ & $\begin{array}{l}6.26 \\
6.31 \\
6.29 \\
6.27 \\
6.25 \\
.--\end{array}$ & $\begin{array}{l}5.91 \\
5.90 \\
5.88 \\
5.85 \\
5.82 \\
5.80\end{array}$ & $\begin{array}{l}5.80 \\
5.79 \\
5.78 \\
5.76 \\
5.74 \\
5.72\end{array}$ & $\begin{array}{l}5.40 \\
5.39 \\
5.38 \\
5.36 \\
5.36 \\
.\end{array}$ \\
\hline $\begin{array}{l}\text { TOTAL } \\
\text { MEAN } \\
\text { MAX } \\
\text { MIN }\end{array}$ & $\begin{array}{l}--- \\
-- \\
-\cdots\end{array}$ & $\begin{array}{l}--- \\
--- \\
--- \\
---\end{array}$ & $\begin{array}{l}--- \\
-- \\
-- \\
\cdots-\end{array}$ & $\begin{array}{l}--- \\
--- \\
--- \\
---\end{array}$ & $\begin{array}{l}--- \\
--- \\
--- \\
---\end{array}$ & $\begin{array}{l}--- \\
--- \\
--- \\
---\end{array}$ & $\begin{array}{l}--- \\
-\ldots \\
-\ldots\end{array}$ & $\begin{array}{l}-- \\
-- \\
--- \\
--\end{array}$ & $\begin{array}{r}185.62 \\
6.19 \\
6.31 \\
6.10\end{array}$ & $\begin{array}{r}188.60 \\
6.08 \\
6.24 \\
5.80\end{array}$ & $\begin{array}{r}178.98 \\
5.77 \\
5.85 \\
5.65\end{array}$ & $\begin{array}{r}164.37 \\
5.48 \\
5.70 \\
5.32\end{array}$ \\
\hline
\end{tabular}


GAGE HEIGHT, FEET, WATER YEAR OCTOBER 1998 TO SEPTEMBER 1999 DAILY MEAN VALUES

\begin{tabular}{|c|c|c|c|c|c|c|c|c|c|c|c|c|}
\hline DAY & OCT & NOV & DEC & JAN & FEB & MAR & APR & MAY & JUN & JUL & AUG & SEP \\
\hline $\begin{array}{l}1 \\
2 \\
3 \\
4 \\
5\end{array}$ & $\begin{array}{l}5.34 \\
5.32 \\
5.30 \\
5.28 \\
5.27\end{array}$ & $\begin{array}{l}5.23 \\
5.22 \\
5.21 \\
5.19 \\
5.19\end{array}$ & $\begin{array}{l}5.19 \\
5.18 \\
5.18 \\
5.18 \\
5.24\end{array}$ & $\begin{array}{l}--- \\
--- \\
--- \\
--- \\
5.19\end{array}$ & $\begin{array}{l}4.78 \\
4.77 \\
4.77 \\
4.76 \\
4.76\end{array}$ & $\begin{array}{l}4.62 \\
4.61 \\
4.61 \\
4.60 \\
4.60\end{array}$ & $\begin{array}{l}4.44 \\
4.44 \\
4.43 \\
4.42 \\
4.42\end{array}$ & $\begin{array}{l}4.27 \\
4.26 \\
4.26 \\
4.25 \\
4.25\end{array}$ & $\begin{array}{l}4.09 \\
4.09 \\
4.08 \\
4.07 \\
4.07\end{array}$ & $\begin{array}{l}3.92 \\
3.91 \\
3.91 \\
3.90 \\
3.90\end{array}$ & $\begin{array}{l}3.74 \\
3.74 \\
3.73 \\
3.72 \\
3.72\end{array}$ & $\begin{array}{l}3.56 \\
3.56 \\
3.55 \\
3.55 \\
3.54\end{array}$ \\
\hline $\begin{array}{r}6 \\
7 \\
8 \\
9 \\
10\end{array}$ & $\begin{array}{l}5.30 \\
5.29 \\
5.28 \\
5.26 \\
5.25\end{array}$ & $\begin{array}{l}5.18 \\
5.17 \\
5.16 \\
5.15 \\
5.20\end{array}$ & $\begin{array}{l}5.24 \\
5.24 \\
5.23 \\
5.22 \\
5.22\end{array}$ & $\begin{array}{l}--- \\
--- \\
--- \\
--- \\
5.20\end{array}$ & $\begin{array}{l}4.75 \\
4.75 \\
4.74 \\
4.73 \\
4.73\end{array}$ & $\begin{array}{l}4.59 \\
4.59 \\
4.58 \\
4.57 \\
4.57\end{array}$ & $\begin{array}{l}4.41 \\
4.41 \\
4.40 \\
4.40 \\
4.39\end{array}$ & $\begin{array}{l}4.24 \\
4.24 \\
4.23 \\
4.22 \\
4.22\end{array}$ & $\begin{array}{l}4.06 \\
4.06 \\
4.05 \\
4.05 \\
4.04\end{array}$ & $\begin{array}{l}3.89 \\
3.88 \\
3.88 \\
3.87 \\
3.87\end{array}$ & $\begin{array}{l}3.71 \\
3.71 \\
3.70 \\
3.70 \\
3.69\end{array}$ & $\begin{array}{l}3.53 \\
3.53 \\
3.52 \\
3.52 \\
3.51\end{array}$ \\
\hline $\begin{array}{l}11 \\
12 \\
13 \\
14 \\
15\end{array}$ & $\begin{array}{l}5.24 \\
5.23 \\
5.22 \\
5.21 \\
5.18\end{array}$ & $\begin{array}{l}5.21 \\
5.21 \\
5.20 \\
5.19 \\
5.18\end{array}$ & $\begin{array}{c}5.21 \\
-- \\
- \\
\cdots- \\
-\end{array}$ & $\begin{array}{l}--- \\
--- \\
--- \\
--- \\
---\end{array}$ & $\begin{array}{l}4.72 \\
4.72 \\
4.71 \\
4.71 \\
4.70\end{array}$ & $\begin{array}{l}4.56 \\
4.56 \\
4.55 \\
4.55 \\
4.54\end{array}$ & $\begin{array}{l}4.38 \\
4.38 \\
4.37 \\
4.37 \\
4.36\end{array}$ & $\begin{array}{l}4.21 \\
4.21 \\
4.20 \\
4.19 \\
4.19\end{array}$ & $\begin{array}{l}4.03 \\
4.03 \\
4.02 \\
4.02 \\
4.01\end{array}$ & $\begin{array}{l}3.86 \\
3.86 \\
3.85 \\
3.84 \\
3.84\end{array}$ & $\begin{array}{l}3.68 \\
3.68 \\
3.67 \\
3.67 \\
3.66\end{array}$ & $\begin{array}{l}3.51 \\
3.50 \\
3.49 \\
3.49 \\
3.48\end{array}$ \\
\hline $\begin{array}{l}16 \\
17 \\
18 \\
19 \\
20\end{array}$ & $\begin{array}{l}5.21 \\
5.31 \\
5.33 \\
5.32 \\
5.31\end{array}$ & $\begin{array}{l}5.18 \\
5.17 \\
5.19 \\
5.21 \\
5.21\end{array}$ & $\begin{array}{l}-- \\
-- \\
-\cdots \\
-\cdots \\
--\end{array}$ & $\begin{array}{c}--- \\
5.20 \\
--- \\
---\end{array}$ & $\begin{array}{l}4.69 \\
4.69 \\
4.68 \\
4.68 \\
4.67\end{array}$ & $\begin{array}{l}4.53 \\
4.53 \\
4.52 \\
4.52 \\
4.51\end{array}$ & $\begin{array}{l}4.36 \\
4.35 \\
4.34 \\
4.34 \\
4.33\end{array}$ & $\begin{array}{l}4.18 \\
4.18 \\
4.17 \\
4.17 \\
4.16\end{array}$ & $\begin{array}{l}4.01 \\
4.00 \\
3.99 \\
3.99 \\
3.98\end{array}$ & $\begin{array}{l}3.83 \\
3.83 \\
3.82 \\
3.82 \\
3.81\end{array}$ & $\begin{array}{l}3.66 \\
3.65 \\
3.64 \\
3.64 \\
3.63\end{array}$ & $\begin{array}{l}3.48 \\
3.47 \\
3.47 \\
3.46 \\
3.45\end{array}$ \\
\hline $\begin{array}{l}21 \\
22 \\
23 \\
24 \\
25\end{array}$ & $\begin{array}{l}5.31 \\
5.29 \\
5.27 \\
5.26 \\
5.27\end{array}$ & $\begin{array}{l}5.21 \\
5.20 \\
5.20 \\
5.20 \\
5.20\end{array}$ & $\begin{array}{l}\cdots \\
\cdots- \\
\cdots \\
-- \\
--\end{array}$ & $\begin{array}{r}--- \\
--- \\
--- \\
5.23 \\
---\end{array}$ & $\begin{array}{l}4.67 \\
4.66 \\
4.65 \\
4.65 \\
4.64\end{array}$ & $\begin{array}{l}4.50 \\
4.50 \\
4.49 \\
4.49 \\
4.48\end{array}$ & $\begin{array}{l}4.33 \\
4.32 \\
4.32 \\
4.31 \\
4.30\end{array}$ & $\begin{array}{l}4.15 \\
4.15 \\
4.14 \\
4.14 \\
4.13\end{array}$ & $\begin{array}{l}3.98 \\
3.97 \\
3.97 \\
3.96 \\
3.95\end{array}$ & $\begin{array}{l}3.80 \\
3.80 \\
3.79 \\
3.79 \\
3.78\end{array}$ & $\begin{array}{l}3.63 \\
3.62 \\
3.61 \\
3.61 \\
3.60\end{array}$ & $\begin{array}{l}3.45 \\
3.44 \\
3.44 \\
3.43 \\
3.43\end{array}$ \\
\hline $\begin{array}{l}26 \\
27 \\
28 \\
29 \\
30 \\
31\end{array}$ & $\begin{array}{l}5.26 \\
5.26 \\
5.26 \\
5.25 \\
5.25 \\
5.25\end{array}$ & $\begin{array}{c}5.20 \\
5.20 \\
5.19 \\
5.19 \\
5.19 \\
-\ldots\end{array}$ & $\begin{array}{l}-\cdots \\
-- \\
--- \\
-- \\
-- \\
-\cdots\end{array}$ & $\begin{array}{l}--- \\
--- \\
--- \\
--- \\
--- \\
5.26\end{array}$ & $\begin{array}{r}4.64 \\
4.63 \\
4.63 \\
\ldots- \\
\ldots \\
\ldots\end{array}$ & $\begin{array}{l}4.48 \\
4.47 \\
4.46 \\
4.46 \\
4.45 \\
4.45\end{array}$ & $\begin{array}{r}4.30 \\
4.29 \\
4.29 \\
4.28 \\
4.28 \\
-.-\end{array}$ & $\begin{array}{l}4.13 \\
4.12 \\
4.11 \\
4.11 \\
4.10 \\
4.10\end{array}$ & $\begin{array}{c}3.95 \\
3.94 \\
3.94 \\
3.93 \\
3.93 \\
-\ldots\end{array}$ & $\begin{array}{l}3.78 \\
3.77 \\
3.76 \\
3.76 \\
3.75 \\
3.75\end{array}$ & $\begin{array}{l}3.60 \\
3.59 \\
3.59 \\
3.58 \\
3.57 \\
3.57\end{array}$ & $\begin{array}{c}3.42 \\
3.41 \\
3.41 \\
3.40 \\
3.40 \\
\ldots\end{array}$ \\
\hline $\begin{array}{l}\text { TOTAL } \\
\text { MEAN } \\
\text { MAX } \\
\text { MIN }\end{array}$ & $\begin{array}{r}163.38 \\
5.27 \\
5.34 \\
5.18\end{array}$ & $\begin{array}{r}155.83 \\
5.19 \\
5.23 \\
5.15\end{array}$ & $\begin{array}{l}--- \\
-- \\
-- \\
-\cdots\end{array}$ & $\begin{array}{l}--- \\
--- \\
--- \\
---\end{array}$ & $\begin{array}{r}131.68 \\
4.70 \\
4.78 \\
4.63\end{array}$ & $\begin{array}{r}140.54 \\
4.53 \\
4.62 \\
4.45\end{array}$ & $\begin{array}{r}130.76 \\
4.36 \\
4.44 \\
4.28\end{array}$ & $\begin{array}{r}129.68 \\
4.18 \\
4.27 \\
4.10\end{array}$ & $\begin{array}{r}120.26 \\
4.01 \\
4.09 \\
3.93\end{array}$ & $\begin{array}{r}118.82 \\
3.83 \\
3.92 \\
3.75\end{array}$ & $\begin{array}{r}113.31 \\
3.66 \\
3.74 \\
3.57\end{array}$ & $\begin{array}{r}104.40 \\
3.48 \\
3.56 \\
3.40\end{array}$ \\
\hline
\end{tabular}


LOCATION.--Lat $42^{\circ} 31^{\prime} 53^{\prime \prime}$, long $88^{\circ} 18^{\prime} 48^{\prime \prime}$, in SE 1/4 NE $1 / 4$ sec.24, T.1 N., R.18 E., Walworth County, Hydrologic Unit 07120006, 1.5 mi southwest of Powers Lake.

PERIOD OF RECORD.--May 1998 to current year.

REMARKS.--Lake sampled near center at deep hole. Water-quality analyses done by Wisconsin State Laboratory of Hygiene.

WATER-QUALITY DATA, APRIL 15 TO AUGUST 04, 1999

(Milligrams per liter unless otherwise indicated)

Lake stage (ft)

Secchi-depth (m)

Chlorophyll a, phytoplankton $(\mu \mathrm{g} / \mathrm{L})$

Depth of sample (m)

water temperature $\left({ }^{\circ} \mathrm{C}\right)$

specific conductance $(\mu \mathrm{s} / \mathrm{cm})$

$\mathrm{pH}$ (units)

Dissolved oxygen ( $\mathrm{mg} / \mathrm{L}$ )

Phosphorus, total (as P)

Phosphorus, ortho, dissolved (as P)

Nitrogen, $\mathrm{NO} 2+\mathrm{NO} 3$ diss. (as N)

Nitrogen, ammonia, dissolved (as N)

Nitrogen, amm. + org., total (as N)

Nitrogen, total (as $\mathrm{N}$ )

Color (Pt-Co. scale)

Turbidity (NTU)

Hardness, (as $\mathrm{CaCO}_{3}$ )

Calcium, dissolved ( $\mathrm{Ca}$ )

Magnesium, dissolved (Mg)

Sodium, dissolved (Na)

Potassium, dissolved ( $\mathrm{K}$

Alkalinity, (as $\mathrm{CaCO}_{3}$ )

Sulfate, dissolved $\left(\mathrm{SO}_{4}\right)$

Chloride, dissolved ( $\mathrm{Cl}$ )

Silica, dissolved $\left(\mathrm{SiO}_{2}\right)$

Solids, dissolved, at $180^{\circ} \mathrm{C}$

Iron, dissolved (Fe) $\mu \mathrm{g} / \mathrm{L}$

Manganese, dissolved (Mn) $\mu \mathrm{g} / \mathrm{L}$

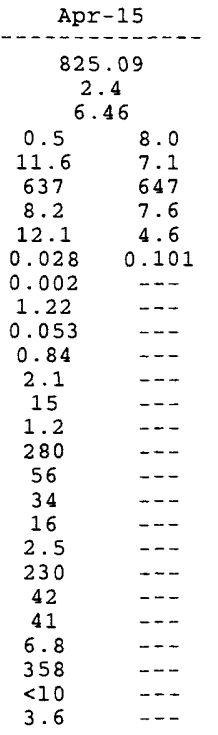

6-10-99

\begin{tabular}{|c|c|c|c|}
\hline \multicolumn{2}{|c|}{ Jun-10 } & \multicolumn{2}{|c|}{ Jul -7 } \\
\hline \multicolumn{2}{|c|}{$\begin{array}{c}826.26 \\
2.3 \\
4.94\end{array}$} & \multicolumn{2}{|c|}{$\begin{array}{c}825.65 \\
1.7 \\
6.72\end{array}$} \\
\hline 0.5 & 8.0 & 0.5 & 8.5 \\
\hline 27.8 & 8.1 & 27.8 & $\begin{array}{l}8.0 \\
672\end{array}$ \\
\hline $\begin{array}{l}563 \\
82\end{array}$ & $\begin{array}{l}668 \\
7.8\end{array}$ & $\begin{array}{l}529 \\
8.3\end{array}$ & $\begin{array}{l}672 \\
7.3\end{array}$ \\
\hline $\begin{array}{l}8.2 \\
9.5\end{array}$ & 0.7 & $\begin{array}{l}8.3 \\
9.8\end{array}$ & 0.4 \\
\hline 0.021 & 0.014 & 0.025 & 0.024 \\
\hline$-\cdots$ & -- & 0.003 & -- \\
\hline--- & -- & 0.283 & $\cdots$ \\
\hline-- & -- & 0.019 & --- \\
\hline$\cdots$ & $\cdots$ & 0.82 & --- \\
\hline-- & -- & 1.1 & --- \\
\hline$\cdots$ & -- & --- & $\ldots$ \\
\hline--- & -- & $\ldots$ & $\cdots$ \\
\hline--- & --- & -- & $\cdots-$ \\
\hline--- & -- & --- & --- \\
\hline--- & --- & -- & --- \\
\hline--- & $\cdots$ & $\cdots$ & --1 \\
\hline-- & -- & --- & $\cdots$ \\
\hline$\cdots$ & -- & $\ldots$ & $\cdots$ \\
\hline-- & $\cdots$ & --- & $\cdots$ \\
\hline--- & $\cdots$ & $\cdots$ & $\cdots$ \\
\hline--- & -- & --- & $\cdots$ \\
\hline-- & --- & $\cdots$ & --- \\
\hline- & -- & --- & $\cdots$ \\
\hline & - & $\cdots$ & \\
\hline
\end{tabular}

$7-07-99$

\begin{tabular}{|c|c|c|}
\hline & Aug -4 & \\
\hline & $\begin{array}{c}825.71 \\
2.3 \\
6.00\end{array}$ & \\
\hline $\begin{array}{c}0.5 \\
27.2 \\
542 \\
8.3 \\
10.8 \\
0.024\end{array}$ & $\begin{array}{c}6.0 \\
9.9 \\
590 \\
7.4 \\
0.1 \\
0.029\end{array}$ & $\begin{array}{l}8.0 \\
8.2 \\
655 \\
7.1 \\
0.0 \\
0.147\end{array}$ \\
\hline$\cdots$ & -- & -- \\
\hline$\cdots$ & -- & $-\cdots$ \\
\hline-- & --- & $\ldots$ \\
\hline-- & -- & $\ldots$ \\
\hline$\ldots$ & --- & --- \\
\hline-- & --- & --- \\
\hline$\ldots$ & $\ldots$ & -- \\
\hline-- & --- & -- \\
\hline--- & --- & --- \\
\hline$\cdots$ & --- & --- \\
\hline--- & --- & -- \\
\hline--- & -- & -- \\
\hline$\cdots$ & $\cdots$ & --- \\
\hline$\cdots$ & -- & -- \\
\hline--- & --- & --- \\
\hline$\ldots$ & -- & --- \\
\hline-- & -- & -- \\
\hline--- & --- & --- \\
\hline
\end{tabular}

8-04-99

DISSOLVED OXYGEN (D.O.), IN MILLIGRAMS PER LITER
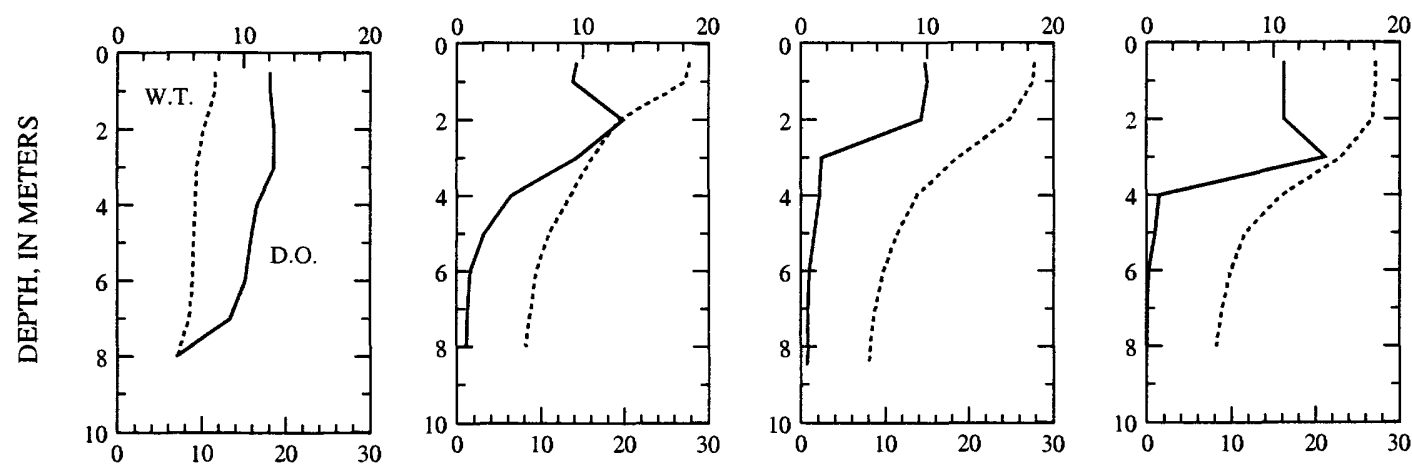

WATER TEMPERATURE (W.T.), IN DEGREES CELSIUS

PH, IN STANDARD UNITS
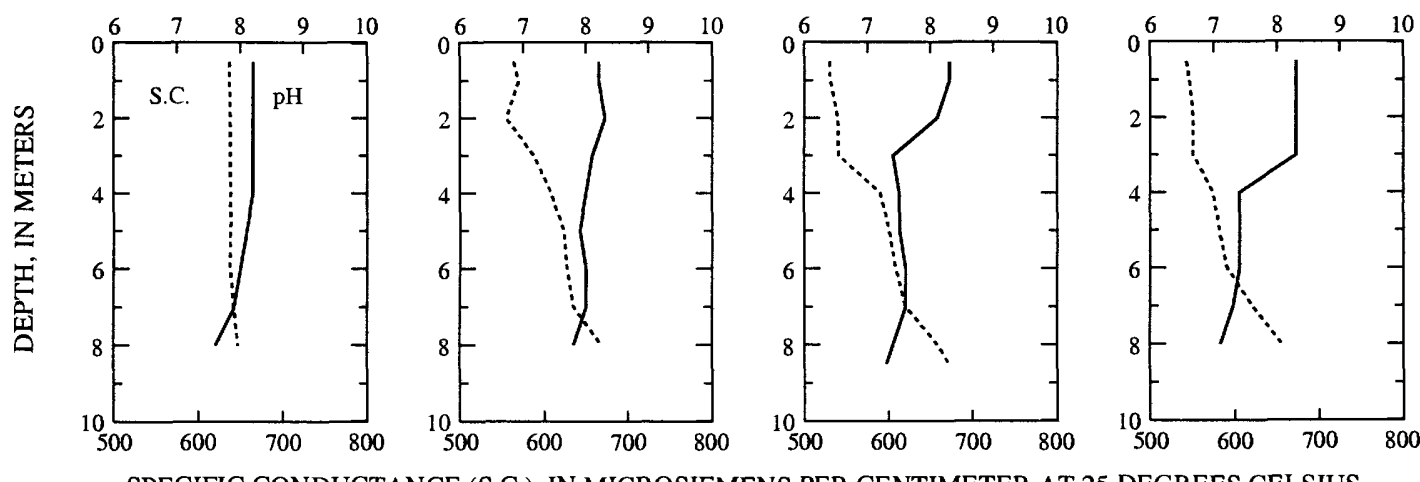

SPECIFIC CONDUCTANCE (S.C.), IN MICROSIEMENS PER CENTIMETER AT 25 DEGREES CELSIUS 

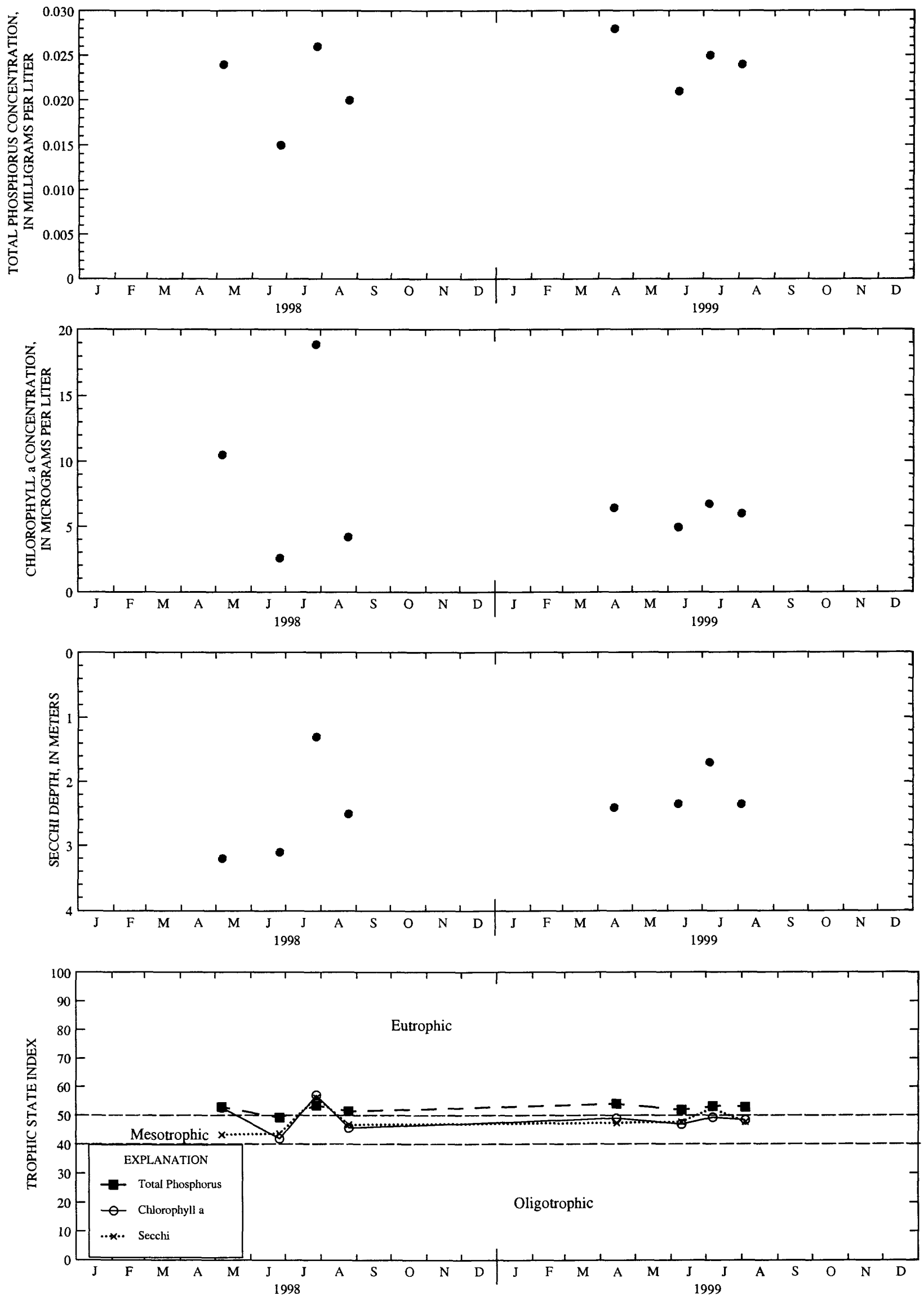

YEAR

Surface total phosphorus, chlorophyll a concentrations, Secchi depths, and TSI data for Tombeau Lake near Powers Lake, Wisconsin. 
LOCATION.--Lat $46^{\circ} 13^{\prime} 21^{\prime \prime}$, long 91 $52^{\prime} 09^{\prime \prime}$, in NW 1/4 SE 1/4 sec.9, T.43 N., R. 12 W., Douglas County, Hydrologic Unit 07030002, near Gordon.

PERIOD OF RECORD.--March 1998 to current year.

REMARKS.--Lake sampled at deepest part of northern basin. Lake ice-covered during March sampling. Water-quality analyses done by Wisconsin State Laboratory of Hygiene.

WATER-QUALITY DATA, MARCH 03 TO AUGUST 16, 1999

(Milligrams per liter unless otherwise indicated)

Lake stage (Et)

Secchi-depth (m)

Chlorophyli a, phytoplankton $(\mu \mathrm{a} / \mathrm{L})$

Depth of sample (m)

Water temperature $\left({ }^{\circ} \mathrm{C}\right)$

Specific conductance $(u S / \mathrm{cm}$ )

$\mathrm{pH}$ (units)

Dissolved oxygen (mg/L)

Phosphorus, total (as P)
Mar-3

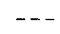

$--$

$--$

0.5

0.6

41
7.4

13.6

$<0.005$

\begin{tabular}{c} 
Apr-14 \\
\hline 4.81 \\
9.1 \\
0.53 \\
0.5 \\
7.2 \\
38 \\
6.9 \\
13.0 \\
$<0.005$
\end{tabular}

\begin{tabular}{c} 
Jun-2 \\
\hline 4.75 \\
8.6 \\
1.81 \\
0.5 \\
16.4 \\
38 \\
7.8 \\
9.4 \\
$<0.005$
\end{tabular}

\begin{tabular}{c} 
Jul-14 \\
\hline 5.08 \\
6.4 \\
1.25 \\
0.5 \\
23.5 \\
39 \\
8.1 \\
8.7 \\
$<0.005$
\end{tabular}

\begin{tabular}{|c|c|}
\hline \multicolumn{2}{|c|}{ Aug-16 } \\
\hline & \\
\hline $\begin{array}{c}0.5 \\
22.0 \\
37 \\
7.7 \\
8.8 \\
<0.005\end{array}$ & $\begin{array}{c}16.0 \\
12.4 \\
64 \\
6.6 \\
0.1 \\
0.044\end{array}$ \\
\hline
\end{tabular}

3-03-99

4-14-99

6-02-99

7-14-99

8-16-99

DISSOLVED OXYGEN (D.O.), IN MILLIGRAMS PER LITER
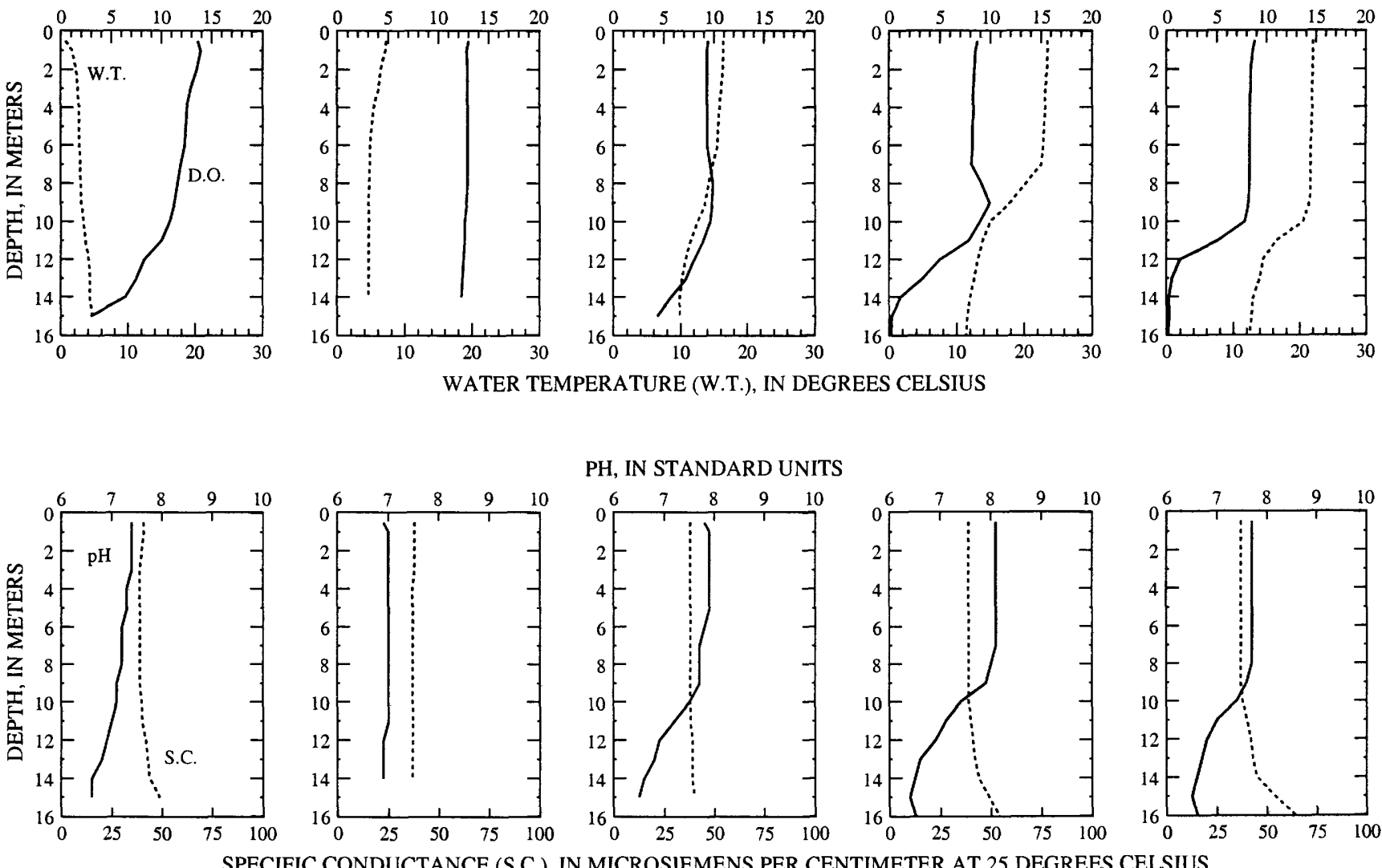

PH, IN STANDARD UNITS
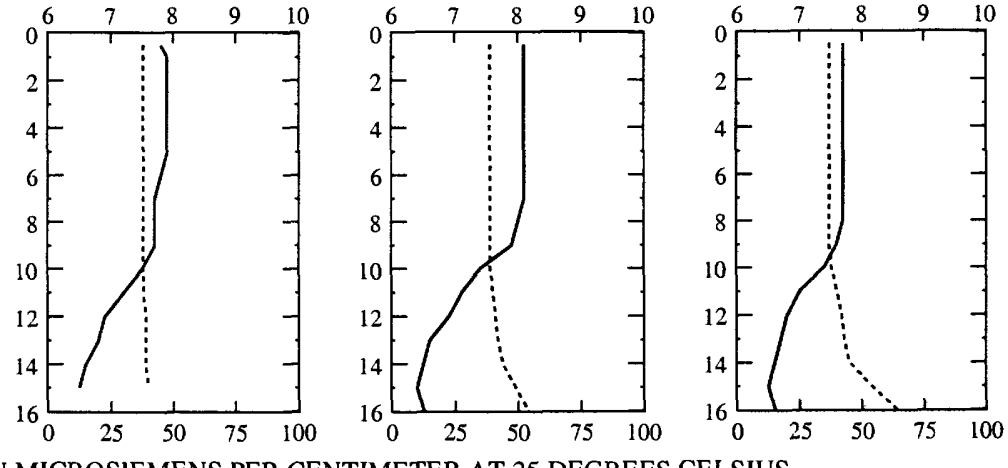

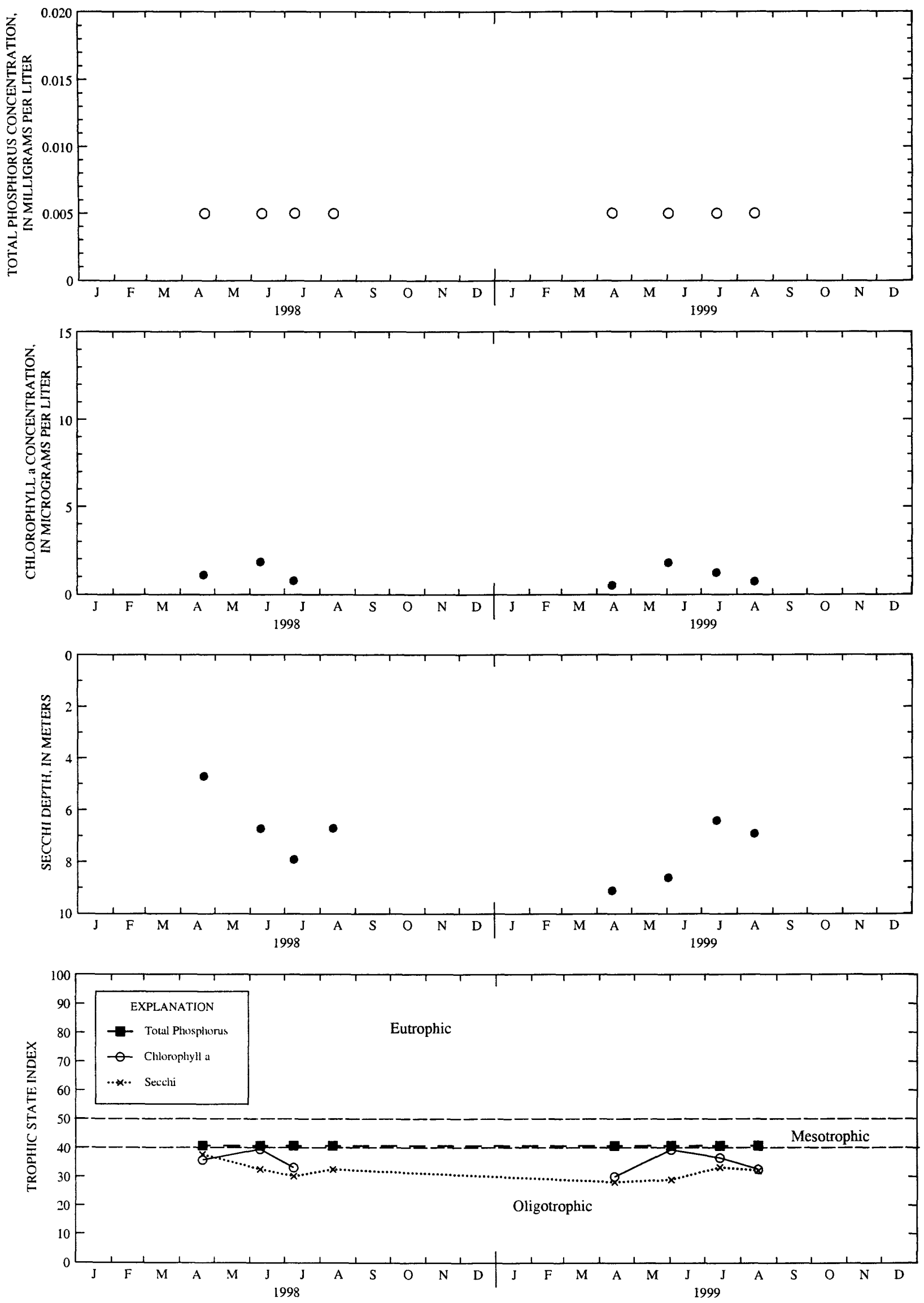

YEAR

Surface total phosphorus, chlorophyll a concentrations, Secchi depths, and TSI data for Whitefish Lake, North Site, near Gordon, Wisconsin. 
LOCATION.--Lat $46^{\circ} 12^{\prime} 12^{\prime \prime}$, long 91 ${ }^{\circ} 52^{\prime} 32^{\prime \prime}$, in SE 1/4 SW 1/4 sec.16, T.43 N., R.12 W., Douglas County, Hydrologic Unit 07030002, near Gordon.

PERIOD OF RECORD.--March 1998 to current year.

REMARKS.--Lake sampled at deepest part of southern basin. Lake ice-covered during March sampling. Water-quality analyses done by Wisconsin State Laboratory of Hygiene.

WATER-QUALITY DATA, MARCH 03 TO AUGUST 16, 1999

(Milligrams per liter unless otherwise indicated)

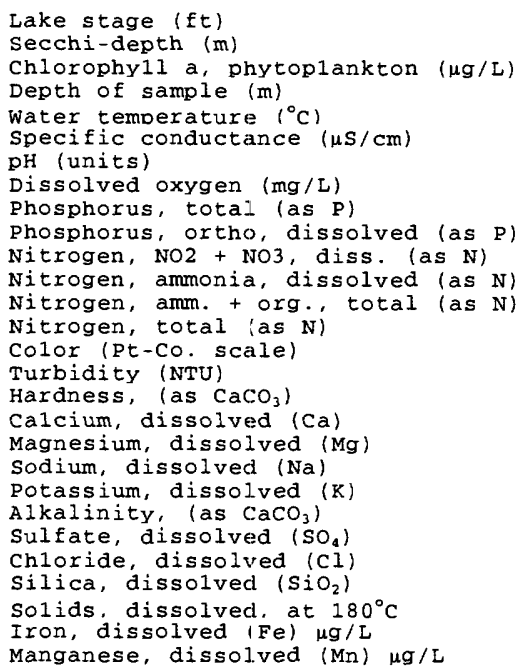

3-03-99

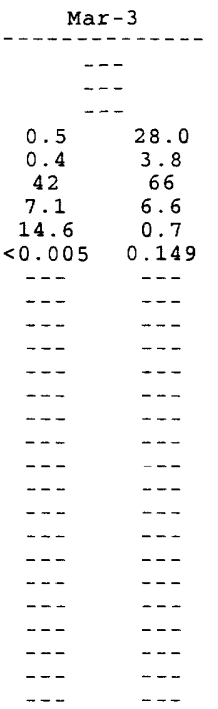

4-14-99

\begin{tabular}{|c|c|}
\hline \multicolumn{2}{|c|}{ Apr -14} \\
\hline \multicolumn{2}{|c|}{$\begin{array}{c}4.81 \\
9.2 \\
0.49\end{array}$} \\
\hline $\begin{array}{l}0.5 \\
6.7\end{array}$ & $\begin{array}{c}27.0 \\
4.2\end{array}$ \\
\hline 37 & $\ldots$ \\
\hline $\begin{array}{c}6.8 \\
12.6\end{array}$ & $\begin{array}{c}6.6 \\
10.7\end{array}$ \\
\hline 0.005 & 0.011 \\
\hline 0.004 & -- \\
\hline 0.045 & $\cdots$ \\
\hline 0.03 & --- \\
\hline 0.29 & $\ldots$ \\
\hline 0.34 & -- \\
\hline$<5$ & -- \\
\hline 0.7 & -- \\
\hline 18 & $\ldots$ \\
\hline 5 & $\cdots$ \\
\hline 1.3 & -- \\
\hline 0.93 & -- \\
\hline 0.6 & $\cdots$ \\
\hline 18 & -- \\
\hline$<2.0$ & $-\cdots$ \\
\hline 0.5 & $\cdots$ \\
\hline 0.9 & --- \\
\hline 28 & -- \\
\hline 30 & $\cdots$ \\
\hline 5.7 & $\cdots$ \\
\hline
\end{tabular}

6-02-99

\begin{tabular}{|c|c|}
\hline & \\
\hline & \\
\hline 0.5 & 27.0 \\
\hline $\begin{array}{c}15.8 \\
38\end{array}$ & $\begin{array}{c}6.3 \\
40\end{array}$ \\
\hline 7.6 & 6.5 \\
\hline $\begin{array}{l}9.2 \\
0.005\end{array}$ & 6.5 \\
\hline 0.005 & 0.011 \\
\hline$-\ldots$ & $\ldots$ \\
\hline & \\
\hline-- & -- \\
\hline$\cdots$ & $\cdots$ \\
\hline$\ldots$ & $\ldots$ \\
\hline & \\
\hline.- & $\cdots$ \\
\hline$\cdots$ & $\ldots$ \\
\hline -.. & -- \\
\hline - & - \\
\hline - & - \\
\hline &.- \\
\hline & 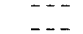 \\
\hline & - - \\
\hline
\end{tabular}

\begin{tabular}{|c|c|}
\hline & \\
\hline 0.5 & 29.0 \\
\hline 23.0 & 6.5 \\
\hline 39 & 44 \\
\hline 7.9 & 6.4 \\
\hline 8.7 & 1.1 \\
\hline$<0.005$ & 0.210 \\
\hline 0.003 & -- \\
\hline$<0.010$ & -- \\
\hline 0.005 & - - \\
\hline 0.32 & -- \\
\hline$\cdots$ & -- \\
\hline$\ldots$ & $-\infty$ \\
\hline-- & -- \\
\hline$\cdots$ & $-\cdots$ \\
\hline-- & -- \\
\hline$\ldots$ & --- \\
\hline-- & $\cdots$ \\
\hline--- & -- \\
\hline-- & -- \\
\hline$\ldots$ & $\ldots$ \\
\hline-- & -- \\
\hline-- & $\cdots$ \\
\hline-- & -- \\
\hline$\ldots$ & -- \\
\hline
\end{tabular}

\begin{tabular}{|c|c|c|}
\hline & Aug -16 & \\
\hline & $\begin{array}{c}5.41 \\
6.3 \\
0.95\end{array}$ & \\
\hline 0.5 & 13.0 & 29.0 \\
\hline 21.8 & 12.4 & 6.6 \\
\hline 37 & 38 & 49 \\
\hline 7.8 & 7.3 & 6.5 \\
\hline 8.6 & 9.5 & 0.1 \\
\hline$<0.005$ & 0.008 & 0.041 \\
\hline$\ldots$ & $\ldots$ & $\ldots$ \\
\hline-- & $\ldots$ & - - - \\
\hline$\ldots$ & --- & -- - \\
\hline$\ldots$ & - - - & --- \\
\hline--- & - - - & --- \\
\hline$\ldots$ & $\ldots$ & --- \\
\hline--- & $\ldots$ & $\ldots$ \\
\hline--- & $\ldots$ & --- \\
\hline$\cdots$ & $\ldots$ & --- \\
\hline$\ldots$ & - - & $\ldots$ \\
\hline--- & $-\ldots$ & -- \\
\hline$\ldots$ & $\ldots$ & --- \\
\hline$\ldots$ & --- & $\ldots$ \\
\hline--- & -- & $-\cdots$ \\
\hline$\ldots$ & - . - & --- \\
\hline$\ldots$ & -- & -- \\
\hline--- & --- & -- \\
\hline$\cdots$ & -- & -- \\
\hline$\cdots$ & -- & -- \\
\hline
\end{tabular}

DISSOLVED OXYGEN (D.O.), IN MILLIGRAMS PER LITER
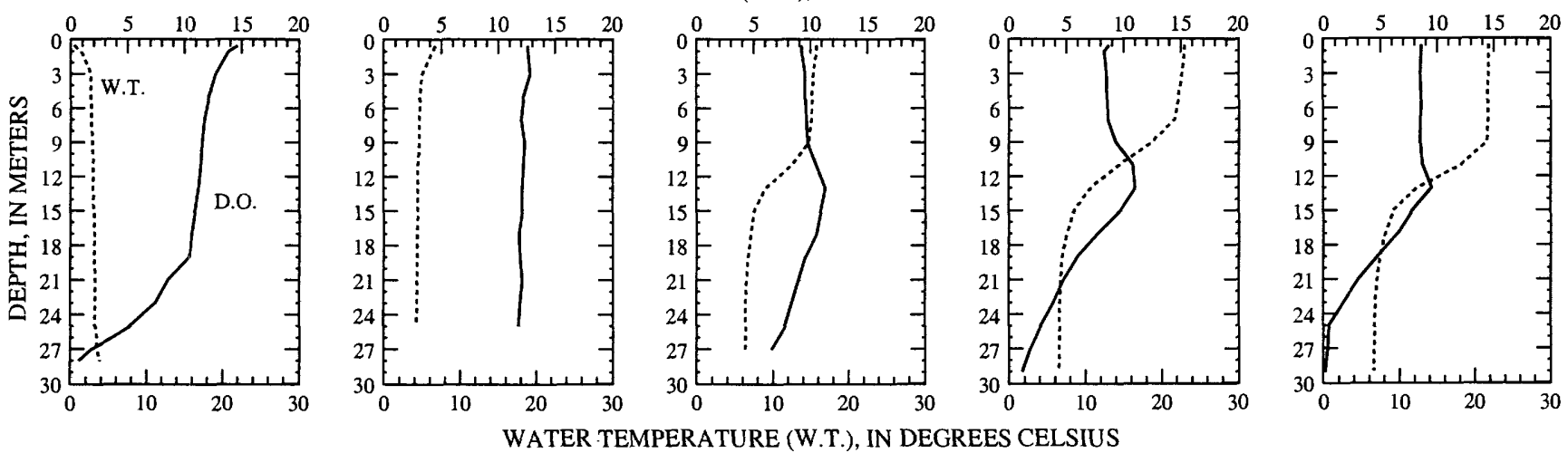

PH, IN STANDARD UNITS
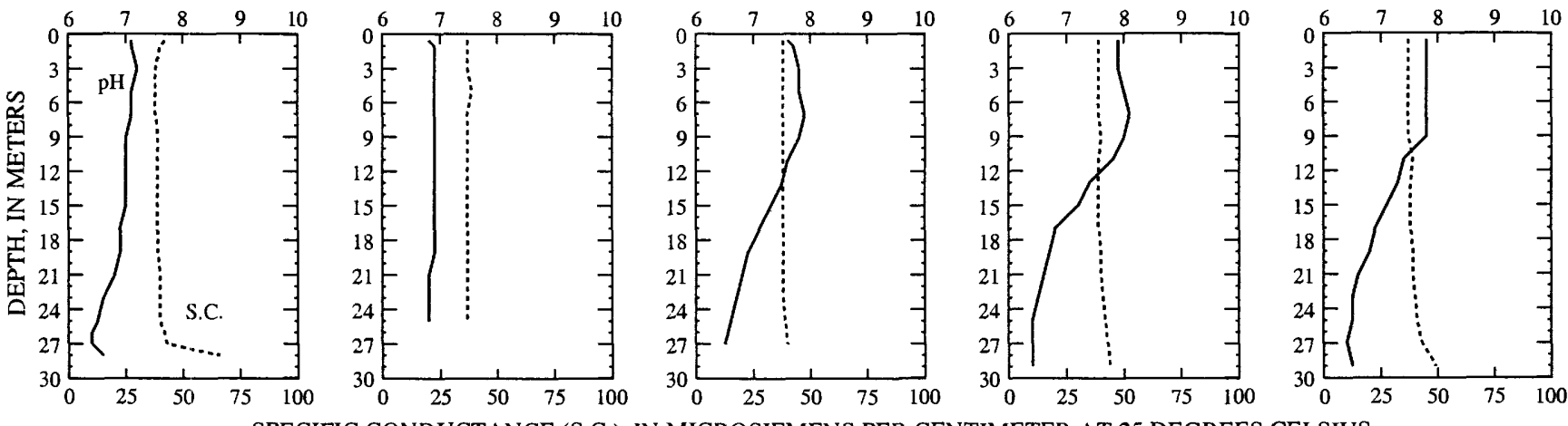

SPECIFIC CONDUCTANCE (S.C.), IN MICROSIEMENS PER CENTIMETER AT 25 DEGREES CELSIUS 

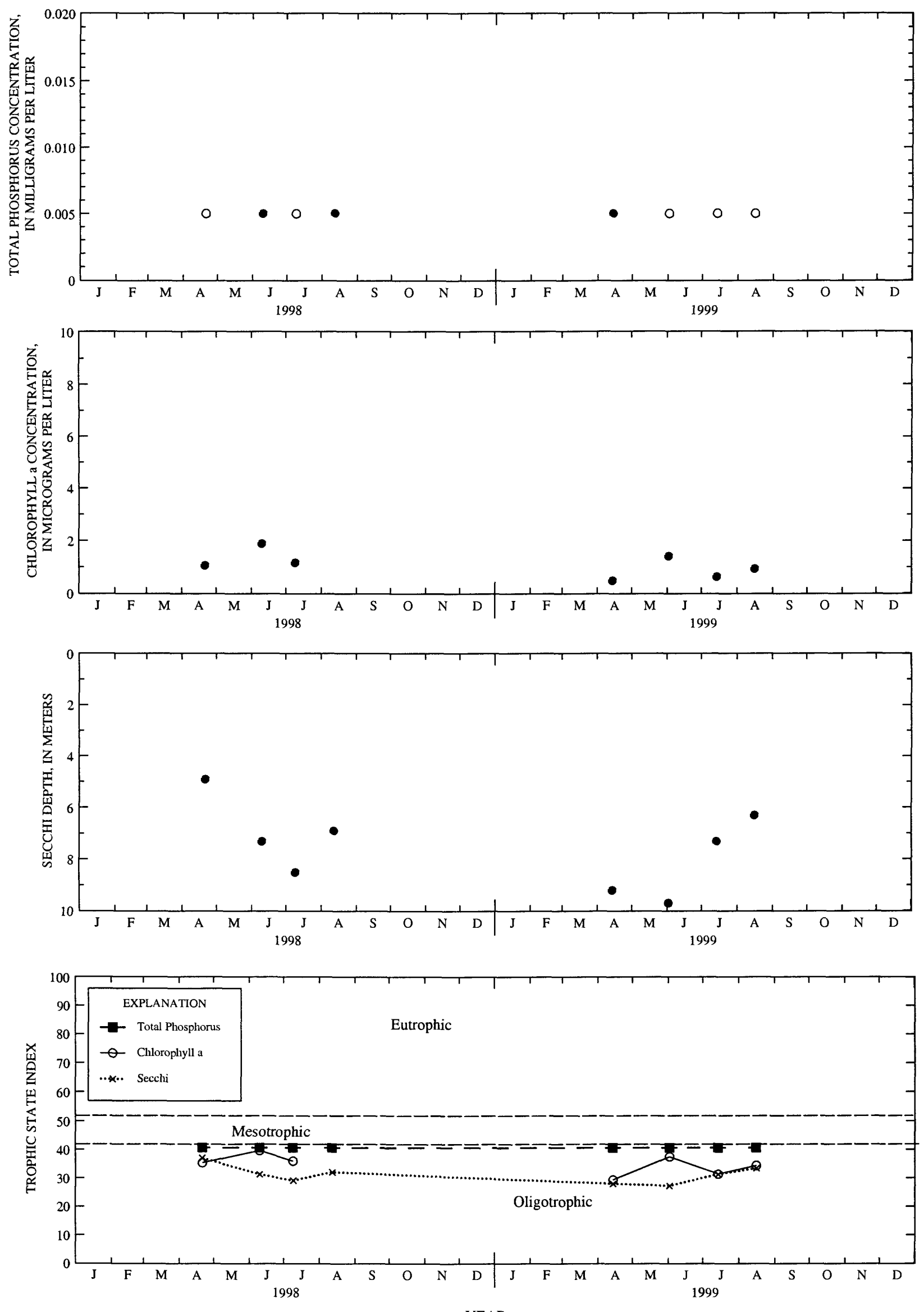

YEAR

Surface total phosphorus, chlorophyll a concentrations, Secchi depths, and TSI data for Whitefish Lake, South Site, near Gordon, Wisconsin.

(Circles on the first three plots indicate laboratory detection limit for selected analyses.Actual concentrations for these particular analyses are less than the plotted circles.) 
LOCATION.--Lat $42^{\circ} 46^{\prime} 08^{\prime \prime}$, long $88^{\circ} 41^{\prime} 48^{\prime \prime}$, in NW 1/4 NW 1/4 sec.35, T.4 N., R.15 E., Walworth County, Hydrologic Unit 07090001, at outlet, $5.0 \mathrm{mi}$ southeast of Whitewater and $10.0 \mathrm{mi}$ north of Delavan.

DRAINAGE AREA.--10.9 $\mathrm{mi}^{2}$, of which $8.5 \mathrm{mi}^{2}$ is non-contributing.

PERIOD OF RECORD.--November 1990 to current year.

GAGE.--Water-stage recorder. Datum of gage is $861.00 \mathrm{ft}$ above sea level, revised, (Wisconsin Department of Natural Resources).

REMARKS.--No estimated daily gage heights. Records good. Point of zero flow of dam crest is $10.97 \mathrm{ft}$. Rainfall data published in 1991 under this station number are now stored under station number 424559088420300.

EXTREMES FOR PERIOD OF RECORD.--Maximum gage height, $11.42 \mathrm{ft}$, June 18, 19, 1996; minimum daily gage height, $8.89 \mathrm{ft}$, Oct. 2 , $3,1991$.

EXTREMES FOR CURRENT YEAR.--Maximum gage height, $11.41 \mathrm{ft}$, Apr. 24, 25; minimum daily gage height, $10.53 \mathrm{ft}$, Sept. $25,26$.

GAGE HEIGHT, FEET, WATER YEAR OCTOBER 1998 TO SEPTEMBER 1999

\section{DAILY MEAN VALUES}

\begin{tabular}{|c|c|c|c|c|c|c|c|c|c|c|c|c|}
\hline DAY & $\mathrm{OCT}$ & NOV & DEC & JAN & FEB & MAR & APR & MAY & JUN & JUL & AUG & SEP \\
\hline $\begin{array}{l}1 \\
2 \\
3 \\
4 \\
5\end{array}$ & $\begin{array}{l}10.92 \\
10.91 \\
10.93 \\
10.94 \\
10.94\end{array}$ & $\begin{array}{l}10.95 \\
11.00 \\
11.01 \\
11.01 \\
11.00\end{array}$ & $\begin{array}{l}11.07 \\
11.08 \\
11.08 \\
11.08 \\
11.08\end{array}$ & $\begin{array}{l}11.08 \\
11.08 \\
11.07 \\
11.07 \\
11.07\end{array}$ & $\begin{array}{l}11.05 \\
11.05 \\
11.04 \\
11.04 \\
11.04\end{array}$ & $\begin{array}{l}11 \cdot 18 \\
11 \cdot 18 \\
11 \cdot 16 \\
11 \cdot 16 \\
11 \cdot 15\end{array}$ & $\begin{array}{l}11.06 \\
11.06 \\
11.09 \\
11.14 \\
11.13\end{array}$ & $\begin{array}{l}11.28 \\
11.32 \\
11.29 \\
11.29 \\
11.27\end{array}$ & $\begin{array}{l}11.14 \\
11.15 \\
11.19 \\
11.19 \\
11.19\end{array}$ & $\begin{array}{l}10.95 \\
10.95 \\
11.09 \\
11.10 \\
11.07\end{array}$ & $\begin{array}{l}10.95 \\
10.92 \\
10.90 \\
10.88 \\
10.87\end{array}$ & $\begin{array}{l}10.77 \\
10.76 \\
10.76 \\
10.75 \\
10.73\end{array}$ \\
\hline $\begin{array}{r}6 \\
7 \\
8 \\
9 \\
10\end{array}$ & $\begin{array}{l}10.99 \\
10.98 \\
11.02 \\
11.02 \\
11.03\end{array}$ & $\begin{array}{l}11.00 \\
11.00 \\
10.94 \\
10.93 \\
11.12\end{array}$ & $\begin{array}{l}11.09 \\
11.12 \\
11.13 \\
11.12 \\
11.11\end{array}$ & $\begin{array}{l}11.07 \\
11.07 \\
11.07 \\
11.08 \\
11.08\end{array}$ & $\begin{array}{l}11.04 \\
11.05 \\
11.05 \\
11.08 \\
11.19\end{array}$ & $\begin{array}{l}11 \cdot 17 \\
11 \cdot 16 \\
11 \cdot 16 \\
11.17 \\
11.17\end{array}$ & $\begin{array}{l}11 \cdot 13 \\
11 \cdot 12 \\
11.12 \\
11.14 \\
11.19\end{array}$ & $\begin{array}{l}11.28 \\
11.28 \\
11.25 \\
11.24 \\
11.22\end{array}$ & $\begin{array}{l}11.19 \\
11.21 \\
11.19 \\
11.17 \\
11.20\end{array}$ & $\begin{array}{l}11.05 \\
11.05 \\
11.05 \\
11.04 \\
11.02\end{array}$ & $\begin{array}{l}10.85 \\
10.83 \\
10.83 \\
10.82 \\
10.82\end{array}$ & $\begin{array}{l}10.72 \\
10.70 \\
10.69 \\
10.67 \\
10.64\end{array}$ \\
\hline $\begin{array}{l}11 \\
12 \\
13 \\
14 \\
15\end{array}$ & $\begin{array}{l}11.04 \\
11.04 \\
11.02 \\
11.02 \\
11.01\end{array}$ & $\begin{array}{l}11.13 \\
11.12 \\
11.11 \\
11.11 \\
11.11\end{array}$ & $\begin{array}{l}11 \cdot 11 \\
11.11 \\
11.11 \\
11.10 \\
11.10\end{array}$ & $\begin{array}{l}11.08 \\
11.08 \\
11.08 \\
11.08 \\
11.08\end{array}$ & $\begin{array}{l}11.22 \\
11.26 \\
11.25 \\
11.24 \\
11.23\end{array}$ & $\begin{array}{l}11 \cdot 17 \\
11 \cdot 17 \\
11 \cdot 16 \\
11 \cdot 16 \\
11.16\end{array}$ & $\begin{array}{l}11 \cdot 18 \\
11 \cdot 17 \\
11 \cdot 16 \\
11 \cdot 16 \\
11.15\end{array}$ & $\begin{array}{l}11.21 \\
11.23 \\
11.22 \\
11.20 \\
11.19\end{array}$ & $\begin{array}{l}11.28 \\
11.25 \\
11.33 \\
11.34 \\
11.30\end{array}$ & $\begin{array}{l}11.00 \\
10.99 \\
10.98 \\
10.96 \\
10.91\end{array}$ & $\begin{array}{l}10.81 \\
10.83 \\
10.83 \\
10.82 \\
10.81\end{array}$ & $\begin{array}{l}10.63 \\
10.62 \\
10.61 \\
10.60 \\
10.58\end{array}$ \\
\hline $\begin{array}{l}16 \\
17 \\
18 \\
19 \\
20\end{array}$ & $\begin{array}{l}11.01 \\
10.95 \\
11.05 \\
11.05 \\
11.07\end{array}$ & $\begin{array}{l}11.10 \\
11.05 \\
10.98 \\
11.02 \\
11.02\end{array}$ & $\begin{array}{l}11.10 \\
11.09 \\
11.10 \\
11.08 \\
11.08\end{array}$ & $\begin{array}{l}11.08 \\
11.07 \\
11.07 \\
11.07 \\
11.07\end{array}$ & $\begin{array}{l}11.23 \\
11.22 \\
11.22 \\
11.21 \\
11.20\end{array}$ & $\begin{array}{l}11 \cdot 16 \\
11 \cdot 16 \\
11 \cdot 14 \\
11 \cdot 13 \\
11 \cdot 12\end{array}$ & $\begin{array}{l}11.16 \\
11.15 \\
11.14 \\
11.13 \\
11.13\end{array}$ & $\begin{array}{l}11.19 \\
11.25 \\
11.28 \\
11.28 \\
11.26\end{array}$ & $\begin{array}{l}11.27 \\
11.04 \\
10.99 \\
10.99 \\
10.98\end{array}$ & $\begin{array}{l}10.90 \\
10.94 \\
10.95 \\
10.94 \\
10.95\end{array}$ & $\begin{array}{l}10.79 \\
10.78 \\
10.78 \\
10.78 \\
10.78\end{array}$ & $\begin{array}{l}10.57 \\
10.56 \\
10.56 \\
10.55 \\
10.57\end{array}$ \\
\hline $\begin{array}{l}21 \\
22 \\
23 \\
24 \\
25\end{array}$ & $\begin{array}{l}11.07 \\
11.06 \\
11.06 \\
11.05 \\
11.05\end{array}$ & $\begin{array}{l}11.01 \\
11.05 \\
11.05 \\
11.05 \\
11.05\end{array}$ & $\begin{array}{l}11.08 \\
11.07 \\
11.07 \\
11.07 \\
11.07\end{array}$ & $\begin{array}{l}11.07 \\
11.07 \\
11.07 \\
11.07 \\
11.07\end{array}$ & $\begin{array}{l}11.19 \\
11.18 \\
11.17 \\
11.17 \\
11.18\end{array}$ & $\begin{array}{l}11 \cdot 12 \\
11 \cdot 11 \\
11 \cdot 11 \\
11 \cdot 10 \\
11.09\end{array}$ & $\begin{array}{l}11 \cdot 14 \\
11 \cdot 20 \\
11 \cdot 35 \\
11 \cdot 38 \\
11.36\end{array}$ & $\begin{array}{l}11 \cdot 23 \\
11.19 \\
11 \cdot 18 \\
11.18 \\
11.19\end{array}$ & $\begin{array}{l}10.98 \\
10.97 \\
10.97 \\
10.97 \\
10.96\end{array}$ & $\begin{array}{l}10.95 \\
10.95 \\
10.98 \\
10.97 \\
10.97\end{array}$ & $\begin{array}{l}10.78 \\
10.77 \\
10.77 \\
10.77 \\
10.76\end{array}$ & $\begin{array}{l}10.57 \\
10.56 \\
10.55 \\
10.54 \\
10.53\end{array}$ \\
\hline $\begin{array}{l}26 \\
27 \\
28 \\
29 \\
30 \\
31\end{array}$ & $\begin{array}{l}11.05 \\
11.06 \\
11.06 \\
11.06 \\
11.06 \\
11.03\end{array}$ & $\begin{array}{r}11.05 \\
11.05 \\
11.05 \\
11.05 \\
11.07 \\
-\end{array}$ & $\begin{array}{l}11.07 \\
11.06 \\
11.06 \\
11.07 \\
11.08 \\
11.08\end{array}$ & $\begin{array}{l}11.07 \\
11.07 \\
11.07 \\
11.06 \\
11.05 \\
11.05\end{array}$ & $\begin{array}{r}11.18 \\
11.18 \\
11.18 \\
--- \\
--- \\
---\end{array}$ & $\begin{array}{l}11.08 \\
11.08 \\
11.08 \\
11.07 \\
11.06 \\
11.07\end{array}$ & $\begin{array}{r}11.38 \\
11.37 \\
11.36 \\
11.33 \\
11.32 \\
-\cdots\end{array}$ & $\begin{array}{l}11.17 \\
11.16 \\
11.15 \\
11.14 \\
11.14 \\
11.14\end{array}$ & $\begin{array}{l}10.96 \\
10.96 \\
10.96 \\
10.95 \\
10.95 \\
---\end{array}$ & $\begin{array}{l}10.98 \\
10.99 \\
10.98 \\
10.98 \\
10.97 \\
10.97\end{array}$ & $\begin{array}{l}10.76 \\
10.76 \\
10.76 \\
10.77 \\
10.77 \\
10.77\end{array}$ & $\begin{array}{r}10.53 \\
10.55 \\
10.67 \\
10.71 \\
10.71 \\
\ldots\end{array}$ \\
\hline $\begin{array}{l}\text { MEAN } \\
\text { MAX } \\
\text { MIN }\end{array}$ & $\begin{array}{l}11.02 \\
11.07 \\
10.91\end{array}$ & $\begin{array}{l}11.04 \\
11.13 \\
10.93\end{array}$ & $\begin{array}{l}11.09 \\
11.13 \\
11.06\end{array}$ & $\begin{array}{l}11.07 \\
11.08 \\
11.05\end{array}$ & $\begin{array}{l}11.15 \\
11.26 \\
11.04\end{array}$ & $\begin{array}{l}11.13 \\
11.18 \\
11.06\end{array}$ & $\begin{array}{l}11.20 \\
11.38 \\
11.06\end{array}$ & $\begin{array}{l}11.22 \\
11.32 \\
11.14\end{array}$ & $\begin{array}{l}11.11 \\
11.34 \\
10.95\end{array}$ & $\begin{array}{l}10.99 \\
11.10 \\
10.90\end{array}$ & $\begin{array}{l}10.81 \\
10.95 \\
10.76\end{array}$ & $\begin{array}{l}10.63 \\
10.77 \\
10.53\end{array}$ \\
\hline
\end{tabular}


LOCATION.--Lat $42^{\circ} 49^{\prime} 15^{\prime \prime}$, long $88^{\circ} 08^{\prime} 39^{\prime \prime}$, in NW 1/4 SW 1/4 sec.9, T.4 N., R.20 E., Racine County, Hydrologic Unit 07120006, at Wind Lake. PERIOD OF RECORD.--February 1985 to current year.

REMARKS.--Lake sampled near center at the deep hole. Lake ice-covered during February sampling. Water-quality analyses done by Wisconsin State Laboratory of Hygiene.

WATER-QUALITY DATA, FEBRUARY 11 TO JUNE 23, 1999

(Milligrams per liter unless otherwise indicated)

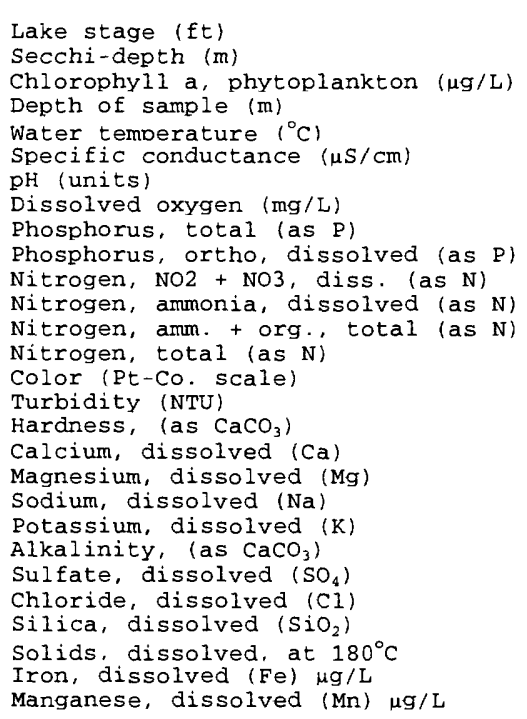

2-11-99

\begin{tabular}{|c|c|c|c|}
\hline \multicolumn{2}{|c|}{ Feb-11 } & \multicolumn{2}{|c|}{$\mathrm{Apr}-8$} \\
\hline & & \multicolumn{2}{|c|}{$\begin{array}{l}7.28 \\
1.2 \\
10.7\end{array}$} \\
\hline 0.5 & 12.5 & 0.5 & \\
\hline $\begin{array}{l}4.9 \\
605\end{array}$ & 3.1 & 10.8 & 10.2 \\
\hline $\begin{array}{l}605 \\
7.7\end{array}$ & 791 & 669 & $\begin{array}{l}680 \\
8.2\end{array}$ \\
\hline 13.5 & $\begin{array}{l}1.4 \\
8.1\end{array}$ & 10.5 & $\begin{array}{l}8.2 \\
9.5\end{array}$ \\
\hline 0.029 & $\ldots$ & 0.032 & -- \\
\hline$\ldots$ & -- & $<0.002$ & -- \\
\hline -.- & -- & 0.31 & -- \\
\hline$\ldots$ & -. & 0.122 & -- \\
\hline -.. & -- & 1.2 & \\
\hline.- & -.. & 1.5 & -. \\
\hline-- & - - & 20 & -- \\
\hline-- & -- & 4.4 & -- \\
\hline$\cdots$ & -- & 250 & -- \\
\hline-- & -- & 54 & -- \\
\hline$-\cdots$ & -- & 28 & -- \\
\hline$\cdots$ &.- & 38 & -- \\
\hline-- & -- & 3.4 & -- \\
\hline-- & -- & 191 & \\
\hline -. & -- & 47 & -- \\
\hline$\cdots$ & 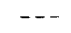 & 78 & $\ldots$ \\
\hline -.. & -- & 0.03 & $\cdots$ \\
\hline -. & --- & 392 & $\cdots$ \\
\hline$\cdots$ & -. & $<10$ & \\
\hline$\ldots$ & $\ldots$ & 0.7 & \\
\hline
\end{tabular}

4-08-99
Jun -23

8.44

1.7

9.61

$5.0 \quad 14.0$

$19.7 \quad 13.6$

$599 \quad 665$

$\begin{array}{ll}7.2 & 7.3\end{array}$

$\begin{array}{ll}1.8 & 0.4\end{array}$

$\begin{array}{lll}8.4 & 1.8 & 0.30 \\ 0.036 & 0.107\end{array}$

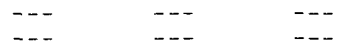

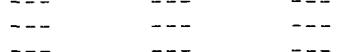

$-$

$-\cdots$

$---$

-

$--$

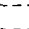

$-+$

-

$--$

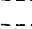

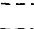

$-1$
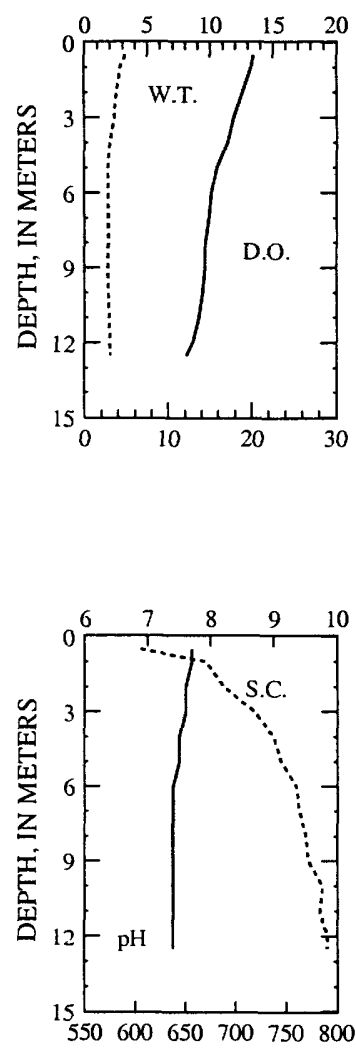

DISSOLVED OXYGEN (D.O.), IN MILLIGRAMS PER LITER
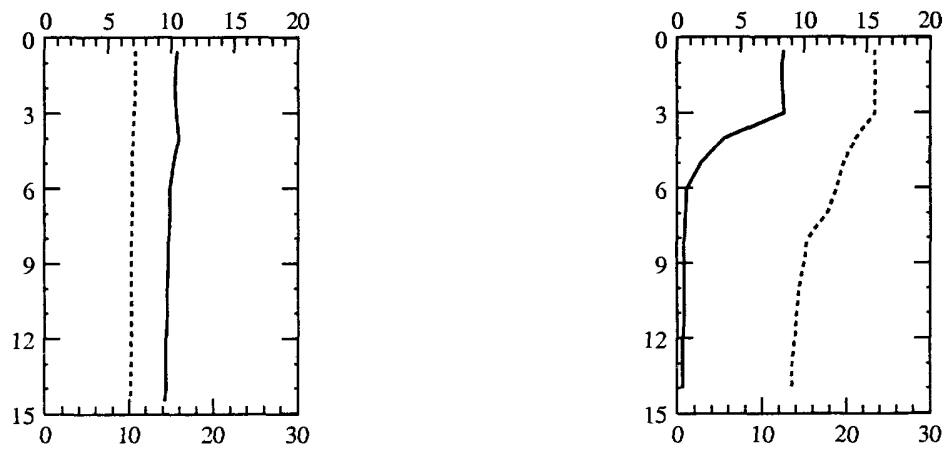

WATER TEMPERATURE (W.T.), IN DEGREES CELSIUS
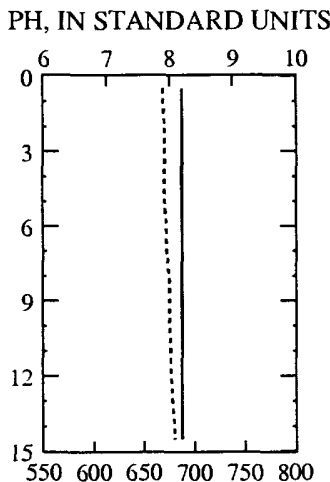

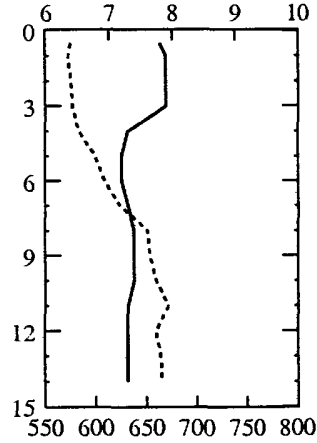


WATER-QUALITY DATA, JULY 27 TO AUGUST 25, 1999

(Milligrams per liter unless otherwise indicated)

Lake stage (ft)

Secchi-depth (m)

Chlorophyll a, phytoplankton ( $\mu \mathrm{g} / \mathrm{L}$ )

Depth of sample (m)

Water temperature $\left({ }^{\circ} \mathrm{C}\right)$

Specific conductance $(\mu \mathrm{S} / \mathrm{cm})$

pH (units)

Dissolved oxygen (mg/L)

Phosphorus, total (as P)

Phosphorus, ortho, dissolved (as P)

Nitrogen, $\mathrm{NO} 2+\mathrm{NO} 3$, diss. (as N)

Nitrogen, ammonia, dissolved (as $\mathrm{N}$ )

Nitrogen, amm. + org., total (as N)

\begin{tabular}{|c|c|c|c|c|}
\hline & & Jul-27 & & \\
\hline & & $\begin{array}{c}8.13 \\
1.1 \\
6.00\end{array}$ & & \\
\hline $\begin{array}{c}0.5 \\
28.0\end{array}$ & $\begin{array}{c}2.0 \\
27.8\end{array}$ & $\begin{array}{c}7.0 \\
20.8\end{array}$ & $\begin{array}{l}11.0 \\
14.2\end{array}$ & $\begin{array}{l}15.0 \\
13.8\end{array}$ \\
\hline 572 & 571 & 605 & 648 & 658 \\
\hline 8.1 & 8.2 & 7.3 & 7.2 & 7.2 \\
\hline 8.5 & 8.4 & 0.0 & 0.0 & 0.0 \\
\hline 0.033 & 0.033 & 0.038 & 0.100 & 0.132 \\
\hline-- & --- & -- & -- & -- \\
\hline--- & --- & $\ldots$ & $\ldots$ & $\ldots$ \\
\hline$\cdots$ & $\ldots$ & $\ldots$ & -.. & $\ldots$ \\
\hline-- & --- & --- & --- & --- \\
\hline
\end{tabular}

\begin{tabular}{|c|c|c|c|c|}
\hline & & Aug-25 & & \\
\hline & & $\begin{array}{c}7.81 \\
1.1 \\
11.0\end{array}$ & & \\
\hline $\begin{array}{c}0.5 \\
22.1\end{array}$ & $\begin{array}{c}7.0 \\
21.3\end{array}$ & $\begin{array}{l}10.0 \\
15.0\end{array}$ & $\begin{array}{l}12.0 \\
14.0\end{array}$ & $\begin{array}{l}14.0 \\
13.8\end{array}$ \\
\hline 577 & 585 & 657 & 671 & 680 \\
\hline 8.1 & 7.7 & 7.0 & 7.0 & 7.0 \\
\hline 7.6 & 2.3 & 0.0 & 0.0 & 0.0 \\
\hline 0.031 & 0.034 & 0.063 & 0.097 & 0.113 \\
\hline 0.003 & --- & -- & -- & $-\cdots$ \\
\hline$<0.010$ & -.. & --. & $\ldots$ & $\cdots$ \\
\hline 0.009 & -.- & -- & -- & --- \\
\hline 1.3 & $\ldots$ & $\ldots$ & --- & -- \\
\hline
\end{tabular}

DISSOLVED OXYGEN (D.O.), IN MILLIGRAMS PER LITER
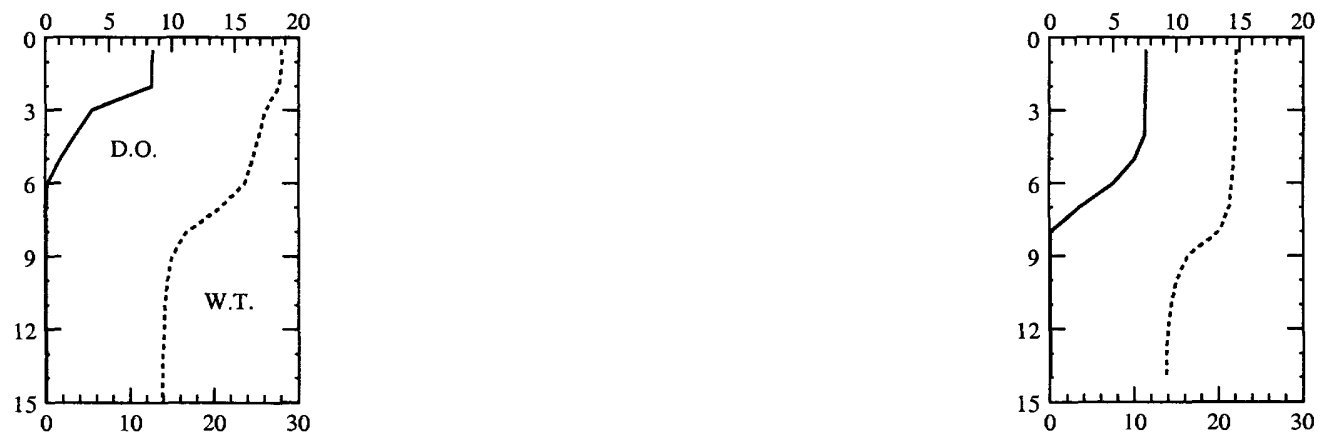

WATER TEMPERATURE (W.T.), IN DEGREES CELCIUS

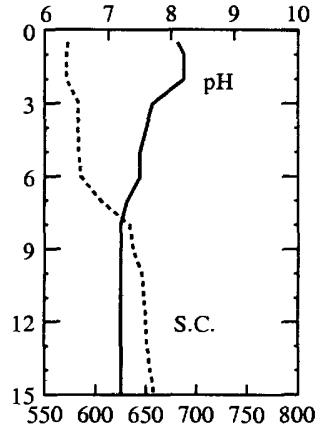

PH, IN STANDARD UNITS

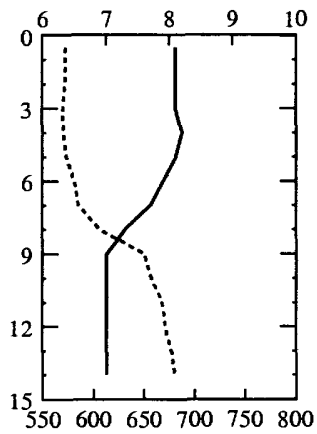

SPECIFIC CONDUCTANCE (S.C.), IN MICROSIEMENS PER CENTIMETER AT 25 DEGREES CELCIUS 

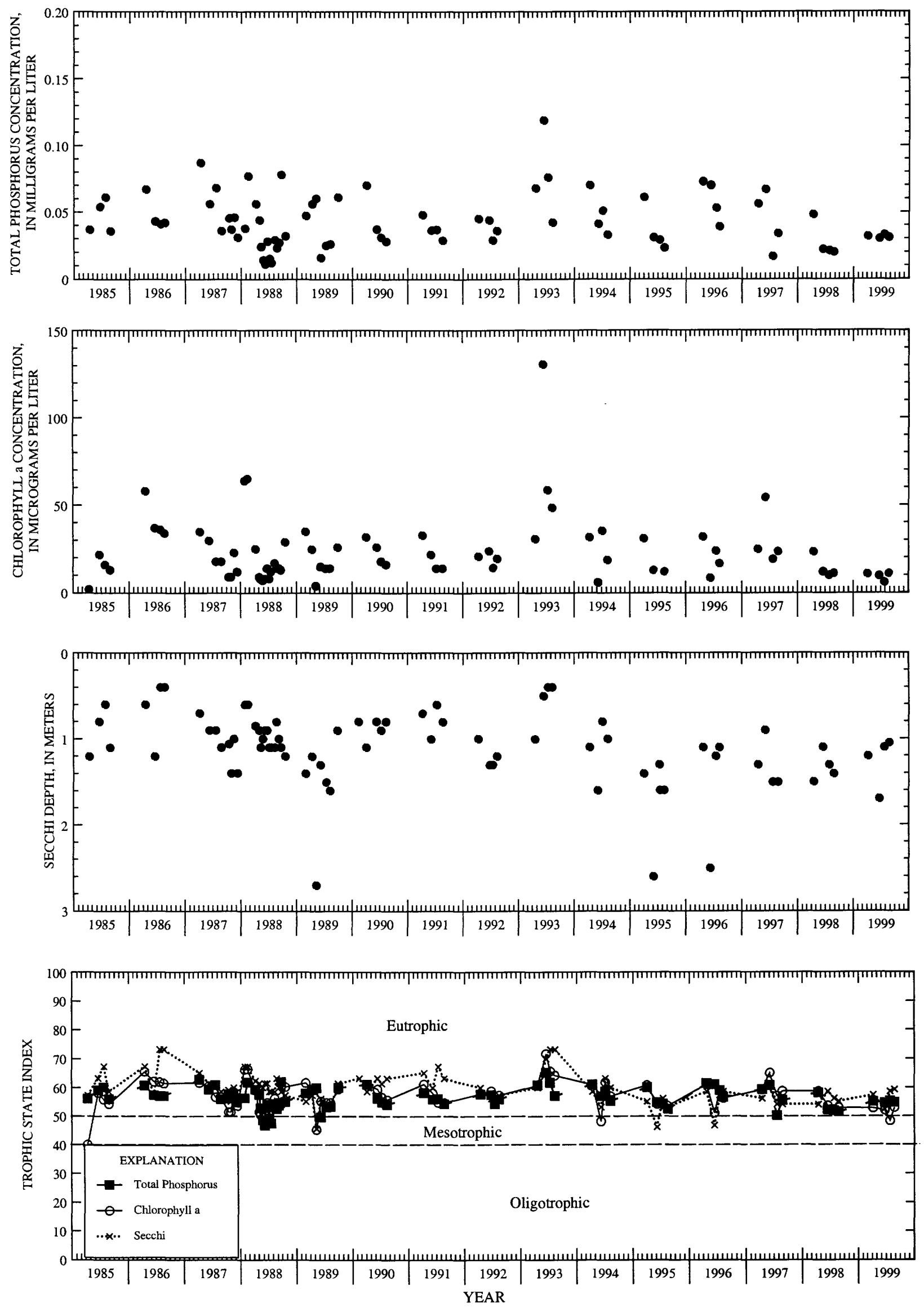

Surface total phosphorus, chlorophyll a concentrations, Secchi depths, and TSI data for Wind Lake, Deep Hole, at Wind Lake, Wisconsin. 
LOCATION.--Lat $42^{\circ} 48^{\prime} 48^{\prime \prime}$ long $88^{\circ} 08^{\prime} 31^{\prime \prime}$, in NE 1/4 NW 1/4 sec.16, T.4 N., R.20 E., Racine County, Hydrologic Unit 07120006, at Wind Lake.

DRAINAGE AREA.--39.6 $\mathrm{mi}^{2}$.

PERIOD OF RECORD.--March 1985 to current year.

REVISED RECORDS.--WDR WI-91-1: 1988(m).

GAGE.--Water-stage recorder and concrete dam. Datum of gage is $760.30 \mathrm{ft}$ above sea level. Prior to Oct. 2, 1987, nonrecording gage at same site and datum.

REMARKS.--No estimated daily gage heights. Records good. Lake level regulated by dam with two 10-foot gates at outlet. Lake ice-covered Dec. 27 to Mar. 17. Prior to October 1987, published as Wind Lake at Wind Lake, Wis.

EXTREMES FOR PERIOD OF RECORD.--Maximum gage height, $8.93 \mathrm{ft}$, June 15, 1999; minimum recorded, $5.95 \mathrm{ft}$, Jan. $2,1996$.

EXTREMES FOR CURRENT YEAR.--Maximum recorded gage height, $8.93 \mathrm{ft}$, June 15; minimum recorded, $7.07 \mathrm{ft}, \mathrm{Mar} .30$.

GAGE HEIGHT, FEET, WATER YEAR OCTOBER 1998 TO SEPTEMBER 1999 DAILY MEAN VALUES

\begin{tabular}{|c|c|c|c|c|c|c|c|c|c|c|c|c|}
\hline DAY & OCT & NOV & DEC & JAN & FEB & MAR & APR & MAY & JUN & JUL & AUG & SEP \\
\hline $\begin{array}{l}1 \\
2 \\
3 \\
4 \\
5\end{array}$ & $\begin{array}{l}8.15 \\
8.13 \\
8.15 \\
8.18 \\
8.21\end{array}$ & $\begin{array}{l}8.19 \\
8.20 \\
8.21 \\
8.21 \\
8.21\end{array}$ & $\begin{array}{l}8.12 \\
7.98 \\
7.85 \\
7.73 \\
7.60\end{array}$ & $\begin{array}{l}7.35 \\
7.42 \\
7.51 \\
7.53 \\
7.55\end{array}$ & $\begin{array}{l}7.33 \\
7.36 \\
7.41 \\
7.42 \\
7.36\end{array}$ & $\begin{array}{l}7.26 \\
7.30 \\
7.35 \\
7.35 \\
7.35\end{array}$ & $\begin{array}{l}7.12 \\
7.15 \\
7.20 \\
7.26 \\
7.28\end{array}$ & $\begin{array}{l}8.21 \\
8.21 \\
8.20 \\
8.13 \\
8.09\end{array}$ & $\begin{array}{l}8.22 \\
8.24 \\
8.19 \\
8.22 \\
8.25\end{array}$ & $\begin{array}{l}8.34 \\
8.34 \\
8.34 \\
8.34 \\
8.32\end{array}$ & $\begin{array}{l}8.05 \\
8.01 \\
7.98 \\
7.96 \\
7.94\end{array}$ & $\begin{array}{l}7.70 \\
7.69 \\
7.68 \\
7.67 \\
7.66\end{array}$ \\
\hline $\begin{array}{r}6 \\
7 \\
8 \\
9 \\
10\end{array}$ & $\begin{array}{l}8.26 \\
8.27 \\
8.25 \\
8.20 \\
8.18\end{array}$ & $\begin{array}{l}8.20 \\
8.19 \\
8.19 \\
8.19 \\
8.34\end{array}$ & $\begin{array}{l}7.50 \\
7.45 \\
7.34 \\
7.24 \\
7.18\end{array}$ & $\begin{array}{l}7.58 \\
7.59 \\
7.61 \\
7.63 \\
7.63\end{array}$ & $\begin{array}{l}7.29 \\
7.20 \\
7.14 \\
7.13 \\
7.14\end{array}$ & $\begin{array}{l}7.35 \\
7.30 \\
7.27 \\
7.26 \\
7.25\end{array}$ & $\begin{array}{l}7.30 \\
7.29 \\
7.28 \\
7.44 \\
7.51\end{array}$ & $\begin{array}{l}8.22 \\
8.31 \\
8.33 \\
8.32 \\
8.28\end{array}$ & $\begin{array}{l}8.29 \\
8.35 \\
8.37 \\
8.38 \\
8.41\end{array}$ & $\begin{array}{l}8.30 \\
8.26 \\
8.23 \\
8.22 \\
8.20\end{array}$ & $\begin{array}{l}7.91 \\
7.90 \\
7.90 \\
7.88 \\
7.89\end{array}$ & $\begin{array}{l}7.64 \\
7.61 \\
7.60 \\
7.57 \\
7.54\end{array}$ \\
\hline $\begin{array}{l}11 \\
12 \\
13 \\
14 \\
15\end{array}$ & $\begin{array}{l}8.18 \\
8.19 \\
8.21 \\
8.20 \\
8.22\end{array}$ & $\begin{array}{l}8.33 \\
8.24 \\
8.16 \\
8.15 \\
8.11\end{array}$ & $\begin{array}{l}7.18 \\
7.17 \\
7.19 \\
7.20 \\
7.21\end{array}$ & $\begin{array}{l}7.61 \\
7.61 \\
7.61 \\
7.60 \\
7.58\end{array}$ & $\begin{array}{l}7.23 \\
7.41 \\
7.41 \\
7.34 \\
7.27\end{array}$ & $\begin{array}{l}7.22 \\
7.19 \\
7.17 \\
7.17 \\
7.16\end{array}$ & $\begin{array}{l}7.48 \\
7.36 \\
7.25 \\
7.19 \\
7.22\end{array}$ & $\begin{array}{l}8.22 \\
8.27 \\
8.31 \\
8.32 \\
8.30\end{array}$ & $\begin{array}{l}8.47 \\
8.46 \\
8.58 \\
8.82 \\
8.91\end{array}$ & $\begin{array}{l}8.18 \\
8.17 \\
8.14 \\
8.12 \\
8.09\end{array}$ & $\begin{array}{l}7.88 \\
7.87 \\
7.88 \\
7.86 \\
7.83\end{array}$ & $\begin{array}{l}7.52 \\
7.51 \\
7.52 \\
7.50 \\
7.49\end{array}$ \\
\hline $\begin{array}{l}16 \\
17 \\
18 \\
19 \\
20\end{array}$ & $\begin{array}{l}8.31 \\
8.41 \\
8.41 \\
8.30 \\
8.25\end{array}$ & $\begin{array}{l}8.04 \\
8.06 \\
8.06 \\
8.08 \\
8.16\end{array}$ & $\begin{array}{l}7.22 \\
7.23 \\
7.22 \\
7.24 \\
7.24\end{array}$ & $\begin{array}{l}7.53 \\
7.49 \\
7.44 \\
7.36 \\
7.27\end{array}$ & $\begin{array}{l}7.22 \\
7.18 \\
7.14 \\
7.14 \\
7.15\end{array}$ & $\begin{array}{l}7.15 \\
7.21 \\
7.28 \\
7.30 \\
7.34\end{array}$ & $\begin{array}{l}7.40 \\
7.53 \\
7.64 \\
7.74 \\
7.76\end{array}$ & $\begin{array}{l}8.30 \\
8.36 \\
8.29 \\
8.24 \\
8.17\end{array}$ & $\begin{array}{l}8.91 \\
8.84 \\
8.71 \\
8.55 \\
8.40\end{array}$ & $\begin{array}{l}8.08 \\
8.13 \\
8.15 \\
8.15 \\
8.15\end{array}$ & $\begin{array}{l}7.81 \\
7.81 \\
7.79 \\
7.82 \\
7.81\end{array}$ & $\begin{array}{l}7.47 \\
7.46 \\
7.45 \\
7.45 \\
7.46\end{array}$ \\
\hline $\begin{array}{l}21 \\
22 \\
23 \\
24 \\
25\end{array}$ & $\begin{array}{l}8.24 \\
8.26 \\
8.28 \\
8.29 \\
8.27\end{array}$ & $\begin{array}{l}8.18 \\
8.18 \\
8.20 \\
8.20 \\
8.21\end{array}$ & $\begin{array}{l}7.26 \\
7.23 \\
7.24 \\
7.26 \\
7.27\end{array}$ & $\begin{array}{l}7.22 \\
7.27 \\
7.40 \\
7.61 \\
7.73\end{array}$ & $\begin{array}{l}7.19 \\
7.19 \\
7.19 \\
7.14 \\
7.15\end{array}$ & $\begin{array}{l}7.37 \\
7.34 \\
7.31 \\
7.25 \\
7.18\end{array}$ & $\begin{array}{l}7.79 \\
7.90 \\
8.32 \\
8.51 \\
8.52\end{array}$ & $\begin{array}{l}8.11 \\
8.15 \\
8.23 \\
8.33 \\
8.38\end{array}$ & $\begin{array}{l}8.29 \\
8.28 \\
8.44 \\
8.48 \\
8.41\end{array}$ & $\begin{array}{l}8.15 \\
8.15 \\
8.16 \\
8.16 \\
8.15\end{array}$ & $\begin{array}{l}7.80 \\
7.79 \\
7.79 \\
7.81 \\
7.81\end{array}$ & $\begin{array}{l}7.44 \\
7.42 \\
7.41 \\
7.40 \\
7.38\end{array}$ \\
\hline $\begin{array}{l}26 \\
27 \\
28 \\
29 \\
30 \\
31\end{array}$ & $\begin{array}{l}8.24 \\
8.17 \\
8.16 \\
8.15 \\
8.16 \\
8.17\end{array}$ & $\begin{array}{l}8.22 \\
8.22 \\
8.21 \\
8.19 \\
8.18 \\
-\end{array}$ & $\begin{array}{l}7.28 \\
7.30 \\
7.30 \\
7.33 \\
7.34 \\
7.35\end{array}$ & $\begin{array}{l}7.71 \\
7.60 \\
7.52 \\
7.41 \\
7.29 \\
7.29\end{array}$ & $\begin{array}{r}7.17 \\
7.20 \\
7.24 \\
-.- \\
--- \\
---\end{array}$ & $\begin{array}{l}7.12 \\
7.11 \\
7.10 \\
7.10 \\
7.09 \\
7.09\end{array}$ & $\begin{array}{l}8.46 \\
8.37 \\
8.30 \\
8.21 \\
8.20 \\
---\end{array}$ & $\begin{array}{l}8.35 \\
8.29 \\
8.25 \\
8.20 \\
8.18 \\
8.18\end{array}$ & $\begin{array}{l}8.32 \\
8.26 \\
8.30 \\
8.34 \\
8.33 \\
---\end{array}$ & $\begin{array}{l}8.14 \\
8.13 \\
8.11 \\
8.08 \\
8.07 \\
8.07\end{array}$ & $\begin{array}{l}7.79 \\
7.78 \\
7.78 \\
7.76 \\
7.73 \\
7.71\end{array}$ & $\begin{array}{l}7.37 \\
7.40 \\
7.66 \\
7.72 \\
7.70 \\
---\end{array}$ \\
\hline $\begin{array}{l}\text { MEAN } \\
\text { MAX } \\
\text { MIN }\end{array}$ & $\begin{array}{l}8.23 \\
8.41 \\
8.13\end{array}$ & $\begin{array}{l}8.18 \\
8.34 \\
8.04\end{array}$ & $\begin{array}{l}7.36 \\
8.12 \\
7.17\end{array}$ & $\begin{array}{l}7.50 \\
7.73 \\
7.22\end{array}$ & $\begin{array}{l}7.24 \\
7.42 \\
7.13\end{array}$ & $\begin{array}{l}7.24 \\
7.37 \\
7.09\end{array}$ & $\begin{array}{l}7.67 \\
8.52 \\
7.12\end{array}$ & $\begin{array}{l}8.25 \\
8.38 \\
8.09\end{array}$ & $\begin{array}{l}8.43 \\
8.91 \\
8.19\end{array}$ & $\begin{array}{l}8.18 \\
8.34 \\
8.07\end{array}$ & $\begin{array}{l}7.85 \\
8.05 \\
7.71\end{array}$ & $\begin{array}{l}7.54 \\
7.72 \\
7.37\end{array}$ \\
\hline
\end{tabular}


LOCATION.--Lat $44^{\circ} 00^{\prime} 35^{\prime \prime}$, long $88^{\circ} 31^{\prime} 38^{\prime \prime}$, in NE $1 / 4$ NE $1 / 4$ sec.25, T.18 N., R.16 E., Winnebago County, Hydrologic Unit 04030203, at 905 Bay Shore Drive, $800 \mathrm{ft}$ east of mouth of the upper Fox River.

DRAINAGE AREA.--5,880 $\mathrm{mi}^{2}$, at lake outlet at Menasha Dam. Area of Lake Winnebago, $215 \mathrm{mi}^{2}$.

PERIOD OF RECORD.--October 1938 to current year in reports of Geological Survey. Records from 1882 to 1938 in files of Geological Survey and U.S. Army Corps of Engineers. A report on Fox River by U.S. Army Corps of Engineers, published as House Document No. 146, 67th Congress, 2nd session, contains semi-monthly records of inflow of Lake Winnebago for the period 1896-1917.

REVISED RECORD.--WDR WI-83-1: Drainage area.

GAGE.--Water-stage recorder. Nonrecording gage read once daily October 1938 to October 1978. Datum of gage is $745.05 \mathrm{ft}$ above mean tide at New York City (levels by U.S. Army Corps of Engineers). Datum of Deuchman gage is $745.00 \mathrm{ft}$ above mean tide at New York City.

REMARKS.--No estimated daily gage heights. Records good. Lake elevations controlled by dams at Menasha and Neenah, which are operated in the interest of navigation. Crests of both dams are at elevation $746.73 \mathrm{ft}$. Present limits of regulation are from $211 / 4 \mathrm{in}$. above the crest of Menasha dam to crest during navigation season, plus additional $18 \mathrm{in.}$. below crest during winter. Oshkosh staff gage gives true level of lake, while Deuchman gage readings are affected by loss of head in the channel between lake and dam. Data-collection platform at station.

EXTREMES FOR PERIOD OF RECORD.--Maximum gage height observed, $5.33 \mathrm{ft}$ (Deuchman gage) Nov. 8, 1881; minimum observed, $-2.00 \mathrm{ft}$ (Deuchman gage) Nov. 28, 1891.

EXTREMES FOR CURRENT YEAR.--Maximum daily mean gage height, $3.22 \mathrm{ft}$, July 22; minimum recorded, $1.65 \mathrm{ft}$, Feb. 11 and Mar. 28.

GAGE HEIGHT, FEET, WATER YEAR OCTOBER 1998 TO SEPTEMBER 1999

DAILY MEAN VALUES

\begin{tabular}{|c|c|c|c|c|c|c|c|c|c|c|c|c|}
\hline DAY & OCT & NOV & DEC & JAN & FEB & MAR & APR & MAY & JUN & JUL & AUG & SEP \\
\hline $\begin{array}{l}1 \\
2 \\
3 \\
4 \\
5\end{array}$ & $\begin{array}{l}2.62 \\
2.63 \\
2.67 \\
2.63 \\
2.65\end{array}$ & $\begin{array}{l}2.79 \\
2.82 \\
2.75 \\
2.69 \\
2.69\end{array}$ & $\begin{array}{l}2.46 \\
2.45 \\
2.43 \\
2.42 \\
2.40\end{array}$ & $\begin{array}{l}2.11 \\
2.12 \\
2.15 \\
2.15 \\
2.14\end{array}$ & $\begin{array}{l}1.85 \\
1.83 \\
1.81 \\
1.80 \\
1.77\end{array}$ & $\begin{array}{l}1.91 \\
1.89 \\
1.90 \\
1.89 \\
1.86\end{array}$ & $\begin{array}{l}1.96 \\
1.98 \\
2.11 \\
2.19 \\
2.29\end{array}$ & $\begin{array}{l}2.63 \\
2.63 \\
2.62 \\
2.62 \\
2.61\end{array}$ & $\begin{array}{l}3.04 \\
3.06 \\
3.09 \\
3.06 \\
3.00\end{array}$ & $\begin{array}{l}2.94 \\
2.98 \\
3.00 \\
3.02 \\
3.02\end{array}$ & $\begin{array}{l}3.04 \\
3.03 \\
3.01 \\
3.01 \\
3.03\end{array}$ & $\begin{array}{l}2.92 \\
2.93 \\
2.92 \\
2.92 \\
2.90\end{array}$ \\
\hline $\begin{array}{r}6 \\
7 \\
8 \\
9 \\
10\end{array}$ & $\begin{array}{l}2.65 \\
2.70 \\
2.73 \\
2.71 \\
2.73\end{array}$ & $\begin{array}{l}2.66 \\
2.66 \\
2.65 \\
2.66 \\
2.51\end{array}$ & $\begin{array}{l}2.38 \\
2.40 \\
2.36 \\
2.35 \\
2.33\end{array}$ & $\begin{array}{l}2.14 \\
2.13 \\
2.11 \\
2.10 \\
2.09\end{array}$ & $\begin{array}{l}1.75 \\
1.73 \\
1.72 \\
1.71 \\
1.70\end{array}$ & $\begin{array}{l}1.85 \\
1.84 \\
1.84 \\
1.82 \\
1.83\end{array}$ & $\begin{array}{l}2.13 \\
2.32 \\
2.40 \\
2.52 \\
2.50\end{array}$ & $\begin{array}{l}2.62 \\
2.64 \\
2.71 \\
2.78 \\
2.78\end{array}$ & $\begin{array}{l}2.99 \\
2.95 \\
2.98 \\
2.97 \\
2.96\end{array}$ & $\begin{array}{l}3.07 \\
3.08 \\
3.07 \\
3.03 \\
3.07\end{array}$ & $\begin{array}{l}3.00 \\
2.94 \\
2.95 \\
2.89 \\
2.87\end{array}$ & $\begin{array}{l}2.91 \\
2.89 \\
2.83 \\
2.82 \\
2.79\end{array}$ \\
\hline $\begin{array}{l}11 \\
12 \\
13 \\
14 \\
15\end{array}$ & $\begin{array}{l}2.74 \\
2.69 \\
2.72 \\
2.75 \\
2.74\end{array}$ & $\begin{array}{l}2.57 \\
2.70 \\
2.68 \\
2.66 \\
2.68\end{array}$ & $\begin{array}{l}2.31 \\
2.31 \\
2.30 \\
2.27 \\
2.26\end{array}$ & $\begin{array}{l}2.08 \\
2.08 \\
2.08 \\
2.07 \\
2.05\end{array}$ & $\begin{array}{l}1.72 \\
1.79 \\
1.80 \\
1.82 \\
1.83\end{array}$ & $\begin{array}{l}1.83 \\
1.83 \\
1.83 \\
1.82 \\
1.82\end{array}$ & $\begin{array}{l}2.49 \\
2.52 \\
2.60 \\
2.64 \\
2.67\end{array}$ & $\begin{array}{l}2.78 \\
2.85 \\
2.85 \\
2.83 \\
2.86\end{array}$ & $\begin{array}{l}2.99 \\
2.98 \\
3.01 \\
3.00 \\
2.97\end{array}$ & $\begin{array}{l}3.05 \\
3.01 \\
2.96 \\
2.90 \\
2.89\end{array}$ & $\begin{array}{l}2.89 \\
2.89 \\
2.93 \\
2.95 \\
2.93\end{array}$ & $\begin{array}{l}2.81 \\
2.76 \\
2.75 \\
2.74 \\
2.75\end{array}$ \\
\hline $\begin{array}{l}16 \\
17 \\
18 \\
19 \\
20\end{array}$ & $\begin{array}{l}2.71 \\
2.74 \\
2.67 \\
2.74 \\
2.75\end{array}$ & $\begin{array}{l}2.69 \\
2.71 \\
2.71 \\
2.55 \\
2.67\end{array}$ & $\begin{array}{l}2.24 \\
2.24 \\
2.20 \\
2.19 \\
2.21\end{array}$ & $\begin{array}{l}2.03 \\
2.03 \\
2.05 \\
2.04 \\
2.02\end{array}$ & $\begin{array}{l}1.85 \\
1.88 \\
1.89 \\
1.88 \\
1.89\end{array}$ & $\begin{array}{l}1.82 \\
1.79 \\
1.84 \\
1.83 \\
1.82\end{array}$ & $\begin{array}{l}2.71 \\
2.67 \\
2.68 \\
2.68 \\
2.66\end{array}$ & $\begin{array}{l}2.87 \\
3.01 \\
3.05 \\
3.12 \\
3.09\end{array}$ & $\begin{array}{l}2.97 \\
2.95 \\
2.93 \\
2.90 \\
2.87\end{array}$ & $\begin{array}{l}2.86 \\
2.91 \\
2.88 \\
2.96 \\
3.05\end{array}$ & $\begin{array}{l}2.89 \\
2.91 \\
2.94 \\
2.96 \\
2.95\end{array}$ & $\begin{array}{l}2.75 \\
2.74 \\
2.72 \\
2.69 \\
2.73\end{array}$ \\
\hline $\begin{array}{l}21 \\
22 \\
23 \\
24 \\
25\end{array}$ & $\begin{array}{l}2.75 \\
2.74 \\
2.74 \\
2.73 \\
2.75\end{array}$ & $\begin{array}{l}2.66 \\
2.60 \\
2.56 \\
2.61 \\
2.55\end{array}$ & $\begin{array}{l}2.09 \\
2.12 \\
2.15 \\
2.14 \\
2.13\end{array}$ & $\begin{array}{l}1.99 \\
2.00 \\
2.03 \\
2.04 \\
2.03\end{array}$ & $\begin{array}{l}1.89 \\
1.89 \\
1.88 \\
1.88 \\
1.89\end{array}$ & $\begin{array}{l}1.84 \\
1.87 \\
1.85 \\
1.90 \\
1.91\end{array}$ & $\begin{array}{l}2.64 \\
2.71 \\
2.70 \\
2.61 \\
2.60\end{array}$ & $\begin{array}{l}3.08 \\
3.10 \\
3.04 \\
3.08 \\
3.05\end{array}$ & $\begin{array}{l}2.85 \\
2.82 \\
2.82 \\
2.85 \\
2.86\end{array}$ & $\begin{array}{l}3.19 \\
3.22 \\
3.17 \\
3.12 \\
3.09\end{array}$ & $\begin{array}{l}2.97 \\
2.97 \\
2.99 \\
3.01 \\
3.02\end{array}$ & $\begin{array}{l}2.71 \\
2.68 \\
2.70 \\
2.69 \\
2.66\end{array}$ \\
\hline $\begin{array}{l}26 \\
27 \\
28 \\
29 \\
30 \\
31\end{array}$ & $\begin{array}{l}2.76 \\
2.77 \\
2.79 \\
2.78 \\
2.75 \\
2.78\end{array}$ & $\begin{array}{l}2.56 \\
2.54 \\
2.52 \\
2.50 \\
2.43 \\
\ldots\end{array}$ & $\begin{array}{l}2.13 \\
2.12 \\
2.12 \\
2.12 \\
2.12 \\
2.12\end{array}$ & $\begin{array}{l}2.01 \\
1.98 \\
1.96 \\
1.93 \\
1.90 \\
1.88\end{array}$ & $\begin{array}{r}1.88 \\
1.89 \\
1.90 \\
-\ldots \\
-- \\
-\end{array}$ & $\begin{array}{l}1.91 \\
1.92 \\
1.89 \\
1.91 \\
1.95 \\
1.94\end{array}$ & $\begin{array}{l}2.59 \\
2.60 \\
2.60 \\
2.58 \\
2.60 \\
.\end{array}$ & $\begin{array}{l}3.04 \\
3.01 \\
3.01 \\
3.00 \\
2.99 \\
2.97\end{array}$ & $\begin{array}{l}2.84 \\
2.86 \\
2.95 \\
2.95 \\
2.93 \\
-\end{array}$ & $\begin{array}{l}3.00 \\
2.97 \\
2.94 \\
2.96 \\
2.98 \\
3.00\end{array}$ & $\begin{array}{l}3.02 \\
3.01 \\
3.01 \\
3.04 \\
2.97 \\
2.92\end{array}$ & $\begin{array}{l}2.64 \\
2.73 \\
2.77 \\
2.77 \\
2.71 \\
\end{array}$ \\
\hline $\begin{array}{l}\text { MEAN } \\
\text { MAX } \\
\text { MIN }\end{array}$ & $\begin{array}{l}2.72 \\
2.79 \\
2.62\end{array}$ & $\begin{array}{l}2.63 \\
2.82 \\
2.43\end{array}$ & $\begin{array}{l}2.25 \\
2.46 \\
2.09\end{array}$ & $\begin{array}{l}2.05 \\
2.15 \\
1.88\end{array}$ & $\begin{array}{l}1.83 \\
1.90 \\
1.70\end{array}$ & $\begin{array}{l}1.86 \\
1.95 \\
1.79\end{array}$ & $\begin{array}{l}2.50 \\
2.71 \\
1.96\end{array}$ & $\begin{array}{l}2.88 \\
3.12 \\
2.61\end{array}$ & $\begin{array}{l}2.95 \\
3.09 \\
2.82\end{array}$ & $\begin{array}{l}3.01 \\
3.22 \\
2.86\end{array}$ & $\begin{array}{l}2.97 \\
3.04 \\
2.87\end{array}$ & $\begin{array}{l}2.78 \\
2.93 \\
2.64\end{array}$ \\
\hline
\end{tabular}


LOCATION.--Lat $44^{\circ} 04^{\prime} 17^{\prime \prime}$, long $88^{\circ} 19^{\prime} 52^{\prime \prime}$, Stockbridge Indian Reservation, Calumet County, Hydrologic Unit 04030203, on east shore of Lake Winnebago, $300 \mathrm{ft}$ south of County Highway E and $1.6 \mathrm{mi}$ west of Stockbridge.

DRAINAGE AREA.--5,880 $\mathrm{mi}^{2}$, at lake outlet at Menasha Dam. Area of Lake Winnebago, $215 \mathrm{mi}^{2}$.

PERIOD OF RECORD.--November 1982 to current year.

GAGE.--Water-stage recorder. Datum of gage is $745.05 \mathrm{ft}$ above mean tide of New York City (levels by U. S. Army Corps of Engineers).

REMARKS.--No estimated daily gage heights. Records good. Lake elevations controlled by dams at Menasha and Neenah, which are operated in the interest of navigation. Crests of both dams are at elevation $746.73 \mathrm{ft}$. Present limits of regulation are from $211 / 4 \mathrm{in}$. above the crest of Menasha dam to crest during navigation season, plus additional 18 in. below crest during winter. Data-collection platform at station.

EXTREMES FOR PERIOD OF RECORD.--Maximum daily mean gage height, $3.85 \mathrm{ft}$, July 9, 11, 1993; minimum observed, $0.30 \mathrm{ft}$, Mar. 1, 1986.

EXTREMES FOR CURRENT YEAR.--Maximum daily mean gage height, $3.21 \mathrm{ft}$, July 9; minimum recorded, $1.60 \mathrm{ft}$, Feb. 8.

GAGE HEIGHT, FEET, WATER YEAR OCTOBER 1998 TO SEPTEMBER 1999

DAILY MEAN VALUES

\begin{tabular}{|c|c|c|c|c|c|c|c|c|c|c|c|c|}
\hline DAY & OCT & NOV & DEC & JAN & $\mathrm{FEB}$ & MAR & APR & MAY & JUN & JUL & AUG & SEP \\
\hline $\begin{array}{l}1 \\
2 \\
3 \\
4 \\
5\end{array}$ & $\begin{array}{l}2.63 \\
2.58 \\
2.48 \\
2.49 \\
2.48\end{array}$ & $\begin{array}{l}2.67 \\
2.55 \\
2.57 \\
2.63 \\
2.64\end{array}$ & $\begin{array}{l}2.50 \\
2.42 \\
2.39 \\
2.37 \\
2.36\end{array}$ & $\begin{array}{l}2.07 \\
2.09 \\
2.13 \\
2.11 \\
2.10\end{array}$ & $\begin{array}{l}1.80 \\
1.79 \\
1.76 \\
1.77 \\
1.73\end{array}$ & $\begin{array}{l}1.87 \\
1.85 \\
1.87 \\
1.84 \\
1.82\end{array}$ & $\begin{array}{l}1.91 \\
1.93 \\
2.00 \\
2.12 \\
2.15\end{array}$ & $\begin{array}{l}2.59 \\
2.59 \\
2.56 \\
2.53 \\
2.49\end{array}$ & $\begin{array}{l}2.96 \\
3.02 \\
3.04 \\
3.00 \\
2.98\end{array}$ & $\begin{array}{l}2.99 \\
3.01 \\
3.03 \\
3.09 \\
3.13\end{array}$ & $\begin{array}{l}3.01 \\
3.00 \\
3.01 \\
3.03 \\
3.02\end{array}$ & $\begin{array}{l}2.88 \\
2.89 \\
2.88 \\
2.87 \\
2.88\end{array}$ \\
\hline $\begin{array}{r}6 \\
7 \\
8 \\
9 \\
10\end{array}$ & $\begin{array}{l}2.65 \\
2.72 \\
2.68 \\
2.69 \\
2.70\end{array}$ & $\begin{array}{l}2.65 \\
2.62 \\
2.62 \\
2.59 \\
2.91\end{array}$ & $\begin{array}{l}2 \cdot 38 \\
2.37 \\
2.38 \\
2.36 \\
2.37\end{array}$ & $\begin{array}{l}2.10 \\
2.08 \\
2.07 \\
2.06 \\
2.06\end{array}$ & $\begin{array}{l}1.71 \\
1.69 \\
1.67 \\
1.68 \\
1.66\end{array}$ & $\begin{array}{l}1.81 \\
1.80 \\
1.78 \\
1.77 \\
1.79\end{array}$ & $\begin{array}{l}2.27 \\
2.32 \\
2.29 \\
2.21 \\
2.33\end{array}$ & $\begin{array}{l}2.65 \\
2.80 \\
2.76 \\
2.74 \\
2.69\end{array}$ & $\begin{array}{l}2.98 \\
3.02 \\
2.97 \\
2.95 \\
2.97\end{array}$ & $\begin{array}{l}3.20 \\
3.17 \\
3.08 \\
3.21 \\
3.18\end{array}$ & $\begin{array}{l}2.95 \\
2.90 \\
2.85 \\
2.86 \\
2.86\end{array}$ & $\begin{array}{l}2.87 \\
2.85 \\
2.86 \\
2.89 \\
2.86\end{array}$ \\
\hline $\begin{array}{l}11 \\
12 \\
13 \\
14 \\
15\end{array}$ & $\begin{array}{l}2.69 \\
2.74 \\
2.78 \\
2.69 \\
2.65\end{array}$ & $\begin{array}{l}2.89 \\
2.71 \\
2.66 \\
2.67 \\
2.70\end{array}$ & $\begin{array}{l}2.36 \\
2.29 \\
2.26 \\
2.28 \\
2.27\end{array}$ & $\begin{array}{l}2.04 \\
2.04 \\
2.04 \\
2.02 \\
2.00\end{array}$ & $\begin{array}{l}1.68 \\
1.77 \\
1.77 \\
1.77 \\
1.79\end{array}$ & $\begin{array}{l}1.80 \\
1.79 \\
1.79 \\
1.78 \\
1.78\end{array}$ & $\begin{array}{l}2.38 \\
2.54 \\
2.59 \\
2.61 \\
2.61\end{array}$ & $\begin{array}{l}2.70 \\
2.68 \\
2.68 \\
2.76 \\
2.81\end{array}$ & $\begin{array}{l}3.00 \\
3.00 \\
3.00 \\
2.99 \\
3.01\end{array}$ & $\begin{array}{l}3.17 \\
3.15 \\
3.14 \\
3.13 \\
3.06\end{array}$ & $\begin{array}{l}2.85 \\
2.85 \\
2.86 \\
2.89 \\
2.90\end{array}$ & $\begin{array}{l}2.75 \\
2.75 \\
2.82 \\
2.79 \\
2.72\end{array}$ \\
\hline $\begin{array}{l}16 \\
17 \\
18 \\
19 \\
20\end{array}$ & $\begin{array}{l}2.67 \\
2.73 \\
2.85 \\
2.81 \\
2.79\end{array}$ & $\begin{array}{l}2.63 \\
2.68 \\
2.63 \\
2.78 \\
2.73\end{array}$ & $\begin{array}{l}2.23 \\
2.24 \\
2.17 \\
2.19 \\
2.14\end{array}$ & $\begin{array}{l}1.99 \\
1.99 \\
2.02 \\
2.00 \\
1.97\end{array}$ & $\begin{array}{l}1.82 \\
1.84 \\
1.84 \\
1.84 \\
1.85\end{array}$ & $\begin{array}{l}1.77 \\
1.79 \\
1.84 \\
1.78 \\
1.78\end{array}$ & $\begin{array}{l}2.55 \\
2.61 \\
2.65 \\
2.65 \\
2.63\end{array}$ & $\begin{array}{l}2.84 \\
2.99 \\
3.09 \\
3.08 \\
3.06\end{array}$ & $\begin{array}{l}2.97 \\
2.96 \\
2.94 \\
2.89 \\
2.86\end{array}$ & $\begin{array}{l}3.03 \\
3.03 \\
3.02 \\
3.09 \\
3.07\end{array}$ & $\begin{array}{l}2.93 \\
2.91 \\
2.86 \\
2.88 \\
2.91\end{array}$ & $\begin{array}{l}2.69 \\
2.69 \\
2.70 \\
2.69 \\
2.66\end{array}$ \\
\hline $\begin{array}{l}21 \\
22 \\
23 \\
24 \\
25\end{array}$ & $\begin{array}{l}2.71 \\
2.73 \\
2.73 \\
2.73 \\
2.71\end{array}$ & $\begin{array}{l}2.66 \\
2.64 \\
2.65 \\
2.58 \\
2.54\end{array}$ & $\begin{array}{l}2.20 \\
2.19 \\
2.11 \\
2.11 \\
2.08\end{array}$ & $\begin{array}{l}1.95 \\
1.96 \\
2.00 \\
2.00 \\
1.99\end{array}$ & $\begin{array}{l}1.85 \\
1.84 \\
1.83 \\
1.83 \\
1.85\end{array}$ & $\begin{array}{l}1.82 \\
1.84 \\
1.87 \\
1.84 \\
1.85\end{array}$ & $\begin{array}{l}2.56 \\
2.40 \\
2.44 \\
2.56 \\
2.57\end{array}$ & $\begin{array}{l}3.06 \\
3.03 \\
3.12 \\
3.13 \\
3.11\end{array}$ & $\begin{array}{l}2.84 \\
2.84 \\
2.86 \\
2.87 \\
2.88\end{array}$ & $\begin{array}{l}3.17 \\
3.19 \\
3.17 \\
3.12 \\
3.06\end{array}$ & $\begin{array}{l}2.94 \\
2.96 \\
2.94 \\
2.91 \\
2.95\end{array}$ & $\begin{array}{l}2.65 \\
2.69 \\
2.65 \\
2.62 \\
2.61\end{array}$ \\
\hline $\begin{array}{l}26 \\
27 \\
28 \\
29 \\
30 \\
31\end{array}$ & $\begin{array}{l}2.72 \\
2.74 \\
2.75 \\
2.70 \\
2.72 \\
2.72\end{array}$ & $\begin{array}{r}2.55 \\
2.50 \\
2.48 \\
2.46 \\
2.50 \\
---\end{array}$ & $\begin{array}{l}2.09 \\
2.08 \\
2.08 \\
2.09 \\
2.09 \\
2.08\end{array}$ & $\begin{array}{l}1.96 \\
1.94 \\
1.92 \\
1.89 \\
1.86 \\
1.83\end{array}$ & $\begin{array}{r}1.83 \\
1.85 \\
1.88 \\
\ldots- \\
- \\
-\end{array}$ & $\begin{array}{l}1.87 \\
1.87 \\
1.91 \\
1.95 \\
1.93 \\
1.94\end{array}$ & $\begin{array}{r}2.55 \\
2.46 \\
2.43 \\
2.52 \\
2.57 \\
-\end{array}$ & $\begin{array}{l}3.03 \\
3.00 \\
2.98 \\
2.98 \\
2.97 \\
2.98\end{array}$ & $\begin{array}{r}2.87 \\
2.87 \\
2.92 \\
3.00 \\
2.97 \\
\ldots\end{array}$ & $\begin{array}{l}3.01 \\
2.95 \\
2.95 \\
2.98 \\
2.97 \\
3.05\end{array}$ & $\begin{array}{l}2.97 \\
2.98 \\
3.00 \\
2.92 \\
2.89 \\
2.88\end{array}$ & $\begin{array}{r}2.64 \\
2.68 \\
2.68 \\
2.74 \\
2.79 \\
\end{array}$ \\
\hline $\begin{array}{l}\text { MEAN } \\
\text { MAX } \\
\text { MIN }\end{array}$ & $\begin{array}{l}2.69 \\
2.85 \\
2.48\end{array}$ & $\begin{array}{l}2.64 \\
2.91 \\
2.46\end{array}$ & $\begin{array}{l}2.24 \\
2.50 \\
2.08\end{array}$ & $\begin{array}{l}2.01 \\
2.13 \\
1.83\end{array}$ & $\begin{array}{l}1.79 \\
1.88 \\
1.66\end{array}$ & $\begin{array}{l}1.83 \\
1.95 \\
1.77\end{array}$ & $\begin{array}{l}2.41 \\
2.65 \\
1.91\end{array}$ & $\begin{array}{l}2.85 \\
3.13 \\
2.49\end{array}$ & $\begin{array}{l}2.95 \\
3.04 \\
2.84\end{array}$ & $\begin{array}{l}3.08 \\
3.21 \\
2.95\end{array}$ & $\begin{array}{l}2.92 \\
3.03 \\
2.85\end{array}$ & $\begin{array}{l}2.76 \\
2.89 \\
2.61\end{array}$ \\
\hline
\end{tabular}




\section{WISCONSIN DISTRICT PUBLICATIONS PERTAINING TO LAKES}

The reports published in a U.S. Geological Survey series are for sale by the U.S. Geological Survey, Box 25425, Federal Center, Denver, CO 80225. Prepayment is required. Remittance should be sent by check or money order payable to the U.S. Geological Survey. Prices can be obtained by writing to the above address or by calling (303) 236-7476. Reprints of journal articles may be obtained by writing directly to the author at U.S. Geological Survey, 8505 Research Way, Middleton, WI 53562.

\section{9}

Lathrop, R.C., Carpenter, S.R., and Robertson, D.M., 1999, Summer water clarity responses to phosphorus, Daphnia grazing, and internal mixing in Lake Mendota, Limnology and Oceanography, Vol. 44 (1), p. 137-146.

Krohelski, J.T., Feinstein, D.T., and Lenz, B.N., 1999, Simulation of stage and hydrologic bidget for Shell Lake, Washburn County, Wisconsin, U.S. Geological Survey Water-Resources Investigations Report 99-4209, 23 p.

Panuska, J.C., and Robertson, D.M., 1999, Estimating phosphorus concentrations following alum treatment using apparent settling velocities, Lakes and Reservoir Management, Vol. 15 (1), p. 28-38.

1998

Hunt, R.J, Anderson, M.P., and Kelson, V.A., 1998, Improving a complex finite-difference ground water flow model through the use of an analytic element screening model, Ground Water, Vol. 36, No. 6, p. 1011-1017.

Hunt, R.J, Anderson, M.P., and Kelson, V.A., 1998, Linking an analytic element flow code to MODFLOW - Implementation and benefits. p. 477-504 in MODFLOW'98: Proceedings of the 3rd International Conference of the International Ground Water Center. Golden, CO: Colorado School of Mines.

Krabbenhoft, D.P., Gilmour, C.C., Benoit, J.M., Babiarz, C.L., Andren, A.W., and Hurley, J.P., 1998, Methyl mercury dyanmics in littoral sediments of a temperate lake, Canadian Journal of Fisheries and Aquatic Sciences, Vol. 55, p. 835-844.

Robertson, D.M., Elder, J.F., Goddard, G.L., and James, W.F., 1998, Dynamics in phosphorus retention in wetlands upstream of Delavan Lake, Wisconsin, Lakes and Reservoir Management, Vol. 14, No. 4, p. 466-477.

Rose, W.J., and Robertson, D.M., 1998, Hydrology, water quality, and phosphorus loading of Kirby Lake, Barron County, Wisconsin, U.S. Geological Survey Fact Sheet FS-066-98, 4 p.

Walker, J.F. and Krabbenhoft, D.P., 1998, Groundwater and surface-water interactions in riparian and lake-dominated systems, in Kendall, C. and McDonnell, J.J. eds., Isotope tracers in catchment hydrology, Elsevier Publishing, New York, 839 P.

1997

Elder, J.F., Manion, B.J., and Goddard, G.L., 1997, Mesocosm experiments to assess factors affecting phosphorus retention and release in an extended Wisconsin wetland: U.S. Geological Survey Water-Resources Investigations Report 97-4272, 14 p.

Goddard, G.L., and Elder, J.F., 1997, Retention of sediments and nutrients in Jackson Creek Wetland near Delavan Lake, Wisconsin, 1993-95, U.S. Geological Survey Water-Resources Investigations Report 97-4014, 22 p. 
Hornewer, N.J., Johnson, G.P., Robertson, D.M. and Hondzo, M., 1997, Field-scale tests for determining mixing patterns associated with coarse-bubble air diffuser configurations, Egan Quarry, Illinois, In: Environmental and Coastal Hydraulics: Protecting the Aquatic Habitat, Proceedings of the International Association of Hydraulic Research, San Francisco, CA, USA, p. 57-63.

Robertson, D.M., 1997, Regionalized loads of sediment and phosphorus to Lakes Michigan and Superior - High flow and long-term average, Journal of Great Lakes Research, Vol. 23, p. 416439.

\section{6}

Anderson, W.L., Robertson, D.M., and Magnuson, J.J., 1996, Evidence of recent warming and El Nino-related variation in ice breakup of Wisconsin lakes, Limnology and Oceanography, Vol. 41, p. 815-821.

Elder, J.F. and Goddard, G.L., 1996, Sediment and nutrient trapping efficiency of a constructed wetland near Delavan Lake, Wisconsin, 1993-1995: U.S. Geological Survey Fact Sheet FS232-96.

Garn, H.S., Olson, D.L., Seidel, T.L., and Rose, W.J., 1996, Hydrology and water quality of Lauderdale Lakes, Walworth County, Wisconsin, 1993-94: U.S. Geological Survey Water-Resources Investigations Report 96-4235, 29 p.

Hunt, R.J. and Krohelski, J.T., 1996, The application of an analytical element model to investigate groundwater-lake interactions at Pretty Lake, Wisconsin, Lake and Reservoir Management, Vol. 12, p. 487-495.

Imberger, J., Robertson, D.M., and Boland, K., 1996, Lake Number: A quantitative indicator of mixing to be used in water quality management. Scientific Impeller, Solna, Sweden, No. 4, p. 9-15.

Kammerer, P.A., Jr., 1996, Hydrology and water quality of Park Lake, Sourth-central Wisconsin: U.S. Geological Survy Fact Sheet FS-197-96.

Robertson, D.M., Field, S.J, Elder, J.F., Goddard, G.L., and James, W.F., 1996, Phosphorus dynamics of Delavan Lake Inlet in southeastern Wisconsin. U.S. Geological Survey Water-Resources Investigations Report 96-4160, $18 \mathrm{p}$.

1995

Assel, R.A. and Robertson, D.M. 1995, Changes in winter air temperatures near Lake Michigan during 1851 - 1993, as determined from regional lake-ice records, Limnology and Oceanography, v. 40, p 165-176.

Assel, R.A., Robertson, D.M., Hoff, M.H., and Selgeby, J.H., 1995, Climatic-change implications from long-term (1823-1994) ice records near the Laurentian Great Lakes: Annals of Glaciology, v. 21 , p. 383-386.

Krabbenhoft, D.P., and Webster, K.E., 1995, Transient hydrogeological controls on the chemistry of a seepage lake: Water Resources Research, v. 31, no. 9, p. 2295-2305.

Krohelski, J.T. and Batten, W.G., 1995, Simulation of stage and the hydrologic budget of Devils Lake, Sauk County, Wisconsin: U.S. Geological Survey Open-File Report 94-348, 22 p.

Wentz, D.A., Rose, W.J., and Webster, K.E., 1995, Long-term hydrologic and biogeochemical responses of a soft water seepage lake in north central Wisconsin: Water Resources Research, v. 31, no. 1, p 199-212. 
Elder, J.F., 1994, Distribution and grain-size partitioning of metals in bottom sediments of an experimentally acidified lake: Water Resources Bulletin, v. 30, no. 2, p. 251-259.

Goddard, G.L., and Field, S.J., 1994, Hydrology and water quality of Whitewater and Rice Lakes in southeastern Wisconsin, 1990-91: U.S. Geological Survey Water-Resources Investigations Report 94-4101, 36 p.

Greb, S.R., and Wentz, D.A., 1994, Chemical budgets, in Klepinger, K.E., ed., RILWAS 1983-86: Wisconsin Regional Integrated Lake Watershed Acidification Study, Volume 1: Madison, Wisconsin Department of Natural Resources, PUBL-RS-909-94, Chapter 7, 20 p.

Hurley, J.P., Krabbenhoft, D.P., Babiarz, C.L., and Andren, A.W., 1994, Cycling processes of mercury across sediment/water interfaces in seepage lakes, in Baker, L.A. ed., Environmental Chemistry of Lakes and Reservoirs: Advances in Chemistry Series, American Chemical Society, Washington, D.C., p. 426-449.

Krabbenhoft, D.P., Bowser, C.J., Kendall, C., and Gat, J.R., 1994, Use of oxygen-19 and deuterium to assess the hydrology of ground-water/lake systems, in Baker, L.A. ed., Environmental Chemistry of Lakes and Reservoirs: Advances in Chemistry Series, American Chemical Society, Washington, D.C., p. 67-90.

Robertson, D.M., Anderson, W., and Magnuson, J.J., 1994, Relations between El Nino/Southern Oscillation events and the climate and ice cover of lakes in Wisconsin. P 48-57, in Greenland, D. ed., EI Nino and Long-Term Ecological Research (LTER) Sites: Publication No. 18. LTER Network Office: University of Washington, Seattle, WA, USA., 57 p.

Robertson, D.M. and Imberger, J. 1994, Lake Number, a quantitative indicator of mixing used to estimate changes in dissolved oxygen, Internationale Revue der gesamten Hydrobiologie, v. 79 , p. $159-176$.

Watras, C.J., Bloom, N.S., Hurley, J.P., Fitzgerald, W.F., Andren, A.W., Krabbenhoft, D.P., and Porcella, D.B., 1994, Sources and fates of mercury and methylmercury in Wisconsin lakes, in Watras and Huckabee eds., Mercury as a Global Pollutant: Intergration and Synthesis, Lewis Pub., Chelsea, Ml., p 153-177.

Wentz, D.A., 1994, Chemistry of snowpack and ground water, in Klepinger, K.E., ed., RILWAS 1983-86: Wisconsin Regional Integrated Lake Watershed Acidification Study, Volume 1: Madison, Wisconsin Department of Natural Resources, PUBL-RS-909-94, Chapter 6, 45 p.

Wentz, D.A., Krohelski, J.T., and Rose, W.J., 1994, Hydrology, in Klepinger, K.E., ed., RILWAS 1983-86: Wisconsin Regional Integrated Lake Watershed Acidification Study, Volume 1: Madison, Wisconsin Department of Natural Resources, PUBL-RS-909-94, Chapter 7, 74 p.

1993

Field, S.J., 1993, Hydrology and water quality of Powers Lake, southeastern Wisconsin: U.S. Geological Survey Water-Resources Investigations Report 90-4126, 36 p.

Field, S.J., 1993, Hydrology and water quality of Wind Lake in southeastern Wisconsin: U.S. Geological Survey Water-Resources Investigations Report 91-4107, 61 p.

House, L.B., 1993, Simulation of the effects of hypothetical residential development on water levels in Graber Pond, Middleton, Wisconsin: U.S. Geological Survey Water-Resources Investigations Report 92-4029, 10 p. 
House, L.B., Waschbusch, R.J., and Hughes, P.E., 1993, Water quality of an urban wet detention pond in Madison, Wisconsin, 1987-88: U.S. Geological Survey Open-File Report 93-172, $57 \mathrm{p}$.

Hughes, P.E., 1993, Hydrology, water quality, trophic status, and aquatic plants of Fowler Lake, Wisconsin: U.S. Geological Survey Water-Resources Investigations Report 91-4076, 44 p.

Rose, W.J., 1993, Hydrology of Little Rock Lake in Vilas County, north-central Wisconsin: U.S. Geological Survey Water-Resources Investigations Report 93-4139, 22 p.

Rose, W.J., 1993, Water and phosphorus budgets and trophic state, Balsam Lake, northwestern Wisconsin, 1987-1989: U.S. Geological Survey Water-Resources Investigations Report $91-4125,28 \mathrm{p}$.

1992

Elder, J.F., Krabbenhoft, D.P, and Walker, J.F., 1992, Water, energy, and biogeochemical budgets (WEBB) program: Data availability and research at the northern temperate lakes site, Wisconsin: U.S. Geological Survey Open-File Report 92-48, 15 p.

Krabbenhoft, D.P., and Babiarz, C.L., 1992, Role of groundwater transport in aquatic mercury cycling: Water Resources Research, vol. 28, no. 12, p. 3119-3128.

Krabbenhoft, D.P., and Krohelski, J.T., 1992, Data on water quality, lake sediment, and lake-level fluctuation, St. Croix Indian Reservation, Wisconsin, 1981-87: U.S. Geological Survey OpenFile Report 92-26, $53 \mathrm{p}$.

Robertson, D.M., Ragotzkie, R.A., and Magnuson, J.J. 1992, Lake ice records used to detect historical and future climatic changes: Climatic Change, v. 21, p. 407-427.

1991

Wentz, D.A., and Rose, W.J., 1991, Hydrology of Lakes Clara and Vandercook in North-Central Wisconsin: U.S. Geological Survey Water-Resources Investigations Report 89-4204, 24 p.

Watras, C.J., Andre, A.W., Bloom, N.S., Fitzgerald, W.F., Hurley, J.P., Krabbenhoft, D.P., Rada, R.G., Wiener, J.G., 1991, Mercury in temperate lakes: a mechanistic field study: Verhandlungen Internat. Verein. Limnologie, 24, p. 2199-2202.

\section{Pre-1990}

Walker, J.F., Pickard, S.A., and Sonzogni, W.C., 1989 Spreadsheet watershed modeling for nonpoint-source pollution management in a Wisconsin basin: Water Resources Bulletin, v. 25, no. 1, p. 139-147.

Wentz, D.A., Garrison, P.J., and Bockheim, J.G., 1989, Section 7-Chemical input-output budgets, in Knauer, D., and Brouwer, S.A., eds., The Wisconsin Regional Integrated Lake-Watershed Acidification Study (RILWAS): 1981-1983: Palo Alto, California, Electric Power Research Institute Report EA-6214, p. 7-1 to 7-30.

Wentz, D.A., and Rose, W.J., 1989, Interrelationships among hydrologic-budget components of a northern Wisconsin seepage lake and implications for acid-deposition modeling: Archives of Environmental Contamination and Toxicology, v. 18, p. 147-155.

Wentz, D.A., Rose, W.J., and Krohelski, J.T., 1989, Section 5-Hydrologic component, in Knauer, D., and Brouwer, S.A., eds., The Wisconsin Regional Integrated Lake-Watershed Acidification Study (RILWAS): 1981-1983: Palo Alto, California, Electric Power Research Institute Report EA-6214, p. 5-1 to 5-77. 
Field, S.J., and Duerk, M.D., 1988, Hydrology and water quality of Delavan Lake in southeastern Wisconsin: U.S. Geological Survey Water-Resources Investigations Report 87-4168, 61 p.

Krug, W.R., Ostenso, N.A., and Krohelski, J.T., 1988, Prediction of the effects of mine dewatering on four lakes near Crandon, Wisconsin, by use of a water-budget model: U.S. Geological Survey Open-File Report 87-471, $63 \mathrm{p}$.

Wentz, D.A., Krohelski, J.T., Rose, W.J., Bockheim, J.G., Garrison, P.J., Knauer, D.R., and Goldstein, R.A., 1987, Hydrologic and chemical budgets of Wisconsin lakes, in Perrry, R., Harrison, R.M., Bel, J.N.B., and Lester, J.N., eds., Acid Rain: Scientific and Technical Advances, Selper Ltd., London, p. 309-316.

House, L.B., 1986, Stage fluctuations of Wisconsin Lakes: Wisconsin Geological and Natural History Survey Information Circular No. 49, 84 p.

House, L.B., 1984, Effects of urbanization on three ponds in Middleton, Wisconsin: U.S. Geological Survey Water-Resources Investigations Report 84-4051, $17 \mathrm{p}$.

Krug, W.R., and House, L.B., 1984, Evaluation of alternative reservoir-management practices in the Rock River basin, Wisconsin: U.S. Geological Survey Water-Resources Investigations Report 83-4186, $21 \mathrm{p}$.

House, L.B., 1981, An assessment of streamflow, water quality, and the effects of construction on impoundment on Bridge Creek at Augusta, Wisconsin: U.S. Geological Survey WaterResources Investigations Open-File Report 81-1192, 25 p.

Krug, W.R., 1981, Hydrologic effects of proposed changes in management practices, Winnebago Pool, Wisconsin: U.S. Geological Survey Water-Resources Investigations 80-107, 19 p.

Batten, W.G., and Hindall, S.M., 1980, Sediment deposition in the White River Reservoir, northwestern Wisconsin: U.S. Geological Survey Water-Supply Paper 2069, 30 p.

Novitzki, R.P., and Holmstrom, B.K., 1979, Monthly and annual water budgets of Lake Wingra, Madison, Wisconsin, 1971-77: U.S. Geological Survey Water-Resources Investigations 79-100, $31 \mathrm{p}$.

Rose, W.J., 1977, Hydrologic considerations associated with dredging spring ponds in Wisconsin: U.S. Geological Survey Water-Resources Investigations 77-18, 35 p.

Oakes, E.L., Hendrickson, G.E., and Zuehls, E.E., 1975, Hydrology of the Lake Wingra basin, Dane County, Wisconsin: U.S. Geological Survey Water-Resources Investigations 17-75, $31 \mathrm{p}$.

Novitzki, R.P., 1971, Hydrologic investigations of Heart Lake, Green Lake County, Wisconsin: U.S. Geological Survey Administrative Report, 9 p. 